\title{
Nov'1 91960
}

SCR-667

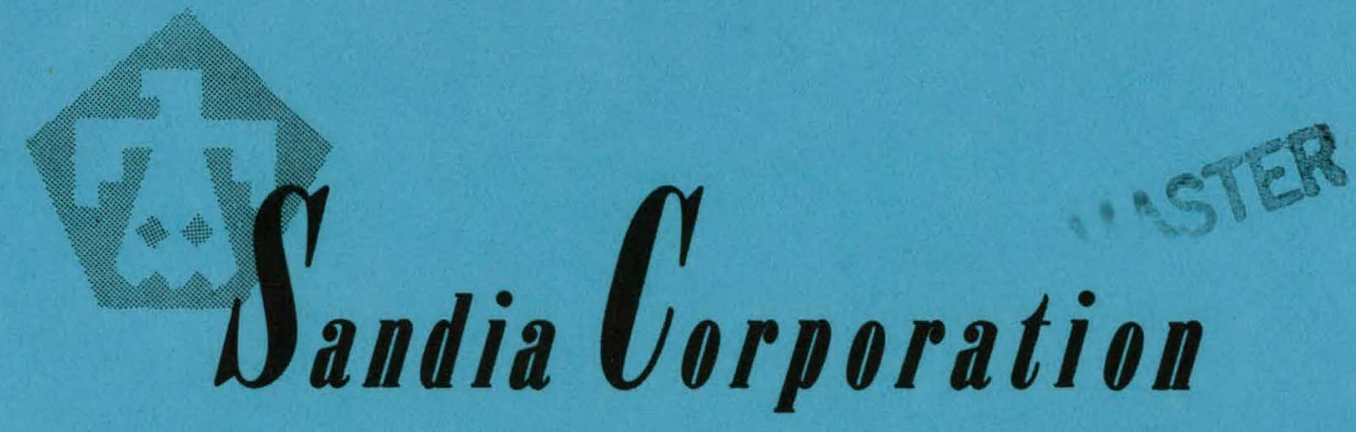

MONOGRAPH

\author{
TABLE OF THE INTEGRALS. \\ $C(h, 0), S(h, 0), E(h, 0), C(h, h), S(h, h)$, AND $E(h, h)$ \\ FOR COMPLEX ARGUMENTS \\ by
}

C W Harrison, Jr.

and

R W P King

OCTOBER 1963 


\section{DISCLAIMER}

This report was prepared as an account of work sponsored by an agency of the United States Government. Neither the United States Government nor any agency Thereof, nor any of their employees, makes any warranty, express or implied, or assumes any legal liability or responsibility for the accuracy, completeness, or usefulness of any information, apparatus, product, or process disclosed, or represents that its use would not infringe privately owned rights. Reference herein to any specific commercial product, process, or service by trade name, trademark, manufacturer, or otherwise does not necessarily constitute or imply its endorsement, recommendation, or favoring by the United States Government or any agency thereof. The views and opinions of authors expressed herein do not necessarily state or reflect those of the United States Government or any agency thereof. 


\section{DISCLAIMER}

Portions of this document may be illegible in electronic image products. Images are produced from the best available original document. 


\section{Published by Sandia Corporation, a prime contractor to the United States Atomic Energy Commission}

\section{LEGAL NOTICE}

This report was prepared as an account of Government sponsored work. Neither the United States, nor the Commission, nor any person acting on behalf of the Commission:

A. Makes any warranty or reprcsentation, expressed or implied, with respect to the accuracy, completeness, or usefulness of the information contained in this report, or that the use of any information, apparatus, method, or process disclosed in this report may not infringe privately owned rights; or

B. Assumes any liablitities with respect to the use of, or for damages resulting from the use of any information, apparatus, method, or process disclosed in this report.

As used in the above, "person acting on behalf of the Commission" includes any employee or contractor of the Commission, or employee of such contractor, to the extent that such employee or contractor of the Commission or employee of such contractor prepares, disseminates, or provides access to, any information pursuant to his employment or contract with the Commission, or his employment pursuant to his employment or contract with the Commission, or his employment with such contractor. 


\section{SANDIA CORPORA TION MONOGRAPH}

TABLE OF THE INTEGRALS $C(h, 0), S(h, 0), E(h, 0)$, $C(h, h), S(h, h)$, AND E $(h, h)$ FOR COMPLEX ARGUMENTS

by

C. W. Harrison, Jr.

Member of the Technical Staff

Sandia Corporation, Albuquerque, New Mexico.

$$
\text { and }
$$

R. W. P. King

Gordon McKay Professor of Applied Physics Harvard University, Cambridge 38, Massachusetts 
The numprical study of the circuit and field characteristics of isulated dipoles or antcnna arrays consisting of parallél nonstagyered lduliators of the oame longth In llssipalios media roquires the evaluation of the integrals $C(h, 0), S(h, 0), E(h, 0), C(h, h), S(h, h)$, and $E(h, h)$ for complex arguments. In this report an extensive tabulation of these integrals is presented. 
TABLE OF THE INTEGRALS $C(h, 0)$, S(h, 0), $E(h, 0)$,

$C(h, h), S(h, h)$, AND $E(h, h)$ FOR COMPLEX ARGUNENTS

\section{Introduction}

Six integrals that have a wide application in the theory of linear antennas in dissipative media* are $C(h, 0), S(h, 0), E(h, 0), C(h, h), S(h, h)$, and $E(h, h)$. where, in general,

$C(h, z)=\int_{0}^{h} \cos k z^{*}\left[\frac{e^{-j k R_{1}}}{R_{1}}+\frac{e^{-j k R_{2}}}{R_{2}}\right] d z^{\prime}$,

$S(h, z)=\int_{0}^{h} \sin k z^{\prime}\left[\frac{e^{-j k R_{1}}}{R_{1}}+\frac{e^{-j k R_{\lambda}}}{R_{2}}\right] d z^{\prime}$

$E(h, z)=\int_{0}^{h}\left[\frac{e^{-j k R_{2}}}{R_{1}}+\frac{c^{-j k R_{2}}}{R_{2}}\right] d z^{\prime}$

with

$$
\begin{aligned}
& R_{1}=\sqrt{\left(z-z^{\prime}\right)^{2}+a^{2}} \\
& R_{2}=\sqrt{\left(z+z^{\prime}\right)^{2}+a^{2}} .
\end{aligned}
$$

In such applications, $h$ is the half-length and $a$ is the radius of each antenna. Alternatively, a may be the distance between centers of identical parallel antennas in an array. The complex wave number $\mathrm{k}$ is

* These functions occur in the quasi-zero-order currents, impedances, and fields of dipoles and arrays of dipoles. Details for dissipationless media are in R. W. P. King's, "Linear Arrays: Currents, Impedances, and Fields, "IRE Transactions on Antennas and Propagation, AP-7, pp S440-S457, December 1959. References to application in dissipative media are in the supplementary list. 


$$
\mathrm{k}=\beta-\mathrm{j} \alpha=\omega \sqrt{\mu \epsilon} \sqrt{1-j \mathrm{p}}=\omega \sqrt{\mu \epsilon}\{\mathrm{f}(\mathrm{p})-\mathrm{jg}(\mathrm{p})\},
$$

$\omega=2 \pi f$ is the radian frequency; $\mu, \sigma$, and $\epsilon$ are the absolute permeability, conductivity, and permittivity of the medium in which the antenna is immersed. The loss tangent of the medium is $\mathrm{p}=\sigma / \omega \epsilon$. The functions $f(p)$ and $g(p)$, which are the real and imaginary parts of $\sqrt{1-j p}$, are defined as follows:

$$
\begin{aligned}
& f(p)=\cosh \left(\frac{1}{2} \sinh ^{-1} p\right) . \\
& g(p)=\sinh \left(\frac{1}{2} \sinh ^{-1} p\right) .
\end{aligned}
$$

Tables of these functions are available in the literature. ${ }^{1}, 2$

All tables were calculated on a CDC-1604 computer at the Sandia Laboratory, Albuquerque, New Mexico. Mr. E. A. Aronson programmed the machine. A table of the integrals $C(h, 0)$, $S(h, 0), E(h, 0), C(h, h), S(h, h)$, and $E(h, h)$ for red arguments $(\alpha=\dot{U})$ hids beril pul,ishicd: 3

\footnotetext{
1 D. W. Gooch, C. W. Harrison, Jr., R. W. P. King, and T. T. Wu, "Impedances and Admittances of Long Antennas in Air and in Dissipative Media with Tables of the Functions $f(p) \pm i g(p)=\sqrt{1 \pm i p}$, "Technical Report No. 353, Cruft Laboratory, Harvard University, January 15, 1962.

2 R. W. P. King, "Fundamental Electromagnetic Theory, "Appendix II, Dover Publications, Inc., 1963.

3 C. W. Harrison, Jr., "Tables of the Integrals $\mathrm{C}(\mathrm{H}, 0), \mathrm{C}(\mathrm{H}, \mathrm{H}), \mathrm{S}(\mathrm{H}, 0), \mathrm{S}(\mathrm{H}, \mathrm{H}), \mathrm{E}(\mathrm{H}, \mathrm{0})$, and $\mathrm{E}(\mathrm{H}, \mathrm{H})$ Commonly Occurring in Antenna Theory, "SCR-580, Sandia Corporation, Albuquerque, New Mexico, November 1962.
} 
NOTES ON THE NUMERICAL EVALUATION OF THE INTEGRALS

(Contributed by E. A. Aronson)

The direct numerical evaluation of the $C(h, z), S(h, z)$, and $E(h, z)$ integrals by use of a digital computer becomes extremely time consuming if the quantity a is small. Considerable machine time can be saved if the functions are integrated once by parts applying the formula

$$
\int_{l_{1}}^{l_{2}} \mathrm{udv}=\left.\mathrm{uv}\right|_{l_{1}} ^{l_{2}}-\int_{l_{1}}^{l_{2} \mathrm{vdu}}
$$

Let $\frac{d z^{\prime}}{R}=d v$, and the rest of the integral be $u$, where $R=\sqrt{\left(z^{\prime} \pm z\right)^{2}+a^{2}}$.

Then,

$$
v=\ln \left(z^{\prime} \pm z+R\right) .
$$

For convenicnce, define

$$
\begin{aligned}
& G_{1}=\ln \left(z^{\prime}-z+R_{1}\right) \exp \left(-j k R_{1}\right) \\
& G_{2}=\ln \left(z^{\prime}+z+R_{2}\right) \exp \left(-j k R_{2}\right) .
\end{aligned}
$$

Integration by parts yields

$C(h, z)=\left.\left(G_{1}+G_{2}\right) \cos k z^{-}\right|_{0} ^{h}+k \int_{0}^{h} G_{1}\left[\frac{j\left(z^{\prime}-z\right)}{R_{1}} \cos k z^{\prime}+\sin k z^{\prime}\right]$

$$
+G_{2}\left[\frac{j\left(z^{\prime}+z\right)}{R_{2}} \cos k z^{\prime}+\sin k z^{\prime}\right] \mid d z^{\prime}
$$

$S(h, z)=\left.\left(G_{1}+G_{2}\right) \sin k z^{\prime}\right|_{0} ^{h}+k \int_{0}^{h}\left\{G_{1}\left[\frac{j\left(z^{\prime}-z\right)}{R_{1}} \sin k z^{\prime}-\cos k z^{\prime}\right]\right.$

$$
\left.+G_{2}\left[\frac{j\left(z^{\prime}+z\right)}{R_{2}} \sin k z^{\prime}-\cos k z^{-}\right]\right\} d z^{\prime}
$$


$E(h, z)=\left.\left(G_{1}+G_{2}\right)\right|_{0} ^{h}+j k \int_{0}^{h}\left\{G_{1} \frac{\left(z^{\prime}-z\right)}{R_{1}}+G_{2} \frac{\left(z^{\prime}+z\right)}{R_{2}}\right\} d z^{\prime}$.

These expressions are evaluated in lieu of the original integrals.

Serious round-off errors occur in the evaluation of $\ln \left(z^{\prime}-z+R_{1}\right)$ when $\left(z^{\prime}-z\right)$ is negative. This condition can be alleviated by using $\ln \left(z^{\prime},-z+R_{1}\right)=\ln \left[a^{2} /\left(z-z^{\prime}+R_{1}\right)\right]$. 'This relation is not to be used when $\left(z^{\prime}-z\right)$ is positive.

Additional time is saved if each integral is broken up into five (or fewer) pieces about $z$, i. e.,

$$
\int_{0}^{h} \equiv \int_{0}^{z-d_{2}}+\int_{z-d_{2}}^{z-d_{2}}+\int_{z-d_{z}}^{z+d / z}+\int_{z+d_{2}}^{z+d} 1 \int_{z+d_{1}}^{h}
$$

$d_{1}, d_{2} \geq 0$.

For the appended computations, $d_{1}=\frac{0.005}{\pi}$, and $d_{2}=\frac{0.0005}{\pi}$. These limits of integration were adjusted such that any limit greater than $\mathrm{h}$ is set to $\mathrm{h}$ and ary limit less than zcro is set to zero.

For the appended computations, with $a / \lambda=0.001191$ and $\alpha / \beta=0$, a time saving of approximately 30:1 was achieved using integration by parts.

All integrals were evaluated using Romberg integration to an accuracy of $\pm 0,000005$.

\section{SUPPLEMENTARY REFERENCES}

R. W. P. King and C. W. Harrison, Jr., "Half-Wave Cylindrical Antenna in a Dissipative Medium: Current and Impedance," Journal of Research NBS-D Radio Propágation, 64 D . No. 4,pp 365-380, July-August 1960.

R. B. Mack and E. W. Mack, "Tables of E(h, z), C(h, z), S(h, z), "Technical Report No. 331 , Cruft Laboratory, Harvard University, Cambridge, "Massachusetts, November 1, 1960.

R. W. P. King, C. W. Harrison, Jr., and D. H. Denton, Jr., "The Electrically Short Antenna as a Probe for Measuring Free Electron Densities and Collision Frequencies in an Ionized Region," Journal of Research NBS-D Radio Propagation, 650, No. 4, pp 371-384, July-August 1960.

R. W. P. King, "Dipoles in Dissipative Media," R. E. Langer, Editor, Electromagnetic Waves, University of Wisconsin Press, pp 199-241, 1962.

D. W. Gooch, C. W. Harrison, Jr., R. W. P. King, and T. T. Wu, "Impedances of Long Antennas in Air and in Dissipative Media," Journal of Research NBS-D, Radio Propagation, 67 D, No. 3, pp 355-360, May-June 1963.

R. W. P. King and C. W. Harrison, Jr., "Electromagnetic Radiation and Antennas," Chs. 3 and 5 (to be published). 
TABLE I

TABLE OF THE INTEGRALS

$C(h, 0), S(h, 0) E(h, 0), C(h, h), S(h, h)$ AND $E(h, h)$ FOR COMPLEX ARGUMENTS

$$
\frac{\mathrm{a}}{\lambda}=0.001191 \quad \frac{\alpha}{\beta}=0.00
$$

Bh

$\mathrm{C}(\mathrm{h}, 0)$

0.01

0.02
0.05

0.05
0.10

0.20

0.50

1.00
1.50

1.50
2.00

2.50
3.00

3.00
3.50

$0.125 \pi$

$0.250 \pi$

$0.375 \pi$

0.625

$0.750 \pi$

$0.875 \pi$

$1.000 \pi$

$1.125 \pi$
$1.250 \pi$

\begin{abstract}
$2.20067-0.02000$
\end{abstract} $3.41858-0.04000$ $5.19358-0.09994$ $6.56408-0.19955$ $7.91853-0.39646$ $9.55044-0.94607$ $10.32909-1.60540$ $10.43120-1.84863$ $10.45827-1.75819$ $10.63237-1.54992$ $10.93666-1.42468$ $11.23556-1.45459$

$9.15689-0.75897$ $10.13656-1.37075$ $10.40060-1.73973$ $10.43135-1.85192$ $10.45239-1.77167$ $10.56474-1.60836$ $10.77497 .-1.46900$ $11.02827-1.41814$ $11.25244-1.46124$ $11.39775-1.55582$

0.01

0.02

0.05

0.20

0.50

1.00

1.50

2.00
2.50

3.00
3.50

$2.20057 \quad-0.02000$ $3.41838-0.03999$ $5.19308-0.09992$ $6.56308-0.19945$ $7.91658-0.39606$ $9.54624-0.94378$ $10.32455-1.59834$ $10.43047-1.83872$ $10.46199-1.74994$ $10.63709-1.54631$ $10.93804-1.42440$ $11.23233-1.45330$

$\begin{array}{lll}0.125 \pi & 9.15336 & -0.75751\end{array}$ $\begin{array}{lll}0.250 \pi & 10.13157 & -1.36576 \\ 0.375 \pi & 10.39707 & -1.73123\end{array}$ $0.500 \pi 10.43133-1.84196$ $0.025 \pi \quad 10.45587-1.76316$ $0.750 \pi \quad 10.56967-1.00335$ $0.875 \pi \quad 10.77845-1.46748$ $1.000 \pi 11.02827-1.41806$ $1.125 \pi \quad 11.02827-1.41806$ $1.125 \pi \quad 11.24896-1.45972$
$S(h, 0)$

$0.01001-0.00010$

$0.02774-0.00040$

$0.08609-0.00250$

$0.18515-0.0099$

$0.38177-0.03973$

$0.93122-0.23981$

$1.59049-0.84737$

$1.83371-1.55618$

$1.53497-2.37660$

$1.40973-2.43700$

$1.43964-2.44640$

$\begin{array}{ll}0.74414 & -0.15030 \\ 1.35585 & -0.55679\end{array}$

$1.72481-1.10366$

$1.83699-1.64826$

$1.75674-2.0735$

$1.59342-2.32578$

$1.45405 \quad-2.42387$

$1.40319-2.43763$

$1.44629-2.44903$

$1.54087-2.51444$
$E(h, 0)$

$2.20071-0.02000$

$3.41874-0.04000$

$5.19477-0.09999$

$6.56898-0.1998$

$7.93820-0.39911$

$9.06643-0.9862$.

$10.69670-1.89215$

$10.96258-2.34934$

$10.86782-3.21079$

$10.26112-3.59720$
$9.95760-3.56621$

$9.23047-0.77869$

$10.39261-1.51794$

$10.84907-2.18188$

$10.96587-2.7415 \mathrm{C}$

$10.88254-3.117702$

$10.68305-3.47946$

$10.42922-3.05093$

$\begin{array}{rr}10.16920 & -3.70383 \\ 9.93946 & -3.05905\end{array}$

$9.76498-3.54334$
$\mathrm{C}(\mathrm{h}, \mathrm{h})$

$1.70934-0.02000$ $2.37737-0.03999$ $3.28256-0.09990$ $3.95854-0.19922$ $4.57776-0.39382$ $4.91357-0.90756$ $3.55876-1.36040$ $1.08991-1.26584$ $-1.66622-0.82462$ $-5.66062-0.21105$

$-5.85226 \quad 1.28059$

$4.94191-0.73974$ $4.33751-1.23750$ $2.75754-1.38212$ $0.70160-1.21881$
$-1.46907-0.86382$ $-3.46734-0.8638$ $\begin{array}{ll}-5.46734 & -0.40134\end{array}$ $-5.86893-0.74608$ $-5.808950 .74608$ $\begin{array}{ll}-5.80855 & 1.32722 \\ -4.80482 & 1.75410\end{array}$

$$
\frac{a}{\lambda}=0.001191 \quad \frac{\alpha}{\beta}=0.005
$$

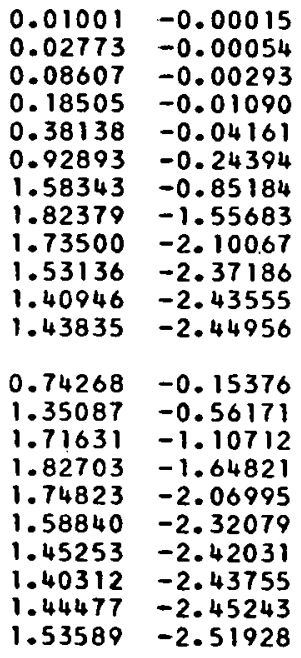

$2.20061-0.62000$

$3.41854-0.62000$

$5.19427-0.09997$

$\begin{array}{ll}3.19427 & -0.09997 \\ 0.56799 & -0.19984 \\ 7.93621 & -0.39891\end{array}$

$\begin{array}{ll}0.56799 & -0.19984 \\ 7.93621 & -0.39891\end{array}$

$0.93621-0.3989$

$10.66830-0.98498$

$10.95262-2.687507$

$10.85874-3.19667$

$10.58761-3.53905$

$10.25967-3.53905$

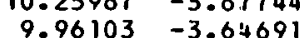

$9.22664-0.77793$

$10.38555-1.51501$

$\begin{array}{ll}10.38555 & -1.51501 \\ 10.83985 & -2.17572\end{array}$

$10.95589-2.73152$

$10.87331-3.10323$

$10.67598-3.40245$

$10.42537-3.63176$

$10.16915-3.68391$

$0.04321-3.6598$

$9.77194-3.52632$

$\begin{array}{rr}1.70924 & -0.02000 \\ 2.37717 & -0.03999 \\ 3.28207 & -0.09986 \\ 3.95755 & -0.19902 \\ 4.57586 & -0.39289 \\ 4.90993 & -0.90109 \\ 3.55719 & -1.33693 \\ 1.09454 & -1.22460 \\ -1.65547 & -0.77292 \\ -4.08613 & -0.16280 \\ -5.63793 & 0.54856 \\ -5.82909 & 1.25560 \\ 4.93866 & -0.73580 \\ 4.33429 & -1.22202 \\ 2.75796 & -1.35191 \\ 0.70715 & -1.17550 \\ -1.45874 & -0.81250 \\ -3.45243 & -0.35021 \\ -5.00286 & 0.18180 \\ -5.84519 & 0.75769 \\ -5.78574 & 1.29833 \\ -4.79045 & 1.67902\end{array}$

(4.

$\begin{array}{lll}0.00877 & -0.00014\end{array}$

$0.02628-0.00053$

$0.10199-0.00301$

$0.26566-0.01129$

$0.65699-0.04269$

$\begin{array}{ll}1.98128 & -0.23882\end{array}$

$3.89819-0.73272$

$4.86687-1.11075$

$4.72100-1.21298$

$1.38803-0.89262$

$\begin{array}{rr}-1.20644 & -0.49019\end{array}$

$1.50208-0.15344$

$3.16633-0.51492$

$4.36848-0.89574$

$4.91397-1.14180$

$4.76889-1.21372$

$3.95863-1.15888$

$2.54550-1.02328$

$0.67523-0.79949$

$\begin{array}{rr}-1.38524 & -0.45501 \\ -3.28494 & 0.00260\end{array}$
$E(h, h)$

$1.70937-0.02000$ $2.37755-0.04000$ 3. $4.63222-0.19955$ $5.34835-0.94607$ $5.43391-1.60540$ $5.13056-1.84863$ $4.86905-1.75818$ $4.82090-1.54991$ $4.94288-1.54467$ $\begin{array}{ll}4.94288 & -1.42467 \\ 5.08763 & -1.45457\end{array}$

$5.19631-0.75897$ $5.48294-1.37075$ $5.34153-1.73973$ $5.08460-1.85192$ $4.88249-1.77167$ $4.81252-1.60835$ $4.88860-1.4689$ $5.08475-1.41813$ $\begin{array}{ll}5.09475 & -1.46123 \\ 5.13470 & -1.55581\end{array}$

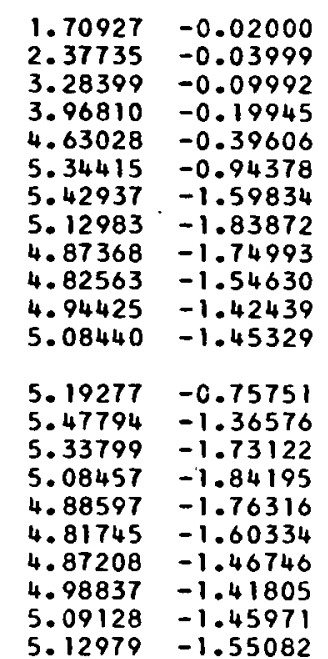




$$
\mathrm{C}(\mathrm{h}, 0)
$$

0.0

0.02

0.10

0.10

0.20

1.00

1.50

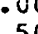

2.50

3.50

$0.125 \pi$

$0.375 \pi 10.39353-1.72278$

$10.43125-1.83208$

$0.625 \div \quad 10.45924-1.7547$

1.000 n $10.78183-1.4658$

$\begin{array}{lll}1.125 \pi & 11.24559 & -1.45810 \\ 1.250 \pi & 11.38808 & -1.54587\end{array}$
$0.250 \% \quad 10.12659-1.36080$

$10.57483-1.59831$

$1.125 \pi-11.24559-1.45810$
$\mathrm{S}(\mathrm{h}, 0)$

$0.01001-0.00020$ $0.02773-0.00068$ $0.08604-0.00336$ $0.18495-0.01182$ $0.38098-0.04348$ $0.92664-0.24806$ $1.81390-1.55752$ $1.72680-2.09698$ $\begin{array}{ll}.40904 & -2.43415 \\ 1.43695 & -2.45261\end{array}$

$0.74122-0.15721$ $1.34590-0.56661$ $1.81715-1.6482$ $1.53337-2.06651$ . 45090 -2.4168 $1.45090-2.41686$ $1.44315-2.45572$ $\begin{array}{ll}1.44315 & -2.45572 \\ 1.53092 & -2.52395\end{array}$ $1.57641-0.85630$ $1.72770-2.09698$ $1.52770-2.36719$

$\ddot{\lambda}=0.001191 \quad \alpha=0.01$

$\mathrm{E}(\mathrm{h}, 0)$

$C(t, h)$

\begin{tabular}{|c|c|}
\hline $\begin{array}{r}2.20051 \\
3.41834 \\
5.19377 \\
6.56699 \\
7.93422 \\
9.65685 \\
10.67991 \\
10.94269 \\
10.84968 \\
10.58160 \\
10.25814 \\
9.96431\end{array}$ & $\begin{array}{l}-0.02000 \\
-0.03999 \\
-0.09996 \\
-0.19979 \\
-0.39871 \\
-0.98376 \\
-1.88298 \\
-2.63084 \\
-3.18264 \\
-3.52123 \\
-3.65777 \\
-3.52776\end{array}$ \\
\hline $\begin{array}{r}9.22282 \\
10.37850 \\
10.83065 \\
10.94593 \\
10.86411 \\
10.66897 \\
10.42148 \\
10.16900 \\
9.94679 \\
9.77868\end{array}$ & $\begin{array}{l}-0.77717 \\
-1.51209 \\
-2.16958 \\
-2.72159 \\
-3.14953 \\
-3.44555 \\
-3.61274 \\
-3.66414 \\
-3.62086 \\
-3.50940\end{array}$ \\
\hline
\end{tabular}
$\begin{array}{ll}1.70914 & -0.020 .00 \\ 2.37697 & -0.03898\end{array}$ $3.28157-0.09981$ $3.95657-0.19831$ $4.57396-0.39195$ $4.90631-0.89462$ $3.55566-1.31351$ $1.09915-1.18344$ $-1.64493-0.72128$ $-4.07003-0.11463$ $-5.61649 \quad 0.57173$

$-5.80774 \quad 1.23089$

$4.93543-0.73186$ $4.33111-1.20658$ $2.75843^{\circ}-1.32176$ $0.71267-1.13227$ $-1.44860-0.76124$ $-3.43798-0.29914$ $\begin{array}{rr}-4.98387 & 0.22058\end{array}$ $-5.82290 \quad 0.76934$ -5.76478
-4.77790 $\frac{a}{\lambda}=0.001191 \quad \frac{\alpha}{\beta}=0.02$

\begin{tabular}{|c|c|c|}
\hline $\begin{array}{l}0.01 \\
0.02 \\
0.05 \\
0.10 \\
0.20 \\
0.50 \\
1.00 \\
1.50 \\
2.00 \\
2.50 \\
3.00 \\
3.50\end{array}$ & $\begin{array}{r}2.20027 \\
3.41778 \\
5.19158 \\
6.56011 \\
7.91075 \\
9.53369 \\
10.31099 \\
10.42807 \\
10.47249 \\
10.65047 \\
10.94190 \\
11.22324\end{array}$ & $\begin{array}{l}-0.01999 \\
-0.03998 \\
-0.09984 \\
-0.19915 \\
-0.39488 \\
-0.93694 \\
-1.57742 \\
-1.80945 \\
-1.72548 \\
-1.53514 \\
-1.42272 \\
-1.44878\end{array}$ \\
\hline $\begin{array}{l}0.125 \pi \\
0.250 \pi \\
0.375 \pi \\
0.500 \pi \\
0.625 \pi \\
0.750 \pi \\
0.875 \pi \\
1.000 \pi \\
1.125 \pi \\
1.250 \pi\end{array}$ & $\begin{array}{r}9.14280 \\
10.11667 \\
10.38645 \\
10.43096 \\
10.46565 \\
10.58363 \\
10.78829 \\
11.02826 \\
11.23918 \\
11.37904\end{array}$ & $\begin{array}{l}-0.75314 \\
-1.35095 \\
-1.70606 \\
-1.81254 \\
-1.73794 \\
-1.58817 \\
-1.46226 \\
-1.41694 \\
-1.45456 \\
-1.53601\end{array}$ \\
\hline
\end{tabular}

(1)

$\begin{aligned} 2.20031 & -0.02000 \\ 3.41794 & -0.03999 \\ 5.19277 & -0.09993 \\ 6.56499 & -0.19968 \\ 7.93026 & -0.39831 \\ 9.64730 & -0.98132 \\ 10.66320 & -1.87387 \\ 10.92291 & -2.61252 \\ 10.83161 & -3.15483 \\ 10.56955 & -3.48598 \\ 10.25486 & -3.61889 \\ 9.97040 & -3.58990 \\ & \\ 9.21519 & -0.77565 \\ 10.36443 & -1.50628 \\ 10.81231 & -2.15737 \\ 10.926110 & -2.701 .89 \\ 10.84576 & -3.12237 \\ 10.65476 & -3.41211 \\ 10.41357 & -3.57512 \\ 10.16841 & -3.62507 \\ 9.95349 & -3.58323 \\ 9.79151 & -3.47587\end{aligned}$

$1.70894-0.01999$ $2.37657-0.03997$ $3.28057-0.09972$ $3.95459-0.19840$ $4.57017-0.39009$ $4.89915-0.88174$ $3.55278-1.26684$ $1.10832-1.10133$ $-1.62447-0.61815$ $-4.03964-0.01848$ $\begin{array}{ll}-5.57734 & 0.61801 \\ -5.77047 & 1.18227\end{array}$

$4.92903-0.72401$ $4.32490-1.17581$ $2.75948-1.26167$ $0.72360-1.04601$ $\begin{array}{ll}-1.42888 & -0.65889\end{array}$ $-3.41047-0.19720$ $\begin{array}{lll}-4.94861 & 0.29796\end{array}$ $\begin{array}{lll}-5.78261 & 0.79276\end{array}$ $\begin{array}{ll}-5.72833 & 1.21344 \\ -4.75824 & 1.45759\end{array}$
$S(h, h)$

J.00877 -0.00019 $3.02628-0.00066$ $0.10196-0.00352$ $0.26556-0.01261$ $0.65660-0.04590$ $\begin{array}{lll}1.97919 & -0.24752\end{array}$ $3.89342-0.74360$ $4.86339-1.11200$ $4.72142-1.19455$ $3.50978-1.07200$ $1.39680-0.81828$ $-1.18946-0.40042$

$1.50070-0.16038$ $3.16239-0.52628$ $4.36365-0.90450$ $4.91098-1.14082$ $4.76902-1.19702$ $3.96159-1.12153$ $2.55145 \quad-0.96269$ $0.68603-0.7187$ $-1.36765-0.36507$ $\begin{array}{ll}-3.26142 & 0.08419\end{array}$
$\mathrm{E}(\mathrm{h}, \mathrm{h})$

$\begin{array}{lll}1.70917 & -0.02000\end{array}$ $2.37715-0.03999$ $3.28349-0.09989$ $3.96711-0.19935$ $4.62833-0.39567$ $5.33996-0.94149$ $5.42484-1.59132$ $5.12907-1.8288$ $4.87729-1.74173$ $4.83022-1.54263$ $\begin{array}{ll}4.94558 & -1.42397 \\ 5.08127 & -1.45189\end{array}$

$5.18925-0.75605$ $5.47296-1.36080$ $5.33445-1.72278$ $5.08450-1.83207$ $4.88934-1.75470$ $4.82224-1.59830$ $4.87546-1.46583$ $4.98837-1.41782$ $5.08791-1.45809$ $5.12504-1.54586$
$0.00877-0.00028$ $0.02627-0.00093$ $0.10191-0.00453$ $\begin{array}{lll}0.26536 & -0.01525\end{array}$ $0.65583-0.05232$ $1.97507-0.2649$ $3.88421-0.76541$ $4.85714-1.11470$ $4.12337-1.15790$ $3.51907-0.98063$ $1.41523-0.67003$

$1.49797-0.17422$ $1.15497-0.17422$ $3.15469-0.54901$ $4.35445-0.92212$ $4.90576-1.13907$ $4.77036-1.16384$ $3.96887-1.04699$ $2.56466-0.84179$ $0.70811-0.55767$ $-1.333 .98-0.18581$

$\begin{array}{ll}1.70897 & -0.01999 \\ 2.37675 & -0.03998 \\ 3.28250 & -0.09984 \\ 3.96513 & -0.19915 \\ 4.82445 & -0.39488 \\ 5.33160 & -0.93694 \\ 5.41581 & -1.57742 \\ 5.12743 & -1.80944 \\ 4.88417 & -1.72547 \\ 4.83901 & -1.53513 \\ 4.94812 & -1.42271 \\ 5.07531 & -1.44876 \\ & \\ 5.18222 & -0.75314 \\ 5.46305 & -1.35094 \\ 5.32738 & -1.70605 \\ 5.08421 & -1.81253 \\ 4.89576 & -1.73793 \\ 4.83141 & -1.58816 \\ 4.88192 & -1.46224 \\ 4.98836 & -1.41692 \\ 5.08150 & -1.45454 \\ 5.11600 & -1.53599\end{array}$


$0.125 \pi$ $\begin{array}{llll}0.250 & \pi & 10.10682 & -1.3411\end{array}$ $\begin{array}{llll}0.375 \pi & 10.37938 & -1.68957\end{array}$ $0.500 \pi-10.43048-1.79331$ $0.625 \pi-10.47165-1.72138$ $0.750 \pi \quad 10.59226-1.57796$ $0.875 \pi \quad 10.79435-1.45827$ $11.02822-1.41549$ $\begin{array}{lll}1.125 \pi & 11.23315 & -1.45064 \\ 1.250: \pi & 11.37058 & -1.52622\end{array}$

\begin{tabular}{|c|c|c|}
\hline $\begin{array}{l}0.01 \\
0.02 \\
0.05 \\
0.10 \\
0.20 \\
0.50 \\
1.00 \\
1.50 \\
2.00 \\
2.50 \\
3.00 \\
3.50\end{array}$ & $\begin{array}{r}2.19967 \\
3.41658 \\
5.18859 \\
6.55417 \\
7.89915 \\
9.50884 \\
10.28413 \\
10.42228 \\
10.49057 \\
10.67381 \\
10.94846 \\
11.20737\end{array}$ & $\begin{array}{l}-0.01999 \\
-0.03995 \\
-0.09969 \\
-0.19856 \\
-0.39253 \\
-0.92346 \\
-1.53672 \\
-1.75291 \\
-1.67780 \\
-1.51148 \\
-1.41588 \\
-1.43706\end{array}$ \\
\hline $\begin{array}{l}0.125 \pi \\
0.250 \pi \\
0.375 \pi \\
0.500 \pi \\
0.625 \pi \\
0.750 \pi \\
0.875 \pi \\
1.000 \pi \\
1.125 \pi \\
1.250 \pi\end{array}$ & $\begin{array}{r}9.12186 \\
10.08727 \\
10.36521 \\
10.42898 \\
10.48244 \\
10.00801 \\
10.80534 \\
11.02804 \\
11.22213 \\
11.35518\end{array}$ & $\begin{array}{l}-0.74451 \\
-1.32197 \\
-1.65726 \\
-1.75573 \\
-1.68887 \\
-1.55733 \\
-1.44919 \\
-1.41106 \\
-1.44174 \\
-1.50683\end{array}$ \\
\hline
\end{tabular}

$S(h, 0)$

$\begin{array}{ll}0.01001 & -0.00040 \\ 0.02771 & -0.00123 \\ 0.08594 & -0.00508 \\ 0.18455 & -0.01550 \\ 0.37941 & -0.05094 \\ 0.91756 & -0.26444 \\ 1.54878 & -0.87402 \\ 1.77538 & -1.56065 \\ 1.69447 & -2.08335 \\ 1.51249 & -2.34984 \\ 1.40598 & -2.42895 \\ 1.43030 & -2.46388 \\ 0.73543 & -0.17095 \\ 1.32630 & -0.58609 \\ 1.67465 & -1.12443 \\ 1.77838 & -1.64868 \\ 1.70645 & -2.05380 \\ 1.56302 & -2.29783 \\ 1.44333 & -2.40404 \\ 1.40054 & -2.43723 \\ 1.43569 & -2.46786 \\ 1.51127 & -2.54116\end{array}$

$\underset{\lambda}{\mathrm{a}}=0.0011 \mathrm{~g} 1 \quad \frac{\alpha}{\bar{\beta}}=0.03$
$E(h, 0)$

$\begin{array}{rr}2.20011 & -0.01999 \\ 3.41754 & -0.03993 \\ 5.19177 & -0.09991 \\ 6.56300 & -0.19958 \\ 7.92629 & -0.39791 \\ 9.63777 & -0.97889 \\ 10.64656 & -1.86483 \\ 10.90324 & -2.59437 \\ 10.81363 & -3.12736 \\ 10.55746 & -3.45123 \\ 10.25129 & -3.58060 \\ 9.97590 & -3.55260 \\ 9.20757 & -0.77414 \\ 10.35042 & -1.50045 \\ 10.79407 & -2.14526 \\ 10.90638 & -2.68238 \\ 10.82750 & -3.09554 \\ 10.64060 & -3.37913 \\ 10.40550 & -3.53807 \\ 10.16745 & -3.58660 \\ 9.95957 & -3.54616 \\ 9.80350 & -3.44275\end{array}$
$C(h, h)$

$1.70874 \quad-0.01999$ $2.37617-0.03996$ 3..27958 -0.09963 $3.95263-0.19799$ -0.38823
-.08 $4.89211-0.06800$ $3.55011-1.22039$ $1.0745-1.01949$ $-1.60483-0.51523$ -5.01168 0.07745 $-5.543120 .66428$ $4.92269-0.71618$ $4.31889-1.14518$ $2.76070-1.20184$ $0.73441-0.96003$ $-1.40990-0.55674$ $-3.38483-0.09546$ $-4.91694 \quad 0.37515$ $-5.748010 .81636$ $\begin{array}{ll}-5.69912 & 1.15826 \\ -4.74577 & 1.31282\end{array}$ - $5.56630-0.19799$
$S(h, h)$

$0.00876-0.00036$ $0.02626-0.00118$ $0.10186-0.00555$ $0.26516-0.01788$ $0.65506-0.05873$ $\begin{array}{lll}1.97102 & -0.28228\end{array}$ $3.87541-0.78726$ $4.85183-1.11765$ $4.72679-1.12152$ $3.53022-0.88946$ $1.43484-0.52223$ $-1.12559-0.04323$ $3.14722-0.57173$ $4.34585-0.93987$ $4.90156-1.13759$ $4.77315-1.13093$ $3.97794-0.97267$ $-1.30200-0.00718$ $\begin{array}{rr}-1.30200 & -0.00718 \\ -3.18094 & 0.41004\end{array}$
$1.49528-0.18805$ $2.57961-0.72118$

\section{$E(h, h)$}

$\begin{array}{ll}1.70877 & -0.01999 \\ 2.37635 & -0.03997 \\ 3.28150 & -0.09979 \\ 3.96315 & -0.19895 \\ 4.62057 & -0.39409 \\ 5.32328 & -0.93241 \\ 5.40681 & -1.56368 \\ 5.12564 & -1.79030 \\ 4.89062 & -1.70940 \\ 4.84728 & -1.52742 \\ 4.95048 & -1.42091 \\ 5.06970 & -1.44523 \\ & \\ 5.17521 & -0.75025 \\ 5.45319 & -1.34119 \\ 5.32030 & -1.08956 \\ 5.08373 & -1.79330 \\ 4.90175 & -1.72137 \\ 4.84004 & -1.57794 \\ 4.88798 & -1.45826 \\ 4.98832 & -1.41547 \\ 5.07547 & -1.45062 \\ 5.10754 & -1.52620\end{array}$

$$
\frac{a}{\lambda}=0.001191 \quad \frac{\alpha}{\beta}=0.05
$$

$\begin{array}{ll}0.01000 & -0.00060 \\ 0.02770 & -0.00179 \\ 0.08584 & -0.00679 \\ 0.18415 & -0.01917 \\ 0.37785 & -0.05837 \\ 0.90861 & -0.28067 \\ 1.52181 & -0.89159 \\ 1.73798 & -1.56435 \\ 1.66287 & -2.07142 \\ 1.49654 & -2.33447 \\ 1.40093 & -2.42446 \\ 1.42212 & -2.47385 \\ 0.72969 & -0.18459 \\ 1.30708 & -0.60534 \\ 1.64235 & -1.13830 \\ 1.74080 & -1.64989 \\ 1.67393 & -2.04271 \\ 1.54239 & -2.28178 \\ 1.43425 & -2.39275 \\ 1.39611 & -2.43711 \\ 1.42680 & -2.47859 \\ 1.49188 & -2.55626\end{array}$

$\begin{array}{rr}1.70834 & -0.01998 \\ 2.37537 & -0.03993 \\ 3.27759 & -0.09945 \\ 3.94871 & -0.19717 \\ 4.55891 & -0.38451 \\ 4.87834 & -0.84336 \\ 3.54540 & -1.12813 \\ 1.13557 & -0.85661 \\ -1.56801 & -0.30989 \\ -3.96301 & 0.26888 \\ -5.48939 & 0.75700 \\ -5.70127 & 1.04226 \\ & \\ 4.91023 & -0.70060 \\ 4.30745 & -1.08434 \\ 2.76367 & -1.08295 \\ 0.75568 & -0.78880 \\ -1.37417 & -0.35296 \\ -3.33909 & 0.10758 \\ -4.86436 & 0.52921 \\ -5.69569 & 0.86427 \\ -5.66214 & 1.05093 \\ -4.74223 & 1.02896\end{array}$

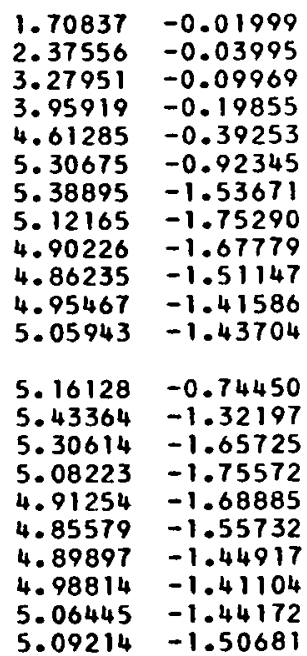


$\beta \mathrm{h}$

$C(h, 0)$

$S(h, 0)$

$E(r, 0)$

$2.19931-0.01999$ $3.41594-0.03996$ $5.18778-0.09980$ $6.55503-0.19917$ $7.91048,-0.3963$ $9.59988-0.96824$ $10.58074-1.82922$ $10.82567-2.52348$ $10.74248-3.02077$ $10.50849-3.31708$ $\begin{array}{rr}10.23423 & -3.43325 \\ 9.99232 & -3.40885\end{array}$

$9.17725-0.76811$ $10.28489-1.47764$ $10.72201-2.0977$ $\begin{array}{ll}10.82862 & -2.60625 \\ 10.75529 & -2.99137\end{array}$ $10.58396-3.25165$ $10.37170-3.39530$ $10.16009-3.43856$ $\begin{array}{ll}9.97814 & -3.40326 \\ 9.84364 & -3.31424\end{array}$

$C(h, h)$

$1.70794-10.01998$ $2.37457-0.03991$ $3.27561-0.09928$ $3.94480-0.19635$ $4.55150-0.38081$ $4.86499-0.81801$ $3.54156-1.03667$ $1.15357-0.69467$ $-1.53444-0.10502$ $-3.92401-0.46007$ $\begin{array}{ll}-5.45510 & 0.85031 \\ -5.69006 & 0.95321\end{array}$

$0.72401-0.19812$ $1.28824-0.62438$ $1.61092-1.15216$ $1.70437-1.05177$ $1.52157-2.26765$ $1.42385-2.38290$ $1.38986-2.43719$ $1.41665-2.48816$ $1.000 \pi \quad 11.02766=1.40481$ $\begin{array}{lll}1.125 \pi & 11.21226 & -1.43160 \\ 1.250 \pi & 11.34153 & -1.48765\end{array}$
$4.89805-0.68511$ $4.29681-1.02402$ $2.76736-0.96502$ $0.77653-0.61347$ $-1.34136-0.14968$ $\begin{array}{ll}-3.30076 & 0.31034\end{array}$ $\begin{array}{lll}-4.82606 & 0.68324\end{array}$ $\begin{array}{lll}-5.65335 & 0.94720\end{array}$ $-4.76698 \quad 0.75103$ $\begin{array}{ll}-5.66567 & 0.91336\end{array}$
$S(h, h)$

$0.00876-0.00071$ $0.02623-0.00224$ $0.10166-0.00961$ $0.26438-0.0284$ $0.65203-0.08432$ $\begin{array}{lll}1.95547 & -0.35148\end{array}$ $\begin{array}{ll}3.84438 & -0.87520\end{array}$ $4.84002-1.1318$ $4.75551-0.97850$ $3.59345-0.52616$ $\begin{array}{rr}1.52487 & 0.06688 \\ -1.01687 & 0.66812\end{array}$

$1.48481-0.24315$ $3.11965-0.66255$ $4.31739-1.0120$

$4.89497-1.13426$

$4.79891-1.00188$ $4.03241-0.67694$ $2.65661-0.24020$ $0.82768 \quad 0.24236$ $-1.19289 \quad 0.7058$ $\begin{array}{ll}-3.08348 & 1.06569\end{array}$

\begin{tabular}{|c|c|c|}
\hline $\begin{array}{l}0.01 \\
0.02 \\
0.05 \\
0.10 \\
0.20 \\
0.50 \\
1.00 \\
1.50 \\
2.00 \\
2.50 \\
3.00\end{array}$ & $\begin{array}{r}2.19867 \\
3.41458 \\
5.18362 \\
6.54431 \\
7.87997 \\
9.46817 \\
10.24015 \\
10.41003 \\
10.51298 \\
10.70365 \\
10.95587 \\
11.18586\end{array}$ & $\begin{array}{l}-0.01997 \\
-0.03991 \\
-0.09944 \\
-0.19757 \\
-0.38866 \\
-0.90157 \\
-1.47209 \\
-1.66428 \\
-1.60191 \\
-1.46906 \\
-1.39607 \\
-1.41107\end{array}$ \\
\hline $\begin{array}{l}0.125 \pi \\
0.250 \pi \\
0.375 \pi \\
0.500 \pi \\
0.625 \pi \\
0.750 \pi \\
0.875 \pi \\
1.000 \pi \\
1.125 \pi \\
1.250 \pi\end{array}$ & $\begin{array}{r}9.08748 \\
10.03934 \\
10.32982 \\
10.42233 \\
10.50300 \\
10.03928 \\
10.82669 \\
11.02661 \\
11.19913 \\
11.32369\end{array}$ & $\begin{array}{l}-0.73042 \\
-1.27556 \\
-1.58027 \\
-1.66670 \\
-1.61098 \\
-1.50503 \\
-1.42111 \\
-1.39251 \\
-1.41436 \\
-1.45916\end{array}$ \\
\hline
\end{tabular}

$0.00999-0.00110$ $0.02766-0.00317$ $0.08559-0.01107$ $\begin{array}{lll}0.18317 & -0.02831\end{array}$ $0.37398-0.07683$ $0.88672-0.32060$ $1.45719-0.93482$ $1.64935-1.57586$ $1.58698-2.04826$ $1.45413-2.30388$ $1.38112-2.41630$ $1.39612-2.49461$ $0.71560-0.21824$ $0.26067-0.65252$ $1.56536-1.17295$ $1.651705-1.65579$ $1.59005-2.02140$ $1.40617-2.24977$ $1.40617-2.37065$ $1.37757-2.43779$ $\begin{array}{ll}1.39942 & -2.50084 \\ 1.44421 & -2.58700\end{array}$

$$
\frac{a}{\lambda}=0.001191 \quad \frac{\alpha}{\beta}=0.10
$$

$\begin{array}{rrrr}2.19871 & -0.01998 & 1.70734 & -0.01997 \\ 3.41475 & -0.03995 & 2.37338 & -0.03987 \\ 5.18479 & -0.09972 & 3.27264 & -0.09901 \\ 6.54907 & -0.19887 & 3.93898 & -0.19513 \\ 7.89866 & -0.39512 & 4.54051 & -0.37528 \\ 9.57171 & -0.96209 & 4.84578 & -0.78032 \\ 10.53215 & -1.80312 & 3.53743 & -0.90090 \\ 10.76861 & -2.47205 & 1.18042 & -0.45328 \\ 10.68995 & -2.94415 & -1.49008 & 0.20188 \\ 10.47133 & -3.22135 & -3.88363 & 0.74732 \\ 10.21878 & -3.32851 & -5.43984 & 0.99226 \\ 9.99927 & -3.30652 & -5.72491 & 0.82484 \\ & & & \\ 9.15467 & -0.76364 & 4.88030 & -0.66208 \\ 10.25379 & -1.46082 & 4.28232 & -0.93451 \\ 10.66891 & -2.06299 & 2.77426 & -0.78978 \\ 10.77144 & -2.55110 & 0.80712 & -0.36431 \\ 10.70202 & -2.91645 & -1.29754 & 0.15474 \\ 10.54149 & -3.16051 & -3.25716 & 0.61172 \\ 10.34490 & -3.29368 & -4.79529 & 0.91527 \\ 10.15116 & -3.33336 & -5.66203 & 0.98973 \\ 9.98651 & -3.30149 & -5.69245 & 0.79719 \\ 9.86621 & -3.22192 & -4.85697 & 0.34108\end{array}$

$E(h, h)$

$\begin{array}{ll}1.70797 & -0.01998 \\ 2.37476 & -0.03993 \\ 3.27752 & -0.09959 \\ 3.95524 & -0.19816 \\ 4.60515 & -0.39097 \\ 5.29037 & -0.91461 \\ 5.37124 & -1.51038 \\ 5.11712 & -1.71662 \\ 4.91232 & -1.64690 \\ 4.87557 & -1.49488 \\ 4.95817 & -1.40905 \\ 5.05022 & -1.42750 \\ & \\ 5.14745 & -0.73882 \\ 5.41431 & -1.30312 \\ 5.29198 & -1.62582 \\ 5.08004 & -1.71928 \\ 4.92182 & -1.65712 \\ 4.86962 & -1.53649 \\ 4.90852 & -1.43877 \\ 4.98776 & -1.40479 \\ 5.05458 & -1.43157 \\ 5.07849 & -1.48762\end{array}$

$\begin{array}{rrrr}0.00875 & -0.00098 & 1.70737 & -0.01997 \\ 0.02620 & -0.00302 & 2.37356 & -0.03991 \\ 0.10152 & -0.01266 & 3.27453 & -0.09944 \\ 0.26379 & -0.03629 & 3.94933 & -0.19756 \\ 0.64980 & -0.10343 & 4.59366 & -0.38865 \\ 1.94451 & -0.40312 & 5.26608 & -0.90156 \\ 3.82540 & -0.94172 & 5.34497 & -1.47208 \\ 4.84108 & -1.14491 & 5.10939 & -1.66426 \\ 4.79306 & -0.87349 & 4.92467 & -1.00189 \\ 3.66056 & -0.25394 & 4.89219 & -1.46904 \\ 1.60435 & 0.50993 & 4.96209 & -1.39604 \\ -0.95150 & 1.20607 & 5.03793 & -1.41104 \\ 1.47731 & -0.28426 & 5.12689 & -0.73041 \\ 3.10135 & -0.73063 & 5.38572 & -1.27555 \\ 4.30221 & -1.06743 & 5.27074 & -1.58026 \\ 4.90081 & -1.13433 & 5.07558 & -1.66668 \\ 4.83381 & -0.90745 & 4.93310 & -1.01096 \\ 4.09256 & -0.45598 & 4.88706 & -1.50501 \\ 2.73237 & 0.12108 & 4.92032 & -1.42108 \\ 0.90655 & 0.72375 & 4.98671 & -1.39248 \\ -1.12992 & 1.24544 & 5.04145 & -1.41433 \\ -3.06447 & 1.56874 & 5.06065 & -1.45913\end{array}$


$\beta h$

$C(h, 0)$

\begin{tabular}{|c|c|c|}
\hline $\begin{array}{l}.01 \\
.02 \\
.05 \\
.10 \\
.20 \\
.50 \\
.00 \\
.50 \\
.00 \\
.50 \\
.00 \\
3.50\end{array}$ & $\begin{array}{r}2.19668 \\
3.41060 \\
5.17371 \\
6.52474 \\
7.84216 \\
9.38948 \\
10.15507 \\
10.37744 \\
10.53408 \\
10.73573 \\
10.95814 \\
11.15205\end{array}$ & $\begin{array}{l}-0.01995 \\
-0.03983 \\
-0.09894 \\
-0.19561 \\
-0.38107 \\
-0.85986 \\
-1.35394 \\
-1.50588 \\
-1.46287 \\
-1.37806 \\
-1.33549 \\
-1.34300\end{array}$ \\
\hline $\begin{array}{l}0.125 \pi \\
0.250 \pi \\
0.375 \pi \\
0.500 \pi \\
0.625 \pi \\
0.750 \pi \\
0.875 \pi \\
1.000 \pi \\
1.125 \pi \\
1.250 \pi\end{array}$ & $\begin{array}{r}9.02055 \\
9.94739 \\
10.25932 \\
10.39868 \\
10.52133 \\
10.07334 \\
10.84723 \\
11.01758 \\
11.16354 \\
11.27813\end{array}$ & $\begin{array}{l}-0.70330 \\
-1.18931 \\
-1.44107 \\
-1.50767 \\
-1.46897 \\
-1.40020 \\
-1.34964 \\
-1.33355 \\
-1.34463 \\
-1.36586\end{array}$ \\
\hline
\end{tabular}

0.0

0.01
0.02
0.05
0.10

0.10

0.20

1.50

2.50

3.00

$2.19269-0.01990$ $3.40265-0.03966$ $5.15405-0.09795$ $6.48617-0.19177$ $7.76870-0.36648$
0.0403 $9.24203-0.78407$ $9.99584-1.15549$ $10.29043-1.25065$ $10.51202-1.23017$ $10.72576-1.19553$ $10.92130-1.18101$

$\begin{array}{llll}0.125 \pi & 8.89372 & -0.65304\end{array}$ $0.250 \pi \quad 9.77770-1.03992$ $0.375 \pi \quad 10.12121-1.21203$ $\begin{array}{llll}0.500 \pi & 10.32309 & -1.25162\end{array}$ $\begin{array}{llll}0.625 \pi & 10.49617 & -1.23293\end{array}$ $0.750 \pi \quad 10.66534-1.20392$ $\begin{array}{llll}0.875 \pi & 10.82645 & -1.18553\end{array}$ $1.000 \pi \quad 10.97132-1.18044$ $1.125 \pi \quad 11.09658-1.18329$ $1.250 \pi \quad 11.20407-1.18807$
$S(h, 0)$

$\begin{array}{ll}0.00997 & -0.00210 \\ 0.02758 & -0.00593 \\ 0.08510 & -0.01960 \\ 0.18121 & -0.04644 \\ 0.36640 & -0.11317 \\ 0.84502 & -0.39780 \\ 1.33904 & -1.01841 \\ 1.49096 & -1.60696 \\ 1.44794 & -2.02567 \\ 1.36313 & -2.27030 \\ 1.32055 & -2.41254 \\ 1.32807 & -2.52693 \\ 0.68849 & -0.28368 \\ 1.17443 & -0.74299 \\ 1.42616 & -1.24195 \\ 1.49275 & -1.67795 \\ 1.45404 & -2.00158 \\ 1.38527 & -2.21421 \\ 1.33470 & -2.34862 \\ 1.31862 & -2.44533 \\ 1.32969 & -2.53493 \\ 1.35092 & -2.63107\end{array}$

$$
\frac{a}{\lambda}=0.001191 \quad \frac{\alpha}{\beta}=0.20
$$

$E(h, 0)$

$\mathrm{C}(\mathrm{h}, \mathrm{h})$

$S(h, h)$

$E(h, h)$

$\begin{array}{rrrr}2.19671 & -0.31996 & 1.70535 & -0.01994 \\ 3.41075 & -0.03989 & 2.36940 & -0.03974 \\ 5.17483 & -0.19945 & 3.26280 & -0.09812 \\ 6.52925 & -0.19786 & 3.91982 & -0.19109 \\ 7.85952 & -0.39119 & 4.50504 & -0.35709 \\ 9.47926 & -0.93877 & 4.78860 & -0.65744 \\ 10.37479 & -1.71968 & 3.53806 & -0.45892 \\ 10.58511 & -2.31077 & 1.27048 & 0.34291 \\ 10.51980 & -2.70782 & -1.39294 & 1.23104 \\ 10.34547 & -2.92981 & -3.90702 & 1.72550 \\ 10.15361 & -3.01170 & -5.70302 & 1.49605 \\ 9.99482 & -2.99612 & -6.29053 & 0.42418 \\ 9.08032 & -0.74897 & 4.82559 & -0.58670 \\ 10.11994 & -1.20656 & 4.24673 & -0.64357 \\ 10.49752 & -1.95277 & 2.81003 & -0.21729 \\ 10.58753 & -2.37857 & 0.90505 & 0.47575 \\ 10.52970 & -2.68510 & -1.19673 & 1.17446 \\ 10.40051 & -2.88207 & -3.23262 & 1.64629 \\ 10.24823 & -2.58551 & -4.92514 & 1.71504 \\ 10.10386 & -3.01527 & -6.00879 & 1.27659 \\ 9.98584 & -2.99258 & -6.27812 & 0.32218 \\ 9.90288 & -2.93787 & -5.62752 & -1.03955\end{array}$

$$
\frac{a}{\lambda}=0.001191 \quad \frac{\alpha}{\beta}=0.40
$$

\begin{tabular}{|c|c|}
\hline $\begin{array}{l}0.00993 \\
0.02742 \\
0.08412 \\
0.17738 \\
0.35181 \\
0.76924 \\
1.14060 \\
1.23574 \\
1.21525 \\
1.18060 \\
1.16609 \\
1.16797\end{array}$ & $\begin{array}{l}-0.00409 \\
-0.01143 \\
-0.03649 \\
-0.08213 \\
-0.18369 \\
-0.54228 \\
-1.17466 \\
-1.69098 \\
-2.04475 \\
-2.27729 \\
-2.44639 \\
-2.58959\end{array}$ \\
\hline $\begin{array}{l}0.63824 \\
1.02504 \\
1.19713 \\
1.23671 \\
1.21801 \\
1.18900 \\
1.17061 \\
1.16551 \\
1.16836 \\
1.17314\end{array}$ & $\begin{array}{l}-0.40755 \\
-0.90970 \\
-1.37709 \\
-1.75055 \\
-2.02376 \\
-2.21922 \\
-2.36641 \\
-2.48861 \\
-2.59891 \\
-2.70213\end{array}$ \\
\hline
\end{tabular}

$\begin{array}{rr}0.00873 & -0.00185 \\ 0.02612 & -0.00564 \\ 0.10102 & -0.02277 \\ 0.26188 & -0.06241 \\ 0.64263 & -0.16669 \\ 1.91209 & -0.57385 \\ 3.78802 & -1.16720 \\ 4.90643 & -1.20216 \\ 5.02089 & -0.53277 \\ 4.00974 & 0.06823 \\ 1.94456 & 2.03974 \\ -0.83125 & 3.10780 \\ & \\ 1.45434 & -0.42008 \\ 3.05460 & -0.95773 \\ 4.28917 & -1.25975 \\ 4.98779 & -1.14889 \\ 5.04998 & -0.60305 \\ 4.41654 & 0.28681 \\ 3.09854 & 1.35735 \\ 1.20910 & 2.39515 \\ -1.03410 & 3.15712 \\ -3.33557 & 3.40794\end{array}$

$\begin{array}{rrrrrr}1.70137 & -0.01987 & 0.00869 & -0.00359 & 1.70139 & -0.01990 \\ 2.36148 & -0.03950 & 0.02597 & -0.01085 & 2.36163 & -0.03965 \\ 3.24339 & -0.09637 & 0.10006 & -0.04284 & 3.24496 & -0.09793 \\ 3.88269 & -0.18313 & 0.25819 & -0.11411 & 3.89120 & -0.19174 \\ 4.43933 & -0.32169 & 0.62947 & -0.29127 & 4.48241 & -0.36644 \\ 4.70500 & -0.42277 & 1.86537 & -0.91032 & 5.03995 & -0.78402 \\ 3.60852 & 0.39250 & 3.82790 & -1.63878 & 5.10068 & -1.15544 \\ 1.46728 & 1.94564 & 5.32967 & -1.37340 & 4.98982 & -1.25058 \\ -1.42645 & 3.42482 & 5.99192 & 0.14978 & 4.92372 & -1.23010 \\ -4.72146 & 3.95290 & 5.35384 & 2.74934 & 4.91432 & -1.19546 \\ -7.80128 & 2.78243 & 3.03083 & 5.78361 & 4.92754 & -1.18094 \\ -9.77421 & -0.44834 & -1.07133 & 8.19132 & 4.93849 & -1.18282 \\ & & & & & \\ 4.73621 & -0.44213 & 1.41742 & -0.68698 & 4.93314 & -0.65300 \\ 4.23402 & -0.08831 & 3.02335 & -1.41607 & 5.12408 & -1.03986 \\ 2.94719 & 0.89983 & 4.43276 & -1.68149 & 5.06215 & -1.21197 \\ 1.09736 & 2.17994 & 5.48470 & -1.23477 & 4.97635 & -1.25155 \\ -1.19601 & 3.33860 & 5.98194 & -0.00269 & 4.92629 & -1.23286 \\ -3.76310 & 3.94702 & 5.69664 & 1.92018 & 4.91315 & -1.20385 \\ -6.33143 & 3.61746 & 4.42335 & 4.26323 & 4.92010 & -1.18546 \\ -8.51650 & 2.07828 & 2.04307 & 6.58315 & 4.93144 & -1.18037 \\ -9.84269 & -0.74283 & -1.41120 & 8.30118 & 4.93892 & -1.18322 \\ -9.79592 & -4.64826 & -5.69378 & 8.78158 & 4.94106 & -1.18799\end{array}$

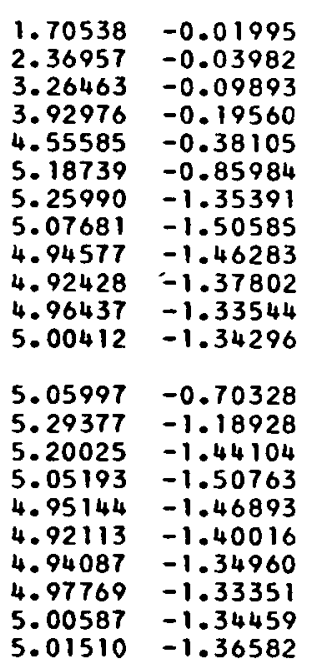

$5.01510-1.36582$ 
$\beta \mathrm{h}$

\begin{tabular}{|c|c|c|}
\hline $\begin{array}{l}01 \\
02 \\
05 \\
10\end{array}$ & $\begin{array}{r}1.76577 \\
2.89082 \\
4.62671 \\
5.99086 \\
7.34368 \\
8.97515 \\
9.75373 \\
9.85583 \\
9.88290 \\
10.05700 \\
10.36130 \\
10.66020\end{array}$ & $\begin{array}{l}-0.0200 \\
-0.0400 \\
-0.0999 \\
-0.1995 \\
-0.3964 \\
-0.9460 \\
-1.6053 \\
-1.8486 \\
-1.7581 \\
-1.5499 \\
-1.4246 \\
-1.4545\end{array}$ \\
\hline $\begin{array}{l}0 \pi \\
5 \pi \\
0 \pi \\
5 \pi \\
0 \pi \\
5 \pi \\
0 \pi \\
5 \pi \\
0 \pi\end{array}$ & $\begin{array}{r}8.58165 \\
9.56121 \\
9.82524 \\
9.85599 \\
9.87702 \\
9.98938 \\
10.19960 \\
10.45291 \\
10.67707 \\
10.82238\end{array}$ & $\begin{array}{l}-0.7589 \\
-1.3707 \\
-1.7397 \\
-1.8519 \\
-1.7716 \\
-1.6083 \\
-1.4689 \\
-1.4181 \\
-1.4612 \\
-1.5558\end{array}$ \\
\hline
\end{tabular}

0.01

0.02

0.05

0.20

0.50

1.00

2.00

2.50

3.00

3.50

$0.125 \%$

$0.250 \pi$

$0.375 \pi$

$0.500 \pi$

$0.750 \pi$

$0.875 \pi$

$0.875 \pi$
$1.000 \pi$

$1.000 \pi$
$S(h, 0)$

$0.00830-0.00010$

$0.02474-0.00040$

$0.08196-0.00250$

$0.18059-0.00998$

$0.37700-0.03973$

$0.92631-0.2398$

$\begin{array}{lll}1.58553 & -0.8473\end{array}$

$1.82873-1.55617$

$1.73827-2.10445$

$1.52999-2.37664$

$1.40475-2.43698$

$1.43466-2.44638$

$0.73926-0.15030$

$.35091-0.55679$
$1.71985-1.10365$

$1.71985-1.10365$

$1.83201-1.64825$

$1.75176-2.07349$

$1.58843-2.32577$

$1.44907-2.42385$

$1.39821-2.43760$

$\begin{array}{lll}1.44131 & -2.44901\end{array}$

$1.53589-2.51442$

$$
\frac{z}{\lambda}=0.001538
$$

$$
E(h, 0)
$$

$1.76579-0.02000$

$2.89097-0.0400$

$4.62787-0.09998$

$\begin{array}{ll}5.99572 & -0.19989\end{array}$

$7.36329-0.3991$

$9.09105 \quad-0.98620$

$10.12124-1.89213$

$10.38711-2.04932$

$10.29234-3.21077$

$10.01812-3.55697$

$9.68563-3.69723$

$8.65515-0.77869$

$9.81717-1.51793$

$10.27361-2.18187$

$10.39039-2.74148$

$10.30706-3.17700$

$10.10757-3.47943$

$9.85373-3.65090$

$9.56371-3.70380$

$9.18950-3.54330$
$C(h, h)$

$1.44546-0.02000$ $2.09626-0.03999$ $2.99620-0.09990$ $3.67232-0.19922$ $4.29539-0.39382$ $4.65992-0.90755$ $3.40123-1.36039$ $1.06708-1.26583$ - 1.06708762 $\begin{array}{ll}-3.87385 & -0.2110 \\ -5.37617 & 0.52538\end{array}$

$-5.58199 \quad 1.28059$

$4.67519-0.73974$ $4.13233-1.2374$ $0.6491-1.3821$ $-1.36128-0.86382$ $-3.26508-0.40133$ $\begin{array}{ll}-4.75791 & 0.14295\end{array}$ $-5.581250 .74608$ $-5.54181 \quad 1.32722$ $\begin{array}{ll}-5.54181 & 1.32722 \\ -4.59964 & 1.75410\end{array}$
Sin, h)

$0.00728-0.00010$ $0.02238-0.00040$ $0.08986-0.00250$ $0.23938-0.00997$ $\begin{array}{lll}0.00260 & -0.03947\end{array}$ $1.84762-0.23010$ $3.56232-0.72185$ $4.58380-1.10955$ $4.45833-1.23147$ $3.32819-1.16361$ $1.32819-1.1636$ $-1.12525-0.58020$

$1.39565-0.14651$ $2.96866-0.50355$ $4.10862-0.88700$ $4.102953-1.14284$ $4.50238-1.23048$ $3.75094-1.19628$ $3.75094-1.19628$ $0.06211-0.88045$ 0.662110 .88045 $-3.10819-0.07912$
$E(h, h)$

$1.44549-0.02000$ $2.09641-0.04000$ $2.99786-0.09994$ $4.34456-0.39645$ $5.06062-0.94607$ $5.14617-1.60538$ $4.84282-1.84861$ $4.58221-1.75816$ $4.53316-1.54989$ $4.65514-1.42465$ $4.79989-1.45456$

$4.90859-0.75896$ $5.19520-1.37074$ $-1.73972$ $4.59475-1.85190$ $4.52478-1.00833$ $4.58086-1.46897$ $4.70063-1.4181$ $4.80701-1.46121$ $4.84696-1.55578$

$$
\frac{\mathrm{a}}{\lambda}=0.001588 \quad \frac{\alpha}{\beta}=0.005
$$

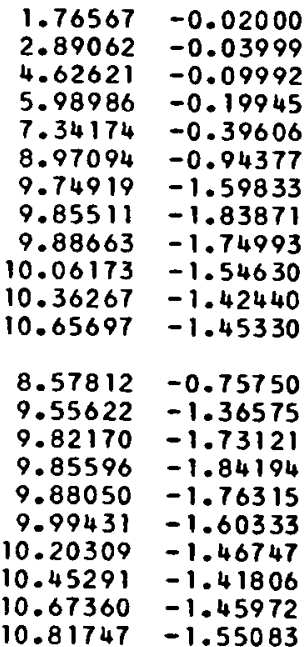

$\begin{array}{ll}10.67360 & -1.45972 \\ 10.81747 & -1.55083\end{array}$

$\begin{aligned} 1.76569 & -0.02000 \\ 2.89077 & -0.04000 \\ 4.62737 & -0.09997 \\ 5.99472 & -0.19983 \\ 7.36131 & -0.39890 \\ 9.08626 & -0.98497 \\ 10.11284 & -1.88754 \\ 10.37715 & -2.64005 \\ 10.28326 & -3.19665 \\ 10.01213 & -3.53902 \\ 9.68419 & -3.67741 \\ 9.38555 & -3.64688 \\ 9.65132 & -0.77792 \\ 9.81011 & -1.51500 \\ 10.26438 & -2.17570 \\ 10.38041 & -2.73150 \\ 10.29783 & -3.16321 \\ 10.10050 & -3.46242 \\ 9.84989 & -3.63173 \\ 9.59366 & -3.68387 \\ 9.36773 & -3.63985 \\ 9.19646 & -3.52628\end{aligned}$

$\begin{array}{rr}1.44536 & -0.02000 \\ 2.09606 & -0.03999 \\ 2.99570 & -0.09986 \\ 3.67133 & -0.19903 \\ 4.29348 & -0.39294 \\ 4.65628 & -0.90141 \\ 3.39965 & -1.33811 \\ 1.07170 & -1.22673 \\ -1.53801 & -0.77553 \\ -3.85712 & -0.16497 \\ -5.35345 & 0.54792 \\ -5.55878 & 1.25732 \\ & \\ 4.67194 & -0.73600 \\ 4.12910 & -1.22280 \\ 2.64557 & -1.35344 \\ 0.70466 & -1.17774 \\ -1.35095 & -0.81510 \\ -3.25075 & -0.35261 \\ -4.73800 & 0.18026 \\ -5.55748 & 0.75765 \\ -5.51896 & 1.30022 \\ -4.58522 & 1.68296\end{array}$

-

$0.00728-0.00014$ $0.02238-0.00051$ $0.08983-0.00295$ $0.23928-0.01116$ $0.60221-0.04241$ $1.84552-0.23819$ $3.65745-0.73193$ $4.58007-1.1105$ $4.45837-1.21414$ $3.33166-1.12062$ $1.34497-0.89686$ $-1.10784-0.49490$ $1.39426-0.15293$ $2.96466-0.51412$ $\begin{array}{lll}4.10364 & -0.89508\end{array}$ $4.62628-1.14177$ $4.50214-1.21476$ $3.75344-1.16123$ $2.43310-1.02689$ $0.67274-0.80398$ $\begin{array}{ll}-1.27744 & -0.45970 \\ -3.08324 & -0.00142\end{array}$

$\begin{array}{ll}1.44539 & -0.02000 \\ 2.09621 & -0.03999 \\ 2.99736 & -0.09992 \\ 3.68065 & -0.19945 \\ 4.34261 & -0.39606 \\ 5.05642 & -0.94377 \\ 5.14163 & -1.59832 \\ 4.84209 & -1.83870 \\ 4.58593 & -1.74992 \\ 4.53789 & -1.54628 \\ 4.65651 & -1.42437 \\ 4.79666 & -1.45327 \\ 4.90505 & -0.75750 \\ 5.19020 & -1.36575 \\ 5.05025 & -1.73121 \\ 4.79683 & -1.84194 \\ 4.59823 & -1.76314 \\ 4.52971 & -1.60332 \\ 4.58434 & -1.46745 \\ 4.70063 & -1.41803 \\ 4.80354 & -1.45969 \\ 4.84205 & -1.55080\end{array}$


$\underset{\ddot{\lambda}}{\stackrel{a}{\lambda}=0.001588 \quad} \quad \frac{\alpha}{\bar{\beta}}=0.01$

Bh

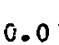

0.01
0.02

0.02

c. 10

0.20

0.50

1.00

2.00

2.50

3.50

$0.125 \pi$

$0.250 \pi$

$0.375 \pi$

$0.500 \pi$

$0.750 \pi$

0.875

1.000

$$
C(h, 0)
$$

$1.76557-0.02000$ $2.89042-0.03999$ $4.02571-0.09989$ $5.98887-0.19935$ $7.33979-0.39567$ $8.96675-0.94748$ $9.74466-1.59131$ $9.85434-1.82888$ $9.89024-1.74173$ $10.06632-1.54263$ $\begin{array}{ll}10.36401 & -1.42399 \\ 10.05384 & -1.45190\end{array}$ $8.57459-0.75604$ $9.55124 \cdot-1.36079$ $9.81817-1.72277$ $9.88387-1.75469$ $9.90910-1.59830$ $10.20647-1.46583$ $10.45291-1.46583$ $10.67023-1.41783$ $10.81272-1.54587$
$S(h, 0)$

$\begin{array}{ll}0.00829 & -0.00018 \\ 0.02473 & -0.00065 \\ 0.08191 & -0.00332 \\ 0.18039 & -0.01178 \\ 0.37621 & -0.04343 \\ 0.92173 & -0.24801 \\ 1.57146 & -0.85624 \\ 1.80899 & -1.55746 \\ 1.72183 & -2.09692 \\ 1.52271 & -2.36712 \\ 1.40406 & -2.43408 \\ 1.43197 & -2.45254 \\ & \\ 0.73634 & -0.15716 \\ 1.34096 & -0.56656 \\ 1.70290 & -1.11052 \\ 1.81217 & -1.64815 \\ 1.73479 & -2.06644 \\ 1.57839 & -2.31586 \\ 1.44592 & -2.41679 \\ 1.39791 & -2.43741 \\ 1.43817 & -2.45565 \\ 1.52594 & -2.52388\end{array}$

$E(h,(1)$

$1.76559-0.02000$ $2.89057-0.03999$ $4.62687-0.09996$ $5.99372-0.19978$ $7.35932-0.39870$ $9.08147-0.98375$ $10.10445-1.88296$ $10.36722-2.63082$ $10.27420-3.18261$ $10.00612-3.52120$ $\begin{array}{ll}9.68266 & -3.65773 \\ 9.38882 & -3.62773\end{array}$

$8.64750-0.77716$ $\begin{array}{ll}-1.51208 \\ 106 & -2.16956\end{array}$ $10.37045-2.72157$ $10.28862-3.14950$ $10.09343-3.44552$ $9.84600-3.01270$ $9.84600-3.61270$ $9.59351-3.66410$

$9.20320-3.50936$
$C(h, h)$

$1.44526-0.02000$ $2.09586-0.03998$ $2.99520-0.09982$ $3.67034 \quad-0.19833$ $4.29158-0.39236$ $\begin{array}{lll}4.65266 & -0.89528\end{array}$ $3.39813-1.31538$ $1.07631-1.18770$ $-1.52745-0.72650$ $-3.84097-0.11807$ $\begin{array}{ll}-5.33191 & 0.57044 \\ -5.53730 & 1.23433\end{array}$

$\begin{array}{lll}4.66871 & -0.73227\end{array}$ $4.12592-1.20814$ $2.64603-1.32484$ $0.71018-1.13675$ $-3.23626-0.30306$ $\begin{array}{rr}-4.71894 & 0.21750\end{array}$ $-5.53508 \quad 0.76925$ $-5.49787 \quad 0.76925$ $\begin{array}{ll}-5.49787 & 1.27354 \\ -4.57255 & 1.61249\end{array}$
$S(h, h)$

$0.00728-0.00017$ $0.02238-0.00062$ $0.08981 \quad-0.00339$ $0.23919-0.01234$ $0.60183-0.04536$ $1.84343-0.24628$ $\begin{array}{ll}3.65267 & -0.74204\end{array}$ $4.57657-1.11165$ $4.45875-1.19687$ $3.33556-1.07769$ $1.35372-0.82678$ $-1.09081-0.40985$

$1.39288-0.15935$ $2.96071-0.52469$ $4.09880-0.00318$ $4.62326-1.14077$ $4.50224-1.19912$ $3.75636-1.12625$ $2.439362-0.96904$ $0.68354-0.72771$ $-1.25979-0.37447$ $\begin{array}{rr}-1.25979 & -0.37447 \\ -3.05960 & 0.07616\end{array}$
$E(h, h)$

$\begin{array}{ll}1.44529 & -0.02000\end{array}$ $\begin{array}{ll}2.09601 & -0.03999\end{array}$ $2.99686-0.09989$ $3.67966-0.19935$ $\begin{array}{ll}4.34067 & -0.39566\end{array}$ $5.13710-1.59131$ $4.84133-1.82887$ $4.58955-1.74172$ $4.54248-1.54261$ $4.65784-1.42395$

$4.90153-0.75604$ $5.18523-1.36079$ $5.04671-1.72276$ $4.79676-1.83205$ $4.53450-1.59828$ $4.58772-1.46581$ $4.70063-1.41780$ $4.80017-1.41780$ $4.83730-1.54584$

$$
\frac{a}{\lambda}=0.001588 \quad \frac{\alpha}{\beta}=0.02
$$

$\begin{array}{lrl}0.01 & 1.76537 & -0.01999 \\ 0.02 & 2.89002 & -0.03998 \\ 0.05 & 4.62471 & -0.09984 \\ 0.10 & 5.98689 & -0.19915 \\ 0.20 & 7.33591 & -0.39488 \\ 0.50 & 8.95839 & -0.93693 \\ 1.00 & 9.73563 & -1.57741 \\ 1.50 & 9.85270 & -1.80944 \\ 2.00 & 9.89713 & -1.72547 \\ 2.50 & 10.07511 & -1.53513 \\ 3.00 & 10.36654 & -1.42271 \\ 3.50 & 10.64788 & -1.44877 \\ 0.125 \pi & 8.56756 & -1.75313 \\ 0.250 \pi & 9.54133 & -1.35094 \\ 0.375 \pi & 9.81109 & -1.70605 \\ 0.500 \pi & 9.85560 & -1.81253 \\ 0.625 \pi & 9.89029 & -1.73793 \\ 0.750 \pi & 10.00827 & -1.58816 \\ 0.875 \pi & 10.21293 & -1.46225 \\ 1.000 \pi & 10.45290 & -1.41693 \\ 1.125 \pi & 10.66382 & -1.45455 \\ 1.250 \pi & 10.80368 & -1.53600\end{array}$

$\begin{array}{ll}0.00829 & -0.00027 \\ 0.02473 & -0.00089 \\ 0.08186 & -0.00413 \\ 0.18019 & -0.01357 \\ 0.37542 & -0.04712 \\ 0.91718 & -0.25617 \\ 1.55755 & -0.86507 \\ 1.78955 & -1.55890 \\ 1.70557 & -2.08983 \\ 1.51522 & -2.35814 \\ 1.40279 & -2.43134 \\ 1.42885 & -2.45831 \\ 0.73343 & -0.16400 \\ 1.33111 & -0.57628 \\ 1.68618 & -1.11740 \\ 1.79264 & -1.64824 \\ 1.71803 & -2.05983 \\ 1.56825 & -2.30649 \\ 1.44233 & -2.41013 \\ 1.39701 & -2.43722 \\ 1.43463 & -2.46186 \\ 1.51607 & -2.53272\end{array}$

$\begin{aligned} 1.76539 & -0.01999 \\ 2.89017 & -0.03999 \\ 4.62587 & -0.09993 \\ 5.99173 & -0.19968 \\ 7.35535 & -0.39830 \\ 9.07192 & -0.98131 \\ 10.08774 & -1.87385 \\ 10.34744 & -2.61249 \\ 10.25613 & -3.15480 \\ 9.99406 & -3.48594 \\ 9.67938 & -3.61885 \\ 9.39492 & -3.58986 \\ & \\ 8.63987 & -0.77564 \\ 9.78900 & -1.50626 \\ 10.23684 & -2.15735 \\ 10.35062 & -2.70186 \\ 10.27028 & -3.12234 \\ 10.07928 & -3.41208 \\ 9.83809 & -3.57508 \\ 9.59293 & -3.52503 \\ 9.37801 & -3.58319 \\ 9.21603 & -3.47583\end{aligned}$

$\begin{array}{rr}1.44506 & -0.01999 \\ 2.09546 & -0.03997 \\ 2.99421 & -0.09973 \\ 3.06837 & -0.19845 \\ 4.28779 & -0.39029 \\ 4.64549 & -0.88306 \\ 3.39522 & -1.27160 \\ 1.08548 & -1.10987 \\ -1.50692 & -0.62861 \\ -3.81038 & -0.02716 \\ -5.29238 & 0.61543 \\ -5.49953 & 1.18916 \\ & \\ 4.66230 & -0.72483 \\ 4.11969 & -1.17894 \\ 2.64706 & -1.26785 \\ 0.72111 & -1.05499 \\ -1.32101 & -0.66931 \\ -3.20859 & -0.20683 \\ -4.68338 & 0.29179 \\ -5.49436 & 0.79260 \\ -5.46093 & 1.22104 \\ -4.55242 & 1.47340\end{array}$

$0.00727-0.00025$ $0.02237-0.00085$ $0.08976-0.00429$ $\begin{array}{lll}0.23899 & -0.01472\end{array}$ $0.60105-0.05124$ $1.83931-0.26242$ $3.64342-0.76228$ $4.57022-1.11401$ $4.46053-1.16256$ $3.34469-0.99203$ $\begin{array}{rr}1.37209 & -0.68704 \\ -1.05789 & -0.24038\end{array}$

$1.39015-0.17217$ $2.95299-0.54582$ $4.08955-0.91949$

$4.61793-1.13898$

$4.50343-1.16804$

$3.76346-1.05645$

$2.45210-0.85630$ $0.70562-0.57569$ $\begin{array}{rr}-1.22583 & -0.20463 \\ -3.01620 & 0.23107\end{array}$

$\begin{array}{ll}1.44509 & -0.01999 \\ 2.09561 & -0.03998 \\ 2.99586 & -0.09984 \\ 3.67768 & -0.19915 \\ 4.33679 & -0.39488 \\ 5.04387 & -0.93693 \\ 5.12807 & -1.57740 \\ 4.83969 & -1.80942 \\ 4.59643 & -1.72545 \\ 4.55127 & -1.53511 \\ 4.66038 & -1.42269 \\ 4.78757 & -1.44874 \\ & \\ 4.89450 & -0.75313 \\ 5.17531 & -1.35093 \\ 5.03964 & -1.70604 \\ 4.79647 & -1.81251 \\ 4.60801 & -1.73791 \\ 4.54367 & -1.58814 \\ 4.59418 & -1.46222 \\ 4.70062 & -1.41690 \\ 4.79376 & -1.45452 \\ 4.82826 & -1.53597\end{array}$


$\ddot{\alpha}=0.001588 \quad \stackrel{\alpha}{\beta}=0.03$

$\beta \mathrm{h}$

$C(h, 0)$

\begin{tabular}{|c|c|c|}
\hline $\begin{array}{l}0.01 \\
0.02 \\
0.05 \\
0.10 \\
0.20 \\
0.50 \\
1.00 \\
1.50 \\
2.00 \\
2.50 \\
3.00 \\
3.50\end{array}$ & $\begin{array}{r}1.76517 \\
2.88962 \\
4.62371 \\
5.98491 \\
7.33204 \\
8.95008 \\
9.72664 \\
9.85092 \\
9.90357 \\
10.08338 \\
10.36890 \\
10.64227\end{array}$ & $\begin{array}{l}-0.01999 \\
-0.03997 \\
-0.09979 \\
-0.19895 \\
-0.39409 \\
-0.093241 \\
-1.56367 \\
-1.79030 \\
-1.70940 \\
-1.52743 \\
-1.42092 \\
-1.44524\end{array}$ \\
\hline $\begin{array}{l}.125 \pi \\
.250 \pi \\
.375 \pi \\
.500 \pi \\
.625 \pi \\
.750 \pi \\
.875 \pi \\
.000 \pi \\
.125 \pi \\
.250 \pi\end{array}$ & $\begin{array}{r}8.56055 \\
9.53147 \\
9.80401 \\
9.85512 \\
9.89629 \\
10.01690 \\
10.21899 \\
10.45286 \\
10.65779 \\
10.79521\end{array}$ & $\begin{array}{l}-0.75024 \\
-1.34118 \\
-1.08956 \\
-1.79329 \\
-1.72137 \\
-1.57795 \\
-1.45826 \\
-1.41548 \\
-1.45063 \\
-1.52621\end{array}$ \\
\hline
\end{tabular}

$\begin{array}{lll}1.125 \pi & 10.65779 & -1.45063 \\ 1.250 \pi & 10.79521 & -1.52621\end{array}$

0.08

$2.88882-0.03995$

$\begin{array}{lll}0.10 & 5.62172 & -0.09969 \\ 0.09 & -0.19855\end{array}$

$0.20 \quad 7.32431-0.39253$

$\begin{array}{lll}0.50 & 8.93355 & -0.92345\end{array}$

$1.00 \quad 9.70877-1.53670$

$\begin{array}{lll}1.50 & 9.84692 & -1.75289\end{array}$

$\begin{array}{lll}2.00 & 9.91521 & -1.67779 \\ 2.50 & 10.09845 & -1.51147\end{array}$

$3.00 \quad 10.37309-1.41587$

$3.50 \quad 10.63200-1.43706$

$\begin{array}{lll}0.125 \pi & 8.54662 & -0.74450\end{array}$

$\begin{array}{lll}0.2507 & 9.51192 & -1.32196\end{array}$

$0.375 \pi \quad 9.78985-1.65725$

$0.500 \pi \quad 9.85362-1.75571$

$\begin{array}{llll}0.625 \pi & 9.90708 & -1.68885\end{array}$

$\begin{array}{llll}0.750 \pi & 10.03265 & -1.55732\end{array}$

$0.875 \pi \quad 10.22998-1.44918$

$1.000 \pi \quad 10.45268-1.41105$

$10.64677-1.44174$

$1.250 \pi \quad 10.77982-1.50683$
$S(h, 0)$

$E(h, G)$

$\begin{array}{ll}0.00829 & -0.00035 \\ 0.02472 & -0.00114 \\ 0.08181 & -0.00495 \\ 0.17999 & -0.01537 \\ 0.37464 & -0.05080 \\ 0.91266 & -0.26429 \\ 1.54382 & -0.37387 \\ 1.77041 & -1.56049 \\ 1.68949 & -2.08319 \\ 1.50751 & -2.34967 \\ 1.40100 & -2.42878 \\ 1.42532 & -2.46371 \\ 0.73054 & -0.17080 \\ 1.32136 & -0.58594 \\ 1.66969 & -1.12428 \\ 1.77340 & -1.64852 \\ 1.70147 & -2.05363 \\ 1.55804 & -2.29766 \\ 1.43835 & -2.40387 \\ 1.39556 & -2.43706 \\ 1.43071 & -2.46769 \\ 1.50629 & -2.54099\end{array}$

$1.43071-2.46769$

$\begin{aligned} 1.70519 & -0.01999 \\ 2.88977 & -0.03998 \\ 4.62487 & -0.09990 \\ 5.98973 & -0.19958 \\ 7.35139 & -0.39790 \\ 9.06239 & -0.97887 \\ 0.07110 & -1.86480 \\ 10.32777 & -2.59434 \\ 10.23915 & -3.12733 \\ 9.98195 & -3.45119 \\ 9.57581 & -3.58056 \\ 9.40041 & -3.55256 \\ 9.63225 & -0.77412 \\ 9.77478 & -1.50047 \\ 10.21860 & -2.14523 \\ 10.33091 & -2.68235 \\ 10.25202 & -3.09550 \\ 10.06512 & -3.37909 \\ 9.83002 & -3.53803 \\ 9.59197 & -3.58656 \\ 9.38409 & -3.54612 \\ 9.22802 & -3.44270\end{aligned}$

$\mathrm{C}(\mathrm{h}, \mathrm{h})$

$S(h, h)$

$\begin{array}{rr}1.44486 & -0.01999 \\ 2.09506 & -0.03996 \\ 2.99321 & -0.09955 \\ 3.66640 & -0.19806 \\ 4.28402 & -0.38854 \\ 4.63843 & -0.87089 \\ 3.39251 & -1.22754 \\ 1.09459 & -1.03232 \\ -1.48717 & -0.53092 \\ -3.78206 & 0.06442 \\ -5.25752 & 0.66040 \\ -5.46858 & 1.14506 \\ & \\ 4.65595 & -0.71742 \\ 4.11365 & -1.14987 \\ 2.64824 & -1.21112 \\ 0.73192 & -0.97350 \\ -1.30193 & -0.57238 \\ -3.18267 & -0.10992 \\ -4.65122 & 0.36590 \\ -5.45905 & 0.81612 \\ -5.43090 & 1.16967 \\ -4.53917 & 1.33657\end{array}$

$0.00727-0.00032$ $0.02236-0.00107$ $0.08971-0.00519$ $0.23879-0.01709$ $0.60029-0.05711$ $\begin{array}{ll}1.83525 & -0.27854\end{array}$ $3.63457-0.78257$ $4.56475-1.11661$ $4.46370-1.12853$ $3.35557-0.90658$ $1.39161-0.54778$ $-1.02647-0.07158$
-.30

$1.38745 \quad-0.18498$ $2.94549-0.56694$ $4.08085-0.9359$ $4.61356-1.13747$ $4.50596-1.13724$ $3.77225-0.98687$ $2.46684-0.74296$ $0.72831-0.42424$ $-1.19361-0.03544$ $\begin{array}{rr}-2.97789 & 0.38587\end{array}$
$\mathrm{E}(\mathrm{h}, \mathrm{h})$

$\begin{array}{ll}1.44489 & -0.01999 \\ 2.09522 & -0.03997 \\ 2.99487 & -0.09979 \\ 3.67570 & -0.19895 \\ 4.33291 & -0.39409 \\ 5.03555 & -0.93240 \\ 5.11907 & -1.56366 \\ 4.83790 & -1.79028 \\ 4.60288 & -1.70938 \\ 4.55954 & -1.52740 \\ 4.66274 & -1.42089 \\ 4.78196 & -1.44521 \\ & \\ 4.88749 & -0.75024 \\ 5.16545 & -1.34117 \\ 5.03256 & -1.68955 \\ 4.79599 & -1.79328 \\ 4.61401 & -1.72135 \\ 4.55230 & -1.57792 \\ 4.60024 & -1.45823 \\ 4.70058 & -1.41545 \\ 4.78773 & -1.45060 \\ 4.81980 & -1.52618\end{array}$

$$
\frac{a}{\lambda}=0.001538 \quad \frac{\alpha}{\beta}=0.05
$$

$\begin{array}{ll}0.00829 & -0.00051 \\ 0.02470 & -0.00164 \\ 0.08171 & -0.00658 \\ 0.17960 & -0.01894 \\ 0.37308 & -0.05814 \\ 0.90370 & -0.28042 \\ 1.51685 & -0.89134 \\ 1.73301 & -1.56409 \\ 1.65789 & -2.07115 \\ 1.49156 & -2.33420 \\ 1.39595 & -2.42419 \\ 1.41714 & -2.47358 \\ & \\ 0.72481 & -0.18434 \\ 1.30214 & -0.60509 \\ 1.63738 & -1.13804 \\ 1.73583 & -1.64962 \\ 1.66895 & -2.04244 \\ 1.53741 & -2.28151 \\ 1.42927 & -2.39248 \\ 1.39113 & -2.43684 \\ 1.42182 & -2.47832 \\ 1.48690 & -2.55589\end{array}$

$\begin{array}{ll}1.76479 & -0.01999 \\ 2.88897 & -0.03997 \\ 4.62287 & -0.09985 \\ 5.98575 & -0.19937 \\ 7.34348 & -0.39710 \\ 9.04340 & -0.97403 \\ 10.03805 & -1.84688 \\ 10.28876 & -2.55855 \\ 10.20242 & -3.07338 \\ 9.95758 & -3.38316 \\ 9.66781 & -3.50575 \\ 9.40970 & -3.47961 \\ & \\ 8.61706 & -0.77110 \\ 9.74712 & -1.48899 \\ 10.18239 & -2.12127 \\ 10.29181 & -2.64390 \\ 10.21575 & -3.04279 \\ 10.03680 & -3.31447 \\ 9.81341 & -3.46557 \\ 9.58897 & -3.51139 \\ 9.39449 & -3.47361 \\ 9.24961 & -3.37766\end{array}$

$1.44446-0.01998$ $2.09426-0.03993$ $2.09122-0.09948$ $3.66248-0.19729$ $4.07652-0.38503$ $4.62461-0.84669$ $3.38768-1.14005$ $1.11268-0.87801$ $-1.44998-0.33607$ $-3.73225 \quad 0.24713$ $-5.20175 \quad 0.75053$ $\begin{array}{ll}-5.42687 & 1.05955\end{array}$

$4.64346-0.70256$ $4.10212-1.09216$ $2.65109-1.09844$ $0.75318-0.81128$ $\begin{array}{lll}-1.26587 & -0.37906\end{array}$ $-3.13604 \quad 0.08345$ $-4.59704 \quad 0.51376$ $-5.40448 \quad 0.86388$ $-5.39125 \quad 1.07003$ $-4.53310 \quad 1.06871$
$0.00727-0.00046$ $0.02234-0.00152$ $0.08961-0.00698$ $0.23840-0.02183$ $0.59876-0.06883$ $1.82731-0.31069$ $3.61804-0.82331$ $4.55645-1.12256$ $4.47421-1.06124$ $3.38259-0.73621$ $1.43402-0.27019$ $\begin{array}{rr}-0.96802 & 0.26493\end{array}$

$1.38214-0.21052$ $2.93115-0.60917$ $4.06515-0.96913$ $4.60766-1.1352$ $4.51508-1.07645$ $3.79494-0.84825$ $2.50123-0.51693$ $0.77553-0.12247$ $\begin{array}{ll}-1.13433 & 0.30191 \\ -2.91625 & 0.69609\end{array}$ $\begin{array}{ll}1.44449 & -0.01998 \\ 2.09442 & -0.03995 \\ 2.99287 & -0.09968 \\ 3.67174 & -0.19855 \\ 4.32519 & -0.39252 \\ 5.01902 & -0.92344 \\ 5.10121 & -1.53669 \\ 4.83391 & -1.75287 \\ 4.61452 & -1.67777 \\ 4.57461 & -1.51144 \\ 4.66694 & -1.41583 \\ 4.77170 & -1.43702 \\ & \\ 4.87356 & -0.74449 \\ 5.14590 & -1.32195 \\ 5.01840 & -1.65723 \\ 4.79449 & -1.75569 \\ 4.62480 & -1.68883 \\ 4.56805 & -1.55729 \\ 4.61124 & -1.44915 \\ 4.70040 & -1.41102 \\ 4.77671 & -1.44170 \\ 4.80440 & -1.50679\end{array}$ 
$\frac{a}{\lambda}=0.001588 \quad \alpha=0.07$

$\beta \mathrm{h}$

$C(h, 0)$

0.01
0.02

0.02

0.05

0.10

0.20

1.00

1.50

2.00

2.50

3.50

$0.125 \pi$

0.250

$0.375 \pi$

$0.500 \pi$

$0.625 \pi$

$0.750 \pi$

$1.000 . \pi$

$1.250: \pi$
$S(h, 0)$

$1.76437-0.01998$ $2.88802-0.03993$ $4.61973-0.09959$ $\begin{array}{ll}4.97700 & -0.19816\end{array}$ $\begin{array}{lll}7.31661 & -0.39097 \\ 8.91717 & -0.91461\end{array}$ $8.91717-0.91461$ $9.69106-1.51038$ $9.84239-1.71662$ $9.92527-1.64690$ $10.37166-1.49489$ $\begin{array}{ll}10.37658 & -1.40907 \\ 10.62279 & -1.42752\end{array}$

$8.53279-0.73882$ $9.49259-1.30312$ $9.77569-1.62582$ $9.85143-1.71928$ $9.91635-1.65712$ $10.04648-1.53649$ $10.23953-1.43878$ $10.45230-1.40480$ $\begin{array}{ll}10.63689 & -1.43159 \\ 10.76617 & -1.48764\end{array}$
$0.00828 \quad-0.00068$ $0.02469-0.00213$ $0.02469-0.00213$ $0.08161-0.00822$ $0.17920-0.02251$ $0.37152-0.06544$ $0.89486-0.29640$ $1.49053-0.90865$ $1.69674-1.56822$ $1.62700-2.06069$ $1.47498-2.32058$ $1.40760-2.48240$

$0.71913-0.1 .9778$ $1.28330-0.62402$ $1.60596-1.15180$ $1.69930-1.65141$ $1.63723-2.03277$ $1.51659-2.26729$ $1.41887-2.38253$ $1.38489-2.43682$ $\begin{array}{ll}1.41167 & -2.48779 \\ 1.46772 & -2.56924\end{array}$
$E(h, 0)$

\begin{tabular}{|c|c|}
\hline $\begin{array}{l}1.76439 \\
2.88817 \\
4.62088 \\
5.98177 \\
7.33558 \\
9.02450 \\
0.00529 \\
0.25020 \\
0.16701 \\
9.93301 \\
9.65875 \\
9.41684\end{array}$ & $\begin{array}{l}-0.01998 \\
-0.03996 \\
-0.09979 \\
-0.19916 \\
-0.39630 \\
-0.96922 \\
-1.82919 \\
-2.52344 \\
-3.02073 \\
-3.31703 \\
-3.43320 \\
-3.40880\end{array}$ \\
\hline $\begin{array}{l}8.60194 \\
9.71946 \\
0.14654 \\
0.25315 \\
0.17982 \\
0.00848 \\
9.79622 \\
9.58461 \\
9.40266 \\
9.26816\end{array}$ & $\begin{array}{l}-1.47762 \\
-2.09768 \\
-2.00621 \\
-2.99133 \\
-3.25160 \\
-3.39526 \\
-3.43851 \\
-3.40321 \\
-3.31419\end{array}$ \\
\hline
\end{tabular}

$C(h, h)$

$\begin{array}{rr}1.44406 & -0.01997 \\ 2.09346 & -0.03991 \\ 2.98924 & -0.09931 \\ 3.65857 & -0.1965 .2 \\ 4.26910 & -0.38154 \\ 4.61119 & -0.82267 \\ 3.38365 & -1.05338 \\ 1.13061 & -0.72465 \\ -1.41585 & -0.14174 \\ -3.69154 & 0.42954 \\ -5.16439 & 0.84122 \\ -5.41168 & 0.97753 \\ 4.63123 & -0.68803 \\ 4.09133 & -1.03493 \\ 2.65459 & -0.98671 \\ 0.77402 & -0.64998 \\ -1.23256 & -0.18629 \\ -3.09637 & 0.27647 \\ -4.55634 & 0.66155 \\ -5.37105 & 0.91281 \\ -5.37845 & 0.97407 \\ -4.55403 & 0.80703\end{array}$

$S(h, h)$

$\begin{array}{rr}0.00726 & -0.00061 \\ 0.02233 & -0.00196 \\ 0.08951 & -0.00876 \\ 0.23800 & -0.02657 \\ 0.59725 & -0.08053 \\ 1.81964 & -0.34275 \\ 3.60306 & -0.86426 \\ 4.55167 & -1.12945 \\ 4.49033 & -0.99491 \\ 3.41663 & -0.56628 \\ 1.48086 & 0.00689 \\ -0.91533 & 0.60141 \\ 1.37696 & -0.23597 \\ 2.91768 & -0.65138 \\ 4.05166 & -1.00282 \\ 4.60557 & -1.13397 \\ 4.52968 & -1.01666 \\ 3.82446 & -0.71021 \\ 2.54215 & -0.29129 \\ 0.82513 & 0.17877 \\ -1.08180 & 0.03934 \\ -2.87419 & 1.00871\end{array}$

$E(h, h)$

$\begin{array}{ll}1.44409 & -0.01998 \\ 2.09362 & -0.03993 \\ 2.99088 & -0.09958 \\ 3.66779 & -0.19815 \\ 4.31749 & -0.39096 \\ 5.00264 & -0.91459 \\ 5.08350 & -1.51036 \\ 4.82938 & -1.71660 \\ 4.62458 & -1.64687 \\ 4.58783 & -1.49485 \\ 4.67043 & -1.40903 \\ 4.76248 & -1.42748 \\ 4.85973 & -0.73881 \\ 5.12657 & -1.30310 \\ 5.00424 & -1.62580 \\ 4.79230 & -1.71925 \\ 4.63408 & -1.65709 \\ 4.58188 & -1.53646 \\ 4.62078 & -1.43875 \\ 4.70003 & -1.40476 \\ 4.76684 & -1.43155 \\ 4.79075 & -1.48760\end{array}$

$$
\frac{\mathrm{a}}{\lambda}=0.001588 \quad \frac{\alpha}{\beta}=0.10
$$

$\begin{array}{ll}0.00828 & -0.00093 \\ 0.02466 & -0.00287 \\ 0.08146 & -0.01066 \\ 0.17861 & -0.02785 \\ 0.36921 & -0.07635 \\ 0.88181 & -0.32011 \\ 1.45223 & -0.93432 \\ 1.64438 & -1.57535 \\ 1.58200 & -2.04775 \\ 1.44915 & -2.30336 \\ 1.37615 & -2.41578 \\ 1.39115 & -2.49410 \\ 0.71072 & -0.21775 \\ 1.25573 & -0.65202 \\ 1.56040 & -1.17244 \\ 1.64680 & -1.05528 \\ 1.59108 & -2.02089 \\ 1.48512 & -2.24925 \\ 1.40119 & -2.37013 \\ 1.37259 & -2.43728 \\ 1.39444 & -2.50032 \\ 1.43923 & -2.58648\end{array}$

$\begin{aligned} 1.76379 & -0.01998 \\ 2.88697 & -0.03994 \\ 4.61788 & -0.09971 \\ 5.97580 & -0.19886 \\ 7.32376 & -0.39510 \\ 8.99633 & -0.96207 \\ 9.95670 & -1.80308 \\ 10.19314 & -2.47201 \\ 10.11447 & -2.94411 \\ 9.89585 & -3.22130 \\ 9.64331 & -3.32846 \\ 9.42380 & -3.30646 \\ & \\ 8.57935 & -0.76361 \\ 9.67834 & -1.46078 \\ 10.09345 & -2.06295 \\ 10.19597 & -2.55105 \\ 10.12654 & -2.91641 \\ 9.96601 & -3.16046 \\ 9.76942 & -3.29363 \\ 9.57569 & -3.33330 \\ 9.41104 & -3.30143 \\ 9.29073 & -3.22187\end{aligned}$

$1.44346-0.01996$ $2.09227-0.03987$ $2.98627-0.09906$ $3.65274-0.19537$ $4.25808-0.37633$ $4.59183-0.78698$ $3.37913-0.92479$ $1.15734-0.49620$ $-1.37029 \quad 0.14925$ $\begin{array}{ll}-3.64750 & 0.70348\end{array}$ $\begin{array}{ll}-5.14257 & 0.97912\end{array}$ $-5.43799 \quad 0.86001$

$\begin{array}{lll}4.61338 & -0.66619\end{array}$ $4.07652-0.95018$ $2.66110-0.82081$ $0.80460-0.40948$ $\begin{array}{ll}-1.18768 & 0.10228\end{array}$ $-3.04992 \quad 0.56611$ $-4.52046 \quad 0.88408$
-5.36012 $-5.36012 \quad 0.98893$ $\begin{array}{ll}-5.40896 & 0.83589 \\ -4.63584 & 0.42212\end{array}$

$\begin{array}{rr}0.00726 & -0.00083 \\ 0.02230 & -0.00263 \\ 0.08936 & -0.01144 \\ 0.23742 & -0.03365 \\ 0.59501 & -0.09802 \\ 1.80858 & -0.39065 \\ 3.58348 & -0.92607 \\ 4.55110 & -1.14148 \\ 4.52521 & -0.89703 \\ 3.48097 & -0.31157 \\ 1.55935 & 0.42358 \\ -0.84685 & 1.10979 \\ 1.36941 & -0.27401 \\ 2.89907 & -0.71467 \\ 4.03557 & -1.05425 \\ 4.60961 & -1.13392 \\ 4.56196 & -0.92865 \\ 3.88170 & -0.50375 \\ 2.61574 & 0.04762 \\ 0.90394 & 0.63215 \\ -1.01536 & 1.14945 \\ -2.84713 & 1.48627\end{array}$

$\begin{array}{ll}1.44349 & -0.01997 \\ 2.09242 & -0.03990 \\ 2.98790 & -0.09943 \\ 3.66188 & -0.19755 \\ 4.30600 & -0.38864 \\ 4.97835 & -0.90154 \\ 5.05724 & -1.47205 \\ 4.82165 & -1.66423 \\ 4.63693 & -1.60186 \\ 4.60446 & -1.46901 \\ 4.67436 & -1.39601 \\ 4.75019 & -1.41101 \\ 4.83917 & -0.73039 \\ 5.09798 & -1.27553 \\ 4.98301 & -1.58023 \\ 4.78784 & -1.66665 \\ 4.64537 & -1.61093 \\ 4.59933 & -1.50498 \\ 4.63259 & -1.42105 \\ 4.69898 & -1.39246 \\ 4.75372 & -1.41430 \\ 4.77291 & -1.45910\end{array}$


Bh

$C(h, 0)$

0.01

0.02

0.10

0.20

0.50

1.00

1.50

2.00

3.00

3.50

$0.125 \pi$

0.250 ii

$0.375 \pi$

$0.500 \pi$

$0.625 \pi$

0.750 i"

$0.875 \pi$

$1.125 \pi$

$1.250 \pi$ $2.88284-0.03982$ $4.60684-0.09893$ $\begin{array}{ll}5.95152-0.19560 \\ 7.26732 & -0.38106\end{array}$ $7.26732-0.38106$ $8.81419-0.85985$ $9.57972-1.35393$ $9.80208-1.50587$ $9.95872-1.46286$ $10.16037-1.37805$ $10.38278-1.33548$ $10.57669-1.34300$

$8.44531-0.70329$ . $9.82332-1.50765$ $10.09798-1.40019$ $10.27187-1.34963$ $10.44222-1.33355$ $10.58818-1.33355$ $10.70277-1.36586$
$1.76177-0.01994$ $9.94597-1.46896$

0.01

0.02
0.05

0.05
0.10

0.20

0.50
1.00

1.50

2.00

2.50

3.00
3.50

$\begin{array}{ll}1.75779-0.01989 \\ 2.87490 & -0.03964\end{array}$ $2.87490-0.0396$ $5.01296-0.10175$ $5.91296-0.19175$ $7.19387-0.36646$ $8.66074-0.78405$ $9.72049-1.15548$ $9.71508-1.25063$ $9.93666-1.23016$ $10.15040-1.19552$ $10.34594-1.18100$

$\begin{array}{llll}0.125 \pi & 8.31849 & -0.65302\end{array}$ $0.250 \pi \quad 9.20236-1.03990$ $0.375 \mathrm{~T} \quad 9.54586-1.2120$ $0.500 \div \quad 9.74774-1.25160$ $\begin{array}{llll}0.625 & 7 & 9.92081 & -1.23292\end{array}$ $0.750 \% \quad 10.08998-1.2039$ $0.875-10.25109-1.18552$ $1.000-10.39596-1.18043$ $\begin{array}{llll}1.125 & \text { T } & 10.52122 & -1.18329\end{array}$ $1.250=10.62871-1.18806$
$S(h, 0)$

$E(h, 0)$

$0.00826-0.00176$ $0.02458-0.00533$ $\begin{array}{lll}0.08097 & -0.01877\end{array}$ $606-0.04553$ $0.36162-0.1122$ $0.84011-0.39681$ $1.33409-1.01741$ $1.48600-1.60595$ $1.44297-2.02466$ $1.35816 \quad-2.26929$ $1.31558-2.4115$ $1.32309-2.5259$

$0.68361-0.28270$ $1.16949-0.74199$ $1.42120-1.24095$ $1.48778-1.67694$ $1.44907-2.00056$ $\begin{array}{lll}1.38030 & -2.21319\end{array}$ $1.32973-2.34760$

$1.31364-2.4443$

$1.32472-2.53392$

$1.34595-2.63005$
$1.76180-0.01995$

$2.88298-0.03988$

$4.60793-0.09943$

$5.95599-0.19783$

$\begin{array}{ll}7.28462 & -0.39115\end{array}$

$8.90389-0.93872$

$9.79934-1.71962$

$\begin{array}{rr}9.00965 & -2.31071 \\ 9.94434 & -2.70775\end{array}$

$9.77008-2.92974$

$9.57814-3.01162$
9.41936

$9.41936-2.99605$

$8.50501-0.74892$ $9.54451-1.40650$ $0.92207-1.95271$ $10.01207-2.37850$

$9.95424-2.68503$ $9.82504-2.88200$ $9.67276-2.98544$ $9.41038-2.99251$ $9.32742 \quad-2.93779$
$C(h, h)$

$\begin{array}{rr}1.44147 & -0.01993 \\ 2.08829 & -0.03975 \\ 2.97643 & -0.09822 \\ 3.63355 & -0.19156 \\ 4.22246 & -0.35918 \\ 4.53372 & -0.57079 \\ 3.37743 & -0.50695 \\ 1.24662 & 0.25611 \\ -1.26604 & 1.12367 \\ -3.64903 & 1.63509 \\ -5.36613 & 1.46871 \\ -5.95165 & 0.49874 \\ 4.55809 & -0.59498 \\ 4.03908 & -0.67501 \\ 2.69455 & -0.27978 \\ 0.90244 & 0.38441 \\ -1.08060 & 1.06750 \\ -3.00833 & 1.54638 \\ -4.61954 & 1.65031 \\ -5.66277 & 1.27492 \\ -5.94228 & 0.40463 \\ -5.35605 & -0.86485\end{array}$

$\frac{a}{\lambda}=0.001583$

$\stackrel{\alpha}{\beta}=0.40$ $\begin{array}{ll}0.00822 & -0.00340 \\ 0.02442 & -0.01023 \\ 0.07999 & -0.03484 \\ 0.17283 & -0.08031 \\ 0.34704 & -0.18178 \\ 0.76433 & -0.54032 \\ 1.13566 & -1.17267 \\ 1.23078 & -1.08898 \\ 1.21029 & -2.04274 \\ 1.17564 & -2.27528 \\ 1.16112 & -2.44438 \\ 1.16300 & -2.58757 \\ 0.63336 & -0.40559 \\ 1.02011 & -0.90772 \\ 1.19218 & -1.37509 \\ 1.23175 & -1.74855 \\ 1.21305 & -2.02175 \\ 1.18403 & -2.21721 \\ 1.16565 & -2.36440 \\ 1.16055 & -2.48660 \\ 1.16340 & -2.59690 \\ 1.16317 & -2.70012\end{array}$

$\begin{array}{ll}1.75781 & -0.01991 \\ 2.87502 & -0.03976 \\ 4.58811 & -0.09888 \\ 5.91667 & -0.19580 \\ 7.20751 & -0.38341 \\ 8.72554 & -0.89426 \\ 9.50447 & -1.56795 \\ 9.67094 & -2.02975 \\ 9.62591 & -2.31076 \\ 9.51500 & -2.45322 \\ 9.40419 & -2.50105 \\ 9.32105 & -2.49325 \\ 8.36052 & -0.72066 \\ 9.29088 & -1.30591 \\ 9.60334 & -1.75548 \\ 9.07273 & -2.07962 \\ 9.63257 & -2.29548 \\ 9.54886 & -2.42382 \\ 9.45746 & -2.48625 \\ 9.37727 & -2.50299 \\ 9.31661 & -2.49149 \\ 9.27714 & -2.46561\end{array}$

$S(h, h)$

$\mathrm{E}(\mathrm{h}, \mathrm{h})$

$0.00724-0.00155$ $0.02222-0.00486$ $0.08887-0.02034$ $0.23550-0.05715$ $0.58781-0.15586$ $\begin{array}{ll}1.77568 & -0.54887\end{array}$ $3.54254-1.13575$ $4.60680-1.19524$ $\begin{array}{lll}4.73717 & -0.58078\end{array}$ $3.81351 \quad 0.54937$ $\begin{array}{ll}1.89356 & 1.85924\end{array}$ $1.34620-0.3995$ $2.85049-0.92572$ $4.01706-1.23321$ $4.68596-1.14809$ $4.76260-0.64626$ $4.18833 \quad 0.18863$ $2.96885 \quad 1.20485$ $\begin{array}{rr}1.20611 & 2.20287\end{array}$ $-3.06876 \quad 3.23018$
$1.44149-0.01994$ $2.08844-0.0398$ $2.97800-0.09892$ $3.64231-0.19558$ $4.26819-0.38103$ $4.89967-0.85981$ $4.97217-1.35388$ $4.78907-1.50581$ $4.65803-1.46279$ $4.63654-1.37799$ $4.67663-1.33541$ $4.71639-1.34292$

$\begin{array}{ll}.77226 & -0.70325\end{array}$ $5.00604-1.18925$ $4.91252-1.44100$ $4.66371-1.46890$ $4.63340-1.40013$ $4.65314-1.34956$ $4.68996-1.33347$ $4.71814-1.34455$ $4.72736-1.36578$

$\begin{array}{rr}1.43749 & -0.01986 \\ 2.08037 & -0.03950 \\ 2.95699 & -0.09655 \\ 3.59628 & -0.18408 \\ 4.15613 & -0.32587 \\ 4.44644 & -0.44960 \\ 3.43845 & 0.29453 \\ 1.44026 & 1.76424 \\ -1.26973 & 3.19280 \\ -4.36906 & 3.74916 \\ -7.28774 & 2.71769 \\ -9.19467 & -0.26136 \\ 4.46634 & -0.45874 \\ 4.01887 & -0.15197 \\ 2.82228 & 0.77140 \\ 1.09437 & 1.98827 \\ -1.05355 & 3.10813 \\ -3.46581 & 3.72476 \\ -5.89095 & 3.46808 \\ -7.97179 & 2.07427 \\ -9.26375 & -0.53520 \\ -9.28272 & -4.18595\end{array}$

$\begin{array}{rr}0.00720 & -0.00299 \\ 0.02207 & -0.00929 \\ 0.08790 & -0.03798 \\ 0.23179 & -0.10359 \\ 0.57454 & -0.26960 \\ 1.72699 & -0.86013 \\ 3.56799 & -1.57465 \\ 4.99034 & -1.35894 \\ 5.64149 & 0.04603 \\ 5.08581 & 2.48148 \\ 2.95308 & 5.35604 \\ -0.85995 & 7.67866 \\ 1.30833 & -0.64583 \\ 2.81187 & -1.35128 \\ 4.13843 & -1.62696 \\ 5.13901 & -1.23309 \\ 5.62943 & -0.09581 \\ 5.39416 & 1.70175 \\ 4.23641 & 3.91126 \\ 2.03835 & 0.12060 \\ -1.17725 & 7.78734 \\ -5.18940 & 8.31119\end{array}$

$\begin{array}{ll}1.43751 & -0.01988 \\ 2.08049 & -0.03963 \\ 2.95834 & -0.09790 \\ 3.60376 & -0.19171 \\ 4.19475 & -0.36640 \\ 4.75224 & -0.78397 \\ 4.81296 & -1.15538 \\ 4.70210 & -1.25053 \\ 4.63600 & -1.23004 \\ 4.62660 & -1.19540 \\ 4.63982 & -1.18088 \\ 4.65077 & -1.18276 \\ 4.64544 & -0.65295 \\ 4.83636 & -1.03981 \\ 4.77443 & -1.21191 \\ 4.68863 & -1.25150 \\ 4.63857 & -1.23281 \\ 4.62543 & -1.20379 \\ 4.63238 & -1.18541 \\ 4.64372 & -1.18031 \\ 4.65120 & -1.18316 \\ 4.65334 & -1.18794\end{array}$


$\underset{\lambda}{a}=0.002381 \quad \stackrel{\alpha}{\beta}=0.00$

$\beta \mathrm{h}$

$C(h, 0)$

0.01
0.02
0.05
0.10
0.20
0.50
1.00
1.50
2.00
2.50
3.00
3.50

$0.125 \pi$ $0.250 \pi$ $0.375 \pi$ $0.500 \pi$ $0.625 \pi$ $0.750 \pi$ $0.875 \pi$ $1.000 \pi$ $1.125 \pi$ $1.250 \pi$ $s(h, 0)$

$E(h, 0)$

$\begin{array}{ll}0.00607 & -0.00010 \\ 0.02003 & -0.00040 \\ 0.07440 & -0.00250 \\ 0.17185 & -0.00998 \\ 0.36764 & -0.03973 \\ 0.91657 & -0.23980 \\ 1.57566 & -0.84735 \\ 1.81881 & -1.55614 \\ 1.72833 & -2.10440 \\ 1.52005 & -2.37658 \\ 1.39480 & -2.43692 \\ 1.42471 & -2.44632 \\ 0.72960 & -0.15030 \\ 1.34108 & -0.55678 \\ 1.70995 & -1.10362 \\ 1.82209 & -1.64821 \\ 1.74182 & -2.07344 \\ 1.57849 & -2.32572 \\ 1.43912 & -2.42379 \\ 1.38827 & -2.43754 \\ 1.43136 & -2.44894 \\ 1.52594 & -2.51436\end{array}$

$C(h, h)$

$\begin{array}{ll}1.25308 & -0.02003 \\ 2.20110 & -0.04000 \\ 3.84136 & -0.09098 \\ 5.19159 & -.3 .19988 \\ 6.55454 & -1.39910 \\ 8.28093 & -0.98613 \\ 9.31091 & -1.89209 \\ 9.57672 & -2.64926 \\ 9.48194 & -3.21070 \\ 9.20771 & -3.55689 \\ 8.87522 & -3.69714 \\ 8.57171 & -3.06007 \\ & \\ 7.84520 & -0.77867 \\ 9.00689 & -1.51789 \\ 9.46325 & -2.18132 \\ 9.58000 & -2.74142 \\ 9.49665 & -3.17692 \\ 9.29716 & -3.47935 \\ 9.04332 & -3.65081 \\ 8.78330 & -3.76370 \\ 8.55357 & -3.65892 \\ 8.37909 & -3.54320\end{array}$

$S(h, h)$

$\begin{array}{rr}1.10053 & -0.02000 \\ 1.70925 & -0.03969 \\ 2.59447 & -0.09950 \\ 3.26957 & -0.19922 \\ 3.89762 & -0.39381 \\ 4.30210 & -0.90753 \\ 3.17820 & -1.36036 \\ 1.03347 & -1.26590 \\ -1.38473 & -0.82459 \\ -3.55233 & -0.21103 \\ -4.97588 & 0.52539 \\ -5.20093 & 1.28057 \\ 4.29911 & -0.73972 \\ 3.84241 & -1.23740 \\ 2.48555 & -1.38208 \\ 0.69413 & -1.21871 \\ -1.21088 & -0.86379 \\ -2.98279 & -0.40132 \\ -4.38561 & 0.14296 \\ -5.17621 & 0.74607 \\ -5.16570 & 1.32720 \\ -4.30971 & 1.75407\end{array}$

$0.00547-0.00010$ $0.01754-0.00040$ $0.07383-0.00250$ $0.20352-0.00997$ $0.52682-0.03947$ $1.65773-0.23010$ $3.32415-0.72183$ $4.18011-1.10952$ $4.08796-1.23144$ $3.08180-1.16358$ $\begin{array}{rr}1.27443 & -0.96707 \\ -0.98782 & -0.58017\end{array}$

$1.24515-0.14650$ $2.68573-0.50354$ $3.73630-0.88698$ $4.22448-1.14281$ $4.12627-1.23045$ $3.46102-1.19625$ $2.26800-1.08391$ $0.65714-0.88042$ $\begin{array}{ll}-1.14512 & -0.54516 \\ -2.82529 & -0.07910\end{array}$

$$
\frac{a}{\lambda}=0.002331 \quad \frac{\alpha}{\beta}=0.005
$$

$\begin{array}{ll}0.00607 & -0.00013 \\ 0.02002 & -0.00050 \\ 0.07438 & -0.00287 \\ 0.17175 & -0.01084 \\ 0.36725 & -0.04153 \\ 0.91428 & -0.24386 \\ 1.56860 & -0.85174 \\ 1.80890 & -1.55671 \\ 1.72008 & -2.10053 \\ 1.51644 & -2.37170 \\ 1.39453 & -2.43540 \\ 1.42342 & -2.44940 \\ 0.72813 & -0.15368 \\ 1.33609 & -0.56162 \\ 1.70145 & -1.10701 \\ 1.81213 & -1.64809 \\ 1.73331 & -2.06981 \\ 1.57348 & -2.32064 \\ 1.43760 & -2.42016 \\ 1.38819 & -2.43739 \\ 1.42985 & -2.45227 \\ 1.52096 & -2.51912\end{array}$

$\begin{array}{rr}1.10043 & -0.02000 \\ 1.70905 & -0.03999 \\ 2.59398 & -0.09986 \\ 3.26858 & -0.19904 \\ 3.89571 & -0.39300 \\ 4.29846 & -0.90186 \\ 3.17662 & -1.33975 \\ 1.03809 & -1.22970 \\ -1.37397 & -0.77918 \\ -3.53558 & -0.16802 \\ -4.95312 & 0.54699 \\ -5.17767 & 1.25970 \\ 4.29587 & -0.73627 \\ 3.83918 & -1.22386 \\ 2.48597 & -1.35558 \\ 0.69969 & -1.18087 \\ -1.20053 & -0.81875 \\ -2.96784 & -0.35599 \\ -4.36566 & 0.17808 \\ -5.15238 & 0.75757 \\ -5.14279 & 1.30286 \\ -4.29524 & 1.68847\end{array}$

$0.00547-0.00013$ $0.01754-0.00049$ $0.07380-0.00287$ $0.20342-0.01098$ $0.52644-0.04204$ $1.65563-0.23731$ $3.31927-0.73082$ $4.17638-1.11029$ $4.08798-1.21573$ $3.08525-1.12458$ $1.28288-0.90281$ $-0.97039-0.50152$

$1.24376-0.15221$ $2.68173-0.51299$ $3.73131-0.89412$ $4.22122-1.14170$ $4.12601-1.21619$ $3.46350-1.16451$ $\begin{array}{ll}3.46350 & -1.16451 \\ 2.27349 & -1.03195\end{array}$ $-1.12700-0.46630$ $\begin{array}{ll}-1.12700 & -0.46630 \\ -2.80029 & -0.00708\end{array}$
$E(h, h)$

$\begin{array}{ll}1.10055 & -0.02000 \\ 1.70937 & -0.03999 \\ 2.59580 & -0.09994 \\ 3.27727 & -0.19955 \\ 3.93958 & -0.39645 \\ 4.65545 & -0.94605 \\ 4.74097 & -1.60535 \\ 4.43761 & -1.84857 \\ 4.17700 & -1.75811 \\ 4.12796 & -1.54984 \\ 4.24994 & -1.42460 \\ 4.39469 & -1.45451 \\ 4.50344 & -0.75895 \\ 4.79000 & -1.37071 \\ 4.64858 & -1.73968 \\ 4.39165 & -1.85185 \\ 4.18954 & -1.77100 \\ 4.11958 & -1.60828 \\ 4.17566 & -1.46892 \\ 4.29543 & -1.41806 \\ 4.40181 & -1.46116 \\ 4.44176 & -1.55574\end{array}$




$$
\frac{a}{\lambda}=0.002381 \quad \frac{\alpha}{\beta}=0.01
$$

$\beta \mathrm{h}$

$C(h, 0)$

0.01

0.02

0.10

0.10

0.20

1.00

1.50

-1
-1.50

2.50

3.50

0.125

$0.250 \pi$

$0.375 \pi$

$0.625 \pi$

.

$1.125 \pi$

$1.250 \pi$

$\begin{array}{ll}1.25286 & -0.02000 \\ 2.20057 & -0.03998 \\ 3.83927 & -0.09989 \\ 5.18486 & -0.19935 \\ 0.53119 & -0.39566 \\ 8.15685 & -0.94746 \\ 8.93459 & -1.59128 \\ 9.04425 & -1.82834 \\ 9.08015 & -1.74169 \\ 9.25624 & -1.54260 \\ 9.55392 & -1.42395 \\ 9.84375 & -1.45188 \\ 7.76485 & -0.75602 \\ 8.74120 & -1.36076 \\ 9.00809 & -1.72273 \\ 9.04580 & -1.83202 \\ 9.07379 & -1.75466 \\ 9.18902 & -1.59827 \\ 9.39638 & -1.46581 \\ 9.64283 & -1.41781 \\ 9.86015 & -1.45808 \\ 10.00263 & -1.54585\end{array}$

$10.00263-1.45808$
S(h, 0!

$0.00607-0.01016$ $0.02002-0.00060$ $0.07435-0.00324$ $0.17166-0.01169$ $0.36686-0.04333$ $0.91199-0.24790$ $1.79907-1.55733$ $1.71189-2.09677$ $1.51277-2.36696$ $1.39411-2.43391$ $1.42203-2.45238$

$0.72668-0.15706$ $1.33113-0.56645$ $1.69300-1.11040$ $1.80225-1.648 \mathrm{C} 1$ $1.72485-2.06630$ $1.56844-2.31570$ $1.43597-2.41663$ $1.38796-2.43725$

$\begin{array}{ll}1.42823 & -2.45549 \\ 1.51599 & -2.52372\end{array}$ $\begin{array}{lll}1.56158 & -0.85612\end{array}$

$$
E(h, 0)
$$

$1.25288-0.02000$ $2.20070-0.03999$ $3.84036-0.09995$ $5.13960-0.19977$ $6.55056-0.39869$ $8.27136 \quad-0.98372$ $9.29412-1.88292$ $9.55683-2.63075$ $9.19571-3.52111$ $\begin{array}{lll}9.19571 & -3.52111 \\ 8.87225 & -3.65764\end{array}$ $\begin{array}{ll}8.87225 & -3.65764 \\ 8.57842 & -3.62762\end{array}$

$7.83756-0.77714$ $8.99277-1.51204$ $9.44482-2.16951$ $9.47822-3.14942$ $9.47322-3.14942$ $9.03559-3.61261$ $8.78311-3.01261$ $8.78311-3.06400$ $\begin{array}{ll}8.56090 & -3.82072 \\ 8.39279 & -3.50925\end{array}$

$$
C(h, h)
$$

$1.10033-0.01799$ $1.70885-10.03998$ $2.59348-0.09982$ $3.26759-0.17836$ $3.89381-0.39219$ $4.29483-0.89619$ $3.17509-1.31919$ $1.04270-1.19368$ $-1.36338-0.73384$ $-3.51935-0.12509$ $-4.93145 \quad 0.50858$ $-5.15602 \quad 1.23911$

$4.29264 \quad-0.73283$ $3.83599-1.21030$ $2.48642-1.32915$ $0.70520-1.14304$ $\begin{array}{ll}-1.19035 & -0.77376\end{array}$ $\begin{array}{lll}-2.95329 & -0.31074\end{array}$ $-4.34650 \quad 0.21314$ $-5.121530 .76710$ $\begin{array}{ll}-5.12153 & 1.27882 \\ -4.282 .40 & 1.62354\end{array}$
$S(h, h)$

$0.00547-0.00015$ $0.01753-0.00058$ $0.07378-0.00323$ $0.20332-0.01199$ $0.52605-0.04461$ $1.65354-0.24452$ $3.31449-0.73982$ $4.17284-1.11112$ $=.08910-1.08560$

$-0.95330-0.42311$

$\begin{array}{lll}1.24238 & -0.15792\end{array}$ $\begin{array}{lll}2.67778 & -0.52243\end{array}$ $3.72645-0.00130$ $\$ .21816-1.14067$ i. $12605-1.20201$ $3.46636-1.13284$ $2.27937-0.98009$ $\begin{array}{lll}0.67857 & -0.74036\end{array}$ $-1.10928-0.38769$ $-2.77649 \quad 0.06482$

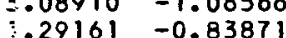

$E(h, h)$

$1.10035-0.02000$ $1.70897-0.03998$ $2.59480-0.09989$ $3.27528-0.19935$ $4.64706-0.39565$ $4.73190-1.59127$ $4.43612-1.82882$ $4.18434-1.74166$ $4.13728-1.54255$ $4.25265-1.42390$ $4.38833-1.45182$

$4.49639-0.75602$ $4.78003-1.36075$ $4.64151-1.72272$ $4.39155-1.83200$ $4.19640-1.75463$ $4.12930-1.59823$ $4.18252-1.46576$ $4.2943-1.41775$

\begin{tabular}{|c|c|}
\hline $\begin{array}{l}0.00606 \\
0.02001 \\
0.07430 \\
0.17146 \\
0.36607 \\
0.90744 \\
1.54768 \\
1.77963 \\
1.69563 \\
1.50527 \\
1.39285 \\
1.41891\end{array}$ & $\begin{array}{l}-0 . \\
-0 . \\
-0 . \\
-0 . \\
-0 . \\
-0 . \\
-0 . \\
-1 . \\
-2 . \\
-2 . \\
-2 . \\
-2 .\end{array}$ \\
\hline $\begin{array}{l}0.72377 \\
1.32128 \\
1.67628 \\
1.78271 \\
1.70809 \\
1.55831 \\
1.43239 \\
1.38707 \\
1.42469\end{array}$ & $\begin{array}{l}-0.16380 \\
-0.57607 \\
-1.11717 \\
-1.04800 \\
-2.05956 \\
-2.30623 \\
-2.40980 \\
-2.43690 \\
-2.46160 \\
-2.5324 .\end{array}$ \\
\hline
\end{tabular}
$4.43210-1.54579$

$$
\frac{a}{\lambda}=0.002381 \quad \frac{\alpha}{\beta}=0.02
$$

$\begin{array}{ll}1.25268 & -0.01999 \\ 2.20030 & -0.03998 \\ 3.83936 & -0.09992 \\ 5.13760 & -0.19967 \\ 6.54660 & -0.39828 \\ 8.26181 & -0.98128 \\ 9.27741 & -1.87380 \\ 9.53705 & -2.61242 \\ 9.44573 & -3.15472 \\ 9.18366 & -3.48585 \\ 8.86897 & -3.61874 \\ 8.58451 & -3.58975 \\ & \\ 7.82993 & -0.77561 \\ 8.97871 & -1.50622 \\ 9.42649 & -2.15729 \\ 9.54024 & -2.70179 \\ 9.45988 & -3.12226 \\ 9.26887 & -3.41198 \\ 9.02768 & -3.57498 \\ 8.78252 & -3.62492 \\ 8.56760 & -3.58308 \\ 8.40563 & -3.47572\end{array}$

$1.10013-0.01999$ $1.70846-0.03997$ $3.26562-0.19850$ $3.89002-0.39057$

$4.28766-0.88489$

$3.17215-1.27825$ $1.05186-1.12185$ $-1.34276-0.64331$ $-3.48846-0.03941$ $-4.89137 \quad 0.61171$ $-5.11755 \quad 1.19873$

$4.28022-0.72596$ $3.82974-1.18328$ $2.48742-1.27650$ $-1.17048 \quad-0.68397$ $-2.92539-0.22041$ $-4.31052 \quad 0.28306$ $-5.08853 \quad 0.79228$ $-5.08388 \quad 1.23163$ $2.59248-0.09974$ $0.71614-1.06759$
$0.00547-0.00021$ $0.01752-0.00075$ $\begin{array}{lll}0.07373 & -0.00397\end{array}$ $0.20312,-0.01400$ $0.52527-0.04974$

$\begin{array}{lll}1.64941 & -0.25892\end{array}$

$3.30519-0.75785$

$4.16636-1.11298$

$4.08987-1.16905$

$3.09800-1.00799$

$\begin{array}{rr}1.30991 & -0.71094 \\ -0.92013 & -0.26696\end{array}$

$\begin{array}{lll}1.23965 & -0.16931\end{array}$ $3.71712-0.91574$ $4.21269-1.13880$ $4.12703-1.17387$ $3.47322,-1.06968$ $2.29227-0.87665$ $0.70064-0.60103$ $-1.07504 \quad-0.23112$ $2.67003-0.54132$
$1.10015-0.01999$ $1.70857-0.03997$ $2.59380-0.09983$ $3.27330-0.19914$ $3.93181-0.39486$ $4.63870-0.93690$ $4.72287-1.57736$

$4.43449-1.80937$

$4.19123-1.72540$

$4.14607-1.53505$

$4.25518 \quad-1.42263$

$-1.44869$

$4.48936 \quad-0.75311$ $4.77012-1.35089$ $4.63443-1.70599$ $4.39126-1.81246$ $4.20281 .-1.73786$ 4. $13847-1.58808$ $4.18898-1.46217$ $4.29542-1.41685$ $\begin{array}{ll}4.38856 & -1.45447 \\ 4.42306 & -1.53592\end{array}$ 
$\beta \mathrm{h}$

0.01
0.02
0.05
0.10
0.20
0.50
1.00
1.50
2.00
2.50
3.00
3.50

0.125 $0.250 \pi$

0.375

0.625

0.750

$0.875 \pi$

$1.125 \pi$

$1.250 \%$

$$
\mathrm{C}(\mathrm{h}, 0)
$$

$1.25246-0.01979$ $2.19977-0.0399$ $5.18089-0.19895$ $6.52343-0.39408$ $8.14018-0.93239$ $8.91657-1.56364$ $9.04083-1.79026$ $9.09349-1.70936$ $9.27329-1.52740$ $9.55882-1.42090$ $9.83219-1.44523$

$\begin{array}{ll}7.75081 & -0.75022 \\ 8.72143 & -1.34115\end{array}$ $8.72143-1.34115$ $8.99393-1.68952$ $9.04503-1.79325$ $9.08620-1.72133$ $9.20682-1.57792$ $9.40891-1.45824$ $9.98513-1.52620$ $3.83727-0.09978$ $9.84771-1.4506$

$\mathrm{S}(\mathrm{h}, 0)$

$\begin{array}{ll}0.00606 & -0.00028 \\ 0.02000 & -0.00100 \\ 0.07425 & -0.00473 \\ 0.17126 & -0.01510 \\ 0.36529 & -0.05052 \\ 0.90292 & -0.26399 \\ 1.53395 & -0.87355 \\ 1.76049 & -1.56016 \\ 1.67956 & -2.08284 \\ 1.49757 & -2.34931 \\ 1.39106 & -2.42842 \\ 1.41538 & -2.46335 \\ 0.72088 & -0.17051 \\ 1.31152 & -0.58563 \\ 1.65979 & -1.12395 \\ 1.76348 & -1.64819 \\ 1.69153 & -2.05329 \\ 1.54810 & -2.29730 \\ 1.42841 & -2.40351 \\ 1.38562 & -2.43670 \\ 1.42076 & -2.46733 \\ 1.49634 & -2.54063\end{array}$

$0.00606-0.00028$ $0.02000-0.00100$ $0.07425-0.00473$ 20.05052 $1.53395-0.87355$ $1.67956-2.08284$ $1.49757-2.34931$ $1.41538-2.46335$

$-0.17051$ $-0.58563$ $.09153-2.05329$ .49634 -2.46733

\begin{tabular}{|c|c|c|c|c|}
\hline $\begin{array}{l}0.01 \\
0.02 \\
0.05 \\
0.10 \\
0.20 \\
0.50 \\
1.00 \\
1.50 \\
2.00 \\
2.50 \\
3.00 \\
3.50\end{array}$ & $\begin{array}{l}1.25206 \\
2.19897 \\
3.83528 \\
5.17694 \\
6.51571 \\
8.12365 \\
8.89870 \\
9.03683 \\
9.10513 \\
9.28836 \\
9.56301 \\
9.82192\end{array}$ & $\begin{array}{l}-0.01998 \\
-0.03995 \\
-0.09968 \\
-0.19854 \\
-0.39252 \\
-0.92343 \\
-1.53667 \\
-1.75285 \\
-1.67775 \\
-1.51145 \\
-1.41585 \\
-1.43704\end{array}$ & $\begin{array}{l}0.00606 \\
0.01999 \\
0.07415 \\
0.17086 \\
0.36372 \\
0.89396 \\
1.50698 \\
1.72309 \\
1.64796 \\
1.48163 \\
1.38601 \\
1.40720\end{array}$ & $\begin{array}{l}-0.00040 \\
-0.00140 \\
-0.00621 \\
-0.01851 \\
-0.05767 \\
-0.27993 \\
-0.89082 \\
-1.56355 \\
-2.07060 \\
-2.33364 \\
-2.42363 \\
-2.47302\end{array}$ \\
\hline $\begin{array}{l}0.125 \pi \\
0.250 \pi \\
0.375 \pi \\
0.500 \pi \\
0.625 \pi \\
0.750 \pi \\
0.875 \pi \\
1.000 \pi \\
1.125 \pi \\
1.250 \pi\end{array}$ & $\begin{array}{l}7.73688 \\
8.70188 \\
8.97977 \\
9.04353 \\
9.09699 \\
9.22256 \\
9.41990 \\
9.64259 \\
9.83668 \\
9.96973\end{array}$ & $\begin{array}{l}-0.74448 \\
-1.32193 \\
-1.65721 \\
-1.75567 \\
-1.68882 \\
-1.55729 \\
-1.44916 \\
-1.41103 \\
-1.44172 \\
-1.50681\end{array}$ & $\begin{array}{l}0.71514 \\
1.29231 \\
1.62749 \\
1.72591 \\
1.65902 \\
1.52748 \\
1.41933 \\
1.38119 \\
1.41188 \\
1.47696\end{array}$ & $\begin{array}{l}-0.18386 \\
-0.60458 \\
-1.13752 \\
-1.04909 \\
-2.04190 \\
-2.28096 \\
-2.39192 \\
-2.43628 \\
-2.47776 \\
-2.55543\end{array}$ \\
\hline
\end{tabular}

E(h, 0:

$\mathrm{C}(\mathrm{h}, \mathrm{h})$

$\begin{array}{ll}1.25248 & -0.01999 \\ 2.19990 & -0.03998 \\ 3.83836 & -3.09989 \\ 5.18561 & -0.19956 \\ 6.54264 & -1.39788 \\ 8.25228 & -0.97884 \\ 9.26077 & -1.86475 \\ 9.51739 & -2.59427 \\ 9.42775 & -3.12724 \\ 9.17155 & -5.45109 \\ 8.86540 & -3.58045 \\ 8.59001 & -3.55244 \\ & \\ 7.82231 & -0.77409 \\ 8.96470 & -1.50042 \\ 9.40824 & -2.14517 \\ 9.52052 & -2.08227 \\ 9.44162 & -3.09541 \\ 9.25472 & -3.37899 \\ 9.01961 & -3.53792 \\ 8.78157 & -3.58645 \\ 8.57369 & -3.54601 \\ 8.41762 & -3.44259\end{array}$

$\frac{\mathrm{a}}{\lambda}=0.002381 \quad \frac{\alpha}{\beta}=0.05$
$S(h, h)$

$1.09993-0.01959$ $1.70806-0.03945$ $2.59149-0.09966$ $3.26365-0.19815$ $3.88624-0.38895$ $4.28057-0.87365$ $3.16939-1.23753$ $1.06095-1.05031$ $-1.32284-0.5530$ $-3.45964 \quad 0.04603$ $\begin{array}{ll}-4.85563 & 0.65483 \\ -5.08545 & 1.15930\end{array}$ $4.27986-0.7191$ $3.82366-1.1564$ $2.48855-1.2241$ $0.72694-0.99242$ $-1.15126-0.59436$ $-2.89908-0.1303$ $\begin{array}{lll}-4.27766 & 0.35278\end{array}$ $-5.05223 \quad 0.81565$ $\begin{array}{rl}-5.05268 & 1.18558\end{array}$ $-4.24724 \quad 1.36984$
$0.00547-0.00026$ $0.01752-0.00093$ $0.07368-0.00471$ $0.20292-0.01602$ $0.52450-0.05486$ $1.64534-0.27329$ $3.29625-0.77594$ $4.16067-1.11508$ $4.09267-1.13828$ $3.10850-0.93056$ $1.32928-0.58367$ $-0.88829-0.11150$

\section{$1.23694 \quad-0.18068$} $2.66249-0.56021$ $3.70830-0.93031$ $4.20807-1.13720$ $4.12920-1.14602$ $3.48162-1.00674$ $2.30671-0.77353$ $0.72332-0.46229$ $-1.04236 \quad-0.07523$ $\begin{array}{ll}-2.69304 & 0.35176\end{array}$
$E(h, h)$

$1.09995-0.01999$ $.70817-0.03996$ $2.59280-0.09978$ $3.27132-0.19894$ $4.63038-0.39407$ $4.71388-1.56362$ $4.43270-1.79023$ $4.19768-1.70932$ $4.15434-1.52734$ $\begin{array}{ll}4.25754 & -1.42083 \\ 4.37677 & -1.44515\end{array}$

$4.48235-0.7502$ $4.76026-1.34114$ $4.62736-1.68950$ $4.39078-1.79322$ $4.20881-1.72129$ $4.14711-1.57786$ $4.19505-1.45818$ $4.29539-1.41539$ $4.38254-1.45054$ $4.41460-1.52612$

\begin{abstract}
$1.25208-0.01998$ $2.19910-0.03996$ $3.83636-0.39983$ $\begin{array}{ll}5.18162 & -0.19935 \\ 0.53472 & -0.39707\end{array}$ $\begin{array}{ll}0.53472 & -0.39707 \\ 8.23329 & -0.07399\end{array}$ $0.23329-0.97399$ $9.22772-1.84681$ $9.47839-2.55847$ $9.39203-3.07328$ $9.1471-3.38304$ $\begin{array}{ll}8.85741 & -3.5 .0563 \\ 8.59931 & -3.47948\end{array}$

$7.80712-0.77106$ $8.93684-1.48893$ $9.37204-2.12120$ $9.48143-2.64381$ $9.40535-3.04269$ $9.22640-3.31436$ $9.00301-3.45546$ $8.77857-3.51126$ $\begin{array}{ll}8.58409 & -3.47348 \\ 8.43921 & -3.3 ? 753\end{array}$
\end{abstract}

$\begin{array}{rr}1.09953 & -0.01998 \\ 1.70726 & -0.03993 \\ 2.58950 & -0.09950 \\ 3.25973 & -0.19744 \\ 3.87874 & -0.38573 \\ 4.26669 & -0.85129 \\ 3.16439 & -1.15673 \\ 1.07897 & -0.90803 \\ -1.28513 & -0.37292 \\ -3.40824 & 0.21641 \\ -4.79699 & 0.74122 \\ -5.04001 & 1.08362 \\ & \\ 4.26732 & -0.70550 \\ 3.81199 & -1.10307 \\ 2.49123 & -1.12012 \\ 0.74820 & -0.84285 \\ -1.11474 & -0.41579 \\ -2.85120 & 0.04940 \\ -4.22125 & 0.49186 \\ -4.99447 & 0.86309 \\ -5.00929 & 1.09663 \\ -4.23760 & 1.12441\end{array}$

$0.00546 \quad-0.00037$ $0.01750-0.00128$ $0.07358-0.00617$ $0.20253-0.02005$ $\begin{array}{lll}0.52298 & -0.06509\end{array}$ $1.63737-0.30196$ $\begin{array}{lll}3.27946 & -0.81227\end{array}$ $4.15166-1.12002$ $4.10201-1.07753$ $3.13430-0.77626$ $1.37125-0.33018$

$1.23162-0.20335$ $2.64801-0.59795$ $3.09219-0.95980$ $4.20138-1.13479$ $4.13718-1.0911$ $3.50303-0.88145$ $2.34015-0.56799$ $\begin{array}{ll}0.77050 & -0.18607\end{array}$ $-0.98158 \quad 0.23535$

$\begin{array}{ll}1.09955 & -0.01998 \\ 1.70737 & -0.03994 \\ 2.59081 & -0.09967 \\ 3.26736 & -0.19853 \\ 3.92021 & -0.39250 \\ 4.61386 & -0.92341 \\ 4.69601 & -1.53664 \\ 4.42871 & -1.75281 \\ 4.20932 & -1.67770 \\ 4.16941 & -1.51138 \\ 4.26174 & -1.41577 \\ 4.36650 & -1.43696 \\ 4.46842 & -0.74446 \\ 4.74071 & -1.32191 \\ 4.61320 & -1.65718 \\ 4.38929 & -1.75563 \\ 4.21961 & -1.68876 \\ 4.16286 & -1.55723 \\ 4.20604 & -1.44908 \\ 4.29521 & -1.41095 \\ 4.37151 & -1.44164 \\ 4.39921 & -1.50672\end{array}$


Bh

$$
\mathrm{C}(\mathrm{h}, 0)
$$

0.01

0.02

0.05

0.10

0.20

1.00

1.50

2.50

2.50

3.50

0.125 0.250 0.375

0.500

0.625

0.750

$0.875 \pi$

$1.000 \pi$

$1.125 \pi$
$1.250 \pi$
$S(h, 0)$

$0.00605-0.00052$ $0.01997-0.00180$ $0.07405-0.00769$ $0.17047-0.02190$

$0.36217-0.06479$

$0.88512-0.29571$

$1.48066-0.90793$

$1.68683-1.56749$

$\begin{array}{lll}1.61708 & -2.05995\end{array}$

$1.46505-2.31983$

$\begin{array}{ll}1.37922 & -2.41955 \\ 1.39767 & -2.48164\end{array}$

$0.70946-0.19710$

$1.27347-0.62332$

$1.59007-1.15108$

$1.68948-1.05068$

$1.62730-2.03203$

$1.50665-2.26653$

$1.40894-2.38178$

$1.37495-2.43606$

$\begin{array}{ll}1.40173 & -2.48704 \\ 1.45778 & -2.56848\end{array}$
$E(h, 0)$

$\begin{array}{ll}1.25168 & -0.01998 \\ 2.19830 & -0.03995 \\ 3.83437 & -0.09977 \\ 5.17764 & -0.19914 \\ 0.52682 & -0.39626 \\ 8.21440 & -0.96916 \\ 0.19496 & -1.82911 \\ 3.43982 & -2.52334 \\ 9.35662 & -3.02061 \\ 9.12262 & -3.31691 \\ 8.84836 & -3.43307 \\ 8.60645 & -3.40867 \\ 7.79200 & -0.76804 \\ 8.90918 & -1.47755 \\ 9.33619 & -2.09759 \\ 9.44277 & -2.60611 \\ 9.36943 & -2.99122 \\ 9.19808 & -3.25148 \\ 8.98583 & -3.39513 \\ 8.77421 & -3.43837 \\ 8.59227 & -3.40308 \\ 8.45777 & -3.31405\end{array}$

$C(h, h)$

$1.09913-0.01997$ $1.70646-0.03990$ $2.58752-13.09934$ $3.25582-0.19673$ $3.87129-0.38252$ $4.25317-0.82913$ $3.16010-1.07674$ $-1.25022-0.19342$ $-3.365130 .38643$ $-4.755130 .38643$ $-5.01922 \quad 1.01140$

$\begin{array}{lll}4.25503 & -0.69199\end{array}$ $3.80099-1.05027$ $2.49446-1.01710$ $0.76902-0.09422$ $\begin{array}{ll}-1.08075 & -0.23780\end{array}$ $\begin{array}{ll}-1.00967 & 0.22870\end{array}$ 4.177190 .63080 4.956250 .91170 $\begin{array}{ll}-4.99086 & 1.01150 \\ -4.25315 & 0.88550\end{array}$
$S(h, h)$

$0.00546-0.00048$ $0.01748-0.00162$ $0.07348-0.00764$ $0.20214-0.02406$ $0.52146-0.07528$ $1.62964-0.33052$ $3.26409-0.84880$ $4.14580-1.12591$

$4.11638-1.01777$

$3.16651 \quad-0.62252$

$1.41742-0.07740$

$1.22641-0.22595$ $2.63434-0.63567$ $\begin{array}{ll}2.63434 & -0.63567 \\ 3.67810 & -0.98976\end{array}$ $4.19811-1.13340$ $4.15006-1.03723$ $3.53063-0.75680$ $2.37962-0.36301$ 0.820040 .08937 $\begin{array}{rr}-0.92679 & 0.54569 \\ -2.58062 & 0.92828\end{array}$
$E(h, h)$

$\begin{array}{ll}1.09915 & -0.01997\end{array}$ $1.70658-0.03992$ $3.5682-0.09957$ $3.91251-0.39094$ $4.59748-0.91455$ $4.67831-1.51031$ $4.42418-1.71053$ $4.21938-1.64680$ $4.18263-1.49478$ $4.18263-1.49478$ $4.26524-1.40896$

$4.45459-0.73877$ $4.72139-1.30305$ $4.59904-1.62574$ $4.38711-1.71919$ $4.22888-1.05702$ $4.17669-1.53639$ $4.21559-1.43868$ $4.29484-1.40469$ $\begin{array}{ll}4.36165 & -1.43148 \\ 4.38556 & -1.48753\end{array}$

$$
\frac{a}{\lambda}=0.002381 \quad \frac{\alpha}{\beta}=0.10
$$

$\begin{array}{ll}0.00605 & -0.00071 \\ 0.01995 & -0.00240 \\ 0.07390 & -0.00990 \\ 0.16987 & -0.02698 \\ 0.35986 & -0.07541 \\ 0.87208 & -0.31912 \\ 1.44237 & -0.93331 \\ 1.63447 & -1.57432 \\ 1.57208 & -2.04670 \\ 1.43922 & -2.30231 \\ 1.36621 & -2.41472 \\ 1.38121 & -2.49304 \\ 0.70105 & -0.21677 \\ 1.24590 & -0.65102 \\ 1.55051 & -1.17142 \\ 1.63689 & -1.65424 \\ 1.58115 & -2.01985 \\ 1.47519 & -2.24820 \\ 1.39126 & -2.36908 \\ 1.36266 & -2.43622 \\ 1.38450 & -2.49926 \\ 1.42930 & -2.58543\end{array}$

(1)

$\begin{array}{ll}1.25108 & -0.01997 \\ 2.19710 & -0.03992 \\ 3.83138 & -0.09968 \\ 5.17168 & -0.19882 \\ 6.51501 & -0.39505 \\ 8.18623 & -0.96199 \\ 9.14638 & -1.80298 \\ 9.38278 & -2.47189 \\ 9.30409 & -2.94397 \\ 9.08546 & -3.22116 \\ 8.83292 & -3.32831 \\ 8.61341 & -3.30631 \\ & \\ 7.76941 & -0.76355 \\ 8.86807 & -1.46069 \\ 9.28310 & -2.06284 \\ 9.38559 & -2.55093 \\ 9.31616 & -2.91627 \\ 9.15563 & -3.16032 \\ 8.95903 & -3.29348 \\ 8.76530 & -3.33315 \\ 8.60065 & -3.30128 \\ 3.48035 & -3.22171\end{array}$

$\begin{array}{rr}1.09853 & -0.01996 \\ 1.70526 & -0.03986 \\ 2.58454 & -0.09916 \\ 3.24998 & -0.19567 \\ 3.86025 & -0.37773 \\ 4.23359 & -0.79622 \\ 3.15502 & -0.95821 \\ 1.12336 & -0.55645 \\ -1.20301 & 0.07515 \\ -3.31599 & 0.64153 \\ -4.72430 & 0.96027 \\ -5.03354 & 0.90889 \\ & \\ 4.23704 & -0.67189 \\ 3.78574 & -0.972 C 4 \\ 2.50043 & -0.86428 \\ 0.79957 & -0.47284 \\ -1.03440 & 0.02845 \\ -2.75922 & 0.49754 \\ -4.13413 & 0.83986 \\ -4.93509 & 0.98734 \\ -5.00929 & 0.88804 \\ -4.32342 & 0.53539\end{array}$

\begin{tabular}{|c|c|c|}
\hline $\begin{array}{l}0.01 \\
0.02 \\
0.05 \\
0.10 \\
0.20 \\
0.50 \\
1.00 \\
1.50 \\
2.00 \\
2.50 \\
3.00 \\
3.50\end{array}$ & $\begin{array}{l}1.25106 \\
2.19698 \\
3.83031 \\
5.16708 \\
6.49653 \\
8.08298 \\
8.85473 \\
9.02458 \\
9.12754 \\
9.31821 \\
9.57043 \\
9.80041\end{array}$ & $\begin{array}{l}-0.01996 \\
-0.03990 \\
-0.09942 \\
-0.19755 \\
-0.38864 \\
-0.90153 \\
-1.47204 \\
-1.06422 \\
-1.60186 \\
-1.46903 \\
-1.39604 \\
-1.41105\end{array}$ \\
\hline $\begin{array}{l}0.125 \pi \\
0.250 \pi \\
0.375 \pi \\
0.500^{\circ} \pi \\
0.625 \pi \\
0.750 \pi \\
0.875 \pi \\
1.000 \pi \\
1.125 \pi \\
1.250 \pi\end{array}$ & $\begin{array}{l}7.70249 \\
8.65397 \\
8.94438 \\
9.03689 \\
9.11755 \\
9.25383 \\
9.44125 \\
9.64116 \\
9.81368 \\
9.93824\end{array}$ & $\begin{array}{l}-0.73038 \\
-1.27551 \\
-1.58022 \\
-1.66604 \\
-1.61093 \\
-1.50499 \\
-1.42107 \\
-1.39249 \\
-1.41434 \\
-1.45914\end{array}$ \\
\hline
\end{tabular}

$\begin{array}{rr}0.00545 & -0.00065 \\ 0.01746 & -0.00215 \\ 0.07333 & -0.00984 \\ 0.20155 & -0.03008 \\ 0.51922 & -0.09052 \\ 1.61847 & -0.37317 \\ 3.24367 & -0.90399 \\ 4.14295 & -1.13643 \\ 4.14751 & -0.92981 \\ 3.22693 & -0.39234 \\ 1.49448 & 0.30223 \\ -0.70098 & 0.97420 \\ & \\ 1.21881 & -0.25968 \\ 2.61530 & -0.69222 \\ 3.66072 & -1.03557 \\ 4.19964 & -1.13311 \\ 4.17868 & -0.95815 \\ 3.58378 & -0.57062 \\ 2.45014 & -0.05551 \\ 0.89872 & 0.50335 \\ -0.85552 & 1.01425 \\ -2.54229 & 1.36987\end{array}$

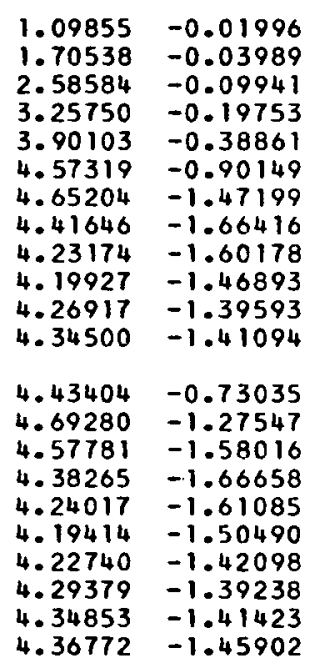


$\stackrel{a}{\grave{\lambda}}=0.002381 \quad \frac{\alpha}{\beta}=0.20$

$\begin{array}{lll}0.01 & 1.24906 & -0.01993 \\ 0.02 & 2.19299 & -0.03930 \\ 0.05 & 3.82041 & -0.09890 \\ 0.10 & 5.14751 & -0.19557 \\ 0.20 & 0.45872 & -0.38103 \\ 0.50 & 8.00430 & -0.85981 \\ 1.00 & 8.76967 & -1.35388 \\ 1.50 & 8.99200 & -1.50583 \\ 2.00 & 9.14864 & -1.46282 \\ 2.50 & 9.35029 & -1.37803 \\ 3.00 & 9.57270 & -1.33546 \\ 3.50 & 9.76660 & -1.34298 \\ 0.125 \pi & 7.63558 & -0.70325 \\ 0.250 \pi & 8.56202 & -1.18925 \\ 0.375 \pi & 8.87390 & -1.44101 \\ 0.500 \pi & 9.01324 & -1.50762 \\ 0.625 \pi & 9.13589 & -1.46893 \\ 0.750 \pi & 9.28790 & -1.40016 \\ 0.875 \pi & 9.46179 & -1.34961 \\ 1.000 \pi & 9.63214 & -1.33353 \\ 1.125 \pi & 9.77810 & -1.34461 \\ 1.250 \pi & 9.89268 & -1.36584\end{array}$

$\operatorname{Sih}, 0)$

$\begin{array}{ll}0.00603 & -0.00131 \\ 0.01987 & -0.00439 \\ 0.07341 & -0.01726 \\ 0.16792 & -0.04378 \\ 0.35227 & -0.11034 \\ 0.83038 & -0.39486 \\ 1.32423 & -1.01541 \\ 1.47610 & -1.00393 \\ 1.43306 & -2.02263 \\ 1.34824 & -2.26725 \\ 1.30566 & -2.40947 \\ 1.31317 & -2.52386 \\ 0.67395 & -0.28076 \\ 1.15966 & -0.74000 \\ 1.41132 & -1.23894 \\ 1.47788 & -1.67491 \\ 1.43916 & -1.99853 \\ 1.37038 & -2.21115 \\ 1.31981 & -2.34556 \\ 1.30372 & -2.44227 \\ 1.31480 & -2.53187 \\ 1.33602 & -2.02800\end{array}$

$E(h, 0)$

$\begin{array}{ll}1.24908 & -0.01994 \\ 2.19311 & -0.03985 \\ 3.82143 & -0.09938 \\ 5.15188 & -3.19776 \\ 6.47588 & -3.39106 \\ 8.09380 & -0.93859 \\ 8.98904 & -1.71947 \\ 9.19931 & -2.31053 \\ 9.13398 & -2.70756 \\ 8.95965 & -2.92954 \\ 8.76779 & -3.01142 \\ 8.60900 & -2.99584 \\ 7.69508 & -0.74881 \\ 8.73425 & -1.40636 \\ 9.11175 & -1.95254 \\ 9.20173 & -2.37832 \\ 9.14388 & -2.68484 \\ 9.01468 & -2.88181 \\ 8.86240 & -2.98524 \\ 8.71804 & -3.01499 \\ 8.60002 & -2.99230 \\ 8.51706 & -2.93759\end{array}$

$C(h, h)$

$\begin{array}{rr}1.09654 & -0.01952 \\ 1.70128 & -0.03974 \\ 2.57469 & -0.09831 \\ 3.23074 & -0.19217 \\ 3.82442 & -0.36200 \\ 4.17421 & -0.68930 \\ 3.15005 & -0.57415 \\ 1.21152 & 0.13425 \\ -1.08888 & 0.97254 \\ -3.28690 & 1.50738 \\ -4.89225 & 1.42930 \\ -5.47410 & 0.60254 \\ & \\ 4.18094 & -0.60640 \\ 3.74570 & -0.71890 \\ 2.53061 & -0.36734 \\ 0.89723 & 0.25613 \\ -0.91859 & 0.91700 \\ -2.69379 & 1.40545 \\ -4.19007 & 1.55853 \\ -5.17580 & 1.27158 \\ -5.46896 & 0.51954 \\ -4.97265 & -0.62007\end{array}$

$S(h, h)$

$0.00543-0.00119$ $0.01738-0.00389$ $0.07284-0.01713$ $0.19963-0.05000$ $0.51198-0.14088$ $1.58489-0.51389$ $3.19777-1.09138$ $18513-1.18505$ $4.33716 \quad-0.64768$ $3.53601 \quad 0.38276$ $\begin{array}{rr}1.82007 & 1.60559 \\ -0.53542 & 2.61552\end{array}$ $\begin{array}{ll}1.19527 & -0.37090\end{array}$ $2.56418-0.88069$ $3.63459-1.19563$ $4.26109-1.14648$ $4.35750-0.70642$ $3.86598 \quad 0.05114$ $2.78476 \quad 0.99079$ $1.20013 \quad 1.93253$ $\begin{array}{ll}-0.70911 & 2.66570 \\ -2.69466 & 2.97929\end{array}$
$E(h, h)$

$\begin{array}{ll}1.09656 & -0.01992 \\ 1.70140 & -0.03979 \\ 2.57594 & -0.09888 \\ 3.23794 & -0.19553 \\ 3.86322 & -0.38097 \\ 4.49452 & -0.85973 \\ 4.56699 & -1.35378 \\ 4.38389 & -1.50571 \\ 4.25286 & -1.46269 \\ 4.23137 & -1.37788 \\ 4.27146 & -1.33530 \\ 4.31122 & -1.34282 \\ & \\ 4.36713 & -0.70318 \\ 4.60086 & -1.18916 \\ 4.50734 & -1.44090 \\ 4.35902 & -1.50750 \\ 4.25853 & -1.46879 \\ 4.22823 & -1.40002 \\ 4.24796 & -1.34945 \\ 4.28478 & -1.33337 \\ 4.31296 & -1.34445 \\ 4.32219 & -1.36568\end{array}$

$$
\frac{a}{\lambda}=0.002381 \quad \frac{\alpha}{\beta}=0.40
$$

$\begin{array}{rr}1.09257 & -0.01984 \\ 1.69337 & -0.03948 \\ 2.55523 & -0.09675 \\ 3.19329 & -0.18530 \\ 3.75726 & -0.33152 \\ 4.08181 & -0.48683 \\ 3.19781 & 0.15747 \\ 1.40053 & 1.50962 \\ -1.05107 & 2.86633 \\ -3.87481 & 3.46147 \\ -6.56596 & 2.62452 \\ -8.37868 & -0.00116 \\ & \\ 4.08592 & -0.48168 \\ 3.71500 & -0.24081 \\ 2.64504 & 0.59146 \\ 1.08840 & 1.71912 \\ -0.85497 & 2.78388 \\ -3.04923 & 3.41134 \\ -5.27244 & 3.25633 \\ -7.20583 & 2.06626 \\ -8.44848 & -0.24596 \\ -8.55856 & -3.53849\end{array}$

$\begin{array}{rr}0.00539 & -0.00227 \\ 0.01722 & -0.00735 \\ 0.07187 & -0.03157 \\ 0.19591 & -0.08927 \\ 0.49855 & -0.23961 \\ 1.53349 & -0.78984 \\ 3.20311 & -1.48420 \\ 4.51307 & -1.33770 \\ 5.14779 & -0.09854 \\ 4.70705 & 2.10611 \\ 2.84114 & 4.75543 \\ -0.56568 & 0.95710 \\ 1.15610 & -0.58833 \\ 2.51533 & -1.26016 \\ 3.72491 & -1.54978 \\ 4.65271 & -1.22975 \\ 5.13286 & -0.22545 \\ 4.96723 & 1.39597 \\ 3.97127 & 3.41736 \\ 2.02894 & 5.47049 \\ -0.85124 & 7.06401 \\ -4.48283 & 7.64758\end{array}$

$1.09258-0.01985$ $1.69346-0.03958$ $2.55629-0.09783$ $3.19940-0.19162$ $3.78980-0.36629$ $4.34711-0.78384$ $4.40781-1.15523$ $4.29695-1.25037$ $4.23086-1.22989$ $4.22146-1.19525$ $\begin{array}{ll}4.23468 & -1.18073 \\ 4.24563 & -1.18261\end{array}$ $4.24034-0.65283$ $4.43122-1.03967$ $4.36928-1.21176$ $4.28349-1.25134$ $4.23343-1.23265$ $4.22028-1.20364$ $4.22724-1.18525$ $4.23858-1.18016$ $\begin{array}{ll}4.23858 & -1.18016 \\ 4.24606 & -1.18301\end{array}$ $4.24819-1.18778$ 
$0.125 \pi$ $0.250 \pi$ $0.375 \pi$ $0.500 \pi$ $0.625 \pi$ $0.750 \pi$ $1.000 \%$ $1.125 \pi$
$1.250 \pi$

$\begin{array}{ll}0.96448 & -0.02000 \\ 1.76579 & -0.03999 \\ 3.29650 & -0.09994 \\ 4.61969 & -0.19954 \\ 5.96159 & -0.39644 \\ 7.58994 & -0.94602 \\ 8.36811 & -1.00530 \\ 8.47017 & -1.84853 \\ 8.49725 & -1.75808 \\ 8.67135 & -1.54984 \\ 8.97565 & -1.42462 \\ 9.27455 & -1.45455 \\ 7.19681 & -0.75893 \\ 8.17567 & -1.37067 \\ 8.43959 & -1.73963 \\ 8.47032 & -1.85181 \\ 8.49137 & -1.77157 \\ 8.60373 & -1.00827 \\ 8.81396 & -1.46893 \\ 9.06726 & -1.41809 \\ 9.29142 & -1.46120 \\ 9.43672 & -1.55579\end{array}$

$E(h, 0)$

$\begin{array}{ll}0.00473 & -0.00010 \\ 0.01659 & -0.00040 \\ 0.06771 & -0.00250 \\ 0.16359 & -0.00998 \\ 0.35852 & -0.03973 \\ 0.90692 & -0.23980 \\ 1.56581 & -0.84732 \\ 1.80889 & -1.55609 \\ 1.71839 & -2.10433 \\ 1.51010 & -2.37650 \\ 1.38485 & -2.43683 \\ 1.41476 & -2.44623 \\ 0.72004 & -0.15029 \\ 1.33128 & -0.55676 \\ 1.70007 & -1.10359 \\ 1.81217 & -1.64816 \\ 1.73188 & -2.07338 \\ 1.56854 & -2.32564 \\ 1.42917 & -2.42370 \\ 1.37832 & -2.43745 \\ 1.42141 & -2.44886 \\ 1.51599 & -2.51427\end{array}$

$C(h, h)$

$0.96450-0.02000$ $1.76589-0.04000$ . $29752-0.09998$ $4.02432-0.19988$ $5.98085-0.39909$ $7.70536-0.98615$ $8.73502-1.89204$ $9.00076-2.64918$ $8.90595-3.21059$ 2.63171 -3.55677 $\begin{array}{ll}8.29923 & -3.69701 \\ 7.99572 & -3.66593\end{array}$ $7.99572-3.60593$

$7.26986-0.77865$ $8.43107-1.51785$ $8.88732-2.18176$ $9.00404 \quad-2.74133$ $8.92067-3.17682$ $3.72117-3.47924$ $8.46732-3.65068$ $8.20730-3.70357$ $\begin{array}{ll}7.9775 B & -3.65878 \\ 7.80310 & -3.54306\end{array}$

$\begin{array}{rr}0.88293 & -0.02000 \\ 1.44523 & -0.03999 \\ 2.31105 & -0.09990 \\ 2.98380 & -0.19921 \\ 3.61484 & -0.39380 \\ 4.04720 & -0.90751 \\ 3.01853 & -1.36032 \\ 1.00815 & -1.26575 \\ -1.26950 & -0.82456 \\ -3.32475 & -0.21101 \\ -4.69168 & 0.52538 \\ -4.92969 & 1.28055 \\ 4.03140 & -0.73970 \\ 3.63541 & -1.23743 \\ 2.37082 & -1.38203 \\ 0.68916 & -1.21873 \\ -1.10534 & -0.86376 \\ -2.78281 & -0.40129 \\ -4.12163 & 0.14296 \\ -4.88842 & 0.74606 \\ -4.89791 & 1.32718 \\ -4.10269 & 1.75404\end{array}$

$S(h, h)$.

$0.00438-0.00010$ $0.01455-0.00040$ $0.06338-0.0025$ $0.17921-0.00997$ $0.47427-0.03947$ $1.52403-0.23009$ $3.08465-0.72181$ $3.89339-1.10948$ $3.82421-1.23139$ $2.90560-1.16352$ $\begin{array}{rr}1.22890 & -0.96702 \\ -0.89152 & -0.58014\end{array}$

$1.13948-0.14650$ $2.48570-0.50352$ $3.47230-0.88695$ $3.93669-1.14277$ $3.85849-1.23040$ $3.25402-1.19619$ $2.15329-1.08380$ $0.65217-0.88038$ $\begin{array}{ll}-1.03958 & -0.54513 \\ -2.02531 & -0.07908\end{array}$
$E(h, h)$

$0.88295-0.02000$ $1.44532-0.03999$ $2.31216-0.09994$ $2.99043-0.19954$ $3.65190-0.39644$ $4.36751-0.94602$ $4.45298-1.60530$ $4.14961-1.84850$ $3.88901-1.75804$ $3.83997-1.54977$ $\begin{array}{ll}3.96195 & -1.42453 \\ 4.10670 & -1.45444\end{array}$

$4.21554-0.75892$ $4.50202-1.37067$ $4.36058-1.73962$ 4.10365 -1.85178 $3.90155-1.77153$ $3.83159-1.60821$ $3.88767-1.46885$ $4.00745-1.41799$ $\begin{array}{ll}4.11383 & -1.46109 \\ 4.15377 & -1.55568\end{array}$

$$
\frac{a}{\lambda}=0.00317 E \quad \frac{\alpha}{\beta}=0.005
$$

$\begin{aligned} 0.00438 & -0.00012 \\ 0.01455 & -0.00047 \\ 0.06336 & -0.00281 \\ 0.17911 & -0.01085 \\ 0.47388 & -0.04178 \\ 1.52193 & -0.23669 \\ 3.07976 & -0.73001 \\ 3.88965 & -1.11006 \\ 3.82422 & -1.21682 \\ 2.90903 & -1.12736 \\ 1.23735 & -0.90700 \\ -0.87408 & -0.50621 \\ & \\ 1.13811 & -0.15170 \\ 2.48170 & -0.51217 \\ 3.46731 & -0.89343 \\ 3.93342 & -1.14162 \\ 3.85822 & -1.21717 \\ 3.25648 & -1.16680 \\ 2.15876 & -1.03551 \\ 0.66281 & -0.81474 \\ -1.02144 & -0.47098 \\ -2.60027 & -0.01111\end{aligned}$

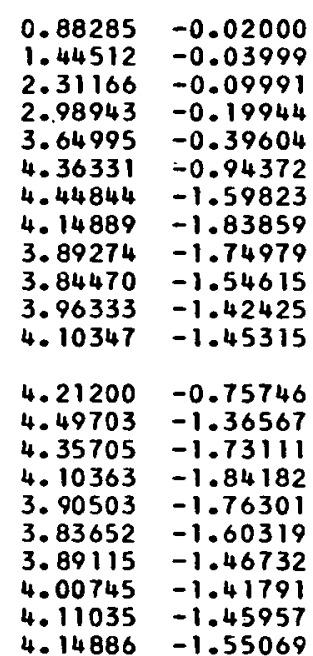

\begin{tabular}{|c|c|c|}
\hline $\begin{array}{l}0.01 \\
0.02 \\
0.05 \\
0.10 \\
0.20 \\
0.50 \\
1.00 \\
1.50 \\
2.00 \\
2.50 \\
3.00\end{array}$ & $\begin{array}{l}0.96438 \\
1.76559 \\
3.29600 \\
4.61870 \\
5.95964 \\
7.58574 \\
8.36357 \\
8.46944 \\
8.50097 \\
8.67608 \\
8.97702 \\
9.27131\end{array}$ & $\begin{array}{l}-0.02000 \\
-0.03999 \\
-0.09991 \\
-0.19944 \\
-0.39604 \\
-0.94372 \\
-1.59824 \\
-1.83861 \\
-1.74984 \\
-1.54623 \\
-1.42434 \\
-1.45326\end{array}$ \\
\hline $\begin{array}{l}0.125 \pi \\
0.250 \\
0.375 \pi \\
0.500 \\
0.625 \pi \\
0.750 \\
0.875 \pi \\
1.000 \pi \\
1.125 \pi \\
1.250 \pi\end{array}$ & $\begin{array}{l}7.19328 \\
8.17068 \\
8.43606 \\
8.47030 \\
8.49485 \\
8.00866 \\
8.81744 \\
9.06726 \\
9.28794 \\
9.43181\end{array}$ & $\begin{array}{l}-0.757 \\
-1.365 \\
-1.731 \\
-1.841 \\
-1.763 \\
-1.603 \\
-1.467 \\
-1.418 \\
-1.459 \\
-1.550\end{array}$ \\
\hline
\end{tabular}

$\begin{array}{ll}0.00473 & -0.00012 \\ 0.01659 & -0.00048 \\ 0.06768 & -0.00284 \\ 0.16349 & -0.01080 \\ 0.35813 & -0.04149 \\ 0.90462 & -0.24380 \\ 1.55875 & -0.85167 \\ 1.79898 & -1.55661 \\ 1.71014 & -2.10041 \\ 1.50649 & -2.37157 \\ 1.38458 & -2.43526 \\ 1.41348 & -2.44927 \\ & \\ 0.71858 & -0.15363 \\ 1.32629 & -0.56155 \\ 1.69157 & -1.10693 \\ 1.80221 & -1.64798 \\ 1.72337 & -2.06970 \\ 1.56353 & -2.32051 \\ 1.42766 & -2.42002 \\ 1.37824 & -2.43720 \\ 1.41990 & -2.45214 \\ 1.51100 & -2.51899\end{array}$

$\begin{array}{ll}0.96440 & -0.02000 \\ 1.76569 & -0.03999 \\ 3.29702 & -0.09996 \\ 4.62332 & -0.19982 \\ 5.97887 & -0.39888 \\ 7.70057 & -0.98492 \\ 8.72662 & -1.88744 \\ 8.99080 & -2.63990 \\ 8.89687 & -3.19647 \\ 8.62572 & -3.53881 \\ 8.29778 & -3.67717 \\ 7.99915 & -3.64663 \\ 7.26604 & -0.77788 \\ 8.42401 & -1.51492 \\ 8.87810 & -2.17559 \\ 8.99405 & -2.73135 \\ 8.91144 & -3.16303 \\ 8.71410 & -3.46221 \\ 8.46348 & -3.63151 \\ 8.20726 & -3.68364 \\ 7.98133 & -3.63961 \\ 7.81006 & -3.52603\end{array}$

$\begin{array}{rr}0.88283 & -0.02000 \\ 1.44503 & -0.03998 \\ 2.31055 & -0.09986 \\ 2.98281 & -0.19904 \\ 3.61294 & -0.39304 \\ 4.04355 & -0.90215 \\ 3.01695 & -1.34088 \\ 1.01277 & -1.23178 \\ -1.25873 & -0.78176 \\ -3.30798 & -0.17019 \\ -4.66888 & 0.54631 \\ -4.90638 & 1.26136 \\ & \\ 4.02815 & -0.73645 \\ 3.63218 & -1.22459 \\ 2.37124 & -1.35707 \\ 0.69471 & -1.18306 \\ -1.09499 & -0.82132 \\ -2.76785 & -0.35839 \\ -4.10166 & 0.17652 \\ -4.86456 & 0.75749 \\ -4.87496 & 1.30469 \\ -4.08818 & 1.69235\end{array}$


$\frac{a}{\lambda}=0.0103175 \quad \frac{\alpha}{\beta}=0.01$

$\beta \mathrm{h}$

0.01
0.02
0.05
0.10
0.20
0.50
1.00
1.50
2.00
2.50
3.00
3.50

$0.125 \pi$

$0.250 \pi$

$0.375 \pi$

$0.625 \pi$

$0.750 \pi$
$0.875 \pi$

$0.875 \pi$

$1.125 \pi$

$1.125 \pi$
$1.250 \pi$
$C(h, 0)$

$0.96428-0.01999$ $1.76539-0.03998$ $3.29551-0.09788$ $4.61771-0.19934$ . $.95770-0.39564$ $8.35904-1.59123$ $8.46868-1.82878$ $8.50459-1.74164$ $8.68067-1.54256$ $8.97836-1.42392$ $9.26819-1.45186$

$7.18975-0.75600$ $8.43252-1.72268$ $8.49822-1.75460$ $8.82082-1.46577$ $9.06726-1.41778$ $9.28458-1.45806$ $9.42706-1.54583$
$S(h, 0)$

$$
\begin{array}{ll}
0.00473 & -0.00015 \\
0.01659 & -0.00057 \\
0.06766 & -0.00317 \\
0.16339 & -0.01161 \\
0.35773 & -0.04324 \\
0.90234 & -0.24780 \\
1.55173 & -0.85600 \\
1.78915 & -1.55718 \\
1.70195 & -2.09660 \\
1.50282 & -2.36678 \\
1.38416 & -2.43373 \\
1.41208 & -2.45219 \\
0.71712 & -0.15696 \\
1.32133 & -0.56634 \\
1.68312 & -1.11026 \\
1.79233 & -1.64786 \\
1.71491 & -2.06613 \\
1.55850 & -2.31552 \\
1.42602 & -2.41644 \\
1.37801 & -2.43706 \\
1.41828 & -2.45530 \\
1.50604 & -2.52354
\end{array}
$$

$$
E(h, 0)
$$

$\begin{array}{ll}0.96430 & -0.01999 \\ 1.76549 & -0.03999 \\ 3.29652 & -0.09995 \\ 4.62232 & -0.19976 \\ 5.97688 & -0.39867 \\ 7.69578 & -0.98369 \\ 8.71823 & -1.88285 \\ 8.98087 & -2.63067 \\ 8.88781 & -3.18242 \\ 8.61972 & -3.52099 \\ 8.29626 & -3.65750 \\ 8.00243 & -3.62748 \\ 7.26222 & -0.77711 \\ 8.41696 & -1.51199 \\ 8.86890 & -2.16944 \\ 8.98410 & -2.72141 \\ 8.90224 & -3.14932 \\ 8.70703 & -3.44531 \\ 8.45959 & -3.61248 \\ 8.20711 & -3.66386 \\ 7.98491 & -3.62057 \\ 7.81681 & -3.50910\end{array}$

$\mathrm{C}(\mathrm{h}, \mathrm{h})$

$\begin{array}{rr}0.88273 & -0.01999 \\ 1.44483 & -0.03998 \\ 2.31005 & -0.09982 \\ 2.98182 & -0.19887 \\ 3.61104 & -0.392 .27 \\ 4.03992 & -0.89681 \\ 3.01541 & -1.32150 \\ 1.01738 & -1.19788 \\ -1.24813 & -0.73903 \\ -3.29170 & -0.12944 \\ -4.64712 & 0.56722 \\ -4.88461 & 1.24244 \\ & \\ 4.02491 & -0.73320 \\ 3.62898 & -1.21130 \\ 2.37169 & -1.33217 \\ 0.70023 & -1.14746 \\ -1.08480 & -0.77894 \\ -2.75326 & -0.31556 \\ -4.08243 & 0.21001 \\ -4.84191 & 0.76894 \\ -4.85357 & 1.28251 \\ -4.07523 & 1.63133\end{array}$

$S(h, h)$

$0.00438-0.00014$ $0.01455-0.00055$ $0.06333-0.00313$ $0.17902-0.01174$ $0.47349-0.04409$ $3.07497-0.7382$ $3.88609-1.11071$ $3.82450-1.20233$ $2.91284-1.09126$ $1.24607-0.84714$ $-0.85695-0.43253$

$1.13673-0.15691$ $2.47774 \quad-0.52083$ $3.46244-0.89994$ $3.93034-1.14055$ $3.85822-1.20402$ $3.25930-1.13747$ $2.16461-0.98725$ $0.67360-0.74931$ $-1.00368-0.3970$

$\begin{array}{rr}-1.003686 & -0.39708 \\ -2.57636 & 0.05674\end{array}$
$E(h, h)$

$0.88275-0.01999$ $1.44492-0.03998$ $2.31116 / /-0.09988$ $2.98844-0.19934$ $3.64801-0.39564$ $4.35912-0.94143$ $4.14813-1.82875$ $3.89635-1.74159$ $3.84929-1.54248$ $3.96466-1.42383$ $4.10034 \quad-1.45175$ $4.20848 \quad-0.75599$ $4.49205-1.36071$ $4.35351-1.72266$ $\begin{array}{ll}4.10356 & -1.83193 \\ 3.90840 & -1.75455\end{array}$ $3.84131-1.59815$ $3.89453-1.46568$ $4.00745-1.41768$ $4.10699-1.45795$ $4.14411-1.54572$

$$
\frac{a}{\lambda}=0.003175 \quad \frac{\alpha}{\beta}=0.02
$$

$\begin{array}{ll}0.00473 & -0.00019 \\ 0.01658 & -0.00073 \\ 0.06761 & -0.00385 \\ 0.16319 & -0.01323 \\ 0.35695 & -0.04674 \\ 0.89779 & -0.25576 \\ 1.53783 & -0.86463 \\ 1.76972 & -1.55842 \\ 1.68569 & -2.08932 \\ 1.49533 & -2.35760 \\ 1.38291 & -2.43080 \\ 1.40896 & -2.45776 \\ 0.71421 & -0.16360 \\ 1.31148 & -0.57585 \\ 1.66640 & -1.11694 \\ 1.77279 & -1.04775 \\ 1.69816 & -2.05931 \\ 1.54837 & -2.30596 \\ 1.42244 & -2.40959 \\ 1.37712 & -2.43668 \\ 1.41474 & -2.46132 \\ 1.49618 & -2.53218\end{array}$

0.8
1
7

$1.49618-2.53218$
$0.88253-0.01893$

$0.96408-0.01999$ $1.76499-0.03997$ $4.01572-0.10914$ $5.61572-0.19974$ $7.57319-0.93688$ $8.57319-0.93688$ $8.45704-1.57732$ $8.46704-1.80934$ $8.68946-1.53506$ $9.26222-1.44874$

$0.125 \pi$ $0.250 \pi$ $0.500 \pi$ $0.625 \pi$ $0.750 \pi$ $0.875 \pi$
$1.000 \pi$

$1.125: \pi$

1.250 in
$7.18272-0.75309$ $8.15579-1.35086$ $8.42545-1.70596$ $8.46994-1.81243$ $8.50463-1.73784$ $8.82728-1.46219$ $9.06725-1.41688$ $9.41802-1.53597$

$\begin{array}{rrrr}0.88253 & -0.01997 & 0.00438 & -0.00019 \\ 1.44443 & -0.03990 & 0.01454 & -0.00069 \\ 2.30906 & -0.09974 & 0.06328 & -0.00376 \\ 2.97985 & -0.19853 & 0.17882 & -0.01352 \\ 3.60724 & -0.39075 & 0.47272 & -0.04870 \\ 4.03274 & -0.88616 & 1.51571 & -0.25645 \\ 3.01245 & -1.28291 & 3.06563 & -0.75469 \\ 1.02653 & -1.13031 & 3.87952 & -1.11219 \\ -1.22743 & -0.05374 & 3.82592 & -1.17357 \\ -3.26060 & -0.04815 & 2.92158 & -1.01925 \\ -4.60667 & 0.60899 & 1.26430 & -0.72786 \\ -4.84565 & 1.20542 & -0.82360 & -0.28583 \\ & & & \\ 4.01849 & -0.72673 & 1.13399 & -0.16729 \\ 3.62271 & -1.18632 & 2.46998 & -0.53813 \\ 2.37266 & -1.28258 & 3.45305 & -0.91305 \\ 0.71116 & -1.07649 & 3.92476 & -1.13860 \\ -1.06487 & -0.69436 & 3.85905 & -1.17793 \\ -2.72520 & -0.23008 & 3.26599 & -1.07898 \\ -4.04615 & 0.27680 & 2.17739 & -0.89103 \\ -4.80018 & 0.79196 & 0.69566 & -0.61898 \\ -4.81544 & 1.23904 & -0.96924 & -0.24994 \\ -4.05395 & 1.51116 & -2.53185 & 0.19218\end{array}$


$\beta h$

0.01

0.02

0.05

0.10

0.20

0.50

1.00

1.50

2.50

3.50

$0.125 \pi$

0.250

$0.500 \pi$

$0.625 \pi$

0.750

0.875

1.000

1.125
$C(h, 0)$

$0.96388-0.01998$ $1.76459-0.03996$ $3.29351-0.09978$ $4.61374-0.19893$ $5.94994-0.39466$ $\begin{array}{ll}7.56487 & -0.93235 \\ 8.34102 & -1.56359\end{array}$ $8.34102-1.56359$ $8.46526-1.79020$ $8.51792-1.70931$ $\begin{array}{ll}8.69773 & -1.52736 \\ 8.98325 & -1.42037\end{array}$ 8.98325
$9.25662-1.42037$ $\begin{array}{ll}7.17572 & -0.75020\end{array}$ $8.14593-1.34111$ $8.41837-1.08946$ $8.46946-1.79320$ $8.51063-1.72128$ $8.03125-1.57787$ $8.83334-1.45820$ $9.06721-1.41543$ $\begin{array}{ll}9.27214 & -1.45059 \\ 9.40956 & -1.52618\end{array}$
$S(h, 0)$

$\begin{array}{ll}0.00473 & -0.00024 \\ 0.01657 & -0.00090 \\ 0.06756 & -0.00452 \\ 0.16299 & -0.01485 \\ 0.35616 & -0.05024 \\ 0.89326 & -0.26369 \\ 1.52410 & -0.87323 \\ 1.75058 & -1.55981 \\ 1.66962 & -2.08247 \\ 1.48763 & -2.34893 \\ 1.38112 & -2.42804 \\ 1.40543 & -2.46297 \\ 0.71132 & -0.17022 \\ 1.30173 & -0.58532 \\ 1.64992 & -1.12362 \\ 1.75357 & -1.04784 \\ 1.68160 & -2.05292 \\ 1.53816 & -2.29692 \\ 1.41846 & -2.40313 \\ 1.37567 & -2.43631 \\ 1.41082 & -2.46695 \\ 1.48640 & -2.54025\end{array}$

$\begin{array}{ll}0.00473 & -0.00024 \\ 0.01657 & -0.00090\end{array}$ $0.06756-0.00452$ $0.16299-0.01485$ $0.89326-0.26369$ $1.52410-0.87323$ $1.75058-1.55981$ $1.48763-2.34893$ $1.380543-2.42804$

$0.71132-0.17022$ $1.30173-0.58532$ 4 $\begin{array}{ll}.758160 & -2.05292\end{array}$ $1.48640-2.54025$

$$
E\left(r_{1}, 0\right)
$$

$0.96390-0.01799$ $1.76469-0.03997$ $3.29452-0.09988$ $4.61833-0.19954$ $\begin{array}{ll}5.96895 & -0.39785 \\ 7.67671 & -0.97879\end{array}$ E. $68488 .-1.86467$ $8.94143-2.59416$ $8.85177-3.12711$ $8.59550-3.45095$ $8.28942-3.58030$ $8.01405-3.55228$

$7.24697-0.77405$
0.38889 B. $38889-1.50036$ $8.83233-2.14508$ $8.94456-2.08216$ $8.86564-3.09529$ $8.67873-3.37885$ $\begin{array}{ll}8.44363 & -3.53778 \\ 8.20558 & -3.58629\end{array}$ $8.20558-3.58629$ $\begin{array}{ll}7.99771 & -3.54585 \\ 7.84165 & -3.44242\end{array}$
$\mathrm{C}(\mathrm{h}, \mathrm{h})$

$0.88233-0.01998$ $1.44403-0.03995$ $2.30806-0.09966$ $2.97788-0.19320$ $3.60346-0.38922$ $4.02564-0.87555$ $3.00964-1.24455$ $1.03561-1.06302$ $\begin{array}{lll}-1.20740 & -0.56860\end{array}$ $-3.23143 \quad 0.03291$ $\begin{array}{ll}-4.57029 & 0.65075 \\ -4.81272 & 1.10945\end{array}$

$4.01212-0.72028$ $3.61660-1.16098$ $2.37376-1.23326$ $0.72196-1.00579$ $-1.04555-0.61000$ $\begin{array}{ll}-6.69861 & -0.14482\end{array}$ $\begin{array}{ll}-4.01280 & 0.34338\end{array}$ $-4.78341$ $\begin{array}{ll}-4.78341 & 1.19671 \\ -4.03880 & 1.39331\end{array}$
$\mathrm{S}(\mathrm{h}, \mathrm{h})$

$0.00438-0.00023$ $0.01453-0.00084$ $0.06323-0.00439$ $0.17862-0.01529$ $0.47195-0.05330$ $\begin{array}{ll}1.51163 & -0.26959\end{array}$ $3.05664-0.77121$ $3.87367-1.11392$ $3.82846-1.14509$ $\begin{array}{ll}2.93181 & -0.94748 \\ 1.28357 & -0.00909\end{array}$ $-0.79146-0.13985$

$1.13128-0.17765$ $2.46241-0.55542$ $3.44415-0.92628$ $3.91997-1.13693$ $3.86097-1.15213$ $3.27411-1.02073$ $2.19161-0.79513$ $2.19161-0.79513$ $-0.93623-0.10350$ $\begin{array}{rr}-0.93023 & -0.10350 \\ -2.49169 & 0.32744\end{array}$
$E(h, h)$

$0.88235-0.01998$ $1.44412-0.03996$ $2.30917-0.09977$ $2.98448-0.19893$ $3.64025-0.39405$ $4.34244-0.93233$ $4.42589-1.56356$ $4.14471-1.79015$ $3.86635-1.52726$ $3.96956-1.42075$ $4.08878-1.44508$

$4.19444 \quad-0.75018$ $4.47228-1.34108$ $4.33937-1.68943$ $3.02082-1.79121$ $3.85912-1.57121$ $3.90706-1.45810$ $4.00740-1.45810$ $4.09455-1.45046$ $4.12661-1.52604$

$$
\frac{a}{\lambda}=0.003175 \quad \frac{\alpha}{\beta}=0.05
$$

\begin{tabular}{|c|c|c|}
\hline $\begin{array}{l}0.01 \\
0.02 \\
0.05 \\
0.10 \\
0.20 \\
0.50 \\
1.00 \\
1.50 \\
2.00 \\
2.50 \\
3.00 \\
3.50\end{array}$ & $\begin{array}{l}0.96348 \\
1.76379 \\
3.29152 \\
4.60979 \\
5.94221 \\
7.54835 \\
8.32316 \\
8.46127 \\
8.52956 \\
8.71280 \\
8.98745 \\
9.24635\end{array}$ & $\begin{array}{l}-0.01998 \\
-0.03994 \\
-0.09967 \\
-0.19853 \\
-0.39250 \\
-0.92339 \\
-1.53662 \\
-1.75280 \\
-1.67770 \\
-1.51141 \\
-1.41582 \\
-1.43702\end{array}$ \\
\hline $\begin{array}{l}0.125 \pi \\
0.250 \pi \\
0.375 \pi \\
0.500 \pi \\
0.625 \pi \\
0.750 \pi \\
0.875 \pi \\
1.000 \pi \\
1.125 \pi \\
1.250 \pi\end{array}$ & $\begin{array}{l}7.16179 \\
8.12639 \\
8.40422 \\
8.46796 \\
8.52142 \\
8.64700 \\
8.84433 \\
9.06703 \\
9.26111 \\
9.39416\end{array}$ & $\begin{array}{l}-0.74445 \\
-1.32188 \\
-1.65716 \\
-1.75562 \\
-1.68877 \\
-1.55725 \\
-1.44912 \\
-1.41100 \\
-1.44170 \\
-1.50679\end{array}$ \\
\hline
\end{tabular}

$\begin{array}{llll}0.00472 & -0.00034 & 0.96350 & -0.01998 \\ 0.01055 & -0.00123 & 1.76390 & -0.03995 \\ 0.06746 & -0.00587 & 3.29253 & -0.09981 \\ 0.16259 & -0.01809 & 4.61435 & -0.19932 \\ 0.35460 & -0.05721 & 5.96104 & -0.39703 \\ 0.88431 & -0.27943 & 7.05772 & -0.97392 \\ 1.49714 & -0.89030 & 8.65184 & -1.84672 \\ 1.71319 & -1.56301 & 8.90244 & -2.55835 \\ 1.63803 & -2.07004 & 8.81605 & -3.07313 \\ 1.47169 & -2.33307 & 8.57120 & -3.38288 \\ 1.37607 & -2.42305 & 8.28144 & -3.50546 \\ 1.39725 & -2.47244 & 8.02333 & -5.47931 \\ 0.70559 & -0.18337 & 7.23179 & -0.77100 \\ 1.28251 & -0.60407 & 8.36103 & -1.48884 \\ 1.61762 & -1.13699 & 8.79612 & -2.12109 \\ 1.71599 & -1.64854 & 8.90547 & -2.64369 \\ 1.64909 & -2.04133 & 8.82938 & -3.04255 \\ 1.51754 & -2.28038 & 8.65042 & -3.31421 \\ 1.40939 & -2.39134 & 8.42703 & -3.46529 \\ 1.37125 & -2.43570 & 8.20260 & -3.51109 \\ 1.40193 & -2.47718 & 8.00812 & -3.47331 \\ 1.46702 & -2.55485 & 7.86324 & -3.37735\end{array}$

$\begin{array}{rr}0.88193 & -0.01997 \\ 1.44323 & -0.03992 \\ 2.30607 & -0.09951 \\ 2.97390 & -0.19752 \\ 3.59595 & -0.38619 \\ 4.01171 & -0.85449 \\ 3.00452 & -1.16845 \\ 1.05359 & -0.92926 \\ -1.16933 & -0.39908 \\ -3.17891 & 0.19450 \\ -4.50963 & 0.73440 \\ -4.76465 & 1.10045 \\ 3.99956 & -0.70746 \\ 3.60484 & -1.11071 \\ 2.37631 & -1.13540 \\ 0.74321 & -0.86518 \\ -1.00871 & -0.44186 \\ -2.04986 & 0.02516 \\ -3.95481 & 0.47614 \\ -4.70317 & 0.86230 \\ -4.73736 & 1.11526 \\ -4.02662 & 1.16372\end{array}$

$0.00437-0.00032$ $0.01451-0.00113$ $0.06313-0.00565$ $0.17823-0.01883$ $0.47042-0.06249$ $1.50363-0.29579$ $3.03966-0.80440$ $3.86415-1.11810$ $3.83697-1.08892$ $2.95673-0.80454$ $1.32521-0.37268$ $-0.73073 \quad 0.15073$

$1.12594-0.19831$ $2.44784-0.58998$ $3.42775-0.95310$ $3.91273-1.13436$ $3.86814-1.10135$ $3.29461-0.90483$ $2.22436-0.60411$ $0.76547-0.23117$ $\begin{array}{ll}-0.87441 & 0.18805 \\ -2.42412 & 0.59828\end{array}$
$0.88195-0.01998$ $1.44333-0.03993$ $2.30717-0.09966$ $2.08052-0.1985$ $3.63253-0.39247$ $4.32592-0.92336$ $4.40803-1.53657$ $3.92133-1.67761$ $3.92133-1.67761$ $3.88743-1.51129$ $3.97376-1.41568$

$4.18051-0.74442$ $4.45274-1.32184$ $4.32521-1.05710$ $4.10130-1.75555$ $3.93162-1.68868$ $3.87487-1.55714$ $3.91806-1.44900$ $4.00723-1.41087$ $4.08353-1.44155$ 
$\frac{a}{\lambda}=0.063175 \quad \stackrel{\alpha}{\beta}=0.07$

$\beta \mathrm{h}$

$C(h, 0)$

$\begin{array}{lll}0.01 & 0.96308 & -0.01997\end{array}$

$\begin{array}{lll}0.02 & 1.76299 & -0.0399 \\ 0.05 & 3.28953 & -0.09956\end{array}$

$\begin{array}{lll}0.05 & 3.28953 & -0.09956 \\ 0.10 & 4.60584 & -0.19813\end{array}$

$0.20 \quad 5.93452-0.39093$

$0.50 \quad 7.53197-0.91454$

$1.00 \quad 8.30546-1.51029$

$\begin{array}{lll}1.50 & 8.45674 & -1.71653\end{array}$

$2.00 \quad 8.53962-1.64682$

$2.50 \quad 8.72602-1.49482$

$\begin{array}{lll}3.00 & 8.99094 & -1.40902 \\ 3.50 & 9.23714 & -1.42748\end{array}$

$0.125 \pi \quad 7.14796 \quad-0.73877$

$0.250 \pi \quad 8.10706-1.30304$

$0.375 \pi \quad 8.39006-1.62573$

$0.500 \pi \quad 8.46578-1.71918$

$0.625 \pi \quad 8.53070-1.65704$

$\begin{array}{llll}0.750 \pi & 8.66083 & -1.53642\end{array}$

$0.875 \pi \quad 8.85388-1.43873$

$\begin{array}{lll}1.000 \pi & 9.06665 & -1.40476\end{array}$

$1.125 \pi \quad 9.25124-1.43155$

$9.38051-1.48761$
$\mathrm{S}(\mathrm{h}, 0)$

$0.00472-0.00043$

$0.01654-0.00156$

$\begin{array}{ll}0.06736 & -0.00722 \\ 0.16220 & -0.02132\end{array}$

$0.16220-0.02132$

$\begin{array}{ll}0.35305 & -0.06414 \\ 0.87547 & -0.29503\end{array}$

$\begin{array}{ll}0.87547 & -0.29503 \\ 1.47082 & -0.90721\end{array}$

$\begin{array}{ll}1.47082 & -0.90721 \\ 1.67602 & -1.56674\end{array}$

$\begin{array}{ll}1.67692 & -1.56674 \\ 1.60715 & -2.05918\end{array}$

$1.60715 \cdot-2.05918$
$1.45511 \cdot-2.31905$

$\begin{array}{ll}1.45511 & -2.31905 \\ 1.36928 & -2.41876\end{array}$

$\begin{array}{ll}1.36928 & -2.41876 \\ 1.38773 & -2.48086\end{array}$

$0.69991-0.19642$

$\begin{array}{lll}1.26368 & -0.62261\end{array}$

$1.58619-1.15036$

$1.67957-1.04993$

$1.61737-2.03126$

$1.49672-2.26576$

$1.39900-2.38100$

$1.36501-2.43528$

$1.39179-2.48625$

$1.44784-2.56770$
$E(h, 0)$

$\begin{array}{ll}0.96310 & -0.01997 \\ 1.76310 & -0.03993 \\ 3.29053 & -0.09975 \\ 4.61037 & -0.19910 \\ 5.95315 & -0.39521 \\ 7.63883 & -0.96909 \\ 8.61909 & -1.82900 \\ 8.86388 & -2.52321 \\ 8.78065 & -3.02046 \\ 8.54664 & -3.31674 \\ 8.27239 & -3.43289 \\ 8.03048 & -3.40848 \\ & \\ 7.21666 & -0.76797 \\ 8.33337 & -1.47745 \\ 8.76029 & -2.09747 \\ 8.86682 & -2.60597 \\ 8.79346 & -2.99106 \\ 8.62211 & -3.25131 \\ 8.40985 & -3.39495 \\ 8.19824 & -3.43819 \\ 8.01630 & -3.40289 \\ 7.88180 & -3.31386\end{array}$

$C(h, h)$

$\begin{array}{rr}0.88153 & -0.01996 \\ 1.44243 & -0.03989 \\ 2.31409 & -0.09935 \\ 2.97005 & -0.19685 \\ 3.58850 & -0.38317 \\ 3.99812 & -0.83361 \\ 3.00005 & -1.09318 \\ 1.07137 & -0.79651 \\ -1.13387 & -0.23011 \\ -3.13411 & 0.35560 \\ -4.46491 & 0.81855 \\ -4.73989 & 1.03508 \\ & \\ 3.98722 & -0.69473 \\ 3.59369 & -1.06099 \\ 2.37937 & -1.03853 \\ 0.76402 & -0.72554 \\ -0.97423 & -0.27436 \\ -2.60701 & 0.19468 \\ -3.90838 & 0.60873 \\ -4.66157 & 0.91059 \\ -4.71 .493 & 1.03772 \\ -4.03834 & 0.94087\end{array}$

$S(h, h)$

$\begin{array}{rr}0.00437 & -0.00041 \\ 0.01450 & -0.00142 \\ 0.06303 & -0.00691 \\ 0.17783 & -0.02237 \\ 0.46890 & -0.07164 \\ 1.49586 & -0.32189 \\ 3.02402 & -0.83778 \\ 3.85754 & -1.12323 \\ 3.85009 & -1.03376 \\ 2.98764 & -0.06221 \\ 1.37089 & -0.13713 \\ -0.67461 & 0.44073 \\ & \\ 1.12071 & -0.21889 \\ 2.43402 & -0.62451 \\ 3.41324 & -0.98039 \\ 3.90862 & -1.13281 \\ 3.87980 & -1.05160 \\ 3.32085 & -0.78963 \\ 2.26281 & -0.41375 \\ 0.81495 & 0.02597 \\ -0.81804 & 0.47913 \\ -2.37311 & 0.87093\end{array}$

$E(h, h)$

$\begin{array}{ll}0.88155 & -0.01997 \\ 1.44253 & -0.03991 \\ 2.30518 & -0.09955 \\ 2.97657 & -0.19811 \\ 3.02483 & -0.39090 \\ 4.30954 & -0.91451 \\ 4.39033 & -1.51023 \\ 4.13619 & -1.71644 \\ 3.93140 & -1.04671 \\ 3.89466 & -1.49469 \\ 3.97726 & -1.40886 \\ 4.06931 & -1.42732 \\ & \\ 4.16669 & -0.73872 \\ 4.43341 & -1.30298 \\ 4.31106 & -1.02566 \\ 4.09912 & -1.71909 \\ 3.94090 & -1.05693 \\ 3.88871 & -1.53628 \\ 3.92761 & -1.43858 \\ 4.00686 & -1.40460 \\ 4.07367 & -1.43139 \\ 4.09758 & -1.48744\end{array}$

$\frac{a}{\lambda}=0.003 i 75 \quad \frac{\alpha}{\beta}=0.10$

$\begin{array}{ll}0.96250 & -0.01996 \\ 1.76190 & -0.03990 \\ 3.28754 & -0.09965 \\ 4.60441 & -0.19877 \\ 5.94133 & -0.39499 \\ 7.61066 & -0.96190 \\ 8.57051 & -1.80285 \\ 8.80684 & -2.47173 \\ 8.72813 & -2.94379 \\ 8.50950 & -3.22097 \\ 8.25696 & -3.32811 \\ 8.03745 & -3.30610 \\ & \\ 7.19408 & -0.76346 \\ 8.29227 & -1.46057 \\ 8.70720 & -2.06270 \\ 8.80966 & -2.55077 \\ 8.74021 & -2.91610 \\ 8.57967 & -3.16013 \\ 8.38307 & -3.29328 \\ 8.18934 & -3.33295 \\ 8.02469 & -3.30107 \\ 7.90439 & -3.22150\end{array}$

$0.88094-0.01995$ $1.44124-0.03985$ $2.30112-0.09912$ $2.96420-0.19585$ $3.57743 \quad-0.37867$ $3.97839-0.80263$ $2.99458-0.98174$ $1.09777-0.59907$ $-1.08550 \quad 0.02256$ $\begin{array}{lll}-3.08137 & 0.59737\end{array}$ $-4.42737 \quad 0.94649$

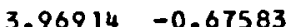
3.57813 2.38494 2.38494
0.79454 $-0.92686$ $-2.55376$ 3.06026 4.63316 $-4.72479$

$-4.10039$
$0.00436-0.00054$ $0.01447-0.00185$ $0.06289-0.00879$ $0.17725-0.02765$ $0.46666-0.08532$ $1.48462-0.36084$ $3.00301-0.88824$ $3.85309-1.13262$ $3.87857-0.95276$ $3.04527-0.44937$ $\begin{array}{rr}1.44691 & 0.21624 \\ -0.59879 & 0.87785\end{array}$

$1.11308-0.24961$ $2.41469-0.67628$ $3.39495-1.02219$ $3.90838-1.13229$ $3.90582-0.97876$ $3.37110-0.01777$ $2.33112-0.12847$ $\begin{array}{lr}2.33112 & -0.12847 \\ 0.89351 & 0.41201\end{array}$ $-0.74340 \quad 0.91815$

$-2.32684 \quad 1.28687$

$\begin{array}{ll}0.88095 & -0.01995 \\ 1.44133 & -0.03988 \\ 2.30220 & -0.09938 \\ 2.97067 & -0.19749 \\ 3.61335 & -0.38856 \\ 4.28526 & -0.90142 \\ 4.36407 & -1.47190 \\ 4.12848 & -1.66405 \\ 3.94376 & -1.60167 \\ 3.91129 & -1.46883 \\ 3.98120 & -1.39583 \\ 4.05703 & -1.41083 \\ & \\ 4.14613 & -0.73029 \\ 4.40483 & -1.27538 \\ 4.28983 & -1.58007 \\ 4.09467 & -1.66647 \\ 3.95220 & -1.61075 \\ 3.90616 & -1.50480 \\ 3.93942 & -1.42087 \\ 4.00581 & -1.39228 \\ 4.06055 & -1.41412 \\ 4.07975 & -1.45892\end{array}$


Bh

$\mathrm{C}(\mathrm{h}, 0)$

0.0

0.02

0.05

0.20

0.50

1.00

1.50
2.00

2.50

3.00
3.50

$0.125 \pi$ $0.250 \pi$ $0.375 \pi$ $0.625 \pi$ 0.750 0.875

$1.000 \pi$

$1.250 \pi$

$\begin{array}{ll}0.96049 & -0.01991 \\ 1.75781 & -0.03977 \\ 3.27665 & -0.09887 \\ 4.58037 & -0.19553 \\ 5.88523 & -0.38098 \\ 7.42902 & -0.85976 \\ 8.19414 & -1.35382 \\ 8.41645 & -1.50577 \\ 8.57308 & -1.46277 \\ 8.77473 & -1.37799 \\ 8.99713 & -1.33543 \\ 9.19104 & -1.34296 \\ 7.06050 & -0.70320 \\ 7.98654 & -1.18919 \\ 8.29836 & -1.44095 \\ 8.43769 & -1.50756 \\ 8.56034 & -1.46888 \\ 8.71235 & -1.40012 \\ 8.88623 & -1.34957 \\ 9.05657 & -1.33350 \\ 9.20253 & -1.34459 \\ 9.31711 & -1.36582\end{array}$

$\frac{a}{\lambda}=c .003175 \quad \frac{\alpha}{\beta}=0.20$

$S(h, 0)$

$E(h, 0)$

$0.00469-0.00104$ $0.01643-0.00370$ $0.06671-0.01592$ $0.15965-0.04213$ $0.34315-0.10851$ $0.82073-0.39291$ $1.31440-1.01340$ $1.46622-1.60189$ $\begin{array}{lll}1.42316 & -2.02057\end{array}$ $1.33833-2.26518$ $\begin{array}{ll}1.29574 & -2.40740 \\ 1.30325 & -2.52179\end{array}$ $0.66440-0.27883$ $1.14988-0.73802$ $1.40147-1.23692$ $1.46800-1.07287$ $1.42926-1.99647$ $1.36048-2.20909$ $1.30990-2.34349$ $1.29381-2.44020$ $1.30488-2.52980$ $1.32610-2.62593$

$\begin{array}{llrr}0.96051 & -0.01992 & 0.87894 & -0.01990 \\ 1.75791 & -0.03981 & 1.43726 & -0.03972 \\ 3.27760 & -0.09931 & 2.29126 & -0.09835 \\ 4.58461 & -0.19767 & 2.94494 & -0.19253 \\ 5.90221 & -0.39094 & 3.54146 & -0.36388 \\ 7.51825 & -0.93843 & 3.91813 & -0.70218 \\ 8.41320 & -1.71926 & 2.98730 & -0.62147 \\ 8.62340 & -2.31030 & 1.18509 & 0.04802 \\ 8.55806 & -2.70731 & -0.96446 & 0.86522 \\ 8.38372 & -2.92928 & -3.03070 & 1.41625 \\ 8.19186 & -3.01115 & -4.55599 & 1.40054 \\ 8.03308 & -2.99556 & -5.13438 & 0.67512 \\ & & & \\ 7.11977 & -0.74866 & 3.91247 & -0.61430 \\ 8.15848 & -1.40617 & 3.53627 & -0.74969 \\ 8.53588 & -1.95233 & 2.41280 & -0.42913 \\ 8.62582 & -2.37809 & 0.89202 & 0.16531 \\ 8.56796 & -2.68459 & -0.80496 & 0.81014 \\ 8.43876 & -2.38154 & -2.47154 & 1.30506 \\ 8.28648 & -2.98497 & -3.88574 & 1.49269 \\ 8.14212 & -3.01472 & -4.83001 & 1.26825 \\ 8.02410 & -2.99202 & -5.13218 & 0.60000 \\ 7.94115 & -2.93731 & -4.69908 & -0.44732\end{array}$

$$
\frac{a}{\lambda}=0.003175
$$$$
\frac{\alpha}{\beta}=0.40
$$

$0.95653-0.01983$ $4.54534-0.19549$ $8.23979-2.31008$ $8.24645-2.29479$ $-3.52552 \quad 2.63456$ $\begin{array}{ll}1.74997 & -0.03963 \\ 3.25781 & -0.09865\end{array}$ $5.82517-0.38301$ $7.34000-0.89374$ $8.11845-1.56734$ $8.28484-2.02909$ $8.12887-2.45251$ $8.01807-2.50034$ $7.93493-2.49253$

$0.97537-0.72018$ $7.90496-1.30533$ $8.21728-1.75485$ $8.28662-2.07896$ $8.16273-2.42312$ $\begin{array}{ll}8.07133 & -2.48554 \\ 7.99115 & -2.50228\end{array}$ $7.93049-2.49078$ $-0.01980$ $1.42935-0.03914$ $2.27179-0.09664$ $2.90738-0.18004$ $3.47374-0.33530$ . $.82217-0.51274$ 0.06095 $1.37064 \quad 1.32948$ $-6.05440 \quad 2.55633$ $-7.79890 \quad 0.1807$ $1.00054 \quad-0.89981$ $1.17250-1.36711$
$1.21202-1.74053$ $1.19330-2.01370$ $1.16427-2.20915$ $\begin{array}{ll}1.14588 & -2.35633\end{array}$

$1.14077-2.47852$

$\begin{array}{ll}1.14362 & -2.58882 \\ 1.14839 & -2.69204\end{array}$

$\begin{array}{rr}3.81520 & -0.49754 \\ 3.49818 & -0.30317 \\ 2.51775 & 0.46449 \\ 1.08245 & 1.52860 \\ -0.71582 & 2.55375 \\ -2.75521 & 3.18818 \\ -4.83464 & 3.10150 \\ -6.66257 & 2.05826 \\ -7.86911 & -0.04351 \\ -8.04247 & -3.09185\end{array}$

$S(h, h)$

$E(h, h)$

$\begin{array}{rr}0.00434 & -0.00097 \\ 0.01439 & -0.00329 \\ 0.06239 & -0.01504 \\ 0.17532 & -0.04514 \\ 0.45939 & -0.13048 \\ 1.45057 & -0.08921 \\ 2.95363 & -1.05976 \\ 3.88573 & -1.17739 \\ 4.05242 & -0.39453 \\ 3.33764 & 0.26511 \\ 1.76619 & 1.42587 \\ -0.41482 & 2.41098 \\ 1.08932 & -0.35074 \\ 2.36180 & -0.84873 \\ 3.36346 & -1.16872 \\ 3.95932 & -1.14486 \\ 4.06920 & -0.74849 \\ 3.63593 & -0.04580 \\ 2.65250 & 0.83935 \\ 1.19416 & 1.74082 \\ -0.57638 & 2.46146 \\ -2.43040 & 2.80043\end{array}$

$0.87896-0.01991$ $1.43735-0.03976$ $0.09231-0.0883$ $.95111-0.19547$ $57556-0.38090$ $.20660-0.8596$ $4.27903-1.35360$ $3.96490-1.46255$ $3.90490-1.46255$ $3.9434-1.37774$ $4.02326-1.34268$

$4.07924-0.70308$ $4.31291-1.18904$ $4.21938-1.44077$ $4.07106-1.50736$ $3.97057-1.46865$ $3.94027-1.39988$ $3.96001-1.34932$ $3.99682-1.33323$ $4.03423-1.36554$

$\begin{array}{rr}0.00430 & -0.00184 \\ 0.01424 & -0.00615 \\ 0.06143 & -0.02739 \\ 0.17159 & -0.07957 \\ 0.44586 & -0.21880 \\ 1.39731 & -0.74026 \\ 2.94490 & -1.41976 \\ 4.17448 & -1.32174 \\ 4.79670 & -0.19975 \\ 4.43660 & 1.84116 \\ 2.75916 & 4.33007 \\ -0.35987 & 6.44467 \\ 1.04926 & -0.54791 \\ 2.30582 & -1.19551 \\ 3.43199 & -1.49453 \\ 4.30759 & -1.22641 \\ 4.77980 & -0.31610 \\ 4.66286 & 1.18044 \\ 3.78099 & 3.06810 \\ 2.01954 & 5.00972 \\ -0.62292 & 0.55022 \\ -3.98432 & 7.17482\end{array}$

$\begin{array}{ll}0.87499 & -0.01982 \\ 1.42943 & -0.03952 \\ 2.27267 & -0.09775 \\ 2.91259 & -0.19151 \\ 3.50216 & -0.36616 \\ 4.05923 & -0.78367 \\ 4.11989 & -1.15504 \\ 4.00903 & -1.25017 \\ 3.94294 & -1.22969 \\ 3.93355 & -1.19504 \\ 3.94676 & -1.18053 \\ 3.95772 & -1.18241 \\ 3.95248 & -0.65266 \\ 4.14331 & -1.03948 \\ 4.08137 & -1.21156 \\ 3.99557 & -1.25114 \\ 3.94551 & -1.23245 \\ 3.93237 & -1.20343 \\ 3.93933 & -1.18505 \\ 3.95067 & -1.17996 \\ 3.95815 & -1.18281 \\ 3.96028 & -1.18758\end{array}$


$C(h, 0)$

$0.78165-0.02000$ $80-0.03999$ $4.18396-0.19954$ $7.14387-0.94598$ $7.92173-1.6052$ $8.02376-1.84845$ $8.22495-1.54978$ $8.82814 \cdot-1.45452$

$6.75101-0.75890$ $7.72935-1.37062$ $7.99319-1.73956$ $8.02391-1.85174$ $8.04496-1.77150$ $8.36756-1.46888$ $8.62086-1.41805$ $8.84501-1.41805$ $8.99032-1.55576$ $\sin , 0)$

$0.00386-0.00010$ $0.01405-0.00040$ $0.06181-0.00250$ $0.15579-0.00998$ $0.34964-0.03973$ $0.89735-0.23979$ $1.55599-0.84729$ $1.79900-1.55602$ $1.70845-2.10425$ $1.50016 \quad-2.37639$ $\begin{array}{ll}1.37491 & -2.43672 \\ 1.40482 & -2.44612\end{array}$

$0.71060-0.15029$ $1.32154-0.55674$ $1.69022-1.10355$ $\begin{array}{ll}1.80226 & -1.04809 \\ 1.72195 & -2.07329\end{array}$ $1.55860-2.32554$ $1.41923-2.42359$ $1.36838-2.43734$ $1.41147-2.44875$ $1.50605-2.51416$
$E(h, 0)$

$0.78166-0.02000$ $1.46790-0.03999$ $2.88987-C .09998$ $4.18844-0.19987$ $5.53689-0.39907$ $7.25897-0.98611$ $8.28823-1.89197$ $8.55388-2.64908$ $8.45904-3.21046$ $8.18478-3.55602$ $7.85230-3.69684$ $7.54879-3.66576$

$\begin{array}{ll}0.82378 & -0.77862 \\ 7.98437 & -1.51779\end{array}$ $8.98437-1.51779$ $8.55715-2.74122$ $8.47375-3.17669$ $8.27424-3.47908$ $8.02040-3.65052$ $7.76038-3.70340$ $7.53066-3.65860$ $\begin{array}{ll}7.53066 & -3.65860 \\ 7.35618 & -3.54287\end{array}$
$C(h, h)$

$0.73394-0.02000$ $1.25034-0.0399$ $2.09326-0.0998$ $2.76259-0.19920$ $3.39544-0.39378$ $3.84898-0.9074$ $2.89375-1.36026$ $0.98740-1.2656$ $-1.18113-0.8245$ $-3.14891-0.21099$ $\begin{array}{ll}-4.47141 & 0.52537 \\ -4.71892 & 1.28052\end{array}$

\section{$3.82336-0.73967$} $3.47408-1.23738$ $2.28082-1.38198$ $0.68419-1.21867$ $-1.02452-0.86371$ $-2.62850-0.40126$ $-3.91732 \quad 0.14298$ $-4.66521 \quad 0.74606$ $\begin{array}{ll}-4.66521 & 0.74606 \\ -4.68979 & 1.32715\end{array}$ $-3.94133 \quad 1.75399$
$\mathrm{S}(\mathrm{h}, \mathrm{h})$

$0.00364-0.00010$ $0.01248-0.00040$ $0.05590-0.00250$ $0.16121-0.00997$ $0.43447-0.03947$ $1.42127-0.23008$ $2.89947-0.72178$ $3.67109-1.10943$ $3.61919-1.23132$ $2.76804-1.16346$ $1.19249-0.96690$ $-0.81787-0.58009$

$1.05851-0.14649$ $2.33132-0.50350$ $3.26796-0.8869$ $3.71348-1.14271$ $3.65038-1.23034$ $3.09268-1.19613$ $2.06329-1.08380$ $0.64721-0.88032$ $-0.95875-0.54508$ $-2.47099-0.07906$
$E(h, h)$

$0.73395-0.02000$ $1.25043-0.03999$ $2.09422-0.09993$ $2.76845-0.19954$ $3.42884-0.39642$ $4.14411-0.94598$ $4.22952-1.60523$ $3.92615-1.84842$ $3.66555-1.75795$ $3.61652-1.54968$ $3.73850-1.42444$ $3.88325-1.45436$ $3.99219 \quad-0.75890$ $4.27857-1.37061$ $4.13712-1.7395$ $3.88019-1.85170$ $3.67809-1.77144$ $3.60814-1.60812$ $3.66422-1.46870$ $3.78399-1.41791$ $\begin{array}{ll}3.89037 & -1.46101 \\ 3.93032 & -1.55559\end{array}$

\begin{tabular}{|c|c|c|}
\hline $\begin{array}{l}0.01 \\
0.02 \\
0.05 \\
0.10 \\
0.20 \\
0.50 \\
1.00 \\
1.50 \\
2.00 \\
2.50 \\
3.00 \\
3.50\end{array}$ & $\begin{array}{l}0.78155 \\
1.46760 \\
2.88843 \\
4.18296 \\
5.51589 \\
7.13967 \\
7.91719 \\
8.02303 \\
8.05457 \\
8.22968 \\
8.53062 \\
8.82491\end{array}$ & $\begin{array}{l}-0.01999 \\
-0.03999 \\
-0.09991 \\
-0.19943 \\
-0.39602 \\
-0.94369 \\
-1.59818 \\
-1.83854 \\
-1.74977 \\
-1.54617 \\
-1.42431 \\
-1.45323\end{array}$ \\
\hline $\begin{array}{l}0.125 \pi \\
0.250 \pi \\
0.375 \pi \\
0.500 \pi \\
0.625 \pi \\
0.750 \pi \\
0.875 \pi \\
1.000 \pi \\
1.125 \pi \\
1.250 \pi\end{array}$ & $\begin{array}{l}6.74748 \\
7.72436 \\
7.98966 \\
8.02389 \\
8.04844 \\
8.16226 \\
8.37104 \\
8.62086 \\
8.84154 \\
8.98540\end{array}$ & $\begin{array}{l}-0.75743 \\
-1.36563 \\
-1.73106 \\
-1.84178 \\
-1.76299 \\
-1.60320 \\
-1.46736 \\
-1.41797 \\
-1.45965 \\
-1.55077\end{array}$ \\
\hline
\end{tabular}

0.01

0.05

.10

0.50

2.00

$$
\frac{a}{\lambda}=0.003969 \quad \frac{\alpha}{\beta}=0.005
$$

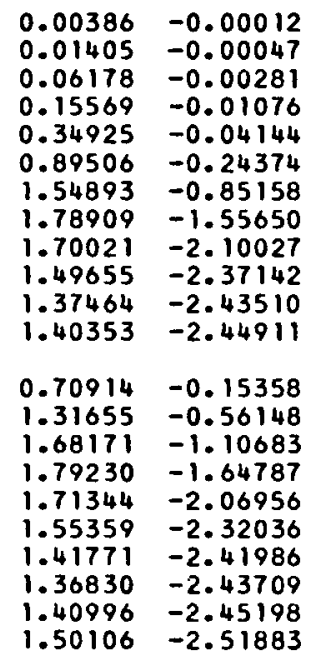

$\begin{array}{llrr}0.78156 & -0.02000 & 0.73384 & -0.01999 \\ 1.46770 & -0.03999 & 1.25014 & -0.03998 \\ 2.88937 & -0.09996 & 2.09276 & -0.09985 \\ 4.18745 & -0.19981 & 2.76160 & -0.19904 \\ 5.53491 & -0.39886 & 3.39353 & -0.39306 \\ 7.25418 & -0.98488 & 3.84533 & -0.90236 \\ 8.27983 & -1.83736 & 2.89217 & -1.34173 \\ 8.54392 & -2.03979 & 0.99202 & -1.23337 \\ 8.44996 & -3.19633 & -1.17036 & -0.78375 \\ 8.17880 & -3.53866 & -3.13213 & -0.17187 \\ 7.85085 & -3.67700 & -4.44859 & 0.54577 \\ 7.55223 & -3.64645 & -4.69558 & 1.26261 \\ & & & \\ 6.81995 & -0.77785 & 3.82011 & -0.73657 \\ 7.97731 & -1.51485 & 3.47084 & -1.22513 \\ 8.43127 & -2.17550 & 2.28123 & -1.35819 \\ 8.54717 & -2.73123 & 0.68974 & -1.18473 \\ 8.46453 & -3.16289 & -1.01417 & -0.82329 \\ 8.26718 & -3.46206 & -2.61353 & -0.36024 \\ 8.01655 & -3.63134 & -3.89733 & 0.17531 \\ 7.76033 & -3.68346 & -4.64133 & 0.75740 \\ 7.53441 & -3.63942 & -4.66681 & 1.30608 \\ 7.36315 & -3.52584 & -3.92679 & 1.69532\end{array}$

$\begin{array}{ll}0.00564 & -0.00012 \\ 0.01248 & -0.00046 \\ 0.05587 & -0.00278 \\ 0.16111 & -0.01076 \\ 0.43408 & -0.04158 \\ 1.41916 & -0.23621 \\ 2.89459 & -0.72937 \\ 3.66734 & -1.10986 \\ 3.61919 & -1.21763 \\ 2.77147 & -1.12947 \\ 1.20093 & -0.91022 \\ -0.80042 & -0.50983 \\ 1.05712 & -0.15131 \\ 2.32732 & -0.51154 \\ 3.26296 & -0.89287 \\ 3.71020 & -1.14153 \\ 3.65010 & -1.21789 \\ 3.09514 & -1.16853 \\ 2.06876 & -1.03823 \\ 0.65784 & -0.81817 \\ -0.94060 & -0.47459 \\ -2.44593 & -0.01423\end{array}$

$\begin{array}{ll}0.73385 & -0.01999 \\ 1.25023 & -0.03999 \\ 2.09372 & -0.09991 \\ 2.76745 & -0.19943 \\ 3.42690 & -0.39602 \\ 4.13991 & -0.94368 \\ 4.22498 & -1.59816 \\ 3.92543 & -1.83850 \\ 3.66928 & -1.74970 \\ 3.62125 & -1.54606 \\ 3.73988 & -1.42416 \\ 3.88002 & -1.45306 \\ 3.98865 & -0.75743 \\ 4.27358 & -1.36562 \\ 4.13359 & -1.73103 \\ 3.88017 & -1.84173 \\ 3.68157 & -1.76292 \\ 3.61307 & -1.60310 \\ 3.66770 & -1.46723 \\ 3.78400 & -1.41782 \\ 3.88690 & -1.45948 \\ 3.92541 & -1.55060\end{array}$


$\frac{a}{\lambda}=0.303969 \quad \frac{\alpha}{\beta}=0.01$

$\beta \mathrm{h}$

$\mathrm{C}(\mathrm{h}, 0)$

$\mathrm{S}(\mathrm{h}, 0)$

$E(h, i))$

$\mathrm{C}(\mathrm{h}, \mathrm{h})$

$\mathrm{S}(\mathrm{h}, \mathrm{h})$

$E(h, h)$

$\begin{array}{lll}0.01 & 0.78145 & -0.01999\end{array}$

$0.02 \quad 1.46740-0.03998$

$\begin{array}{lll}0.05 & 2.88793 & -0.09988\end{array}$

$\begin{array}{ll}4.18197 & -0.19933\end{array}$

$5.51395-0.395 .33$

$7.13548,-0.94140$

$7.91266-1.5911$

$8.02227-1.8287$

$8.05818-1.74158$

$8.23427-1.54250$

$8.53196-1.42389$

$8.82178-1.45183$

3.00
3.50

$\begin{array}{lll}0.125 \pi & 6.74396 & -0.75597 \\ 0.250 \pi & 7.71938 & -1.36006\end{array}$

$\begin{array}{llll}0.250 \pi & 7.71938 & -1.36066\end{array}$

$\begin{array}{llll}0.375 \pi & 7.98612 & -1.72261\end{array}$

$\begin{array}{llll}0.500 \pi & 8.02382 & -1.83190\end{array}$

$\begin{array}{llll}0.625 \pi & 8.05181-1.75454\end{array}$

$\begin{array}{llll}0.750 \pi & 8.16705 & -1.59817\end{array}$

$\begin{array}{llll}0.875 \pi & 8.37442 & -1.46573\end{array}$

$\begin{array}{llll}1.000 \pi & 8.62086 & -1.41774\end{array}$

$\begin{array}{llll}1.125 \pi & 8.83817 & -1.45303\end{array}$

$1.250 \pi \quad 8.98065-1.54581$

$0.00386-0.00014$

$0.01404-0.00054$

$0.06176-0.00312$

$0.15559-0.01153$

$0.34886-0.04315$

$0.89277-0.24769$

$\begin{array}{lll}1.54192 & -0.35587\end{array}$

$1.77926-1.5570$

$1.69202-2.0964$

$1.49289-2.36658$

$1.37422-2.43352$

$1.40214 \quad-2.45198$

$0.70768-0.15680$

$1.31159-0.56622$

$1.67327-1.11012$

$1.78242-1.64769$

$1.70499-2.06594$

$1.54856-2.31532$

$\begin{array}{ll}1.41609 & -2.41623\end{array}$

$1.36807-2.43685$

$1.40834-2.45509$

$\begin{array}{llrr}0.78146 & -0.01999 & 0.73374 & -0.01999 \\ 1.46750 & -0.03998 & 1.24994 & -0.03997 \\ 2.88887 & -0.09994 & 2.09226 & -0.09982 \\ 4.18645 & -0.19975 & 2.76061 & -0.19888 \\ 5.53292 & -0.39865 & 3.39163 & -0.39233 \\ 7.24940 & -0.98364 & 3.84170 & -0.89726 \\ 8.27144 & -1.88277 & 2.89063 & -1.32326 \\ 8.53399 & -2.63055 & 0.99663 & -1.20111 \\ 8.44090 & -3.18228 & -1.15974 & -0.74304 \\ 8.17280 & -3.52082 & -3.11581 & -0.13233 \\ 7.84933 & -3.65732 & -4.42675 & 0.56613 \\ 7.55551 & -3.62729 & -4.67372 & 1.24498 \\ & & & \\ 6.81613 & -0.77707 & 3.81687 & -0.73347 \\ 7.97026 & -1.51192 & 3.46765 & -1.21293 \\ 8.42207 & -2.16934 & 2.28168 & -1.33447 \\ 8.53721 & -2.72129 & 0.69526 & -1.15086 \\ 8.45533 & -3.14918 & -1.00396 & -0.78294 \\ 8.26011 & -3.44515 & -2.59891 & -0.31929 \\ 8.01267 & -3.61230 & -3.87804 & 0.20757 \\ 7.76019 & -3.66368 & -4.61860 & 0.76877 \\ 7.53800 & -3.62038 & -4.64533 & 1.28532 \\ 7.36989 & -3.50891 & -3.91375 & 1.03731\end{array}$

$0.00364-0.00014$

$0.01247-0.00052$

$0.05585-0.00305$

$0.16101-0.01156$

$0.43369-0.04369$

$\begin{array}{ll}1.41707 & -0.24233 \\ 2.88978 & -0.73697\end{array}$

$2.88978-0.73697$

$3.66376-1.11035$

$3.61944-1.20402$

$2.77525-1.09555$

$\begin{array}{rr}1.20964 & -0.85364 \\ -0.78325 & -0.43982\end{array}$

$1.05575-0.15613$

$2.32336-0.51957$

$3.25808-0.89886$

$3.70710-1.14042$

$3.65008-1.20552$

$3.09792-1.14100$

$2.07458-0.99276$

$0.66804-0.75622$

$\begin{array}{lll}-0.92280 & -0.40435\end{array}$

$\begin{array}{ll}-2.42192 & 0.05046\end{array}$
$0.73375-0.01999$ $1.25003-0.03998$ $2.09322-0.09988$ $2.76646-0.19933$ $3.42495-0.39562$ $4.13572-0.94139$ $4.22045-1.59114$ $3.92467-1.82866$ $3.67289-1.74149$ $3.62584-1.54238$ $3.74121-1.42373$ $3.87689-1.45165$

$3.98513-0.75596$ $4.26861-1.36065$ $4.13005-1.72258$ $3.88010-1.83184$ $3.68495-1.75446$ $3.61786-1.59805$ $3.67108-1.46559$ $3.78400-1.41759$ $3.88354-1.45786$ $3.92066-1.54563$

\begin{tabular}{|c|c|c|}
\hline $\begin{array}{l}0.01 \\
0.02 \\
0.05 \\
0.10 \\
0.20 \\
0.50 \\
1.00 \\
1.50 \\
2.00 \\
2.50 \\
3.00 \\
3.50\end{array}$ & $\begin{array}{l}0.78125 \\
1.46700 \\
2.88693 \\
4.17999 \\
5.51007 \\
7.12713 \\
7.90363 \\
8.02063 \\
8.06506 \\
8.24306 \\
8.53449 \\
8.81582\end{array}$ & $\begin{array}{l}-0.01999 \\
-0.03997 \\
-0.09982 \\
-0.19913 \\
-0.39483 \\
-0.93684 \\
-1.57726 \\
-1.80927 \\
-1.72532 \\
-1.53501 \\
-1.42262 \\
-1.44871\end{array}$ \\
\hline $\begin{array}{l}0.125 \pi \\
0.250 \pi \\
0.375 \pi \\
0.500 \pi \\
0.625 \pi \\
0.750 \pi \\
0.875 \pi \\
1.000 \pi \\
1.125 \pi \\
1.250 \pi\end{array}$ & $\begin{array}{l}6.73693 \\
7.70947 \\
7.97905 \\
8.02353 \\
8.05823 \\
8.17621 \\
8.38088 \\
8.62084 \\
8.83176 \\
8.97161\end{array}$ & $\begin{array}{l}-0.75306 \\
-1.35081 \\
-1.70589 \\
-1.81236 \\
-1.73778 \\
-1.58803 \\
-1.46214 \\
-1.41685 \\
-1.45449 \\
-1.53594\end{array}$ \\
\hline
\end{tabular}

$\frac{\bar{\lambda}}{\bar{\lambda}}=0.003960 \quad \frac{\alpha}{\beta}=0.02$

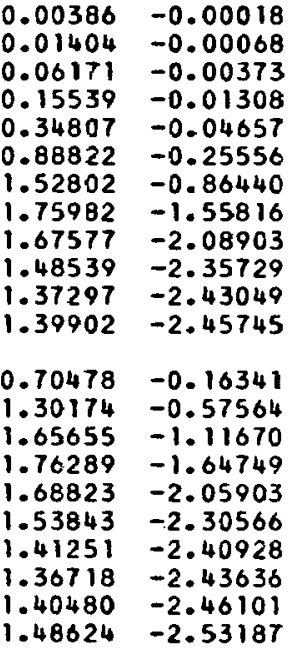

$\begin{array}{ll}0.78126 & -0.01999 \\ 1.46710 & -0.03997 \\ 2.88787 & -0.09990 \\ 4.18445 & -0.19964 \\ 5.52896 & -0.39823 \\ 7.23985 & -0.98118 \\ 8.25473 & -1.87364 \\ 8.51422 & -2.61220 \\ 8.42284 & -3.15445 \\ 8.16075 & -3.48554 \\ 7.84606 & -3.61841 \\ 7.56161 & -3.58940 \\ & \\ 6.80851 & -0.77553 \\ 7.95620 & -1.50608 \\ 8.40374 & -2.15711 \\ 8.51739 & -2.70156 \\ 8.43699 & -3.12199 \\ 8.24597 & -3.41169 \\ 8.00477 & -3.57466 \\ 7.75962 & -3.62458 \\ 7.54470 & -3.58273 \\ 7.38274 & -3.47536\end{array}$

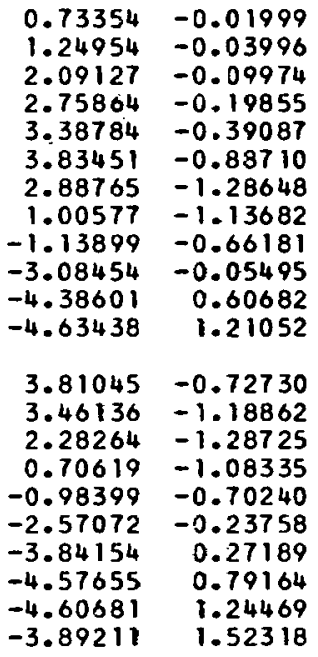

$\begin{array}{rr}0.00364 & -0.00017 \\ 0.01246 & -0.00065 \\ 0.05580 & -0.00361 \\ 0.16081 & -0.01316 \\ 0.43292 & -0.04791 \\ 1.41294 & -0.25454 \\ 2.88042 & -0.75222 \\ 3.65712 & -1.11152 \\ 3.62074 & -1.17700 \\ 2.78386 & -1.02790 \\ 1.22782 & -0.74093 \\ -0.74978 & -0.30046 \\ 1.05301 & -0.16574 \\ 2.31558 & -0.53564 \\ 3.24866 & -0.91093 \\ 3.70145 & -1.13839 \\ 3.65079 & -1.18101 \\ 3.10449 & -1.08612 \\ 2.08726 & -0.90211 \\ 0.69069 & -0.63286 \\ -0.88821 & -0.26453 \\ -2.37706 & 0.17959\end{array}$

$\begin{array}{ll}0.73355 & -0.01999 \\ 1.24963 & -0.03997 \\ 2.09223 & -0.09982 \\ 2.76448 & -0.19912 \\ 3.42107 & -0.39482 \\ 4.12737 & -0.93682 \\ 4.21142 & -1.57722 \\ 3.92303 & -1.80921 \\ 3.67978 & -1.72522 \\ 3.63463 & -1.53487 \\ 3.74375 & -1.42246 \\ 3.87094 & -1.44852 \\ 3.97810 & -0.75304 \\ 4.25870 & -1.35078 \\ 4.12298 & -1.70584 \\ 3.87981 & -1.81229 \\ 3.69137 & -1.73768 \\ 3.62703 & -1.58791 \\ 3.67755 & -1.46199 \\ 3.78399 & -1.41667 \\ 3.87713 & -1.45430 \\ 3.91162 & -1.53575\end{array}$




\begin{tabular}{|c|c|c|}
\hline $\begin{array}{l}0.01 \\
0.02 \\
0.05 \\
0.10 \\
0.20 \\
0.50 \\
1.00 \\
1.50 \\
2.00 \\
2.50 \\
3.00 \\
3.50\end{array}$ & $\begin{array}{l}0.78105 \\
1.46661 \\
2.88593 \\
4.17801 \\
5.50619 \\
7.11881 \\
7.89465 \\
8.01885 \\
8.07151 \\
8.25133 \\
8.53685 \\
8.81021\end{array}$ & $\begin{array}{l}-0.01998 \\
-0.03995 \\
-0.09977 \\
-0.19892 \\
-0.39404 \\
-0.93231 \\
-1.56352 \\
-1.79013 \\
-1.70924 \\
-1.52730 \\
-1.42083 \\
-1.44518\end{array}$ \\
\hline $\begin{array}{l}0.125 \pi \\
0.250 \pi \\
0.375 \pi \\
0.500 \pi \\
0.625 \pi \\
0.750 \pi \\
0.875 \pi \\
1.000 \pi \\
1.125 \pi \\
1.250 \pi\end{array}$ & $\begin{array}{l}6.72993 \\
7.69961 \\
7.97198 \\
8.02305 \\
8.06423 \\
8.18485 \\
8.38694 \\
8.62081 \\
8.82573 \\
8.96315\end{array}$ & $\begin{array}{l}-0.75016 \\
-1.34105 \\
-1.68940 \\
-1.79313 \\
-1.72122 \\
-1.57782 \\
-1.45816 \\
-1.41540 \\
-1.45056 \\
-1.52615\end{array}$ \\
\hline
\end{tabular}

$\frac{\mathrm{a}}{\lambda}=0.063069 \quad \frac{\alpha}{\beta}=0.03$

$E(h, 0)$

$C(h, h)$

$0.00385-0.00022$ $0.01403-0.00082$ $0.06166-0.00435$ $0.15519-0.01462$ $0.34729-0.04997$ $0.88370-0.26339$ $1.51429-0.87290$ $1.74069-1.55945$ $1.65970-2.08208$ $1.47770-2.34853$ $1.37118-2.42763$ $1.39550-2.46256$

$0.70189-0.16993$ $1.29199-0.58500$ $1.64007-1.12328$ $1.74367-1.64747$ $1.67168-2.05253$ $1.52822-2.29653$ $1.40853-2.40272$ $1.36574-2.43591$ $1.40088-2.46654$ $1.47646-2.53984$

$\begin{array}{ll}0.78106 & -0.01998 \\ 1.46670 & -0.03996 \\ 2.88687 & -0.09987 \\ 4.18246 & -0.19952 \\ 5.52499 & -0.39782 \\ 7.23032 & -5.97873 \\ 8.23810 & -1.86457 \\ 8.49456 & -2.59403 \\ 8.40487 & -3.12695 \\ 8.14865 & -3.45077 \\ 7.84250 & -3.58010 \\ 7.56712 & -3.55208 \\ & \\ 6.80089 & -1.77400 \\ 7.94220 & -1.50027 \\ 8.38550 & -2.14497 \\ 8.49768 & -2.68203 \\ 8.41874 & -3.09513 \\ 8.23182 & -3.37868 \\ 7.99671 & -3.53759 \\ 7.75867 & -3.58609 \\ 7.55080 & -3.54564 \\ 7.39474 & -3.44221\end{array}$

$\mathrm{S}(\mathrm{h}, \mathrm{h})$

$0.00364-0.00021$ $0.01246-0.00077$ $0.05575-0.00417$ $0.16061-0.01475$ $0.43215-0.05212$ $1.40885-0.26674$ $2.87139-0.76752$ $3.65115-1.11294$ $3.62309-1.15027$ $2.79388-0.96049$ $\begin{array}{ll}2.79388 & -0.96049 \\ 1.24702 & -0.62873\end{array}$ $-0.71742-0.16184$

$1.05029-0.17533$ $2.30799-0.55170$ 2.307990 .05170 $3.69652-1.13664$ $3.69652-1.13604$ $3.65251-1.15678$ $2.10131-0.81180$ $2.10131-0.81180$ $-0.85495-0.12543$ $-2.33632 \quad 0.30852$
$E(h, h)$

$\begin{array}{ll}0.73335 & -0.01998 \\ 1.24923 & -0.03995 \\ 2.09123 & -0.09976 \\ 2.76250 & -0.19891 \\ 3.41720 & -0.39402 \\ 4.11905 & -0.93228 \\ 4.20243 & -1.56348 \\ 3.92125 & -1.79005 \\ 3.68624 & -1.70913 \\ 3.64291 & -1.52716 \\ 3.74612 & -1.42065 \\ 3.86534 & -1.44498 \\ 3.97110 & -0.75014 \\ 4.24884 & -1.34101 \\ 4.11591 & -1.68934 \\ 3.87933 & -1.79305 \\ 3.69737 & -1.72111 \\ 3.63567 & -1.57768 \\ 3.68362 & -1.45799 \\ 3.78396 & -1.41521 \\ 3.87111 & -1.45036 \\ 3.90317 & -1.52594\end{array}$

$$
\frac{a}{\lambda}=0.003969 \quad \frac{\alpha}{B}=0.05
$$

$0.00385-0.00029$ $0.01401 \cdot-0.00110$ $0.06156-0.00558$ $0.15479-0.01770$ $0.34572-0.05676$ $0.87475-0.27894$ $1.48733-0.88977$ $1.70330-1.56245$ $1.62811-2.06945$ $1.46176-2.33246$ $\begin{array}{ll}1.36614 & -2.42244 \\ 1.38732 & -2.47183\end{array}$

$0.69615-0.18289$

$1.27277-0.00356$

$1.60777-1.13645$

$1.70610-1.64797$

$1.63917-2.04075$

$1.50761-2.27979$

$1.39946-2.39073$

$1.36132-2.43509$

$1.39200-2.47657$

$\begin{array}{ll}0.78066 & -0.01997 \\ 1.46590 & -0.03994 \\ 2.88488 & -0.09979 \\ 4.17848 & -0.19929 \\ 5.51708 & -0.39698 \\ 7.21134 & -0.97384 \\ 8.20506 & -1.84660 \\ 8.45557 & -2.55819 \\ 8.36916 & -3.07296 \\ 8.12429 & -3.38269 \\ 7.83453 & -3.50525 \\ 7.57643 & -3.47909 \\ & -1 \\ 0.78571 & -0.77094 \\ 7.91434 & -1.58871 \\ 8.34930 & -2.12096 \\ 8.45860 & -2.64353 \\ 8.38249 & -3.04237 \\ 8.20352 & -3.31401 \\ 7.98013 & -3.46509 \\ 7.75569 & -3.51088 \\ 7.56121 & -3.47309 \\ 7.41634 & -3.37713\end{array}$

$0.73294 \quad-0.01997$ $0.24835-0.03991$
2.0885 $\begin{array}{ll}1.24835 & -0.03991 \\ 2.08828 & -0.09951\end{array}$ $2.75275-0.19758$ $3.37654-0.38652$ $3.37654-0.38652$ $2.87961-1.17745$ $\begin{array}{ll}2.87961 & -1.17745 \\ 1.03279 & -0.94564\end{array}$ $-1.03279-0.94564$ $-3.00172-0.4774$ $-4.286920 .72895$ $\begin{array}{ll}-4.28692 & 0.72895 \\ -4.55070 & 1.11328\end{array}$ $3.79149-0.70892$ $3.44339-1.11655$ $2.28616-1.14715$ $0.73823-0.88242$ $-0.92750-0.46204$ $-2.49450 \quad 0.00632$ $-3.74861 \quad 0.46384$ $\begin{array}{ll}-4.47726 & 0.86150\end{array}$ $-4.52603 \quad 1.12949$ $-3.86219 \quad 1.19399$
$0.00363-0.00028$ $0.01244-0.00102$ $0.05565-0.00528$ $0.16022-0.01793$ $0.43062-0.0605$ $\begin{array}{lll}1.40084 & -0.29104\end{array}$ $2.85427-0.7982$ $3.64125-1.11652$ $3.63095-1.0976$ $2.81813-0.82631$ $\begin{array}{rr}1.28840 & -0.40553 \\ -0.65597 & 0.11394\end{array}$

$1.04495-0.19444$ $2.29335-0.58379$ $3.22308-0.94786$ $3.68885-1.13392$ $3.65906-1.10914$ $3.13218-0.9228$ $2.13353-0.63198$ $0.76045-0.26607$ $\begin{array}{ll}-0.79234 & 0.15136 \\ -2.26686 & 0.56600\end{array}$
$0.73295-0.01997$ $1.24843-0.03993$ $2.08924-0.09964$ $2.75854-0.19849$ $3.40947-0.39244$ $4.10253-0.92330$ $4.18458-1.53648$ $3.91726-1.75262$ $3.69789-1.67750$ $3.65799-1.51118$ $3.85508-1.43676$

$3.95717-0.74437$ $4.22930-1.32177$ $4.10176-1.65701$ $3.87785-1.75544$ $3.70817-1.68856$ $3.65143-1.55703$ $3.69462-1.44889$ $3.78379-1.41076$ $3.86009-1.44144$ $3.88778-1.50653$ 

$1.46501-0.03990$ $2.88195-0.09954$ $4.17010-0.19810$ $5.49077-0.39090$ $\begin{array}{ll}7.08591 & -0.91450\end{array}$ $7.85909-1.51022$ $8.01034-1.71646$ $8.09322-1.64675$ $8.27902-1.49477$ $8.54454-1.40898$ $8.79073-1.42745$ $6.70217-0.73872$ $7.66075-1.30298$ $7.94367-1.62560$ $8.01938-1.71911$ $8.08430-1.65697$ $8.21443-1.53637$ $8.40748-1.43868$ $8.02025-1.40472$ $\begin{array}{ll}8.80484 & -1.43152 \\ 8.93410 & -1.48758\end{array}$

$\begin{array}{ll}0.00385 & -0.00037 \\ 0.01400 & -0.00138 \\ 0.06146 & -0.00680 \\ 0.15440 & -0.02077 \\ 0.34417 & -0.06352 \\ 0.86591 & -0.29434 \\ 1.46102 & -0.90649 \\ 1.66704 & -1.56598 \\ 1.59723 & -2.05840 \\ 1.44519 & -2.31825 \\ 1.35935 & -2.41796 \\ 1.37780 & -2.48005 \\ 0.69047 & -0.19575 \\ 1.25394 & -0.62190 \\ 1.57635 & -1.14962 \\ 1.66968 & -1.64916 \\ 1.60746 & -2.03048 \\ 1.48680 & -2.26496 \\ 1.38908 & -2.38019 \\ 1.35509 & -2.43448 \\ 1.38187 & -2.48545 \\ 1.43791 & -2.56690\end{array}$

$0.78026-0.01996$ $1.46510-0.03992$ $2.88288-0.09972$ $4.17450-0.19906$ $5.50919-0.39615$ $7.19245-0.96899$ $8.17231-1.82886$ $8.41702-2.52304$ $8.33376-3.02026$ 8.09975-3.31652 $\begin{array}{ll}7.82549 & -3.43266 \\ 7.58358 & -3.40824\end{array}$

$6.77059 \quad-0.76789$ $7.88669-1.47733$ $8.41990-2.60579$ $8.34658-2.99086$ $8.17522-3.25110$

$.96295-3.39472$ $7.75135-3.43796$ $\begin{array}{ll}7.56940 & -3.40265 \\ 7.43491 & -3.31361\end{array}$

$\begin{array}{rr}0.73254 & -0.01996 \\ 1.24755 & -0.03988 \\ 2.08630 & -0.09935 \\ 2.74883 & -0.19693 \\ 3.36908 & -0.38364 \\ 3.79980 & -0.83700 \\ 2.87499 & -1.10580 \\ 1.05052 & -0.81948 \\ -1.04466 & -0.25854 \\ -2.95563 & 0.33170 \\ -4.23985 & 0.81090 \\ -4.52286 & 1.05315 \\ & \\ 3.77912 & -0.69679 \\ 3.43214 & -1.06918 \\ 2.28907 & -1.05501 \\ 0.75903 & -0.74973 \\ -0.89266 & -0.30268 \\ -2.45064 & 0.16823 \\ -3.70035 & 0.59145 \\ -4.43303 & 0.90948 \\ -4.50050 & 1.05775 \\ -3.87094 & 0.98353\end{array}$
$0.05555-0.0063$ $0.15983-0.0211$ $0.42910-0.0688$ $1.39304-0.31523$ $2.83842-0.82920$ $3.63406-1.12103$ $3.64311-1.04597$ $2.84802-0.69280$ $1.33369-0.1833$ $-0.59878 \quad 0.38896$

$1.03971-0.21348$ $2.27943-0.61586$ $3.20824-0.97306$ $3.66977-1.06255$ $3.15737-0.81488$ $2.17117-0.45291$ $0.80987-0.02310$ $\begin{array}{ll}-0.73477 & 0.4274\end{array}$ $-2.21300 \quad 0.82630$
$0.00363-0.00035$ $0.01242-0.0012$ $3.68411-1.13222$ $8.31347-2.09732$

$\begin{array}{ll}0.73255 & -0.01996 \\ 1.24763 & -0.03990 \\ 2.08725 & -0.09953 \\ 2.75459 & -0.19808 \\ 3.40178 & -0.39086 \\ 4.08616 & -0.91443 \\ 4.16688 & -1.51013 \\ 3.91275 & -1.71633 \\ 3.70796 & -1.04659 \\ 3.67122 & -1.49457 \\ 3.75382 & -1.40875 \\ 3.84587 & -1.42720 \\ & \\ 3.94334 & -0.73866 \\ 4.20998 & -1.30290 \\ 4.08761 & -1.62555 \\ 3.87567 & -1.71898 \\ 3.71746 & -1.65681 \\ 3.66527 & -1.53617 \\ 3.70418 & -1.43846 \\ 3.78342 & -1.40448 \\ 3.85023 & -1.43127 \\ 3.87414 & -1.48732\end{array}$

$$
\frac{a}{\lambda}=0.003969 \quad \frac{\alpha}{\beta}=0.10
$$

$\begin{array}{ll}0.77967 & -0.01995 \\ 1.46390 & -0.03989 \\ 2.87990 & -0.09961 \\ 4.16854 & -0.19871 \\ 5.49738 & -0.39491 \\ 7.16429 & -0.96178 \\ 8.12375 & -1.80268 \\ 8.35999 & -2.47153 \\ 8.28126 & -2.94357 \\ 8.06262 & -3.22072 \\ 7.81008 & -3.32785 \\ 7.59057 & -3.30583 \\ & \\ 6.74801 & -0.76335 \\ 7.84559 & -1.46043 \\ 8.26040 . & -2.06252 \\ 8.36280 & -2.55056 \\ 8.29333 & -2.91587 \\ 8.13278 & -3.15989 \\ 7.93619 & -3.29303 \\ 7.74246 & -3.33269 \\ 7.57781 & -3.30080 \\ 7.45752 & -3.22123\end{array}$

$\begin{array}{ll}0.00362 & -0.00046 \\ 0.01240 & -0.00164 \\ 0.05540 & -0.00805 \\ 0.15924 & -0.02585 \\ 0.42685 & -0.08138 \\ 1.38174 & -0.35134 \\ 2.81696 & -0.87598 \\ 3.62837 & -1.12948 \\ 3.66954 & -0.97028 \\ 2.90348 & -0.49332 \\ 1.40888 & 0.14974 \\ -0.52066 & 0.80311 \\ & \\ 1.03204 & -0.24187 \\ 2.25986 & -0.66392 \\ 3.18925 & -1.01172 \\ 3.68250 & -1.13146 \\ 3.69380 & -0.99448 \\ 3.20537 & -0.65404 \\ 2.23776 & -0.18480 \\ 0.88831 & 0.34131 \\ 0.65754 & 0.84359 \\ -2.16063 & 1.22228\end{array}$

1.22228
$-3.92660$

$\begin{array}{rr}0.73194 & -0.01994 \\ 1.24635 & -0.03984 \\ 2.08333 & -0.09912 \\ 2.74299 & -0.19596 \\ 3.35799 & -0.37934 \\ 3.77996 & -0.80749 \\ 2.86922 & -0.99981 \\ 1.07680 & -0.63198 \\ -0.99540 & -0.01822 \\ -2.90011 & 0.56295 \\ -4.19728 & 0.93548 \\ -4.52207 & 0.96917 \\ & \\ 3.76097 & -0.67878 \\ 3.41634 & -0.99910 \\ 2.29434 & -0.91855 \\ 0.78952 & -0.55233 \\ -0.84451 & -0.06458 \\ -2.39523 & 0.41073 \\ -3.64832 & 0.78329 \\ -4.39903 & 0.98414 \\ -4.50372 & 0.95673 \\ -3.92660 & 0.67758\end{array}$

0.67758

$\begin{array}{ll}0.73195 & -0.01994 \\ 1.24643 & -0.03986 \\ 2.08427 & -0.09936 \\ 2.74869 & -0.19745 \\ 3.39030 & -0.38851 \\ 4.06187 & -0.90134 \\ 4.14063 & -1.47178 \\ 3.90504 & -1.66393 \\ 3.72032 & -1.60154 \\ 3.68786 & -1.46869 \\ 3.75776 & -1.39570 \\ 3.83359 & -1.41070 \\ 3.92280 & -0.73021 \\ 4.18140 & -1.27528 \\ 4.06639 & -1.57995 \\ 3.87123 & -1.66634 \\ 3.72876 & -1.61062 \\ 3.68273 & -1.50466 \\ 3.71599 & -1.42074 \\ 3.78238 & -1.39215 \\ 3.83712 & -1.41399 \\ 3.85631 & -1.45879\end{array}$

$\begin{array}{lll}0.01 & 0.77965 & -0.01994 \\ 0.02 & 1.46381 & -0.03986 \\ 0.05 & 2.87897 & -0.09938 \\ 0.10 & 4.16420 & -0.19750 \\ 0.20 & 5.47929 & -0.38857 \\ 0.50 & 7.06163 & -0.90143 \\ 1.00 & 7.83283 & -1.47191 \\ 1.50 & 8.00262 & -1.66409 \\ 2.00 & 8.10557 & -1.60175 \\ 2.50 & 8.29625 & -1.46894 \\ 3.00 & 8.54846 & -1.39597 \\ 3.50 & 8.77844 & -1.41099 \\ 0.125 \pi & 0.68162 & -0.73030 \\ 0.250 \pi & 7.63217 & -1.27540 \\ 0.375 \pi & 7.92245 & -1.58009 \\ 0.500 \pi & 8.01492 & -1.66652 \\ 0.625 \pi & 8.09559 & -1.61082 \\ 0.750 \pi & 8.23187 & -1.50490 \\ 0.875 \pi & 8.41928 & -1.42100 \\ 1.000 \pi & 8.61920 & -1.39242 \\ 1.125 \pi & 8.79171 & -1.41428 \\ 1.250 \pi & 8.91626 & -1.45909\end{array}$

$\begin{array}{ll}0.00384 & -0.00048 \\ 0.01397 & -0.00180 \\ 0.06131 & -0.00864 \\ 0.15381 & -0.02537 \\ 0.34188 & -0.07360 \\ 0.85287 & -0.31717 \\ 1.42272 & -0.93127 \\ 1.61470 & -1.57222 \\ 1.55225 & -2.04456 \\ 1.41937 & -2.30014 \\ 1.34636 & -2.41254 \\ 1.36136 & -2.49086 \\ 0.68207 & -0.21486 \\ 1.22638 & -0.64902 \\ 1.53080 & -1.16936 \\ 1.61710 & -1.65213 \\ 1.56133 & -2.01770 \\ 1.45535 & -2.24603 \\ 1.37141 & -2.36690 \\ 1.34281 & -2.43404 \\ 1.36465 & -2.49708 \\ 1.40944 & -2.58325\end{array}$

$\begin{array}{ll}7.45752 & -3.30080\end{array}$ 
$\frac{a}{\lambda}=0.003969 \quad \frac{\alpha}{\ddot{\beta}}=0.20$

$\mathrm{C}(\mathrm{h}, 0)$

$S(h, 0)$

$E(h, 0)$

$\mathrm{C}(\mathrm{h}, \mathrm{h})$

$S(h, h)$

$E(h, h)$

$\begin{array}{ll}0.77767 & -0.01990 \\ 1.45992 & -0.03978 \\ 2.86996 & -0.09924 \\ 4.14875 & -0.19756 \\ 5.45827 & -0.39080 \\ 7.07190 & -0.93823 \\ 7.96646 & -1.71900 \\ 8.17660 & -2.31001 \\ 8.11123 & -2.70700 \\ 7.93689 & -2.92895 \\ 7.74503 & -3.01081 \\ 7.58625 & -2.99522 \\ & \\ 6.67372 & -0.74847 \\ 7.71183 & -1.40593 \\ 8.08911 & -1.95206 \\ 8.17901 & -2.37779 \\ 8.12113 & -2.68428 \\ 7.99192 & -2.88122 \\ 7.83964 & -2.98463 \\ 7.69528 & -3.01437 \\ 7.57727 & -2.99168 \\ 7.49432 & -2.93696\end{array}$

$0.72995-0.01988$ $1.24238-0.039 .69$ $2.07348 \quad-0.09836$ $2.72371-0.1927$ $3.32193-0.36524$ $3.71903-0.71175$ $2.86015 \quad-0.65734$ $1.16344-0.01858$ $-0.86910 \quad 0.7820$ $-2.83284 \quad 1.34527$ $-4.29553 \quad 1.37753$ $\begin{array}{ll}-4.87058 & 0.73053\end{array}$

$3.70388-0.6202^{4}$ $3.37307-0.77327$ $2.32039-0.47672$ $0.88682 \quad 0.09513$ $-0.71797 \quad 0.72734$ $-2.30013 \quad 1.22703$ $-3.65032 \quad 1.44113$ $-4.56198 \quad 1.2649$ $-4.870620 .66150$ $\begin{array}{ll}-4.48601 & -0.31420\end{array}$
$0.00360-0.00082$ $0.01232-0.00288$ $0.05491-0.01354$ $0.15732-0.04155$ $0.41957-0.12260$ $1.34735-0.47020$ $2.76493-1.03516$ $3.65368-1.1711$ $3.83118-0.73033$ $3.18287 \quad 0.17444$ $1.72313 \quad 1.28687$ $\begin{array}{ll}-0.32265 & 2.25232\end{array}$

$1.00813-0.33527$ $2.20564-0.82397$ $3.15367-1.14768$ $3.72535-1.14324$ $3.84524-0.78060$ 3.456720 .72040 2.54879 $-0.474812 .30300$ $\begin{array}{ll}-0.47481 & 2.30300 \\ -2.22663 & 2.66125\end{array}$

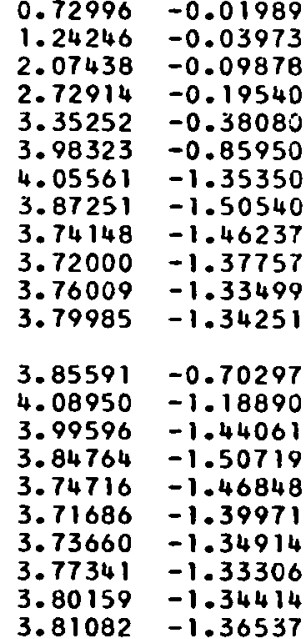

$$
\frac{a}{\lambda}=0.003969 \quad \frac{\alpha}{\beta}=0.40
$$

$\begin{array}{ll}0.77371 & -0.01979 \\ 1.45199 & -0.03956 \\ 2.85019 & -0.09851 \\ 4.10952 & -0.19529 \\ 5.38128 & -0.38275 \\ 0.89372 & -0.89339 \\ 7.67181 & -1.56691 \\ 7.83814 & -2.02863 \\ 7.79307 & -2.030960 \\ 7.68215 & -2.45202 \\ 7.57135 & -2.49984 \\ 7.48821 & -2.49203 \\ & \\ 6.52938 & -0.71985 \\ 7.45840 & -1.30493 \\ 7.77061 & -1.75441 \\ 7.83991 & -2.67849 \\ 7.79973 & -2.29431 \\ 7.71601 & -2.42263 \\ 7.62461 & -2.48505 \\ 7.54443 & -2.50178 \\ 7.48377 & -2.49028 \\ 7.44431 & -2.46440\end{array}$

$\begin{array}{rr}0.72598 & -0.01977 \\ 1.23447 & -0.03940 \\ 2.05400 & -0.09685 \\ 2.68608 & -0.18652 \\ 3.25381 & -0.33805 \\ 3.62036 & -0.53241 \\ 2.89127 & -0.01324 \\ 1.34618 & 1.19037 \\ -0.78020 & 2.45496 \\ -3.25608 & 3.09651 \\ -5.05861 & 2.50191 \\ -7.34923 & 0.31946 \\ 3.60491 & -0.50949 \\ 3.32932 & -0.35094 \\ 2.41798 & 0.36670 \\ 1.07650 & 1.38140 \\ -0.60940 & 2.37547 \\ -2.52867 & 3.01473 \\ -4.49636 & 2.98568 \\ -6.24198 & 2.05028 \\ -7.41966 & 0.11115 \\ -7.64100 & -2.73030\end{array}$

$0.00356-0.00154$ $0.01216-0.00532$ $0.05394-0.02439$ $0.15358-0.07238$ $0.40596-0.20303$ $1.29268-0.70207$ $2.74543-1.36965$ $3.91226-1.30869$ $4.52416-0.27710$ $4.22581 \quad 1.63701$ $\begin{array}{ll}4.09371 \quad 4.00123 \\ -0.20278 & 6.04743\end{array}$ $\begin{array}{rr}2.0020278 & 6.04743\end{array}$

$0.96740-0.51688$ $2.14423-1.14543$ $3.20547-1.45137$

.04024 - 1.22307

$4.50579-0.38528$

$4.42600 \quad 1.01461$
.6306

$3.63196 \quad 2.79849$

$2.01016 \quad 4.65323$

$\begin{array}{ll}-0.44840 & 6.15185 \\ -3.60038 & 6.80718\end{array}$

$-7.64100-2.73030$ $\begin{array}{ll}0.72599 & -0.01978 \\ 1.23454 & -0.03946 \\ 2.05476 & -0.09765 \\ 2.69064 & -0.19137 \\ 3.27915 & -0.36599 \\ 3.83591 & -0.78346 \\ 3.89654 & -1.15480 \\ 3.78567 & -1.24992 \\ 3.71959 & -1.22943 \\ 3.71019 & -1.19479 \\ 3.72341 & -1.18028 \\ 3.73436 & -1.18216 \\ 3.72920 & -0.65246 \\ 3.91996 & -1.03925 \\ 3.85801 & -1.21132 \\ 3.77221 & -1.25089 \\ 3.72215 & -1.23220 \\ 3.70901 & -1.20318 \\ 3.71597 & -1.18480 \\ 3.72731 & -1.17971 \\ 3.73479 & -1.18256 \\ 3.73692 & -1.18733\end{array}$

$\begin{array}{lll}1.125 \pi & 8.68918 & -1.18321 \\ 1.250 \pi & 8.79667 & -1.18800\end{array}$

$\begin{array}{ll}1.13376 & -2.58475 \\ 1.13853 & -2.68797\end{array}$ 
$0.65613-0.02000$ $\begin{array}{ll}0.65613 & -0.02000 \\ 1.25228 & -0.03999\end{array}$ $\begin{array}{lll}0.02 & 1.25228 & -0.03999 \\ 0.05 & 2.56887 & -0.09993 \\ 0.10 & 3.83205 & -0.09953\end{array}$ $\begin{array}{lll}0.05 & 2.56887 & -0.09993 \\ 0.10 & 3.83205 & -0.19953\end{array}$ $0.20 \quad 5.15635-0.3964 \mathrm{C}$ $\begin{array}{lll}0.20 & 5.15635 & -0.39640 \\ 0.50 & 6.77957 & -0.94594\end{array}$ $\begin{array}{lll}0.50 & 0.77957 & -0.94594 \\ 1.50 & 7.55704 & -1.60517\end{array}$ $\begin{array}{lll}.50 & 7.65903-1.84837\end{array}$ $\begin{array}{lll}7.00 & 7.68612-1.75794\end{array}$ $\begin{array}{lll}2.50 & 7.86024 & -1.54972 \\ 3.00 & 8.16454 & -1.42453\end{array}$ $3.50 \quad 8.46342-1.45448$

$0.125 \pi \quad 6.38704 \quad-0.75836$ $\begin{array}{llll}0.250 \pi & 7.36473 & -1.37055\end{array}$ $\begin{array}{lll}0.375 \pi & 7.62848 & -1.73948\end{array}$ $0.500 \pi \quad 7.65918-1.85165$ $0.625 \pi \cdot 7.68023-1.77142$ $0.750 \pi \quad 7.79261-1.60814$ $0.8751 \pi \quad 8.00284-1.46882$ $\begin{array}{llll}1.000 \pi & 8.25615 & -1.41801\end{array}$ $\begin{array}{lll}1.125 \pi & 8.48030 & -1.46113 \\ 1.250 \pi & 8.62559 & -1.55573\end{array}$
$E(h, 0)$

$\begin{array}{ll}0.65614 & -0.02600 \\ 1.25236 & -0.03899\end{array}$ $\begin{array}{ll}1.25236 & -0.03999 \\ 2.56975 & -0.09997\end{array}$ $\begin{array}{ll}2.56975 & -0.09997 \\ 3.83640 & -0.19986\end{array}$ $\begin{array}{ll}3.83640 & -0.19986 \\ 5.17517 & -0.39905\end{array}$ $5.17517-0.39905$ $0.89430-0.98007$ $7.92307-1.89188$ $8.18861-2.04895$ $8.09372-3.21030$ $7.81746-3.55 .343$ $\begin{array}{ll}7.48697 & -3.69663 \\ 7.18347 & -3.66554\end{array}$ $0.45948-0.77858$ $\begin{array}{ll}7.61932 & -1.51772\end{array}$ $8.07527-2.18157$ $8.19187-2.74109$ $8.10844-3.17654$ $\begin{array}{ll}7.90892 & -3.47891\end{array}$ $7.65507-3.65032$ $7.39505-3.70319$ $7.16533-3.65838$ $6.99087-3.54265$
$\mathrm{C}(\mathrm{h}, \mathrm{h})$

$0.62617-i .02000$ $1.09975-0.03999$ $1.91734-0.09939$ 1.58232-0.19918 $.21614-0.39377$ 3.08601 -0.90743 $2.79105-1.36019$ $0.03955-1.26562$ $-1.10976-0.82446$ $-3.00579-0.21096$ $-4.29158 \quad 0.52537$ $-4.54640 \quad 1.28049$ $3.65307-0.7396$ $3.34163-1.23732$ $2.20045-1.38790$ $0.67922-1.21860$ $-0.95932-0.86365$ $-2.50307-0.40123$ $\begin{array}{ll}-3.75075 & 0.14299\end{array}$ $-4.48285 \quad 0.74605$ $\begin{array}{ll}-4.51940 & 1.32712 \\ -3.80886 & 1.75394\end{array}$
$S(h, h)$

$2.00311-0.00010$ $-0.00040$ $0.05020-0.00250$ $0.14713-0.00997$ $0.40270-0.03946$ $1.33805-0.23007$ $2.74865-0.72174$ $3.48952-1.10937$ $3.45131-1.23125$ $2.65494-1.16338$ $-0.75855-0.58004$

$0.99312-0.14649$ $2.20581-0.50348$ $3.10134-0.8868$ $3.53111-1.14265$ $3.48000-1.23026$ $2.96024-1.19605$ $1.98893-1.08372$ $0.64225-0.88025$ $-0.89354-c .54503$ $-2.34555-0.07903$
$E(h, h)$

$0.62618-0.02000$ $-0.03999$ $1.91820-0.09993$ $2.58759-0.19953$ $3.24668-0.39640$ $3.96153-0.94594$ $4.04686-1.60515$ $3.74349-1.84832$ $3.48289-1.75784$ $3.43387-1.54956$ $3.55585-1.42433$ $3.70060-1.45425$

$3.80966-0.75880$ $\begin{array}{ll}4.09593 & -1.37055\end{array}$ $3.95446-1.73945$ $3.09753-1.85159$ $3.49543-1.77132$ $3.42549-1.60800$ $3.48157-1.46865$ $3.60135-1.41780$ $3.70772-1.46090$ $3.74767-1.55549$ $\frac{a}{\lambda}=0.004763 \quad \frac{\alpha}{\beta}=0.005$

$\begin{array}{rr}0.62607 & -0.01999 \\ 1.09955 & -0.03998 \\ 1.91684 & -0.09985 \\ 2.58133 & -0.19904 \\ 3.21423 & -0.39306 \\ 3.68290 & -0.96252 \\ 2.78947 & -1.34240 \\ 0.97417 & -1.23463 \\ -1.09898 & -0.78535 \\ -2.98900 & -0.17324 \\ -4.26874 & 0.54531 \\ -4.52303 & 1.26361 \\ 3.64982 & -1.73665 \\ 3.33840 & -1.22555 \\ 2.20686 & -1.35908 \\ 0.68477 & -1.18607 \\ -0.94897 & -0.32489 \\ -2.48809 & -0.36175 \\ -3.73074 & 0.17431 \\ -4.45895 & 0.75731 \\ -4.49640 & 1.30719 \\ -3.79430 & 1.69771\end{array}$

$0.00311-0.00012$ $0.01093-0.00045$ $0.05018-0.00275$ $0.14703-0.01069$ $0.40232-0.04142$ $\begin{array}{ll}1.33595 & -0.23581\end{array}$ $2.74376-0.72883$ $3.48577-1.10967$ $3.45130-1.21826$ $2.65836-1.13116$ $\begin{array}{rr}1.17029 & -0.91282 \\ -0.74109 & -0.51277\end{array}$

\section{$0.99173-0.15049$} $2.20180-0.51101$ $3.09635-0.89240$ $3.52782-1.14143$ $3.47972-1.21845$ $2.96268-1.16991$ $\begin{array}{ll}1.96268 & -1.10991\end{array}$ $0.65289-0.82094$ $-0.87539-0.47752$ $-2.32046-0.01679$ $\begin{array}{ll}0.62608 & -0.01999 \\ 1.09962 & -0.03998 \\ 1.91770 & -0.09990 \\ 2.58659 & -0.19942 \\ 3.24473 & -0.39600 \\ 3.95733 & -0.94363 \\ 4.04232 & -1.59808 \\ 3.74276 & -1.83840 \\ 3.48662 & -1.74958 \\ 3.43860 & -1.54594 \\ 3.55723 & -1.42405 \\ 3.69737 & -1.45295 \\ 3.80613 & -0.75739 \\ 4.09094 & -1.36555 \\ 3.95093 & -1.73094 \\ 3.69750 & -1.84162 \\ 3.49892 & -1.76281 \\ 3.43042 & -1.60298 \\ 3.48505 & -1.46712 \\ 3.60135 & -1.41771 \\ 3.70425 & -1.45938 \\ 3.74276 & -1.55049\end{array}$

$1.000 \pi \quad 8.25615-1.41793$

$1.250 \pi \quad 8.62068-1.45962$

$1.35837-2.43691$

$\begin{array}{ll}1.40002 & -2.45179 \\ 1.49113 & -2.51865\end{array}$ 
$\frac{\mathrm{a}}{\lambda}=0.00 \leqslant 763 \quad \frac{\alpha}{\beta}=0.01$

$\beta \mathrm{h}$

$\mathrm{C}(\mathrm{h}, 0)$

0.0
0.02
0.05
0.10
0.20
0.50
1.00
1.50
2.00
2.50
3.00
3.50

$0.125 \pi$

$0.375 \pi$

$0.500 \pi$

$0.625 \pi$

$0.750 \pi$

$0.875 \pi$

$1.000 \pi$
$1.125 \pi$
$1.250 \pi$
Sịh, 0)

$\begin{array}{ll}0.65593 & -0.01999 \\ 1.25188 & -0.03998 \\ 2.56787 & -0.09987 \\ 3.83007 & -0.19932 \\ 5.15246 & -0.39561 \\ 6.77118 & -0.94135 \\ 7.54798 & -1.59109 \\ 7.65755 & -1.82862 \\ 7.69346 & -1.74149 \\ 7.86956 & -1.54244 \\ 8.16724 & -1.42384 \\ 8.45706 & -1.45179 \\ 6.37999 & -0.75593 \\ 7.35477 & -1.36060 \\ 7.62141 & -1.72253 \\ 7.65909 & -1.83181 \\ 7.68709 & -1.75446 \\ 7.80233 & -1.59810 \\ 8.00971 & -1.46567 \\ 8.25615 & -1.41770 \\ 8.47345 & -1.45799 \\ 8.61593 & -1.54578\end{array}$

$0.65593-0.01999$ $1.25188-0.03998$ $6.77118-0.94135$ $7.65755-1.82862$ $7.69346-1.74149$ $8.86956-1.54244$ $6.37999-0.75593$ -0.75593
-1.36060 $1.65909-1.83181$ $7.08709-1.75446$ $8.00971-1.46567$ $8.61593-1.54578$
$E(h, 0)$

$\begin{array}{ll}6.65594 & -0.01999 \\ 1.25196 & -6.03998 \\ 2.56875 & -0.09993 \\ 3.83440 & -6.19974 \\ 5.17120 & -0.39863 \\ 6.88473 & -0.98359 \\ 7.90629 & -1.88257 \\ 8.16873 & -2.63041 \\ 8.07559 & -3.18211 \\ 7.80747 & -3.52063 \\ 7.48401 & -3.65710 \\ 7.19019 & -3.62706 \\ & \\ 6.45184 & -0.77703 \\ 7.60521 & -1.51164 \\ 8.05685 & -2.16923 \\ 8.17194 & -2.72115 \\ 8.09002 & -3.14901 \\ 7.89479 & -3.44496 \\ 7.64734 & -3.61210 \\ 7.39487 & -3.66346 \\ 7.17268 & -3.62016 \\ 7.00458 & -3.50869\end{array}$

$\mathrm{C}(\mathrm{h}, \mathrm{h})$

$\begin{array}{rr}0.62597 & -0.01999 \\ 1.09935 & -0.03997 \\ 1.91634 & -0.09981 \\ 2.58034 & -0.19883 \\ 3.21233 & -0.39235 \\ 3.67934 & -0.89761 \\ 2.73792 & -1.32460 \\ 0.97877 & -1.20372 \\ -1.08836 & -0.74630 \\ -2.97265 & -0.13560 \\ -4.24684 & 0.56522 \\ -4.50109 & 1.24700 \\ & \\ 3.64659 & -0.73367 \\ 3.33520 & -1.21382 \\ 2.20730 & -1.33632 \\ 0.69029 & -1.15361 \\ -0.93875 & -0.78618 \\ -2.47344 & -0.32234 \\ -3.71140 & 0.20555 \\ -4.43615 & 0.76861 \\ -4.47484 & 1.28757 \\ -3.78118 & 1.64215\end{array}$

$\mathrm{S}(\mathrm{h}, \mathrm{h})$

$\begin{array}{rr}0.00311 & -0.00013 \\ 0.01093 & -0.00051 \\ 0.05015 & -0.00300 \\ 0.14693 & -0.01142 \\ 0.40193 & -0.04337 \\ 1.33386 & -0.24155 \\ 2.73896 & -0.73594 \\ 3.48218 & -1.11002 \\ 3.45153 & -1.20535 \\ 2.06211 & -1.09900 \\ 1.17899 & -0.85891 \\ -0.72389 & -0.44575 \\ 0.99035 & -0.15549 \\ 2.19784 & -0.51854 \\ 3.09146 & -0.89796 \\ 3.52471 & -1.14027 \\ 3.47967 & -1.20671 \\ 2.90544 & -1.14384 \\ 2.00019 & -0.99722 \\ 0.66368 & -0.76183 \\ -0.85756 & -0.41027 \\ -2.29639 & 0.04533\end{array}$

$E(h, h)$

$\begin{array}{ll}0.62598 & -0.01999 \\ 1.09942 & -0.03998 \\ 1.91720 & -0.09987 \\ 2.58560 & -0.19931 \\ 3.24279 & -0.39560 \\ 3.95314 & -0.94134 \\ 4.03780 & -1.59106 \\ 3.74201 & -1.82855 \\ 3.49024 & -1.74137 \\ 3.44319 & -1.54227 \\ 3.55856 & -1.42362 \\ 3.69425 & -1.45154 \\ 3.80261 & -0.75592 \\ 4.08597 & -1.36058 \\ 3.94739 & -1.72248 \\ 3.69743 & -1.83173 \\ 3.50229 & -1.75434 \\ 3.43521 & -1.59794 \\ 3.48844 & -1.46547 \\ 3.60135 & -1.41747 \\ 3.70089 & -1.45774 \\ 3.73801 & -1.54552\end{array}$

$$
\frac{a}{\lambda}=0.0047 E 3 \quad \frac{\alpha}{\beta}=0.02
$$

$\begin{array}{rr}0.62577 & -0.01998 \\ 1.09895 & -0.03996 \\ 1.91535 & -0.09973 \\ 2.57837 & -0.19856 \\ 3.20854 & -0.39096 \\ 3.67214 & -0.88784 \\ 2.78493 & -1.28934 \\ 0.98791 & -1.14210 \\ -1.06757 & -0.65838 \\ -2.94125 & -0.06052 \\ -4.20586 & 0.60499 \\ -4.46144 & 1.21461 \\ & \\ 3.64016 & -0.72773 \\ 3.32891 & -1.19046 \\ 2.20825 & -1.29101 \\ 0.70122 & -1.08891 \\ -0.91874 & -0.70894 \\ -2.44515 & -0.24372 \\ -3.67472 & 0.26785 \\ -4.39383 & 0.79132 \\ -4.43601 & 1.24923 \\ -3.75924 & 1.53291\end{array}$

$0.00311-0.00016$ $0.01092-0.00062$ $0.05010-0.00350$ $0.14674-0.01288$ $0.40115-0.04728$ $\begin{array}{ll}0.32972 & -0.25299 \\ 2.72958 & -0.750 .18\end{array}$ $2.72958-0.75018$ $3.47548-1.11093$ $3.45273-1.17974$ $2.67062-1.03490$ $\begin{array}{rr}1.19713 & -0.75155 \\ -0.69031 & -0.31240\end{array}$ $0.98761-0.16448$ $2.19006-0.53360$ $3.08200-0.90917$ $3.51899-1.13817$ $3.48029-1.18345$ $2.97190-1.09189$ $2.01279-0.91111$ $0.68573-0.64416$ $-0.82285-0.27643$ $-2.251240 .16928$

$\begin{array}{ll}0.62578 & -0.01998 \\ 1.09902 & -0.03996 \\ 1.91620 & -0.09981 \\ 2.58362 & -0.19910 \\ 3.23891 & -0.39479 \\ 3.94479 & -0.93676 \\ 4.02877 & -1.57713 \\ 3.74037 & -1.80909 \\ 3.49713 & -1.72510 \\ 3.45198 & -1.53475 \\ 3.56111 & -1.42234 \\ 3.68829 & -1.44840 \\ 3.79558 & -0.75299 \\ 4.07606 & -1.35070 \\ 3.94033 & -1.70574 \\ 3.69715 & -1.81218 \\ 3.50871 & -1.73756 \\ 3.44438 & -1.58778 \\ 3.49490 & -1.46187 \\ 3.60134 & -1.41656 \\ 3.69448 & -1.45418 \\ 3.72898 & -1.53563 \ldots\end{array}$


$\begin{array}{lll}0.02 & 1.25553 & -0.01998 \\ 0.02 & 0.05108 & -0.03995\end{array}$

$\begin{array}{lll}0.05 & 2.56588 & -0.09976\end{array}$

$3.82610-0.19390$

$5.14471-0.39402$

$6.75451-0.93226$

$7.52996-1.56345$

$7.65413-1.79004$

$7.70680-1.70916$

$7.88662-1.52724$

$8.17214-1.42078$

$8.44550-1.44514$

3.50

$\begin{array}{llll}0.125 \pi & 0.36596 & -0.75012\end{array}$

$0.250 \pi \quad 7.33500-1.34098$

$\begin{array}{lll}0.375 \pi & 7.60727 & -1.68931\end{array}$

$0.500 \pi \quad 7.65833-1.79304$

$0.625 \pi \quad 7.69951-1.72114$

$0.750 \pi \quad 7.82014-1.57775$

$0.875 \pi \quad 8.02223-1.45810$

$1.000 \pi-8.25600-1.41535$

$\begin{array}{lll}1.125 \pi & 8.46102 & -1.45053 \\ 1.250 \pi & 8.59843 & -1.52612\end{array}$
$E(h, 0)$

$\begin{array}{ll}0.00325 & -0.00020 \\ 0.01210 & -0.00076 \\ 0.05647 & -0.00419 \\ 0.14784 & -0.01440 \\ 0.33865 & -0.04971 \\ 0.87423 & -0.26310 \\ 1.50452 & -0.87256 \\ 1.73081 & -1.55907 \\ 1.64979 & -2.08168 \\ 1.46778 & -2.34810 \\ 1.36126 & -2.42720 \\ 1.38557 & -2.46213 \\ & \\ 0.69257 & -0.16964 \\ 1.28230 & -0.58468 \\ 1.63025 & -1.12293 \\ 1.73379 & -1.64709 \\ 1.66177 & -2.05213 \\ 1.51830 & -2.29610 \\ 1.39860 & -2.40229 \\ 1.35581 & -2.43547 \\ 1.39096 & -2.46611 \\ 1.46653 & -2.53941\end{array}$

$\begin{array}{ll}0.65554 & -0.01998 \\ 1.25116 & -0.03995 \\ 2.56675 & -0.09985 \\ 3.83041 & -0.19950 \\ 5.16328 & -0.39778 \\ 6.86566 & -0.97865 \\ 7.87295 & -1.86445 \\ 8.12930 & -2.59387 \\ 8.03957 & -3.12676 \\ 7.78334 & -3.45055 \\ 7.47719 & -3.57987 \\ 7.20181 & -3.55183 \\ & \\ 6.43660 & -0.77393 \\ 7.57715 & -1.50017 \\ 8.02029 & -2.14483 \\ 8.13242 & -2.68186 \\ 8.05344 & -3.09494 \\ 7.86651 & -3.37847 \\ 7.63140 & -3.53736 \\ 7.39336 & -3.58585 \\ 7.18549 & -3.54539 \\ 7.02944 & -3.44196\end{array}$

$C(h, h)$

$S(h, h)$

$\begin{array}{rr}0.62557 & -0.01998 \\ 1.09855 & -0.03994 \\ 1.91435 & -0.09965 \\ 2.57640 & -0.19824 \\ 3.20476 & -0.38956 \\ 3.66503 & -0.87812 \\ 2.78208 & -1.25425 \\ 0.99698 & -1.03078 \\ -1.04738 & -0.59068 \\ -2.91160 & 0.01432 \\ -4.16860 & 0.04474 \\ -4.42736 & 1.18327 \\ & \\ 3.63373 & -0.72182 \\ 3.32275 & -1.16725 \\ 2.20929 & -1.24597 \\ 0.71202 & -1.02445 \\ -0.89928 & -0.63193 \\ -2.41818 & -0.16533 \\ -3.64068 & 0.32994 \\ -4.35585 & 0.3142 \\ -4.40281 & 1.21204 \\ -3.74297 & 1.42603\end{array}$

$0.00311-0.00019$ $0.01091-0.00073$ $0.05005-0.00400$ $0.14654-0.01433$ $\begin{array}{ll}0.40038 & -0.05117\end{array}$ $1.32563-0.26442$ $2.72051-0.76448$ $3.46941-1.11209$ $3.45492-1.1544$ $2.68047-0.97104$ $1.21626-0.64472$ $0.65777-0.17979$ $2.18245-0.54866$ $3.07298-0.92050$ $3.51396-1.13634$ $3.48186-1.16049$ $2.97962-1.04017$ $2.02671-0.82534$ $0.70838-0.52712$ $-0.78939-0.14334$ $\begin{array}{ll}-2.21002 & 0.29302\end{array}$
$E(h, h)$.

$\begin{array}{ll}0.62558 & -0.01998 \\ 1.09862 & -0.03995 \\ 1.91521 & -0.09975 \\ 2.58164 & -0.19889 \\ 3.23503 & -0.39399 \\ 3.93647 & -0.93222 \\ 4.01978 & -1.56338 \\ 3.73859 & -1.78993 \\ 3.50359 & -1.70900 \\ 3.46026 & -1.52703 \\ 3.56347 & -1.42053 \\ 3.68269 & -1.44485 \\ & \\ 3.78858 & -0.75008 \\ 4.06621 & -1.34093 \\ 3.93325 & -1.68923 \\ 3.69668 & -1.79293 \\ 3.51472 & -1.72098 \\ 3.45303 & -1.57755 \\ 3.50097 & -1.45787 \\ 3.60131 & -1.41509 \\ 3.68846 & -1.45024 \\ 3.72052 & -1.52582\end{array}$

$$
\frac{a}{\lambda}=0.004763 \quad \frac{\alpha}{\beta}=0.05
$$

$\begin{array}{ll}0.00324 & -0.00026 \\ 0.01209 & -0.00100 \\ 0.05637 & -0.00532 \\ 0.14745 & -0.01733 \\ 0.33709 & -0.05633 \\ 0.86528 & -0.27845 \\ 1.47756 & -0.88924 \\ 1.69343 & -1.56187 \\ 1.61820 & -2.06884 \\ 1.45184 & -2.33184 \\ 1.35623 & -2.42181 \\ 1.37740 & -2.47120 \\ 0.68684 & -0.18242 \\ 1.26308 & -0.60305 \\ 1.59796 & -1.13590 \\ 1.69622 & -1.64739 \\ 1.62927 & -2.04014 \\ 1.49770 & -2.27917 \\ 1.38954 & -2.39011 \\ 1.35140 & -2.43446 \\ 1.38208 & -2.47594 \\ 1.44716 & -2.55361\end{array}$

$\begin{array}{ll}0.65514 & -0.01997 \\ 1.25036 & -0.03993 \\ 2.56476 & -0.09977 \\ 3.82643 & -0.19925 \\ 5.15537 & -0.39693 \\ 0.84668 & -0.97375 \\ 7.83991 & -1.84645 \\ 8.09032 & -2.55801 \\ 8.00387 & -3.07274 \\ 7.75899 & -3.38245 \\ 7.46923 & -3.50499 \\ 7.21113 & -3.47882 \\ & \\ 6.42142 & -0.77086 \\ 7.54930 & -1.48862 \\ 7.98410 & -2.12080 \\ 8.09334 & -2.64334 \\ 8.01720 & -3.04216 \\ 7.83822 & -3.31378 \\ 7.61482 & -3.46484 \\ 7.39039 & -3.51062 \\ 7.19592 & -3.47282 \\ 7.05105 & -3.37685\end{array}$

$\begin{array}{cc}0.62517 & -0.01996 \\ 1.09775 & -0.03991 \\ 1.91237 & -0.09950 \\ 2.57248 & -0.19701 \\ 3.19724 & -0.38676 \\ 3.65103 & -0.85832 \\ 2.77679 & -1.18471 \\ 1.01489 & -0.95895 \\ -1.00881 & -0.43588 \\ -2.85751 & 0.16343 \\ -4.10511 & 0.72436 \\ -4.37558 & 1.12360 \\ 3.62111 & -0.71007 \\ 3.31086 & -1.12124 \\ 2.21167 & -1.15667 \\ 0.73325 & -0.89644 \\ -0.86200 & -0.47851 \\ -2.36822 & -0.00011 \\ -3.58050 & 0.45370 \\ -4.29269 & 0.86071 \\ -4.35303 & 1.14794 \\ -3.72721 & 1.21354\end{array}$

$\begin{array}{ll}0.62518 & -0.01996 \\ 1.09782 & -0.03992 \\ 1.91322 & -0.09963 \\ 2.57768 & -0.19846 \\ 3.22731 & -0.39240 \\ 3.91996 & -0.92323 \\ 4.00193 & -1.53637 \\ 3.73461 & -1.75249 \\ 3.51524 & -1.67736 \\ 3.47535 & -1.51104 \\ 3.56768 & -1.41544 \\ 3.67244 & -1.43663 \\ 3.77465 & -0.74431 \\ 4.04667 & -1.32167 \\ 3.91911 & -1.65689 \\ 3.69519 & -1.75531 \\ 3.52553 & -1.68843 \\ 3.46879 & -1.55689 \\ 3.51198 & -1.44875 \\ 3.60115 & -1.41062 \\ 3.67745 & -1.44131 \\ 3.70514 & -1.50640\end{array}$

$\begin{array}{lll}0.01 & 0.65513 & -0.01997 \\ 0.02 & 1.25029 & -0.03992 \\ 0.05 & 2.56389 & -0.09964 \\ 0.10 & 3.82215 & -0.19849 \\ 0.20 & 5.13698 & -0.39244 \\ 0.50 & 0.73799 & -0.92329 \\ 1.00 & 7.51211 & -1.53647 \\ 1.50 & 7.65014 & -1.75264 \\ 2.00 & 7.71844 & -1.67756 \\ 2.50 & 7.90169 & -1.51124 \\ 3.00 & 8.17633 & -1.41573 \\ 3.50 & 8.43523 & -1.43695 \\ 0.125 \pi & 6.35203 & -0.74437 \\ 0.250 \pi & 7.31546 & -1.32175 \\ 0.375 \pi & 7.59312 & -1.65700 \\ 0.500 \pi & 7.65684 & -1.75546 \\ 0.025 \pi & 7.71030 & -1.68862 \\ 0.750 \pi & 7.83589 & -1.55712 \\ 0.875 \pi & 8.03322 & -1.44702 \\ 1.000 \pi & 8.25592 & -1.41093 \\ 1.1251 \pi & 8.44999 & -1.44163 \\ 1.2501 \pi & 8.58303 & -1.50673\end{array}$

$\begin{array}{rr}0.00310 & -0.00026 \\ 0.01089 & -0.00094 \\ 0.04995 & -0.00499 \\ 0.14614 & -0.01723 \\ 0.39885 & -0.05894 \\ 1.31761 & -0.28718 \\ 2.70328 & -0.79322 \\ 3.45920 & -1.11513 \\ 3.45227 & -1.10458 \\ 2.70417 & -0.84397 \\ 1.25742 & -0.43229 \\ -0.59576 & 0.08388 \\ 0.97955 & -0.19131 \\ 2.16775 & -0.57874 \\ 3.05620 & -0.94352 \\ 3.50595 & -1.13347 \\ 3.48790 & -1.11538 \\ 2.99885 & -0.93737 \\ 2.05848 & -0.65464 \\ 0.75544 & -0.29451 \\ -0.72613 & 0.12138 \\ -2.13903 & 0.54064\end{array}$


$\frac{a}{\lambda}=0.004763 \quad \frac{\alpha}{\beta}=0.07$ $\beta \mathrm{h}$

$\mathrm{C}(\mathrm{h}, 0)$

0.01

$3.81820-0.19808$

$5.12929-0.39087$

$\begin{array}{lll}0.50 & 6.72162 & -0.91444\end{array}$

$7.49441-1.51014$

$\begin{array}{lll}1.50 & 7.64562 & -1.71637\end{array}$

$2.00 \quad 7.72851-1.64668$

$7.91491-1.49471$

3.00

3.50

$\begin{array}{llll}0.250 \pi & 7.29614 & -1.30290\end{array}$

$\begin{array}{lll}0.250 \pi & 7.29614 & -1.30290 \\ 0.375 \pi & 7.57897 & -1.02557\end{array}$

$\begin{array}{lll}0.500 \pi & 7.57897 & -1.62557 \\ 0.65466 & -1.71903\end{array}$

$0.625 \pi \quad 7.71959-1.65689$

$0.625 \pi \quad 7.79959-1.05689$

$0.750 \pi \pi \quad 7.84972-1.53630$

$1.000 \pi \quad 8.25554-1.43863$

$8.255012-1.40468$

$\begin{array}{lll}1.125 \pi & 8.44012 & -1.43148 \\ 1.250 \pi & 8.56938 & -1.48755\end{array}$
$2.56190-0.09952$

$\begin{array}{llll}0.125 \pi & 6.33821 & -0.73867\end{array}$
$S(h, 0)$

$\begin{array}{ll}0.00324 & -0.00033 \\ 0.01207 & -0.00125 \\ 0.05627 & -0.00644 \\ 0.14705 & -0.02026 \\ 0.33553 & -0.06291 \\ 0.85644 & -0.29367 \\ 1.45125 & -0.90576 \\ 1.65718 & -1.56521 \\ 1.58734 & -2.05759 \\ 1.43528 & -2.31743 \\ 1.34944 & -2.41713 \\ 1.36788 & -2.47923 \\ 0.68116 & -0.19509 \\ 1.24426 & -0.62119 \\ 1.56654 & -1.14887 \\ 1.65981 & -1.64838 \\ 1.59756 & -2.02968 \\ 1.47689 & -2.26414 \\ 1.37917 & -2.37937 \\ 1.34517 & -2.43365 \\ 1.37195 & -2.48462 \\ 1.42799 & -2.56607\end{array}$

$E(h, 0)$

$\begin{array}{ll}0.65474 & -0.01995 \\ 1.24957 & -0.03990 \\ 2.56277 & -0.09969 \\ 3.82246 & -0.19901 \\ 5.14747 & -0.39608 \\ 6.82779 & -0.96888 \\ 7.80717 & -1.82869 \\ 8.05177 & -2.52285 \\ 7.96848 & -3.02002 \\ 7.73445 & -3.31626 \\ 7.46020 & -3.43238 \\ 7.21830 & -3.40795 \\ 6.40630 & -0.76779 \\ 7.52166 & -1.47718 \\ 7.94828 & -2.09714 \\ 8.05471 & -2.60558 \\ 7.98130 & -2.99063 \\ 7.80993 & -3.25084 \\ 7.59766 & -3.39445 \\ 7.38606 & -3.53767 \\ 7.20412 & -3.4 .0236 \\ 7.06963 & -3.31332\end{array}$

$\mathrm{C}(\mathrm{h}, \mathrm{h})$

$0.02477-0.01995$ $1.09696-0.03987$ $2.56856=0.04934$ $\begin{array}{ll}2.56856 & -0.049698 \\ 3.18977 & -0.38399\end{array}$ $3.63735-0.83970$ $2.77206-1.11601$ $1.77206-1.11601$ $-0.07261 \quad-0.28175$ $-2.81036 \cdot 0.31205$ $-4.05612 \quad 0.80446$ $\begin{array}{ll}-4.05612 & 0.80446 \\ -4.34523 & 1.06767\end{array}$

$3.60878-0.69842$ $3.29951-1.07577$ $\begin{array}{ll}3.29951 & -1.07577 \\ 2.21447 & -1.06837\end{array}$ $0.75404-0.76940$ $\begin{array}{lll}-0.82687 & -0.32579\end{array}$ $\begin{array}{ll}.32354 & 0.14657\end{array}$ $\begin{array}{lll}-3.53075 & 0.57720\end{array}$ $-4.24634 \quad 0.90837$ $\begin{array}{ll}-4.32497 & 1.07388 \\ -3.73352 & 1.01813\end{array}$
$S(h, h)$

$0.00310-0.00032$ $0.01088-0.00116$ $0.04986-0.00599$ $0.14575-0.02012$ $0.39734-0.06668$ $1.30979-0.30983$ $2.68726-0.82216$ $3.45154-1.11912$ $3.47364-1.05578$ $2.73323-0.71761$

$0.97430-0.20909$ $4-0.60879$ $2.15374-0.60879$ $3.50069-1.13161$ $3.49784-1.07134$ $3.02317-0.83534$ $2.09546-0.48476$ $0.80480-0.06310$ $-0.66759 \quad 0.38530$ $-2.08287 \quad 0.78972$ $1.30239-0.22093$
$-0.53770-0.34666$
$E(h, h)$.

$0.62478-0.01995$ $1.09703-0.03989$ $1.91123-0.09951$ $2.57374-0.19804$ $3.29962-0.39081$ $3.90359-0.91435$ $3.98424-1.51001$ $3.53010-1.71619$ . $\begin{array}{ll}3.57119 & -1.40860 \\ 3.66324 & -1.42706\end{array}$

$3.76083-0.73859$ $4.02735-1.30279$ $3.90496-1.62542$ $3.69303-1.71884$ $3.53482-1.65666$ $3.48263-1.53603$ $3.52154-1.43832$ $3.60078-1.40434$ $\begin{array}{ll}3.66759 & -1.43113 \\ 3.69151 & -1.48718\end{array}$ $3.57110-1.40860$ $\frac{a}{\lambda}=0.004763 \quad \frac{\alpha}{\beta}=0.10$

$\begin{array}{llll}0.00323 & -0.00042 & 0.05415 & -0.01994 \\ 0.01205 & -0.00161 & 1.24837 & -0.03987 \\ 0.05613 & -0.00812 & 2.55978 & -0.07957 \\ 0.14646 & -0.02463 & 3.81650 & -0.19865 \\ 0.33322 & -0.07273 & 5.13567 & -0.39482 \\ 0.84340 & -0.31621 & 6.79964 & -0.96164 \\ 1.41296 & -0.93024 & 7.75862 & -1.80248 \\ 1.60484 & -1.57115 & 7.99476 & -2.47130 \\ 1.54236 & -2.04346 & 7.91599 & -2.94330 \\ 1.40947 & -2.29902 & 7.69734 & -3.22043 \\ 1.33646 & -2.41142 & 7.44480 & -3.32754 \\ 1.35145 & -2.48973 & 7.22530 & -3.30552 \\ & & & \\ 0.67276 & -0.21391 & 6.38373 & -0.76323 \\ 1.21670 & -0.64801 & 7.48057 & -1.46025 \\ 1.52100 & -1.16832 & 7.89522 & -2.06231 \\ 1.60724 & -1.65106 & 7.99756 & -2.55032 \\ 1.55144 & -2.01660 & 7.92807 & -2.91560 \\ 1.44545 & -2.24492 & 7.76751 & -3.15060 \\ 1.36151 & -2.36578 & 7.57091 & -3.29273 \\ 1.33290 & -2.43291 & 7.37718 & -3.33238 \\ 1.35474 & -2.49596 & 7.21254 & -3.30048 \\ 1.39952 & -2.58212 & 7.09225 & -3.22691\end{array}$

$1.39952-2.58212$
$0.62418-0.01993$ $1.09576-0.03982$ $1.90741-0.09911$ $2.56271-0.19604$ $3.61742-0.81137$ $2.76604-1.01442$ $1.05876-0.65875$ $-0.92264-0.05150$ $-2.75260 \quad 0.53471$ $\begin{array}{ll}-4.00946 & 0.92621 \\ -4.33907 & 0.99014\end{array}$

$3.59058-0.68112$ $3.28353-1.00855$ $2.21949-0.93769$ $0.78450-0.58052$ $-0.77809-0.09772$ $-2.266390 .37963$ $-3.47555 \quad 0.76280$ $-4.20777 \quad 0.98255$ $\begin{array}{ll}-4.32277 & 0.98002 \\ -3.78395 & 0.72767\end{array}$ $3.17867-0.37985$ $\begin{array}{ll}0.00309 & -0.00041\end{array}$ $0.01085-0.00149$ $0.04971-0.00748$ $0.14517-0.02445$ $0.39508-0.07823$ $1.29844-0.34363$ $2.66544-0.86593$ $3.44485-1.12678$ $3.49840-0.98437$ $2.78692-0.52899$ 1.376880 .09557 $-0.457730 .74204$

$0.96661-0.23561$ $2.13400-0.65383$ $3.02155-1.00309$ $3.49797-1.13062$ $3.52024-1.00711$ $3.00933-0.68344$ $2.16064-0.23062$ 0.883110 .28367 $\begin{array}{ll}-0.58829 & 0.78265 \\ -2.02555 & 1.16933\end{array}$
$0.62419-0.01993$ $1.09583-0.03984$ $2.56783-0.19741$ $3.20814-0.38845$ $3.87931-0.90124$ $3.95800-1.47165$

$3.72240-1.66377$

$3.53769-1.60138$

$3.50523-1.46853$

$\begin{array}{ll}3.57514 & -1.39554 \\ 3.65097 & -1.41055\end{array}$

$3.74028-0.73013$ $3.99878-1.27516$ $3.68859-1.66619$ $3.54613-1.61046$ $3.50010-1.50450$ $3.53336-1.42058$ $3.59975-1.39199$ $3.65449-1.41384$ $1.90825-0.09932$ $3.88375-1.57980$ $3.67369-1.45863$

$\begin{array}{lll}1.000 \pi & 8.05458 & -1.42094 \\ 1.125449 & -1.39238\end{array}$

$\begin{array}{lll}1.125 \pi & 8.42700 & -1.41425 \\ 1.250 \pi & 8.55154 & -1.45906\end{array}$

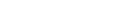


$\beta \mathrm{h}$

$$
C(h, 0)
$$

$S(h, 0)$

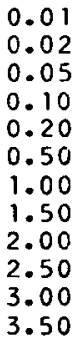

$0.125 \pi$ $0.250 \pi$ $0.375 \pi$ $0.500 \pi$ $0.025 \pi$ $0.750 \pi$ $0.875 \pi$ $1.000 \mathrm{~T}$ $1.125 \pi$
$1.250 \pi$
$E(., 0)$

$\begin{array}{ll}0.65216 & -0.01988 \\ 1.24439 & -0.03974 \\ 2.54985 & -0.09916 \\ 3.79672 & -0.19745 \\ 5.09658 & -0.39064 \\ 6.70728 & -0.93860 \\ 7.60137 & -1.71870 \\ 7.81142 & -2.30966 \\ 7.74602 & -2.70662 \\ 7.57167 & -2.92855 \\ 7.37981 & -3.01040 \\ 7.22104 & -2.99480 \\ 0.30945 & -0.74826 \\ 7.34684 & -1.40566 \\ 7.72397 & -1.95174 \\ 7.81382 & -2.37744 \\ 7.75592 & -2.63391 \\ 7.62671 & -2.88083 \\ 7.47443 & -2.93423 \\ 7.33067 & -3.01397 \\ 7.21206 & -2.99126 \\ 7.12911 & -2.93655\end{array}$

$C(h, h)$

$0.00321-0.00075$ $0.01197-0.00281$ $0.05563-0.01370$ $0.14451-0.03909$ $0.32564-0.10490$ $0.80172-0.38906$ $1.29487-1.00938$ $1.29487-1.00938$ $1.40342-2.01641$ $1.31857-2.2009$ $1.27597-2.40320$ $1.28347-2.51759$ $0.64566-0.27505$ $\begin{array}{ll}1.13048 & -0.73405\end{array}$ $1.38187-1.23286$ $1.44830-1.66875$ $1.40952-1.99231$ $1.29013-2.33929$ $1.29013-2.33929$ $1.27403-2.43599$ $\begin{array}{ll}1.28510 & -2.52559 \\ 1.30631 & -2.62172\end{array}$

$$
\frac{\mathrm{a}}{\lambda}=0.004763 . \quad \frac{\alpha}{\beta}=0.40
$$

$\begin{array}{rr}0.62219 & -0.01986 \\ 1.09179 & -0.03966 \\ 1.89756 & -0.09834 \\ 2.54342 & -0.19293 \\ 3.14253 & -0.36628 \\ 3.55596 & -0.71975 \\ 2.75553 & -0.68728 \\ 1.14482 & -0.07276 \\ -0.79211 & 0.71411 \\ -2.67139 & 1.28704 \\ -4.08301 & 1.35819 \\ -4.65481 & 0.77506 \\ & \\ 3.53316 & -0.62495 \\ 3.23913 & -0.79229 \\ 2.24407 & -0.51532 \\ 0.88163 & 0.03801 \\ -0.64783 & 0.65975 \\ -2.16086 & 1.16308 \\ -3.45849 & 1.39862 \\ -4.34313 & 1.25158 \\ -4.65663 & 0.71101 \\ -4.31121 & -0.20628\end{array}$

$S(h, h)$

$\begin{array}{rr}0.00307 & -0.00072 \\ 0.01078 & -0.00257 \\ 0.04922 & -0.01240 \\ 0.14324 & -0.03873 \\ 0.38779 & -0.11630 \\ 1.26377 & -0.45477 \\ 2.61127 & -1.01499 \\ 3.46422 & -1.16570 \\ 3.65010 & -0.75914 \\ 3.05567 & 0.10083 \\ 1.68692 & 1.17362 \\ -0.24846 & 2.12270 \\ 0.94257 & -0.32275 \\ 2.07870 & -0.80376 \\ 2.98265 & -1.13035 \\ 3.53426 & -1.14160 \\ 3.66196 & -0.80640 \\ 3.30967 & -0.18087 \\ 2.46315 & 0.32728 \\ 1.18224 & 1.47143 \\ -0.39292 & 2.17352 \\ -2.06111 & 2.54718\end{array}$

$E(h, h)$

$0.62220 \quad-0.01987$ $1.09186-0.03969$ $1.89836-0.09872$ $.54829-0.19532$
3 $-17037-0.38070$ $3.80069-0.85935$ $-1.3533$ $3.68991-1.50520$ $3.53740-1.37736$ $3.53740-1.37736$ $3.57749-1.33479$ $3.67342-0.70283$ $3.67342-0.70283$ $3.90691-1.18872$ $3.81335-1.44041$ $3.66503-1.50698$ $3.56455-1.46827$ $3.53425-1.39950$ $3.55400-1.34894$ $3.59081-1.33286$ $\begin{array}{ll}3.61899 & -1.34394 \\ 3.62822 & -1.36516\end{array}$
0.01
0.02
0.05
0.10
0.20
0.50
1.00
1.50
2.00
2.50
3.00
3.50

$0.64819-0.01975$ $1.23641-0.03942$ $2.52941-0.09763$ $3.75424-0.19143$ $5.00665-0.36612$ $0.47134-0.78370$ $7.22401-1.15516$ $7.51844-1.25037$ $7.73998-1.22995$ $7.95370-1.19535$ $8.14921 .-1.1808$ $8.31430-1.18277$

$\begin{array}{llll}0.125 \pi & 0.12404 & -0.65268\end{array}$ 0.250 पा $7.00608-1.03956$ $0.375 \pi \quad 7.34929-1.21171$ $\begin{array}{llll}0.500 \pi & 7.55109 & -1.25135\end{array}$ $\begin{array}{llll}0.625 \pi & 7.72413 & -1.2327\end{array}$ $\begin{array}{llll}0.750 \pi & 7.89328 & -1.20373\end{array}$ $\begin{array}{llll}0.875 \pi & 8.05437 & -1.18538\end{array}$ $\begin{array}{llll}1.000 \pi & 8.19923-1.18031\end{array}$ $\begin{array}{lll}1.125 \pi & 8.32448 & -1.18317\end{array}$
$0.00317-0.00138$ $0.01181-0.00518$ $0.05466-0.02470$ $0.14069-0.06743$ $0.31108-0.16734$ $0.72600-0.52485$ $1.09656-1.15678$ $1.19147-1.67291$ $1.17090-2.02659$ $1.13622-2.25908$ $\begin{array}{ll}1.12168 & -2.42816 \\ 1.12354 & -2.57135\end{array}$

$0.59546-0.39033$ $0.98121-0.89195$ $1.15298-1.35912$ $1.19243-1.73247$ $1.17367-2.00560$ $1.14462-2.20103$ $1.12621-2.34820$ $1.12110-2.47037$ $\begin{array}{ll}1.12393 & -2.58067 \\ 1.12869 & -2.08389\end{array}$

$\begin{array}{llrr}0.64819 & -0.01975 & 0.61823 & -0.01973 \\ 1.23647 & -0.03948 & 1.08389 & -0.03931 . \\ 2.53011 & -0.09836 & 1.87869 & -0.09685 \\ 3.75753 & -0.19507 & 2.50575 & -0.186 .34 \\ 5.01964 & -0.38245 & 3.07411 & -0.34015 \\ 6.52918 & -0.89297 & 3.45513 & -0.54813 \\ 7.30683 & -1.56642 & 2.78074 & -0.07331 \\ 7.47308 & -2.02809 & 1.32516 & 1.07723 \\ 7.42799 & -2.30903 & -0.68546 & 2.30836 \\ 7.31707 & -2.45144 & -3.03712 & 2.96550 \\ 7.20627 & -2.49926 & -5.33604 & 2.45621 \\ 7.12313 & -2.49144 & -6.98185 & 0.43053 \\ & & & \\ 6.16519 & -0.71946 & 3.43285 & -0.51858 \\ 7.09350 & -1.30446 & 3.19079 & -0.38948 \\ 7.40559 & -1.75389 & 2.33563 & 0.28736 \\ 7.47485 & -2.07795 & 1.07057 & 1.26159 \\ 7.43465 & -2.29375 & -0.52368 & 2.22908 \\ 7.35093 & -2.42206 & -2.34481 & 2.87273 \\ 7.25953 & -2.48446 & -4.22103 & 2.88774 \\ 7.17935 & -2.50119 & -5.89897 & 2.04231 \\ 7.11869 & -2.48969 & -7.05239 & 0.23559 \\ 7.07923 & -2.46380 & -7.31202 & -2.44522\end{array}$

$0.00303-0.00133$ $0.01062-0.00470$ $0.04825-0.02212$ $0.13950-0.06675$ $0.37413-0.19043$ $1.20800-0.67110$ $2.58310-1.32861$ $3.69834-1.29748$ $4.30131-0.339 .36$ $4.05275 \quad 1.47133$ $\begin{array}{rr}2.63871 & 3.73345 \\ -0.07649 & 5.72306\end{array}$ $0.90133-0.49179$ $2.01294-1.10458$ $3.02096-1.41584$ $3.82206-1.21972$ $4.2817 .8-0.44088$ $4.23185 \quad 0.88022$ $3.50902 \quad 2.57927$ $2.00080 \quad 4.36270$ $-0.30790 \quad 5.82648$ $\begin{array}{ll}-3.28891 & 0.50603\end{array}$

$\begin{array}{ll}0.61824 & -0.01974 \\ 1.08395 & -0.03940 \\ 1.87876 & -0.09753 \\ 2.50982 & -0.19122 \\ 3.09704 & -0.36580 \\ 3.65342 & -0.78321 \\ 3.71400 & -1.15452 \\ 3.60313 & -1.24963 \\ 3.53705 & -1.22914 \\ 3.52765 & -1.19450 \\ 3.54087 & -1.17998 \\ 3.55182 & -1.18186 \\ & \\ 3.54675 & -0.65223 \\ 3.73742 & -1.03897 \\ 3.67546 & -1.21103 \\ 3.58967 & -1.25060 \\ 3.53962 & -1.23190 \\ 3.52647 & -1.20289 \\ 3.53343 & -1.18450 \\ 3.54477 & -1.17941 \\ 3.55225 & -1.18226 \\ 3.55439 & -1.18704\end{array}$


$C(h, 0)$

$\begin{array}{llll}0.01 & 0.64229 & -0.01956\end{array}$

$0.02 \quad 1.22465-0.03900$

$0.05 \quad 2.50042-0.09596$

$3.69795-0.18563$

0.50

1.00

1.50

2.00

2.50

3.00

3.50

$0.125 \pi$

$0.250 \pi$

$0.375 \pi$
$0.500 \pi$

$0.625 \pi$

$0.750 \pi$

0.875

$1.000 \pi$

1.125

1.250 $0.27233-0.68638$ $7.01003-0.93001$ $7.36274-0.97747$ $7.62653-0.97080$ $7.84691-0.96175$ $\begin{array}{ll}8.03171 & -0.95808 \\ 8.18744 & -0.95913\end{array}$

$5.94985-0.58602$ $0.78218-0.86190$ $\begin{array}{lll}7.15381 & -0.95962\end{array}$ $\begin{array}{lll}7.40348 & -0.97789\end{array}$ $\begin{array}{ll}7.60889 & -0.97164\end{array}$ $7.78741-0.96370$ $7.94299-0.95968$ $8.07844 .-0.95879$ $8.19724-0.95918$ 8.30282 .0 .95970
Sin, o)

$0.00311-0.00233$ $0.01158-0.00869$ $0.05322-0.04084$ $0.13516 \quad-0.10854$ $0.29064-0.25574$ $0.62894-0.70659$ $0.87168-1.35322$ $0.91884-1.81098$ $0.91202-2.12237$ $\begin{array}{lll}0.90288 & -2.34817\end{array}$ $0.89995 \quad-2.52794$ $0.90016-2.68048$

$0.52906-0.54739$ $0.80381-1.09839$ $0.90115-1.53702$ $0.91922-1.86244$ $0.91287-2.10317$ $0.90485-2.28921$ $0.90078 \quad-2.44187$ $0.89985-2.57344$ $0.90020-2.69018$ $0.90070-2.79529$ $\frac{a}{\lambda}=0.001763 \cdot \frac{\alpha}{\beta}=0.70$

$E(h, 0)$

C $(h, h)$

$S(h, h)$

$E(h, h)$

$\begin{array}{ll}0.64230 & -j .01957 \\ 1.22469 & -3.03911 \\ 2.50080 & -j .09717 \\ 3.69962 & -1.19156 \\ 4.90722 & -0.37055 \\ 6.27755 & -0.83069 \\ 6.90924 & -1.37106 \\ 7.02644 & -1.69034 \\ 7.00062 & -1.85786 \\ 6.94424 & --.931117 \\ 6.89554 & -1.95255 \\ 6.86399 & -1.94978 \\ & \\ 5.95897 & -1.67894 \\ 6.74551 & -1.17048 \\ 6.98065 & -1.50637 \\ 7.02756 & -1.72179 \\ 7.00429 & -1.84943 \\ 6.96058 & -1.91698 \\ 6.91804 & -1.94626 \\ 6.88482 & -1.95333 \\ 6.86245 & -1.94917 \\ 6.84949 & -1.94074\end{array}$

$0.61234-0.01954$

$1.07217-0.03886$

$1.84944-0.09400$

$2.45146-0.17805$

$2.98066-0.30328$

$3.35569-0.31419$

$2.94258 \quad 0.797=1$

$1.66298 \quad 2.906 \equiv 2$

$-0.89741 \quad 5.239 \geq 5$

$-5.14936 \quad 0.58736$

$\begin{array}{ll}-10.95621 & 5.29782\end{array}$

$\begin{array}{lll}-17.07851 & -0.45384\end{array}$

$3.31639-0.37356$ $3.21780 \quad 0.16502$

$2.60105 \quad 1.46633$

$1.39039 \quad 3.2458$

$-0.656325 .08482$

$\begin{array}{ll}-3.74521 & 0.39021\end{array}$

$\begin{array}{ll}-7.89064 & 0.39063\end{array}$

$-12.74773 \quad 4.19658$

$-17.44929-1.05175$

$-20.49195-9.92900$
$0.00297-0.00223$

$0.01039-0.00785$

$2.72586-1.84711$

$\begin{array}{rr}4.57275 & -1.61796 \\ 6.34192 & 0.34668\end{array}$

$\begin{array}{ll}4.34192 & 0.34668 \\ 7.10754 & 4.41160\end{array}$

$5.37528 \quad 10.30423$

$-0.6295816 .63721$

$0.85479-0.73493$

$2.01394-1.56749$

$3.35951-1.92746$

$\begin{array}{rr}4.84280 & -1.45789 \\ 0.23192 & 0.13396\end{array}$

$7.06834 \quad 3.02654$

$6.66764 \quad 7.17113$

$4.18312 \quad 12.14854$

$\begin{array}{rr}-1.23824 & 17.02385 \\ -10.18775 & 20.24280\end{array}$
$0.04684-0.03633$

$0.13418-0.10743$

$0.35531-0.29706$

$1.15433-0.98528$
$0.61234-0.01955$ $1.07220-0.03896$ $1.84981-0.09578$ $2.45361-0.18528$ $2.99231-0.34485$ $3.45462-0.68553$ $3.50031-0.92893$ $3.44777-0.97628$ $3.42396-0.96953$ $3.42125-0.96043$ $3.42377-0.95753$ $3.42536-0.95776$

$3.37276-0.58524$ $3.51378-0.86090$ $3.48029-0.95849$ $3.44241-0.97660$ $3.42474-0.97037$ $3.42098-0.96239$ $3.42244-0.95835$ $3.42439-0.95744$ $3.42541-0.95781$ $3.42565-0.95832$

$\begin{array}{lll}0.01 & 0.63645 & -0.01938 \\ 0.02 & 1.21302 & -0.03858 \\ 0.05 & 2.47192 & -0.09431 \\ 0.10 & 3.64335 & -0.18007 \\ 0.20 & 4.80273 & -0.32628 \\ 0.50 & 6.09590 & -0.00546 \\ 1.00 & 6.82178 & -0.76638 \\ 1.50 & 7.20191 & -0.79020 \\ 2.00 & 7.48084 & -0.78805 \\ 2.50 & 7.70314 & -0.78569 \\ 3.00 & 7.88590 & -0.78514 \\ 3.50 & 8.04026 & -0.78519 \\ 0.125 \pi & 5.79246 & -0.52853 \\ 0.250 \pi & 0.58824 & -0.72617 \\ 0.375 \pi & 0.97515 & -0.78191 \\ 0.500 \pi & 7.24587 & -0.79037 \\ 0.625 \pi & 7.46266 & -0.78830 \\ 0.750 \pi & 7.64385 & -0.78614 \\ 0.875 \pi & 7.79827 & -0.78527 \\ 1.000 \pi & 7.93211 & -0.78513 \\ 1.125 \pi & 8.05001 & -0.78519 \\ 1.250 \pi & 8.15538 & -0.78526\end{array}$

$\begin{array}{ll}0.00306 & -0.00325 \\ 0.01135 & -0.01212 \\ 0.05183 & -0.05656 \\ 0.12985 & -0.14804 \\ 0.27176 & -0.33840 \\ 0.54828 & -0.86583 \\ 0.70831 & -1.52401 \\ 0.73184 & -1.95427 \\ 0.72954 & -2.25046 \\ 0.72708 & -2.47432 \\ 0.72647 & -2.65612 \\ 0.72648 & -2.81001 \\ & \\ 0.47185 & -0.68774 \\ 0.66835 & -1.27494 \\ 0.72371 & -1.69818 \\ 0.73198 & -2.00250 \\ 0.72980 & -2.23181 \\ 0.72755 & -2.41516 \\ 0.72663 & -2.56896 \\ 0.72645 & -2.70213 \\ 0.72648 & -2.81975 \\ 0.72652 & -2.92507\end{array}$

$$
\frac{a}{\lambda}=0.004733 \quad \frac{\alpha}{\beta}=1.00
$$

\begin{tabular}{|c|c|}
\hline $\begin{array}{l}0.63645 \\
1.21302 \\
2.47184 \\
3.64274 \\
4.79828 \\
6.04302 \\
6.55701 \\
6.63974 \\
6.62495 \\
6.59624 \\
6.57479 \\
6.56280\end{array}$ & $\begin{array}{l}-0.01940 \\
-0.03873 \\
-0.09599 \\
-0.18812 \\
-0.35912 \\
-0.77412 \\
-1.20850 \\
-1.42971 \\
-1.52979 \\
-1.56760 \\
-1.57717 \\
-1.57619\end{array}$ \\
\hline $\begin{array}{l}5.76417 \\
6.43131 \\
6.60860 \\
6.64045 \\
6.62697 \\
6.60413 \\
6.58431 \\
6.57052 \\
6.56226 \\
6.55800\end{array}$ & $\begin{array}{l}-0.54139 \\
-1.05487 \\
-1.30619 \\
-1.44956 \\
-1.52514 \\
-1.560174 \\
-1.57449 \\
-1.57748 \\
-1.575998 \\
-1.57323\end{array}$ \\
\hline
\end{tabular}

$0.60651-0.01935$ $1.06059-0.03839$ $1.82145-0.09243$ $2.39973-0.16964$ $2.89755-0.26893$ $3.31409-0.10097$ $3.26731 \quad 1.67770$ 3.26731
$2.14222 \quad 5.13826$ $\begin{array}{rr}2.14222 & 5.13826 \\ -1.03967 & 9.60639\end{array}$ $-10.19694 \quad 13.08113$ $-25.07614 \quad 11.20155$ $\begin{array}{lll}-45.02608 & -3.43541\end{array}$

$3.23764-0.24117$ $3.36391 \quad 0.69260$ $3.05258 \quad 2.73536$ $1.81480 \quad 5.73701$ $-7.22797 \quad 9.27829$ $-16.72001 \quad 13.38410$ $-16.79001 \quad 13.20376$ $\begin{array}{ll}-30.38788 & 8.74685\end{array}$ $\begin{array}{ll}-40.43954 & -5.14701 \\ -60.53422 & -33.31867\end{array}$
$0.00292-0.00311$ $0.01016-0.01093$ $0.04547-0.05012$ $0.12917-0.14658$ $0.33985-0.39882$ $\begin{array}{lll}1.13186 & -1.29377\end{array}$ $\begin{array}{ll}3.08382 & -2.44730\end{array}$ $6.19593-2.10260$ $10.21263 \quad 1.34268$ $13.32846 \quad 9.85035$ $11.23419 \quad 24.81160$ $-3.4962244 .87163$

$0.82429-0.96931$ $2.12280-2.06080$ $4.04971-2.56783$

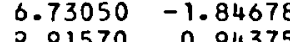
$9.91570 \quad 0.94375$
12.72019 $12.72019 \quad 0.77624$ $13.32659 \quad 16.47566$ 8.7426130 .15473 $\begin{array}{rr}-5.21094 & 40.29216 \\ -33.39793 & 60.45736\end{array}$
$0.60651-0.01937$ $1.06059-0.03853$ $1.82137-0.09406$ $2.39914-0.17956$ $2.89347-0.32547$ $3.27851-0.60425$ $3.31247-0.76489$ $3.28740-0.78859$ $3.27876-0.78636$ $3.27798-0.78395$ $3.27847-0.78337$

$3.21566-0.52743$ $3.32023-0.72478$ $3.30207-0.78037$ $3.28526-0.78874$ $3.27900-0.78662$ $3.27792-0.7844$ $3.27823-0.78352$ $3.27856-0.78335$ $-3.27873-0.78346$ 


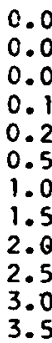

0.01
0.02
0.05
0.10
0.20
0.50
1.00
1.50
2.00
2.50
3.00
3.50

$\begin{array}{lll}0.49570 & -0.01999\end{array}$ $0.96381-0.03999$ $2.09597-0.09992$ $3.28845 \quad-0.19950$ $4.58936-0.39636$ $6.20548-0.94583$ $6.98199-1.60498$ $\begin{array}{ll}7.08388 & -1.84815\end{array}$ $7.11098-1.75773$ $7.28512-1.54955$ $7.58942-1.42441$ $7.88829-1.45439$

$\begin{array}{lll}0.125 \pi & 5.81380 & -0.75877\end{array}$ $0.250 \pi \quad 0.78986-1.37039$ $0.375 \pi \quad 7.05336-1.73928$ $0.500 \pi \quad 7.08403-1.85143$ $\begin{array}{lll}0.625 \pi & 7.10509-1.77121\end{array}$ $0.750 \pi \quad 7.21749 .-1.60796$ $0.875 \pi-7.42773-1.46868$ $1.000 \pi \quad 7.68103-1.41790$ $1.125 \pi \quad 7.90517-1.46105$ $1.125 \pi-8.90517-1.46105$

$$
\frac{a}{\lambda}=0.006350 \quad \frac{\alpha}{\beta}=0.00
$$

$E(h, 0)$

$\mathrm{C}(\mathrm{h}, \mathrm{h})$

$S(h, h)$

$E(h, h)$

$\begin{array}{ll}0.00247 & -0.00010 \\ 0.00946 & -0.00040 \\ 0.04806 & -0.00250 \\ 0.13504 & -0.00998 \\ 0.32445 & -0.03972 \\ 0.86922 & -0.23975 \\ 1.52678 & -0.84715 \\ 1.76942 & -1.55575 \\ 1.67874 & -2.10386 \\ 1.47040 & -2.37593 \\ 1.34515 & -2.43624 \\ 1.37506 & -2.44564 \\ 0.68303 & -0.15026 \\ 1.29263 & -0.55665 \\ 1.66084 & -1.10336 \\ 1.77265 & -1.64780 \\ 1.69223 & -2.07291 \\ 1.52885 & -2.32509 \\ 1.38947 & -2.42312 \\ 1.33862 & -2.43686 \\ 1.38171 & -2.44827 \\ 1.47627 & -2.51369\end{array}$

$0.49577-0.01999$ $0.96387-0.03999$ $2.09673-0.09996$ $3.29250-0.19984$ $4.60767-0.39901$ $6.31938-0.98595$ $7.34690-1.89165$ $7.61215-2.64863$ $7.51716 \quad-3.20989$ $7.2428 t-3.55596$ $\begin{array}{ll}6.91037 & -3.69611 \\ 0.60689 & -3.60498\end{array}$

$5.88549-0.77849$ $7.04343-1.51754$ $7.49896-2.13131$ $7.61539-2.74076$ $\begin{array}{lll}7.53187 & -3.17613\end{array}$ $7.33233-3.47845$ $7.07847-3.64982$ $6.81845-3.70265$ $6.58875-3.65783$ $6.41430-3.54208$

$0.48193-0.01999$ $0.88220-0.0$ $1.64554-0.09988$ $2.29941-0.19917$ $2.93337-0.39372$ $3.42963-0.90732$ $2.62734-1.36002$ $0.93932-1.26545$ $-0.99911-0.82432$ $-2.78134 \quad-0.21089$ $-4.00828 \quad 0.52537$ $-4.27361 \quad 1.2804$

$3.38384-0.73955$ $3.13131-1.237 .17$ $2.08724-1.32172$ $0.66931-1.21843$ $-0.85844-0.8635 \mathrm{t}$ $-2.30674 \quad-0.40114$ $-4.19528 \quad 0.14306$ $-4.24991 \quad 1.32703$

$-3.59848 \quad 1.75380$
$0.00240-0.00010$ $0.00876-0.00040$ $0.04201-0.00250$ $0.12625-0.00996$ $0.35427-0.03946$ $\begin{array}{lll}1.20852 & -0.23005\end{array}$ $2.51189-0.72165$ $3.20334-1.10921$ $3.18571-1.23105$ $2.47493-1.16317$ $1.11148-0.96670$ $\begin{array}{lll}-0.66694 & -0.57990\end{array}$

$0.89174 \quad-0.14647$ $2.00927-0.5034$ $2.83937-0.88675$ $3.24351-1.14248$ $2.74992-1.19584$ $1.86976-1.08352$ $0.63237-0.88008$ $-0.79263-0.54490$ $-2.14921-0.07895$

$\begin{array}{ll}0.48194 & -0.01999 \\ 0.88226 & -0.03999 \\ 1.64625 & -0.09992 \\ 2.30384 & -0.19950 \\ 2.95964 & -0.39636 \\ 3.67345 & -0.94583 \\ 3.75858 & -1.60495 \\ 3.45519 & -1.84805 \\ 3.19461 & -1.75755 \\ 3.14560 & -1.54928 \\ 3.26759 & -1.42405 \\ 3.41234 & -1.45398 \\ 3.52172 & -0.75877 \\ 3.80770 & -1.37038 \\ 3.66616 & -1.73923 \\ 3.40923 & -1.85133 \\ 3.20715 & -1.77104 \\ 3.13722 & -1.60771 \\ 3.19331 & -1 . .46836 \\ 3.31309 & -1.41752 \\ 3.41946 & -1.46063 \\ 3.45940 & -1.55522\end{array}$

$$
\frac{a}{\lambda}=0.006350 \quad \frac{\alpha}{\beta}=0.005
$$

$\begin{array}{rr}0.48183 & -0.01959 \\ 0.88200 & -0.03997 \\ 1.64505 & -0.09984 \\ 2.29843 & -0.19902 \\ 2.93147 & -0.39396 \\ 3.42598 & -0.90271 \\ 2.62576 & -1.34337 \\ 0.94394 & -1.23655 \\ -0.98833 & -0.78792 \\ -2.76454 & -0.17539 \\ -3.98542 & 0.54459 \\ -4.25021 & 1.26511 \\ 3.38059 & -0.73671 \\ 3.12808 & -1.22613 \\ 2.08766 & -1.366 \equiv 9 \\ 0.67486 & -1.188 .0 \\ -0.84808 & -0.82735 \\ -2.29175 & -0.36410 \\ -3.46884 & 0.172 .2 \\ -4.17135 & 0.75713 \\ -4.22087 & 1.30337 \\ -3.58386 & 1.70139\end{array}$

$0.00240 \quad-0.00011$ $0.00875-0.00044$ $\begin{array}{lll}0.04199 & -0.00271\end{array}$ $0.12616-0.01059$ $0.35389-0.04118$ $\begin{array}{lll}1.20642 & -0.23518\end{array}$ $2.50701-0.72795$ $3.19958-1.10928$ $3.18569-1.219 .15$ $2.47834-1.13372$ $1.11992-0.91683$ $-0.64746-0.51736$ $0.89036 \quad-0.15049$ $2.00527-0.51016$ $2.83437-0.89160$ $3.24022-1.14118$ $2.75235-1.17198$ $1.87521-1.04377$ $0.74300-0.82523$ $\begin{array}{ll}-0.77446 & -0.48211 \\ -2.12408 & -0.02080\end{array}$

$\begin{array}{ll}0.48184 & -0.01999 \\ 0.88206 & -0.03998 \\ 1.64575 & -0.09988 \\ 2.30284 & -0.19939 \\ 2.95769 & -0.39595 \\ 3.66925 & -0.94352 \\ 3.75405 & -1.59787 \\ 3.45447 & -1.83813 \\ 3.19834 & -1.74929 \\ 3.15034 & -1.54565 \\ 3.26897 & -1.42377 \\ 3.40911 & -1.45268 \\ 3.51819 & -0.75729 \\ 3.80271 & -1.36537 \\ 3.66263 & -1.73070 \\ 3.40921 & -1.84135 \\ 3.21064 & -1.76251 \\ 3.14215 & -1.60269 \\ 3.19680 & -1.46683 \\ 3.31309 & -1.41743 \\ 3.41599 & -1.45910 \\ 3.45449 & -1.55021\end{array}$


$\frac{a}{\lambda}=0.006350 \quad \frac{\alpha}{\beta}=0.01$

$C(h, 0)$

$\begin{array}{lll}0.01 & 0.49556 & -0.01999 \\ 0.02 & 0.96341 & -0.03997 \\ 0.05 & 2.09497 & -0.09985 \\ 0.10 & 3.28646 & -0.19929 \\ 0.20 & 4.58547 & -0.39555 \\ 0.50 & 6.19709 & -0.94124 \\ 1.00 & 6.97293 & -1.59090 \\ 1.50 & 7.08240 & -1.82840 \\ 2.00 & 7.11832 & -1.74129 \\ 2.50 & 7.29444 & -1.54228 \\ 3.00 & 7.59213 & -1.42372 \\ 3.50 & 7.88194 & -1.45170\end{array}$

$\begin{array}{lll}0.125 \pi & 5.80675 & -0.7558\end{array}$ $\begin{array}{llll}0.250 \pi & 0.77990 & -1.36043\end{array}$ $0.375 \pi \quad 7.04630-1.72232$ $0.500 \pi \quad 7.08394-1.83159$ $0.625 \pi \quad 7.11195-1.75425$ $0.750 \pi \quad 7.22721-1.59792$ $0.875 \pi \quad 7.43459-1.4655$ $1.000 \pi \quad 7.68103=1.41759$ $1.125 \pi \quad 7.89832-1.45791$ $1.250 \pi 8.04078=1.54570$
Sịn, 0)

$\begin{array}{ll}0.00246 & -0.00012 \\ 0.00945 & -0.00049 \\ 0.04801 & -0.00298 \\ 0.13485 & -0.01132 \\ 0.32366 & -0.04289 \\ 0.86464 & -0.24737 \\ 1.51271 & -0.85543 \\ 1.74969 & -1.55645 \\ 1.66231 & -2.09573 \\ 1.46314 & -2.36582 \\ 1.34447 & -2.43274 \\ 1.37238 & -2.45121 \\ 0.68011 & -0.15656 \\ 1.28269 & -0.56583 \\ 1.64390 & -1.10964 \\ 1.75283 & -1.64711 \\ 1.67528 & -2.06527 \\ 1.51882 & -2.31458 \\ 1.38634 & -2.41546 \\ 1.33832 & -2.43607 \\ 1.37858 & -2.45432 \\ 1.46633 & -2.52257\end{array}$

$E(h, 0)$

$\begin{array}{ll}0.49557 & -1.01999 \\ 0.96347 & -0.03997 \\ 2.09573 & -0.09991 \\ 3.29051 & -0.19970 \\ 4.60371 & -0.39856 \\ 0.30981 & -0.98345 \\ 7.33012 & -1.88242 \\ 7.59228 & -0.63006 \\ 7.49904 & -3.18168 \\ 7.23089 & -3.52013 \\ 6.90742 & -3.65656 \\ 6.61362 & -3.62648 \\ 5.87785 & -0.77692 \\ 7.02933 & -1.51164 \\ 7.48055 & -2.16894 \\ 7.59547 & -2.72079 \\ 7.51348 & -3.14858 \\ 7.31821 & -3.44448 \\ 7.07076 & -3.61157 \\ 6.81829 & -3.66290 \\ 6.59611 & -3.61958 \\ 6.42802 & -3.50808\end{array}$

$\mathrm{C}(\mathrm{h}, \mathrm{h})$

$\begin{array}{rr}0.48173 & -0.01949 \\ 0.88180 & -0.03996 \\ 1.64455 & -0.09980 \\ 2.29744 & -0.19886 \\ 2.92957 & -0.39239 \\ 3.42235 & -0.89810 \\ 2.62421 & -1.32677 \\ 0.94854 & -1.20773 \\ -0.97769 & -0.75137 \\ -2.74814 & -0.13997 \\ -3.96343 & 0.56371 \\ -4.22815 & 1.25009 \\ & \\ 3.37736 & -0.73394 \\ 3.12488 & -1.21514 \\ 2.08809 & -1.33913 \\ 0.68038 & -1.15785 \\ -0.83785 & -0.79124 \\ -2.27707 & -0.32714 \\ -3.44943 & 0.20235 \\ -4.14844 & 0.76827 \\ -4.20519 & 1.29101 \\ -3.57065 & 1.64965\end{array}$

$S(h, h)$

$0.00240-0.00012$

$0.00875-0.00049$

$0.04196-0.00292$

$0.12606-0.01121$

$0.35350-0.04289$

$1.20432-0.24032$

$2.50219-0.73426$

$3.19597-1.10942$

$3.18589-1.20732$

$2.48205-1.10433$

$\begin{array}{rr}1.12860 & -0.86713 \\ -0.63223 & -0.45507\end{array}$

$0.88898-0.15451$ $2.00131-0.51690$ $2.82947-0.89647$ $3.23709-1.13995$ $3.21017-1.20846$ $2.75507-1.14819$ $1.88098-1.00413$ $0.65379-0.77059$ $\begin{array}{lll}-0.75659 & -0.41957\end{array}$ $-2.09990 ; 0.03721$
$E(h, h)$

$0.48174 \quad-0.0199$ $0.88186-0.03997$ $1.64525-0.09985$ $2.30185-0.19928$ $2.95575-0.39554$ $3.66506-0.94121$ $3.74952-1.59084$ $3.45371-1.82828$ $3.20196-1.74108$ $3.15493-1.54197$ $3.27031-1.42333$ $3.40599-1.45126$

$3.51467-0.75582$ $3.79773-1.36039$ $3.65910-1.72224$ $3.40914-1.83145$ $3.21401-1.75404$ $3.14695-1.59764$ $3.20018-1.46518$ $3.31310-1.41719$ $3.41263-1.45746$ $3.44975-1.54523$

$$
\frac{a}{\lambda}=0.006350 \quad \frac{\alpha}{\beta}=0.02
$$

$\begin{array}{rr}0.48153 & -0.01998 \\ 0.88140 & -0.03994 \\ 1.64355 & -0.09971 \\ 2.29547 & -0.19855 \\ 2.92577 & -0.39106 \\ 3.41515 & -0.88893 \\ 2.62120 & -1.29374 \\ 0.95768 & -1.15031 \\ -0.95683 & -0.67869 \\ -2.71654 & -0.06934 \\ -3.92208 & 0.60198 \\ -4.18801 & 1.22087 \\ 3.37092 & -0.72836 \\ 3.11857 & -1.19325 \\ 2.08902 & -1.29682 \\ 0.69131 & -1.09757 \\ -0.81778 & -0.71920 \\ -2.24862 & -0.25341 \\ -3.41246 & 0.26141 \\ -4.10572 & 0.79066 \\ -4.16587 & 1.25620 \\ -3.54824 & 1.54807\end{array}$

$0.00240-0.00015$ $0.00874-0.00057$ $0.04191-0.00333$ $\begin{array}{lll}0.12586 & -0.01246\end{array}$ $0.35272-0.04631$ $\begin{array}{lll}1.20018 & -0.25056\end{array}$ $2.49278-0.74692$ $3.18918-1.10988$ $3.18694-1.18389$ $2.49040-1.04576$ $\begin{array}{lll}2.14668 & -0.76818\end{array}$ $-0.59848-0.33119$

$0.88624-0.16252$ $1.99351-0.53038$ $2.81997-0.90632$ $3.23127-1.13770$ $3.21064-1.18715$ $2.76136-1.10080$ $1.89344-0.92513$ $0.67582-0.66186$ $-0.72170-0.29518$ $-2.054310 .15296$

$\begin{array}{ll}0.48154 & -0.01998 \\ 0.88146 & -0.03995 \\ 1.64426 & -0.09978 \\ 2.29987 & -0.19906 \\ 2.95187 & -0.39473 \\ 3.65671 & -0.93663 \\ 3.74050 & -1.57690 \\ 3.45209 & -1.80881 \\ 3.20886 & -1.72479 \\ 3.16373 & -1.53444 \\ 3.27286 & -1.42204 \\ 3.40004 & -1.44811 \\ 3.50764 & -0.75288 \\ 3.78783 & -1.35051 \\ 3.65204 & -1.70549 \\ 3.40887 & -1.81189 \\ 3.22044 & -1.73725 \\ 3.15613 & -1.58747 \\ 3.20665 & -1.46157 \\ 3.31309 & -1.41626 \\ 3.40623 & -1.45389 \\ 3.44072 & -1.53534\end{array}$


Bh

$C(h, 0)$

\begin{tabular}{|c|c|}
\hline $\begin{array}{l}0.49516 \\
0.96261 \\
2.09293 \\
3.28250 \\
4.57772 \\
6.18943 \\
6.95493 \\
7.07899 \\
7.13166 \\
7.31150 \\
7.59702 \\
7.87037\end{array}$ & $\begin{array}{l}-0.01997 \\
-0.03993 \\
-0.09973 \\
-0.19886 \\
-0.39395 \\
-0.93213 \\
-1.56325 \\
-1.78982 \\
-1.70896 \\
-1.52708 \\
-1.42056 \\
-1.44505\end{array}$ \\
\hline $\begin{array}{l}5.79273 \\
.76014 \\
.03217 \\
.08319 \\
.12438 \\
.24502 \\
.44712 \\
.68098\end{array}$ & $\begin{array}{l}-0.75001 \\
-1.34081 \\
-1.68910 \\
-1.79282 \\
-1.72093 \\
-1.57757 \\
-1.45796 \\
-1.41524 \\
-1.45044 \\
-1.52604\end{array}$ \\
\hline
\end{tabular}

0.01

0.02

0.05

0.16

0.20

0.50
1.00

1.50

2.00

3.00

3.50

$0.250 \pi$

$0.375 \pi$

$0.500 \pi$

$0.750 \pi$

$0.875 \pi$

$1.000 \pi$

$1.125 \pi$
$1.250 \pi$
$S(h, 0)$

$E(h, 0)$

$0.00246-0.00017$

$0.00943-0.00068$

$0.04791-0.00393$

$0.13445-0.01399$

$\begin{array}{ll}0.32209 & -0.04921 \\ 0.85557 & -0.26250\end{array}$

$\begin{array}{ll}0.85557 & -0.26250 \\ 1.48510 & -0.87187\end{array}$

$\begin{array}{ll}1.48510 & -0.87187 \\ 1.71114 & -1.55828\end{array}$

$1.63001-2.08081$

$1.44797-2.34718$

$1.34145-2.42626$

$1.36576-2.46119$

$0.67431-0.16967$

$1.26307-0.58403$

$1.61071-1.12220$

$1.71409-1.04628$

$1.64200-2.05126$

$1.49851-2.29519$

$1.37880-2.40135$

$1.33601-2.43453$

$\begin{array}{ll}1.37115 & -2.46517 \\ 1.44671 & -2.53848\end{array}$

$0.00246-0.00022$

$0.00942-0.00087$

$0.04781-0.00489$

$0.13405-0.01666$

$0.32053-0.05549$

$0.84662-0.27745$

$\begin{array}{ll}0.84662 & -0.27745 \\ 1.45815 & -0.88815\end{array}$

$\begin{array}{ll}1.45815 & -0.88815 \\ 1.67377 & -1.56068\end{array}$

$\begin{array}{ll}1.59845 & -2.06758\end{array}$

$\begin{array}{ll}1.59845 & -2.06758 \\ 1.43206 & -2.33052\end{array}$

$1.33643-2.42048$

$\begin{array}{ll}1.33643 & -2.42048 \\ 1.35760 & -2.46987\end{array}$

$0.06858-0.18148$

$1.24389-0.60201$

$1.57843-1.13477$

$1.67654-1.64619$

$1.60952-2.03868$

$1.47792-2.27780$

$1.36975-2.38878$

$1.33161-2.43313$

$\begin{array}{ll}1.36226 & -2.47461 \\ 1.42735 & -2.55229\end{array}$
$C(h, h)$

$S(h, h)$

$E(h, h)$

$\begin{array}{ll}0.49517 & -0.01997 \\ 0.96268 & -0.03994 \\ 2.09374 & -0.09981 \\ 3.28652 & -0.19943 \\ 4.59578 & -0.39768 \\ 6.29075 & -0.97847 \\ 7.29680 & -1.08414 \\ 7.55287 & -2.59346 \\ 7.46304 & -3.12627 \\ 7.20678 & -3.44999 \\ 0.90063 & -3.57927 \\ 5.02526 & -3.55120 \\ & \\ 5.86262 & -0.77378 \\ 7.00128 & -1.49991 \\ 7.44401 & -2.14449 \\ 7.55597 & -2.68144 \\ 7.47692 & -3.09446 \\ 7.28996 & -3.37793 \\ 7.05484 & -3.53678 \\ 0.81680 & -3.58524 \\ 0.60894 & -3.54476 \\ 0.45291 & -3.44132\end{array}$

$0.48133-0.01897$ $0.83100-0.03992$ $1.64256-1.009703$ $2.29350-0.19824$ $\begin{array}{ll}2.92199 & -0.33973 \\ 3.40802 & -0.87480\end{array}$ $3.40802-0.87980$ $2.01832-1.26093$ $0.96672-1.05319$ $-0.93654-0.60622$ $-2.686550 .00105$ $-3.88420 \quad 0.64023$ $-4.15311 \quad 1.19271$

$3.35454-0.72281$ $3.11239-1.17151$ $2.09003-1.25477$ $0.70210-1.03757$ $-0.79823-0.54740$ $-2.22139-0.1799$ $\begin{array}{ll}-3.37795 & 0.32525\end{array}$ $-4.06704 \quad 0.21325$ $-4.13186 \quad 1.22255$ $-3.53118 \quad 1.44887$
$0.00239-0.03017$ $0.00873-0.00066$ $0.04185-0.00375$ $0.12566-0.01370$ $\begin{array}{ll}0.35195 & -0.04973 \\ 1.19608 & -0.26078\end{array}$ $2.48366-0.75963$ $3.18297-1.11060$ $2.49998-0.98745$ $\begin{array}{lll}1.16570 & -0.06977\end{array}$ $-0.56567-0.20807$

$\begin{array}{ll}0.88352 & -0.17052\end{array}$ $1.98587-0.54384$ $2.81086-0.91629$ $3.22607-1.13572$ $3.21196-1.16613$ $2.76880-1.05364$ $1.99715-0.84649$ $0.69845-0.55379$ $\begin{array}{rr}-0.68793 & -0.17155 \\ -2.01235 & 0.26847\end{array}$ $3.18888-1.16074$
$0.48134-0.01997$ $0.88106-0.03993$ $1.64326-0.09972$ $2.29789-0.19884$ $2.94300-0.39391$ $3.64840-0.93207$ $3.73152-1.56313$ $3.4503 .1-1.78964$ $3.21532-1.70868$ $3.17201-1.52671$ $\begin{array}{ll}3.27523 & -1.42022 \\ 3.39445 & -1.44455\end{array}$

$3.50064-0.74996$ $3.77798-1.34072$ $3.64498-1.68897$ $3.40840-1.79262$ $3.22645-1.72066$ $3.16477-1.57723$ $3.21273-1.45755$ $3.31307-1.41478$ $3.40022-1.44994$ $3.43227-1.52552$

$$
\frac{a}{\lambda}=0.006350 \quad \frac{\alpha}{\beta}=0.05
$$

$\begin{array}{ll}0.49477 & -0.01995 \\ 0.96188 & -0.03991 \\ 2.09174 & -0.09972 \\ 3.28254 & -0.19917 \\ 4.58788 & -0.39680 \\ 6.27178 & -0.97352 \\ 7.26378 & -1.84610 \\ 7.51391 & -2.55755 \\ 7.42737 & -3.07220 \\ 7.18246 & -3.38184 \\ 6.89269 & -3.50434 \\ 6.63461 & -3.47814 \\ 5.84745 & -0.77066 \\ 6.97344 & -1.48831 \\ 7.40783 & -2.12041 \\ 7.51691 & -2.04287 \\ 7.44070 & -3.04162 \\ 7.26169 & -3.31319 \\ 7.03829 & -3.46421 \\ 6.81386 & -3.50996 \\ 6.61940 & -3.47214 \\ 6.47455 & -3.37617\end{array}$

$0.00239-0.00022$ $0.00872-0.00084$ $0.04176-0.00458$ $0.12527-0.01618$

$0.35042-0.05653$

$\begin{array}{ll}1.18804 & -0.28114 \\ 2.46626 & -0.78521\end{array}$

$2.46626-0.78521$

$3.17227-1.11275$

$\begin{array}{ll}3.19541 & -1.11527 \\ 2.52281 & -0.87150\end{array}$

$\begin{array}{ll}2.52281 & -0.87150 \\ 1.20651 & -0.47426\end{array}$

$\begin{array}{ll}-0.50278 & 0.03652\end{array}$

$0.87816-0.18644$ $1.97109-0.57076$ $2.79381-0.93657$ $3.21753-1.13254$ $3.21721-1.12493$ $1.78713-0.96000$ $1.93822-0.69009$ $-0.7454-0.33919$ $\begin{array}{ll}-0.62368 & 0.07411 \\ -1.93898 & 0.49951\end{array}$

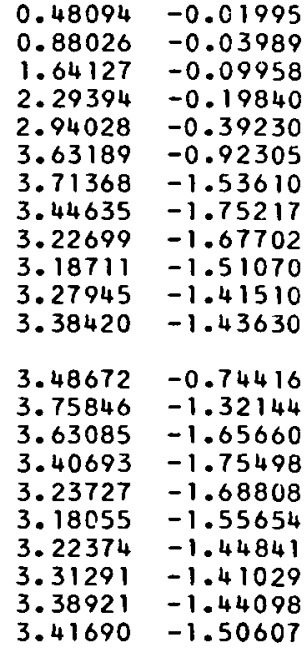


$\frac{a}{\lambda}=0.006350 \quad \frac{\alpha}{\beta}=0.07$

$\beta \mathrm{h}$

$\mathrm{C}(\mathrm{h}, 0)$

$\begin{array}{lll}0.01 & 0.49436 & -0.01994 \\ 0.02 & 0.96102 & -0.03996 \\ 0.05 & 2.08900 & -0.09947 \\ 0.10 & 3.27460 & -0.19801 \\ 0.20 & 4.56231 & -0.39078 \\ 0.50 & 6.14755 & -0.91429 \\ 1.00 & 6.91939 & -1.50993 \\ 1.50 & 7.07050 & -1.71615 \\ 2.00 & 7.15339 & -1.64643 \\ 2.50 & 7.33980 & -1.49455 \\ 3.00 & 7.60471 & -1.40881 \\ 3.50 & 7.85089 & -1.42732 \\ 0.125 \pi & 5.76498 & -0.73854 \\ 0.250 \pi & 6.72130 & -1.30271 \\ 0.375 \pi & 7.00389 & -1.62535 \\ 0.500 \pi & 7.07954 & -1.71880 \\ 0.625 \pi & 7.14446 & -1.65669 \\ 0.750 \pi & 7.27461 & -1.53613 \\ 0.875 \pi & 7.46766 & -1.43849 \\ 1.000 \pi & 7.68042 & -1.40457 \\ 1.125 \pi & 7.86499 & -1.43130 \\ 1.250 \pi & 7.99425 & -1.48747\end{array}$

$\sin , 0)$

$\begin{array}{ll}0.00245 & -0.00027 \\ 0.00940 & -0.00106 \\ 0.04772 & -0.00584 \\ 0.13366 & -0.01932 \\ 0.31898 & -0.06174 \\ 0.83779 & -0.29232 \\ 1.43184 & -0.90428 \\ 1.63753 & -1.56362 \\ 1.56759 & -2.05593 \\ 1.41551 & -2.31572 \\ 1.32966 & -2.41540 \\ 1.34810 & -2.47750 \\ & \\ 0.66291 & -0.19378 \\ 1.22506 & -0.61977 \\ 1.54702 & -1.14735 \\ 1.64014 & -1.64679 \\ 1.57782 & -2.02802 \\ 1.45713 & -2.26244 \\ 1.35939 & -2.37765 \\ 1.32539 & -2.43192 \\ 1.35216 & -2.48289 \\ 1.40819 & -2.56434\end{array}$

$E(h, 0)$

$\begin{array}{ll}0.49437 & -c .01994 \\ 0.96108 & -c .03987 \\ 2.08975 & -1.09962 \\ 3.27857 & -0.19890 \\ 4.57999 & -0.39592 \\ 6.25290 & -0.96801 \\ 7.23105 & -1.82829 \\ 7.47539 & -2.52232 \\ 7.39200 & -3.01943 \\ 7.15795 & -3.31561 \\ 6.88369 & -3.43168 \\ 6.64180 & -3.40723 \\ & \\ 5.83233 & -0.76755 \\ 6.94581 & -1.47683 \\ 7.37203 & -2.09609 \\ 7.47830 & -2.60506 \\ 7.40482 & -2.9 .9004 \\ 7.23342 & -3.25020 \\ 7.02115 & -3.39378 \\ 6.80955 & -3.43697 \\ 0.62763 & -3.40164 \\ 6.49315 & -3.31259\end{array}$

$\mathrm{C}(\mathrm{h}, \mathrm{h})$

$\begin{array}{rr}0.48053 & -0.01993 \\ 0.87941 & -0.03985 \\ 1.03859 & -0.09931 \\ 2.02566 & -0.19702 \\ 2.90700 & -0.38446 \\ 3.38025 & -0.84373 \\ 2.60801 & -1.13183 \\ 1.00220 & -0.86733 \\ -0.86091 & -0.31823 \\ -2.58259 & 0.28087 \\ -3.76674 & 0.79389 \\ -4.06442 & 1.09000 \\ & \\ 3.33949 & -0.70084 \\ 3.08893 & -1.08593 \\ 2.09491 & -1.08918 \\ 0.74408 & -0.80021 \\ -0.72506 & -0.36210 \\ -2.12463 & 0.11231 \\ -3.26416 & 0.55442 \\ -3.95198 & 0.90614 \\ -4.04740 & 1.09872 \\ -3.51532 & 1.07213\end{array}$

$S(h, h)$ $\begin{array}{ll}0.00239 & -0.00027 \\ 0.00870 & -0.00161\end{array}$ $0.04166-0.00541$ $0.12487-0.01866$ $\begin{array}{ll}0.34890 & -0.06331 \\ 1.18019 & -0.30139\end{array}$ $\begin{array}{ll}1.18019 & -0.30139 \\ 2.44998 & -0.81097\end{array}$ $\begin{array}{ll}2.44998 & -0.81097 \\ 3.16388 & -1.11584\end{array}$ $3.16388-1.11584$ $3.20557-1.07085$ $2.55055-0.75633$ $\begin{array}{rr}1.25094 & -C .27998 \\ -0.44339 & 0.27997\end{array}$

$0.87289-0.20228$ $1.95696-0.59764$ $2.77831-0.95732$ $3.21146-1.13036$ $3.22596-1.08480$ $2.81010-0.86718$ $1.97415-0.53462$ $0.79468-0.12597$ $-0.56366 \quad 0.31875$ $-1.879230 .73176$
$E(h, h)$

$0.48054-0.01994$ $0.87947-0.03986$ $1.63928-0.09945$ $2.29000-0.19796$ $2.93260-0.39069$ $3.61553-0.91414$ $3.69600-1.50971$ $3.44185-1.71584$ $3.23708-1.64607$ $3.20035-1.49406$ $3.28296-1.40824$ $3.37501-1.42671$

$3.47290-0.73841$ $3.73915-1.30253$ $3.61671-1.62510$ $3.40478-1.71849$ $3.24658-1.65630$ $3.19440-1.53566$ $3.23332-1.43796$ $3.31256-1.40398$ $3.37937-1.43077$ $3.40328-1.48683$

$$
\frac{a}{\lambda}=0.006350 \quad \frac{\alpha}{\beta}=0.10
$$

$\begin{array}{rrrr}0.47994 & -0.01991 & 0.00238 & -0.00034 \\ 0.87822 & -0.03979 & 0.00868 & -0.00127 \\ 1.63562 & -0.09907 & 0.04152 & -0.00665 \\ 2.27981 & -0.19610 & 0.12429 & -0.02236 \\ 2.89588 & -0.38055 & 0.34665 & -0.07342 \\ 3.36019 & -0.81724 & 1.16877 & -0.33158 \\ 2.60162 & -1.03712 & 2.42761 & -0.84998 \\ 1.02823 & -0.70066 & 3.15563 & -1.12216 \\ -0.80984 & -0.10392 & 3.22771 & -1.00605 \\ -2.52133 & 0.48992 & 2.00144 & -0.58468 \\ -3.71367 & 0.91101 & 1.32429 & 0.01051 \\ -4.04980 & 1.02240 & -0.36059 & 0.64574 \\ & & & \\ 3.32120 & -0.68461 & 0.86518 & -0.22588 \\ 3.07266 & -1.02314 & 1.93694 & -0.63792 \\ 2.09954 & -0.96751 & 2.75790 & -0.98928 \\ 0.77449 & -0.62470 & 3.20703 & -1.12891 \\ -0.67532 & -0.14990 & 3.24580 & -1.02648 \\ -2.06477 & 0.33042 & 2.85335 & -0.72922 \\ -3.20401 & 0.73005 & 2.03707 & -0.30237 \\ -3.90627 & 0.97935 & 0.87274 & 0.19305 \\ -4.03668 & 1.01592 & -0.48117 & 0.68654 \\ -3.55749 & 0.80587 & -1.81420 & 1.08543\end{array}$

$0.47994 \quad-0.01991$ $0.87827-0.03980$ $1.63631-0.09925$ $2.28410-0.19730$ $2.92112-0.38829$ $3.59126-0.90100$ $3.66978-1.4713$ $3.43417-1.66339$ $3.24947-1.60098$ $3.21702-1.46813$ $3.28693-1.39515$ $3.36276-1.41016$ $3.45237-0.72991$ $3.71060-1.27486$ $3.59552-1.57945$ $3.40036-1.66580$ $3.25791-1.61006$ $3.21189-1.50410$ $3.24516-1.42018$ $3.31155-1.39160$ $\begin{array}{ll}3.36629 & -1.41345 \\ 3.38548 & -1.45825\end{array}$ 
$\beta h$

$\mathrm{C}(\mathrm{h}, 0)$

\begin{tabular}{|c|c|c|}
\hline $\begin{array}{l}0.01 \\
0.02 \\
0.05 \\
0.10 \\
0.20 \\
0.50 \\
1.00 \\
1.50 \\
2.00 \\
2.50 \\
3.00 \\
3.50\end{array}$ & $\begin{array}{l}0.49178 \\
0.95583 \\
2.07615 \\
3.24917 \\
4.51308 \\
6.04468 \\
6.80818 \\
7.03029 \\
7.18690 \\
7.38854 \\
7.01092 \\
7.80481\end{array}$ & $\begin{array}{l}-0.01983 \\
-0.03963 \\
-0.09865 \\
-0.19527 \\
-0.38039 \\
-0.85939 \\
-1.35341 \\
-1.50538 \\
-1.46245 \\
-1.37773 \\
-1.33523 \\
-1.34279\end{array}$ \\
\hline $\begin{array}{l}0.125 \pi \\
0.250 \pi \\
0.375 \pi \\
0.500 \pi \\
0.625 \pi \\
0.750 \pi \\
0.875 \pi \\
1.000 \pi \\
1.125 \pi \\
1.250 \pi\end{array}$ & $\begin{array}{l}5.67760 \\
6.00088 \\
6.91229 \\
7.05153 \\
7.17415 \\
7.32616 \\
7.50003 \\
7.67036 \\
7.81630 \\
7.93087\end{array}$ & $\begin{array}{l}-0.70285 \\
-1.18879 \\
-1.44054 \\
-1.50718 \\
-1.46855 \\
-1.39985 \\
-1.34935 \\
-1.33331 \\
-1.34442 \\
-1.36567\end{array}$ \\
\hline
\end{tabular}

0.01

0.02

0.10

0.10

0.50

1.00

1.50

2.00

2.50
3.00

3.50

0.125

$0.250 \pi$

$0.375 \pi$

$0.500 \pi$

$0.750 \pi$

$0.875 \pi$

$0.875 \pi$

$1.000 \pi$

$1.125 \pi$
$1.250 \pi$
$\mathrm{S}(\mathrm{h}, 0)$

$E(h, 0)$

$0.00243-0.00059$ $0.00930-0.00227$

$0.04707-0.01198$

$0.13112-0.03640$

$0.30909-0.10166$

$0.78309-0.38526$

$\begin{array}{ll}1.27553 & -1.00535 \\ 1.42697 & -1.59362\end{array}$

$1.38377-2.01218$

$1.29890-2.25672$

$1.25629-2.39892$

$1.26377-2.51330$

$0.62742-0.27135$

$1.11134-0.73010$

$1.36242-1.22878$

$1.42872-1.66458$

$1.38989-1.98809$

$1.32105-2.20064$

$1.27045-2.3350$

$1.25435-2.4317$

$1.26540-2.52130$

$1.28660-2.61743$
$0.49179-0.01983$ $0.05591-0.03966$ $2.07685-0.09989$ $\begin{array}{ll}3.25287 & -0.19718\end{array}$ $4.52914-0.39026$ $\begin{array}{lll}0.13247 & -0.93744 \\ 7.02538 & -1.71797\end{array}$ $7.02538-1.71797$ $7.23518-2.30882$ $7.66972-2.70571$ $6.99535-2.92759$ $\begin{array}{ll}6.80349 & -3.00940 \\ 6.64473 & -2.99379\end{array}$

$5.73556-0.74775$ $6.77110-1.40499$ $7.14786-1.95096$ $7.23757-2.37659$ $7.17962-2.68299$ $7.05039-2.87987$ $6.39811-2.98325$ $6.75375-3.01296$ $6.63575-2.89025$ $6.55281-2.93553$
$C(h, h)$

$0.47795-0.01983$ $0.87425-0.03960$ $1.62578-0.09827$ $.26052-0.19308$ $2.85965-0.36773$ $.29792-0.73163$ $.58883-0.73306$ $1.11332-0.15766$ $-0.67281 \quad 0.00711$ $-2.41967 \quad 1.19466$ $\begin{array}{ll}-3.74853 & 1.32646 \\ -4.31397 & 0.34359\end{array}$

$3.26329-0.63204$ $3.02651-0.82170$ $2.12180-0.57555$ $0.87128-0.05158$ $\begin{array}{ll}-0.53935 & 0.55332\end{array}$ $\begin{array}{ll}-1.94304 & 1.06192 \\ -3.15716 & 1.33067\end{array}$ $-3.99835 \quad 1.2549$ $-4.31852 \quad 0.78738$ $-4.03392-0.03775$
$S(h, h)$

$0.00236-0.00058$ $0.00860-0.00213$ $0.04103-0.01076$ $0.12236-0.03456$ $0.33934-0.10668$ $1.13370-0.43067$ $2.37016-0.98304$ $3.16575-1.15653$ $3.36381-0.3035$ $2.85339-0.01416$

$\begin{array}{rr}1.62743 & 0.99579 \\ -0.13402 & 1.91829\end{array}$

$0.84095-0.30329$ $1.88000-0.77193$ $2.71387-1.10269$ $3.23308-1.13832$ $3.37229-0.84608$ $3.07633-0.27512$ $2.32602 \quad 0.47824$ $1.17036 \quad 1.2812$ $\begin{array}{rr}-0.26635 & 1.96927 \\ -1.80234 & 2.36645\end{array}$

$$
\frac{a}{\lambda}=0.006350 \quad \frac{\alpha}{\beta}=0.40
$$

$\begin{array}{rr}0.47400 & -0.01963 \\ 0.86638 & -0.03921 \\ 1.60633 & -0.09667 \\ 2.22281 & -0.13717 \\ 2.79080 & -0.34311 \\ 3.19380 & -0.57211 \\ 2.60480 & -0.16677 \\ 1.28963 & 0.89989 \\ -0.53887 & 2.07750 \\ -2.69458 & 2.75778 \\ -4.82924 & 2.38131 \\ -6.40256 & 0.60237 \\ & \\ 3.16096 & -0.53329 \\ 2.97104 & -0.44933 \\ 2.20385 & 0.16356 \\ 1.05877 & 1.07374 \\ -0.39129 & 2.00096 \\ -2.05772 & 2.64816 \\ -3.78925 & 2.731 .33 \\ -5.35951 & 2.026+3 \\ -6.47313 & 0.427+1 \\ -6.79107 & -2.00059\end{array}$

$\begin{array}{rr}0.00232 & -0.00104 \\ 0.00844 & -0.00383 \\ 0.04006 & -0.01884 \\ 0.11863 & -0.05840 \\ 0.32562 & -0.17120 \\ 1.07628 & -0.62274 \\ 2.32862 & -1.26363 \\ 3.36175 & -1.27853 \\ 3.94949 & -0.43536 \\ 3.77797 & 1.21261 \\ 2.54852 & 3.31320 \\ 0.11794 & 5.21197 \\ 0.79896 & -0.45281 \\ 1.80760 & -1.04028 \\ 2.73126 & -1.35922 \\ 3.47863 & -1.21304 \\ 3.92823 & -0.52645 \\ 3.92425 & 0.67081 \\ 3.31245 & 2.23599 \\ 1.98215 & 3.90625 \\ -0.09111 & 5.31367 \\ -2.80284 & 6.02939\end{array}$

$E(h, h)$

$\begin{array}{lll}0.47796 & -0.01983\end{array}$ $0.87430-0.03962$ $1.62643-0.09859$ $2.26457-0.19513$ $2.88338-0.38044$ $3.51269-0.85899$ $3.58486-1.35285$ $3.40175-1.50470$ $3.27073-1.46160$ $3.24926-1.37685$ $\begin{array}{ll}3.28935 & -1.33428 \\ 3.32911 & -1.34180\end{array}$

$3.38555-0.70250$ $3.61879-1.18829$ $3.52519-1.43994$ $3.37688-1.50648$ $3.27641-1.46770$ $3.24611-1.39899$ $3.26586-1.3484$ $3.30267-1.33235$ $3.33085-1.34343$ $3.34008-1.36466$
$0.00239-0.00107$ $0.00914-0.00411$ $0.04610-0.02127$ $0.29455-0.16069$ $0.70742-0.51731$ $1.07732-1.14886$ $1.17205-1.06483$ $\begin{array}{ll}1.15141 & -2.01844 \\ 1.11669 & -2.25090\end{array}$ $\begin{array}{ll}1.11669 & -2.25090 \\ 1.10213 & -2.41096\end{array}$ $1.10397-2.56313$

$0.57726-0.38296$ $0.96214-0.88415$ $1.17298-1.72438$ $1.17299-1.72438$ $1.12510-2.19285$ $1.10667-2.34000$ $1.10155-2.46217$ $1.10437-2.57245$ $1.10912-2.67567$

$\begin{array}{ll}0.48784 & -0.01967 \\ 0.94802 & -0.03933 \\ 2.05717 & -0.09803 \\ 3.21376 & -0.19456 \\ 4.45233 & -0.38174 \\ 5.95456 & -0.89200 \\ 6.73110 & -1.56522 \\ 6.89715 & -2.02678 \\ 6.85201 & -2.30766 \\ 6.74108 & -2.45003 \\ 6.63029 & -2.49783 \\ 6.54716 & -2.49001 \\ & \\ 5.59148 & -0.71856 \\ 6.51801 & -1.30334 \\ 6.82975 & -1.75265 \\ 6.89891 & -2.07663 \\ 6.85868 & -2.29238 \\ 6.77495 & -2.42066 \\ 0.68355 & -2.48304 \\ 6.60337 & -2.49977 \\ 6.54272 & -2.48825 \\ 6.50327 & -2.46237\end{array}$

$0.47401 \quad-0.01967$ $0.86642-0.03926$ $1.60688-0.09728$ $2.22616 \quad-0.19087$ $2.81014-0.36534$ $3.36555-0.78261$ $3.42601-1.15383$ $3.31514-1.24892$ $3.24906-1.22842$ $3.23967-1.19378$ $3.25289-1.17927$ $\begin{array}{ll}3.25289 & -1.17927 \\ 3.26384 & -1.18115\end{array}$

$3.25900-0.65167$ $3.44946-1.03832$ $3.38748-1.03832$ $3.30168-1.24988$ $3.25163-1.23118$ $3.23849-1.20217$ $3.25679-1.17869$ $3.25679-1.17869$ $3.26640-1.18632$ 
$\frac{\mathrm{a}}{\lambda}=0.006350 \quad \frac{\alpha}{\beta}=0.70$

$\beta \mathrm{h}$

$\mathrm{C}(\mathrm{h}, 0)$

$S(r, 0)$

$E(h ; 0)$

$\mathrm{C}(\mathrm{h}, \mathrm{h})$

$\mathrm{S}(\mathrm{h}, \mathrm{h})$

$0.00233-0.00178$ $0.00891-0.00681$ $0.04468-0.03484$ $0.12179-0.09915$ $\begin{array}{ll}0.27415 & -0.2441 \\ 0.61047 & -0.69345\end{array}$ $0.85260-1.33952$ $0.89962-1.79710$ $0.89274-2.10841$ $0.88356-2.33416$ $0.88356-2.33416$ $0.88080-2.06643$

$0.51095-0.53454$ $0.78489-1.08486$ $0.88200-1.52324$ $0.89998-1.84854$ $0.89359-2.0892$ $0.88554-2.27521$ $0.88145-2.42785$ $0.88050-2.55940$ $0.88085-2.5540$ $0.88133-2.78123$

$\begin{array}{ll}0.48197 & -0.01944 \\ 0.93630 & -0.03884 \\ 2.02800 & -0.09661 \\ 3.15606 & -0.19071 \\ 4.34022 & -0.36939 \\ 5.70337 & -0.82913 \\ 0.33405 & -1.36924 \\ 6.45110 & -1.08841 \\ 6.42525 & -1.85589 \\ 6.36887 & -1.92918 \\ 6.32017 & -1.95055 \\ 6.28863 & -1.94778 \\ 5.38566 & -0.67748 \\ 6.17052 & -1.16874 \\ 6.40538 & -1.50450 \\ 6.45221 & -1.71986 \\ 6.42893 & -1.84746 \\ 6.38522 & -1.91499 \\ 6.34268 & -1.94426 \\ 6.30946 & -1.95132 \\ 6.28709 & -1.94717 \\ 6.27413 & -1.93874\end{array}$

$0.46815-0.01941$ $0.85471-0.03864$ $1.57774-0.09438$ $2.16839-0.17865$ $2.69615-0.30853$ $3.08516-0.35678$ $2.74023 \quad 0.62508$ 1.615442 .55879 $-0.66462 \quad 4.74749$ $\begin{array}{ll}-4.49949 & 0.09789\end{array}$ $-15.52463 \quad 0.05945$

$\begin{array}{lll}3.03885 & -0.39888\end{array}$ $2.97797 \quad 0.05724$ $2.97172 \quad 1.23380$ $\begin{array}{ll}2.44172 & 1.23380 \\ 1.37418 & 2.87372\end{array}$ $-0.44879 \quad 4.60021$ $-3.22768 \quad 5.87697$ $-6.99619 \quad 6.00107$ $-11.466714 .15325$ $-15.87748-0.47237$ $-18.88166-8.43959$
$0.00226-0.00173$ $0.03866-0.03059$ $0.11330-0.09282$ $0.30710-0.26336$ $1.01801-0.89971$ $2.43323-1.72768$ $4.12266-1.58103$ $5.78303 \quad 0.14198$ $6.58610 \quad 3.80174$ $5.17120 \quad 9.19371$ $-0.10816 \quad 15.10742$

$\begin{array}{lll}0.75033 & -0.66626\end{array}$ $1.78986-1.45156$ $3.00934-1.82135$ $4.37261-1.44485$ $5.67748-0.04730$ $6.51372 \quad 2.54774$ $6.26033 \quad 6.31588$ $4.13873 \quad 10.90018$ $-0.65011 \quad 15.47528$ $-8.6853918 .64628$
$E(h, h)$

$0.46815-0.01942$ $0.85473-0.03872$ $1.57803-0.09535$ $2.17011-0.18469$ $2.70561-0.34412$ $3.16703-0.68462$ $3.21263-0.92794$ $3.16009 \quad-0.97527$ $3.13628-0.96853$ $\begin{array}{ll}3.13357 & -0.95943 \\ 3.13609 & -0.95652\end{array}$ $3.13768-0.95670$

$3.08526-0.58437$ $3.22611-0.85993$ $3.19261-0.95749$ $3.15473-0.97566$ $3.13707-0.96937$ $3.13330-0.96134$ $3.13476-0.95734$ $3.13671-0.95643$

$3.13774-0.95681$

$3.13797-0.95732$

$$
\frac{a}{\lambda}=0.006350 \quad \frac{\alpha}{\beta}=1.00
$$

$\begin{array}{ll}0.47618 & -0.01920 \\ 0.92473 & -0.03836 \\ 1.99925 & -0.09521 \\ 3.09951 & -0.18694 \\ 4.23172 & -0.35751 \\ 5.46944 & -0.77201 \\ 5.98253 & -1.20613 \\ 6.06515 & -1.42725 \\ 6.05033 & -1.52729 \\ 6.02163 & -1.56508 \\ 6.00018 & -1.57465 \\ 5.98819 & -1.57367 . \\ 5.19142 & -0.63940 \\ 5.85699 & -1.05257 \\ 0.03411 & -1.30377 \\ 6.06585 & -1.44709 \\ 6.05236 & -1.52263 \\ 6.02952 & -1.55822 \\ 6.00970 & -1.57198 \\ 5.99591 & -1.57496 \\ 5.98765 & -1.57346 \\ 5.98339 & -1.57071 .\end{array}$

$0.46236 \cdot-0.01917$ $0.84321-0.03808$ $1.54983-0.09213$ $2.11644-0.17053$ $2.61116-0.27649$ $3.02905-0.16303$ $3.02142 \quad 1.41236$ $2.07329 \quad 4.55229$ $-1.23741 \quad 8.67755$ $-8.86776 \quad 12.02976$ $-22.32241 \quad 10.7$ $-40.66139-1.97372$ $2.95124-0.27781$ $3.09174 \quad 0.53119$ $2.84673 \quad 2.36653$ $1.79066 \quad 5.1002$ $-0.87386 \quad 8.3711$ $-6.11880 \quad 11.32292$ $-14.80634 \quad 12.30820$ $-27.16941 \quad 8.63566$ $-41.97750-3.48055$ $-55.37783-28.51194$
$0.00221-0.00240$ $0.00798-0.00875$ $0.03730-0.04193$ $0.10829-0.1257$ $0.29085-0.35060$ $0.98828-1.16934$ $2.72832-2.26336$ 0.546060 $9.24666 \quad 0.9560$ $12.26185 \quad 8.54026$ $\begin{array}{rr}10.74376 & 22.07247 \\ -2.03165 & 40.51547\end{array}$

$0.71653-0.87020$ $1.86858-1.88738$ $3.59744-2.3996$ $6.03189-1.8245$ $8.96945 \quad 0.60439$ $11.63844 \quad 5.78625$ $12.42319 \quad 14.50935$ 8.6310126 .9491 $-3.54142 \quad 41.83825$ $-28.58718 \quad 55.30524$
$0.46236-0.01918$ $0.84320-0.03820$ . $54975-0.09347$ $11586-0.1787$ $.60705-0.32447$ $.99126-0.60306$ $.02517-0.76364$ -0.78732
$.00009-0.78510$ $\begin{array}{ll}2.09146 & -0.78510\end{array}$ $2.99068-0.78269$ $2.09139-0.78214$

$2.92850-0.52629$ $3.03293-0.72354$ $3.01476-0.77911$ $2.99796-0.78748$ $2.99170-0.78536$ $2.99062-0.78315$ $2.99092-0.78226$ $2.99126-0.78209$ $\begin{array}{ll}2.99140 & -0.78214 \\ 2.99143 & -0.78220\end{array}$ 
$\frac{a}{\lambda}=0.009525 \quad \frac{\alpha}{\beta}=0.00$

$\beta h$

$C(h, 0)$

$S(h, 0)$

$E(h, 0)$

$\mathrm{C}(\mathrm{h}, \mathrm{h})$

$S(h, h)$

$\begin{array}{ll}0.00166 & -0.00010 \\ 0.00649 & -0.00040 \\ 0.03618 & -0.00250 \\ 0.11285 & -0.00998 \\ 0.29408 & -0.03971 \\ 0.83298 & -0.23967 \\ 1.48834 & -0.84686 \\ 1.73023 & -1.55520 \\ 1.63926 & -2.10307 \\ 1.43084 & -2.37500 \\ 1.30559 & -2.43525 \\ 1.33549 & -2.44466 \\ & \\ 0.64792 & -0.15021 \\ 1.25482 & -0.55646 \\ 1.62205 & -1.10298 \\ 1.73341 & -1.64721 \\ 1.65277 & -2.07214 \\ 1.48930 & -2.32419 \\ 1.34991 & -2.42214 \\ 1.29905 & -2.43586 \\ 1.34214 & -2.44729 \\ 1.43667 & -2.51273\end{array}$

$0.33205-0.01999$ $0.65529-0.03998$ $1.51693-0.09993$ $2.56384-0.19977$ $3.81840-0.39887$ $5.50945-0.98562$ $0.53341-1.89101$ $6.79786-2.64770$ $0.70257-3.20873$ $\begin{array}{ll}0.42817 & -3.55460 \\ 0.09567 & -3.09461\end{array}$ $\begin{array}{ll}6.09567 & -3.69461 \\ 5.79222 & -3.66340\end{array}$ $\begin{array}{ll}5.07821 & -0.77823\end{array}$ $6.23076-1.51703$ $6.68509-2.18056$ $6.80104-2.73980$ $6.7173-3.17499$ $6.51765-3.47715$ $6.00376-3.64839$ $5.77409-3.70113$ $\begin{array}{ll}5.77409 & -3.65624 \\ 5.59968 & -3.54045\end{array}$
$0.00163-0.00010$ $0.00622-0.00040$ $0.03202-0.00250$ $0.09988-0.30996$ $0.29024-0.33945$ $1.03045-0.22997$ $2.18103-0.72138$ $2.80021-1.10876$ $2.80886-1.23048$ $2.21654-1.16258$ $\begin{array}{ll}2.21654 & -1.16258 \\ 1.03477 & -0.96617\end{array}$ $\begin{array}{ll}-0.54318 & -0.57951\end{array}$

$0.75347-0.14642$ $1.73600-0.50324$ $2.47210-0.88641$ $2.83800-1.14201$ $2.83844-1.14201$ $2.82844-1.22950$ $2.44934-1.19525$ $0.69642-1.08295$ $0.61266-0.87958$ $\begin{array}{ll}-0.65570 & -0.54451 \\ -1.87647 & -0.07873\end{array}$

$\begin{array}{lll}0.01 & 0.33194 & -0.01998 \\ 0.02 & 0.65505 & -0.03996 \\ 0.05 & 1.51584 & -0.09985 \\ 0.10 & 2.55934 & -0.19932 \\ 0.20 & 3.79927 & -0.39581 \\ 0.50 & 5.39340 & -0.94321 \\ 1.00 & 6.16682 & -1.59737 \\ 1.50 & 0.27223 & -1.83760 \\ 2.00 & 6.30382 & -1.74890 \\ 2.50 & 6.47902 & -1.54547 \\ 3.00 & 6.77997 & -1.42379 \\ 3.50 & 7.07420 & -1.45284 \\ 0.125 \pi & 5.00478 & -0.75704 \\ 0.250 \pi & 5.97475 & -1.36493 \\ 0.375 \pi & 6.23901 & -1.73017 \\ 0.500 \pi & 6.27309 & -1.84084 \\ 0.625 \pi & 6.29769 & -1.76211 \\ 0.750 \pi & 6.41158 & -1.60244 \\ 0.875 \pi & 0.62040 & -1.46675 \\ 1.000 \pi & 6.87020 & -1.41750 \\ 1.125 \pi & 7.09083 & -1.45927 \\ 1.250 \pi & 7.23464 & -1.55044\end{array}$

$\begin{array}{ll}0.00166 & -0.00011 \\ 0.00649 & -0.00043 \\ 0.03616 & -0.00268 \\ 0.11275 & -0.01054 \\ 0.29368 & -0.04114 \\ 0.83069 & -0.24330 \\ 1.48129 & -0.85081 \\ 1.72034 & -1.55533 \\ 1.63103 & -2.09875 \\ 1.42725 & -2.36968 \\ 1.30533 & -2.43328 \\ 1.33421 & -2.44730 \\ 0.64646 & -0.15319 \\ 1.24984 & -0.56086 \\ 1.61356 & -1.10592 \\ 1.72346 & -1.64664 \\ 1.64428 & -2.06806 \\ 1.48431 & -2.31867 \\ 1.34840 & -2.41807 \\ 1.29899 & -2.43527 \\ 1.34063 & -2.45017 \\ 1.43170 & -2.51705\end{array}$

$\begin{array}{rr}0.32754 & -0.01998 \\ 0.62504 & -0.03996 \\ 1.28089 & -0.09980 \\ 1.90480 & -0.19895 \\ 2.53314 & -0.39297 \\ 3.06107 & -0.90279 \\ 2.39009 & -1.34444 \\ 0.89553 & -1.23897 \\ -0.83760 & -0.79111 \\ -2.45156 & -0.17836 \\ -3.58679 & 0.5434 C \\ -3.86349 & 1.2670 C \\ & \\ 2.99913 & -0.73672 \\ 2.82750 & -1.2267 C \\ 1.91425 & -1.36195 \\ 0.65508 & -1.19065 \\ -0.71120 & -0.83066 \\ -2.01906 & -0.3673 \\ -3.10182 & 0.1704 i \\ -3.76585 & 0.75673 \\ -3.84462 & 1.31100 \\ -3.28307 & 1.70628\end{array}$

$\begin{array}{ll}0.00163 & -0.00011 \\ 0.00621 & -0.00043 \\ 0.03199 & -0.00266 \\ 0.09979 & -0.01045 \\ 0.28985 & -0.04084 \\ 1.02835 & -0.23428 \\ 2.17615 & -0.72656 \\ 2.79645 & -1.10848 \\ 2.80883 & -1.22005 \\ 2.21993 & -1.13696 \\ 1.04320 & -0.92219 \\ -0.52569 & -0.52364 \\ & \\ 0.75209 & -0.14978 \\ 1.73199 & -0.50887 \\ 2.46710 & -0.89028 \\ 2.83470 & -1.14055 \\ 2.82813 & -1.21997 \\ 2.45176 & -1.17454 \\ 1.70187 & -1.04816 \\ 0.62330 & -0.83101 \\ -0.63751 & -0.48839 \\ -1.85130 & -0.02642\end{array}$

$E(h, h)$

$\begin{array}{ll}0.32765 & -0.01999 \\ 0.62528 & -0.03997 \\ 1.28192 & -0.09988 \\ 1.90920 & -0.19944 \\ 2.55588 & -0.39622 \\ 3.26671 & -0.94551 \\ 3.35129 & -1.60437 \\ 3.04784 & -1.84731 \\ 2.78731 & -1.75673 \\ 2.73935 & -1.54846 \\ 2.86036 & -1.42326 \\ 3.00509 & -1.45321 \\ & \\ 3.11538 & -0.75852 \\ 3.40052 & -1.36990 \\ 3.25883 & -1.73857 \\ 3.00188 & -1.85056 \\ 2.79984 & -1.77023 \\ 2.72995 & -1.00689 \\ 2.78607 & -1.46756 \\ 2.90585 & -1.41674 \\ 3.01222 & -1.45986 \\ 3.05215 & -1.55445\end{array}$

$\begin{array}{ll}0.33195 & -0.01998 \\ 0.65510 & -0.03996 \\ 1.51643 & -0.09989 \\ 2.56284 & -0.19969 \\ 3.81642 & -0.39863 \\ 5.50467 & -0.98434 \\ 6.52503 & -1.88635 \\ 6.78792 & -2.63836 \\ 6.69352 & -3.19454 \\ 6.42221 & -3.53659 \\ 6.09426 & -3.67473 \\ 5.79569 & -3.64404 \\ & \\ 5.07439 & -0.77742 \\ 6.22371 & -1.51404 \\ 6.67588 & -2.17433 \\ 6.79107 & -2.72975 \\ 0.70811 & -3.16113 \\ 6.51061 & -3.46007 \\ 6.25995 & -3.62916 \\ 0.00375 & -3.68114 \\ 5.77797 & -3.63701 \\ 5.60687 & -3.52337\end{array}$

$$
\frac{a}{\lambda}=0.009525 \quad \frac{\alpha}{\beta}=0.005
$$

$\begin{array}{ll}0.32755 & -0.01998 \\ 0.62508 & -0.03996 \\ 1.28142 & -0.09984 \\ 1.90821 & -0.19932 \\ 2.55393 & -0.09580 \\ 3.26251 & -0.94318 \\ 3.34676 & -1.59727 \\ 3.04713 & -1.83736 \\ 2.79105 & -1.744846 \\ 2.74309 & -1.54482 \\ 2.86174 & -1.42296 \\ 3.00188 & -1.45189 \\ 3.11186 & -0.75702 \\ 3.39554 & -1.36488 \\ 3.25531 & -1.73003 \\ 3.00187 & -1.84057 \\ 2.80334 & -1.76169 \\ 2.73489 & -1.00185 \\ 2.78956 & -1.46601 \\ 2.90586 & -1.41663 \\ 3.00875 & -1.45831 \\ 3.04724 & -1.54943 \\ & \end{array}$


$\frac{a}{\lambda}=0.009525 \quad \frac{\alpha}{\beta}=0.01$

$\beta \mathrm{h}$

$C(h, 0)$

$S(h, 0)$

$\mathrm{E}(\mathrm{h}, 0$ :

$C(h, h)$

$S(h, h)$

$E(h, h)$

$\begin{array}{ll}0.33185 & -0.01998 \\ 0.65490 & -0.03995 \\ 1.51593 & -0.09786 \\ 2.56184 & -0.19961 \\ 3.81444 & -0.39839 \\ 5.49989 & -0.98306 \\ 6.51666 & -1.88171 \\ 0.77801 & -2.62907 \\ 6.68448 & -3.18044 \\ 0.41623 & -3.51870 \\ 3.09276 & -3.65500 \\ 5.79899 & -3.62484 \\ 5.07058 & -0.77660 \\ 0.21667 & -1.51106 \\ 6.66670 & -2.16813 \\ 0.78114 & -2.71976 \\ 0.69893 & -3.14736 \\ 0.50357 & -3.44311 \\ 0.25609 & -3.61007 \\ 0.00363 & -3.66132 \\ 5.78149 & -3.61792 \\ 5.61344 & -3.50640\end{array}$

$0.32744-0.01997$ $0.62484-0.03974$ $1.28039-0.09975$ $1.90382-0.198 .30$ $2.53124-0.39234$ $3.05744-0.89858$ $2.38854-1.32941$ $0.90013-1.21307$ $-0.82693-0.75836$ $-2.43509-0.14611$ $\begin{array}{ll}-3.56468 & 0.56142 \\ -3.84127 & 1.25411\end{array}$ $1.30493-2.43135$

$0.64500-0.15615$ $1.24488-0.56526$ $1.60512-1.10886$ $1.71360-1.64612$ $1.63584-2.06409$ $1.47930-2.31328$ $1.34679-2.41409$ $1.29878-2.43468$

$1.42675-2.52121$
$0.00163-0.00012$ $0.00621-0.00046$ $0.03197-0.00281$ $0.09969-0.01094$ $0.28947-0.04224$ $1.02626-0.23858$ . $.79281-1.10826$ $2.80899-1.20969$ $2.22360-1.11142$ $2.22360-1.11142$ $-0.50841-0.46803$

$0.75071-0.15313$ $2.83154-1.13917$ 2.83154 -1.131051 $2.45442-1.15389$ $1.70759-1.01347$ $0.63408-0.78264$ $0.61850-0.43252$ $\begin{array}{rr}-0.61958 & -0.43252 \\ -1.82697 & 0.02577\end{array}$ $1.72803-0.51450$ $.72803-0.51450$
$0.32745 \quad-0.01998$ $0.62488-0.03995$ $1.28092-0.09980$ $1.90722-0.19919$ $\begin{array}{ll}2.55199 & -0.39538\end{array}$ $3.25833-0.94080$ $3.34224-1.59022$ $3.04638-1.82750$ $2.79468-1.74023$ $.74769-1.5411$ $2.86309-1.42251$ $2.99876-1.45046$

$3.10834-0.75553$ $3.39057-1.35988$ $3.25178-1.72155$ $.00182-1.83066$ $.80672-1.75320$ 2.73970 - 1.59679 $2.90588-1.41637$ $3.00540-1.45666$ $3.00540-1.45666$
$3.04251-1.54444$

$$
\frac{a}{\lambda}=0.009525 \quad \frac{\alpha}{\beta}=0.02
$$

$\begin{array}{ll}0.00165 & -0.00013 \\ 0.00648 & -0.00053 \\ 0.03608 & -0.00322 \\ 0.11245 & -0.01221 \\ 0.29251 & -0.04543 \\ 0.82386 & -0.25414 \\ 1.46041 & -0.86259 \\ 1.69111 & -1.55594 \\ 1.60664 & -2.08647 \\ 1.41615 & -2.35452 \\ 1.30371 & -2.42763 \\ 1.32974 & -2.45461 \\ 0.64210 & -0.16206 \\ 1.23504 & -0.57400 \\ 1.58843 & -1.11474 \\ 1.69410 & -1.64521 \\ 1.61912 & -2.05649 \\ 1.46920 & -2.30293 \\ 1.34325 & -2.40645 \\ 1.29792 & -2.43351 \\ 1.33552 & -2.45817 \\ 1.41692 & -2.52905\end{array}$

\begin{tabular}{|c|c|}
\hline $\begin{array}{l}0.33165 \\
0.65450 \\
1.51493 \\
2.55985 \\
3.81048 \\
5.49036 \\
6.49998 \\
6.75828 \\
6.66648 \\
6.40424 \\
6.08955 \\
5.80515\end{array}$ & $\begin{array}{l}-0.01996 \\
-0.03993 \\
-0.09979 \\
-0.19945 \\
-0.39791 \\
-0.98051 \\
-1.37247 \\
-2.51061 \\
-3.15250 \\
-3 .+8332 \\
-3.01599 \\
-3.58685\end{array}$ \\
\hline $\begin{array}{l}5.06297 \\
3.20264 \\
3.64840 \\
3.76136 \\
3.68064 \\
3.48948 \\
0.24825 \\
3.00311 \\
5.78825\end{array}$ & $\begin{array}{l}-0.17498 \\
-1.50512 \\
-2.15578 \\
-2.69991 \\
-3.12007 \\
-3.40953 \\
-3.57233 \\
-3.62212 \\
-3.58018 \\
-3.47275\end{array}$ \\
\hline
\end{tabular}

$\begin{array}{rrrr}0.32724 & -0.01996 & 0.00163 & -0.00013 \\ 0.02444 & -0.03992 & 0.00620 & -0.00052 \\ 1.27940 & -0.09966 & 0.03192 & -0.00313 \\ 1.90185 & -0.19849 & 0.09949 & -0.01193 \\ 2.52745 & -0.39109 & 0.28869 & -0.04502 \\ 3.05024 & -0.89020 & 1.02211 & -0.24716 \\ 2.38551 & -1.29953 & 2.16188 & -0.74216 \\ 0.90926 & -1.16151 & 2.78591 & -1.10802 \\ -0.80599 & -0.69302 & 2.80984 & -1.18920 \\ -2.40322 & -0.08183 & 2.23172 & -1.06052 \\ -3.52282 & 0.59741 & 1.06984 & -0.79119 \\ -3.80047 & 1.22916 & -0.47444 & -0.35751 \\ & & & \\ 2.98946 & -0.72904 & 0.74797 & -0.15981 \\ 2.81798 & -1.19683 & 1.72021 & -0.52575 \\ 1.91558 & -1.30459 & 2.45263 & -0.90205 \\ 0.67153 & -1.10942 & 2.82560 & -1.13661 \\ -0.68081 & -0.73345 & 2.82828 & -1.19182 \\ -1.97565 & -0.26704 & 2.46048 & -1.11279 \\ -3.04495 & 0.25211 & 1.71987 & -0.94439 \\ -3.69952 & 0.78934 & 0.05609 & -0.68646 \\ -3.78278 & 1.26547 & -0.58444 & -0.32147 \\ -3.24661 & 1.56883 & -1.78078 & 0.12982\end{array}$

$\begin{array}{ll}0.33164 & -0.01996 \\ 0.65445 & -0.03992 \\ 1.51435 & -0.09973 \\ 2.55637 & -0.19897 \\ 3.79345 & -0.39458 \\ 5.38087 & -0.93632 \\ 6.15330 & -1.57643 \\ 6.26986 & -1.80833 \\ 6.31434 & -1.72445 \\ 6.49241 & -1.53431 \\ 6.78384 & -1.42211 \\ 7.06512 & -1.44832 \\ & \\ 4.99425 & -0.75263 \\ 5.95989 & -1.35009 \\ 6.22844 & -1.70499 \\ 6.27275 & -1.81142 \\ 6.30750 & -1.73690 \\ 6.42555 & -1.58728 \\ 6.63024 & -1.46154 \\ 6.87018 & -1.41638 \\ 7.08105 & -1.45410 \\ 7.22086 & -1.53561\end{array}$


0.0

0.02

$0.65406-0.03990$

$0.10 \quad 2.55439-0.19874$

$\begin{array}{lll}0.10 & 3.75439 & -0.19874 \\ 0.50 & 5.38959 & -0.39376 \\ 1.00 & 6.14257 & -0.93177\end{array}$

$\begin{array}{lll}0.50 & 5.37257 & -0.93177 \\ 1.00 & 6.14433 & -1.56268\end{array}$

$1.50 \quad 6.26810-1.78918$

$\begin{array}{lll}2.00 & 6.32080 & -1.70838\end{array}$

$2.50 \quad 6.50068-1.52662$

$\begin{array}{lll}3.00 & 6.78620 & -1.42032 \\ 3.50 & 7.05951 & -1.44478\end{array}$

$\begin{array}{llll}0.125 \pi & 4.98725 & -0.74977\end{array}$

$0.250 \pi \quad 5.95006-1.34031$

$0.375 \pi \quad 0.22138-1.68849$

$0.500 . \pi \quad 6.27229-1.79218$

$\begin{array}{lll}0.625 \pi & 6.31351 & -1.72034 \\ 0.750 \pi & 6.43419 & -1.57707\end{array}$

0.875 " $0.63630-1.45756$

$1.000 \pi \quad 6.87015-1.41492$

$1.125 \pi \quad 7.07503-1.4492$

$\begin{array}{lll}1.125 \pi & 7.07503 & -1.45018 \\ 1.250 \pi & 7.21240 & -1.52581\end{array}$
$0.00165-0.00015$

$0.00647-0.00059$

$0.03603-0.00358$

$0.11225-0.01332$

$0.29172-0.048 .27$

$0.81934-0.25132$

$1.44669-0.87040$

$\begin{array}{ll}1.67201 & -1.55653 \\ 1.59061 & -2.07883\end{array}$

$1.40848-2.34506$

$1.30195-2.42409$

$1.32624-2.45902$

$0.63921-0.16795$

$1.22530-0.58268$

$1.57197-1.12063$

$1.67490-1.64450$

$1.60260-2.04930$

$1.45903-2.29311$

$1.33930-2.39920$

$1.29650-2.43236$

$1.33163-2.46301$

$0.33145 \quad-0.01995$

$0.05410-0.03990$

$1.51394-0.09973$

$2.55786-0.19928$

$3.80652-0.39742$

$5.48085-0.97797$

$6.48337-1.86330$

$6.73866-2.59233$

$6.64855-3.12490$

$6.39219-3.44844$

$6.08604-3.57758$

$5.81071-3.54943$

$\begin{array}{ll}5.05537 & -0.77336\end{array}$

$6.18866-1.49921$

$6.63020-2.14354$

$0.66244-3.08027$

$6.47539-3.37642$

$6.24024-3.53516$

$6.00222-3.58353$

$5.79439-3.5435$

$5.63840-3.43952$

$\begin{array}{rr}0.32704 & -0.01995 \\ 0.62404 & -0.03989 \\ 1.27840 & -0.09957 \\ 1.89988 & -0.19818 \\ 2.52367 & -0.33985 \\ 3.04310 & -0.88186 \\ 2.38258 & -1.26936 \\ 0.91829 & -1.11023 \\ -0.78556 & -0.62792 \\ -2.37276 & -3.01779 \\ -3.48409 & 0.63338 \\ -3.76443 & 1.20527\end{array}$

$-3.76443 \quad 1.2052$

$2.98308-0.72394$ $2.81177-1.17709$ $1.91654-1.26669$ $0.68232-1.05560$ $-0.06113-0.56897$ $-1.94807-0.20050$ $-3.00978 \quad 0.30625$ $-3.65989 \quad 0.81130$ $\begin{array}{ll}-3.22843 & 1.43025\end{array}$
(1.00163 -0.00015 $0.00619-0.0005$ $0.03187-0.00345$ $0.09929-0.0129$ $0.28792-0.04780$ $1.01801-0.25572$ $2.15269-0.75262$ $2.77950-1.1080$ $2.81142-1.16900$ $2.24092-1.00989$ $1.08870-0.70458$ $\begin{array}{ll}-0.44127 & -0.24778\end{array}$

$0.74525-0.16647$ $1.71255-0.53699$ $2.44341-0.91065$ $2.820 .18-1.13432$ $2.82927-1.17343$ $2.46753-1.07194$ $1.73327-0.87568$ $\begin{array}{lll}0.67866 & -0.59097\end{array}$ $-0.55027-0.2112$

$-1.73780 \quad 0.23361$
$0.32705-0.01995$ $0.62408-0.03990$ $1.27893-0.0996$ $1.90326-0.1987$ $2.54425-0.39370$ $3.24169-0.93165$ $3.32428-1.56245$ $3.04302-1.78879$ $2.80807-1.70778$ $2.76481-1.52580$ $2.86804-1.41933$ $2.98725-1.44368$

$3.09433-0.74960$ $3.37085-1.34013$ $3.23769-1.08821$ $3.00111-1.79177$ $2.81920-1.71976$ $2.75756-1.57632$ $2.80553-1.45660$ $2.90588-1.41390$ $2.99302-1.44907$ $3.02506-1.52466$

$$
\frac{a}{\lambda}=10.009525 \quad \frac{\alpha}{\beta}=0.05
$$

$\begin{array}{ll}0.33105 & -0.01993 \\ 0.65330 & -0.03985 \\ 1.51195 & -0.09960 \\ 2.55389 & -0.198 .96 \\ 3.79863 & -0.39646 \\ 5.46190 & -0.97290 \\ 6.45040 & -1.84512 \\ 6.59976 & -2.55628 \\ 6.61294 & -3.07069 \\ 6.36795 & -3.38016 \\ 6.07818 & -3.50253 \\ 5.82013 & -3.47626 \\ 5.04021 & -0.77013 \\ 6.16085 & -1.48748 \\ 6.59407 & -2.11932 \\ 6.70270 & -2.64156 \\ 6.62629 & -3.04013 \\ 6.44719 & -3.31155 \\ 6.22377 & -3.46246 \\ 5.99935 & -3.50812 \\ 5.80493 & -3.47025 \\ 5.66011 & -3.37425\end{array}$

$\begin{array}{rr}0.32665 & -0.01993 \\ 0.62325 & -0.03984 \\ 1.27642 & -0.09939 \\ 1.89595 & -0.19756 \\ 2.51615 & -0.38737 \\ 3.02903 & -0.36534 \\ 2.37703 & -1.21117 \\ 0.93609 & -1.00853 \\ -0.74618 & -0.49835 \\ -2.31610 & 0.10965 \\ -3.41585 & 0.76540 \\ -3.70638 & 1.16060 \\ 2.97043 & -0.71383 \\ 2.79968 & -1.13803 \\ 1.91855 & -1.19168 \\ 0.70351 & -0.94878 \\ -0.62314 & -0.54067 \\ -1.89611 & -0.06804 \\ -2.94573 & 0.41405 \\ -3.59144 & 0.85591 \\ -3.69152 & 1.18225 \\ -3.20652 & 1.30961\end{array}$

-

$0.00162-0.00018$ $0.00618-0.00071$ $0.03177-0.00408$ $0.09890-0.01486$ $0.28639-0.05333$ . $00995-0.27275$ $\begin{array}{lll}2.13508 & -0.77369\end{array}$ $2.76815-1.10876$ $2.81684-1.12942$ $2.26252-0.90935$ $.12898-0.53276$ $-0.37720-0.03015$

$0.73988-0.17973$ $1.69766-0.55945$ $2.42601-0.9264$ $2.81091-1.13053$ $2.83342-1.13748$ $2.48460-0.99093$ $1.76334-0.73922$ $0.72550-0.40164$

$-0.48470 \quad 0.00755$

$-3.206521 .30961$

$\begin{array}{ll}0.32665 & -0.01993 \\ 0.02329 & -0.03984 \\ 1.27695 & -0.09948 \\ 1.89931 & -0.19823 \\ 2.53654 & -0.39203 \\ 3.22520 & -0.92256 \\ 3.30647 & -1.53535 \\ 3.03909 & -1.75127 \\ 2.81978 & -1.07606 \\ 2.77993 & -1.50974 \\ 2.87229 & -1.41416 \\ 2.97704 & -1.43537 \\ 3.08043 & -0.74374 \\ 3.35135 & -1.32078 \\ 3.22360 & -1.05578 \\ 2.99968 & -1.75406 \\ 2.83006 & -1.68712 \\ 2.77336 & -1.55558 \\ 2.81658 & -1.44746 \\ 2.90575 & -1.40935 \\ 2.98205 & -1.44006 \\ 3.00972 & -1.50515\end{array}$


$\frac{\mathrm{a}}{\lambda}=0.009525 \quad \frac{\alpha}{\beta}=0.07$

$\beta \mathrm{h}$

$$
C(h, 0)
$$

$S(h, 0)$

0.01

0.02
0.05

0.10

0.20

.50

1.00

1.50
2.00

2.50

3.00
3.50

$0.125 \pi$ $0.250 \pi$ $0.375 \pi$
$0.500 \pi$ $0.525 \pi$ $0.625 \pi$
$0.750 \pi$ $0.750 \pi$
$0.875 \pi$

$1.000 \pi$

$1.125 \cdot \pi$

$1.250 \pi$

$\begin{array}{ll}0.33065 & -0.01990 \\ 0.65246 & -0.03980 \\ 1.50938 & -0.09934 \\ 2.54651 & -0.19782 \\ 3.77420 & -0.39051 \\ 5.33973 & -0.91386 \\ 6.10885 & -1.50933 \\ 0.25966 & -1.71551 \\ 6.34255 & -1.64591 \\ 6.52899 & -1.49410 \\ 6.79389 & -1.40847 \\ 7.04004 & -1.42706 \\ 4.95955 & -0.73817 \\ 5.91126 & -1.30217 \\ 6.19316 & -1.62472 \\ 6.26869 & -1.71817 \\ 6.33363 & -1.65612 \\ 6.46380 & -1.53564 \\ 6.65685 & -1.43810 \\ 0.86960 & -1.40425 \\ 7.05411 & -1.43113 \\ 7.18337 & -1.48723\end{array}$

$0.00164-0.00021$ $0.00644-0.00085$ $0.03583-0.00500$ $0.28861-0.05959$ $1.39349-0.90124$ $1.52828-2.05237$ $1.52828-2.05237$ $1.30866-2.47376$

$0.62781-0.19125$ $1.18730-0.61688$ $1.50834-1.14420$ $1.60103-1.64341$ $1.53852-2.02447$ $1.41774 \quad-2.25878$ $1.31998-2.37393$ $1.28597-2.42818$ $1.31272-2.47916$ $1.36872-2.56061$ $0.11145-0.01775$ $0.80158-0.28966$ $1.37611-2.311203$

$$
E(h, 0)
$$

c. $33065-0.01990$ $0.65251-0.03981$ $1.50996-0.09946$ $2.54992-3.19864$ $\begin{array}{ll}3.79075 & -0.39550\end{array}$ $5.44305-3.96787$ $0.41772-1.32718$ $6.66129-2.52091$ $0.57765-3.01778$ $6.34351-3.31379$ $6.06926-3.42975$ $5.82740-3.40523$

$\begin{array}{lll}5.02512 & -0.76692\end{array}$ 6. $13326-1.47587$ $6.55832 \quad-2.09540$ $6.66415-2.60361$ $6.59048 \quad-2.98841$ $0.41900-3.24843$ $6.20671-3.39190$ $5.99512-3.43501$ $\begin{array}{ll}5.81323 & -5.39963 \\ 5.67879 & -3.31055\end{array}$

$$
\frac{a}{\lambda}=0.009 .525 \quad \frac{\alpha}{\beta}=0.10
$$

0.01

0.02

0.05

0.10

0.50

1.00

1.50

2.00

2.50

3.50

$0.125 \pi$

$0.250 \pi$

$0.375 \pi$

$0.625 \pi$

$0.750 \%$

$0.750 \pi$
$0.875 \pi$

$1.000 \pi$

$1.125 \pi$

$0.33005 \quad-0.01987$ $\begin{array}{lll}0.65127 & -0.03972\end{array}$ $1.50641-0.09911$ $2.54061-0.19713$ $3.76274-0.38810$ $5.31549-0.90073$ $6.08265-1.47099$ $6.25200-1.66314$ $6.35494-1.60092$ $6.54563-1.46828$ $6.79783-1.39546$ $7.02775-1.41059$

$4.93903-0.72967$ $5.88274-1.27454$ $6.17199-1.57913$ $6.26429-1.66558$ $6.34495-1.60998$ $0.66860-1.42042$ $\begin{array}{ll}6.66866 & -1.42042 \\ 6.86855 & -1.39195\end{array}$ $6.86855-1.39195$ $7.16554-1.45873$
$0.00164-0.00026$ $0.00641-0.00104$ $0.03569-0.00607$ $0.11087-0.02106$ $0.28630-0.06799$ $0.78855-0.31054$ $1.35525-0.92397$ $1.54621-1.56445$ $1.48339-2.03646$ $1.35040-2.29185$ $1.27734-2.40419$ $1.29230-2.48250$

$0.61942-0.20845$ $1.15978-0.64196$ $1.46286-1.16188$

$1.54854-1.64431$

$1.49249-2.00962$

$.30241-2.35858$

$1.27378-2.42568$

$1.27378-2.42568$

$1.34033-2.57489$ $0.65131-0.03973$ $\begin{array}{lll}1.50698 & -0.0992 .7\end{array}$ $2.54399-0.19815$ $5.41496-0.96039$ $6.36926-1.80069$ $6.60440-2.46909$ $6.52530-2.94079$ $6.30655-3.21769$ $6.05402-3.32466$ $\begin{array}{ll}6.03457 & -3.32466\end{array}$

$\begin{array}{lll}5.00260 & -0.76213\end{array}$ $6.09225-1.45867$ $6.50536-2.06035$ $6.60713-2.54807$ $6.53739-2.91311$ $6.37674-3.15693$ $6.18012 \cdot-3.28991$ $5.98641-3.32946$ $5.82181-3.29751$ $\begin{array}{ll}5.82181 & -3.29751 \\ 5.70156 & -3.21790\end{array}$
$0.33006 \quad-0.01987$ $3.77898-0.39406$

$\mathrm{C}(\mathrm{h}, \mathrm{h})$

$0.32625-0.01990$ $0.62245-0.03979$ $1.27444-0.09920$ $1.89205-0.19695$ $2.50868-0.38491$ $3.01522-0.84900$ $2.37190-1.15333$ $0.95359-0.90790$ $-0.70877-0.36955$ $\begin{array}{rr}-2.26506 & 0.23646\end{array}$ $\begin{array}{ll}-3.35972 & 0.7778\end{array}$ $2.95796-0.70381$ $2.78803-1.09952$ $1.92103-1.11769$ $0.72423-0.84300$. $-0.58695-0.41315$ $-1.848420 .06375$ $\begin{array}{ll}-2.89067 & 0.5214\end{array}$ $-3.53710 \quad 0.90167$ $\begin{array}{ll}-3.65390 & 1.13213 \\ -3.20354 & 1.1466\end{array}$

$0.32565-0.01986$ $0.62126 \quad-0.0397$ $\begin{array}{lll}1.27148 & -0.09893\end{array}$ $1.88621-0.19603$ $2.49756 \quad-0.38124$ $2.99499-0.82484$ $2.36499-1.06807$ $0.97938-0.75884$ $-0.65624-0.1775$ $-2.19900 \quad 0.42607$ $-3.29775 \quad 0.42679$

$-3.64007 .1 .00560$

$2.93958 \quad-0.68897$ $2.77137-1.04275$ $1.925 .12,-1.00851$ $0.75454^{\circ}-0.68616$ $-0.53593-0.22314$ $\begin{array}{ll}-1.78486 & 0.26064\end{array}$ $\begin{array}{ll}-2.82367 & 0.68267\end{array}$ $\begin{array}{ll}-3.48143 & 0.97296\end{array}$ 1.06425 $-3.23401 \quad 0.91380$
$S(h, h)$
$0.00162-0.00021$ $0.00616 \quad-0.00083$ $0.03167-0.0047$ $0.09851-0.0168$ $0.28487-0.05884$ $\begin{array}{lll}1.00206 & -0.28968\end{array}$ $2.11846-0.79495$ $2.82529-1.0909$ $2.28842-0.8096$ $1.17261-0.36237$

$\begin{array}{lll}0.73460 & -0.1929\end{array}$ $1.68337-0.58189$ $2.40997-0.94322$ $2.80374-1.12775$ $2.84051-1.10262$ $2.50566-0.9108$ $1.79776-0.60382$ $0.77452-0.21393$

$\begin{array}{ll}-0.42268 & 0.22495 \\ -1.59648 & 0.64932\end{array}$ $2.75877-1.11043$ $-0.31606 \quad 0.18604$

$E(h, h)$

$\begin{array}{ll}0.32625 & -0.01990 \\ 0.62249 & -0.03979 \\ 1.27496 & -0.09932 \\ 1.89538 & -0.19775 \\ 2.52887 & -0.39037 \\ 3.20886 & -0.91359 \\ 3.28882 & -1.50889 \\ 3.03463 & -1.71487 \\ 2.82990 & -1.04505 \\ 2.79321 & -1.49304 \\ 2.87583 & -1.40724 \\ 2.96788 & -1.42572 \\ & \\ 3.06663 & -0.73793 \\ 3.33207 & -1.30180 \\ 3.20950 & -1.62422 \\ 2.99756 & -1.71751 \\ 2.83940 & -1.65528 \\ 2.78725 & -1.53464 \\ 2.82619 & -1.43694 \\ 2.90543 & -1.40299 \\ 2.97223 & -1.42979 \\ 2.99614 & -1.48584\end{array}$

$0.00161-0.00026$ $0.00614-0.00101$ $0.03152-0.00565$ $0.09792-0.01971$ $0.28262-0.06704$ $0.99056-0.31488$ $2.09535-0.82718$ $2.74839-1.11461$ $2.84380-1.03506$ $2.33534-0.66155$

$1.24424-0.10829$ $-0.22946 \quad 0.51004$
0

$0.72685 .-0.21252$ $1.66299-0.61548$ \begin{tabular}{ll}
$.38840-0.96926$ \\
\hline
\end{tabular} \begin{tabular}{ll}
$.79695-1.12538$ \\
\hline
\end{tabular} - 1.05226 $\begin{array}{ll} & 0.79208\end{array}$ 1.85744 -0.40209 $\begin{array}{ll}0.85208 & 0.06616\end{array}$ 0.55100 $-1.52085 \quad 0.96007$
$0.32566-0.01987$ $0.62130-0.03972$ $1.27199-0.09908$ $\begin{array}{ll}.88949 & -0.19703 \\ .51741 & -0.38789\end{array}$ $3.18463-0.90035$ $3.26265-1.47039$ $3.02701-1.66233$ $2.84235-1.59987$ $2.80993-1.46703$ $2.87985-1.39406$ $2.95568-1.40908$

$3.04613 \quad-0.72934$ $3.30357-1.27403$ $3.18837-1.57846$ $2.99320-1.66473$ -1.6089
-1.5029 $-1.5029$ . $83808-1.41909$ $2.90447^{\circ}-1.3905$ $2.97839-1.45717$ 
$\begin{array}{llll}0.125 \pi \quad 4.87228 & -0.70227\end{array}$ $0.250 \pi \quad 5.79101-1.18811$ $\begin{array}{llll}0.375 \pi & 6.10172 & -1.43935\end{array}$ $0.24082-1.50655$ $\begin{array}{llll}0.625 \pi & 6.36341 & -1.46800\end{array}$ $\begin{array}{llll}0.750 \pi & 6.51540 & -1.39939\end{array}$ $\begin{array}{lll}0.875 \pi & 6.68925 & -1.34897\end{array}$ $\begin{array}{lll}1.000 \pi & 0.85955 & -1.3329\end{array}$ $\begin{array}{lll}1.125 \pi & 7.00547-1.34415\end{array}$ $1.250 \pi \quad 7.12002-1.36542$

(1)

$\begin{array}{rr}0.32367 & -0.01974 \\ 0.61731 & -0.03945 \\ 1.26166 & -0.09803 \\ 1.86693 & -0.19301 \\ 2.46123 & -0.36925 \\ 2.93169 & -0.74715 \\ 2.34909 & -0.79575 \\ 1.06298 & -0.27577 \\ -0.51050 & 0.45663 \\ -2.06854 & 1.06292 \\ -3.27879 & 1.27828 \\ -3.83188 & 0.93546 \\ & \\ 2.88107 & -0.64103 \\ 2.72287 & -0.86147 \\ 1.94412 & -0.65859 \\ 0.85067 & -0.17641 \\ -0.39235 & 0.40375 \\ -1.64095 & 0.91838 \\ -2.73560 & 1.23238 \\ -3.51319 & 1.24165 \\ -3.84003 & 0.89025 \\ -3.63842 & 0.19498\end{array}$

$0.00160-0.00042$ $0.00606-0.00162$ $0.03104-0.00876$ $0.09600-0.02927$ $0.27530-0.09393$ $0.95499-0.39732$ $2.03350-0.93753$ $2.74580-1.14176$ $2.95815-0.86326$ $\begin{array}{ll}1.53697 & 0.74724 \\ 0.02021 & 1.63019\end{array}$

$0.70241-0.27661$ $1.60391-0.72712$ $2.33743-1.06274$ $2.80890-1.13167$ $2.96208 \quad-0.89917$ $2.74337-0.40483$ $2.12688 \quad 0.27093$ $\begin{array}{lll}1.14672 & 1.01470\end{array}$ $-0.09499 \quad 1.08121$ $-1.44374 \quad 2.10938$ $\begin{array}{ll}2.56348 & -0.17316 \\ 1.53697 & 0.74724\end{array}$



$$
\frac{a}{\lambda}=0.009525 \quad \frac{\alpha}{\beta}=0.40
$$

\begin{tabular}{|c|c|c|}
\hline $\begin{array}{l}0.01 \\
0.02 \\
0.05 \\
0.10 \\
0.20 \\
0.50 \\
1.00 \\
1.50 \\
2.00 \\
2.50 \\
3.00 \\
3.50\end{array}$ & $\begin{array}{l}0.32415 \\
0.63948 \\
1.47709 \\
2.48281 \\
3.65191 \\
5.08996 \\
5.83901 \\
6.13293 \\
6.35431 \\
6.56794 \\
6.76339 \\
6.92843\end{array}$ & $\begin{array}{l}-0.01951 \\
-0.03898 \\
-0.09682 \\
-0.19041 \\
-0.36501 \\
-0.78254 \\
-1.15409 \\
-1.24949 \\
-1.22925 \\
-1.19480 \\
-1.18042 \\
-1.18239\end{array}$ \\
\hline $\begin{array}{l}0.125 \pi \\
0.250 \pi \\
0.375 \pi \\
0.500 \pi \\
0.625 \pi \\
0.750 \pi \\
0.875 \pi \\
1.000 \pi \\
1.125 \pi \\
1.250 \pi\end{array}$ & $\begin{array}{l}4.74584 \\
5.62176 \\
5.96401 \\
6.16554 \\
6.33847 \\
6.50755 \\
6.66858 \\
6.81339 \\
6.93860 \\
7.04606\end{array}$ & $\begin{array}{l}-0.65151 \\
-1.03844 \\
-1.21071 \\
-1.25050 \\
-1.23200 \\
-1.20314 \\
-1.18488 \\
-1.17988 \\
-1.18280 \\
-1.18762\end{array}$ \\
\hline
\end{tabular}

$\begin{array}{ll}0.00158 & -0.00074 \\ 0.00618 & -0.00292 \\ 0.03423 & -0.01650 \\ 0.10514 & -0.05314 \\ 0.26425 & -0.14845 \\ 0.07142 & -0.50259 \\ 1.03942 & -1.13307 \\ 1.13362 & -1.04861 \\ 1.11279 & -2.00200 \\ 1.07799 & -2.23436 \\ 1.06336 & -2.40337 \\ 1.06517 & -2.54651 \\ 0.54233 & -0.36874 \\ 0.92474 & -0.86868 \\ 1.09549 & -1.33515 \\ 1.13452 & -1.70812 \\ 1.11557 & -1.98103 \\ 1.08641 & -2.17634 \\ 1.06794 & -2.32343 \\ 1.06277 & -2.44557 \\ 1.06556 & -2.55583 \\ 1.07028 & -2.05903\end{array}$

$\begin{array}{ll}0.32416 & -0.01951 \\ 0.63952 & -0.03901 \\ 1.47754 & -0.09731 \\ 2.48539 & -0.19338 \\ 3.66353 & -0.38002 \\ 5.14545 & -0.88948 \\ 5.91882 & -1.56210 \\ 6.08431 & -2.02334 \\ 6.03903 & -2.30403 \\ 5.92808 & -2.44630 \\ 5.81730 & -2.49404 \\ 5.73419 & -2.48619 \\ & \\ 4.78491 & -0.71626 \\ 5.70639 & -1.30043 \\ 6.01718 & -1.74939 \\ 6.08604 & -2.07315 \\ 6.04570 & -2.28876 \\ 5.96194 & -2.41695 \\ 5.87055 & -2.47928 \\ 5.79038 & -2.49597 \\ 5.72975 & -2.48444 \\ 5.69031 & -2.45855\end{array}$

$0.31975-0.01950$ $0.60943-0.03893$ $1.24230-0.09624$ $1.82926-0.18712$ $2.39197-0.34630$ $2.82328-0.60365$ $2.35223-0.29495$ $1.23299 \quad 0.05315$ $-0.34003 \quad 1.75298$ $-2.21927 \quad 2.4619$ $\begin{array}{ll}-4.11997 & 2.4619 ! \\ -4.56809 & 0.26785\end{array}$ $-5.586090 .8320^{\circ}$

$2.77620-0.5516$ $2.65771-0.53003$ $2.01274-0.00731$ $\begin{array}{rr}-0.21242 & 0.81194\end{array}$ $\begin{array}{ll}-0.21242 & 1.67927 \\ -1.66083 & 2.32989\end{array}$ $-3.18727 \quad 2.5054$ $-4.60304 \quad 1.99485$ $-4.60304 \quad 1.99485$ $\begin{array}{rr}-5.65626 & 0.68557 \\ -6.05066 & -1.38743\end{array}$
$0.00156-0.00074$ $0.00590-0.00281$ $0.03008-0.01483$ $0.09227-0.04783$ $0.26153-0.14570$ $0.89551-0.55588$ $1.97390-1.17130$ $2.88933-1.24828$ $3.45241-0.56463$ $3.38538 \quad 0.85501$ $2.41174 \quad 2.72647$

$0.65953-0.39940$ $1.52271-0.94992$ $2.32637-1.27770$ $2.99617-1.19969$ $3.42898-0.64116$ $3.48665 \quad 0.38265$ $3.02791 \quad 1.75885$ $\begin{array}{ll}1.94510 & 3.26753 \\ 0.20123 & 4.59156\end{array}$ $\begin{array}{rr}1.20123 & 4.59156 \\ -2.13175 & 5.35262\end{array}$

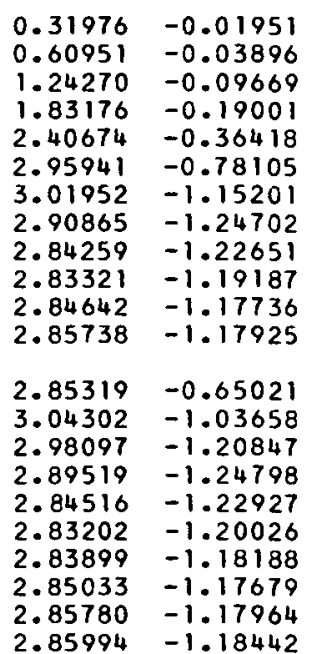


$\beta \mathrm{h}$

$\mathrm{C}(\mathrm{h}, 0)$

0.01

0.02

0.05

0.10

0.20

1.00

2.00

2.50

3.50

$0.125 \pi$

$0.250 \pi$

$0.375 \pi$

$0.500 \pi$

$0.625 \pi$

$0.750 \pi$

1.000

1.2501

$\frac{a}{\lambda}=0.009525 \quad \frac{\alpha}{\beta}=0.70$

$E(h, 0)$

$0.31836-0.01916$ $0.62793-0.03831$

$1.44868-0.09540$

$2.42821-C .18873$

$3.55218-0.36654$

$4.89540-0.82515$

$5.52320-1.36454$

$5.63983-1.68341$

$5.61390-1.85075$

$5.55751-1.92398$

$\begin{array}{ll}5.50883 & -1.94532 \\ 5.47730 & -1.94254\end{array}$

$4.58013-0.67379$

$5.36024-1.16427$

$5.59430-1.49967$

$5.64092-1.71483$

$5.61757-1.84233$

$5.57386-1.90980$

$5.53133-1.93904$

$5.47575-1.94193$

$\begin{array}{ll}5.47575 & -1.94193 \\ 5.46280 & -1.93350\end{array}$
$C(h, h)$

$S(h, h)$

$E(h, h)$

$0.31397-0.01915$ $0.59794-0.03816$ $1.21390-0.09364$ $1.77489-0.17865$ $2.29605-0.31428$ $2.70250-0.41293$ $2.45090 \quad 0.38912$ $.53995-2.07606$
$-0.35059 \quad 4.05780$ $-0.35059 \quad 4.05788$
$-3.60253 \quad 5.40289$ $-3.60253 \quad 5.40289$ $-13.21441 \quad 4.80149$ c. 74462 $2.64685-0.43144$ $2.63715-0.0889$ $2.21166 \quad 0.91324$ $1.34211 \quad 2.35611$ $-0.16994 \quad 3.92114$ $-2.51609 \quad 5.15153$ 0.9784350 $-13.67175 \quad 0.30466$ $\begin{array}{rr}-13.67175 & 0.30466 \\ -16.60388 & -6.39355\end{array}$
$0.00150-0.00110$ $0.00568-0.00454$ $0.02869-0.02358$ $0.08698-0.07434$ $0.24286-0.21873$ $0.83140-0.78155$ $2.02710-1.5584$ -1.52241
$-09406-13322$ $4.09747-0.13322$ $5.84486 \quad 2.96120$ . 7.64850 $0.58818 \quad 12.96253$

$0.60835-0.57230$ $1.48011-1.28900$ $2.52214-1.66906$ $3.71539-1.41899$ $4.89859-0.28994$ $5.72873 \quad 1.89057$ $5.67362 \quad 5.1308$ $4.05087 \quad 9.15943$ 0.1389713 .30282 $-6.6212316 .38834$
$0.31397-0.01915$ $0.59795-0.03822$ $1.21411-0.09437$ $1.77609-0.18327$ $2.30273-0.34220$ $.76160-0.68227$ $2.80695-0.92537$ $-0.97266$ $2.73062-0.96591$ $2.72791-0.95681$ $2.73043-0.9539$ $.08012-0.58213$ $2.82046-0.85742$ $2.78693-0.95490$ $2.74906-0.97305$ $2.73140-0.96675$ $2.72764-0.95877$ $2.72910-0.95473$ $2.73105-0.95382$ $\begin{array}{ll}2.73207 & -0.95419 \\ 2.73231 & -0.95470\end{array}$ $\frac{a}{\lambda}=0.009525 \quad \frac{\alpha}{\beta}=1.00$

$6.09559-0.78738$

$6.31770-0.78515$

$\begin{array}{ll}6.505461 & -0.78469 \\ 6.65461 & 0.780\end{array}$

3.50

$\begin{array}{llll}0.125 \pi & 4.41546 & -0.52654\end{array}$

$\begin{array}{llll}0.250 \pi & 5.20495 & -0.72469\end{array}$

$\begin{array}{llll}0.375 \pi & 5.59065 & -0.78080\end{array}$

$\begin{array}{llll}0.500 \pi & 5.86090 & -0.78952\end{array}$

$\begin{array}{lll}0.625 \pi & 6.07743 & -0.78762\end{array}$

$\begin{array}{llll}0.750 \pi & 6.25845 & -0.78557\end{array}$

$\begin{array}{llll}0.875 \pi & 0.41276 & -0.78478\end{array}$

$\begin{array}{lll}1.000 \pi & 6.54652 & -0.78470\end{array}$

$1.125 \pi \quad 6.66436 \quad-0.78481$

$\begin{array}{lll}1.250 \pi & 6.76968 & -0.78492\end{array}$
$0.67554-1.895$

$0.67042-2.41516$

$0.66972-2.59684$

$0.66966-2.75065$

$0.41942-0.63426$ $0.61308-1.21794$ $0.66768-1.64004$ $0.67564-1.94384$ $0.67328-2.17287$ $0.67092-2.35605$ $0.66992-2.50974$ $0.66968-2.64282$ $0.66967-2.86565$ $5.20031-1.56549$
$0.31267-0.01882$

$0.61656-0.03761$

$1.42038-0.09353$

$2.37240-0.18420$

$3.44476-0.35362$

$4.66301-0.76671$

$5.17350-1.20006$

$5.25580-1.42091$

$5.24094-1.52085$

$5.21224-1.55861$

$\begin{array}{ll}5.19080 & -1.56816 \\ 5.17882 & -1.56718\end{array}$

$0.30828 . \quad-0.01880$ $0.58662-0.03742$ $1.18628-0.09111$ $1.72296-0.17060$ $2.20901-0.28487$ $2.02722-0.24488$ $2.67153 \quad 1.04927$ $1.96434 \quad 3.74069$ $\begin{array}{ll}-0.69757 & 7.37990\end{array}$ $\begin{array}{ll}-7.04202 \quad 10.5428 \\ -18.50582 & 9.98063\end{array}$ $\begin{array}{rr}-18.50582 & 9.98063 \\ -34.56718 & -0.03062\end{array}$

$4.38731-0.63444$ $5.04844-1.04673$ $5.22491-1.29757$ $5.25649-1 .+4073$ $5.24297-1.51620$ $5.22012-1.55176$ $\begin{array}{ll}5.18653 & -1.56847 \\ 5.17828 & -1.56697\end{array}$ $5.17402-1.56422$

$\begin{array}{rr}2.54783 & -0.32502 \\ 2.70667 & 0.31242 \\ 2.55098 & 1.85917 \\ 1.74309 & 4.21709 \\ -0.40054 & 7.10455 \\ -4.73782 & 9.82890 \\ -12.06796 & 11.02438 \\ -22.69948 & 8.41657 \\ -35.74390 & -1.25490 \\ -48.11622 & -21.93710\end{array}$

$0.00144 \quad-0.00163$ $0.00546-0.00620$ $0.02735-0.03192$ $0.08200-0.0993$ $0.22635-0.2867$ $0.79246-0.9978$ $2.23743-2.00340$ $4.63809-1.94229$ $7.89568 \quad 0.43783$ $10.75280 \quad 6.74155$ $10.00681 \quad 18.27670$ $-0.08458 \quad 34.43348$

$0.57054-0.73475$ $1.51879-1.64477$ $2.97146 \quad-2.15908$ $5.06047-1.78084$ $7.64680 \quad 0.15150$ $10.11470 \quad 4.43250$ $11.12785 \quad 11.79564$ 8.4111122 .49755 $-1.31153 \quad 35.61631$ $-22.00668 \quad 48.04982$

$\begin{array}{ll}0.30828 & -0.01881 \\ 0.58661 & -0.03749 \\ 1.18620 & -0.09210 \\ 1.72238 & -0.17681 \\ 2.20489 & -0.32198 \\ 2.58675 & -0.60003 \\ 2.62047 & -0.76042 \\ 2.59540 & -0.78408 \\ 2.58677 & -0.78185 \\ 2.58599 & -0.77945 \\ 2.58648 & -0.77886 \\ 2.58671 & -0.77889 \\ & \\ 2.52422 & -0.52336 \\ 2.62825 & -0.72036 \\ 2.61006 & -0.77588 \\ 2.59327 & -0.78424 \\ 2.58701 & -0.78211 \\ 2.58593 & -0.77990 \\ 2.58624 & -0.77901 \\ 2.58657 & -0.77885 \\ 2.58671 & -0.77890 \\ 2.58674 & -0.77895\end{array}$


$\frac{a}{\lambda}=0.1 \quad \frac{\alpha}{\beta}=0.00$ $\beta \mathrm{h}$

C̣, $(h, 0)$

0.01

0.02

0.05

0.10

0.50

1.00

1.50

2.00

3.00

3.50

$0.125 \pi$

$0.250 \pi$

$0.375 \pi$
$0.500 \pi$

$0.625 \pi$

$0.750 \pi$

$0.875 \pi$

$1.000 \pi$

1.125

(

$0.125553-0.09350$

$1.08607-0.88472$

$1.52028=1.49989$

$1.60897-1.64314$

$1.79350-1.45760$

$2.09820-1.35694$

$2.38940-1.40405$

$1.40511-1.28123$

$1.56156-1.62480$

$1.57435-1.72876$

$1.60214-1.65539$

$1.72371-1.50876$

$1.93765-1.38940$

$2.18818-1.35626$

$2.54380-1.51223$
$0.02575-0.01871$

$0.49958-0.37086$

$1.57458-1.72573$

$0.90589-0.70985$
$S(h, 0)$

$E(h, G)$

$0.00013-0.00009$ $0.00051-0.00037$ $0.00321-0.00234$ $0.01275-0.00934$ $0.04952-0.03717$ $0.25821-0.22421$ $0.631 .85-0.79090$ $0.74867-1.44832$ $0.60639-1.95112$ $0.38364-2.19450$

$0.25769 .-2.24426$

$0.28653-2.25515$

$0.17226-0.14056$ $0.49145-0.52014$ $0.70913-1.02918$ $0.74117-1.53326$ $0.62205-1.92300$ $0.44398-2.14995$ $0.30175-2.23442$ $0.25126-2.24445$ . $29293-2.25809$ $0.38271-2.32741$
$\mathrm{C}(\mathrm{h}, \mathrm{h})$

$\begin{array}{ll}1.02575 & -0.01871 \\ 0.05149 & -0.03742 \\ 3.12856 & -0.09354 \\ 1.25595 & -0.18699 \\ 0.50287 & -0.37334 \\ 1.12768 & -0.92224 \\ 1.70615 & -1.76733 \\ 1.83553 & -2.46975 \\ 1.68792 & -2.98456 \\ 1.39569 & -3.29370 \\ 1.06254 & -3.40692 \\ 0.76688 & -3.35842 \\ 0.92796 & -0.72829 \\ 1.52421 & -1.41869 \\ 1.79387 & -2.03677 \\ 1.82303 & -2.55476 \\ 1.70500 & -2.85388 \\ 1.48802 & -3.22579 \\ 1.22947 & -3.37292 \\ 0.97226 & -3.40761 \\ 0.74944 & -3.35039 \\ 0.58361 & -3.22736\end{array}$

$\mathrm{S}(\mathrm{h}, \mathrm{h})$

$\begin{array}{rr}0.02575 & -0.01871 \\ 0.05145 & -0.03741 \\ 0.12792 & -0.09346 \\ 0.25102 & -0.13636 \\ 0.46813 & -0.36833 \\ 0.81855 & -0.84773 \\ 0.68979 & -1.26430 \\ 0.20273 & -1.16731 \\ -0.35082 & -0.74883 \\ -0.90616 & -0.17185 \\ -1.36069 & 0.52209 \\ -1.49684 & 1.23464 \\ 0.74311 & -0.69138 \\ 0.81866 & -1.15330 \\ 0.53491 & -1.28184 \\ 0.12563 & -1.12223 \\ -0.30995 & -0.78577 \\ -0.74940 & -0.35075 \\ -1.15743 & 0.16140 \\ -1.44117 & 0.73043 \\ -1.48974 & 1.27653 \\ -1.25162 & 1.6777\end{array}$

$0.00013-0.00009$ $0.00051-0.00037$ $0.00320-0.00234$ $0.01253-0.00932$ $0.04656-0.0369$ $.20691-0.21489$ $0.42475-0.37056$ $0.48032-1.02157$ $3.46839-1.12140$ $0.41289-1.04961$ $0.41289-1.04961$

$0.14568-0.13691$ $0.35148-0.46905$ $0.46002-0.82168$ $0.48026-1.05064$ $0.48026-1.05064$ $0.46992-1.12140$ $0.43905-1.08167$ $0.10419-0.78424$ $0.10419-0.78424$ $-0.58030-0.03628$ $-0.19973-0.50423$
$E(h, h)$

$0.02575-0.01871$ $0.05145-0.03742$ $0.12798-0.09350$ $0.25143-0.18667$ $0.85307-0.37369$ $0.84396-1.49228$ $0.28010-1.59976$ $0.24069-1.39141$ $0.36636-1.27138$ $0.36636-1.27138$

$0.76210 \quad-0.70934$ $0.91402-1.27738$ $0.74401-1.61290$ $0.48131-1.7038$ $0.23004-1.4489$ $0.23004-1.44899$ $0.41180-1.26634$ $0.51642-1.31237$ $0.55372-1.40766$ $0.53127-1.70346$

$$
\frac{a}{\lambda}=0.1 \quad \frac{\alpha}{\beta}=0.005
$$

$\begin{array}{llll}0.00013 & -0.00009 & 0.02567 & -0.01865 \\ 0.00051 & -0.00038 & 0.05133 & -0.03730 \\ 0.00319 & -0.00235 & 0.12816 & -0.09324 \\ 0.01266 & -0.00937 & 0.25514 & -0.18640 \\ 0.04918 & -0.03729 & 0.50126 & -0.37215 \\ 0.25622 & -0.22465 & 1.12384 & -0.91907 \\ 0.62583 & -0.79002 & 1.09955 & -1.76008 \\ 0.74057 & -1.44317 & 1.82810 & -2.45758 \\ 0.60041 & -1.94171 & 1.68189 & -2.96767 \\ 0.38229 & -2.18442 & 1.39307 & -3.27326 \\ 0.25939 & -2.23764 & 1.06459 & -3.38493 \\ 0.28696 & -2.25299 & 0.77377 & -3.33725 \\ 0.17099 & -0.14091 & 0.92488 & -0.72587 \\ 0.48716 & -0.52024 & 1.51860 & -1.41329 \\ 0.70196 & -1.02703 & 1.78673 & -2.02779 \\ 0.73312 & -1.52741 & 1.82066 & -2.54189 \\ 0.61579 & -1.91381 & 1.09880 & -2.93731 \\ 0.44126 & -2.13958 & 1.48425 & -3.20620 \\ 0.30236 & -2.22567 & 1.22908 & -3.35142 \\ 0.25307 & -2.23920 & 0.97571 & -3.38561 \\ 0.29311 & -2.25616 & 0.75664 & -3.32937 \\ 0.37938 & -2.32670 & 0.59391 & -3.20865\end{array}$

$\begin{array}{rrrr}0.02566 & -0.01365 & 0.00013 & -0.00009 \\ 0.05129 & -0.03730 & 0.00051 & -0.00038 \\ 0.12752 & -0.09315 & 0.00317 & -0.00235 \\ 0.25022 & -0.18575 & 0.01244 & -0.00936 \\ 0.46660 & -0.36707 & 0.04622 & -0.03702 \\ 0.81573 & -0.84389 & 0.20509 & -0.21498 \\ 0.68932 & -1.25616 & 0.42074 & -0.66856 \\ 0.20812 & -1.15625 & 0.47782 & -1.01677 \\ -0.33991 & -0.73729 & 0.46958 & -1.11553 \\ -0.88989 & -0.16085 & 0.41716 & -1.04197 \\ -1.33891 & 0.52756 & 0.21098 & -0.85234 \\ -1.47491 & 1.22610 & -0.18262 & -0.48773 \\ 0.74055 & -0.68856 & 0.14448 & -0.13709 \\ 0.81650 & -1.14658 & 0.34811 & -0.46824 \\ 0.53636 & -1.27198 & 0.45608 & -0.81857 \\ 0.13186 & -1.11104 & 0.47824 & -1.04558 \\ -0.29940 & -0.77425 & 0.47086 & -1.11557 \\ -0.73474 & -0.33934 & 0.44246 & -1.07486 \\ -1.13821 & 0.17002 & 0.34028 & -1.96421 \\ -1.41845 & 0.73277 & 0.11497 & -0.77013 \\ -1.46819 & 1.26878 & -0.21343 & -0.45499 \\ -1.23851 & 1.65339 & -0.55620 & -0.02208\end{array}$

$-0.55620-0.02208$
$0.02566-0.01865$ $0.05129-0.03730$ $0.12757-0.09320$ $0.25063-0.18608$ $0.53230-1.69246$ $\begin{array}{ll}0.36906 & -1.27020 \\ 0.50764 & -1.30321\end{array}$

$0.75930-0.70664$ $0.91033-1.27095$ $0.74212-1.60310$ $0.48785-1.69281$ $0.29695-1.60432$ $0.23648-1.44319$ $0.29589-1.31053$ $0.41313-1.26530$ $0.51431-1.30980$ $0.55027-1.40158$ $0.46965-0.36950$ $0.84978-0.88004$ $0.84095-1.48383$ $0.28548-1.59068$ $0.24687-1.3870$

$2.18819-1.35618$

$\begin{array}{lll}1.125 \pi & 2.40224 & -1.40981 \\ 1.250 \pi & 2.53914 & -1.50700\end{array}$

$0.37938-2.32670$

$\begin{array}{rr}-1.46819 & 1.26878 \\ -1.23851 & 1.65339\end{array}$ 
$\beta \mathrm{h}$

$C(h, 0)$

$S(h, 0)$

$\begin{array}{lll}0.01 & 0.02559 & -0.01859\end{array}$

$\begin{array}{llll}0.02 & 0.05117 & -0.03718\end{array}$

$\begin{array}{lll}0.05 & 0.12770 & -0.09291\end{array}$

$0.10 \quad 0.25393-0.18550$

$\begin{array}{lll}0.20 & 0.49645 & -0.36844 \\ 0.50 & 1.07943 & -0.87786\end{array}$

$1.00 \quad 1.51389-1.48442$

$1.50 \quad 1.57559-1.70597$

$\begin{array}{lll}2.00 & 1.61785 & -1.62749 \\ 2.50 & 1.80335 & -1.45107\end{array}$

$3.00 \quad 2.10095-1.35642$

$\begin{array}{lll}0.125 \pi & 0.90026 & -0.7047\end{array}$ $0.250 \pi$ $0.375 \pi$ $0.500 \pi$ $0.625 \pi$

$0.750 \pi$

$0.875 \pi$

$1.000 \pi$

$1.250 \pi$ $1.39756-1.26935$ $1.55723-1.00696$ $1.57664-1.70906$ $1.61061-1.63919$ $\begin{array}{ll}.94472 & -1.38674 \\ 2.18820 & -1.35595\end{array}$ $2.18820-1.35595$ $2.53464-1.50181$
$3.50 \quad 2.38321-1.40101$ $1.73422-1.49955$
$0.00013-0.00009$ $0.00317-0.00235$ $0.01257-0.00941$ $0.04884-0.03742$ $0.25425-0.22507$ $0.61987-0.78915$ c. $73256-1.43809$ $0.59446-1.93243$ $0.38086-2.17447$ $0.26094-2.23107$ $0.28728-2.25073$

$0.16973-0.14127$ $0.48290-0.52032$ $0.69485-1.02490$ $0.72514-1.52164$ $0.60956-1.90474$ $0.43851-2.12935$ $0.30285-2.21703$ $0.25473-2.23394$ $0.29320-2.25412$ $0.37608-2.32583$ $0.00051-0.00038$

$$
\frac{a}{\lambda}=0.1 \quad \frac{\alpha}{\beta}=0.01
$$

$E(h, 0)$

$C(h, h)$

$S(h, h)$

$0.02559-0.01859$ $0.05117-0.03718$ $0.12776-0.09295$ $0.25434-0.18581$ $0.49966-0.37097$ $1.12001-0.91591$ $1.69298-1.75282$ $1.82070-2.44548$ $1.67588-2.95089$ $1.39044-3.25297$ $1.06655-3.36311$

$0.92181-0.72345$ $1.51301-1.40791$ $1.77962-2.01885$ $1.81332-2.52910$ $1.69262-2.92084$ $1.48048-3.18674$ $1.22864-3.33008$ $0.97905-3.36378$ $0.76366-3.30850$ $0.60397-3.19005$

$$
\frac{a}{\lambda}=0.1 \quad \frac{\alpha}{\beta}=0.02
$$

0.01

$0.02543-0.01848$

$\begin{array}{llll}0.02 & 0.05084 & -0.03695\end{array}$

$0.05 \quad 0.12690-0.09233$

$0.10 \quad 0.25233-0.18432$

$0.20 \quad 0.49334-0.36603$

$\begin{array}{lll}0.50 & 1.07284 & -0.87105\end{array}$

$1.00 \quad 1.57641-1.68651$

$1.57641-1.6865$

$2.00 \quad 1.62625-1.61199$

$3.00 \quad 2.10356-1.35533$

$\begin{array}{lll}3.00 & 2.10356 & -1.35533 \\ 3.50 & 2.37743 & -1.39757\end{array}$

$\begin{array}{llll}0.125 \pi & 0.89467 & -0.69960\end{array}$ $0.250 \pi \quad 1.39007-1.2576$ $0.375 \pi \quad 1.55286-1.58938$ $0.500 \pi \quad 1.57871-1.68967$ $0.625 \pi \quad 1.61863-1.62316$ $0.750 \pi \quad 1.74418-1.49022$

$0.875 \% \quad 1.95141-1.38363$

$1.000 \pi \quad 2.18824-1.35505$

$\begin{array}{lll}1.125 \pi & 2.39276 & =1.40411 \\ 1.250 \pi & 2.52611 & =1.49151\end{array}$
$0.00013-0.00009$ $0.00050-0.00038$ $0.00312-0.00237$ $0.01240-0.00947$ $0.04816-0.03766$ $0.25033-0.22591$ $0.60808-0.78740$ $0.71674-1.42808$ $0.37781-2.15499$ $0.26360-2.21806$

$0.16722-0.14196$ $0.47447-0.52048$ $0.68083-1.02069$ $0.70940-1.51028$ $0.59723-1.88698$ $0.43290-2.10933$ $0.30348-2.20006$ $0.25759-2.22344$ $\begin{array}{ll}0.29307 & -2.24973 \\ 0.36955 & -2.32365\end{array}$ $0.58266-1.91424$
$0.02543-0.01848$ $0.05085-0.03695$ $0.12695-0.09237$ $0.25274-0.18465$ $0.49648-0.36860$ $1.11238-0.90963$ $1.67992-1.73838$ $1.80599-2.42148$ $1.66392-2.91764$ $1.38510-3.21283$ $1.07022-3.31998$

$0.91571-0.71865$ $1.50189-1.39722$ $1.79873-2.5037$ $1.68031-2.83821$ $1.47291-3.14824$ $1.22761-3.237 .90$ $0.98542-3.32063$ $1.76548-2.00109$ $\begin{array}{ll}0.77721 & -3.257 .22 \\ 0.62343 & -3.15318\end{array}$

$\begin{array}{rr}0.02558 & -0.01859 \\ 0.05113 & -0.03718 \\ 0.12712 & -0.09287 \\ 0.24942 & -0.18517 \\ 0.46507 & -0.36582 \\ 0.81293 & -0.84008 \\ 0.08883 & -1.24757 \\ 0.21344 & -1.14527 \\ -0.32909 & -0.72581 \\ -0.87375 & -0.14992 \\ -1.31749 & 0.53303 \\ -1.45356 & 1.21785 \\ 0.73800 & -0.68576 \\ 0.81435 & -1.13990 \\ 0.53779 & -1.26220 \\ 0.13803 & -1.09992 \\ -0.28896 & -0.76278 \\ -0.72021 & -0.32799 \\ -1.11924 & 0.17979 \\ -1.39618 & 0.73516 \\ -1.44722 & 1.25937 \\ -1.22594 & 1.63069\end{array}$

$0.00013-0.00009$ $0.00051-0.00038$ $0.00315-0.00235$ $0.01235-0.00939$ $0.04588-0.03713$ $0.20329-0.21507$ $0.41677-0.66658$ $0.47535-1.01205$ $0.47076-1.10974$ $0.42144-1.03440$ $0.21979-0.83992$ $-0.16565-0.47146$

$0.14329-0.13727$ $0.34476-0.46745$ $0.45218-0.81550$ $0.47625-1.04061$ $0.47178-1.10983$ $0.44588-1.06811$ $0.34639-0.95451$ $0.12577-0.75620$ $.19583-0.43867$ $-0.53252-0.00798$

$\begin{array}{rrrr}0.02542 & -0.01848 & 0.00013 & -0.00009 \\ 0.05081 & -0.03695 & 0.00050 & -0.00038 \\ 0.12632 & -0.09228 & 0.00311 & -0.00237 \\ 0.24784 & -0.18398 & 0.01218 & -0.00945 \\ 0.46203 & -0.36332 & 0.04521 & -0.03733 \\ 0.80736 & -0.83250 & 0.19973 & -0.21523 \\ 0.68784 & -1.23058 & 0.40895 & -0.66270 \\ 0.22389 & -1.12351 & 0.47047 & -1.00282 \\ -0.30775 & -0.70298 & 0.47309 & -1.09838 \\ -0.84197 & -0.12821 & 0.43008 & -1.01946 \\ -1.27573 & 0.54398 & 0.23755 & -0.81553 \\ -1.41257 & 1.20225 & -0.13210 & -0.43959 \\ 0.73294 & -0.68018 & 0.14093 & -0.13761 \\ 0.81006 & -1.12667 & 0.33814 & -0.46587 \\ 0.54057 & -1.24284 & 0.44450 & -0.80948 \\ 0.15015 & -1.07789 & 0.47230 & -1.03089 \\ -0.26835 & -0.73999 & 0.47359 & -1.09858 \\ -0.69155 & -0.30544 & 0.45274 & -1.05479 \\ -1.08202 & 0.19799 & 0.35877 & -0.93540 \\ -1.35292 & 0.74013 & 0.14744 & -0.72890 \\ -1.40701 & 1.24150 & -0.16110 & -0.40669 \\ -1.20235 & 1.58626 & -0.48633 & 0.01997\end{array}$

$E(h, h)$

$0.02558-0.01859$ $0.05113-0.03718$ $0.12717-0.0929$ $0.24983-0.18548$ $0.46809-0.36833$ $0.84649-0.8764$ $0.53328-1.68155$ $0.29074-1.58165$ $0.25291-1.38255$ $0.37173-1.26887$ $0.50588-1.3007$

$0.75650-0.70396$ $0.90666-1.26455$ $0.74024-1.59337$ $0.48953-1.68189$ $0.30199-1.59502$ $0.24277-1.43738$ $0.30065-1.30806$ $0.41446-1.26412$ $0.51232-1.30713$ $0.54698-1.39553$

$\begin{array}{ll}0.02542 & -0.01848 \\ 0.05081 & -0.03695 \\ 0.12637 & -0.09232 \\ 0.24824 & -0.18430 \\ 0.46499 & -0.36587 \\ 0.83996 & -0.86919 \\ 0.83196 & -1.45882 \\ 0.53511 & -1.05999 \\ 0.30091 & -1.56374 \\ 0.26460 & -1.37348 \\ 0.37695 & -1.26581 \\ 0.50266 & -1.29559 \\ 0.75094 & -0.69861 \\ 0.89937 & -1.25186 \\ 0.73645 & -1.57412 \\ 0.49271 & -1.66032 \\ 0.31171 & -1.57659 \\ 0.25495 & -1.42569 \\ 0.30987 & -1.30281 \\ 0.41711 & -1.26134 \\ 0.50866 & -1.30151 \\ 0.54086 & -1.38353\end{array}$


$\mathrm{C}(\mathrm{H}, \mathrm{O})$

\begin{tabular}{|c|c|c|}
\hline $\begin{array}{l}0.01 \\
0.02 \\
0.05 \\
0.10 \\
0.20 \\
0.50 \\
1.00 \\
1.50 \\
2.00 \\
2.50 \\
3.00 \\
3.50\end{array}$ & $\begin{array}{l}0.02527 \\
0.05053 \\
0.12611 \\
0.25075 \\
0.49025 \\
1.06630 \\
1.50118 \\
1.57705 \\
1.63420 \\
1.82150 \\
2.10603 \\
2.37204\end{array}$ & $\begin{array}{l}-0.01836 \\
-0.03672 \\
-0.09175 \\
-0.18315 \\
-0.36363 \\
-0.86430 \\
-1.45410 \\
-1.06736 \\
-1.59665 \\
-1.43726 \\
-1.35369 \\
-1.39373\end{array}$ \\
\hline $\begin{array}{l}0.125 \pi \\
0.250 \pi \\
0.375 \pi \\
0.500 \pi \\
0.625 \pi \\
0.750 \pi \\
0.875 \pi \\
1.000 \pi \\
1.125 \pi \\
1.250 \pi\end{array}$ & $\begin{array}{l}0.88912 \\
1.38262 \\
1.54847 \\
1.58055 \\
1.62620 \\
1.75360 \\
1.95773 \\
2.18830 \\
2.38697 \\
2.51816\end{array}$ & $\begin{array}{l}-0.69454 \\
-1.24600 \\
-1.57205 \\
-1.67058 \\
-1.60730 \\
-1.48076 \\
-1.38009 \\
-1.35360 \\
-1.39987 \\
-1.48132\end{array}$ \\
\hline
\end{tabular}

$\mathrm{SIH}, \mathrm{O}:$

$\begin{array}{ll}0.00012 & -0.00010 \\ 0.00049 & -0.00038 \\ 0.00308 & -0.00239 \\ 0.01223 & -0.00954 \\ 0.04749 & -0.03790 \\ 0.24645 & -0.22673 \\ 0.59647 & -0.73567 \\ 0.70121 & -1.41829 \\ 0.57100 & -1.89655 \\ 0.37451 & -2.13605 \\ 0.26571 & -2.20523 \\ 0.28751 & -2.24076 \\ 0.16475 & -0.14264 \\ 0.46616 & -0.52062 \\ 0.66703 & -1.01654 \\ 0.69394 & -1.49919 \\ 0.58506 & -1.86970 \\ 0.42715 & -2.08989 \\ 0.30367 & -2.18349 \\ 0.25988 & -2.21296 \\ 0.29258 & -2.24496 \\ 0.36312 & -2.32094\end{array}$

$E\{H, O)$

$\begin{array}{ll}0.02527 & -0.01836 \\ 0.05053 & -0.03672 \\ 0.12616 & -0.09179 \\ 0.25115 & -0.18348 \\ 0.49332 & -0.30626 \\ 1.10481 & -0.90338 \\ 1.66696 & -1.72407 \\ 1.79141 & -2.39773 \\ 1.65203 & -2.88483 \\ 1.37968 & -3.17327 \\ 1.07356 & -3.27751 \\ 0.80577 & -3.23375 \\ 0.90964 & -0.71387 \\ 1.49085 & -1.38661 \\ 1.75146 & -1.93350 \\ 1.78427 & -2.47861 \\ 1.66808 & -2.85600 \\ 1.46531 & -3.11029 \\ 1.22639 & -3.24636 \\ 0.99138 & -3.27815 \\ 0.79011 & -3.22654 \\ 0.04201 & -3.11675\end{array}$

$\frac{a}{\lambda}=0.1$

$\begin{array}{ll}0.00012 & -0.00010 \\ 0.00048 & -0.00039 \\ 0.00300 & -0.00242 \\ 0.01190 & -0.00967 \\ 0.04617 & -0.03837 \\ 0.23884 & -0.22831 \\ 0.57330 & -0.78225 \\ 0.67098 & -1.39935 \\ 0.54811 & -1.86260 \\ 0.36721 & -2.09971 \\ 0.26838 & -2.18010 \\ 0.28623 & -2.22954 \\ 0.15987 & -0.14395 \\ 0.44988 & -0.52085 \\ 0.04015 & -1.00843 \\ 0.66385 & -1.47774 \\ 0.56120 & -1.83651 \\ 0.41534 & -2.05261 \\ 0.30283 & -2.15151 \\ 0.26290 & -2.19212 \\ 0.29056 & -2.23444 \\ 0.35049 & -2.31409\end{array}$

$\begin{array}{ll}0.02495 & -0.01813 \\ 0.04990 & -0.03626 \\ 0.12458 & -0.09064 \\ 0.24800 & -0.18118 \\ 0.48706 & -0.36161 \\ 1.08981 & -0.39103 \\ 1.64136 & -1.09582 \\ 1.76261 & -2.35099 \\ 1.62847 & -2.82045 \\ 1.36861 & -3.09588 \\ 1.07928 & -3.19453 \\ 0.82860 & -3.15367 \\ 0.89763 & -0.70441 \\ 1.46903 & -1.36564 \\ 1.72377 & -1.94880 \\ 1.75571 & -2.42924 \\ 1.64387 & -2.79280 \\ 1.45006 & -3.03597 \\ 1.22338 & -3.16514 \\ 1.00209 & -3.19513 \\ 0.81401 & -3.14696 \\ 0.67667 & -3.04519\end{array}$

$\mathrm{C}(\mathrm{H}, \mathrm{H})$

$\begin{array}{rr}0.02526 & -0.01836 \\ 0.05049 & -0.03671 \\ 0.12553 & -0.09170 \\ 0.24627 & -0.13230 \\ 0.45902 & -1.036084 \\ 0.80184 & -1.82500 \\ 0.08681 & -1.21383 \\ 0.23410 & -1.16203 \\ -0.28679 & -0.06036 \\ -0.81083 & -0.10672 \\ -1.23539 & 0.55497 \\ -1.37376 & 1.18781 \\ 0.72791 & -0.67466 \\ 0.80580 & -1.11361 \\ 0.54324 & -1.22377 \\ 0.16199 & -1.05613 \\ -0.24813 & -0.71741 \\ -0.66342 & -0.23310 \\ -1.04577 & 0.21602 \\ -1.31135 & 0.74535 \\ -1.36900 & 1.22491 \\ -1.18078 & 1.54435\end{array}$

$S(H, H)$

$0.00012-0.00010$

$0.00049-0.00038$

$0.00307-0.00239$

$0.01201-0.00951$

$0.04455-0.03754$

$0.19621-0.21538$

$0.40128-0.65890$

$0.46568 \quad-0.99387$

$0.47538-1.08732$

$0.43882-1.00478$

$\begin{array}{rr}0.25548 & -0.79172 \\ -0.09904 & -0.40854\end{array}$

$0.13859-0.13794$ $0.33164-0.46431$ $0.43697-0.80362$ $0.46843-1.02147$ $0.47537-1.08763$ $\begin{array}{lll}0.45962 & -1.04173\end{array}$ c. $.37134-0.91667$ $0.16918-0.70229$ $-0.12693-0.37553$ $\begin{array}{lll}-0.44164 & 0.04762\end{array}$
$E(H, H)$

$0.02526-0.01836$ $0.05049-0.03672$ $0.12558-0.09174$ $0.24666-0.18313$ $0.46190-0.36344$ $0.83348-0.86204$ $0.82602-1.44241$ $0.53678 \cdot-1.63876$ $0.31062-1.54604$ $0.27577-1.36421$ $0.38200-1.26223$ $0.49981-1.29004$ $0.74543-0.69330$ $0.89214-1.23931$ $0.73266-1.55514$ $0.49569-1.63907$ $0.32101-1.55837$ $0.26658-1.41392$ $0.31869-1.29717$ $0.41976-1.25803$ $0.50540-1.29556$ $0.53532-1.37164$

\begin{tabular}{|c|c|c|}
\hline $\begin{array}{l}0.01 \\
0.02 \\
0.05 \\
0.10 \\
0.20 \\
0.50 \\
1.00 \\
1.50 \\
2.00 \\
2.50 \\
3.00 \\
3.50\end{array}$ & $\begin{array}{l}0.02495 \\
0.04990 \\
0.12453 \\
0.24762 \\
0.48413 \\
1.05336 \\
1.48856 \\
1.57779 \\
1.64877 \\
1.83771 \\
2.11053 \\
2.36227\end{array}$ & $\begin{array}{l}-0.01813 \\
-0.03620 \\
-0.09060 \\
-0.18084 \\
-0.35390 \\
-0.85093 \\
-1.42457 \\
-1.62994 \\
-1.56642 \\
-1.42257 \\
-1.34894 \\
-1.38501\end{array}$ \\
\hline $\begin{array}{l}0.125 \pi \\
0.250 \pi \\
0.375 \pi \\
0.500 \pi \\
0.625 \pi \\
0.750 \pi \\
0.875 \pi \\
1.000 \pi \\
1.125 \pi \\
1.250 \pi\end{array}$ & $\begin{array}{r}0.87814 \\
1.36787 \\
1.53959 \\
1.58358 \\
1.64007 \\
1.77093 \\
1.96930 \\
2.18839 \\
2.37648 \\
2.50378\end{array}$ & $\begin{array}{l}-0.68453 \\
-1.22318 \\
-1.53815 \\
-1.63327 \\
-1.57612 \\
-1.46157 \\
-1.37183 \\
-1.34919 \\
-1.39040 \\
-1.46123\end{array}$ \\
\hline
\end{tabular}

$\frac{\alpha}{\beta}=0.05$

$\begin{array}{rr}0.02495 & -0.01813 \\ 0.04986 & -0.0362 \epsilon \\ 0.12395 & -0.09055 \\ 0.24316 & -0.1804 \epsilon \\ 0.45305 & -0.35594 \\ 0.79092 & -0.81025 \\ 0.68467 & -1.18105 \\ 0.25380 & -1.0599 \\ -0.24601 & -0.63572 \\ -0.75047 & -0.06433 \\ -1.15877 & 0.57713 \\ -1.30238 & 1.16233 \\ 0.71797 & -0.66375 \\ 0.79736 & -1.08792 \\ 0.54828 & -1.13644 \\ 0.18489 & -1.01342 \\ -0.20879 & -0.67288 \\ -0.60872 & -0.23902 \\ -0.97608 & 0.25185 \\ -1.23301 & 0.75662 \\ -1.29930 & 1.19541 \\ -1.14347 & 1.46768\end{array}$

$\begin{array}{rr}0.00012 & -0.00010 \\ 0.00048 & -0.00039 \\ 0.00298 & -0.00242 \\ 0.01168 & -0.00964 \\ 0.04325 & -0.03793 \\ 0.18933 & -0.21565 \\ 0.38636 & -0.65156 \\ 0.45637 & -0.97679 \\ 0.47985 & -1.06609 \\ 0.45656 & -0.97626 \\ 0.29179 & -0.74574 \\ -0.03431 & -0.34868 \\ 0.13401 & -0.13858 \\ 0.31896 & -0.46125 \\ 0.42235 & -0.79236 \\ 0.46088 & -1.00350 \\ 0.47886 & -1.06664 \\ 0.47350 & -1.01639 \\ 0.39699 & -0.88034 \\ 0.21283 & -0.65103 \\ -0.06018 & -0.31539 \\ -0.35638 & 0.10225\end{array}$

$\begin{array}{ll}0.02495 & -0.01813 \\ 0.04986 & -0.03626 \\ 0.12400 & -0.09059 \\ 0.24353 & -0.18080 \\ 0.45579 & -0.35861 \\ 0.82068 & -0.84791 \\ 0.81424 & -1.41023 \\ 0.53964 & -1.59727 \\ 0.32875 & -1.51121 \\ 0.29664 & -1.34516 \\ 0.39162 & -1.25367 \\ 0.49508 & -1.27791 \\ 0.73452 & -0.68282 \\ 0.87786 & -1.21462 \\ 0.72503 & -1.51799 \\ 0.50105 & -1.59757 \\ 0.33834 & -1.52260 \\ 0.28831 & -1.39024 \\ 0.33524 & -1.28482 \\ 0.42500 & -1.24996 \\ 0.49993 & -1.28270 \\ 0.52577 & -1.34819\end{array}$




\begin{tabular}{|c|c|c|}
\hline $\begin{array}{l}0.01 \\
0.02 \\
0.05 \\
0.10 \\
0.20 \\
0.50 \\
1.00 \\
1.50 \\
2.00 \\
2.50 \\
3.00 \\
3.50\end{array}$ & $\begin{array}{l}0.02464 \\
0.049 .27 \\
0.12298 \\
0.24453 \\
0.47809 \\
1.04060 \\
1.47601 \\
1.57786 \\
1.06168 \\
1.85211 \\
2.111444 \\
2.35363\end{array}$ & $\begin{array}{l}-0.01790 \\
-0.03581 \\
-0.08946 \\
-0.17856 \\
-0.35422 \\
-0.83790 \\
-1.39581 \\
-1.59367 \\
-1.53682 \\
-1.40713 \\
-1.34240 \\
-1.37500\end{array}$ \\
\hline $\begin{array}{l}0.125 \pi \\
0.250 \pi \\
0.375 \pi \\
0.500 \pi \\
0.625 \pi \\
0.750 \pi \\
0.875 \pi \\
1.000 \pi \\
1.125 \pi \\
1.250 \pi\end{array}$ & $\begin{array}{l}0.86730 \\
1.35332 \\
1.53060 \\
1.58579 \\
1.65233 \\
1.78635 \\
1.97952 \\
2.18840 \\
2.36722 \\
2.49114\end{array}$ & $\begin{array}{l}-0.67468 \\
-1.20086 \\
-1.50520 \\
-1.59710 \\
-1.54563 \\
-1.44204 \\
-1.36218 \\
-1.34295 \\
-1.37975 \\
-1.44145\end{array}$ \\
\hline
\end{tabular}

0.01

0.02

0.05

0.10

0.20
0.50

1.50

1.50

1.50
2.00

2.00

3.50
3.00

3.50

$0.125 \pi$

$0.250 \pi$

$0.375 \pi$

$0.500 \pi$

$0.625 \pi$

$0.750 \pi$

$0.875 \pi$

1.000

$1.125 \pi$ $\frac{\mathrm{a}}{\lambda}=0.1$

$E(h, 0$ :

$0.00012-0.00010$

$0.00047-0.00039$

$0.00292-0.00245$

$0.01157-0.00979$

$0.04487-0.03882$

$0.23141-0.22982$

$0.55184-0.77887$

$0.64184-1.38121$

$0.52577-1.83044$

$0.35911-2.06532$

$\begin{array}{ll}0.26921 & -2.15569 \\ 0.28362 & -2.21732\end{array}$

$0.15511-0.14521$

$0.43406-0.52100$

$0.61416-1.00055$

$0.63485-1.45725$

$0.53797-1.80507$

$\begin{array}{lll}0.40314 & -2.01738\end{array}$

$0.30053-2.12104$

$0.26403-2.17151$

$0.28731-2.22282$

$0.33811-2.30563$
$C(h, h)$

$\begin{array}{ll}0.02464 & -1.01790 \\ 0.04927 & -1.03581 \\ 0.12302 & -0.08951 \\ 0.24489 & -0.17891 \\ 0.48088 & -0.35702 \\ 1.07502 & -0.87884 \\ 1.61617 & -1.06804 \\ 1.73431 & -2.30524 \\ 1.60521 & -2.75772 \\ 1.35726 & -3.02072 \\ 1.08380 & -3.11408 \\ 0.84912 & -3.07592 \\ & \\ 0.88578 & -0.69508 \\ 1.44754 & -1.34499 \\ 1.69654 & -1.91475 \\ 1.72764 & -2.38094 \\ 1.61998 & -2.73119 \\ 1.43472 & -2.96373 \\ 1.21966 & -3.08635 \\ 1.01130 & -3.11464 \\ 0.83554 & -3.06967 \\ 0.70818 & -2.97535\end{array}$

$\frac{a}{\lambda}=0.1$

$\frac{\alpha}{\beta}=0.10$ $\frac{\alpha}{\beta}=0.07$

$\begin{array}{rr}0.02464 & -0.01790 \\ 0.04923 & -0.03580 \\ 0.12240 & -0.08941 \\ 0.24009 & -0.178 .6 \\ 0.44716 & -0.351: 0 \\ 0.78017 & -0.79575 \\ 0.68241 & -1.14918 \\ 0.27261 & -1.01889 \\ -0.20669 & -0.59189 \\ -0.69260 & -0.02268 \\ -1.08734 & 0.59969 \\ -1.23882 & 1.14122 \\ 0.70818 & -0.65306 \\ 0.78902 & -1.06302 \\ 0.55295 & -1.15018 \\ 0.20680 & -0.97177 \\ -0.17087 & -0.62913 \\ -0.55608 & -0.19573 \\ -0.91005 & 0.28682 \\ -1.16078 & 0.76912 \\ -1.23750 & 1.17064 \\ -1.11354 & 1.39980\end{array}$

$\mathrm{S}(\mathrm{h}, \mathrm{h})$

$0.00012-0.00010$ $0.00047-0.00039$ $0.00290-0.00245$ $0.01135-0.00975$ $0.04198-0.03831$ $\begin{array}{lll}0.18263 & -0.21587\end{array}$ $0.37201-0.64454$ $0.44743-0.96075$ $0.48433-1.04602$ $0.47467-0.94886$ $0.32866-0.70182$ $0.02875-0.29138$

$0.12954-0.13917$ $0.30671-0.45828$ $0.40832-0.78169$ $0.45363-0.98663$ $0.48231-1.04682$ $0.48755-0.99210$ $0.42330-0.84549$ $0.25670-0.60216$ $0.25670-0.60216$

$\begin{array}{rr}0.027606 & 0.15632\end{array}$
$E(h, h)$

$\begin{array}{ll}0.02464 & -0.01790 \\ 0.04924 & -0.03581 \\ 0.12245 & -0.08946 \\ 0.24044 & -0.17851 \\ 0.44976 & -0.35385 \\ 0.80808 & -0.83402 \\ 0.80260 & -1.37886 \\ 0.54190 & -1.55704 \\ 0.34525 & -1.47716 \\ 0.31565 & -1.32551 \\ 0.40058 & -1.24344 \\ 0.49144 & -1.26457 \\ 0.72377 & -0.67250 \\ 0.86382 & -1.19047 \\ 0.71736 & -1.48187 \\ 0.50565 & -1.55732 \\ 0.35409 & -1.48767 \\ 0.30811 & -1.36639 \\ 0.35036 & -1.27121 \\ 0.43008 & -1.24015 \\ 0.49565 & -1.26873 \\ 0.51796 & -1.32510\end{array}$

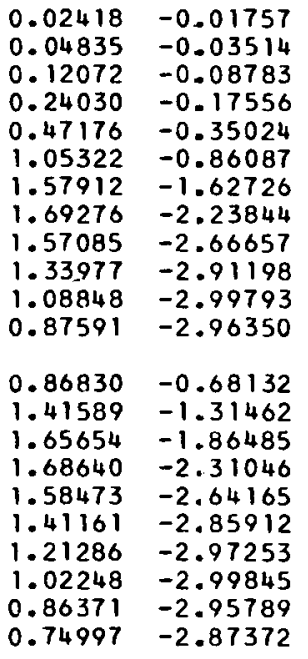

$\begin{array}{rr}0.02418 & -0.01757 \\ 0.04831 & -0.03513 \\ 0.12011 & -0.08773 \\ 0.23555 & -0.17475 \\ 0.43847 & -0.34397 \\ 0.76437 & -0.77457 \\ 0.67885 & -1.10304 \\ 0.29927 & -0.95935 \\ -0.15034 & -0.52769 \\ -0.61037 & 0.03859 \\ -0.98936 & 0.63458 \\ -1.15699 & 1.11736 \\ 0.69377 & -0.03734 \\ 0.77671 & -1.02675 \\ 0.55931 & -1.09773 \\ 0.23792 & -0.91124 \\ -0.11654 & -0.56512 \\ -0.48087 & -0.13206 \\ -0.81757 & 0.33901 \\ -1.06310 & 0.79037 \\ -1.15846 & 1.14189 \\ -1.08166 & 1.31316\end{array}$

$\begin{array}{ll}0.00011 & -0.00010 \\ 0.00045 & -0.00040 \\ 0.00278 & -0.00249 \\ 0.01088 & -0.00992 \\ 0.04012 & -0.03884 \\ 0.17291 & -0.21613 \\ 0.35148 & -0.63458 \\ 0.43471 & -0.93853 \\ 0.49105 & -1.01801 \\ 0.50257 & -0.90989 \\ 0.38488 & -0.63949 \\ 0.12063 & -0.20933 \\ 0.12305 & -0.13998 \\ 0.28910 & -0.45396 \\ 0.38832 & -0.76672 \\ 0.44333 & -0.96329 \\ 0.48752 & -1.01920 \\ 0.50904 & -0.95767 \\ 0.46391 & -0.79590 \\ 0.32283 & -0.53282 \\ 0.09880 & -0.17508 \\ 0.16359 & 0.23720\end{array}$

$\begin{array}{ll}0.02418 & -0.01757 \\ 0.04832 & -0.03514 \\ 0.12015 & -0.08778 \\ 0.23588 & -0.17512 \\ 0.44087 & -0.34683 \\ 0.78956 & -0.81363 \\ 0.78543 & -1.33329 \\ 0.54424 & -1.49897 \\ 0.36714 & -1.42754 \\ 0.34097 & -1.29518 \\ 0.41278 & -1.22544 \\ 0.48756 & -1.24262 \\ 0.70795 & -0.65731 \\ 0.84320 & -1.15523 \\ 0.70580 & -1.42956 \\ 0.51124 & -1.49923 \\ 0.37498 & -1.43686 \\ 0.33447 & -1.33047 \\ 0.37057 & -1.24881 \\ 0.43729 & -1.22270 \\ 0.49097 & -1.24599 \\ 0.50887 & -1.29101\end{array}$


$\frac{a}{\lambda}=0.1 \quad \frac{\alpha}{\beta}=0.20$.

$E(h, 0)$

$C(h, t)$

$S(h, h)$

$E(h, h)$ $0.44257-0.32857$

$1.71207-1.35914$

$1.90919-1.29562$

$\begin{array}{lll}3.00 & 2.12532 & -1.27004 \\ 3.50 & 2.31262 & -1.28871\end{array}$

$\begin{array}{llll}0.125 \pi & 0.80039 & -0.61432\end{array}$

$\begin{array}{llll}0.250 \pi & 1.26327 & -1.06743\end{array}$

$\begin{array}{llll}0.375 \pi & 1.47020 & -1.31243\end{array}$

$0.500 \pi \quad 1.58349-1.38704$

0.625 ष $1.69965-1.36367$

$0.750 \pi \quad 1.84826-1.31190$

$0.875 \pi \quad 2.01776-1.27639$

$1.000 \pi \quad 2.18282-1.27183$

$1.125 \pi \quad 2.32371-1.29091$

$1.250 \pi \quad 2.43426-1.31749$
$0.02271-0.01050$ $0.04541-0.03300$ $0.11336-0.08248$ $0.22561-0.16482$ $0.98369-0.80361$

$1.46187-1.49968$

$1.56167-2.03058$

$1.46099 \quad-2.38669$

$1.27822-2.58154$

$\begin{array}{ll}1.08863 & -2.64681 \\ 0.93578 & -2.62237\end{array}$

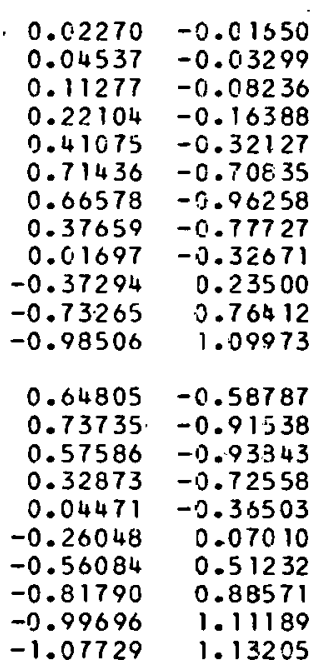

$0.81249-0.63738$

$1.31542-1.21834$

$1.53020-1.70821$

$1.55630-2.08160$

$1.33412-2.54040$

$1.18133-2.62783$

$1.04039-2.64720$

$\begin{array}{ll}0.92725 & -2.61844 \\ 0.84022 & -2.50085\end{array}$
$0.84922-2.50085$
$0.00010-0.00011$ $0.00039-0.00042$ $0.00241-0.0026$ $0.00938-0.01040$ $0.03433-0.04037$ $0.14330-0.21637$ $0.29104-0.60576$ $0.39828-0.87836$ $0.51548-0.94156$ $0.60249-0.79810$ $\begin{array}{r}0.57985 \\ 0.41090\end{array}-0.45684$

$0.10307-0.1420$ $0.23652-0.44069$ $0.32993-0.72476$ $0.41414-0.90033$ $0.50697-0.94407$ $0.58556-0.86005$ $0.60867-0.65197$ $0.5469 .5-0.32788$ $0.39411 \quad 0.08194$ $\frac{\alpha}{\beta}=0.40$

$\begin{array}{ll}0.02002 & -0.01455 \\ 0.04001 & -0.02909 \\ 0.09942 & -0.07258 \\ 0.19468 & -0.14412 \\ 0.36060 & -0.23038 \\ 0.62543 & -0.59397 \\ 0.63721 & -0.73384 \\ 0.49335 & -0.47866 \\ 0.27759 & 0.02273 \\ -0.03606 & 0.61498 \\ 0.47030 & 1.11195 \\ -1.01112 & 1.31303 \\ 0.56636 & -0.50099 \\ 0.66634 & -0.73054 \\ 0.59465 & -0.68049 \\ 0.46746 & -0.41923 \\ 0.29625 & -0.01898 \\ 0.06597 & 0.44558 \\ 0.23684 & 0.88700 \\ -0.61463 & 1.20717 \\ -1.05059 & 1.31145 \\ -1.49922 & 1.12655\end{array}$ $\begin{array}{ll}0.02270 & -0.01650 \\ 0.04537 & -0.03299 \\ 0.11280 & -0.08241 \\ 0.22129 & -0.16428 \\ 0.41248 & -0.32442 \\ 0.73094 & -0.74934 \\ 0.73049 & -1.19335 \\ 0.54432 & -1.32341 \\ 0.41936 & -1.27419 \\ 0.40200 & -1.19003 \\ 0.44306 & -1.14948 \\ 0.48216 & -1.15812 \\ 0.65771 & -0.60917 \\ 0.05771 & -1.04580 \\ 0.77815 & -1.27020 \\ 0.66706 & -1.32360 \\ 0.52020 & -1.28043 \\ 0.42461 & -1.28043 \\ 0.39803 & -1.21167 \\ 0.41940 & -1.16265 \\ 0.45628 & -1.14799 \\ 0.48384 & -1.15978 \\ 0.49243 & -1.18106\end{array}$

$\begin{array}{lll}0.01 & 0.02003 & -0.01455 \\ 0.02 & 0.04005 & -0.02910 \\ 0.05 & 0.09995 & -0.07267 \\ 0.10 & 0.19878 & -0.14482 \\ 0.20 & 0.38891 & -0.28553 \\ 0.50 & 0.85398 & -0.65192 \\ 1.00 & 1.28206 & -1.01383 \\ 1.50 & 1.51257 & -1.12859 \\ 2.00 & 1.71260 & -1.13198 \\ 2.50 & 1.91343 & -1.11706 \\ 3.00 & 2.09917 & -1.11654 \\ 3.50 & 2.25699 & -1.12791 \\ 0.125 \pi & 0.70839 & -0.53273 \\ 0.250 \pi & 1.13880 & -0.89613 \\ 0.375 \pi & 1.37425 & -1.07586 \\ 0.500 \pi & 1.54101 & -1.13298 \\ 0.625 \pi & 1.69789 & -1.13309 \\ 0.750 \pi & 1.85633 & -1.12038 \\ 0.875 \pi & 2.00892 & -1.11471 \\ 1.000 \pi & 2.14688 & -1.11904 \\ 1.1251 & 2.26675 & -1.12884 \\ 1.250 \pi & 2.37014 & -1.13890\end{array}$

$\begin{array}{ll}0.00007 & -0.00011 \\ 0.00028 & -0.00045 \\ 0.00177 & -0.00282 \\ 0.00701 & -0.01121 \\ 0.02692 & -0.04399 \\ 0.13122 & -0.24483 \\ 0.27386 & -0.72739 \\ 0.28609 & -1.16664 \\ 0.23239 & -1.48055 \\ 0.18155 & -1.09304 \\ 0.15645 & -1.85180 \\ 0.14997 & -1.98887 \\ & \\ 0.09010 & -0.15874 \\ 0.22838 & -0.51440 \\ 0.29137 & -0.89652 \\ 0.28046 & -1.21883 \\ 0.23678 & -1.46164 \\ 0.19374 & -1.63941 \\ 0.16590 & -1.77624 \\ 0.15334 & -1.89200 \\ 0.14986 & -1.99785 \\ 0.14922 & -2.09762\end{array}$

$\begin{array}{ll}0.02003 & -0.01455 \\ 0.04005 & -0.02910 \\ 0.09996 & -0.07273 \\ 0.19886 & -0.14528 \\ 0.38951 & -0.28917 \\ 0.85818 & -0.70031 \\ 1.25363 & -1.27223 \\ 1.33064 & -1.07531 \\ 1.26191 & -1.92182 \\ 1.14752 & -2.04472 \\ 1.03955 & -2.08238 \\ 0.96046 & -2.07005 \\ 0.71142 & -0.55783 \\ 1.13573 & -1.04683 \\ 1.30685 & -1.43522 \\ 1.32678 & -1.71910 \\ 1.26935 & -1.90846 \\ 1.18137 & -2.01979 \\ 1.09103 & -2.07178 \\ 1.01377 & -2.08259 \\ 0.95629 & -2.06813 \\ 0.91955 & -2.04116\end{array}$

$\begin{array}{ll}0.00007 & -0.00011 \\ 0.00028 & -0.00045 \\ 0.00176 & -0.00281 \\ 0.00682 & -0.01109 \\ 0.02447 & -0.04242 \\ 0.09511 & -0.21440 \\ 0.20028 & -0.56390 \\ 0.35147 & -0.80707 \\ 0.58480 & -0.85421 \\ 0.84287 & -0.64803 \\ 1.01899 & -0.16040 \\ 0.98742 & 0.56050 \\ 0.06999 & -0.14369 \\ 0.15496 & -0.41828 \\ 0.24473 & -0.06888 \\ 0.38001 & -0.82708 \\ 0.56584 & -0.85871 \\ 0.77183 & -0.73597 \\ 0.94839 & -0.43974 \\ 1.03675 & 0.02465 \\ 0.97442 & 0.61531 \\ 0.70401 & 1.25113\end{array}$

$\begin{array}{ll}0.02002 & -0.01455 \\ 0.04001 & -0.02910 \\ 0.09943 & -0.07264 \\ 0.19476 & -0.14458 \\ 0.36109 & -0.28386 \\ 0.62682 & -0.63612 \\ 0.63096 & -0.96091 \\ 0.51977 & -1.04119 \\ 0.45742 & -1.01779 \\ 0.44980 & -0.98369 \\ 0.46326 & -0.96995 \\ 0.47397 & -0.97213 \\ 0.56786 & -0.52341 \\ 0.66339 & -0.85955 \\ 0.59069 & -1.00990 \\ 0.50688 & -1.04129 \\ 0.45978 & -1.02058 \\ 0.44832 & -0.99182 \\ 0.45582 & -0.97413 \\ 0.46711 & -0.96951 \\ 0.47438 & -0.97253 \\ 0.47636 & -0.97730\end{array}$


$\frac{a}{\lambda}=0.1$

$E(h, 0)$

$0.00004-0.00012$ $0.00016-0.00047$ $0.00101-0.00295$ $0.00400-0.01173$ $0.01522-0.04578$ $0.06922-0.24635$ $0.12051-0.68423$ $0.10186-1.05165$ $0.06603-1.32009$ $0.04010-1.52315$ $0.02555-1.68924$ $0.01711-1.83254$

$0.04897-0.16197$ $0.10930-0.49736$ $0.11913-0.82746$ $0.09680-1.09505$ $0.06843-1.30314$ $0.04620-1.46949$ $0.03170-1.60928$ $0.01664-1.8417$ $0.01187-1.94151$ $0.02276-1.73178$
$0.01659-0.01205$ $0.03317-0.02410$ $\begin{array}{lll}0.08277 & -0.06022\end{array}$ $0.16457-0.12023$ $0.32161-0.23874$ $0.69942-0.56984$ $0.99707-0.99709$ $1.04928-1.26332$ $1.01049-1.40548$ $0.95376-1.46714$ $0.90729-1.48365$ $0.87781-1.47924$ $1.03366-1.10892$ $1.04694-1.28995$ $1.01447-1.39833$ $0.96971-1.4553$ $0.92861-1.47023$ $0.87638-1.47858$ $0.86451-1.46993$
$0.58294 \quad-0.45678$ $0.89722-1.48374$

$\frac{\alpha}{\beta}=0.70$

\author{
$C(h, h)$
}

$0.01658-0.01205$ $0.03313-0.02407$ $0.08230-0.06005$ $0.16095-0.11895$ $0.29693-0.2288$ $0.51564-0.4590$ $0.59662-0.4879:$ $0.61890-0.1523$ $0.55902 \quad 0.4648$ $0.24730 \quad 1.24495$ $-0.52385 \quad 1.96250$

$-1.88919 \quad 2.2448$

$0.46459-0.39581$ $0.57780-0.5257 \mathrm{i}$ $0.60757-0.40575$ $0.61840-0.0801 \mathrm{~L}$ $0.56942 \quad 0.41235$ $0.37514 \quad 1.0145$ $-0.84845 \quad 2.10678$ $-2.00428 \quad 2.23588$ $-3.46800 \quad 1.7568 \epsilon$

$\frac{\alpha}{\beta}=1.00$

$\begin{array}{lll}0.01 & 0.01374 & -0.00998 \\ 0.02 & 0.02747 & -0.01996 \\ 0.05 & 0.06858 & -0.04981 \\ 0.10 & 0.13652 & -0.09904 \\ 0.20 & 0.26813 & -0.19366 \\ 0.50 & 0.60562 & -0.42236 \\ 1.00 & 0.99429 & -0.61084 \\ 1.50 & 1.27830 & -0.67213 \\ 2.00 & 1.51180 & -0.09612 \\ 2.50 & 1.70750 & -0.71100 \\ 3.00 & 1.87302 & -0.72227 \\ 3.50 & 2.01542 & -0.73091 \\ 0.125 \pi & 0.49589 & -0.35159 \\ 0.250 \pi & 0.84680 & -0.55374 \\ 0.375 \pi & 1.10295 & -0.64080 \\ 0.500 \pi & 1.31398 & -0.67669 \\ 0.625 \pi & 1.49613 & -0.69481 \\ 0.750 \pi & 1.65464 & -0.70717 \\ 0.875 \pi & 1.79320 & -0.71698 \\ 1.000 \pi & 1.91542 & -0.72496 \\ 1.125 \pi & 2.02449 & -0.73142 \\ 1.250 \pi & 2.12294 & -0.73666 \\ & & \end{array}$

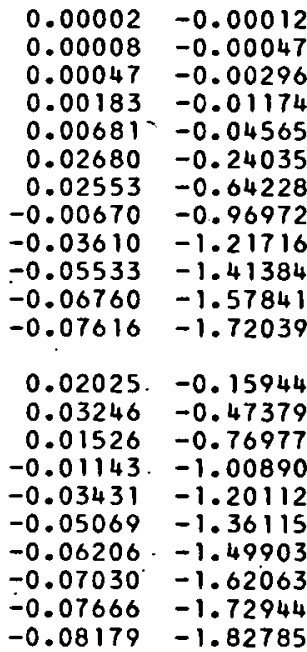

$\begin{array}{ll}0.01374 & -0.00998 \\ 0.02747 & -0.01996 \\ 0.06854 & -0.04987 \\ 0.13619 & -0.09949 \\ 0.26555 & -0.19711 \\ 0.57016 & -0.46379 \\ 0.79441 & -0.78330 \\ 0.82983 & -0.95940 \\ 0.80790 & -1.04152 \\ 0.77973 & -1.07250 \\ 0.75969 & -1.07975 \\ 0.74868 & -1.07817 \\ 0.47770 & -0.37407 \\ 0.73285 & -0.06606 \\ 0.81957 & -0.86005 \\ 0.82841 & -0.97559 \\ 0.81004 & -1.03768 \\ 0.78725 & -1.06695 \\ 0.76852 & -1.07791 \\ 0.75575 & -1.07979 \\ 0.74819 & -1.07795 \\ 0.74435 & -1.07517\end{array}$
$0.02744-0.01995$ $0.06814-0.04969$ $0.13310-0.09812$ $0.42831-0.35749$ $0.56455-0.32143$ 0.724690 .0822 $0.79183 \quad 0.88804$ $0.39105 \quad 2.09545$ $-4.22285 \quad 4.24005$ $0.38293-0.31428$ $0.50798-0.38233$ $0.61865-0.22059$ $0.79686 \quad 0.81482$ $0.58525 \quad 1.7138$ $-0.15881 \quad 2.78905$ $-1.75927,3.79803$ $\begin{array}{ll}-4.51454 & 4.23658 \\ -8.53858 & 3.25017\end{array}$
$0.01373-0.00998$ $0.24480-0.18702$ $-1.06276 \quad 3.46689$ $0.74598 \quad 0.17076$
$S(h, h)$

$E(h, h)$

$0.00004 \quad-0.00012$ $0.00016-0.00047$ $0.00100-0.00294$ $0.00385-0.01156$ $0.01324-0.04349$ $0.04421-0.20700$ $0.11696-0.52673$ $0.33533-0.77891$ $0.76142-0.84247$ $1.35306-0.54749$ $\begin{array}{ll}1.93140 & 0.28259 \\ 2.14015 & 1.73939\end{array}$ $0.03401-0.14148$ $0.07523-0.39171$ $0.17265-0.62962$ $0.38284-0.80282$ $0.72352-0.8476$ $1.17331-0.68004$ $1.66044-0.21023$ $2.04809 \quad 0.63162$ $2.12845 \quad 1.86094$ $1.63559 \quad 3.39428$
$0.01658-0.01205$ $0.03313-0.02409$ $0.08228-0.06011$ $0.16081-0.11937$ $0.29577-0.23234$ $0.49853-0.49855$ $0.50524-0.70274$ $0.45364-0.74183$ $0.43155-0.73413$ $\begin{array}{ll}0.42934 & -0.72528 \\ 0.43188 & -0.72256\end{array}$ $0.43343-0.72283$ $0.45594-0.41727$ $0.52347-0.64497$ $0.48486-0.72769$ $0.44861-0.74187$ $0.43225-0.73497$ $0.42901-0.72716$ $0.43057-0.7233$ $0.43249-0.72249$ $0.43348-0.72288$ $0.43370-0.72339$
$0.00002-0.00012$ $0.00007-0.00047$ $0.00046-0.00294$ $0.00171-0.01153$ $0.00531-0.04283$ $0.01147-0.19628$ $0.07301-0.50758$ $0.36727-0.80875$ $1.04172-0.93166$ $2.14550-0.52202$ 3.456540 .97091 4.206434 .17319 $0.01003-0.13558$ $0.01003-0.13558$ $0.14242-0.62134$ $0.43696-0.84298$ $0.97753-0.9352$ $1.78942-0.72336$ $2.80367 \quad 0.0455$ $3.77789 \quad 1.67990$ $\begin{array}{ll}4.20237 & 4.46747 \\ 3.21537 & 8.51676\end{array}$
$0.01373-0.00998$ $0.02744-0.01995$ $0.06809-0.04975$ $0.13277-0.09856$ $0.24230-0.19020$ $0.39720-0.39165$ $0.40395-0.52076$ $0.37984-0.53988$ $\begin{array}{ll}0.37196 & -0.53733\end{array}$ $0.37133-0.53502$ 53448 $0.37204-0.53451$

$0.36643-0.33303$ $0.41421-0.48780$ $0.39362-0.53347$ $0.37788-0.53989$ $0.37217-0.53758$ $0.37158-0.53461$ $0.37158-0.53461$ $0.37191-0.53447$ $\begin{array}{ll}0.37204 & -0.53452 \\ 0.37207 & -0.53457\end{array}$ 
0.02

0.05

0.10

0.20

0.50

1.50

2.00

2.50

3.00
3.50

$0.125 ; \pi$ $0.250 \pi$

0.375.

$0.625 \%$

$0.750:$

$0.875 \pi$

$1.000 \pi$

$1.250 \pi$

$0.00983-0.03027$

c. $04885-0.15102$

$0.20823-0.71483$

$0.26549-1.20783$

$0.23756-1.38567$

$0.28659-1.32464$

$0.48967-1.20103$

$0.78735-1.16656$

$1.05205-1.25899$

$0.17415-0.57381$ $0.25988-1.03333$ $0.25671-1.30671$ $0.23656-1.38800$ $0.27788-1.33351$ $0.41675-1.23243$ $0.63369-1.16724$ $0.87105-1.18145$ $1.06621-1.26854$
$0.01 \quad 0.00492-0.01514$

$0.02455-0.07564$

$0.09573-0.29997$

$$
\frac{a}{\lambda}=0.2
$$

$E(h, 0)$

$0.00002-0.00008$ $0.00010-0.00030$ $0.00061-0.00189$ $0.00951-0.03006$ $0.09236-1.63466$ $-0.01417-1.15029$ $-0.25953-1.52817$ $-0.50874-1.69299$ $-0.63306-1.71415$ $-0.60761-1.72892$

$0.03319-0.11357$ $0.08640-0.41870$ $0.07482-0.82323$ $-0.04280-1.21559$ $-0.23956-1.50781$ $-0.44564-1.66558$ $-0.59076-1.71317$ $-0.63906-1.71322$ $-0.60203-1.73268$ $0.00244-0.00755$ $0.04944-0.18103$ $-0.52687-1.81174$

$0.00492-0.01514$ $0.00983-0.03027$ $0.02456-0.07567$ $0.04893-0.15127$ $0.09536-0.30190$ $0.21618-0.74510$ $0.28306-1.42205$ $0.17703-1.97317$ $-0.07574-2.35 ? 89$ $-0.40056-2.56794$ $-0.72653-2.60831$ $99520-2.51385$

$0.17841-0.58871$ $0.27990-1.14390$ $0.26842-1.63526$ $0.14837-2.03852$ $-0.05395 \quad-2.33768$ $-0.30405 \quad-2.52622$ $-0.56673 \quad-2.60739$ $-0.81051-2.59337$ $\begin{array}{ll}-1.01042 & -2.50359\end{array}$ $\begin{array}{ll}-1.14982 & -2.36227\end{array}$

$$
\frac{a}{\lambda}=0.2 \quad \frac{\alpha}{\beta}=0.005
$$

\begin{tabular}{|c|c|c|}
\hline $\begin{array}{l}0.01 \\
0.02 \\
0.05 \\
0.10 \\
0.20 \\
0.50 \\
1.00 \\
1.50 \\
2.00 \\
2.50 \\
3.00 \\
3.50\end{array}$ & $\begin{array}{l}0.00489 \\
0.00977 \\
0.02440 \\
0.04854 \\
0.09515 \\
0.20720 \\
0.26577 \\
0.24128 \\
0.29299 \\
0.49524 \\
0.78875 \\
1.04917\end{array}$ & $\begin{array}{l}-0.01504 \\
-0.03008 \\
-0.07517 \\
-0.15007 \\
-0.29808 \\
-0.71016 \\
-1.19945 \\
-1.37647 \\
-1.31825 \\
-1.19897 \\
-1.16664 \\
-1.25704\end{array}$ \\
\hline $\begin{array}{l}0.125 \pi \\
0.250 \pi \\
0.375 \pi \\
0.500 \pi \\
0.625 \pi \\
0.750 \pi \\
0.875 \pi \\
1.000 \pi \\
1.125 \pi \\
1.250 \pi\end{array}$ & $\begin{array}{l}0.17319 \\
0.25925 \\
0.25808 \\
0.24079 \\
0.28418 \\
0.42298 \\
0.63746 \\
0.87107 \\
1.06314 \\
1.17872\end{array}$ & $\begin{array}{l}-0.57012 \\
-1.02636 \\
-1.297 .61 \\
-1.37900 \\
-1.32682 \\
-1.22919 \\
-1.16672 \\
-1.18138 \\
-1.26632 \\
-1.37875\end{array}$ \\
\hline
\end{tabular}

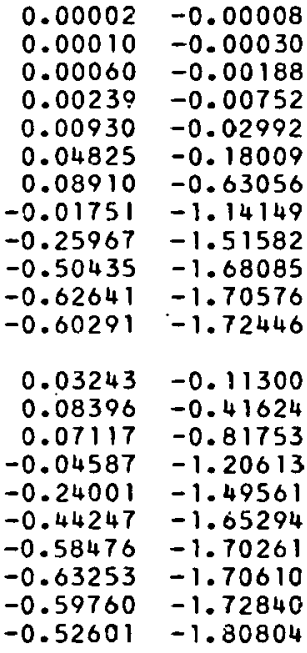

$\begin{array}{rr}0.00489 & -0.01504 \\ 0.00977 & -0.03008 \\ 0.02441 & -0.07520 \\ 0.04862 & -0.15032 \\ 0.09575 & -0.30008 \\ 0.21489 & -0.74032 \\ 0.28617 & -1.41232 \\ 0.17614 & -1.95859 \\ -0.07390 & -2.34119 \\ -0.39455 & -2.54661 \\ -0.71563 & -2.58640 \\ -0.97968 & -2.49360 \\ 0.17727 & -0.58497 \\ 0.27807 & -1.13631 \\ 0.26670 & -1.02377 \\ 0.14777 & -2.02330 \\ -0.05237 & -2.31924 \\ -0.29935 & -2.50546 \\ -0.55832 & -2.58550 \\ -0.79823 & -2.57171 \\ -0.99402 & -2.48353 \\ -1.13131 & -2.34497\end{array}$

$C(h, h)$

$S(h, h)$

$E(h, h)$

$\begin{array}{rrrr}0.00492 & -0.01514 & 0.00002 & -0.00008 \\ 0.00983 & -0.03027 & 0.00010 & -0.00030 \\ 0.02445 & -0.07560 & 0.00061 & -0.00189 \\ 0.04810 & -0.15074 & 0.00240 & -0.00754 \\ 0.09000 & -0.29773 & 0.00894 & -0.02984 \\ 0.13963 & -0.58212 & 0.03333 & -0.17279 \\ -0.03177 & -1.00081 & -0.01719 & -0.52896 \\ -0.28789 & -0.89619 & -0.77711 & -0.77964 \\ -0.48207 & -0.54132 & -0.28398 & -0.82017 \\ -0.64283 & -0.06584 & -0.28350 & -0.73905 \\ -0.75312 & 0.51021 & -0.28302 & -0.58784 \\ -0.67951 & 1.10410 & -0.34874 & -0.30001 \\ & & & \\ 0.13658 & -0.55747 & 0.02617 & -0.11035 \\ 0.06850 & -0.92051 & 0.02390 & -0.37369 \\ -0.12612 & -1.00521 & -0.06925 & -0.64159 \\ -0.31953 & -0.85661 & -0.19896 & -0.79728 \\ -0.46966 & -0.57198 & -0.28041 & -0.82275 \\ -0.59818 & -0.21318 & -0.28967 & -0.76906 \\ -0.71101 & 0.20986 & -0.27558 & -0.67515 \\ -0.75718 & 0.68422 & -0.29613 & -0.52380 \\ -0.66475 & 1.14035 & -0.35416 & -0.27374 \\ -0.40909 & 1.46265 & -0.39700 & 0.07605\end{array}$

$0.00492-0.01514$ $0.00983-0.03027$ $0.02446-0.07564$ $0.04818-0.1509$ $0.09059-0.29972$ $0.14403-0.71102$ $-0.03787-1.17994$ $-0.36326-1.30415$ $-0.58416-1.1662$ $-0.59581-0.95997$ $-0.46045-0.85591$ $-0.32274 \quad-0.90232$

$0.13995-0.57195$ $0.07419-1.01926$ $-0.15203-1.2631$ $-0.40526-1.29669$ $-0.57491-1.18113$ $-0.61284-1.0145$ $-0.53825-0.88898$ $-0.41542-0.85522$ $-0.31649-0.90960$

\begin{tabular}{|c|c|c|c|c|c|}
\hline $\begin{array}{r}0.00489 \\
0.00977 \\
0.02430 \\
0.04780 \\
0.08944 \\
0.13800 \\
-0.02938 \\
-0.28062 \\
-0.47108 \\
-0.62842 \\
-0.73507 \\
-0.66262\end{array}$ & $\begin{array}{r}-0.01504 \\
-0.03008 \\
-0.07513 \\
-0.14979 \\
-0.29583 \\
-0.67740 \\
-0.99293 \\
-0.88891 \\
-0.53658 \\
-0.06359 \\
0.50732 \\
1.09100\end{array}$ & $\begin{array}{r}0.00002 \\
0.00010 \\
0.00060 \\
0.00235 \\
C .00874 \\
C .03230 \\
-C .01884 \\
-c .17622 \\
-c .27948 \\
-c .27689 \\
-0.27349 \\
-0.33315\end{array}$ & $\begin{array}{l}-0.00008 \\
-0.00030 \\
-0.00188 \\
-0.00751 \\
-0.02969 \\
-0.17176 \\
-0.52472 \\
-0.77290 \\
-0.81427 \\
-0.73483 \\
-0.58331 \\
-0.29601\end{array}$ & $\begin{array}{r}0.00489 \\
0.00977 \\
0.02431 \\
0.04788 \\
0.09001 \\
0.14309 \\
-0.03695 \\
-0.35782 \\
-0.57471 \\
-0.58617 \\
-0.45461 \\
-0.32135\end{array}$ & $\begin{array}{l}-0.01504 \\
-0.03008 \\
-0.07516 \\
-0.15004 \\
-0.29781 \\
-0.70616 \\
-1.17060 \\
-1.29320 \\
-1.15787 \\
-0.95637 \\
-0.85515 \\
-0.89999\end{array}$ \\
\hline $\begin{array}{r}0.13583 \\
0.06915 \\
-0.12198 \\
-0.31164 \\
-0.45890 \\
-0.58484 \\
-0.69463 \\
-0.73867 \\
-0.64829 \\
-0.40105\end{array}$ & $\begin{array}{r}-0.55374 \\
-0.91359 \\
-0.97712 \\
-0.84966 \\
-0.56706 \\
-0.21013 \\
0.21016 \\
0.67890 \\
1.12645 \\
1.44017\end{array}$ & $\begin{array}{r}0.02546 \\
0.02219 \\
-0.07035 \\
-0.19753 \\
-3.27613 \\
-0.28357 \\
-0.26787 \\
-0.28516 \\
-0.33811 \\
-0.37672\end{array}$ & $\begin{array}{r}-0.10974 \\
-0.37099 \\
-0.63616 \\
-0.79045 \\
-0.81671 \\
-0.76453 \\
-0.67098 \\
-0.51909 \\
-0.26994 \\
0.07570\end{array}$ & $\begin{array}{r}0.13903 \\
0.07388 \\
-0.14968 \\
-0.39912 \\
-0.56566 \\
-0.60278 \\
-0.53015 \\
-0.41097 \\
-0.31533 \\
-0.28702\end{array}$ & $\begin{array}{l}-0.56816 \\
-1.01165 \\
-1.25273 \\
-1.28586 \\
-1.17248 \\
-1.00959 \\
-0.88727 \\
-0.85448 \\
-0.90703 \\
-1.00038\end{array}$ \\
\hline
\end{tabular}
$-0.28711-1.00657$

$\begin{array}{rr}0.00489 & -0.01504 \\ 0.00977 & -0.03008 \\ 0.02431 & -0.07516 \\ 0.04788 & -0.15004 \\ 0.09001 & -0.29781 \\ 0.14309 & -0.70616 \\ -0.03695 & -1.17060 \\ -0.35782 & -1.29320 \\ -0.57471 & -1.15787 \\ -0.58617 & -0.95637 \\ -0.45461 & -0.85515 \\ -0.32135 & -0.89999 \\ 0.13903 & -0.56816 \\ 0.07388 & -1.01165 \\ -0.14968 & -1.25273 \\ -0.39912 & -1.28586 \\ -0.56566 & -1.17248 \\ -0.00278 & -1.00959 \\ -0.53015 & -0.88727 \\ -0.41097 & -0.85448 \\ -0.31533 & -0.90703 \\ -0.28702 & -1.00038\end{array}$ 


\begin{tabular}{|c|c|c|}
\hline $\begin{array}{l}0.01 \\
0.02 \\
0.05 \\
0.10 \\
0.20 \\
0.50 \\
1.00 \\
1.50 \\
2.00 \\
2.50 \\
3.00 \\
3.50\end{array}$ & $\begin{array}{l}0.00436 \\
0.00971 \\
0.02424 \\
0.04824 \\
0.09457 \\
0.20617 \\
0.26604 \\
0.24492 \\
0.29925 \\
0.50069 \\
0.79015 \\
1.04643\end{array}$ & $\begin{array}{l}-0.01495 \\
-0.02989 \\
-0.07469 \\
-0.14913 \\
-0.29020 \\
-0.70553 \\
-1.19114 \\
-1.36734 \\
-1.31188 \\
-1.19081 \\
-1.16657 \\
-1.25500\end{array}$ \\
\hline $\begin{array}{l}0.125 \pi \\
0.250 \pi \\
0.375 \pi \\
0.500 \pi \\
0.625 \pi \\
0.750 \pi \\
0.875 \pi \\
1.000 \pi \\
1.125 \pi \\
1.250 \pi\end{array}$ & $\begin{array}{l}0.17224 \\
0.25862 \\
0.25943 \\
0.24492 \\
0.29035 \\
0.42968 \\
0.64115 \\
0.87112 \\
1.06021 \\
1.17504\end{array}$ & $\begin{array}{l}-0.56646 \\
-1.01940 \\
-11.28859 \\
-1.37006 \\
-1.32016 \\
-1.22584 \\
-1.16000 \\
-1.18115 \\
-1.26401 \\
-1.37297\end{array}$ \\
\hline
\end{tabular}

0.0

0.02

0.05

0.10

0.50

1.00

1.50

2.00

2.50

3.00

0.125

$0.250 \pi$

0.375

$0.625 \pi$

$0.750 \pi$

0.875

$1.000 \pi$

$1.125 \pi$
$1.250 \pi$

$0.00480-0.01476$ $0.00959-0.02952$ $0.02394-0.07376$ $0.04764-0.14726$ $0.09342-0.29247$ $0.20412-0.69636$ $0.26653-1.17472$ $0.25197-1.34929$ $0.31138-1.29918$ $0.51125-1.19224$ $0.79291-1.16598$ $1.04130-1.25067$
$0.00002-0.00027$ $0.00009-0.00030$ $0.00059-0.00187$ $0.00233-0.00748$ $0.00909-0.02978$ $0.04708-0.17915$ $0.08588-0.62650$ $-0.02079-1.13278$ $-0.25982-1.50362$ $-0.50008-1.66886$ $\begin{array}{ll}-0.61993 & -1.69739 \\ -0.59832 & -1.71990\end{array}$ $0.03167-0.11244$ $0.08154-0.41380$ $0.06758-0.81180$ $-0.04890-1.19679$ $-0.24048-1.48356$ $-0.43940-1.64046$ $-0.57892-1.69215$ $-0.62618-1.69897$ $-0.52512-1.80420$

$0.00002-0.00007$ $0.00009-0.00030$ $0.00056-0.00186$ $0.00223-0.00741$ $0.00869-0.02949$ $0.04479-0.17729$ $0.07957-0.61848$ $-0.02723-1.11565$ $-0.26015-1.47968$ $-0.49187-1.64530$ $-0.60750-1.68075$ $-0.58947-1.71049$

\section{$0.03019-0.11132$} $0.07679-0.40897$ $\begin{array}{rr}0.07679 & -0.40897 \\ -0.05483 & -1.8007\end{array}$ $-0.24142-1.45941$ $-0.43350-1.61596$ $-0.56771-1.67152$ $-0.61398-1.07458$ $-0.58491-1.08458$ $\begin{array}{ll}-0.61398 & -1.68458 \\ -0.52326 & -1.79614\end{array}$

$$
\frac{\mathrm{a}}{\lambda}=0.2
$$

$E(h, 0)$

$0.00486-0.01495$ $0.00971-0.02989$ $0.02425-0.07473$ $0.04832-0.14938$ $0.09515-0.29819$ $0.21342-0.73550$ $0.28429-1.40260$ $0.17526-1.94412$ $-0.07208-2.32265$ $-0.38862-2.52547$ $-0.70489-2.56460$ $-0.96439-2.47352$

$0.17614 \quad-0.58125$ $0.27625-1.12877$ $0.26498-1.01235$ $0.14717-2.0081$ $\begin{array}{ll}-0.05 C 80 & -2.30090\end{array}$ $-0.29472-2.48488$ $-0.55003-2.56380$ $-0.78613-2.55025$ $-0.97906 \quad-2.46363$ $\begin{array}{lll}-1.11310 & -2.32778\end{array}$

$$
\frac{a}{\lambda}=0.2
$$

$\begin{aligned} 0.00480 & -0.01476 \\ 0.00959 & -0.02952 \\ 0.02395 & -0.07379 \\ 0.04771 & -0.14751 \\ 0.09396 & -0.29445 \\ 0.21069 & -0.72615 \\ 0.28057 & -1.38354 \\ 0.17350 & -1.91552 \\ -0.06852 & -2.28603 \\ -0.37700 & -2.48375 \\ -0.68386 & -2.52186 \\ -0.93450 & -2.43384 \\ 0.17391 & -0.57387 \\ 0.27261 & -1.11384 \\ 0.26158 & -1.58977 \\ 0.14597 & -1.97833 \\ -0.04774 & -2.26484 \\ -0.28562 & -2.44425 \\ -0.53378 & -2.52098 \\ -0.76245 & -2.50788 \\ -0.94863 & -2.424 .32 \\ -1.07751 & -2.29372\end{aligned}$

$\frac{\alpha}{\beta}=0.01$

$\mathrm{C}(\mathrm{h}, \mathrm{h})$

$0.00486-0.01405$ $0.00970-0.02989$ $0.02415-0.07466$ $0.04750-0.14884$ $0.03890-0.29394$ $0.13738-0.67272$ $-0.02702-0.98512$ $0.27345-0.88169$ $-0.46022 \quad-0.53185$ $-0.61416-0.06133$ $\begin{array}{ll}-0.64612 & 1.07825\end{array}$

$0.13509-0.55004$ $0.06979-0.90673$ $-0.11791-0.98910$ $-0.30385-0.84275$ $-0.44827-0.56215$ $-0.57164-0.20708$ $-0.67845 \quad 0.21047$ $-0.12048 \quad 0.67369$ $\begin{array}{ll}-0.03221 & 1.11292 \\ -0.39325 & 1.41836\end{array}$
$S(h, h)$

$0.00002-0.00007$ $0.00009-0.00030$ $0.00058-0.00187$ $0.00230-0.00747$ $0.00853-0.02954$ $0.03127-0.17073$ $-0.02047-0.52053$ $-0.17534-0.76625$ $-0.27507-0.80845$ $-0.27035-0.73066$ $-0.26402-0.57887$ $-0.31777-0.29215$ $0.02477-0.10913$ $0.02050-0.36832$ $-0.07142-0.63079$ $-0.19612-0.78372$ $-0.27193-0.81074$ $-0.27755-0.76004$ $0.26022-0.66687$ $-0.27427-0.51450$ $\begin{array}{rr}-0.27427 & -0.51450 \\ -0.32228 & -0.26627 \\ & 0.07537\end{array}$
$E(h, h)$

$0.00486-0.01495$ $0.00971-0.02989$ $0.02416-0.07469$ 0.14909
0.29592 $0.08944-0.29592$ $0.14215-0.70133$ $-0.03604-1.16133$ $-0.35245-1.28235$ 0.565420 .1495 $-0.57669-0.95272$ $\begin{array}{ll}-0.44882 & -0.85425 \\ -0.31987 & -0.89759\end{array}$ $0.13812-0.56439$ $0.07358-1.00410$ $-0.14736-1.24244$ $-0.39 .307-1.27512$ $-0.5955-1.16389$ $-0.52217-1.00462$ $-0.40652-0.8854$ $-0.31406-0.85360$ $\begin{array}{ll}-0.31406 & -0.90437 \\ -0.28678 & -0.99426\end{array}$ $\frac{\alpha}{\beta}=0.02$

$\begin{array}{rr}0.09480 & -0.01476 \\ 0.00958 & -0.02952 \\ 0.02385 & -0.07373 \\ 0.04691 & -0.14698 \\ 0.08781 & -0.29020 \\ 0.13614 & -0.66346 \\ -0.02240 & -0.96970 \\ -0.25940 & -0.86739 \\ -0.43886 & -0.52241 \\ -0.58611 & -0.05682 \\ -0.68262 & 0.49903 \\ -0.61423 & 1.05376 \\ & \\ 0.13360 & -0.54270 \\ 0.07102 & -0.89317 \\ -0.10994 & -0.97326 \\ -0.28860 & -0.82907 \\ -0.42738 & -0.55236 \\ -0.54566 & -0.20099 \\ -0.64671 & 0.21112 \\ -0.68506 & 0.06364 \\ -0.60115 & 1.08694 \\ -0.37836 & 1.37067\end{array}$

$\begin{array}{rr}0.00002 & -0.00007 \\ 0.00009 & -0.00030 \\ 0.00056 & -0.00186 \\ 0.00219 & -0.00740 \\ 0.00814 & -0.02925 \\ 0.02926 & -0.16871 \\ -0.02364 & -0.51228 \\ -0.17362 & -0.75321 \\ -0.26647 & -0.79702 \\ -0.25748 & -0.72243 \\ -0.24527 & -0.57027 \\ -0.28762 & -0.28480 \\ 0.02340 & -0.10793 \\ 0.01721 & -0.36305 \\ -0.07352 & -0.62023 \\ -0.19337 & -0.77054 \\ -0.26375 & -0.79904 \\ -0.26574 & -0.75120 \\ -0.24507 & -0.65882 \\ -0.25275 & -0.50567 \\ -0.29127 & -0.25930 \\ -0.31814 & 0.07479\end{array}$

$\begin{aligned} 0.00480 & -0.01476 \\ 0.00958 & -0.02952 \\ 0.02386 & -0.07376 \\ 0.04698 & -0.14722 \\ 0.08831 & -0.29216 \\ 0.14029 & -0.09177 \\ -0.03426 & -1.14301 \\ -0.34193 & -1.26093 \\ -0.54726 & -1.13313 \\ -0.55817 & -0.94523 \\ -0.43737 & -0.85206 \\ -0.31664 & -0.89252 \\ & \\ 0.13632 & -0.55692 \\ 0.07298 & -0.98916 \\ -0.14281 & -1.22212 \\ -0.38122 & -1.25394 \\ -0.53876 & -1.14686 \\ -0.57357 & -0.99460 \\ -0.50652 & -0.88148 \\ -0.39764 & -0.85144 \\ -0.31123 & -0.89884 \\ -0.28588 & -0.98217\end{aligned}$


$\frac{a}{\lambda}=0.2 \quad \frac{\alpha}{\beta}=0.03$

$C(h, 0)$

0.01

8.02

0.05

0.10

0.50

1.00

1.50

2.00

3.00

3.50

$0.125 \pi$

0.250

$0.375 \pi$

$0.625 \pi$

$0.750 \%$

$0.875 \pi$

$1.000 \pi$

$1.125 \pi$

$0.00474-0.01458$

$0.00247-0.02915$

$0.02364-0.07284$

$\begin{array}{ll}0.04705 & -0.14542 \\ 0.09229 & -0.28879\end{array}$

$0.20209-0.08731$

$0.26696-1.15856$

$0.26696-1.15856$

$0.32300-1.28655$

$0.32300-1.28655$

$0.79562-1.10484$

$\begin{array}{ll}0.79562 & -1.16484 \\ 1.03662 & -1.24603\end{array}$

$0.16847-0.55203$

$0.25607-0.99206$

$0.26446-1.25324$

$0.26063-1.33497$

$0.31374-1.29372$

$0.45215-1.21212$

$0.07163-1.17887$

$0.87163-1.17587$

$\begin{array}{ll}1.04971 & -1.25409 \\ 1.16175 & -1.35037\end{array}$
$\mathrm{S}(\mathrm{h}, 0)$

$0.00002-0.00007$

$0.00009-0.00029$

$0.00054-0.00134$

$0.00213-0.00735$

$0.04254-0.17546$

$0.04254-0.17546$

$-0.03348-1.09889$

$-0.26054-1.45635$

$-0.26054-1.45635$

$-0.59572-1.66424$

$\begin{array}{ll}-0.59572 & -1.06424 \\ -0.58105 & -1.70073\end{array}$

$0.02874 \quad-0.11021$

$0.07216-0.40421$

$0.05368-0.78981$

$-0.06059-1.16043$

$-0.24238-1.43685$

$-0.42792-1.59206$

$-0.55709-1.65126$

$-0.60243-1.67038$

$\begin{array}{ll}-0.57693 & -1.70545 \\ -0.52129 & -1.78762\end{array}$
$E(h, 0)$

$0.00474-0.01458$

$0.02365-0.02915$

$0.04712-0.07287$

$\begin{array}{ll}0.04712 & -0.14567 \\ 0.09278 & -0.29076\end{array}$

$0.09278-0.29076$

$0.27690-1.36468$

$0.17170-1.88734$

$-0.06507-2.25000$

$-0.08507-2.25000$

$-0.06341-2.44275$

$\begin{array}{ll}-0.06341 & -2.47978 \\ -0.90550 & -2.39482\end{array}$

$0.17171-0.56659$

$0.26809-1.09910$

$0.25822-1.56751$

$0.14477-1.94891$

$-0.04476-2.22930$

$-0.27677-2.40431$

$-0.51796-2.47892$

$-0.73944-2.46626$

$\begin{array}{ll}-0.91911 & -2.38564 \\ -1.04304 & -2.26010\end{array}$

$$
\frac{a}{\lambda}=0.2
$$

$\begin{array}{rr}0.00462 & -0.01421 \\ 0.00924 & -0.02843 \\ 0.02306 & -0.07106 \\ 0.04595 & -0.14205 \\ 0.09047 & -0.28351 \\ 0.20272 & -0.09861 \\ 0.26970 & -1.32773 \\ 0.16832 & -1.83224 \\ -0.05845 & -2.17971 \\ -0.34394 & -2.36289 \\ -0.02422 & -2.39784 \\ -0.85007 & -2.31868 \\ 0.16738 & -0.55231 \\ 0.26212 & -1.07022 \\ 0.25164 & -1.52393 \\ 0.14237 & -1.89142 \\ -0.03908 & -2.15996 \\ -0.25976 & -2.32649 \\ -0.48761 & -2.39703 \\ -0.69537 & -2.38520 \\ -0.86270 & -2.31016 \\ -0.97727 & -2.19414\end{array}$

$\mathrm{C}(\mathrm{h}, \mathrm{h})$

$0.00474-0.01458$ $0.00946-0.02915$ $0.02355-C .07230$ $0.04633-0.14513$ $0.08673-0.28051$ $0.13490-0.65433$ $-0.01793-0.95454$ $-0.24575-0.85331$ $-0.41800-0.51302$ $-0.55868-0.05231$ $-0.64903 \quad 0.49385$

$0.13214-0.53547$ $0.07220 \quad-0.87983$ $-0.10220 \cdot-0.95769$ $-0.27376-0.21557$ $-0.40698-0.54263$ $-0.52023 .-0.19490$ 0.2118 0.65089 - 0.65408 $\begin{array}{ll}-0.57150 & 1.06238 \\ -0.36437 & 1.33746\end{array}$
$S(h, h)$

$0.00002-0.30007$ $0.00009-0.30029$ c. $00053-0.00184$ $0.00209-0.00733$ $0.00775-0.02897$ $0.02730-0.16670$ $-0.02671-0.50420$ $-0.17195-0.74053$ $-0.25815-0.78587$ $-0.24487-0.7858$ $0.24487-0.71436$ $\begin{array}{ll}-0.22678 & -0.56203 \\ -0.25823 & -0.27793\end{array}$ $0.02206-0.10674$ $0.01400-0.35787$ $-0.07555-0.60993$ $-0.19072-0.75771$ $-0.25585-0.78764$ $-0.25423-0.74252$ $-0.23015 .0 .65099$ $-0.23157-0.49727$ $\begin{array}{rr}-0.26109 & -0.25278 \\ -0.28086 & 0.07433\end{array}$
$E(h, h)$

$0.00474 \quad-0.01458$ $0.00946-0.02915$ $0.02356-0.07283$ $0.04639-0.14538$ $0.08719-0.28845$ $0.13845-0.68234$ $-0.03253-1.12500$ $-0.33171-1.23989$ $\begin{array}{lll}-0.52968 & -1.11687\end{array}$ $-0.54023-0.93753$ $-0.42611-0.84936$ $-0.31306-0.88715$

$0.13454-0.54955$ $0.07238-0.97446$ $-0.13839-1.20216$ $-0.36972-1.23313$ $-0.52152-1.13005$ $-0.55488-0.98448$ $-0.49131-0.87712$ $-0.38880-0.84878$ $\begin{array}{ll}-0.30802 & -0.89303 \\ -0.28449 & -0.97028\end{array}$

$\begin{array}{lllll}0.01 & 0.00462 & -0.01421 & 0.00002 & -0.00007 \\ 0.02 & 0.00923 & -0.02843 & 0.00008 & -0.00029 \\ 0.05 & 0.02306 & -0.07103 & 0.00049 & -0.00180 \\ 0.10 & 0.04589 & -0.14181 & 0.00194 & -0.00721 \\ 0.20 & 0.09007 & -0.28157 & 0.00752 & -0.02866 \\ 0.50 & 0.19810 & -0.06959 & 0.03820 & -0.17185 \\ 1.00 & 0.26762 & -1.12699 & 0.06160 & -0.59521 \\ 1.50 & 0.27141 & -1.29684 & -0.04547 & -1.06645 \\ 2.00 & 0.34476 & -1.26148 & -0.26142 & -1.41140 \\ 2.50 & 0.54020 & -1.17665 & -0.46979 & -1.57785 \\ 3.00 & 0.80089 & -1.16104 & -0.57403 & -1.63167 \\ 3.50 & 1.02845 & -1.23590 & -0.56537 & -1.68030 \\ & & & & \\ 0.125 \pi & 0.16479 & -0.53798 & 0.02594 & -0.10802 \\ 0.250 \pi & 0.25347 & -0.96552 & 0.06323 & -0.39487 \\ 0.375 \pi & 0.26898 & -1.21902 & 0.04052 & -0.76854 \\ 0.500 \pi & 0.27505 & -1.30095 & -0.07164 & -1.12568 \\ 0.625 \pi & 0.33518 & -1.26764 & -0.24435 & -1.39244 \\ 0.750 \pi & 0.47324 & -1.19755 & -0.41767 & -1.54602 \\ 0.875 \pi & 0.66806 & -1.15647 & -0.53752 & -1.61187 \\ 1.000 \pi & 0.87251 & -1.17458 & -0.58113 & -1.64179 \\ 1.125 \pi & 1.04093 & -1.24313 & -0.56206 & -1.68555 \\ 1.250 \pi & 1.15051 & -1.32852 & -0.51714 & -1.76938\end{array}$

$\begin{array}{rr}0.00462 & -0.01421 \\ 0.00923 & -0.02842 \\ 0.02297 & -0.07099 \\ 0.04518 & -0.14151 \\ 0.08462 & -0.27926 \\ 0.13246 & -0.03646 \\ -0.00940 & -0.92500 \\ -0.21956 & -0.02575 \\ -0.37773 & -0.49433 \\ -0.50563 & -0.04323 \\ -0.58494 & 0.48432 \\ -0.52679 & 0.98802 \\ 0.12926 & -0.52129 \\ 0.07441 & -0.85379 \\ -0.08740 & -0.92735 \\ -0.24529 & -0.73913 \\ -0.36761 & -0.52333 \\ -0.47100 & -0.13275 \\ -0.55631 & 0.21351 \\ -0.58605 & 0.63641 \\ -0.51616 & 1.01729 \\ -0.33893 & 1.26600\end{array}$

$0.00002-0.00007$ $\begin{array}{ll}0.00008 & -0.000029\end{array}$ $0.00049-0.00180$ $0.00190-0.00719$ $0.00700-0.02840$ $0.02352-0.16277$ $-0.03256-0.48854$ $-0.03256-0.48854$ $-0.16878-0.71616$ $-0.24234-0.76443$ $-0.22045-0.09874$ $\begin{array}{ll}-0.19056 & -0.54656 \\ -0.20165 & -0.26542\end{array}$

$0.0 .1948-0.1044$ $0.00787-0.34778$ $-0.07938-0.59065$ $-0.18567-0.73308$ $-0.24083-0.7657$ $-0.23209-0.72573$ $-0.20097 .-0.63600$ $-0.19022-0.48170$ $\begin{array}{ll}-0.20305 & -0.24094 \\ -0.2101 .1 & 0.07380\end{array}$
$0.00462-0.01421$ $0.00923-0.02843$ $0.02297-0.07102$ $0.04524-0.14176$ $0.08499-0.28118$ $0.13485-0.66386$ $-0.02923-1.08986$ $-0.31211-1.19892$ $-0.49615-1.08495$ $-0.50602-0.92155$ $-0.40416-0.84260$ $-0.30505-0.87555$

$0.13106-0.53511$ $0.07119-0.9457$ $-0.12988-1.16325$ $-0.34768-1.19260$ $-0.48864-1.09707$ $-0.51926-0.96402$ $\begin{array}{ll}-0.46212 & -0.86730\end{array}$ $-0.37125-0.84208$ $-0.30068-0.88064$ $-0.28038-0.94704$ 
0.01

0.02

0.05
0.10

0.20

0.50
1.00

1.50

2.00

2.50

3.50

$0.125 \pi$

$0.250 \pi$

$0.375 \pi$

$0.625 \pi$

$0.750 \pi$

$0.875 \pi$

$1.000 \pi$
$1.125 \pi$

$1.250 \pi$ $\frac{a}{\lambda}=0.2$

$0.00450-0.01386$ $0.00901-0.02772$ $0.02249-0.06927$ 0.044700 .13828 $0.19418-0.05234$ $0.19418-0.05234$ $0.28302-1.26322$ $0.28302-1.26322$ $0.36407-1.23570$ $0.55740-1.15541$ $1.02161-1.221474$

$0.16119-0.52430$ $0.25084-0.93977$ $0.27301-1.18590$ $0.28820-1.26795$ $0.35481-1.24194$ $0.49246-1.18231$ $0.67987-1.14916$ $0.87363-1.16451$ $\begin{array}{ll}1.03357 & -1.23125 \\ 1.14098 & -1.30730\end{array}$ $-0.45697-1.53544$ $-0.24637-1.35018$ $-0.40852-1.50218$
$S(h, 0)$

$E(h, 0)$

$0.00002-0.00007$ $0.00007-0.00028$ $0.00044-0.00177$ $0.00175-0.00707$ $0.00679-0.02812$ $0.03406-0.16832$ $0.05039-0.58031$ $-0.26243-1.36805$ $\begin{array}{ll}-0.55459 & -1.59973 \\ -0.55114 & -1.65890\end{array}$ $0.02326-0.10588$ $0.05474-0.38580$ $0.02306-0.74805$ $-0.08207-1.09245$ $-0.51998-1.57392$ $-0.54854-1.66460$ $-0.51281-1.74980$ $-0.56203-1.61334$

$\mathrm{C}(\mathrm{h}, \mathrm{h})$

$0.00450-0.01380$ $0.04481-0.13852$ $-0.65377-2.30694$

$\begin{array}{lllll}0.01 & 0.00434 & -0.01335 & 0.00002 & -0.00007 \\ 0.02 & 0.00867 & -0.02670 & 0.00006 & -0.00028 \\ 0.05 & 0.02165 & -0.06670 & 0.00037 & -0.00172 \\ 0.10 & 0.04312 & -0.13316 & 0.00148 & -0.00688 \\ 0.20 & 0.08474 & -0.26432 & 0.00575 & -0.02732 \\ 0.50 & 0.18844 & -0.62734 & 0.02819 & -0.16316 \\ 1.00 & 0.26825 & -1.05228 & 0.03465 & -0.55886 \\ 1.50 & 0.29857 & -1.21474 & -0.07257 & -0.99123 \\ 2.00 & 0.39132 & -1.20012 & -0.26411 & -1.30835 \\ 2.50 & 0.58029 & -1.14557 & -0.44019 & -1.47537 \\ 3.00 & 0.81280 & -1.14404 & -0.52913 & -1.55308 \\ 3.50 & 1.01333 & -1.20635 & -0.53225 & -1.62550 \\ 0.125 \pi & 0.15594 & -0.50445 & 0.01947 & -0.10275 \\ 0.250 \pi & 0.24684 & -0.90254 & 0.04278 & -0.37265 \\ 0.375 \pi & 0.27820 & -1.13819 & 0.01063 & -0.71867 \\ 0.500 \pi & 0.30599 & -1.22029 & -0.09662 & -1.04529 \\ 0.625 \pi & 0.38109 & -1.20413 & -0.24941 & -1.29056 \\ 0.750 \pi & 0.51808 & -1.15341 & -0.39661 & -1.44028 \\ 0.875 \pi & 0.69564 & -1.13564 & -0.49700 & -1.51961 \\ 1.000 \pi & 0.87551 & -1.15657 & -0.53699 & -1.57111 \\ 1.125 \pi & 1.02462 & -1.21196 & -0.53053 & -1.63176 \\ 1.250 \pi & 1.12923 & -1.27639 & -0.50615 & -1.71858\end{array}$
$0.00901-0.02772$ $\therefore .02249-0.06930$ $0.08822-0.27645$ $0.19758-0.68083$ $0.26208-1.29179$ $0.16493-1.77878$ $-0.05221-2.11169$ $-0.32334-2.28578$ $\begin{array}{ll}-0.58719 & -2.31877 \\ -0.79789 & -2.24501\end{array}$

$0.16317-0.53839$ $0.25533-1.04209$ $0.24522-1.48157$ $0.13999-1.83566$ $-0.03373-2.09285$ $-0.24364-2.25131$ $-0.45889-2.31800$ $\begin{array}{ll}-0.80961 & -2.23710 \\ -0.91554 & -2.12988\end{array}$

$$
\frac{a}{\lambda}=0.2 \quad \frac{\alpha}{\beta}=0.10
$$

$\begin{array}{rr}0.00450 & -0.01396 \\ 0.00900 & -0.02772 \\ 0.02240 & -0.06923 \\ 0.04407 & -0.13798 \\ 0.03256 & -0.27220 \\ 0.13006 & -0.61910 \\ -0.00158 & -0.89645 \\ -0.19480 & -0.79901 \\ -0.33931 & -0.47599 \\ -0.45496 & -0.03422 \\ -0.52476 & 0.47596 \\ -0.47469 & 0.95022 \\ 0.12644 & -0.50750 \\ 0.07642 & -0.82859 \\ -0.07345 & -0.89804 \\ -0.21834 & -0.76342 \\ -0.33003 & -0.50430 \\ -0.42391 & -0.17065 \\ -0.49986 & 0.21551 \\ -0.52560 & 0.62066 \\ -0.46567 & 0.97727 \\ -0.31605 & 1.20311\end{array}$

$0.00434 \quad-0.01335$ $0.00867-0.02670$ $0.02166-0.06673$ $0.04315-0.13339$ $0.08494-0.26618$ $0.19010-0.65502$ $0.25251-1.23970$ $0.15995-1.70155$ $\begin{array}{lll}-0.04351 & -2.01377\end{array}$ $-0.29444-2.17505$ $-0.72530-2.13890$

$0.15704-0.51816$ $0.24547-1.00129$ $0.13645-1.75515$ $-0.02629-1.99620$ $-0.41866-2.20460$ $\begin{array}{ll}-0.41860 & -2.20460 \\ -0.59572 & -2.19461\end{array}$ $-0.73578-2.13190$ $-0.82996-2.03664$ $-0.53545 \quad-2.20532$ $-0.22102-2.14330$

$1.02462-1.2 .1196$

$$
S(h, h)
$$

$0.00002 \quad-0.00007$ $0.00007-0.00028$ $0.0044-0.00177$ $0.00172-0.05706$ $0.01928-0.02785$ $0.01992-0.15895$ $-0.03803-0.47354$ $-0.16579-0.69307$ $-0.22754-0.74408$ $-0.19705-0.68384$ $-0.15538-0.5323$ $0.01703-0.10212$ $0.00209-0.33804$ $-0.08294-0.57108$ $-0.18093-0.70975$ $-0.22678-C .74491$ $-0.21103-0.70968$ $-0.17268-0.62189$ $-0.15021-0.46761$ $\begin{array}{rr}-0.14789 & -0.23045 \\ -0.14390 & 0.07388\end{array}$
$E(h, h)$

$0.00450-0.01380$ $0.00900-0.0277$ $0.02240-0.06926$ $0.04411-0.13822$ $0.08285-0.27408$ $0.13134-0.64589$ $-0.02611-1.05585$ $-0.29360-1.15939$ $-0.46468-1.05378$ $-0.47391-0.90492$ $-0.38300-0.8342$ $-0.29610-0.86295$

$0.12760 \quad-0.52105$ $0.07000-0.91783$ $-0.12 .182-1.12560$ $-0.32689-1.15347$ $-0.45777-1.06494$ $-0.48588-0.94334$ $-0.43452-0.85620$ $-0.35396-0.83375$ $\begin{array}{ll}-0.29232 & -0.86736 \\ -0.27480 & -0.92443\end{array}$

$\begin{array}{rrrr}0.00434 & -0.01335 & 0.00002 & -0.00007 \\ 0.00867 & -0.02669 & 0.00006 & -0.00028 \\ 0.02157 & -0.06667 & 0.00037 & -0.00172 \\ 0.04244 & -0.13285 & 0.00145 & -0.00686 \\ 0.07957 & -0.26194 & 0.00526 & -0.02704 \\ 0.12652 & -0.59399 & 0.01487 & -0.15339 \\ 0.00974 & -0.85542 & -0.04558 & -0.45217 \\ -0.16017 & -0.76039 & -0.16160 & -0.06066 \\ -0.28500 & -0.44891 & -0.20704 & -0.71552 \\ -0.39323 & -0.02057 & -0.16372 & -0.66287 \\ -0.44129 & 0.46562 & -0.10459 & -0.51327 \\ -0.40472 & 0.90177 & -0.07152 & -0.23990 \\ & & & \\ 0.12232 & -0.48752 & 0.01356 & -0.09879 \\ 0.07912 & -0.79228 & -0.00596 & -0.32406 \\ -0.05402 & -0.85592 & -0.08779 & -0.54424 \\ -0.18060 & -0.72622 & -0.17436 & -0.67706 \\ -0.27708 & -0.47634 & -0.20734 & -0.71574 \\ -0.35711 & -0.15261 & -0.18134 & -0.68705 \\ -0.42058 & 0.21922 & -0.13190 & -0.60238 \\ -0.44243 & 0.60058 & -0.09261 & -0.44894 \\ -0.39801 & 0.92603 & -0.06993 & -0.21673 \\ -0.28860 & 1.12318 & -0.05188 & 0.07529\end{array}$

$0.00434-0.01335$ $0.00867-0.02669$ $0.02157-0.06669$ $0.04247-0.13309$ $0.07974 \quad-0.26378$ $0.12625-0.61985$ $-0.02176-1.00688$ $-0.26772-1.10266$ $-0.42108-1.00847$ $-0.42941-0.87900$ $-0.35275-0.81908$ $-0.28141-0.84248$

$0.12274-0.50064$ $0.06822-0.87757$ $-0.11051-1.07165$ $-0.29786-1.09731$ $-0.41498-1.01832$ $-0.43970-0.91206$ $-0.39593-0.83754$ $-0.32869-0.81870$ $-0.27835-0.84605$ $-0.26432-0.89152$ 


$$
\frac{a}{\lambda}=0.2 \quad \frac{\alpha}{\beta}=0.20
$$

\section{$0.125 \pi$
$0.250 \pi$} $0.250 \pi$

$0.500 \pi$

$0.500 \pi$

$0.625 \pi$

$0.875 \pi$

$1.000 \mathrm{r}$

$1.125 \%$

1.250,

$0.00382-0.01177$ $0.00765-0.02354$ $0.01910-2.05882$ $0.03806-0.1174$ $0.07502-0.23293$ $0.17049-0.55100$ $0.26586-0.91928$ $0.45674-1.08402$ $0.63524-1.07143$ $0.82951-1.08816$

$0.13965-0.44369$ $0.23314-0.78973$ $0.28910-0.99471$ $0.34955-1.07615$ $0.44569-1.08493$ $0.57997-1.07337$ $0.73321-1.07487$ $0.88069-1.09933$ $1.10347-1.17891$
$0.00001-0.00006$ $0.00003-0.00025$ $0.00018-0.00157$ $0.00072-0.00625$ $0.00275-0.02482$ $0.01144-0.14713$ $-0.00943-0.49438$ $-0.11594-0.80263$ $-0.27025-1.13597$ $-0.40043-1.30195$ $-0.46874-1.40950$ $-0.48582-1.50994$

\section{$0.00858-0.08295$} $0.00894-0.33253$ $\begin{array}{lll}-0.03777 & -0.63142\end{array}$ $-0.13659-0.90842$ $-0.25903-1.12001$ $-0.44244-1.35907$ $-0.47745-1.43708$ $-0.48594-1.51731$

$-0.48493-1.60683$
$E(h, 0)$

$\mathrm{C}(\mathrm{h}, \mathrm{h})$

$\begin{aligned} 0.00382 & -0.01177 \\ 0.00765 & -0.02354 \\ 0.01910 & -0.05885 \\ 0.03805 & -0.11762 \\ 0.07487 & -0.23462 \\ 0.16717 & -0.57583 \\ 0.22134 & -1.08084 \\ 0.14418 & -1.40793 \\ -0.01961 & -1.72003 \\ -0.21349 & -1.84508 \\ -0.39171 & -1.86777 \\ -0.52589 & -1.82115 \\ 0.13824 & -0.45606 \\ 0.21529 & -1.87649 \\ 0.20730 & -1.23374 \\ 0.12491 & -1.51188 \\ -0.00599 & -1.70614 \\ -0.15762 & -1.82091 \\ -0.30635 & -1.86721 \\ -0.43496 & -1.86009 \\ -0.53310 & -1.81629 \\ -0.59674 & -1.75207\end{aligned}$
$0.00764-0.02354$ $0.01903-0.05878$ $0.03745-0.11710$ $0.07036-0.23049$ $0.11531-0.51769$ $0.03992-0.73287$ $-0.06363-0.64405$ $-0.12983-0.36414$ $\begin{array}{lll}-0.17798 & 0.02547\end{array}$ $\begin{array}{ll}-0.21292 & 0.45015\end{array}$ $-0.228 .20 \quad 0.80151$

$0.10955-0.42657$ $0.08558-0.68322$ $-0.00054-0.73025$ $-0.07507-0.01367$ $-0.12587-0.38906$ $-0.16514-0.09402$ $-0.19765 \quad 0.23849$ $-0.21915$ $-0.228710 .81989$ $-0.23406 \quad 0.95809$
$S(h, h)$

$0.00001-0.00000$ $0.00003-0.00025$ $0.00018-0.01156$ $0.00070-0.00623$ $0.00234-0.02448$ $0.00063-0.13635$ $-0.06567-0.39977$ $-0.14954-0.56944$ $-0.15056-0.63578$ $0.04895-0.46569$ $0.00371-0.08849$ $-0.02801-0.28237$ $-0.10017-0.46709$ $-0.15594 \quad-0.58531$ $-0.15391-0.63431$ $-0.09609-0.62401$ $-0.00935-0.55088$ $0.08055-0.40357$ $0.15761-0.18182$ 0.209620 .09296
$\mathrm{E}(\mathrm{h}, \mathrm{h})$

$0.00382-0.01177$ $0.00764-0.02354$ $0.01902-0.05881$ $0.03743-0.11731$ $0.07018-0.23215$ $0.11067-0.54042$ $-0.00981-0.86002$ $-0.19585-0.93389$ $-0.30239-0.86954$ $-0.30826-0.78820$ $-0.26481-0.75368$ $-0.22783-0.76548$

$0.10765-0.43824$ $0.06246-0.75594$ $-0.07881 \quad-0.91046$ $-0.21748 \quad-0.93005$ $-0.29837-0.87604$ $-0.31449-0.80823$ $-0.28878-0.76398$ $-0.25196-0.75346$ $-0.22633-0.76722$ $-0.21963-0.78856$

$\begin{array}{lll}0.01 & 0.00298 & -0.00916 \\ 0.02 & 0.00595 & -0.01831 \\ 0.05 & 0.01486 & -0.04575 \\ 0.10 & 0.02966 & -0.09129 \\ 0.00 & 0.05882 & -0.18097 \\ 0.50 & 0.13948 & -0.42009 \\ 1.00 & 0.25108 & -0.70714 \\ 1.50 & 0.36700 & -0.83436 \\ 2.00 & 0.51263 & -0.88159 \\ 2.50 & 0.67590 & -0.90922 \\ 3.00 & 0.83238 & -0.94106 \\ 3.50 & 0.96823 & -0.97530 \\ 0.125 \pi & 0.11204 & -0.34376 \\ 0.250 \pi & 0.20530 & -0.60794 \\ 0.375 \pi & 0.28971 & -0.76703 \\ 0.500 \pi & 0.38576 & -0.84459 \\ 0.625 \pi & 0.50110 & -0.87935 \\ 0.750 \pi & 0.62865 & -0.90120 \\ 0.875 \pi & 0.75588 & -0.92433 \\ 1.000 \pi & 0.87316 & -0.95086 \\ 1.125 \pi & 0.97673 & -0.97754 \\ 1.250 \pi & 1.06766 & -1.00082\end{array}$

$-0.00000-0.00005$ $-0.00001-0.00021$ $-0.00009-0.00129$ $-0.00035-0.00516$ $-0.00147-0.02043$ $-0.01169-0.11982$ $\begin{array}{lll}-0.06710 & -0.39176\end{array}$ $\begin{array}{ll}-0.16942 & -0.67095\end{array}$ $\begin{array}{ll}-0.27919 & -0.88807\end{array}$ $-0.36164-1.04735$ $-0.41027-1.17656$ $-0.43698-1.29507$

$-0.00055-0.07606$ $-0.03649-0.26668$ $-0.09977-0.26068$ $-0.18557-0.70593$ $-0.27184-0.87448$ $-0.34161-1.0058$ $-0.38954-1.11385$ $-0.41940-1.21075$ $\begin{array}{ll}-0.43840 & -1.30300 \\ -0.45276 & -1.3920\end{array}$

$$
\frac{\mathrm{a}}{\lambda}=0.2 \quad \frac{\alpha}{\beta}=0.40
$$

$\begin{aligned} 0.00297 & -0.00916 \\ 0.00595 & -0.01831 \\ 0.01485 & -0.04577 \\ 0.02953 & -0.09146 \\ 0.05817 & -0.18229 \\ 0.12927 & -0.44502 \\ 0.17011 & -0.82186 \\ 0.11647 & -1.09385 \\ 0.01027 & -1.25828 \\ -0.10551 & -1.33348 \\ -0.20303 & -1.34624 \\ -0.27009 & -1.32321 \\ 0.10711 & -0.35330 \\ 0.16562 & -0.67170 \\ 0.16006 & -0.93151 \\ 0.10351 & -1.12340 \\ 0.01879 & -1.24959 \\ -0.07318 & -1.31948 \\ -0.15743 & -1.34590 \\ -0.22531 & -1.34229 \\ -0.27351 & -1.32090 \\ -0.30257 & -1.29171\end{aligned}$

$\begin{array}{rr}-0.00000 & -0.00005 \\ -0.00001 & -0.00021 \\ -0.00009 & -0.00129 \\ -0.00037 & -0.00513 \\ -0.00174 & -0.02004 \\ -0.01825 & -0.10812 \\ -0.08811 & -0.29670 \\ -0.13007 & -0.44435 \\ -0.07249 & -0.53277 \\ 0.08472 & -0.54021 \\ 0.30361 & -0.42297 \\ 0.52185 & -0.15063 \\ -0.00964 & -0.07106 \\ -0.05501 & -0.21737 \\ -0.11165 & -0.35582 \\ -0.12861 & -0.46063 \\ -0.08037 & -0.52860 \\ 0.03107 & -0.54883 \\ 0.18925 & -0.50007 \\ 0.36885 & -0.36203 \\ 0.53469 & -0.12625 \\ 0.64178 & 0.19788\end{array}$

$0.00297-0.00916$ $0.00594-0.01831$ $0.01479-0.04573$ $0.02909 \quad-0.09114$ $0.05438-0.17981$ $0.08505-0.41093$ $0.00513-0.62914$ $-0.10151-0.67312$ $-0.15304-0.64303$ $-0.15586-0.61085$ $-0.14187-0.59937$

$0.08281-0.33585$ $0.05176-0.56170$ $-0.03659-0.65974$ $-0.11265-0.67114$ $-0.15128-0.64586$ $-0.15813-0.61821$ $-0.14926-0.60258$ $-0.13820-0.59931$ $-0.13155-0.60279$ $-0.13002-0.60750$ 
$\beta \mathrm{h}$

$C(h, 0)$

$S(h, 0)$

$0.00204-0.00625$

$0.00408-0.01253$

$0.01020-0.03133$

$0.02041-0.06261$

$0.04086-0.12405$

$0.10310-0.29145$

$0.21700-0.48602$

$0.35240-0.58992$

$0.50748-0.64944$

$0.64795-0.69273$

$\begin{array}{ll}0.78192 & -0.72822 \\ 0.90162 & -0.75710\end{array}$

$0.08059-0.23531$

$0.16578-0.41601$

$0.26275-0.53096$

$0.37306-0.60011$

$0.49051-0.64584$

$0.60685-0.68122$

$0.71646-0.71125$

$0.81721-0.73706$

$\begin{array}{lll}1.125 \pi & 0.90934 & -0.75885 \\ 1.250 \pi & 0.99402 & -0.77700\end{array}$ $\frac{a}{\lambda}=0.2 \quad \frac{\alpha}{\beta}=0.70$

$E(h, 0)$

$\mathrm{C}(\mathrm{h}, \mathrm{h})$

$S(h, h)$

$E(h, h)$

$\begin{array}{lll}-0.00001 & -0.00004\end{array}$

$-0.00005-0.00015$

$-0.00029-0.00096$

$-0.00118-0.00384$

$-0.00470-0.01520$

$-0.02881-0.08834$

$-0.10496-0.28394$

$-0.19724-0.48599$

$-0.27315-0.65798$

$-0.33256-0.80379$

$\begin{array}{ll}-0.37118 & -0.93291 \\ -0.39910 & -1.05025\end{array}$

$-0.01794-0.05628$

$-0.06814-0.19454$

$\begin{array}{ll}-0.13787 & -0.35846\end{array}$

$-0.27115-0.64644$

$-0.31849-0.76390$

$-0.35353-0.86972$

$-0.37991-0.96723$

$\begin{array}{ll}-0.40075 & -1.05791 \\ -0.41800 & -1.14238\end{array}$
$0.00204-0.00623$

$0.00408-0.0125 s$

$0.01016-0.03134$

$\begin{array}{ll}0.02005 & -0.06232 \\ 0.03509 & -0.12182\end{array}$

$\begin{array}{ll}0.03509 & -0.12182 \\ 0.07150 & -0.26370\end{array}$

$\begin{array}{ll}0.07150 & -0.26370 \\ 0.09065 & -0.35104\end{array}$

$\begin{array}{ll}0.09665 & -0.35104 \\ 0.16148 & -0.2820^{\circ}\end{array}$

$\begin{array}{ll}0.16148 & -0.2820^{\circ} \\ 0.27075 & -0.07592\end{array}$

$\begin{array}{rr}0.27075 & -0.07592 \\ 0.35980 & 0.26403\end{array}$

$\begin{array}{ll}0.35980 & 0.26403 \\ 0.31230 & 0.71069\end{array}$

$\begin{array}{rr}0.001419 & 0.71069\end{array}$

$0.06310-0.22052$

$0.08466-0.33518$

$0.11311-0.34262$

$0.17512-0.26118$

$0.26234-0.09559$

$0.34226 \quad 0.15333$

$0.36198 \quad 0.47662$

$0.25427 \quad 0.84664$

$\begin{array}{ll}-0.05015 & 1.20589 \\ -0.50342 & 1.45730\end{array}$
$-0.00001-0.00004$ $-0.00005-0.00015$ $-0.00029-0.00090$ $-0.00119-0.00381$ $\begin{array}{lll}-0.00481 & -0.01479\end{array}$ $-0.03081-0.07699$ $-0.09545-0.20823$ $-0.10483-0.34103$ $0.00359-0.46098$ $0.25803-0.53123$ $0.64857-0.43254$ $1.04308-0.04806$

$-0.01904-0.05128$ $-0.06912-0.15153$ $-0.10934-0.25520$ $-0.09758-0.35995$ $-0.00909-0.45890$ $0.16946-0.52433$ $0.43840-0.51002$ $0.77496-0.35794$ $1.12149-0.00832$ 1.376810 .58369
$0.00204-0.00628$ $0.00408-0.01256$ $0.01014-0.03135$ $0.01992-0.06242$ $0.03708-0.12258$ $0.05732-0.27268$ $0.01402-0.39620$ $-0.032 .49-0.41644$ $-0.04990-0.40677$ $-0.05080-0.39872$ $-0.04823-0.3965$ $-0.04683-0.39690$

$0.05589-0.22539$ $0.03819-0.36099$ $-0.00561-0.41066$ $-0.03661-0.41571$ $-0.04940-0.40758$ $-0.05130-0.40036$ $-0.04949-0.39707$ $-0.04767-0.39650$ $-0.04678-0.39694$ $-0.04662-0.39743$

$\begin{array}{lll}0.01 & 0.00140 & -0.00431 \\ 0.02 & 0.00280 & -0.00861 \\ 0.05 & 0.00701 & -0.02152 \\ 0.10 & 0.01405 & -0.04295 \\ 0.20 & 0.02842 & -0.08513 \\ 0.50 & 0.07616 & -0.20073 \\ 1.00 & 0.18019 & -0.34088 \\ 1.50 & 0.31064 & -0.42719 \\ 2.00 & 0.44906 & -0.48542 \\ 2.50 & 0.58152 & -0.52900 \\ 3.00 & 0.70310 & -0.56282 \\ 3.50 & 0.81370 & -0.58934 \\ 0.125 \pi & 0.05804 & -0.16178 \\ 0.250 \pi & 0.13152 & -0.28888 \\ 0.375 \pi & 0.22450 & -0.37629 \\ 0.500 \pi & 0.33019 & -0.43668 \\ 0.625 \pi & 0.43904 & -0.48175 \\ 0.750 \pi & 0.54445 & -0.51760 \\ 0.875 \pi & 0.64346 & -0.54688 \\ 1.000 \pi & 0.73549 & -0.57098 \\ 1.125 \pi & 0.82092 & -0.59094 \\ 1.250 \pi & 0.90043 & -0.60762\end{array}$

$\begin{array}{ll}-0.00001 & -0.00003 \\ -0.00006 & -0.00011 \\ -0.00036 & -0.00071 \\ -0.00145 & -0.00284 \\ -0.00575 & -0.01125 \\ -0.03384 & -0.06534 \\ -0.11159 & -0.21117 \\ -0.19264 & -0.36897 \\ -0.25687 & -0.51476 \\ -0.30360 & -0.64712 \\ -0.33802 & -0.76784 \\ -0.36442 & -0.87813 \\ -0.02145 & -0.04164 \\ -0.07569 & -0.14409 \\ -0.14170 & -0.26810 \\ -0.20292 & -0.39048 \\ -0.25283 & -0.50459 \\ -0.29166 & -0.61032 \\ -0.32196 & -0.70859 \\ -0.34617 & -0.80009 \\ -0.36601 & -0.88533 \\ -0.38259 & -0.96482\end{array}$

$\frac{\alpha}{\beta}=1.00$

$\frac{\mathrm{a}}{\lambda}=0.2$

$0.00140-0.00431$ $0.00280-0.00862$ $0.00699-0.02153$ $0.01391-0.04300$ $0.02728-0.08549$ $0.05979-0.20547$ $0.07728-0.36223$ $0.05923-0.45681$ $0.03019-0.50258$ $0.00543-0.51900$ $-0.01060-0.52126$

$-0.01901-0.51848$

$0.04983-0.16426$ $0.07546-0.30265$ $0.07360-0.40269$ $0.05529-0.46580$ $0.03228-0.50046$ $0.01171-0.51628$ $-0.00364-0.52119$ $-0.01365-0.52073$ $-0.01837-0.51823$ $\begin{array}{ll}-0.01937 & -0.51823 \\ -0.02215 & -0.51548\end{array}$
$0.00140-0.00431$ $0.00280-0.00861$ $0.00697-0.02149$ $0.01378-0.04270$ $0.02640-0.08323$ $0.05329-0.17772$ $0.09702-0.23296$ $0.20179-0.17558$ 0.370210 .02059 $0.51748 \quad 0.42148$ 0.51748
-0.17099

$0.04534-0.14937$ $0.07379-0.22365$

$\begin{array}{lll}0.12550 & -0.22557\end{array}$

$0.22271-0.15750$

0.357020 .00025

0.486410 .28101

0.526790 .71717 $\begin{array}{rr}0.34432 & 1.31403\end{array}$ $\begin{array}{ll}-1.44767 & 2.61195\end{array}$

$\begin{array}{ll}-0.00001 & -0.00003 \\ -0.00006 & -0.00011 \\ -0.00036 & -0.00071 \\ -0.00145 & -0.00282 \\ -0.00576 & -0.01086 \\ -0.03305 & -0.05531 \\ -0.08825 & -0.15450 \\ -0.08225 & -0.28819 \\ 0.05999 & -0.45681 \\ 0.42159 & -0.58524 \\ 1.06222 & -0.48852 \\ 1.92287 & 0.15154 \\ -0.02128 & -0.03710 \\ -0.06750 & -0.10918 \\ -0.09697 & -0.19698 \\ -0.07223 & -0.31071 \\ 0.04321 & -0.44439 \\ 0.29046 & -0.56082 \\ 0.70496 & -0.58193 \\ 1.29141 & -0.37944 \\ 1.98381 & 0.22569 \\ 2.59073 & 1.44177\end{array}$

$0.00140-0.00431$ $0.00280-0.00861$ $0.00695-0.02150$ $0.01364-0.04275$ $0.02529-0.08357$ $\begin{array}{ll}0.03864 & -0.1811 \\ 0.01510 & -0.25129\end{array}$ $-0.00530-0.26063$ $-0.01122-0.25751$ $-0.01149-0.25548$ $-0.01101-0.25505$ $-0.01082-0.25510$

$0.03773-0.15132$ $0.02764-0.23290$ $0.00585-0.25814$ $-0.00682-0.26036$ $-0.01160-0.25585$ $-0.01123-0.25585$ $-0.01123-0.25515$ $0.01093-0.25505$ $\begin{array}{ll}-0.01081 & -0.25511 \\ -0.01079 & -0.25516\end{array}$ 
$S(h, 0)$

$$
\frac{\mathrm{a}}{\lambda}=0.3
$$

$E(h, 0)$

\begin{tabular}{|c|c|c|}
\hline $\begin{array}{l}0.01 \\
0.02 \\
0.05 \\
0.10 \\
0.20 \\
0.50 \\
1.00 \\
1.50 \\
2.00 \\
2.50 \\
3.00 \\
3.50\end{array}$ & $\begin{array}{r}-0.00328 \\
-0.00656 \\
-0.01640 \\
-0.03281 \\
-0.06572 \\
-0.16542 \\
-0.32199 \\
-0.40734 \\
-0.34876 \\
-0.14318 \\
0.12371 \\
0.33698\end{array}$ & $\begin{array}{l}-0.01009 \\
-0.02018 \\
-0.05042 \\
-0.10067 \\
-0.19988 \\
-0.47503 \\
-0.79605 \\
-0.90666 \\
-0.87539 \\
-0.83606 \\
-0.88914 \\
-1.03822\end{array}$ \\
\hline $\begin{array}{l}0.125 \pi \\
0.250 \pi \\
0.375 \pi \\
0.500 \pi \\
0.625 \pi \\
0.750 \pi \\
0.875 \pi \\
1.000 \pi \\
1.125 \pi \\
1.250 \pi\end{array}$ & $\begin{array}{r}-0.12964 \\
-0.25870 \\
-0.36500 \\
-0.40890 \\
-0.35855 \\
-0.21365 \\
-0.01046 \\
0.19381 \\
0.34755 \\
0.42746\end{array}$ & $\begin{array}{l}-0.38178 \\
-0.68376 \\
-0.85843 \\
-0.90802 \\
-0.87961 \\
-0.84077 \\
-0.84820 \\
-0.92399 \\
-1.05022 \\
-1.18281\end{array}$ \\
\hline
\end{tabular}

\begin{tabular}{|c|c|c|}
\hline $\begin{array}{l}0.01 \\
0.02 \\
0.05 \\
0.10 \\
0.20 \\
0.50 \\
1.00 \\
1.50 \\
2.00 \\
2.50 \\
3.00 \\
3.50\end{array}$ & $\begin{array}{l}-0.00325 \\
-0.00650 \\
-0.01624 \\
-0.03250 \\
-0.06509 \\
-0.16365 \\
-0.31753 \\
-0.39999 \\
-0.34043 \\
-0.13724 \\
0.12503 \\
0.33470\end{array}$ & $\begin{array}{l}-0.01000 \\
-0.01999 \\
-0.04995 \\
-0.09972 \\
-0.19800 \\
-0.47059 \\
-0.78897 \\
-0.96002 \\
-0.87189 \\
-0.83593 \\
-0.88962 \\
-1.03560\end{array}$ \\
\hline $\begin{array}{l}0.125 \pi \\
0.250 \pi \\
0.375 \pi \\
0.500 \pi \\
0.625 \pi \\
0.750 \pi \\
0.875 \pi \\
1.000 \pi \\
1.125 \pi \\
1.250 \pi\end{array}$ & $\begin{array}{r}-0.12832 \\
-0.25551 \\
-0.35944 \\
-0.40124 \\
-0.35019 \\
-0.20669 \\
-0.00672 \\
0.19385 \\
0.34514 \\
0.42507\end{array}$ & $\begin{array}{l}-0.37821 \\
-0.67749 \\
-0.85112 \\
-0.90169 \\
-0.87583 \\
-0.83984 \\
-0.84885 \\
-0.92393 \\
-1.04729 \\
-1.17624\end{array}$ \\
\hline
\end{tabular}

$\begin{array}{ll}-0.00002 & -0.00005 \\ -0.00007 & -0.00020 \\ -0.00041 & -0.00126 \\ -0.00164 & -0.00504 \\ -0.00662 & -0.02002 \\ -0.04336 & -0.12008 \\ -0.19267 & -0.41461 \\ -0.45730 & -0.73188 \\ -0.77429 & -0.93669 \\ -1.03002 & -0.99440 \\ -1.14290 & -0.97759 \\ -1.12348 & -0.99699 \\ -0.02622 & -0.07548 \\ -0.11375 & -0.27570 \\ -0.27520 & -0.53347 \\ -0.50141 & -0.76983 \\ -0.75181 & -0.92702 \\ -0.96898 & -0.99015 \\ -1.10580 & -0.98822 \\ -1.14795 & -0.97521 \\ -1.11932 & -1.00172 \\ -1.06845 & -1.09109\end{array}$

$-0.00328-0.01009$ $-0.00656-0.02018$ $-0.03286-0.10083$ $-0.06616 \quad-0.20121$ $-0.17282-0.49509$ $\begin{array}{lll}-0.39142 & -0.93530\end{array}$ $-0.67238-1.27477$ $-0.99810-1.48405$ $-1.32965-1.55440$ $\begin{array}{ll}-1.62245 & -1.49835 \\ -1.83881 & -1.34648\end{array}$

$-0.13312-0.39168$ $-0.29007-0.75633$ $-0.48462-1.06970$ $\begin{array}{ll}-0.48462 & -1.06970 \\ -0.71653 & -1.31275\end{array}$ $-0.97350-1.47350$ $-1.23634-1.54805$ $-1.23634-1.54805$ $-1.09279-1.46338$ $-1.85017-1.33358$ $-1.94548-1.17265$ $-0.01640-0.05045$
$-0.00328-0.0100$ $-0.03303-0.10044$ $\begin{array}{ll}-0.06743 & -0.19807\end{array}$ $-0.18747-0.44369$ $-0.41999-0.631059$ $\begin{array}{ll}-0.56564 & -0.51902\end{array}$ $-0.57270-\mathrm{j} .25590$ $\begin{array}{ll}-0.41786 & 0.48468 \\ -0.19573 & 0.90933\end{array}$

$-0.14127-0.358 .51$ $-0.32184-0.59321$ $-0.48849-0.61827$ $-0.57398-0.48760$ $\begin{array}{rr}-0.557535 & -0.27770 \\ -0.47691 & 0.02704\end{array}$ $-0.37112 \quad 0.26095$ $-0.17448 \quad 0.03468$ $0.11314 \quad 1.14713$ $-0.00656-0.02018$ $-0.01642-0.05040$ $-0.51672 \quad 0.07560$ $-0.57535-0.27770$

$\begin{array}{ll}-0.00002 & -0.00005 \\ -0.00007 & -0.00020 \\ -0.00041 & -0.00126 \\ -0.00165 & -0.00502 \\ -0.00679 & -0.01984 \\ -0.04861 & -0.11346 \\ -0.22929 & -0.33058 \\ -0.47610 & -0.44374 \\ -0.61626 & -0.40644 \\ -0.59560 & -0.31699 \\ -0.50819 & -0.21532 \\ -0.41454 & -0.03097 \\ -0.02844 & -0.07289 \\ -0.13520 & -0.23968 \\ -0.31862 & -0.39000 \\ -0.50581 & -0.44603 \\ -0.61199 & -0.41224 \\ -0.61366 & -0.34297 \\ -0.55370 & -0.27105 \\ -0.48303 & -0.17502 \\ -0.40687 & -0.01380 \\ -0.29011 & 0.21497\end{array}$

$-0.00002-0.00005$ $-0.00007-0.00020$ $65-0.0050$ $-0.00679-0.01984$ $-0.22929-0.33058$ $-0.47610-0.44374$ $-0.59560-0.31699$ $-0.50819-0.21532$

$-0.02844-0.07289$ $-0.50581-0.44603$ $\begin{array}{ll}-0.61366 & -0.34297\end{array}$ $-0.48303-0.17502$ $\begin{array}{lll}-0.29011 & 0.21497\end{array}$

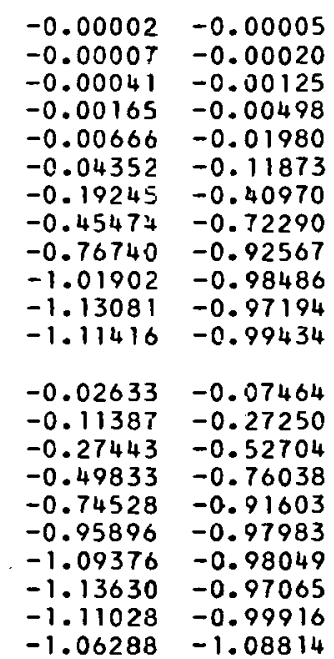

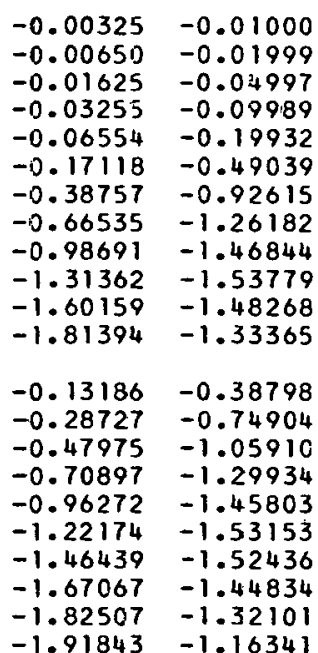

$-0.00325-0.01000$ $-0.00650-0.01999$ $-0.01625-0.04997$ $-0.03255-0.08989$ $-0.06554-0.19932$ $-0.17118-0.49039$ $-0.38757-0.92615$ $-0.66535-1.26182$ $-0.98691-1.46844$ $-1.31362-1.53779$ $-1.60159-1.48268$ $-1.81394-1.33365$

$-0.13186-0.38798$ $-0.28727-0.74904$ $-0.47975-1.05910$ $-0.70897-1.29934$ $-0.96272-1.45803$ $-1.22174-1.53153$ $-1.46439-1.52436$ $-1.67067-1.44834$ $\begin{array}{ll}-1.82507 & -1.32101 \\ -1.91843 & -1.16341\end{array}$

$\frac{\alpha}{\beta}=0.005$

$\begin{array}{ll}-0.00325 & -0.01000 \\ -0.00650 & -0.01999 \\ -0.01627 & -0.04892 \\ -0.03271 & -0.09949 \\ -0.06678 & -0.19620 \\ -0.18544 & -0.44439 \\ -0.41428 & -0.02469 \\ -0.55651 & -0.51546 \\ -0.56227 & -0.25622 \\ -0.50533 & 0.07219 \\ -0.40529 & 0.47634 \\ -0.18597 & 0.89320 \\ & \\ -0.13981 & -0.36509 \\ -0.31785 & -0.58750 \\ -0.48136 & -0.61281 \\ -0.56454 & -0.48458 \\ -0.56497 & -0.27768 \\ -0.52451 & -0.02957 \\ -0.46483 & 0.26446 \\ -0.35867 & 0.59949 \\ -0.16519 & 0.91799 \\ 0.11421 & 1.12557\end{array}$

$-0.00002-0.00005$ $-0.00007-0.00020$ $-0.00041-0.00125$ $-0.00166-0.00497$ $-0.00683-0.01962$ $-0.04866-0.11212$ $-0.22802-0.32618$ $-0.47120-0.43790$ $-0.60817-0.40278$; $-0.58680-0.31648 /$ $\begin{array}{ll}-0.49861 & -0.21650 \\ -0.40193 & -0.03487\end{array}$

$-0.02851-0.07205$ $-0.13481-0.23663$ $-0.31625-0.38471$ $-0.50034-0.44029$ $-0.60404-0.40834$ $-0.60490-0.34168$ $-0.54477-0.2715$ $-0.47276-0.17668$ $-0.39404 \quad-0.01802$ $\begin{array}{ll}-0.27587 & 0.20583\end{array}$
$-0.00328-0.01009$ $-0.00656-0.02018$ $-0.01643-0.05042$ $\begin{array}{ll}-0.03308 & -0.1006\end{array}$ $-0.06788-0.19939$ $-0.19571-0.46765$ $-0.49905-0.74203$ $-0.81122-0.74917$ $-0.97803-0.57056$ $\begin{array}{lll}-0.94781 & -0.37454\end{array}$ $-0.80068 \quad-0.29767$ $-0.67396-0.36260$

$-0.14503-0.37816$ $-0.35826 \quad-0.65638$ $-0.84639-0.77402$ $-0.97274-0.58633$ $-0.97400-0.42297$ $-0.88066-0.31691$ $-0.75716-0.3038$ $\begin{array}{ll}-0.75716 & -0.30381 \\ -0.66887 & -0.3705\end{array}$ $-0.65255-0.46886$
$-0.00325-0.01000$ $-0.00650-0.01999$ $-0.01628 \quad-0.04994$ $0.03277-0.09960$ $-0.06724-0.19751$ $-0.19378-0.46308$ $\begin{array}{lll}-0.49345 & -0.73422\end{array}$ $-0.80079-0.74134$ $-0.96439-0.56628$ $-0.93497-0.37496$ $\begin{array}{ll}-0.79206 & -0.30023 \\ -0.66954 & -0.36296\end{array}$ $-0.14363-0.37452$ $\begin{array}{lll}-0.35448 & -0.04967\end{array}$ $-0.61087-0.76577$ $-0.83534-0.72417$ $\begin{array}{ll}-0.96048 & -0.58171 \\ -0.062215\end{array}$ $-0.86966-0.31891$ $-0.74992-0.30618$ $\begin{array}{ll}-0.66462 & -0.37059\end{array}$ $-0.64890-0.46522$ 
$\beta \mathrm{h}$

$\mathrm{C}(\mathrm{h}, 0)$

$\begin{array}{lll}0.01 & -0.00322 & -0.00990\end{array}$

$0.02 \quad-0.00644-0.01980$

$-0.01609-0.04948$

$0.10-0.03219-0.09879$

$0.20-0.06446-0.19614$

$\begin{array}{lll}1.00 & -0.31312 & -0.78196\end{array}$

$\begin{array}{lll}.50 & -0.39275 & -0.89342\end{array}$

$\begin{array}{lll}2.00 & -0.33225 & -0.86836 \\ 2.50 & -0.13140 & -0.83568\end{array}$

$\begin{array}{lll}3.00 & 0.12638 & -0.88994 \\ 3.50 & 0.33257 & -1.03293\end{array}$

$0.125 \pi \quad-0.12701-0.37467$

$\begin{array}{llll}0.250 \pi & -0.25235 & -0.67128\end{array}$

$0.375 \pi \quad-0.35395 \quad-0.84387$

$0.500 \pi \quad-0.39370-0.89540$

$\begin{array}{llll}0.625 \pi & -0.34197 & -0.87203\end{array}$

$0.750 \pi \quad-0.19985-0.838 \varepsilon 2$

$0.875 \pi \quad-0.00302-0.84935$

$\begin{array}{lll}1.000 \pi & 0.19396 & -0.92373\end{array}$

$\begin{array}{lll}1.125 \pi & 0.34289 & -1.04433 \\ 1.250 \pi & 0.42282 & -1.16977\end{array}$ $\frac{a}{\lambda}=0.3$

$S(h, 0)$

$E(h, 0)$

$-0.00002-0.00005$

$-0.00007-0.00020$

$-0.00041-0.00123$

$-0.00166-0.00493$

$0.00669-0.01958$

$-0.04367-0.11740$

$0.19223-0.40485$

$-0.45220-0.71405$

$-0.76002-0.91480$

$-1.00822-0.97543$

$-1.11894-0.96627$

$-0.02645-0.07381$

$-0.11397-0.26935$

$-0.27365-0.52070$

$-0.49528-0.75107$

$-0.73883-0.90519$

$-0.94912-0.96965$

$-1.08194-0.97282$

$-1.12485-0.96604$

$-1.05728-1.08507$ $\frac{\alpha}{\beta}=0.01$

$\begin{array}{ll}-0.00322 & -0.00990 \\ -0.00644 & -0.01980 \\ -0.01610 & -0.04950 \\ -0.03225 & -0.09895 \\ -0.06492 & -0.19745 \\ -0.16955 & -0.48574 \\ -0.38375 & -0.91710 \\ -0.65840 & -1.24900 \\ -0.97584 & -1.45300 \\ -1.29779 & -1.52135 \\ -1.58101 & -1.46718 \\ -1.78942 & -1.32094 \\ -0.13062 & -0.38431 \\ -0.28449 & -0.74183 \\ -0.47494 & -1.04861 \\ -0.70149 & -1.28607 \\ -0.95198 & -1.44273 \\ -1.20731 & -1.51519 \\ -1.44615 & -1.50814 \\ -1.64886 & -1.43345 \\ -1.80034 & -1.30854 \\ -1.89177 & -1.15421\end{array}$

$-0.00322-0.00930$

$-0.00644 \quad-0.019 .30$

$-0.01612-0.049+5$

$-0.03240-0.09856$

$-0.06613-0.19435$

$-0.18343-0.44012$

$-0.40862-0.61885$

$-0.54752-0.51102$

$-0.55197-0.25640$

$-0.49418 \quad 0.0687$

$-0.39293 \quad 0.408 i 5$

$-0.17643 \quad 0.87725$

$-0.13836-0.361 \epsilon$

$-0.31391-0.581 \varepsilon 5$

$-0.47433-0.6074$

$-0.55524-0.48156$

$-0.55472-0.27760$

$-0.51366-0.032 a 3$

$\begin{array}{lll}-0.45291 & 0.25906\end{array}$

$\begin{array}{lll}-0.34645 & 0.5893\end{array}$

$\begin{array}{rr}-0.15612 & 0.9017 \\ 0.11527 & 1.10463\end{array}$
$S(h, h)$

$\begin{array}{ll}-0.00002 & -0.00005\end{array}$

$-0.00007-0.00020$

$-0.00042-0.00123$

$-0.00167-0.00491$

$-0.00686-0.01940$

$-0.04372-0.11079$

$-0.22676-0.32184$

$-0.46037-0.43216$

$-0.60021-0.39916$

$-0.57813-0.31596$

$-0.48914 \quad-0.21768$

$-0.38956-0.03876$

$-0.02858-0.0712$

$-0.13442-0.23362$

$-0.31390-0.3794$

$-0.49495-0.43465$

$-0.59623-0.40449$

$\begin{array}{ll}-0.59627 & -0.34039\end{array}$

$\begin{array}{ll}-0.53595 & -0.27197\end{array}$

$-0.46263-0.17835$

$-0.38145-0.02223$

$\begin{array}{ll}-0.26197 & 0.19686\end{array}$
$E(h, h)$

$\begin{array}{ll}-0.00322 & -0.00990 \\ -0.00644 & -0.01980 \\ -0.01612 & -0.04948 \\ -0.03246 & -0.09872 \\ -0.06660 & -0.19564 \\ -0.19187 & -0.45855 \\ -0.48792 & -0.72650 \\ -0.79050 & -0.73359 \\ -0.95095 & -0.56201 \\ -0.92230 & -0.37528 \\ -0.78351 & -0.30264 \\ -0.66503 & -0.36323 \\ -0.14224 & -0.37091 \\ -0.35074 & -0.04303 \\ -0.60366 & -0.75760 \\ -0.82443 & -0.71673 \\ -0.94588 & -0.57710 \\ -0.94716 & -0.42125 \\ -0.85879 & -0.32077 \\ -0.74270 & -0.30840 \\ -0.66029 & -0.37059 \\ -0.64514 & -0.46165\end{array}$

$\begin{array}{lll}0.01 & -0.00316 & -0.00972 \\ 0.02 & -0.00632 & -0.01943 \\ 0.05 & -0.01579 & -0.04856 \\ 0.10 & -0.03159 & -0.09694 \\ 0.20 & -0.06323 & -0.19248 \\ 0.50 & -0.15847 & -0.45755 \\ 1.00 & -0.30450 & -0.76812 \\ 1.50 & -0.37861 & -0.88035 \\ 2.00 & -0.31633 & -0.86121 \\ 2.50 & -0.11999 & -0.83482 \\ 3.00 & 0.12918 & -0.89017 \\ 3.50 & 0.32872 & -1.02747 \\ 0.125 \pi & -0.12443 & -0.36769 \\ 0.250 \pi & -0.24617 & -0.65904 \\ 0.375 \pi & -0.34322 & -0.82958 \\ 0.500 \pi & -0.37899 & -0.88294 \\ 0.625 \pi & -0.32597 & -0.86436 \\ 0.750 \pi & -0.18653 & -0.83647 \\ 0.875 \pi & 0.00428 & -0.84992 \\ 1.000 \pi & 0.19439 & -0.92295 \\ 1.125 \pi & 0.33881 & -1.03830 \\ 1.250 \pi & 0.41873 & -1.15712\end{array}$

$\begin{array}{ll}-0.00002 & -0.00005 \\ -0.00007 & -0.00019 \\ -0.00042 & -0.00121 \\ -0.00168 & -0.00482 \\ -0.00676 & -0.01915 \\ -0.04395 & -0.11477 \\ -0.19174 & -0.39534 \\ -0.44718 & -0.09672 \\ -0.74735 & -0.89353 \\ -0.98718 & -0.95689 \\ -1.09585 & -0.95489 \\ -1.08690 & -0.98566 \\ -0.02665 & -0.07217 \\ -0.11414 & -0.26316 \\ -0.27207 & -0.50826 \\ -0.48926 & -0.73284 \\ -0.72621 & -0.88399 \\ -0.92993 & -0.94967 \\ -1.05894 & -0.95762 \\ -1.10256 & -0.95667 \\ -1.08383 & -0.99072 \\ -1.04601 & -1.07858\end{array}$

$\begin{array}{ll}-0.00316 & -0.00972 \\ -0.00632 & -0.01943 \\ -0.01580 & -0.04858 \\ -0.03165 & -0.09710 \\ -0.06371 & -0.19375 \\ -0.16635 & -0.47657 \\ -0.37622 & -0.89926 \\ -0.64471 & -1.22375 \\ -0.95408 & -1.42260 \\ -1.26672 & -1.48902 \\ -1.54066 & -1.43666 \\ -1.74142 & -1.29584 \\ -0.12816 & -0.37708 \\ -0.27902 & -0.72760 \\ -0.46545 & -1.0279 .4 \\ -0.68676 & -1.25993 \\ -0.93087 & -1.41261 \\ -1.17898 & -1.48305 \\ -1.41036 & -1.47623 \\ -1.60612 & -1.40413 \\ -1.75191 & -1.28393 \\ -1.83962 & -1.13592\end{array}$

$-0.00002-0.00005$ $\begin{array}{ll}-0.00007 & -0.00019\end{array}$ $-0.00042-0.00121$ $-0.00169-0.00481$ $-0.00692-0.01897$ $-0.04880-0.108 .18$ $-0.22426-0.31334$ $-0.45691-0.42093$ $-0.58471-0.39206$ $-0.56118-0.31486$ $-0.47056-0.22005$ $-0.36549-0.04651$

$-0.02871-0.06958$ $-0.13363-0.22771$ $-0.30926-0.36929$ $-0.48442-0.42362$ $\begin{array}{lll}-0.58100 & -0.39693\end{array}$ $-0.57942-0.33779$ $-0.51864-0.27282$ $-0.44279-0.1817$ $-0.35699-0.03058$

$\begin{array}{ll}-0.00316 & -0.00972 \\ -0.00632 & -0.01943 \\ -0.01582 & -0.04855 \\ -0.03186 & -0.09688 \\ -0.06535 & -0.19196 \\ -0.18811 & -0.44963 \\ -0.47704 & -0.71130 \\ -0.77033 & -0.71833 \\ -0.92465 & -0.55351 \\ -0.89749 & -0.37563 \\ -0.76658 & -0.30698 \\ -0.65579 & -0.36353 \\ -0.13951 & -0.36380 \\ -0.34338 & -0.62996 \\ -0.58949 & -0.74152 \\ -0.80306 & -0.70206 \\ -0.91981 & -0.56796 \\ -0.92109 & -0.41927 \\ -0.83744 & -0.32406 \\ -0.72830 & -0.31238 \\ -0.65139 & -0.37037 \\ -0.63731 & -0.45469\end{array}$


$\frac{a}{\lambda}=0.3$

$E(h, 0)$

$S(h, 0)$

$-0.00002-0.00005$ $-0.00007-0.00019$ $-0.00042-0.00118$ $-0.00170-0.0047$ $-0.04420-0.11220$ $-0.04420-0.11220$ $-0.19121-0.38606$ $-0.73446-0.07287$ $-0.934469-0.07287$ $-0.96889-0.93877$ $\begin{array}{ll}-1.07359 & -0.94347 \\ -1.06931 & -0.97931\end{array}$

$\begin{array}{ll}-0.02685 & -0.07057\end{array}$ $-0.11426-0.25711$ $-0.27047-0.49614$ $-0.48334-0.71512$ $-0.71395-0.86340$ $-0.91139-0.93020$ $-1.03678-0.94264$ $-1.08103-0.94712$

$-1.06672-0.98452$

$-1.03467-1.07166$

$\frac{\alpha}{\beta}=0.03$

$\mathrm{C}(\mathrm{h}, \mathrm{h})$

$\mathrm{Sth}, \mathrm{h})$

$E(h, h)$

\begin{tabular}{|c|c|}
\hline $\begin{array}{l}-0.00310 \\
-0.00620 \\
-0.01550 \\
-0.03105 \\
-0.06252 \\
-0.16320 \\
-0.36885 \\
-0.63131 \\
-0.93281 \\
-1.23641 \\
-1.50139 \\
-1.69477\end{array}$ & $\begin{array}{l}-0.00954 \\
-0.01907 \\
-0.04767 \\
-0.09529 \\
-0.19013 \\
-0.46757 \\
-0.88176 \\
-1.19901 \\
-1.39285 \\
-1.45738 \\
-1.40678 \\
-1.27117\end{array}$ \\
\hline $\begin{array}{l}-0.12575 \\
-0.27365 \\
-0.45615 \\
-0.67235 \\
-0.91022 \\
-1.15132 \\
-1.37548 \\
-1.56452 \\
-1.70485 \\
-1.78898\end{array}$ & $\begin{array}{l}-0.36999 \\
-0.71365 \\
-1.00767 \\
-1.23432 \\
-1.38313 \\
-1.45159 \\
-1.44501 \\
-1.37540 \\
-1.25973 \\
-1.11778\end{array}$ \\
\hline
\end{tabular}

$-0.00310-0.00954$ $-0.00620-0.01907$ $\begin{array}{ll}-0.01552 & -0.04762\end{array}$ $-0.03120-0.09491$ $-0.06362-0.18713$ $-0.17561-1.42349$ $-0.38679-0.59600$ $-0.51284-0.49786$ $-0.51214 \quad-0.25661$ $-0.45075 \quad 0.05593$ $-0.34555 \quad 0.43681$ $-0.14037 \quad 0.81820$

$-0.13274-0.34803$ $-0.29863-0.5598$ $-0.44719-0.58627$ $-0.51939-0.40949$ $-0.51510-0.27683$ $-0.47156-0.04116$ $\begin{array}{ll}-0.40691 & 0.23840\end{array}$ $-0.29984 \quad 0.55067$ $\begin{array}{rr}-0.12184 & 0.84055 \\ 0.11933 & 1.02683\end{array}$

$$
\frac{a}{\lambda}=0.3 \quad \frac{\alpha}{\beta}=0.05
$$

\begin{tabular}{|c|c|c|}
\hline $\begin{array}{l}0.01 \\
0.02 \\
0.05 \\
0.10 \\
0.20 \\
0.50 \\
1.00 \\
1.50 \\
2.00 \\
2.50 \\
3.00 \\
3.50\end{array}$ & $\begin{array}{r}-0.00298 \\
-0.00597 \\
-0.01492 \\
-0.02984 \\
-0.05968 \\
-0.14859 \\
-0.27998 \\
-0.33884 \\
-0.27180 \\
-0.08790 \\
0.13815 \\
0.32011\end{array}$ & $\begin{array}{l}-0.00918 \\
-0.01837 \\
-0.04589 \\
-0.09161 \\
-0.18191 \\
-0.43256 \\
-0.72818 \\
-0.84227 \\
-0.83916 \\
-0.82961 \\
-0.88775 \\
-1.01008\end{array}$ \\
\hline $\begin{array}{l}0.125 \pi \\
0.250 \pi \\
0.375 \pi \\
0.500 \pi \\
0.625 \pi \\
0.750 \pi \\
0.875 \pi \\
1.000 \pi \\
1.125 \pi \\
1.250 \pi\end{array}$ & $\begin{array}{r}-0.11701 \\
-0.22849 \\
-0.31281 \\
-0.33765 \\
-0.28122 \\
-0.14921 \\
0.02528 \\
0.119708 \\
0.332958 \\
0.40930\end{array}$ & $\begin{array}{l}-0.34755 \\
-0.62370 \\
-0.78825 \\
-0.84648 \\
-0.84091 \\
-0.82732 \\
-0.84846 \\
-0.91784 \\
-1.01946 \\
-1.12120\end{array}$ \\
\hline
\end{tabular}

$\begin{array}{ll}-0.00002 & -0.00005 \\ -0.00007 & -0.00018 \\ -0.00043 & -0.00113 \\ -0.00172 & -0.00451 \\ -0.00693 & -0.01792 \\ -0.04463 & -0.10724 \\ -0.19002 & -0.36818 \\ -0.43252 & -0.84753 \\ -0.70976 & -0.83327 \\ -0.92838 & -0.90376 \\ -1.03140 & -0.92057 \\ -1.03547 & -0.96545 \\ -0.02718 & -0.06747 \\ -0.11437 & -0.24544 \\ -0.26718 & -0.47284 \\ -0.47178 & -0.68113 \\ -0.69042 & -0.82394 \\ -0.87613 & -0.89273 \\ -0.99482 & -0.91333 \\ -1.04016 & -0.92758 \\ -1.03374 & -0.97091 \\ -1.01191 & -1.05672\end{array}$

$\begin{array}{ll}-0.00002 & -0.00005 \\ -0.00007 & -0.00019\end{array}$ $\begin{array}{ll}-0.00007 & -0.00019\end{array}$ $-0.00342-0.00118$ $-0.00171-0.00470$ $-0.00697-0.01855$ $-0.04886-0.10563$ $-0.22178-0.30509$ $\begin{array}{lll}-0.44771 & -0.41005\end{array}$ $\begin{array}{ll}-0.56972 & -0.38514\end{array}$ $-0.54472-0.31370$ $-0.45245-0.22242$ $-0.34230-0.05421$

$-0.02883-0.06798$ $-0.13282-0.22195$ $\begin{array}{lll}-0.30471 & -0.35939\end{array}$ $-0.47419-0.41294$ $-0.56629-0.3895$ $-0.56311-0.33517$ $-0.50179-0.27361$ $-0.42350-0.1851$ $\begin{array}{rr}-0.20948 & 0.1627\end{array}$
$-0.00310-0.00954$ $-0.00620 \quad-0.01907$ $-0.01553-0.04764$ $\begin{array}{lll}-0.03126 & -0.09506\end{array}$ $-0.06412-0.18835$ $\begin{array}{ll}-0.18442 & -0.44088\end{array}$ $-0.46640-0.69643$ $\begin{array}{ll}-0.75069 & -0.70339 \\ -0.89913 & -0.54507\end{array}$ $-0.87338-0.37561$ $-0.74989-0.31073$ $\begin{array}{ll}-0.74989 & -0.31073 \\ -0.64629 & -0.36351\end{array}$ $\begin{array}{lll}-0.13682 & -0.35682\end{array}$ $-0.33618-0.61716$ $-0.57566-0.72580$ $-0.78226-0.68770$ $\begin{array}{ll}-0.89449 & -0.55889\end{array}$ $-0.89577-0.41704$ $-0.81659-0.32682$ $\begin{array}{lll}-0.71399 & -0.31579\end{array}$ $\begin{array}{ll}-0.64220 & -0.36987 \\ -0.62913 & -0.44794\end{array}$

$\begin{array}{llll}-0.00298 & -0.00918 & -0.00298 & -0.00918 \\ -0.00597 & -0.01837 & -0.00597 & -0.01836 \\ -0.01493 & -0.04591 & -0.01494 & -0.04586 \\ -0.02990 & -0.09176 & -0.03004 & -0.09139 \\ -0.06020 & -0.18308 & -0.06120 & -0.18017 \\ -0.15709 & -0.45008 & -0.16813 & -0.40750 \\ -0.35453 & -0.84779 & -0.36611 & -0.57414 \\ -0.00534 & -1.15104 & -0.48016 & -0.48400 \\ -0.89170 & -1.33522 & -0.47439 & -0.25608 \\ -1.17799 & -1.39615 & -0.40947 & 0.04420 \\ -1.42591 & -1.34887 & -0.30129 & 0.40771 \\ -1.60535 & -1.22313 & -0.10735 & 0.76456 \\ & & & \\ -0.12106 & -0.35621 & -0.12734 & -0.33496 \\ -0.26322 & -0.08654 & -0.28410 & -0.53865 \\ -0.43811 & -0.96833 & -0.42157 & -0.56586 \\ -0.64444 & -1.18467 & -0.48560 & -0.45747 \\ -0.87032 & -1.32601 & -0.47758 & -0.27537 \\ -1.09798 & -1.39070 & -0.43150 & -0.04925 \\ -1.30837 & -1.38456 & -0.36346 & 0.21917 \\ -1.48466 & -1.31967 & -0.25656 & 0.51489 \\ -1.61466 & -1.21256 & -0.09049 & 0.78528 \\ -1.09207 & -1.08201 & 0.12336 & 0.9577\end{array}$

$\begin{array}{ll}-0.00002 & -0.00005 \\ -0.00007 & -0.00018 \\ -0.00043 & -0.00113 \\ -0.00173 & -0.00450 \\ -0.00707 & -0.01774 \\ -0.04893 & -0.10072 \\ -0.21637 & -0.28926 \\ -0.43008 & -0.38929 \\ -0.54123 & -0.37178 \\ -0.51324 & -0.31126 \\ -0.41765 & -0.22714 \\ -0.29838 & -0.06937 \\ -0.02902 & -0.06489 \\ -0.13114 & -0.21089 \\ -0.29586 & -0.34046 \\ -0.45462 & -0.39255 \\ -0.53830 & -0.37540 \\ -0.53198 & -0.32993 \\ -0.46940 & -0.27503 \\ -0.38650 & -0.19198 \\ -0.28890 & -0.05509 \\ -0.16149 & 0.13136\end{array}$

$\begin{array}{ll}-0.00298 & -0.00918 \\ -0.00597 & -0.01836 \\ -0.01495 & -0.04588 \\ -0.03010 & -0.09154 \\ -0.06173 & -0.18133 \\ -0.17726 & -0.42389 \\ -0.44585 & -0.66761 \\ -0.71296 & -0.67444 \\ -0.85028 & -0.52834 \\ -0.82714 & -0.37455 \\ -0.71726 & -0.31661 \\ -0.62668 & -0.36258 \\ -0.13161 & -0.34327 \\ -0.32222 & -0.59234 \\ -0.54899 & -0.69535 \\ -0.74233 & -0.65984 \\ -0.84604 & -0.54100 \\ -0.84732 & -0.41188 \\ -0.77636 & -0.33089 \\ -0.68569 & -0.32106 \\ -0.62314 & -0.36808 \\ -0.61187 & -0.43501\end{array}$



$-0.00575-0.01769$ $-0.01437-0.04419$ $\begin{array}{ll}-0.01437 & -0.04419 \\ -0.05742 & -0.08822 \\ -0.14235 & -0.17519\end{array}$ $-0.14235-0.41668$ $-0.26470-0.70278$ $-0.31439-0.81779$ $-0.24466-0.82405$ $-0.06820-0.82422$ $0.14445-0.88386$ $0.31639-0.99775$

$0.1251 \pi$ $-0.11232-0.33474$ $-0.21741-0.60125$ $-0.29390-0.76192$ $-0.31226-0.82294$ $-0.25394 .0 .82294$ $-0.12643-0.81968$ $0.03851-0.81968$ $0.03851-0.84517$ $0.32551-1.00631$ $\begin{array}{ll}0.32551 & -1.00631 \\ 0.40498 & -1.09867\end{array}$ $\begin{array}{ll}-0.88245 & -0.87033 \\ -0.99209 & -0.89772\end{array}$

$\begin{array}{ll}-0.00287 & -0.00884 \\ -0.00575 & -0.01768 \\ -0.01439 & -0.04416 \\ -0.02892 & -0.08801 \\ -0.05887 & -0.17348 \\ -0.16097 & -0.39212 \\ -0.34653 & -0.55306 \\ -0.44937 & -0.47037 \\ -0.43865 & -0.25491 \\ -0.37028 & 0.03354 \\ -0.25988 & 0.38078 \\ -0.07702 & 0.71608 \\ -0.12217 & -0.32239 \\ -0.27028 & -0.51831 \\ -0.39736 & -0.54617 \\ -0.45376 & -0.44555 \\ -0.44206 & -0.27332 \\ -0.39343 & -0.05638 \\ -0.32245 & 0.20136 \\ -0.21632 & 0.48213 \\ -0.06171 & 0.73541 \\ 0.12722 & 0.89654\end{array}$

$-0.00002-0.00004$ $-0.00007-0.00017$ $-0.00044-0.00108$ $-0.00175 \quad-0.00431$ $-0.00702-0.01714$ $-0.04497-0.10249$ $-0.18866-0.35118$ $-0.42306-0.61695$ $-0.88245-0.7958$

$-1.00333-0.95032$

$-0.02746-0.06451$ $-0.11432-0.23432$ $-0.26380-0.45072$ $-0.46060-0.64900$ $-0.66815-0.78667$ $-0.84315-0.85715$ $-0.95576-0.88489$ $-1.00198-0.90762$ $-1.00234-0.95599$ $-0.98918-1.04056$

$\begin{array}{ll}-0.00287 & -0.00884 \\ -0.00575 & -0.01769 \\ -0.01438 & -0.04421 \\ -0.02880 & -0.08836 \\ -0.05797 & -0.17630 \\ -0.15121 & -0.43324 \\ -0.34076 & -0.81513 \\ -0.58045 & -1.10500 \\ -0.85243 & -1.28000 \\ -1.12240 & -1.033753 \\ -1.35436 & -1.26336 \\ -1.52086 & -1.17676 \\ -0.11655 & -0.34294 \\ -0.25319 & -0.66047 \\ -0.42078 & -0.93054 \\ -0.01769 & -1.13703 \\ -0.83218 & -1.27128 \\ -1.04716 & -1.33240 \\ -1.24462 & -1.32667 \\ -1.40902 & -1.26619 \\ -1.52945 & -1.16701 \\ -1.60069 & -1.04693\end{array}$

$\frac{a}{\lambda}=0.3$

$\frac{\alpha}{\beta}=0.10$

$-0.00002-0.00004$ $-0.00007-0.00016$ $-0.00044-0.00101$ $-0.00178-0.00403$ $-0.00713-0.01603$ $-0.04532-0.09577$ $-0.18636-0.32723$ $-0.40932-0.57412$ $-0.65369-0.74358$ $-0.84292-0.8229$ $\begin{array}{ll}-0.93800 & -0.86374 \\ -0.95811 & -0.92584\end{array}$

$-0.02776-0.06030$ $-0.11397-0.21860$ $-0.25858-0.41963$ $-0.44448 \quad-0.60404$ $-0.63689-0.73459$ $-0.79754-0.80706$ $-0.90208-0.84388$ $-0.94929-0.87724$ $\begin{array}{ll}-0.95803 & -0.93176 \\ -0.95547 & -1.01464\end{array}$

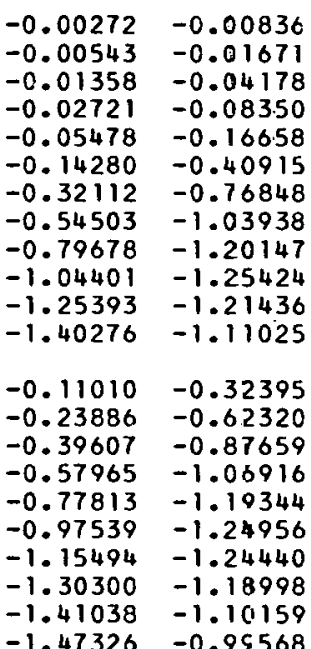

$\begin{array}{ll}-1.403038 & -1.19159 \\ -1.47326 & -0.94568\end{array}$

$\begin{array}{ll}-0.00002 & -0.00004 \\ -0.00007 & -0.00017 \\ -0.00044 & -0.00108 \\ -0.00176 & -0.00430 \\ -0.00715 & -0.01696 \\ -0.04892 & -0.09603 \\ -0.21205 & -0.27430 \\ -0.41340 & -0.36980 \\ -0.51457 & -0.35910 \\ -0.48359 & -0.30869 \\ -0.38469 & -0.23181 \\ -0.25749 & -0.08412 \\ -0.02915 & -0.06195 \\ -0.12941 & -0.20039 \\ -0.28731 & -0.32262 \\ -0.43616 & -0.37340 \\ -0.51211 & -0.36196 \\ -0.50278 & -0.32471 \\ -0.43873 & -0.27627 \\ -0.35156 & -0.19883 \\ -0.24749 & -0.07082 \\ -0.11737 & 0.10245\end{array}$

$E(h, h)$

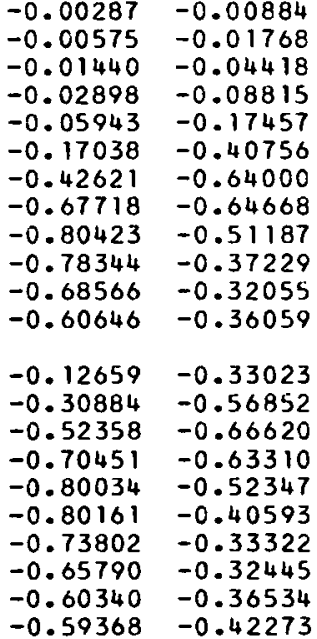

$-0.00272-0.00836$ $-0.00543-0.01671$ $-0.01360-0.04174$ $\begin{array}{ll}-0.02732 & -0.08316 \\ -0.05555 & -0.16390\end{array}$ $-0.15080-0.37016$ $-0.31909-0.52294$ $-0.40647-0.45038$ $-0.38858-0.25212$ $-0.31526 \quad-0.0194$ $-0.20272 \quad 0.34426$ $-0.03592 \quad 0.65210$ $-0.11480-0.30442$ $-0.25080-0.48930$ $-0.36356-0.51793$ $-0.40940-0.42789$ $-0.39235-0.26929$ $\begin{array}{ll}-0.33992 & -0.06544\end{array}$ $\begin{array}{ll}-0.26524 & 0.17716\end{array}$ $-0.16112 \quad 0.43785$

$\begin{array}{rr}-0.02273 & 0.66974 \\ 0.13283 & 0.81768\end{array}$ $\begin{array}{ll}-0.00002 & -0.00004\end{array}$ $-0.00007-0.00016$ $-0.00044 \quad-0.00101$ $-0.00178-0.00402$ $-0.00725-0.01585$ $-0.048785-0.08940$ $-0.39001-0.34275$ $-0.47776-0.34126$ $-0.44235-0.30474$ $-0.33854-0.23870$ $-0.20125-0.10533$

$-0.02925-0.05777$ $-0.12670-0.18564$ $-0.27503-0.29778$ $-0.41036-0.34683$ $-0.47593-0.34312$ $-0.46229-0.31705$ $\begin{array}{lll}-0.39581 & -0.2779\end{array}$ $-0.30281-0.2090$ $\begin{array}{ll}-0.19062 & -0.0933\end{array}$ $\begin{array}{ll}-0.05739 & 0.06338\end{array}$
$-0.00272-0.00836$ $-0.00543-0.0167$ $-0.01361-0.04175$ $-0.02739-0.08329$ $-0.05614-0.16491$ $-0.16056-0.38424$ $-0.39839-0.60073$ $-0.62697-0.60718$ $-0.74004-0.48767$ $-0.72232-0.36699$ $-0.64024-0.32332$

$-0.11943-0.31160$ $-0.28982-0.53458$ $-0.48770-0.62478$ $\begin{array}{lll}-0.65150 & -0.59499\end{array}$ $-0.73663-0.49784$ $\begin{array}{lll}-0.73786 & -0.39577\end{array}$ $-0.68392-0.3339$ $-0.61735-0.32653$ $-0.57303-0.35969$ $-0.56524-0.40524$ 
$\beta \mathrm{h}$

$\mathrm{C}(\mathrm{h}, 0)$

$S(h, p)$

$-0.00002-0.00003$

$-0.00007-0.00013$

$-0.00045-0.00081$

$\begin{array}{ll}-0.00182 & -0.00323\end{array}$

$-0.00727-0.01282$

$-0.04532-0.07638$

$-0.17680-0.25916$

$-0.36704-0.45422$

$-0.56129-0.59768$

$-0.70836-0.68683$

$-0.79163-0.75603$

$-0.82972-0.83687$

$-0.02799-0.04816$

$-0.11081-0.17363$

$\begin{array}{ll}-0.24028 & -0.33180 \\ -0.39571 & -0.47837\end{array}$

$-0.54824-0.58933$

$-0.67266-0.66501$

$-0.75724-0.72135$

$-0.80565-0.77692$

$-0.83149-0.84318$

$-0.84993-0.92134$
$E(h, 0)$

$\frac{a}{\lambda}=0.3 \quad \frac{\alpha}{\beta}=0.20$

$\mathrm{C}(\mathrm{h}, \mathrm{h})$

$S(h, h)$

$E(h, h)$
$-0.00225-0.00692$ $-0.00450-0.0133$ $-0.01126-0.03456$ $-0.02259-0.06886$ $-0.04577-0.13564$ $-0.12136-0.30562$ $-0.24218-0.43438$ $-0.28820-0.3882$ $-0.24913 .-0.2366$ $\begin{array}{lll}-0.16148 & -0.01397\end{array}$ $-0.04789 \quad 0.25210$ $0.07205 \quad 0.50070$ $-0.19549-0.40423$ $-0.26964-0.43410$ $-0.28708-0.37191$ $-0.25405-0.25032$ $-0.18997-0.08418$ $-0.01324 \quad 0.11604$ 0.079610 .51515 0.150250 .64303
$-0.00002-0.00003$ $-0.00007-0.00013$ $-0.00045-0.0008$ $-0.00182-0.0032$ $-0.00734-0.01265$ $-0.04739-0.07043$ $-0.18257-0.14516$ $-0.32407-0.26880$ $-0.37774-0.2913$ $-0.32910-0.29223$ $-0.21037-0.26028$ $-0.04886-0.16693$

$\begin{array}{ll}-0.02892 & -0.04576\end{array}$ $-0.11704-0.14405$ $-0.23837 \quad-0.22923$ $-0.37756-0.29056$ $-0.35184-0.29404$ $-0.27673-0.28248$ $-0.16805-0.24088$ $-0.03678-0.15801$ $0.10413-0.03600$
$-0.00225-0.00692$ $-0.00450-0.01384$ $-0.01127-0.03458$ $-0.02268-0.06896$ $-0.04643-0.13639$ $-0.13173-0.31572$ $-0.31825-0.48660$ $-0.48571-0.4922$ $-0.56240-0.41219$ $-0.55202-0.33784$ $-0.50621-0.31302$

$-0.09835-0.25676$ $-0.23453-0.43550$ $-0.50282-0.48370$ $-0.56020-0.41875$ $-0.56125-0.35495$ $-0.53006-0.31885$ $-0.49416 \quad-0.31470$ $-0.47190-0.33119$

$-0.46819-0.35230$

$\begin{array}{lll}0.01 & -0.00154 & -0.00475 \\ 0.02 & -0.00308 & -0.00949 \\ 0.05 & -0.00771 & -0.02373 \\ 0.10 & -0.01537 & -0.04738 \\ 0.20 & -0.03041 & -0.09417 \\ 0.50 & -0.07029 & -0.22576 \\ 1.00 & -0.10258 & -0.39586 \\ 1.50 & -0.07524 & -0.50266 \\ 2.00 & 0.00570 & -0.57443 \\ 2.50 & 0.11460 & -0.63762 \\ 3.00 & 0.22602 & -0.70024 \\ 3.50 & 0.32704 & -0.75811 \\ 0.125 \pi & -0.05724 & -0.18068 \\ 0.250 \pi & -0.09534 & -0.33140 \\ 0.375 \pi & -0.09998 & -0.44005 \\ 0.500 \pi & -0.06657 & -0.51418 \\ 0.625 \pi & -0.00151 & -0.56970 \\ 0.750 \pi & 0.08209 & -0.61952 \\ 0.875 \pi & 0.17089 & -0.66898 \\ 1.000 \pi & 0.25589 & -0.71739 \\ 1.125 \pi & 0.33351 & -0.76175 \\ 1.2501 \pi & 0.40409 & -0.79979\end{array}$

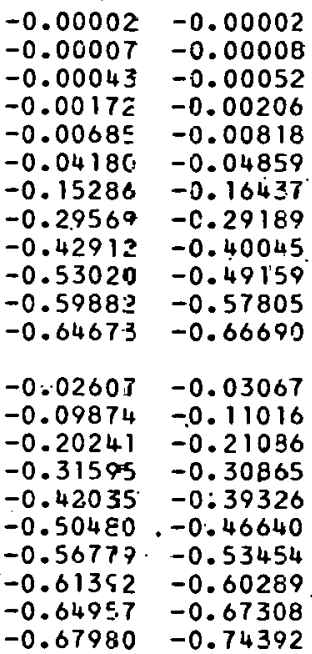

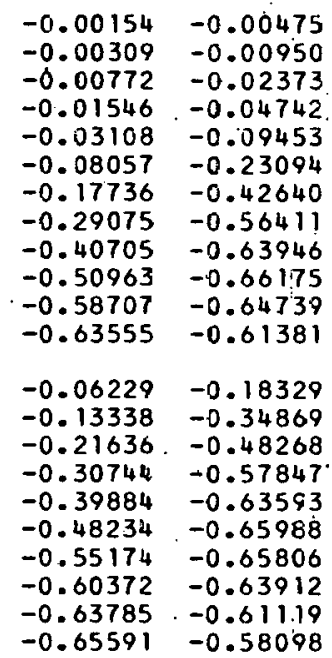

$\frac{\alpha}{E}=0.40$ $\begin{array}{ll}-0.00002 & -0.00002\end{array}$ $-0.00007-0.00008$ $-0.00043 .-0.00052$ $-0.00172-0.00205$ $\begin{array}{lll}-0.00687 & -0.00803\end{array}$ $-0.04197 \rightarrow 0.04367$ 0.17402 $-0.23160-0.17402$ $-0.1831-0.27601$ $-0.04084-0.28792$ $\begin{array}{rr}-0.04084 & -0.29792 \\ 0.15789 & -0.25375\end{array}$ $\begin{array}{lll}-0.02621 & -0.02865\end{array}$ $-0.09684-0.08730$ $-0.18060-0.13970$ $-0.23902-0.18117$ $-0.25053-0.22170$ $-0.21036-0.26282$ $-0.12101-0.29286$ $0.01107-0.29364$ $0.17266-0.2474$ $0.34077-0.14143$
$-0.00154-0.00475$ $-0.00309-0.00949$ $-0.00773-0.02371$ $-0.01554 \quad-0.04727$ $-0.03175 \quad-0.09329$ $-0.08868-0.21320$ $-0.20352-0.31973$ $-0.29353-0.32369$ $-0.32887-0.28776$ $-0.32533-0.25948$ $\begin{array}{ll}-0.31103 & -0.25145 \\ -0.30234 & -0.25557\end{array}$

$-0.06669-0.17434$ $-0.15372-0.28924$ $-0.24117-0.32994$ $-0.30186-0.31956$ $-0.32796-0.2904$ $-0.32858-0.26553$ $-0.31814 \quad-0.25322$ $-0.30769-0.25192$ $\begin{array}{ll}-0.30122 & -0.26054\end{array}$ 
$-0.00088-0.00270$ $-0.00175-0.00539$ $-0.00437-0.01348$ $-0.00871-0.02693$ $-0.01708-0.05363$ $-0.03713-0.13024$ $-0.04070-0.23919$ $0.00121-0.32509$ $0.06885-0.39668$ $\begin{array}{ll}0.15208 & -0.45939 \\ 0.23712 & -0.51390\end{array}$ $0.31940-0.55990$

$-0.03111-0.10362$ $-0.04455-0.19560$ $\begin{array}{rr}-0.03123 & -0.27201 \\ 0.00728 & -0.33590\end{array}$ $0.06310-0.39179$ $0.12761-0.44218$ $0.19459-0.48758$ $0.26078-0.52778$ $0.32491-0.56275$ $0.38669-0.59294$
$-0.00001-0.00001$ $-0.00006-0.00004$ $-0.00035-0.00026$ $-0.00138-0.00104$ $-0.00549-0.00413$ $-0.03295-0.02460$ $-0.11532-0.08556$ $-0.21338-0.16052$ $-0.30191-0.23852$ $-0.37280-0.31810$ $\begin{array}{ll}-0.42837 & -0.39908 \\ -0.47317 & -0.47983\end{array}$

$-0.02069-0.01553$ $-0.07603-0.05654$ $-0.15013-0.11152$ $-0.22686-0.17147$ $-0.29603-0.23277$ $-0.35418-0.29504$ $-0.40208-0.35831$ $-0.47594-0.42206$ $-0.50539-0.54710$ $\frac{a}{\lambda}=0.3$

$E(h, 0)$

$-0.00088-0.00270$ $-0.00175-0.00539$ $-0.00438-0.01348$ $-0.00878-0.02693$ $-0.01764-0.05365$ $-0.04546-0.13036$ $\begin{array}{ll}-0.09802 & -0.23671\end{array}$ $-0.15547-0.30675$ $-0.20924-0.34181$ $-0.25185-0.35123$ $-0.28045-0.34605$ $-0.29626-0.33520$

$-0.03524-0.10371$ $-0.07450-0.19513$ $-0.11831-0.26600$ $-0.16352-0.31367$ $-0.20562-0.34025$ $-0.24100-0.35048$ $-0.26784-0.34984$ $\begin{array}{lll}-0.29696 & -0.33441\end{array}$ $-0.30215-0.32579$ $-0.28610-0.34324$
$-0.00088-0.0027 c$ $-0.02175-0.00539$ $-0.00438-0.01347$ $-0.00875-0.02682$ $-0.01741-0.05277$ $-0.04125-0.11864$ $\begin{array}{lll}-0.05837 & -0.17662\end{array}$ $-0.02553-0.18109$ $0.06181-0.13612$ 0.299150 .16404

$-0.03331-0.09762$ $-0.05607-0.15934$ $-0.05308-0.18361$ $-0.01632-0.17793$ $0.05382-0.14133$ $0.14854-0.06625$ $0.31904 \quad 0.23456$ 0.318520 .23456 $0.19661 \quad 0.72475$
$S(h, h)$

$-0.00001-0.0000$ $-0.00006-0.00004$ $-0.00138-0.00103$ $-0.00546-0.06402$ $-0.03181 \quad-0.02128$ $-0.09874-0.05795$ $-0.14846-0.17683$ $-0.07215-0.27137$ $0.10317-0.34860$ $0.38240-0.33841$

$-0.02026-0.0141$ $-0.06927-0.04201$ $-0.12073-0.07214$ $-0.15252-0.11228$ $-0.10336-0.1705$ $0.00172-0.31566$ $0.17235-0.35833$ $0.40472-0.33223$ $0.07496-0.19064$
$E(h, h)$

$-0.00088-0.00270$ $-0.00175-0.00539$ $-0.00439-0.01347$ $-0.01796-0.05278$ $-0.04901-0.11835$ $-0.10462-0.17090$ $-0.15063-0.15550$ $-0.14812-0.1540$ $-0.14695-0.15454$

$-0.03725 \quad-0.09757$ $-0.08176-0.15684$ $-0.12050-0.17524$ $-0.15108-0.1628$ $-0.15131-0.15677$ $-0.14928-0.15431$ $-0.14763-0.15408$ $-0.14692-0.15458$ $-0.14682-0.15504$
0.01

0.02

0.05

0.10

0.20

0.50

1.00

1.50

2.00

3.00

3.50

$0.125 \pi$ $0.375 \pi$

$0.500 \%$

$0.625 \pi$

$0.750: \pi$

$1.000 \pi$

$\begin{array}{cc}-0.00050 & -0.00153 \\ -0.00100 & -0.00306 \\ -0.00248 & -0.00766 \\ -0.00493 & -0.01531 \\ -0.00961 & -0.03058 \\ -0.01967 & -0.07562 \\ -0.01406 & -0.14676 \\ 0.02194 & -0.21251 \\ 0.07865 & -0.27266 \\ 0.14529 & -0.32610 \\ 0.21527 & -0.37217 \\ 0.28537 & -0.41721 \\ -0.01696 & -0.05968 \\ -0.02040 & -0.11690 \\ -0.00437 & -0.17078 \\ 0.02900 & -0.22138 \\ 0.07407 & -0.26848 \\ 0.12557 & -0.31148 \\ 0.17997 & -0.34996 \\ 0.23520 & -0.38390 \\ 0.29013 & -0.41366 \\ 0.34412 & -0.43975\end{array}$

$$
\frac{a}{\lambda}=0.3 \quad \frac{\alpha}{\beta}=1.00
$$

$\begin{array}{ll}-0.00001 & -0.00001 \\ -0.00004 & -0.00002 \\ -0.00025 & -0.00013 \\ -0.00101 & -0.00052 \\ -0.00402 & -0.00206 \\ -0.02405 & -0.01252 \\ -0.08320 & -0.04630 \\ -0.15307 & -0.09491 \\ -0.21789 & -0.15391 \\ -0.27311 & -0.21979 \\ -0.31936 & -0.28911 \\ -0.35826 & -0.35901 \\ -0.01514 & -0.00782 \\ -0.05512 & -0.02964 \\ -0.10796 & -0.06219 \\ -0.16275 & -0.10271 \\ -0.21347 & -0.14932 \\ -0.25822 & -0.20035 \\ -0.29716 & -0.25407 \\ -0.33106 & -0.30894 \\ -0.36070 & -0.36377 \\ -0.38672 & -0.41777\end{array}$

\begin{tabular}{|c|c|c|c|}
\hline $\begin{array}{l}-0.00050 \\
-0.00100 \\
-0.00249 \\
-0.00495 \\
-0.00976 \\
-0.02170 \\
-0.02272 \\
0.01667 \\
0.10899 \\
0.24779 \\
0.38628 \\
0.40944\end{array}$ & $\begin{array}{l}-0.00153 \\
-0.00306 \\
-0.00765 \\
-0.01524 \\
-0.02999 \\
-0.06786 \\
-0.10552 \\
-0.11750 \\
-0.09522 \\
-0.00221 \\
0.22176 \\
0.03679\end{array}$ & $\begin{array}{l}-0.00001 \\
-0.00004 \\
-0.00025 \\
-0.00101 \\
-0.00398 \\
-0.02257 \\
-0.06709 \\
-0.10071 \\
-0.09747 \\
-0.01623 \\
0.20388 \\
0.62084\end{array}$ & $\begin{array}{l}-0.00001 \\
-0.00002 \\
-0.00013 \\
-0.00051 \\
-0.00198 \\
-0.01034 \\
-0.03066 \\
-0.071141 \\
-0.15401 \\
-0.27833 \\
-0.40239 \\
-0.41448\end{array}$ \\
\hline $\begin{array}{r}-0.01803 \\
-0.02619 \\
-0.01419 \\
0.02639 \\
0.10042 \\
0.20482 \\
0.32199 \\
0.41172 \\
0.40212 \\
0.18323\end{array}$ & $\begin{array}{r}-0.05564 \\
-0.09289 \\
-0.11260 \\
-0.11697 \\
-0.09864 \\
-0.03942 \\
0.08867 \\
0.31806 \\
0.67295 \\
1.14669\end{array}$ & $\begin{array}{r}-0.01454 \\
-0.04775 \\
-0.08158 \\
-0.10320 \\
-0.09971 \\
-0.05089 \\
0.07182 \\
0.30026 \\
0.05726 \\
1.13467\end{array}$ & $\begin{array}{l}-0.00688 \\
-0.02084 \\
-0.04150 \\
-0.08024 \\
-0.14638 \\
-0.23972 \\
-0.34509 \\
-0.42426 \\
-0.40657 \\
-0.18240\end{array}$ \\
\hline
\end{tabular}

$\begin{array}{ll}-0.00050 & -0.00153 \\ -0.00100 & -0.00306 \\ -0.00249 & -0.00765 \\ -0.00501 & -0.01522 \\ -0.01016 & -0.02986 \\ -0.02710 & -0.06573 \\ -0.05410 & -0.09172 \\ -0.06825 & -0.09277 \\ -0.07175 & -0.08947 \\ -0.07162 & -0.08789 \\ -0.07117 & -0.08762 \\ -0.07102 & -0.08768 \\ -0.02081 & -0.05461 \\ -0.04360 & -0.08522 \\ -0.06080 & -0.09356 \\ -0.06921 & -0.09230 \\ -0.07168 & -0.08967 \\ -0.07176 & -0.08816 \\ -0.07136 & -0.08767 \\ -0.07110 & -0.08763 \\ -0.07101 & -0.08769 \\ -0.07100 & -0.08773\end{array}$


$\beta \mathrm{h}$

$C(h, 0)$

\begin{tabular}{|c|c|c|}
\hline $\begin{array}{r}.01 \\
.02 \\
.05 \\
.05 \\
.10 \\
.20\end{array}$ & $\begin{array}{l}-0.00644 \\
-0.01288 \\
-0.03218 \\
-0.06429 \\
-0.12801 \\
-0.31018 \\
-0.54961 \\
-0.65444 \\
-0.59816 \\
-0.42117 \\
-0.21834 \\
-0.08230\end{array}$ & $\begin{array}{l}-0.00468 \\
-0.00935 \\
-0.02337 \\
-0.04664 \\
-0.09249 \\
-0.21794 \\
-0.35546 \\
-0.39502 \\
-0.39436 \\
-0.43918 \\
-0.57454 \\
-0.077002\end{array}$ \\
\hline $\begin{array}{l}.125 \pi \\
.250 \pi \\
.375 \pi \\
.500 \pi \\
.625 \pi \\
.750 \pi \\
.875 \pi \\
.000 \pi \\
125 \pi \\
.250 \pi\end{array}$ & $\begin{array}{l}-0.24715 \\
-0.46002 \\
-0.60490 \\
-0.65611 \\
-0.60725 \\
-0.47945 \\
-0.31678 \\
-0.17018 \\
-0.07670 \\
-0.04490\end{array}$ & $\begin{array}{l}-0.17584 \\
-0.30932 \\
-0.37914 \\
-0.39536 \\
-0.39382 \\
-0.41765 \\
-0.49538 \\
-0.02683 \\
-0.78367 \\
-0.92401\end{array}$ \\
\hline
\end{tabular}

0.01 $-0.01271-0.00924$ $-0.06348-0.02308$ $0.20-0.06348-0.04606$ $0.50-0.12641-0.09134$ $1.00-0.30619-0.21533$ $1.50-0.54204-0.35192$ $-0.64477-0.39289$ $2.50-0.58916-0.39495$ $-0.21729-0.47545$ $3.50-0.08360-0.76680$

$0.125 \pi \quad-0.24401-0.17370$ $0.250 \pi \quad-0.45389-0.30590$ $\begin{array}{lll}0.375 \pi & -0.59640 & -0.37585\end{array}$ $0.500 \pi-0.64634-0.39358$ $\begin{array}{llll}0.625 \pi & -0.59810 & -0.3942\end{array}$ $\begin{array}{lll}0.750 \pi & -0.47274 & -0.41968\end{array}$ $\begin{array}{lll}0.875 \pi & -0.31358 & -0.497 \mathrm{~L}\end{array}$ $1.000 \pi-0.17012-0.6267$ $\begin{array}{lll}1.125 \pi & -0.07802 & -0.78012\end{array}$ $1.250 \pi \quad-0.04520-0.91702$

$$
\frac{a}{\lambda}=0.4 \quad \frac{\alpha}{\beta}=0.00
$$

$S(h, 0)$

$E(h, 0)$

$-0.00003-0.00002$ $-0.00013-0.00009$ $-0.00080-0.00058$ $-0.00322-0.00233$ $-0.01285-0.00926$ $-0.07941-0.05478$ $-0.30295-0.17965$ $-0.61831-0.28768$ $-0.93438-0.31339$ $\begin{array}{ll}-1.15697 & -0.26375\end{array}$ $-1.24415-0.21089$

$-1.23319-0.23363$

$-0.04924-0.03465$ $-0.19169-0.12272$ $-0.40833-0.22463$ $-0.66542-0.29706$ $-0.91350-0.31462$ $-1.10644-0.23228$ $-1.21676-0.23222$ $-1.24765-0.20722$ $\begin{array}{ll}-1.23098 & -0.23901 \\ -1.212 .01 & -0.33277\end{array}$

$-0.00003-0.00002$ $-0.00013-0.00009$ $-0.00080 \quad-0.00057$ $\begin{array}{ll}-0.00319 & -0.00229 \\ -0.01273 & -0.00908\end{array}$ $-0.07867-0.0537$ $-0.29978-0.17609$ $-0.61096-0.2820$ $-0.92224-0.30786$ $\begin{array}{ll}-1.14165 & -0.26102\end{array}$ $-1.22909-0.21185$ $-1.22148-0.23627$

$-0.04879-0.03397$ $-0.18979-0.12030$ $-0.40434-0.22016$ $-0.65738-0.29123$ $-0.90169-0.30898$ $-1.09174-0.27853$

$-1.20110-0.23140$

$-1.23331-0.20899$

$-1.21955-0.24163$

$-1.20355-0.33389$

$C(h, h)$

$S(h, h)$

$E(h, h)$

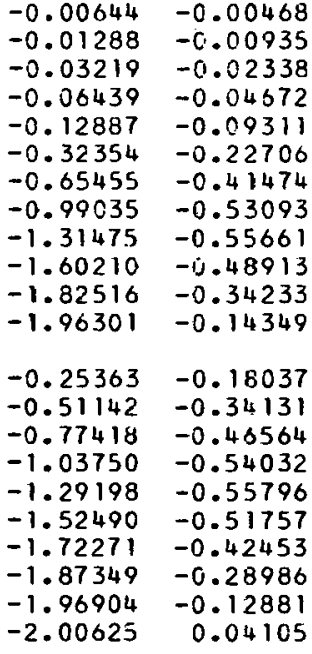
$-0.00644 \quad-0.00468$ $-0.01288-0.00935$ $-0.03213-\mathrm{C}-02335$ $-0.06433-0.04648$ $-0.31379-0.19904$ $-0.55472-0.23830$ $-0.02824-0.12550$ $-0.54159 \quad 0.03502$ $-0.33633$ $-0.386330 .21128$ $0.10040 \quad 0.67934$
$-0.24918-0.16637$ $-0.46723-0.24481$ $-0.60285-0.21031$ $-0.62383-0.10361$ $-0.55129 \quad 0.02308$ $-0.43417 \quad 0.15664$ 0.296180 .31780 0.118330 .51166 $0.41314 \quad 0.78496$

$-0.00003-0.00002$ $-0.00013-0.00009$ $\begin{array}{ll}-0.00080 & -0.00058\end{array}$ $-0.00322-0.00232$ $-0.01288-0.00913$ $-0.08020-0.05003$ $-0.29955-0.12079$ $-0.54417-0.09458$ $\begin{array}{ll}-0.66042 & 0.01470\end{array}$ $\begin{array}{ll}-0.61620 & 0.10377\end{array}$ $-0.49502 \quad 0.14812$ $\begin{array}{ll}-0.33929 & 0.22036\end{array}$

$\begin{array}{lll}-0.04961 & -0.03278\end{array}$ $-0.19303-0.09719$ $-0.39269-0.1254$ $-0.57102-0.08194$ $\begin{array}{ll}-0.65796 & 0.00627\end{array}$ $-0.64109 \quad 0.08429$ $\begin{array}{lll}-0.56025 & 0.12775\end{array}$ 0.16248 $-0.32637 \quad 0.2277$ $\begin{array}{ll}-0.14584 & 0.32606\end{array}$
$-0.00644-0.00468$ $-0.01288-0.00935$ $-0.03220-0.02336$ $-0.06444-0.04655$ $\begin{array}{ll}-0.06444 & -0.04655 \\ -0.32728 & -0.09180 \\ -0.0020737\end{array}$ $-0.32728-0.20737$ $\begin{array}{ll}-0.65737 & -0.2783 \\ -0.91258 & -0.17116\end{array}$ $-1.00342 \quad 0.03608$ $-0.02470 \quad 0.20714$ $\begin{array}{ll}-0.76954 & 0.24619\end{array}$ $-0.66146 \quad 0.15951$

$-0.25571-0.17066$ $-0.51875-0.27016$ $-0.76245-0.25878$ $-0.93674-0.14493$ 0.02052 0.960650 .16892 $-0.84890 \quad 0.24528$ $-0.658020 .23152$ $\begin{array}{ll}-0.65802 & 0.15100 \\ -0.05935 & 0.05347\end{array}$

$$
\frac{a}{\lambda}=0.4 \quad \frac{\alpha}{\beta}=0.005
$$

$\begin{array}{ll}-0.00636 & -0.00462 \\ -0.01272 & -0.00924 \\ -0.03179 & -0.02309 \\ -0.06359 & -0.04614 \\ -0.12726 & -0.09195 \\ -0.31948 & -0.22420 \\ -0.64616 & -0.40944 \\ -0.97728 & -0.52402 \\ -1.29675 & -0.54932 \\ -1.57930 & -0.48298 \\ -1.79825 & -0.33890 \\ -1.93332 & -0.14411 \\ -0.25045 & -0.17811 \\ -0.50494 & -0.33699 \\ -0.76416 & -0.45964 \\ -1.02374 & -0.53327 \\ -1.27434 & -0.55065 \\ -1.50344 & -0.51092 \\ -1.69774 & -0.41954 \\ -1.84563 & -0.28746 \\ -1.93921 & -0.12975 \\ -1.97560 & 0.03634\end{array}$

$-0.00636-0.00462$ $\begin{array}{lll}-0.01271 & -0.00924\end{array}$ $-0.03173-0.02306$ $-0.06352-0.04590$ $-0.12671-0.09005$ $-0.30969-0.19663$ $-0.54677-0.23626$ $-0.61861-0.1265$ $-0.53298$ 0.20418 $\begin{array}{ll}-0.18274 & 0.42890 \\ 0.10262 & 0.66314\end{array}$ $-0.24598 \quad-0.16432$ $-0.46080-0.2421$ $-0.59395-0.20903$ $-0.61421-0.10515$ $-0.54256 \quad 0.01873$ $-0.42631 \quad 0.15027$ $\begin{array}{lll}-0.28923 & 0.30912\end{array}$ $-0.11258 \quad 0.4993$ 0.1258 n 0.67618 $0.40722 \quad 0.76655$ $\begin{array}{ll}-0.00003 & -0.00002\end{array}$ $-0.00013-0.00009$ $-0.00080-0.00057$ $\begin{array}{lll}-0.00319 & -0.00228\end{array}$ $-0.01276-0.00895$ $\begin{array}{lll}-0.07941 & -0.0490\end{array}$ $-0.29592-0.11800$ $-0.53643-0.09218$ $-0.65043 \quad 0.01375$ $-0.60714 \quad 0.09932$ $\begin{array}{ll}-0.48725 & 0.14176\end{array}$ $-0.33140 \quad 0.21111$

$-0.04914 \quad-0.03213$ $-0.19088-0.09507$ $-0.38760-0.12237$ $\begin{array}{ll}-0.56277 & -0.07987\end{array}$ $-0.64802 \quad 0.0056$ $-0.63156 \quad 0.08068$ $-0.44768 \quad 0.15555$ $\begin{array}{lll}-0.31848 & 0.21815\end{array}$ $-0.13920 \quad 0.31224$
$-0.00636-0.00462$ $-0.01272-0.00924$ $-0.03179-0.02307$ $-0.06363-0.04597$ $-0.12757-0.09065$ $-0.32308-0.20472$ $-0.64837-0.27466$ $-0.89912 \quad-0.16945$ $-0.98809 \quad 0.03336$ $-0.911430 .20010$ $\begin{array}{ll}-0.76088 & 0.23805 \\ -0.65647 & 0.15437\end{array}$ $-0.25247-0.16849$ $\begin{array}{lll}-0.51187 & -0.2666\end{array}$ $-0.75172-0.25546$ $-0.92281-0.14373$ $-0.98780 \quad 0.0181$ $\begin{array}{lll}-0.94640 & 0.16292\end{array}$ $-0.83780 \quad 0.23716$ $-0.72224 \quad 0.22386$ $\begin{array}{lll}-0.65316 & 0.14617\end{array}$ $-0.65441 \quad 0.05240$ 
$\beta \mathrm{h}$

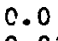

0.0
0.02
0.05

0.05

0.10

0.20

0.50

1.00

2.00

3.00

3.50

$0.125 \pi$

$0.250 \pi$

$0.375 \pi$

(1)

$0.750 \pi$

$0.7575 \pi$

$0.875 \pi$

$1.125 \pi$

$1.250 \pi$

$$
C(h, 0)
$$

$-0.00628-0.00456$ $-0.01256-0.00912$ $\begin{array}{ll}-0.03138 & -0.02279\end{array}$ $-0.12482-0.04549$ $-0.30226-0.21275$ $-0.53458-0.34841$ $-0.41025-0.44356$ $-0.21615-0.57625$ $-0.08474-0.76359$

$-0.24090-0.17158$ $-0.58796-0.37258$ $-0.63673-0.39178$ $-0.46611-0.42158$ $-0.31036-0.49928$ $-0.16994-0.62664$ $-0.07919-0.77660$ $-0.04539-0.91019$ $-0.63525-0.39074$ $-0.58029-0.39544$ $-0.44784-0.30251$
$S(h, 0)$

$-0.00003-0.00002$ $-0.00013-0.00009$ $-0.00079-0.00056$ $-0.01262-0.00890$ $-0.07793-0.05266$ $-0.29664-0.17259$ $-0.60371-0.27644$ $-1.12660-0.25030$ $-1.12660-0.25830$ $-1.20988-0.23872$

$-0.04834-0.03331$ $\begin{array}{ll}-0.18790 & -0.11793 \\ -0.39990 & -0.21577\end{array}$ $-0.64947-0.28552$ $\begin{array}{ll}-0.89008 & -0.30344\end{array}$ $-1.07728-0.27483$ $\begin{array}{lll}-1.18571 & -0.23053\end{array}$ $\begin{array}{ll}-1.21919 & -0.21060\end{array}$ $-1.20822-0.24407$ $\begin{array}{ll}-1.19507 & -0.33487\end{array}$ $-0.60371-0.27644$ $-0.18790-0.11793$

$$
\frac{a}{\lambda}=0.4 \quad \frac{\alpha}{\beta}=0.01
$$

$E(h, 0)$

$C(h, h)$

$-0.00628-0.00456$

$-0.01256-0.00912$

$-0.06279-0.02280$

$-0.12567-0.09080$

$-0.31546-0.22139$

$-0.63789-0.22139$

$-0.96438-0.40421$

$\begin{array}{ll}-0.637838 & -0.51720 \\ -1.27900 & -0.5428\end{array}$

$-1.55682-0.47691$

$-1.77174-0.33549$

$-1.90408-0.14467$

$-0.24731-0.17587$

$-0.49853-0.33272$

$-0.75428-0.45373$

$-1.01016-0.52631$

$-1.25694 \quad-0.54343$

$-1.48228-0.50437$

$-1.67313-0.4146$

$-1.81819-0.28507$

$\begin{array}{rr}-1.90985 & -0.13062 \\ -1.94544 & 0.03177\end{array}$
$S(h, h)$

$-0.00628-0.00456$ $-0.01256-0.00912$

$-0.03738-0.0227$

$-0.12512-0.08893$

$-0.30564-0.19425$

$-0.53894-0.23400$

$-0.00913-0.12745$

$\begin{array}{lll}-0.52449 & 0.02608\end{array}$

$\begin{array}{ll}-0.17646 & 0.41827\end{array}$

$0.10478 \quad 0.64734$

$-0.24282-0.16230$ $-0.45446-0.23943$ $-0.58519-0.20774$ $-0.60475-0.10665$ $\begin{array}{lll}-0.53395 & 0.01457\end{array}$ $-0.419130 .14404$ $-0.28238 \quad 0.35063$ $\begin{array}{rr}-0.12745 & 0.48727 \\ 0.12747 & 0.66008\end{array}$ $0.40149 \quad 0.74868$
$-0.00003-0.00002$ $-0.00013-0.00009$ $-0.02079-0.00056$ $-0.00316-0.00224$ $-0.01265-0.00878$ $-0.07862-0.0480$ $-0.29234-0.11527$ $-0.52881-0.08983$ $-0.64063 \quad 0.0128$

$-0.04867-0.03148$ $-0.18877-0.09300$ . $-0.55466-0.07784$ $\begin{array}{lll}-0.63826 & 0.00497\end{array}$ $0.62220 \quad 0.07716$ 0.11684 $-0.44009 \quad 0.14873$ $\begin{array}{ll}-0.31079 & 0.20876 \\ -0.13276 & 0.29875\end{array}$ $-0.47960 \quad 0.13550$

$-0.323690 .13550$
$\mathrm{E}(\mathrm{h}, \mathrm{h})$

$-0.00628-0.00456$ $-0.01256-0.00912$ $\begin{array}{lll}-0.03140-0.02278 & -0.04\end{array}$ $-0.06283-0.04540$ $-0.12597-0.08951$ $-0.31894 \quad-0.2021$ $\begin{array}{ll}-0.63950 & -0.27106\end{array}$ $-0.88587-0.1677$ $\begin{array}{lll}-0.97301 & 0.03072\end{array}$ $\begin{array}{ll}-0.75228 & 0.23013 \\ -0.65141 & 0.14935\end{array}$

$-0.24926-0.16636$ $-0.50508-0.26315$ $-0.74114-0.25218$ 0.072720 .14254 0.972720 .01589 $-0.82682 \quad 0.15707$ $-0.71490 \quad 0.21640$ $-0.71490 \quad 0.21640$ $\begin{array}{ll}-0.64822 & 0.14145 \\ -0.64940 & 0.05130\end{array}$ $-0.75228 \quad 0.19326$

$\begin{array}{lll}0.01 & -0.00612 & -0.00445 \\ 0.02 & -0.01224 & -0.00890 \\ 0.05 & -0.03060 & -0.02223 \\ 0.10 & -0.06113 & -0.04436 \\ 0.20 & -0.12171 & -0.08799 \\ 0.50 & -0.29454 & -0.20769 \\ 1.00 & -0.51997 & -0.34150 \\ 1.50 & -0.61665 & -0.38642 \\ 2.00 & -0.56297 & -0.39617 \\ 2.50 & -0.39953 & -0.44734 \\ 3.00 & -0.21363 & -0.57747 \\ 3.50 & -0.08657 & -0.75719 \\ 0.125 \pi & -0.23482 & -0.16743 \\ 0.250 \pi & -0.43598 & -0.29586 \\ 0.375 \pi & -0.57146 & -0.36611 \\ 0.500 \pi & -0.61795 & -0.38811 \\ 0.625 \pi & -0.57146 & -0.39488 \\ 0.750 \pi & -0.45310 & -0.42495 \\ 0.875 \pi & -0.30386 & -0.50257 \\ 1.000 \pi & -0.16927 & -0.62606 \\ 1.125 \pi & -0.08107 & -0.76961 \\ 1.250 \pi & -0.04548 & -0.89696\end{array}$

$-0.00003-0.00002$ $-0.00078-0.00054$ $-0.00310-0.00216$ $-0.01239-0.00856$ $-0.07648-0.05062$ $-0.29046-0.16579$ $-0.58953-0.26562$ $-0.88699-0.29182$ $-1.09728 \quad-0.25290$ $-1.18537-0.21408$ $\begin{array}{lll}-1.18701 & -0.24309\end{array}$

$\begin{array}{lll}-0.04746 & -0.03203\end{array}$ $-0.18419-0.11331$ $-0.39119-0.20724$ $-0.63397-0.27441$ $-0.86738-0.29264$ $-1.04913-0.26756$ $\begin{array}{ll}-1.15572 & -0.22864\end{array}$ $-1.19158-0.21342$ $\begin{array}{ll}-1.18586 & -0.24840 \\ -1.17802 & -0.33645\end{array}$ $-0.00012-0.00009$

$$
\frac{a}{\lambda}=0.4 \quad \frac{\alpha}{\beta}=0.02
$$

$\begin{array}{ll}-0.00612 & -0.00445 \\ -0.01224 & -0.00890 \\ -0.03061 & -0.02223 \\ -0.06124 & -0.04443 \\ -0.12255 & -0.08854 \\ -0.30758 & -0.21586 \\ -0.62165 & -0.39395 \\ -0.93910 & -0.50382 \\ -1.24422 & -0.52802 \\ -1.51284 & -0.46499 \\ -1.71992 & -0.32876 \\ -1.84696 & -0.14563 \\ -0.24115 & -0.17149 \\ -0.48596 & -0.32434 \\ -0.73489 & -0.44213 \\ -0.98355 & -0.51267 \\ -1.22286 & -0.52928 \\ -1.44085 & -0.49150 \\ -1.62500 & -0.40492 \\ -1.76456 & -0.28030 \\ -1.85248 & -0.13217 \\ -1.88652 & 0.02307\end{array}$
$-0.01224-0.00889$ $-0.03060-0.0222$ $-0.06117-0.04420$ $-0.12199-0.08673$ $-0.29771-0.18957$ $-0.52362-0.22980$ $\begin{array}{lll}-0.59061 & -0.12919\end{array}$ $-0.50784 \quad 0.01763$ $\begin{array}{ll}-0.35767 & 0.18373\end{array}$ $\begin{array}{rr}-0.16423 & 0.3976 \\ 0.10894 & 0.61690\end{array}$

$-0.23661-0.15834$ $-0.44204-0.23418$ $-0.56807-0.20516$ $-0.58625-0.10942$ $-0.51708 \quad 0.00654$ $-0.26895 \quad 0.28409$ $-0.09603 \quad 0.46386$ $0.13069 \quad 0.62906$ $0.39058 \quad 0.71447$ $-0.40444 \quad 0.13199$
$-0.00003-0.00002$ $-0.00012-0.00009$ $-0.00311-0.00215$ $-0.01242-0.00844$ $-0.07707-0.04606$ $-0.28531-0.10996$ $-0.51396-0.08528$ $-0.02156 \quad 0.01106$ $-0.58086 \quad 0.08663$ $-0.46464 \quad 0.12332$ $\begin{array}{ll}-0.30882 & 0.18426\end{array}$

$-0.04775-0.03023$ $-0.18460-0.08895$ $-0.37278-0.11365$ $-0.53885-0.07392$ $\begin{array}{ll}-0.61927 & 0.00376\end{array}$ $-0.60396 \quad 0.07040$ $-0.29595 \quad 0.19044$ $-0.120490 .2727$ $-0.00078-0.0005$ $-0.52804 \quad 0.10640$

$\begin{array}{ll}-0.00612 & -0.00445 \\ -0.01225 & -0.00889 \\ -0.03062 & -0.02221 \\ -0.06127 & -0.04427 \\ -0.12283 & -0.08728 \\ -0.31082 & -0.19697 \\ -0.02211 & -0.26401 \\ -0.85996 & -0.16438 \\ -0.94353 & 0.02569 \\ -0.87271 & 0.18013 \\ -0.73523 & 0.21493 \\ -0.64108 & 0.13965 \\ & \\ -0.24298 & -0.16217 \\ -0.49177 & -0.25633 \\ -0.72043 & -0.24575 \\ -0.88228 & -0.14015 \\ -0.94326 & 0.01154 \\ -0.90492 & 0.14587 \\ -0.80524 & 0.21410 \\ -0.70024 & 0.20209 \\ -0.63812 & 0.13232 \\ -0.63918 & 0.04899\end{array}$


$S(h, 0)$

$\begin{array}{ll}-0.00003 & -0.00002 \\ -0.00012 & -0.00008 \\ -0.00076 & -0.00152 \\ -0.00305 & -0.00207 \\ -0.01217 & -0.00323 \\ -0.07505 & -0.04365 \\ -0.28442 & -0.15723 \\ -0.57573 & -0.25519 \\ -0.86444 & -0.28160 \\ -1.06897 & -0.24755 \\ -1.15738 & -0.21504 \\ -1.16458 & -0.24680 \\ -0.04658 & -0.03078 \\ -0.18054 & -0.10886 \\ -0.38269 & -0.15902 \\ -0.61891 & -0.26371 \\ -0.84541 & -0.26223 \\ -1.02193 & -0.26045 \\ -1.12675 & -0.26657 \\ -1.16479 & -0.25571 \\ -1.16390 & -0.25208 \\ -1.16094 & -0.33755\end{array}$

$$
\frac{a}{\lambda}=0.4 \quad \frac{\alpha}{\beta}=0.03
$$

$E(h, 0)$

$C(h, h)$

$-0.00597-0.00434$ $-0.01194-0.00868$ $-0.02985-0.02168$ $-0.05972-0.04333$ $-0.11950-0.08634$ $-0.29090 \quad-0.21047$ $-0.60582-0.38395$ $-1.21039-0.51428$ $-1.47012 \quad-0.45336$ $\begin{array}{ll}-1.66964 & -0.32214 \\ -1.79157 & -0.14639\end{array}$

$-0.23514-0.16722$ $-0.47370-0.31617$ $-0.71601-0.43083$ $-0.95764-0.49938$ $-1.18971-0.51550$ $-1.40060-0.47896$ $-1.57827-0.39544$ $-1.71255-0.27556$ $\begin{array}{rr}-1.79628 & -0.13350 \\ -1.82944 & 0.01492\end{array}$ $-0.91447-0.49679$
$S(h, h)$

$-0.00003-0.00002$ $-0.00012-0.00008$ $-0.00076-0.0005$ $-0.00305-0.00207$ $-0.01219-0.00811$ $-0.07555-0.04418$ $-0.27346-0.10487$ $-0.56405 \quad 0.07868$ $-0.45013 \quad 0.11156$ $-0.29462 \quad 0.16710$

$-0.04684-0.02902$ $-0.18053-0.08506$ $-0.38325-0.10814$ $-0.52357-0.07016$ $\begin{array}{ll}-0.60097 & 0.00263\end{array}$ $\begin{array}{ll}-0.59633 & 0.06398\end{array}$ $-0.51264 \quad 0.0963$ $-0.41095 \quad 0.12253$ $\begin{array}{ll}-0.28181 & 0.17274 \\ -0.10893 & 0.24789\end{array}$ $-0.49960-0.08092$ $-0.60317 \quad 0.00940$
$E(h, h)$

$-0.00597-0.00434$ $-0.01194-0.00867$ $-0.02986-0.02166$ $-0.05975-0.04317$ $-0.11979-0.08510$ $-0.30291-0.19197$ $-0.83482-0.16107$ $\begin{array}{lll}-0.91498 & 0.02097\end{array}$ 0.16770 $-0.23685-0.15808$ $-0.47882-0.24969$ $-0.70030-0.23948$ $\begin{array}{ll}-0.85627 & -0.13778\end{array}$ $-0.91472 \quad 0.00746$ $-0.87830 \quad 0.13526$ $-0.78415 \quad 0.19977$ $-0.68565 \quad 0.1885$ $\begin{array}{ll}-0.62777 & 0.12360\end{array}$ $-0.60520-0.25714$ $\begin{array}{ll}-0.03052 & 0.20056\end{array}$

$$
\frac{a}{\lambda}=0.4 \quad \frac{\alpha}{\beta}=0.05
$$

$\begin{array}{ll}-0.00568 & -0.00413 \\ -0.01136 & -0.00825 \\ -0.02839 & -0.02062 \\ -0.05679 & -0.04120 \\ -0.11364 & -0.08210 \\ -0.28510 & -0.20008 \\ -0.57537 & -0.36470 \\ -0.86716 & -0.48573 \\ -1.14549 & -0.48786 \\ -1.38829 & -0.43096 \\ -1.57352 & -0.30920 \\ -1.68590 & -0.014733 \\ -0.22357 & -0.15899 \\ -0.45012 & -0.30044 \\ -0.67968 & -0.40908 \\ -0.90785 & -0.47383 \\ -1.12609 & -0.48901 \\ -1.32346 & -0.45483 \\ -1.48887 & -0.37710 \\ -1.61316 & -0.26618 \\ -1.69075 & -0.13551 \\ -1.72053 & 0.00014\end{array}$

$\begin{array}{rr}-0.00568 & -0.00412 \\ -0.01135 & -0.00825 \\ -0.02838 & -0.02059 \\ -0.05672 & -0.04099 \\ -0.11306 & -0.08046 \\ -0.27514 & -0.17621 \\ -0.48031 & -0.21738 \\ -0.53836 & -0.13320 \\ -0.46051 & -0.00499 \\ -0.31721 & 0.14647 \\ -0.13005 & 0.34029 \\ 0.12036 & 0.53420 \\ -0.21895 & -0.14702 \\ -0.40685 & -0.21909 \\ -0.51973 & -0.19736 \\ -0.53404 & -0.11623 \\ -0.46917 & -0.01490 \\ -0.36240 & 0.09897 \\ -0.23089 & 0.23811 \\ -0.06570 & 0.39922 \\ 0.13955 & 0.54494 \\ 0.36190 & 0.62316\end{array}$

$-0.00003-0.00002$ $-0.00012-0.00008$ $-0.00074-0.00048$ $-0.00294-0.00191$ $-0.01175-0.00748$ $\begin{array}{lll}-0.07259 & -0.04062\end{array}$ $-0.47227-0.09527$ $-0.568350 .0727$ $-0.53205 \quad 0.00638$ $-0.42247 \quad 0.0892$ $\begin{array}{ll}-0.42247 & 0.08929 \\ -0.26812 & 0.13451\end{array}$

$-0.04507-0.02673$ $-0.17266-0.07772$ $-0.34501-0.09778$ $-0.49454-0.06313$ $\begin{array}{ll}-0.56629 & 0.00057\end{array}$ $\begin{array}{ll}-0.55287 & 0.05210\end{array}$ $-0.48325 \quad 0.07763$ $\begin{array}{ll}-0.25546 & 0.13917 \\ -0.08777 & 0.20162\end{array}$ $\begin{array}{ll}-0.38375 & 0.09802\end{array}$ $\begin{array}{ll}-0.00568 & -0.00412\end{array}$ $-0.02839-0.02060$ $\begin{array}{ll}-0.05682 & -0.04105 \\ -0.01387 & -0.08092\end{array}$ $-0.28708-0.08092$ $-0.57275-0.24393$ $-0.78676-0.15460$ $-0.86050 \quad 0.01237$

$-0.68540 \quad 0.17484$

$\begin{array}{ll}-0.68540 & 0.17413 \\ -0.60885 & 0.11319\end{array}$

$-0.22506-0.15022$ $-0.45392-0.23691$ $-0.66173-0.22742$ $-0.80658-0.13309$ $-0.86027 \quad 0.00007$ $\begin{array}{ll}-0.82739 & 0.11576\end{array}$ $\begin{array}{ll}-0.65673 & 0.16361 \\ -0.60648 & 0.10733\end{array}$ $\begin{array}{ll}-0.60648 & 0.10733 \\ -0.60723 & 0.04152\end{array}$ $-0.01136-0.00825$ $-0.80005 \quad 0.14484$ $-0.656730 .17341$

$\begin{array}{lll}0.028417 & -0.50915 \\ 1.000 \pi & -0.16501 & -0.62228 \\ 1.125 \pi & -0.08364 & -0.74902\end{array}$

$\begin{array}{rrr}1.125 \pi & -0.08364 & -0.74902 \\ 1.250 \pi & -0.04365 & -0.86026\end{array}$

$\begin{array}{rr}-1.72053 & 0.00014\end{array}$ 
$0.125 \pi$ $0.250 \pi$ $0.375 \pi$ $0.500 \pi$ $0.625 \pi$ $0.875 \pi$ $1.000 \pi$ $-0.01080-0.00784$ $-0.02699-0.01960$ $-0.10727-0.07766$ $-0.25884-0.18416$ $-0.45289-0.30888$ $-0.53184-0.36437$ . $-0.34880-0.45828$ $-0.19740-0.57714$ $-0.08848-0.72555$

$-0.20663-0.14814$ $-0.38136-0.26473$ $-0.49589-0.33517$ $-0.53238-0.36878$ $-0.27100-0.51111$ $-0.16067-0.61824$ $-0.08323-0.7355$ $-0.04102-0.83789$
$-0.00540-0.00392$ $-0.05390-0.03913$ $-0.39261-0.39227$

$$
\frac{\mathrm{a}}{\lambda}=0.4 \quad \frac{\alpha}{\beta}=0.07
$$

$E(h, J)$ $-0.00011-0.00007$ $-0.00071-0.00044$ $-0.01731-0.00701$ $-0.26154-0.13525$ $-0.26154-0.13525$ $-0.78118-0.2174$ $-0.96505-0.22675$ $-0.96505-0.22675$ $\begin{array}{ll}-1.05399 & -0.21542 \\ -1.07930 & -0.25602\end{array}$

$-0.04324-0.02622$ $-0.16668-0.09254$ $-0.35067-0.16901$ $-0.56279-0.22474$ $-0.76427-0.2441$ $-0.92202-0.23368$ $-1.02029-0.21684$ $-1.06529-0.22048$ $\begin{array}{ll}-1.08013 & -0.26115 \\ -1.09279 & -0.33793\end{array}$
$-0.00003-0.00002$ $0.00283-0.00177$

$\begin{array}{ll}-0.00540 & -0.00392 \\ -0.01080 & -0.00785 \\ -0.02700 & -0.01961 \\ -0.05400 & -0.03918 \\ -0.10806 & -0.07807 \\ -0.27103 & -0.19021 \\ -0.54645 & -0.34642 \\ -0.82229 & -0.44195 \\ -1.08409 & -0.46280 \\ -1.31106 & -0.40966 \\ -1.48303 & -0.29667 \\ -1.58659 & -0.14759 \\ -0.21257 & -0.15117 \\ -0.42770 & -0.28549 \\ -0.64520 & -0.38844 \\ -0.86066 & -0.44959 \\ -1.06589 & -0.46388 \\ -1.25061 & -0.43192 \\ -1.40459 & -0.35958 \\ -1.51964 & -0.25693 \\ -1.59104 & -0.13675 \\ -1.61828 & -0.01277\end{array}$

$C(h, h)$

$\begin{array}{ll}-0.00540 & -0.00392\end{array}$ $-0.01080-0.0078$ $-0.02699-0.01958$ $-0.05393-0.03899$ $-0.26106-0.16784$ $-0.45350-0.16784$ $-0.43106-0.1349$ $-0.29189 \quad 0.12414$ $\begin{array}{ll}-0.10917 & 0.30575\end{array}$ $0.12727 \quad 0.48557$

$\begin{array}{ll}-0.20792 & -0.13994\end{array}$ $-0.38499-0.20957$ $-0.48987-0.19213$ $-0.50181-0.11976$ $-0.43938-0.02718$ $-0.33606 \quad 0.07937$ $-0.20728 \quad 0.21032$ $-0.04743 \quad 0.36047$ $\begin{array}{ll}0.14495 & 0.49557 \\ 0.34582 & 0.57082\end{array}$ $-0.50612-0.13499$
$S(h, h)$

$\begin{array}{ll}-0.00003 & -0.00002\end{array}$ $-0.00011-0.0000$ $-0.00284-0.00176$ $-0.01133-0.00690$ $-0.06975-0.03730$ $-0.25282-0.08643$ $-0.44669-0.06531$ $-0.535950 .00370$ $-0.396540 .05052$ $\begin{array}{ll}-0.24391 & 0.10416\end{array}$

$-0.04336-0.02459$ $-0.16515-0.0709$ $-0.32779-0.08826$ $-0.46740-0.05670$ $\begin{array}{ll}-0.53403 & -0.00123\end{array}$ $\begin{array}{lll}-0.52164 & 0.04138\end{array}$ $-0.35836 \quad 0.07513$ $\begin{array}{ll}-0.06882 & 0.15953\end{array}$ $-0.00071-0.00044$ $\begin{array}{ll}-0.45565 & 0.0604\end{array}$
$E(h, h)$

$\begin{array}{ll}-0.00540 & -0.00392 \\ -0.01080 & -0.00784 \\ -0.02700 & -0.01959 \\ -0.05403 & -0.03904 \\ -0.10827 & -0.07693 \\ -0.27322 & -0.17321 \\ -0.54204 & -0.23140 \\ -0.74152 & -0.14834 \\ -0.80936 & 0.00481 \\ -0.75496 & 0.12442 \\ -0.65338 & 0.15051 \\ -0.58669 & 0.09759 \\ -0.21385 & -0.14275 \\ -0.43033 & -0.22479 \\ -0.62531 & -0.21596 \\ -0.75982 & -0.12847 \\ -0.80914 & -0.00639 \\ -0.77947 & 0.09834 \\ -0.70454 & 0.14986 \\ -0.62827 & 0.14130 \\ -0.58465 & 0.099 .254 \\ -0.58523 & 0.03631\end{array}$

$$
\frac{a}{\lambda}=0.4 \quad \frac{\alpha}{\beta}=0.10
$$

$\begin{array}{ll}-0.00501 & -0.00364 \\ -0.01001 & -0.00727 \\ -0.02503 & -0.01816 \\ -0.05001 & -0.03616 \\ -0.09962 & -0.07099 \\ -0.24130 & -0.15603 \\ -0.41614 & -0.19795 \\ -0.46135 & -0.13654 \\ -0.38985 & -0.03480 \\ -0.25632 & 0.09411 \\ -0.08040 & 0.25900 \\ 0.13683 & 0.42119 \\ & \\ -0.19241 & -0.12994 \\ -0.35443 & -0.19605 \\ -0.44836 & -0.18429 \\ -0.45704 & -0.12358 \\ -0.39773 & -0.04297 \\ -0.29903 & 0.05323 \\ -0.17436 & 0.17265 \\ -0.02234 & 0.30828 \\ 0.15253 & 0.43033 \\ 0.32560 & 0.50250\end{array}$

0.32560

$\begin{array}{ll}-0.00003 & -0.00002 \\ -0.00011 & -0.00006 \\ -0.00067 & -0.00039 \\ -0.00268 & -0.00156 \\ -0.01071 & -0.00610 \\ -0.06567 & -0.03276 \\ -0.23528 & -0.07444 \\ -0.41133 & -0.05532 \\ -0.49151 & 0.00022 \\ -0.46085 & 0.03269 \\ -0.36073 & 0.04032 \\ -0.21139 & 0.06261 \\ -0.04090 & -0.02166 \\ -0.15451 & -0.06165 \\ -0.30376 & -0.07542 \\ -0.42995 & -0.04809 \\ -0.48977 & -0.00355 \\ -0.47869 & 0.02720 \\ -0.41745 & 0.03718 \\ -0.32350 & 0.04369 \\ -0.19925 & 0.06527 \\ -0.04382 & 0.10336\end{array}$

$-0.04382$

$\begin{array}{ll}-0.00501 & -0.00364 \\ -0.01001 & -0.00727 \\ -0.02504 & -0.01817 \\ -0.05010 & -0.03620 \\ -0.10037 & -0.07132 \\ -0.25288 & -0.16035 \\ -0.49907 & -0.21381 \\ -0.67856 & -0.13932 \\ -0.73842 & -0.00479 \\ -0.69200 & 0.09783 \\ -0.60727 & 0.11978 \\ -0.55304 & 0.07694 \\ -0.19808 & -0.13223 \\ -0.39722 & -0.20777 \\ -0.57443 & -0.19984 \\ -0.69481 & -0.12168 \\ -0.73823 & -0.01451 \\ -0.71279 & 0.07569 \\ -0.64966 & 0.11922 \\ -0.58669 & 0.11224 \\ -0.55141 & 0.07291 \\ -0.55181 & 0.02849\end{array}$

\begin{tabular}{|c|c|c|}
\hline $\begin{array}{l}0.01 \\
0.02 \\
0.05 \\
0.10 \\
0.20 \\
0.50 \\
1.00 \\
1.50 \\
2.00 \\
2.50 \\
3.00 \\
3.50\end{array}$ & $\begin{array}{l}-0.00501 \\
-0.01001 \\
-0.02503 \\
-0.04999 \\
-0.09945 \\
-0.23956 \\
-0.41700 \\
-0.48690 \\
-0.44197 \\
-0.32071 \\
-0.18565 \\
-0.08529\end{array}$ & $\begin{array}{l}-0.00364 \\
-0.00728 \\
-0.01818 \\
-0.03629 \\
-0.07206 \\
-0.17135 \\
-0.29079 \\
-0.35095 \\
-0.39120 \\
-0.45958 \\
-0.57267 \\
-0.70664\end{array}$ \\
\hline $\begin{array}{l}0.125 \pi \\
0.250 \pi \\
0.375 \pi \\
0.500 \pi \\
0.025 \pi \\
0.750 \pi \\
0.875 \pi \\
1.000 \pi \\
1.125 \pi \\
1.250 \pi\end{array}$ & $\begin{array}{l}-0.19138 \\
-0.35201 \\
-0.45562 \\
-0.48709 \\
-0.44852 \\
-0.35981 \\
-0.25143 \\
-0.15249 \\
-0.08017 \\
-0.03550\end{array}$ & $\begin{array}{l}-0.13766 \\
-0.24766 \\
-0.31770 \\
-0.35661 \\
-0.38778 \\
-0.43523 \\
-0.51099 \\
-0.61024 \\
-0.71554 \\
-0.80679\end{array}$ \\
\hline
\end{tabular}

$\begin{array}{ll}-0.00003 & -0.00002 \\ -0.00011 & -0.00006 \\ -0.00067 & -0.00039 \\ -0.00268 & -0.00156 \\ -0.01071 & -0.00621 \\ -0.06572 & -0.03662 \\ -0.24566 & -0.11943 \\ -0.48910 & -0.19227 \\ -0.72532 & -0.21932 \\ -0.89585 & -0.21190 \\ -0.98456 & -0.21287 \\ -1.01988 & -0.25822 \\ -0.04088 & -0.02319 \\ -0.15698 & -0.08176 \\ -0.32859 & -0.14927 \\ -0.52464 & -0.19915 \\ -0.70979 & -0.21891 \\ -0.85544 & -0.21527 \\ -0.94929 & -0.20848 \\ -0.99799 & -0.22038 \\ -1.02154 & -0.26324 \\ -1.04267 & -0.33484\end{array}$

$\begin{array}{ll}-0.00501 & -0.00364 \\ -0.01001 & -0.00728 \\ -0.02504 & -0.01818 \\ -0.05008 & -0.03633 \\ -0.10020 & -0.07240 \\ -0.25123 & -0.17631 \\ -0.50577 & -0.32070 \\ -0.75932 & -0.40855 \\ -0.99813 & -0.42762 \\ -1.20328 & -0.37964 \\ -1.35712 & -0.27864 \\ -1.44873 & -0.14688 \\ -0.19707 & -0.14014 \\ -0.39616 & -0.26446 \\ -0.59673 & -0.35940 \\ -0.79444 & -0.41554 \\ -0.98160 & -0.42859 \\ -1.14885 & -0.39968 \\ -1.28716 & -0.33474 \\ -1.38962 & -0.24337 \\ -1.45264 & -0.13735 \\ -1.47647 & -0.02902\end{array}$


$\mathrm{C}(\mathrm{h}, 0)$

$\begin{array}{lll}0.01 & -0.00389 & -0.00283 \\ 0.02 & -0.00779 & -0.00566 \\ 0.05 & -0.01946 & -0.01414 \\ 0.10 & -0.03887 & -0.02824 \\ 0.20 & -0.07727 & -0.05614 \\ 0.50 & -0.18517 & -0.13481 \\ 1.00 & -0.31725 & -0.23765 \\ 1.50 & -0.36378 & -0.30659 \\ 2.00 & -0.32696 & -0.36711 \\ 2.50 & -0.23953 & -0.44447 \\ 3.00 & -0.14266 & -0.54213 \\ 3.50 & -0.06244 & -0.04337 \\ 0.125 \pi & -0.14827 & -0.10782 \\ 0.250 \pi & -0.26984 & -0.19839 \\ 0.375 \pi & -0.34432 & -0.26512 \\ 0.500 \pi & -0.36313 & -0.31494 \\ 0.025 \pi & -0.33187 & -0.36233 \\ 0.750 \pi & -0.26734 & -0.41981 \\ 0.875 \pi & -0.19032 & -0.49129 \\ 1.000 \pi & -0.11771 & -0.57141 \\ 1.125 \pi & -0.05774 & -0.04990 \\ 1.250 \pi & -0.00975 & -0.71737\end{array}$

$S(h, 0)$

$\begin{array}{ll}-0.00002 & -0.00001\end{array}$ $-0.00009-0.00004$ $-0.00056-0.00026$ $-0.00223-0.00102$ $-0.00888-0.00406$ $-0.19964-0.02389$ $\begin{array}{ll}-0.09964 & -0.07779 \\ -0.39043 & -0.12701\end{array}$ $-0.57227-0.15339$ $-0.70835-0.15339$ $-0.70835-0.16772$ $\begin{array}{ll}-0.79368 & -0.19423 \\ -0.84776 & -0.24773\end{array}$

$\begin{array}{ll}-0.03382 & -0.01514\end{array}$ $-0.12853-0.05325$ $-0.26530-0.09742$ $\begin{array}{ll}-0.26530 & -0.09742 \\ -0.41787 & -0.13217\end{array}$ $-0.56027-0.15214$ $-0.67480-0.16341$ $-0.75631-0.17805$ $\begin{array}{lll}-0.81103 & -0.20648\end{array}$ $-0.85098-0.25239$ $-0.88757-0.31208$

$$
\frac{a}{\lambda}=0.4 \quad-\frac{\alpha}{\beta}=0.20
$$

$E(h, 0)$

$-0.00389-0.00283$ $-0.00779-0.00566$ $-0.01947-0.01414$ $-0.03895-0.02826$ $-0.07792-0.05629$ $\begin{array}{ll}-0.19507 & -0.13691\end{array}$ $\begin{array}{ll}-0.39082 & -0.24799\end{array}$ $-0.58227-0.31441$ $-0.80457-0.32854$ $-0.90457-0.29446$ $-1.01068-0.22497$

$\begin{array}{lll}-0.15312 & -0.10889\end{array}$ $-0.30687-0.20491$ $-0.45998-0.27742$ $-0.60843-0.31962$ $-0.74609-0.32928$ $-0.86619-0.30859$ $-0.96291-0.26325$ $-1.03254-0.20125$ $-1.07410-0.13145$ $-1.08936-0.06236$
$\mathrm{C}(\mathrm{h}, \mathrm{h})$

$\begin{array}{ll}-0.00389 & -0.00233 \\ -0.00779 & -0.00566 \\ -0.01946 & -0.01413 \\ -0.03888 & -0.02813 \\ -0.07734 & -0.05527 \\ -0.18571 & -0.12238 \\ -0.31295 & -0.16357 \\ -0.33882 & -0.13443 \\ -0.27563 & -0.07225 \\ -0.15718 & 0.01913 \\ -0.00289 & 0.14049 \\ 0.16438 & 0.26549 \\ -0.14865 & -0.10154 \\ -0.26935 & -0.15698 \\ -0.33431 & -0.15882 \\ -0.33445 & -0.12717 \\ -0.28246 & -0.07767 \\ -0.19555 & -0.01062 \\ -0.08389 & 0.07679 \\ 0.04472 & 0.17723 \\ 0.17529 & 0.27319 \\ 0.28399 & 0.34613\end{array}$

$S(h, h)$

$-0.00002-0.00001$ $-0.00009-0.00004$ $-0.0 C 056-0.00026$ $-0.00223-0.00102$ $-0.00887-0.00397$ $-0.05367-0.02079$ $-0.18573-0.04375$ $-0.31527-0.03047$ $-0.37304-0.00812$ $-0.35000-0.01159$ $\begin{array}{ll}-0.26477 & -0.03367\end{array}$ $-0.12826-0.04610$

$\begin{array}{ll}-0.03362 & -0.01390\end{array}$ $-0.12388-0.03701$ $-0.23688-0.04292$ $-0.32865-0.02674$ $-0.37175-0.00908$ $0.36379-0.00759$ $0.33102-0.03924$ $-0.23102-0.03924$ $\begin{array}{rr}-0.11724 & -0.04608 \\ 0.01968 & -0.03644\end{array}$
$E(h, h)$

$-0.00389-0.00283$ $-0.00779-0.00566$ $-0.01947-0.01413$ $-0.03896-0.02815$ $-0.07798-0.05542$ $-0.19541-0.12399$ $-0.37905-0.16428$ $-0.50534-0.11248$ $-0.54480-0.02510$ $\begin{array}{lll}-0.51741 & 0.03649\end{array}$ $\begin{array}{lll}-0.47111 & 0.04883\end{array}$ $\begin{array}{ll}-0.44388 & 0.02764\end{array}$

$-0.15344-0.10246$ $-0.30422-0.15981$ $-0.43310-0.15429$ $0.51627-0.10062$ $-0.54468-0.03118$ $-0.52944 \quad 0.02367$ 0.460510 .04849 $-0.46051 \quad 0.04495$ $\begin{array}{ll}-0.44311 & 0.02573 \\ -0.44320 & 0.00549\end{array}$

$\begin{array}{lllll}0.01 & -0.00236 & -0.00171 & -0.00002 & -0.00000 \\ 0.02 & -0.00471 & -0.00342 & -0.00006 & -0.00002 \\ 0.05 & -0.01177 & -0.00856 & -0.00038 & -0.00010 \\ 0.10 & -0.02350 & -0.01710 & -0.00152 & -0.00038 \\ 0.20 & -0.04666 & -0.03410 & -0.00605 & -0.00152 \\ 0.50 & -0.11089 & -0.08359 & -0.03670 & -0.00898 \\ 1.00 & -0.18511 & -0.15842 & -0.13260 & -0.02983 \\ 1.50 & -0.20558 & -0.22651 & -0.25462 & -0.05251 \\ 2.00 & -0.17945 & -0.29637 & -0.37103 & -0.07511 \\ 2.50 & -0.12698 & -0.37184 & -0.46594 & -0.10396 \\ 3.00 & -0.06651 & -0.44865 & -0.53959 & -0.14501 \\ 3.50 & -0.00705 & -0.51943 & -0.59958 & -0.19816 \\ 0.125 \pi & -0.08913 & -0.06622 & -0.02274 & -0.00569 \\ 0.250 \pi & -0.15944 & -0.12751 & -0.08610 & -0.02016 \\ 0.375 \pi & -0.19865 & -0.18302 & -0.17494 & -0.03804 \\ 0.500 \pi & -0.20431 & -0.23612 & -0.27201 & -0.05562 \\ 0.625 \pi & -0.18252 & -0.29107 & -0.36318 & -0.07334 \\ 0.750 \pi & -0.14354 & -0.34969 & -0.44106 & -0.09458 \\ 0.875 \pi & -0.09708 & -0.41038 & -0.50484 & -0.12272 \\ 1.0001 \pi & -0.04942 & -0.46959 & -0.55762 & -0.15900 \\ 1.1251 \pi & -0.00306 & -0.52392 & -0.60338 & -0.20214 \\ 1.250 \pi & 0.04200 & -0.57154 & -0.64500 & -0.24942\end{array}$

$\begin{array}{ll}-0.00236 & -0.00171 \\ -0.00477 & -0.00342 \\ -0.01178 & -0.00856 \\ -0.02356 & -0.01709 \\ -0.04711 & -0.03404 \\ -0.11762 & -0.08256 \\ -0.23333 & -0.14830 \\ -0.34255 & -0.18628 \\ -0.43787 & -0.17408 \\ -0.51257 & -0.17683 \\ -0.56306 & -0.14393 \\ -0.58097 & -0.10555 \\ -0.09244 & -0.06574 \\ -0.18415 & -0.12303 \\ -0.27337 & -0.16532 \\ -0.35707 & -0.18916 \\ -0.43154 & -0.19446 \\ -0.49349 & -0.18385 \\ -0.54079 & -0.16175 \\ -0.57295 & -0.13320 \\ -0.59104 & -0.10295 \\ -0.59730 & -0.07483\end{array}$

$\begin{array}{ll}-0.00236 & -0.00171 \\ -0.00471 & -0.00342 \\ -0.01177 & -0.00855 \\ -0.02350 & -0.01703 \\ -0.04663 & -0.03352 \\ -0.11027 & -0.07542 \\ -0.17829 & -0.11056 \\ -0.18263 & -0.11352 \\ -0.12891 & -0.09608 \\ -0.03059 & -0.05334 \\ 0.09312 & 0.01999 \\ 0.20850 & 0.12163 \\ & \\ -0.08886 & -0.06208 \\ -0.15640 & -0.10063 \\ -0.18723 & -0.11427 \\ -0.17825 & -0.11228 \\ -0.13452 & -0.09811 \\ -0.06254 & -0.06867 \\ 0.02954 & -0.02069 \\ 0.12847 & 0.04616 \\ 0.21500 & 0.12945 \\ 0.26702 & 0.22416\end{array}$

$\begin{array}{ll}-0.00002 & -0.00000 \\ -0.00005 & -0.00002 \\ -0.00038 & -0.00010 \\ -0.00152 & -0.00038 \\ -0.00602 & -0.00147 \\ -0.03567 & -0.00707 \\ -0.11728 & -0.01094 \\ -0.19244 & -0.00615 \\ -0.22766 & -0.01728 \\ -0.21452 & -0.05829 \\ -0.14950 & -0.11781 \\ -0.03095 & -0.17004 \\ -0.02255 & -0.00491 \\ -0.07997 & -0.01103 \\ -0.14725 & -0.00920 \\ -0.20022 & -0.00615 \\ -0.22669 & -0.01546 \\ -0.22343 & -0.04371 \\ -0.18889 & -0.08702 \\ -0.12122 & -0.13464 \\ -0.02102 & -0.17258 \\ 0.10514 & -0.18549\end{array}$

$\begin{array}{ll}-0.00236 & -0.00171 \\ -0.00471 & -0.00342 \\ -0.01178 & -0.00855 \\ -0.02355 & -0.01702 \\ -0.04708 & -0.03345 \\ -0.11669 & -0.07415 \\ -0.21894 & -0.09704 \\ -0.28153 & -0.07196 \\ -0.29870 & -0.03504 \\ -0.28915 & -0.01281 \\ -0.27530 & -0.00891 \\ -0.26842 & -0.01410 \\ -0.09207 & -0.06152 \\ -0.17853 & -0.09458 \\ -0.24674 & -0.09193 \\ -0.28648 & -0.06660 \\ -0.29865 & -0.03742 \\ -0.29317 & -0.01711 \\ -0.28176 & -0.00903 \\ -0.27248 & -0.00994 \\ -0.26825 & -0.01453 \\ -0.26822 & -0.01874\end{array}$




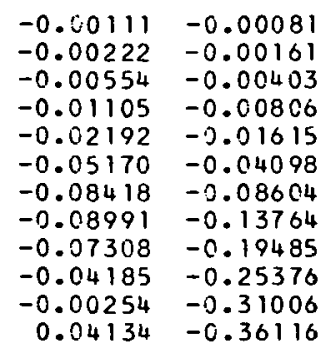

$E(h, 0)$

$C(h, h)$

$S(h, h)$

$\begin{array}{ll}-0.00111 & -0.00081 \\ -0.00222 & -0.00161 \\ -0.00554 & -0.00402 \\ -0.01108 & -0.00804 \\ -0.02215 & -0.01600 \\ -0.05506 & -0.03866 \\ -0.10772 & -0.06860 \\ -0.15475 & -0.08502 \\ -0.19282 & -0.08821 \\ -0.22004 & -0.08201 \\ -0.23663 & -0.07127 \\ -0.24454 & -0.06008 \\ & \\ -0.04336 & -0.03084 \\ -0.08561 & -0.05724 \\ -0.12530 & -0.07608 \\ -0.16074 & -0.08621 \\ -0.19039 & -0.08836 \\ -0.21334 & -0.08447 \\ -0.22953 & -0.07694 \\ -0.23963 & -0.06801 \\ -0.24483 & -0.05937 \\ -0.24647 & -0.05207\end{array}$

$-0.00111-0.0008$ $-0.00222--0.0016$ $-0.00554-0.00402$ $-0.01104-0.00802$ $-0.02186-0.0158 \mathrm{~L}$ $-0.05076-0.0366$ $-0.07803-0.0605$ $-0.07171-0.07646$ $-0.02843-0.0846$ $0.05178-0.0746$ $0.15726-0.0267$ $0.25726 \quad 0.08299$

$-0.04123-0.02977$ $-0.07014-0.05172$ $-0.07984-0.06086$ $-0.06789-0.07817$ $-0.03288-0.08448$ $0.02530-0.08039$ $0.10254-0.05701$ $0.18801-0.0029$ 0.262710 .09329 $0.29912 \quad 0.23807$ $\begin{array}{lll}0.01 & -0.00052 & -0.00038\end{array}$ $0.02 \quad-0.00104-0.00076$ $\begin{array}{lll}0.05 & -0.00261 & -0.00190\end{array}$ $-0.00520-0.00380$ $-0.01031-0.00766$ $-0.02428-0.02019$ $-0.03919-0.04675$ $-0.04060-0.08173$ $\begin{array}{lll}-0.02936 & -0.12272\end{array}$ $-0.00819-0.16571$ $3.00 \quad 0.02030-0.20758$ $\begin{array}{lll}3.50 & 0.05398 & -0.24667\end{array}$

$\begin{array}{llll}0.125 \pi & -0.01959 & -0.01549\end{array}$ $0.250 \pi \quad-0.03434-0.03432$ $0.375 \pi \quad-0.04127-0.05830$ $0.500 \pi \quad-0.03972-0.08726$ $0.625 \pi \quad-0.03056-0.11961$ $0.750 \pi \quad-0.01514-0.15335$ $0.875 \pi \quad 0.00522-0.18683$ $\begin{array}{llll}1.000 \pi & 0.02939 & -0.21897\end{array}$

$\begin{array}{llll}-0.00000 & 0.00000 & -0.00052 & -0.00038 \\ -0.00002 & 0.00000 & -0.00104 & -0.00076 \\ -0.00011 & 0.00002 & -0.00261 & -0.00189 \\ -0.00045 & 0.00007 & -0.00521 & -0.00378 \\ -0.00179 & 0.00028 & -0.01041 & -0.00752 \\ -0.01091 & 0.00154 & -0.02578 & -0.01810 \\ -0.04003 & 0.00365 & -0.04974 & -0.03174 \\ -0.07973 & 0.00108 & -0.07000 & -0.03884 \\ -0.12338 & -0.01003 & -0.08521 & -0.04015 \\ -0.16709 & -0.03039 & -0.09514 & -0.03791 \\ -0.20891 & -0.05847 & -0.10060 & -0.03441 \\ -0.24786 & -0.09207 & -0.10292 & -0.03114 \\ & & & \\ -0.00681 & 0.00101 & -0.02034 & -0.01447 \\ -0.02577 & 0.00300 & -0.03980 & -0.02664 \\ -0.05336 & 0.00354 & -0.05747 & -0.03503 \\ -0.08579 & 0.00008 & -0.07248 & -0.03934 \\ -0.12016 & -0.00890 & -0.08428 & -0.04020 \\ -0.15465 & -0.02366 & -0.09279 & -0.03878 \\ -0.18822 & -0.04354 & -0.09834 & -0.03621 \\ -0.22026 & -0.06752 & -0.10151 & -0.03342 \\ -0.25041 & -0.09452 & -0.10300 & -0.03095 \\ -0.27853 & -0.12366 & -0.10344 & -0.02905\end{array}$

$\begin{array}{rr}-0.00052 & -0.00038 \\ -0.00104 & -0.00076 \\ -0.00260 & -0.00189 \\ -0.00519 & -0.00378 \\ -0.01026 & -0.00750 \\ -0.02353 & -0.01789 \\ -0.03485 & -0.03302 \\ -0.02737 & -0.04858 \\ 0.00687 & -0.06370 \\ 0.07627 & -0.06562 \\ 0.18068 & -0.02392 \\ 0.29628 & 0.10992 \\ -0.01921 & -0.01432 \\ -0.03196 & -0.02668 \\ -0.03468 & -0.03838 \\ -0.02437 & -0.05089 \\ 0.00326 & -0.06279 \\ 0.05243 & -0.06782 \\ 0.12457 & -0.05299 \\ 0.21424 & 0.00219 \\ 0.30321 & 0.12390 \\ 0.35268 & 0.33805\end{array}$

$\begin{array}{rr}-0.00000 & 0.00000 \\ -0.00002 & 0.00000 \\ -0.00011 & 0.00002 \\ -0.00045 & 0.00007 \\ -0.00177 & 0.00029 \\ -0.01016 & 0.00187 \\ -0.03203 & 0.00588 \\ -0.05548 & 0.00185 \\ -0.07541 & -0.02504 \\ -0.07884 & -0.08651 \\ -0.03581 & -0.18422 \\ 0.10107 & -0.29535 \\ -0.00651 & 0.00115 \\ -0.02207 & 0.00432 \\ -0.04046 & 0.00614 \\ -0.05867 & -0.00024 \\ -0.07427 & -0.02202 \\ -0.08093 & -0.06489 \\ -0.06583 & -0.13123 \\ -0.00894 & -0.21627 \\ 0.11529 & -0.30207 \\ 0.33215 & -0.34980\end{array}$

$E(h, h)$



$-0.00111-0.00081$ $-0.00161$ $-0.01107-0.00800$ $-0.02208-0.01569$ $-0.09641-0.04411$ $-0.11831-0.03564$ $-0.12128-0.02061$ $-0.04280-0.02862$ $-0.08037-0.04311$ $-0.11982-0.03401$ $-0.12324-0.02604$ $-0.11999-0.01995$ $-0.11810-0.02060$ $-0.11810-0.02100$

$\begin{array}{ll}-0.00052 & -0.00038 \\ -0.00104 & -0.00076 \\ -0.00261 & -0.00189 \\ -0.00521 & -0.00376 \\ -0.01035 & -0.00736 \\ -0.02487 & -0.01587 \\ -0.04261 & -0.02007 \\ -0.05030 & -0.01721 \\ -0.05172 & -0.01438 \\ -0.05131 & -0.01332 \\ -0.05094 & -0.01320 \\ -0.05082 & -0.01328 \\ -0.01990 & -0.01332 \\ -0.03624 & -0.01967 \\ -0.04640 & -0.01939 \\ -0.05076 & -0.01671 \\ -0.05172 & -0.01453 \\ -0.05146 & -0.01349 \\ -0.05109 & -0.01321 \\ -0.05088 & -0.01322 \\ -0.05082 & -0.01328 \\ -0.05082 & -0.01332\end{array}$


$\beta \mathrm{h}$

$C(h, 0)$

\begin{tabular}{|c|c|c|}
\hline $\begin{array}{l}0.01 \\
0.02 \\
0.05 \\
0.10 \\
0.20 \\
0.50 \\
1.00 \\
1.50 \\
2.00 \\
2.50 \\
3.00 \\
3.50\end{array}$ & $\begin{array}{l}-0.00637 \\
-0.01273 \\
-0.03182 \\
-0.06355 \\
-0.12639 \\
-0.30394 \\
-0.52729 \\
-0.61786 \\
-0.57583 \\
-0.45765 \\
-0.34709 \\
-0.30510\end{array}$ & $\begin{array}{r}0.00000 \\
0.00000 \\
0.00000 \\
0.00003 \\
0.00027 \\
0.00387 \\
0.02322 \\
0.04332 \\
0.01967 \\
-0.08774 \\
-0.27545 \\
-0.48797\end{array}$ \\
\hline $\begin{array}{l}0.125 \pi \\
0.250 \pi \\
0.375 \pi \\
0.500 \pi \\
0.025 \pi \\
0.750 \pi \\
0.875 \pi \\
1.000 \pi \\
1.125 \pi \\
1.250 \pi\end{array}$ & $\begin{array}{l}-0.24299 \\
-0.44563 \\
-0.57605 \\
-0.01921 \\
-0.58242 \\
-0.49456 \\
-0.39673 \\
-0.32693 \\
-0.30514 \\
-0.32593\end{array}$ & $\begin{array}{r}0.00194 \\
0.01309 \\
0.03220 \\
0.04381 \\
0.02393 \\
-0.04739 \\
-0.17360 \\
-0.33642 \\
-0.50150 \\
-0.03261\end{array}$ \\
\hline
\end{tabular}

0.0

0.02

0.05

0.10

0.20

0.50

1.50

2.00

2.50

3.50

$0.125 \pi$

$0.125 \pi$

$0.250 \pi$

$0.500 \pi$

$0.625 \pi$

$0.750 \pi$

$0.875 \pi$

$1.000 \pi$

$1.250 \pi$

$\begin{array}{lr}-0.00627 & 0.00000 \\ -0.01253 & 0.00000 \\ -0.03132 & 0.00000 \\ -0.06255 & 0.00003 \\ -0.12442 & 0.00025 \\ -0.29918 & 0.00368 \\ -0.51904 & 0.02194 \\ -0.60853 & 0.04004 \\ -0.56828 & 0.01475 \\ -0.45379 & -0.09224 \\ -0.34652 & -0.27672 \\ -0.30506 & -0.48450 \\ -0.23920 & 0.00185 \\ -0.43864 & 0.01241 \\ -0.56710 & 0.03027 \\ -0.60996 & 0.04024 \\ -0.57466 & 0.01903 \\ -0.48957 & -0.05230 \\ -0.39473 & -0.17681 \\ -0.32686 & -0.33640 \\ -0.30501 & -0.49772 \\ -0.32371 & -0.62599\end{array}$

$S(t, 0)$

$\begin{array}{ll}-0.00003 & 0.00000\end{array}$

$-0.00013 \quad 0.00000$

$-0.00080 \quad 0.00000$

$\begin{array}{lll}-0.00318 & 0.00000\end{array}$

$-0.01268 \quad 0.00004$

$-0.07743 \quad 0.00152$

$\begin{array}{lll}-0.28469 & 0.02151\end{array}$

$-0.55350 \quad 0.08841$

$\begin{array}{lll}-0.79638 & 0.20680\end{array}$

$-0.947010 .33698$

$\begin{array}{ll}-0.99611 & 0.41342 \\ -0.99492 & 0.39038\end{array}$

$-0.04827$

$-0.18341$

$-0.37786$

$-0.78124$

$-0.91496$

$-0.98210$

$-0.99762$

$-1.01064$

0.00059

0.00872

0.03887

0.10248

0.30210

0.38564

0.41774
0.38504

0.29813
$E(h, 0)$

$\begin{array}{ll}-0.00637 & 0.00000 \\ -0.01273 & 0.00000 \\ -0.03183 & 0.00000 \\ -0.06365 & 0.00003 \\ -0.12724 & 0.00027 \\ -0.31693 & 0.00417 \\ -0.62483 & 0.03227 \\ -0.91145 & 0.10282 \\ -1.16052 & 0.22479 \\ -1.35396 & 0.39533 \\ -1.47615 & 0.60012 \\ -1.51847 & 0.81611 \\ -0.24934 & 0.00203 \\ -0.49447 & 0.01591 \\ -0.73013 & 0.05183 \\ -0.94942 & 0.11690 \\ -1.14400 & 0.21413 \\ -1.30503 & 0.34194 \\ -1.42444 & 0.49427 \\ -1.49638 & 0.66129 \\ -1.51842 & 0.83066 \\ -1.49225 & 0.93897\end{array}$

$C(h, h)$

$\begin{array}{ll}-0.00637 & 0.00000 \\ -0.01273 & 0.00000 \\ -0.03181 & 0.00002 \\ -0.06351 & 0.00013 \\ -0.12613 & 0.00106 \\ -0.29901 & 0.01535 \\ -0.49063 & 0.09233 \\ -0.50909 & 0.19645 \\ -0.39354 & 0.25198 \\ -0.22690 & 0.30212 \\ -0.02216 & 0.37199 \\ 0.25805 & 0.44569 \\ -0.24095 & 0.00771 \\ -0.42803 & 0.05172 \\ -0.51729 & 0.13049 \\ -0.49881 & 0.26837 \\ -0.40449 & 0.25876 \\ -0.27749 & 0.28934 \\ -0.13240 & 0.33195 \\ 0.04887 & 0.39647 \\ 0.27982 & 0.44826 \\ 0.53184 & 0.43290\end{array}$

$\mathrm{S}(\mathrm{h}, \mathrm{h})$

$-0.00003$ $-0.00013$ $-0.00080$ $-0.01265$ $-0.07629$ $-0.26275$ $-0.43928$ $-0.49203$ $-0.42071$ $-0.29964$

$-0.14900$

$-0.04785$ $-0.17560$ $-0.33421$ $-0.45574$ $-0.49312$ $-0.44954$ $-0.36306$ $-0.26149$ $-0.13647$

0.03624

0.00000
0.00000
0.00000
0.00001
0.00012
0.00439
0.05551
0.18896
0.34207
0.41631
0.40501
0.37900
0.00172
0.02383
0.09457
0.21202
0.33269
0.40580
0.41731
0.39635
0.37781
0.36265

$\mathbf{E}(\mathrm{h}, \mathrm{h})$

$\begin{array}{ll}-0.00637 & 0.00000 \\ -0.01273 & 0.00000 \\ -0.03183 & 0.00002 \\ -0.06362 & 0.00013 \\ -0.12697 & 0.00107 \\ -0.31241 & 0.01613 \\ -0.58026 & 0.11239 \\ -0.73808 & 0.30006 \\ -0.74124 & 0.50807 \\ -0.61801 & 0.63570 \\ -0.46497 & 0.62921 \\ -0.38460 & 0.52194 \\ -0.24724 & 0.00795 \\ -0.47471 & 0.05845 \\ -0.05252 & 0.17097 \\ -0.74819 & 0.33065 \\ -0.74612 & 0.49448 \\ -0.06154 & 0.61191 \\ -0.53836 & 0.64921 \\ -0.43152 & 0.60560 \\ -0.38327 & 0.51314 \\ -0.40554 & 0.42082\end{array}$

$\begin{array}{ll}-0.00003 & 0.00000 \\ -0.00013 & 0.00000 \\ -0.00078 & 0.00000 \\ -0.00313 & 0.00002 \\ -0.01248 & 0.00010 \\ -0.07621 & 0.00186 \\ -0.28007 & 0.02233 \\ -0.54425 & 0.08863 \\ -0.78304 & 0.20452 \\ -0.93200 & 0.33092 \\ -0.98265 & 0.40431 \\ -0.98486 & 0.38115 \\ -0.04751 & 0.00081 \\ -0.18047 & 0.00939 \\ -0.37166 & 0.03966 \\ -0.58152 & 0.10247 \\ -0.76813 & 0.19500 \\ -0.90010 & 0.29716 \\ -0.96749 & 0.37781 \\ -0.98504 & 0.40823 \\ -0.98511 & 0.37594 \\ -1.00281 & 0.29149\end{array}$

$$
\frac{\mathrm{a}}{\lambda}=0.5 \quad \frac{\alpha}{\beta}=0.005
$$

$\begin{array}{ll}-0.00627 & 0.00000 \\ -0.01253 & 0.00000 \\ -0.03133 & 0.00000 \\ -0.06266 & 0.00003 \\ -0.12525 & 0.00027 \\ -0.31197 & 0.00411 \\ -0.61493 & 0.03175 \\ -0.89675 & 0.10112 \\ -1.14138 & 0.22090 \\ -1.33112 & 0.38817 \\ -1.45080 & 0.58873 \\ -1.49219 & 0.79990 \\ -0.24544 & 0.00200 \\ -0.48669 & 0.01566 \\ -0.71850 & 0.05099 \\ -0.93406 & 0.11495 \\ -1.12517 & 0.21045 \\ -1.28316 & 0.33584 \\ -1.40018 & 0.48511 \\ -1.47059 & 0.64857 \\ -1.49214 & 0.81410 \\ -1.46660 & 0.96860\end{array}$

$\begin{array}{ll}-0.00627 & 0.00000 \\ -0.01252 & 0.00000 \\ -0.03132 & 0.00002 \\ -0.06252 & 0.00013 \\ -0.12416 & 0.00104 \\ -0.29488 & 0.01498 \\ -0.48273 & 0.08990 \\ -0.50122 & 0.19083 \\ -0.38830 & 0.25421 \\ -0.22420 & 0.29348 \\ -0.02156 & 0.36140 \\ 0.25391 & 0.43232 \\ -0.23716 & 0.00753 \\ -0.42117 & 0.05042 \\ -0.50901 & 0.12695 \\ -0.49122 & 0.20284 \\ -0.39903 & 0.25109 \\ -0.27417 & 0.28093 \\ -0.13064 & 0.32257 \\ 0.04854 & 0.38503 \\ 0.27517 & 0.43477 \\ 0.52067 & 0.41998\end{array}$

$\begin{array}{llll}-0.00003 & 0.00000 & -0.00627 & 0.00000 \\ -0.00013 & 0.00000 & -0.01253 & 0.00000 \\ -0.00078 & 0.00000 & -0.03133 & 0.00002 \\ -0.00313 & 0.00002 & -0.06263 & 0.00013 \\ -0.01245 & 0.00018 & -0.12499 & 0.00106 \\ -0.07506 & 0.00468 & -0.30747 & 0.01587 \\ -0.25818 & 0.05561 & -0.57069 & 0.11045 \\ -0.43121 & 0.18662 & -0.72540 & 0.29436 \\ -0.48327 & 0.33562 & -0.72854 & 0.49754 \\ -0.41443 & 0.40694 & -0.60867 & 0.62176 \\ -0.29609 & 0.39474 & -0.46040 & 0.61552 \\ -0.14730 & 0.36714 & -0.38283 & 0.51207 \\ -0.04709 & 0.00192 & -0.24334 & 0.00783 \\ -0.17264 & 0.02420 & -0.46703 & 0.05748 \\ -0.32825 & 0.09411 & -0.64158 & 0.16792 \\ -0.44735 & 0.20915 & -0.73530 & 0.32429 \\ -0.48428 & 0.32653 & -0.73330 & 0.48430 \\ -0.44238 & 0.39701 & -0.65097 & 0.59865 \\ -0.35829 & 0.40737 & -0.53142 & 0.63487 \\ -0.25848 & 0.38583 & -0.42808 & 0.59271 \\ -0.13492 & 0.36576 & -0.38155 & 0.50360 \\ 0.03481 & 0.34814 & -0.40293 & 0.41494\end{array}$


Bh
$C(h, 0)$

$S(h, 0)$

$E(h, 0)$

0.01
0.02

0.02

0.05

0.20

0.50

1.00

2.00

2.50

3.50

$0.125 \pi$ $0.250 \pi$
$0.375 \pi$

$0.500 \pi$

$0.625 \pi$

$0.850 \pi$

$1.000 \pi$

$1.125 \pi$
$1.250 \pi$

$\begin{array}{lr}-0.00617 & 0.00000 \\ -0.01234 & 0.00000 \\ -0.03083 & 0.00000 \\ -0.06158 & 0.00003 \\ -0.12248 & 0.00024 \\ -0.29451 & 0.00350 \\ -0.51093 & 0.02069 \\ -0.59935 & 0.03686 \\ -0.56083 & 0.00999 \\ -0.44992 & -0.09657 \\ -0.34582 & -0.27792 \\ -0.30488 & -0.48111 \\ -0.23546 & 0.00176 \\ -0.43177 & 0.01176 \\ -0.55829 & 0.02839 \\ -0.60086 & 0.03678 \\ -0.56700 & 0.01438 \\ -0.48459 & -0.05703 \\ -0.39265 & -0.17989 \\ -0.32664 & -0.33634 \\ -0.30474 & -0.49402 \\ -0.32145 & -0.61956\end{array}$

$-0.00003 \quad 0.00000$ -0.00012
-0.00077 $-0.00308$ $-0.01228$ -0.01228
-0.0750 $-0.07501$ $-0.27553$ $-0.76095$ 0.91728 0.96940

$-0.97486$

$-0.04676$ $-0.17758$ $-0.36556$ $-0.57180$ $-0.85553$ 0.95315 $-0.97263$ -0.97533
-0.99492 0.00000 0.00003 0.00016 0.00219 0.02312 0.08883 0.20228 0.32500 0.37218 0.00102 0.01004 0.04042 0.10243 0.29233 0.29233 0.39900 0.39900 0.36709
0.28502 $-0.01234 \quad 0.00000$ $-0.061680 .00003$ $-0.30709 \quad 0.00404$ $-0.60519$ 0.09944 0.01709 $-1.30868$ $\begin{array}{ll}-1.42589 & 0.57755\end{array}$

$\begin{array}{ll}-1.46636 & 0.78400\end{array}$

$\begin{array}{ll}-0.24161 & 0.00197\end{array}$ $-0.47903 \quad 0.01541$ $-0.70705 \quad 0.05016$ 0.1189404 $-1.106640 .20682$ $-1.261650 .32984$ $-1.376330 .47612$ $\begin{array}{ll}-1.44525 & 0.63610 \\ -1.46631 & 0.73787\end{array}$ $\begin{array}{rr}-1.44140 & 0.94866\end{array}$ $-0.03085 \quad 0.00000$ $-0.12330 \quad 0.00026$
$C(h, h)$

$\begin{array}{rl}-0.00617 & 0.00000 \\ -0.01234 & 0.00000 \\ -0.03083 & 0.00002 \\ -0.06155 & 0.00013 \\ -0.12222 & 0.00101 \\ -0.29022 & 0.01461 \\ -0.47497 & 0.08753 \\ -0.49348 & 0.18535 \\ -0.38310 & 0.24663 \\ -0.22156 & 0.28498 \\ -0.02095 & 0.35103 \\ 0.24986 & 0.41929 \\ -0.23342 & 0.00735 \\ -0.41443 & 0.04914 \\ -0.50085 & 0.12349 \\ -0.48373 & 0.19696 \\ -0.39362 & 0.24360 \\ -0.27086 & 0.27270 \\ -0.12888 & 0.31337 \\ 0.04822 & 0.37385 \\ 0.27068 & 0.42164 \\ 0.50985 & 0.40744\end{array}$

$\mathrm{S}(\mathrm{h}, \mathrm{h})$ $-0.00012$ $-0.00077$ $-0.00308$ $-0.01226$ $-9.07384$ $-0.25369$ $-0.42329$ $-0.47469$ $-0.40825$

$-0.29258$

$-0.14566$

$-0.04634$ $-0.16974$ $-0.32240$

$-0.43912$

$-0.47562$

$-0.43534$

$-0.35359$

$-0.25551$

-0.13344
0.03337
0.00000
0.00000
0.00001
0.00004
0.00024
0.00496
0.05569
0.18431
0.32930
0.39779
0.38468
0.35554
0.00212
0.02456
0.09363
0.20632
0.32050
0.38843
0.39765
0.37552
0.35398
0.33403
$E(h, h)$

$-0.00617$ $0.03084 \quad 0.00000$ $-0.06165 \quad 0.00013$ $-0.12304 \quad 0.0010$ $-0.30260 \quad 0.01562$ $-0.56128 \quad 0.10854$ $-0.71295 \quad 0.28878$ $-0.71606 \quad 0.48724$ $-0.59946 \quad 0.60813$ $\begin{array}{ll}-0.45580 & 0.60213 \\ -0.38095 & 0.50237\end{array}$

$-0.23951 \quad 0.00770$ $\begin{array}{ll}-0.45947 & 0.05652\end{array}$ $-0.63083 \quad 0.16492$ $-0.72263 \quad 0.31805$ $-0.72070 \quad 0.47433$ $-0.64055 \quad 0.58568$ $-0.52454 \quad 0.62085$ $-0.42458 \quad 0.58010$ $\begin{array}{ll}-0.37972 & 0.49422 \\ -0.40023 & 0.40907\end{array}$

$$
\frac{a}{\lambda}=0.5 \quad \frac{\alpha}{\beta}=0.02
$$

$\begin{array}{llr}0.01 & -0.00598 & 0.00000 \\ 0.02 & -0.01196 & 0.00000 \\ 0.05 & -0.02988 & 0.00000 \\ 0.10 & -0.05967 & 0.00003 \\ 0.20 & -0.11869 & 0.00022 \\ 0.50 & -0.28537 & 0.00315 \\ 1.00 & -0.49509 & 0.01830 \\ 1.50 & -0.58141 & 0.03079 \\ 2.00 & -0.54619 & 0.00094 \\ 2.50 & -0.44212 & -0.10474 \\ 3.00 & -0.34405 & -0.28006 \\ 3.50 & -0.30412 & -0.47454 \\ 0.125 \pi & -0.22816 & 0.00158 \\ 0.250 \pi & -0.41836 & 0.01050 \\ 0.375 \pi & -0.54108 & 0.02480 \\ 0.500 \pi & -0.58307 & 0.03017 \\ 0.625 \pi & -0.55196 & 0.00545 \\ 0.750 \pi & -0.47469 & -0.06599 \\ 0.875 \pi & -0.38826 & -0.18564 \\ 1.000 \pi & -0.32580 & -0.33609 \\ 1.125 \pi & -0.30382 & -0.48688 \\ 1.250 \pi & -0.31681 & -0.00724\end{array}$

$\begin{array}{ll}-0.00003 & 0.00000 \\ -0.00012 & 0.00000 \\ -0.00075 & 0.00002 \\ -0.00299 & 0.00006 \\ -0.01190 & 0.00027 \\ -0.07266 & 0.00282 \\ -0.26667 & 0.02461 \\ -0.51750 & 0.08914 \\ -0.74451 & 0.19790 \\ -0.88866 & 0.31358 \\ -0.94348 & 0.37848 \\ -0.95502 & 0.35500 \\ & \\ -0.04531 & 0.00143 \\ -0.17194 & 0.01126 \\ -0.35368 & 0.04184 \\ -0.55288 & 0.10230 \\ -0.73028 & 0.18907 \\ -0.85721 & 0.28299 \\ -0.92520 & 0.35554 \\ -0.94830 & 0.38131 \\ -0.95590 & 0.35014 \\ -0.97902 & 0.27253\end{array}$

$\begin{array}{ll}-0.00598 & 0.00000 \\ -0.01196 & 0.00000 \\ -0.02989 & 0.00000 \\ -0.05977 & 0.00003 \\ -0.11948 & 0.00025 \\ -0.29755 & 0.00392 \\ -0.58617 & 0.03024 \\ -0.85405 & 0.09617 \\ -1.08584 & 0.20965 \\ -1.26492 & 0.36748 \\ -1.37735 & 0.55584 \\ -1.41606 & 0.75317 \\ & \\ -0.23412 & 0.00191 \\ -0.46406 & 0.01492 \\ -0.68470 & 0.04855 \\ -0.88945 & 0.10930 \\ -1.07051 & 0.19976 \\ -1.21972 & 0.31817 \\ -1.32986 & 0.45864 \\ -1.39589 & 0.61188 \\ -1.41601 & 0.76640 \\ -1.39230 & 0.91001\end{array}$

$-0.00598$ $-0.02987 \quad 0.00000$

$-0.05964 \quad 0.00012$

$\begin{array}{ll}-0.11843 & 0.00097\end{array}$

$\begin{array}{lll}-0.28112 & 0.01391\end{array}$

$-0.45983 \quad 0.08296$

$-0.47835 \quad 0.17479$

$-0.37285 \quad 0.23201$

$\begin{array}{ll}-0.21615 & 0.26853\end{array}$

$\begin{array}{rr}-0.01972 & 0.33092 \\ 0.24208 & 0.39426\end{array}$

0.39426

$\begin{array}{ll}-0.22614 & 0.00700 \\ -0.40127 & 0.04667\end{array}$

$-0.48495 \quad 0.0466$

$-0.46910 \quad 0.18563$

$-0.38296 \quad 0.22916$

$-0.26426 \quad 0.25678$

$-0.12535 \cdot 0.2565$

$0.04763 \quad 0.35221$

$0.26200 \quad 0.39642$

$0.48921 \quad 0.38347$

$\begin{array}{llll}-0.00003 & 0.00000 & -0.00598 & 0.00000 \\ -0.00012 & 0.00000 & -0.01196 & 0.00000 \\ -0.00075 & 0.00002 & -0.02989 & 0.00002 \\ -0.00298 & 0.00007 & -0.05974 & 0.00013 \\ -0.01188 & 0.00035 & -0.11922 & 0.00101 \\ -0.07148 & 0.00549 & -0.29309 & 0.01512 \\ -0.24496 & 0.05580 & -0.54292 & 0.10482 \\ -0.40794 & 0.17979 & -0.68868 & 0.27792 \\ -0.45804 & 0.31707 & -0.69173 & 0.46728 \\ -0.39620 & 0.38011 & -0.58142 & 0.58179 \\ -0.28569 & 0.36517 & -0.44656 & 0.57624 \\ -0.14254 & 0.33306 & -0.37685 & 0.48347 \\ -0.04487 & 0.00249 & -0.23203 & 0.00746 \\ -0.16408 & 0.02522 & -0.44473 & 0.05465 \\ -0.31102 & 0.09266 & -0.60986 & 0.15908 \\ -0.42315 & 0.20078 & -0.69794 & 0.30594 \\ -0.45882 & 0.30881 & -0.69615 & 0.45500 \\ -0.42164 & 0.37185 & -0.62020 & 0.56059 \\ -0.34435 & 0.37884 & -0.51095 & 0.59376 \\ -0.24969 & 0.35551 & -0.41742 & 0.55568 \\ -0.13065 & 0.33117 & -0.37571 & 0.47592 \\ 0.03047 & 0.30694 & -0.39459 & 0.39738\end{array}$


$\frac{a}{\lambda}=0.5 \quad \frac{\alpha}{\beta}=0.03$

Bh

$C(h, 0)$

0.01
0.02
0.05
0.10
0.20
0.50
1.00
1.50
2.00
2.50
3.00
3.50

$0.125 \pi$

$0.250 \pi$

$0.375 \pi$

$0.500 \pi$

-10
0.750

$0.875 \pi$

$1.000 \pi$

$1.250 \pi$

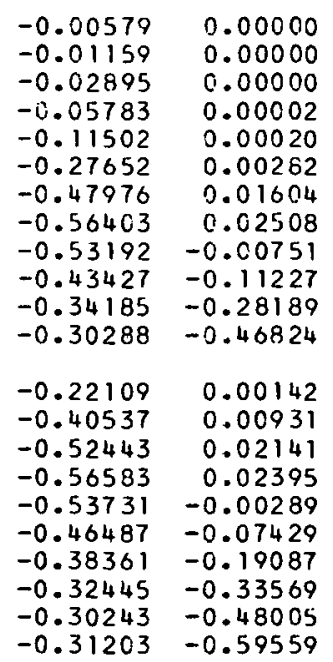

$S(h, h)$

$E(h, h)$
$S(h, 0)$

$E(h, 0)$

-0.00003
-0.00012

$-0.00012 \quad 0.00000$

0.00000

$\begin{array}{ll}-0.00289 & 0.00009\end{array}$

$-0.01153 \quad 0.00038$

$0.07039 \quad 0.00341$

$-0.50047 \quad 0.08935$

$\begin{array}{ll}-0.72003 & 0.19364\end{array}$

$\begin{array}{ll}-0.86109 & 0.30271\end{array}$

$\begin{array}{ll}-0.96109 & 0.30271 \\ -0.93542 & 0.36242\end{array}$

$\begin{array}{lll}-0.04390 & 0.00182\end{array}$

$-0.16649$

0.01240

0.10209

0.70623

$-0.82995$

-0.92461
-0.93668

0.27405

0.34164

0.36459

0.26064
$-0.96297$
$-0.00579$

0.00000

$\begin{array}{ll}-0.05793 & 0.00003\end{array}$

$-0.115790 .00025$

$-1.05033 \quad 0.20246$

$\begin{array}{ll}-1.22263 & 0.35430\end{array}$

$\begin{array}{ll}-1.33048 & 0.53496 \\ -1.36750 & 0.72356\end{array}$

$\begin{array}{ll}-10.22686 & 0.00185\end{array}$

$-0.44956 \quad 0.01445$

$\begin{array}{lll}-0.66306 & 0.04699\end{array}$

$-0.86090 \quad 0.10568$

$\begin{array}{rr}-1.03555 & 0.17293 \\ -1.17919 & 0.30691\end{array}$

$\begin{array}{ll}-1.17919 & 0.3369 \\ -1.28497 & 0.44180\end{array}$

$-1.34822 \quad 0.58858$

$\begin{array}{ll}-1.36746 & 0.73618 \\ -1.34488 & 0.87296\end{array}$
0.00380

$\begin{array}{ll}-0.82672 & 0.09301\end{array}$
$C(h, h)$

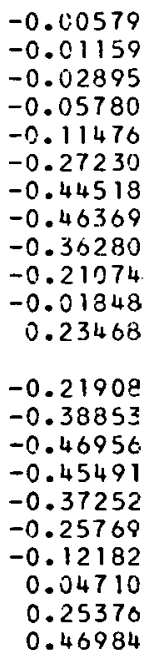

3.00000 0.00000 0.00001 0.00012 0.00092 0.01324 0.07860 0.16475 0.21807 0.25276 0.31165 0.370 .51

0.00667 0.04431 0.11047 0.17485 0.2154 0.24155 0.2783 0.3315 0.3725 0.36089
$-0.00003$ $-0.00012$ $-0.00072$ . $-0.01151$ $-0.23654$ $-0.44205$ $-0.38453$ $-0.27897$ $-0.13962$

$-0.04346$ $-0.15862$ $-0.30008$ $-0.40783$ $-0.33536$ $-0.24404$ $-0.12805$ 0.02757
0.00000 0.00000 C. 00002 0.00009 c. 00045 0.00598 0.17539 0.30535 0.36323 0.34647 0.31155

0.00284 0.02580 0.09166 0.19542 0.29762 0.35602 0.36085 0.33630 0.30934 0.28130

$\begin{array}{ll}-0.00579 & 0.00000 \\ -0.01159 & 0.00000 \\ -0.02896 & 0.00002 \\ -0.05789 & 0.00012 \\ -0.11552 & 0.00098 \\ -0.28388 & 0.01464 \\ -0.52516 & 0.10123 \\ -0.66524 & 0.26748 \\ -0.66824 & 0.44816 \\ -0.56387 & 0.55661 \\ -0.43727 & 0.55149 \\ -0.37235 & 0.46521 \\ -0.22478 & 0.00723 \\ -0.43045 & 0.05284 \\ -0.58960 & 0.15346 \\ -0.67411 & 0.29429 \\ -0.67244 & 0.43648 \\ -0.60047 & 0.53661 \\ -0.49758 & 0.56788 \\ -0.41007 & 0.53229 \\ -0.37130 & 0.45822 \\ -0.38868 & 0.38578\end{array}$

$$
\frac{\mathrm{a}}{\lambda}=0.5 \quad \frac{Q}{\beta}=0.05
$$

$\begin{array}{llll}-0.00003 & 0.00000 & -0.00544 & 0.00000 \\ -0.00011 & 0.00001 & -0.01088 & 0.00000 \\ -0.00068 & 0.00003 & -0.02720 & 0.00000 \\ -0.00272 & 0.00014 & -0.05440 & 0.00003 \\ -0.01083 & 0.00057 & -0.10873 & 0.00023 \\ -0.06605 & 0.00447 & -0.27068 & 0.00356 \\ -0.24184 & 0.02840 & -0.53263 & 0.02745 \\ -0.46821 & 0.08950 & -0.77466 & 0.08699 \\ -0.07378 & 0.18548 & -0.98276 & 0.18883 \\ -0.80894 & 0.28245 & -1.14226 & 0.32935 \\ -0.87034 & 0.33284 & -1.24151 & 0.49552 \\ -0.89703 & 0.30898 & -1.27537 & 0.06782 \\ & & & \\ -0.04121 & 0.00251 & -0.21301 & 0.00173 \\ -0.15611 & 0.01444 & -0.42192 & 0.01356 \\ -0.32044 & 0.04535 & -0.62180 & 0.04401 \\ -0.50012 & 0.10143 & -0.80654 & 0.09881 \\ -0.66079 & 0.17787 & -0.96904 & 0.17998 \\ -0.77843 & 0.25732 & -1.10214 & 0.28558 \\ -0.84701 & 0.31592 & -1.19971 & 0.40997 \\ -0.87911 & 0.33386 & -1.25776 & 0.54463 \\ -0.89894 & 0.30470 & -1.27533 & 0.07931 \\ -0.93084 & 0.23854 & -1.25486 & 0.30338\end{array}$

$\begin{array}{ll}-0.00003 & 0.00000 \\ -0.00011 & 0.00001 \\ -0.00068 & 0.00003 \\ -0.00272 & 0.00014 \\ -0.01080 & 0.00064 \\ -0.06484 & 0.00685 \\ -0.22059 & 0.05577 \\ -0.36541 & 0.16692 \\ -0.41196 & 0.28339 \\ -0.36233 & 0.33172 \\ -0.26604 & 0.31136 \\ -0.13431 & 0.27126 \\ -0.04075 & 0.00346 \\ -0.14825 & 0.02680 \\ -0.27939 & 0.08957 \\ -0.37898 & 0.18516 \\ -0.41236 & 0.27661 \\ -0.38338 & 0.32648 \\ -0.31811 & 0.32720 \\ -0.23321 & 0.30021 \\ -0.12338 & 0.26852 \\ 0.02186 & 0.23404\end{array}$

?

$\begin{array}{ll}-0.00544 & 0.00000 \\ -0.01088 & 0.00000 \\ -0.02720 & 0.00001 \\ -0.05437 & 0.00012 \\ -0.10847 & 0.00092 \\ -0.26632 & 0.01372 \\ -0.49138 & 0.09441 \\ -0.02076 & 0.24776 \\ -0.62364 & 0.41224 \\ -0.53021 & 0.50955 \\ -0.41864 & 0.50517 \\ -0.36234 & 0.43056 \\ -0.21096 & 0.00678 \\ -0.40327 & 0.04947 \\ -0.55107 & 0.14279 \\ -0.62888 & 0.27232 \\ -0.62743 & 0.40169 \\ -0.56281 & 0.49173 \\ -0.47155 & 0.51954 \\ -0.39494 & 0.48840 \\ -0.36143 & 0.42457 \\ -0.37616 & 0.36293\end{array}$

0.02186

$\begin{array}{ll}-0.00544 & 0.00000 \\ -0.01088 & 0.00000 \\ -0.02719 & 0.00001 \\ -0.05427 & 0.00011 \\ -0.10775 & 0.00084 \\ -0.25550 & 0.01198 \\ -0.41729 & 0.07047 \\ -0.43569 & 0.14605 \\ -0.34330 & 0.19217 \\ -0.19999 & 0.22316 \\ -0.01593 & 0.27549 \\ 0.22103 & 0.32660 \\ -0.20563 & 0.00601 \\ -0.36428 & 0.0399: \\ -0.44028 & 0.09865 \\ -0.42777 & 0.15485 \\ -0.35230 & 0.18980 \\ -0.24470 & 0.21305 \\ -0.11475 & 0.24614 \\ 0.04622 & 0.29281 \\ 0.23858 & 0.32835 \\ 0.43462 & 0.31960\end{array}$

$\begin{array}{llr}0.01 & -0.00544 & 0.00000 \\ 0.02 & -0.01088 & 0.00000 \\ 0.05 & -0.02719 & 0.00000 \\ 0.10 & -0.05431 & 0.00002 \\ 0.20 & -0.10801 & 0.00016 \\ 0.50 & -0.25964 & 0.00221 \\ 1.00 & -0.45053 & 0.01189 \\ 1.50 & -0.53087 & 0.01466 \\ 2.00 & -0.50442 & -0.02276 \\ 2.50 & -0.41849 & -0.12560 \\ 3.00 & -0.33630 & -0.28469 \\ 3.50 & -0.29912 & -0.45630 \\ 0.125 \pi & -0.20761 & 0.00111 \\ 0.250 \pi & -0.38061 & 0.00712 \\ 0.375 \pi & -0.49270 & 0.01520 \\ 0.500 \pi & -0.53291 & 0.01264 \\ 0.625 \pi & -0.50912 & -0.01796 \\ 0.750 \pi & -0.44550 & -0.08908 \\ 0.875 \pi & -0.37362 & -0.19990 \\ 1.000 \pi & -0.32045 & -0.33440 \\ 1.125 \pi & -0.29841 & -0.46715 \\ 1.250 \pi & -0.30214 & -0.57405\end{array}$


$\beta \mathrm{h}$

0.01

0.02

0.05

.10

0.50

1.00

.50

2.50
3.00
3.50

3.50

$0.125 \pi$ $0.250 \pi$

$0.500 \pi$

$0.625 \pi$

$0.750 \pi$

$1.000 \pi$

$1.125 \pi$

$1.250 \pi$
$\mathrm{C}(\mathrm{h}, 0)$

$\begin{array}{lr}-0.00511 & 0.00000 \\ -0.01022 & 0.00000 \\ -0.02554 & 0.00000 \\ -0.05100 & 0.00002 \\ -0.10143 & 0.00012 \\ -0.24380 & 0.00166 \\ -0.42314 & 0.00820 \\ -0.49973 & 0.00551 \\ -0.47828 & -0.03598 \\ -0.40269 & -0.13681 \\ -0.32946 & -0.28642 \\ -0.29393 & -0.44511 \\ -0.19495 & 0.00085 \\ -0.35739 & 0.00517 \\ -0.46295 & 0.00969 \\ -0.50198 & 0.00270 \\ -0.48237 & -0.03105 \\ -0.42654 & -0.10167 \\ -0.36291 & -0.20720 \\ -0.31498 & -0.33253 \\ -0.29301 & -0.45513 \\ -0.29189 & -0.55455\end{array}$

$S(h, 0)$

$\begin{array}{ll}-0.00003 & 0.00000 \\ -0.00010 & 0.00001 \\ -0.00064 & 0.00004 \\ -0.00255 & 0.00018 \\ -0.01017 & 0.00074 \\ -0.06200 & 0.00538 \\ -0.22663 & 0.03042 \\ -0.43821 & 0.08932 \\ -0.63090 & 0.17775 \\ -0.76050 & 0.26401 \\ -0.82518 & 0.30633 \\ -0.85981 & 0.28234 \\ -0.03868 & 0.00311 \\ -0.14640 & 0.01619 \\ -0.30013 & 0.04714 \\ -0.46804 & 0.10051 \\ -0.61866 & 0.17086 \\ -0.73065 & 0.24198 \\ -0.79923 & 0.29273 \\ -0.83603 & 0.30637 \\ -0.86224 & 0.27840 \\ -0.89829 & 0.21849\end{array}$

$$
\frac{\mathrm{a}}{\lambda}=0.5 \quad \frac{\alpha}{\beta}=0.07
$$

$E(h, 0$ :

$-0.01022 \quad 2.00000$

$\begin{array}{ll}-0.02555 & 0.00000 \\ -0.05108 & 0.00003\end{array}$

$-0.10210 \quad 0.00022$

$\begin{array}{lll}-0.25413 & 0.00334\end{array}$

$-0.49968 \quad 0.02573$

$\begin{array}{ll}-0.72588 & 0.08136\end{array}$

$\begin{array}{ll}-0.91954 & 0.17611 \\ -1.06720 & 0.30617\end{array}$

$\begin{array}{ll}-1.06720 & 0.30617 \\ -1.15853 & 0.45901\end{array}$

$-1.18950 \quad 0.61642$

$-0.20000 \quad 0.00163$

$-0.39597 \quad 0.01272$

$\begin{array}{ll}-0.5831 .2 & c .04123 \\ -0.75560 & c .09238\end{array}$

$\begin{array}{ll}-0.75560 & C .09238 \\ -0.90681 & 0.16790\end{array}$

$-1.03015 \quad 0.26574$

$-1.12014 \quad 0.38045$

$-1.173410 .50399$

$\begin{array}{ll}-1.18946 & 0.62687 \\ -1.17092 & 0.73941\end{array}$
$-0.00511 \quad 0.00070$

$0.01022 \quad 0.00000$

$-0.02553 \quad 0.0000$

$\begin{array}{ll}-0.10118 & 0.000^{76}\end{array}$

$-0.23974 \quad 0.01083$

$-0.39120 \quad 0.06307$

$-0.40938 \quad 0.12922$

$-0.13937 \quad 0.196015$

$0.01330 \quad 0.242 \equiv$

0.20882

$-0.19300$

$-0.34157$

$-0.41286$

$-0.40223$

$-0.33295$

$-0.23196$

$-0.10773$

0.04560

0.22500

0.40362

0.00547

0.03588

0.08792

0.13676

0.16671

0.18700

0.21649

0.25743

0.28864
0.2829
$S(h, h)$ $-0.00010$ -0.00064
-0.00255 $-0.00255$ $-0.01014$ $-0.06077$ $-0.20577$ $-0.33977$ $-0.38420$ $-0.34158$

$-0.25379$

$-0.12959$

$-0.03822$

$-0.13858$

-0.26021
-0.35238

$-0.38438$

$-0.36010$

$-0.30182$

$-0.22302$

$-0.11928$

0.01640
$E(h, h)$

$-0.02554$

$-0.051050 .0001$

$-0.101850 .00080$

$\begin{array}{ll}-0.24984 & 0.01286\end{array}$

$-0.459770 .08806$

0.582030 .22951

$-0.498390 .46655$

$-0.40007 \quad 0.4628$

$-0.19798$

$-0.37780$

$-0.51507$

$-0.58671$

$-0.58546$

$-0.52743$

$-0.44649$

$-0.37942$

$-0.35046$

$-0.36294$

0.19166

0.39829

0.25200

0.3697

0.47541

0.39315

0.34071
0.00000

0.00636

0.04619

0.13287

0.45067

$\begin{array}{llr}0.01 & -0.00465 & 0.00000 \\ 0.02 & -0.00930 & 0.00000 \\ 0.05 & -0.02324 & 0.00000 \\ 0.10 & -0.04641 & 0.00001 \\ 0.20 & -0.09230 & 0.00007 \\ 0.50 & -0.22185 & 0.00095 \\ 1.00 & -0.38521 & 0.00343 \\ 1.50 & -0.45654 & -0.00617 \\ 2.00 & -0.44149 & -0.05248 \\ 2.50 & -0.37920 & -0.15015 \\ 3.00 & -0.31737 & -0.28723 \\ 3.50 & -0.28405 & -0.42941 \\ & & \\ 0.125 \pi & -0.17740 & 0.00050 \\ 0.250 \pi & -0.32523 & 0.00264 \\ 0.375 \pi & -0.42176 & 0.00261 \\ 0.500 \pi & -0.45903 & -0.00994 \\ 0.625 \pi & -0.44478 & -0.04740 \\ 0.750 \pi & -0.39900 & -0.11694 \\ 0.875 \pi & -0.34589 & -0.21529 \\ 1.000 \pi & -0.30462 & -0.32869 \\ 1.125 \pi & -0.28289 & -0.43838 \\ 1.250 \pi & -0.27609 & -0.52833\end{array}$

$$
\frac{\mathrm{a}}{\lambda}=0.5 \quad \frac{\alpha}{\beta}=0.10
$$

$\begin{array}{ll}-0.00002 & 0.00000 \\ -0.00009 & 0.00001 \\ -0.00058 & 0.00006 \\ -0.00232 & 0.00023 \\ -0.00925 & 0.00095 \\ -0.05637 & 0.00652 \\ -0.20568 & 0.03280 \\ -0.39708 & 0.08851 \\ -0.57233 & 0.16690 \\ -0.69417 & 0.23932 \\ -0.76240 & 0.27152 \\ -0.80630 & 0.24754 \\ -0.03518 & 0.00386 \\ -0.13298 & 0.01830 \\ -0.27219 & 0.04912 \\ -0.42408 & 0.09869 \\ -0.56110 & 0.16094 \\ -0.66533 & 0.22126 \\ -0.73345 & 0.26204 \\ -0.77568 & 0.27039 \\ -0.80934 & 0.24401 \\ -0.85028 & 0.19185\end{array}$

$\begin{array}{ll}-0.00465 & 0.00000 \\ -0.00930 & 0.00000 \\ -0.02325 & 0.00000 \\ -0.04649 & 0.00002 \\ -0.09291 & 0.00020 \\ -0.23118 & 0.00304 \\ -0.45404 & 0.02335 \\ -0.65841 & 0.07360 \\ -0.83228 & 0.15863 \\ -0.96381 & 0.27442 \\ -1.04443 & 0.40926 \\ -1.07151 & 0.54670 \\ -0.18197 & 0.001448 \\ -0.36001 & 0.01155 \\ -0.52955 & 0.03737 \\ -0.68518 & 0.08352 \\ -0.82089 & 0.15128 \\ -0.93091 & 0.23853 \\ -1.01063 & 0.34012 \\ -1.05747 & 0.44868 \\ -1.07148 & 0.55578 \\ -1.05548 & 0.65301\end{array}$

$\begin{array}{ll}-0.00465 & 0.00000 \\ -0.00930 & 0.00000 \\ -0.02323 & 0.00001 \\ -0.04638 & 0.00008 \\ -0.09206 & 0.00065 \\ -0.21793 & 0.0092 \varepsilon \\ -0.35514 & 0.0532 C \\ -0.37287 & 0.10696 \\ -0.29806 & 0.13756 \\ -0.17382 & 0.15961 \\ -0.00923 & 0.19781 \\ 0.19298 & 0.23499 \\ -0.17551 & 0.00469 \\ -0.31017 & 0.03050 \\ -0.37498 & 0.07366 \\ -0.36671 & 0.11284 \\ -0.30555 & 0.13603 \\ -0.21345 & 0.15215 \\ -0.09736 & 0.17654 \\ 0.04517 & 0.21024 \\ 0.20740 & 0.23642 \\ 0.36398 & 0.23530\end{array}$

.

$\begin{array}{ll}-0.00465 & 0.00000 \\ -0.00930 & 0.00000 \\ -0.02324 & 0.00001 \\ -0.04646 & 0.00010 \\ -0.09266 & 0.00078 \\ -0.22702 & 0.01167 \\ -0.41614 & 0.07932 \\ -0.52221 & 0.20463 \\ -0.52479 & 0.33470 \\ -0.45395 & 0.40890 \\ -0.37260 & 0.40597 \\ -0.33315 & 0.35406 \\ -0.18000 & 0.00578 \\ -0.34259 & 0.04176 \\ -0.46546 & 0.11927 \\ -0.52873 & 0.22434 \\ -0.52774 & 0.32651 \\ -0.47836 & 0.39555 \\ -0.41075 & 0.41630 \\ -0.35581 & 0.39413 \\ -0.33254 & 0.34999 \\ -0.34227 & 0.30883\end{array}$


$\begin{array}{lll}0.01 \quad-0.00340 & -0.00000\end{array}$ $0.02 .-0.00679-0.00000$ $\begin{array}{lll}0.05 & -0.01697 & -0.00000\end{array}$ $\begin{array}{lll}0.10 & -0.03390 & -0.00000\end{array}$ $\begin{array}{lll}0.20 & -0.06742 & -0.00004 \\ 0.50 & -0.16206 & -0.00067\end{array}$ $\begin{array}{lll}0.50 & -0.16206 & -0.00667\end{array}$ $\begin{array}{lll}1.00 & -0.28219 & -0.00718\end{array}$ 1.00
$2.00-0.33762-0.03120$ $2.50-0.33762-0.08535$ $3.00-0.26883-0.27807$ $3.50 \quad-0.24130-6.38261$

$0.125 \pi \quad-0.12958-0.00031$ $0.250 \pi-0.23780-0.00306$ $0.375 \pi \quad-0.30983 \quad-0.01298$ $0.500 \pi \quad-0.34145 \quad-0.03682$ $\begin{array}{lll}0.625 \pi & -0.33912 & -0.08024\end{array}$ $\begin{array}{llll}0.750 \pi & -0.31608 & -0.14423\end{array}$ $0.875 \pi \quad-0.28645-0.22363$ $\begin{array}{lll}1.000 \pi & -0.25999 & -0.30874\end{array}$

$\begin{array}{lll}1.125 \pi & -0.23973 & -0.38924 \\ 1.250 \pi & -0.22300 & -0.45799\end{array}$

\section{$-0.00340$}

$-0.00679 \quad 0.00000$

$-0.01693 \quad 0.00000$

$-0.03395 \quad 0.00002$

$-0.16864 \quad 0.00022$

$-0.16864 \quad 0.00222$

$-0.329940 .01690$

$-0.59705 \quad 0.11197$

$-0.08649 \quad 0.19060$

$-0.73968 \quad 0.27938$

$\begin{array}{ll}-0.73968 & 0.27938 \\ -0.75701 & 0.36685\end{array}$

$\begin{array}{ll}-0.13280 & 0.00108\end{array}$

$-0.26211 \quad 0.00839$
-0.38409

$-0.38409 .0 .02695$

$\begin{array}{ll}-0.49454 & 0.05968 \\ -0.58919 & 0.10690\end{array}$

$-0.66437 \quad 0.16647$

$\begin{array}{ll}-0.71759 & 0.23422 \\ -0.74809 & 0.30479\end{array}$

$-0.74809 \cdot 0.30479$

$\begin{array}{ll}-0.75699 & 0.37251 \\ -0.74721 & 0.43224\end{array}$
$-0.00340 \quad 0.00050$

0.0067970 .00000

$\begin{array}{ll}-0.001697 & 0.000001 \\ -11.03387 & 0.00005\end{array}$

$0.03387-0.00005$

$0.06720 \quad 0.00039$

$-0.158640 .00539$

$-0.257720 .02897$

0.273190 .05305

$-0.12685 \quad 0.06883$

$0.00492 \quad 0.08689$

0.15749

$-0.12790$

$-0.22518$

$-0.27254$

$-0.22764$

$-0.15825$

$-0.06534$

0.04728

0.16786

0.27614 0.12499
$-0.00002$ $-3.00007$ $-0.00042$ $-0.00169$ $-0.00673$ $-0.03990$ $-0.11452$ $-0.21452$ $-0.24886$ $-0.23664$ $-0.18974$ $-0.10751$

$-n .02521$ $-0.08967$ $-0.16520$ $-0.22266$ $-0.24811$ $-0.24402$ $-0.21741$ $-0.17025$ $-0.10050$ $-0.00923$
0.00000 0.00001 0.00009 0.00140 0.01002 0.05012 0.11551 0.16783 0.12623 0.06251 0.00585 0.02855 0.07212 0.12464 0.16344 0.17249 0.15127 0.10954 0.05780 0.00359
$-0.00340$ $-0.00679$ $-0.01698$ $-0.03393$ $-0.06762$ $-0.16497$ $-0.29853$ $-0.36984$ $-0.37183$ $-0.33108$ $-0.26844$ $-0.13105$ $-0.24727$ $-0.33218$ $-0.37404$ $-0.37361$ $-0.34477$ $-0.30764$ $-0.27939$ -0.26815
-0.27241
0.00000 0.00000 0.00001 0.00057 0.00845 0.05599 0.13969 0.22106 0.26422 0.26294
0.23780 0.00419 0.02984 0.08324 0.15240 0.21612 0.25673 0.26827 0.25700 0.23592 0.21756

$$
\frac{a}{\lambda}=0.5 \quad \frac{\alpha}{\beta}=0.40
$$

\begin{tabular}{|c|c|c|}
\hline $\begin{array}{l}0.01 \\
0.02 \\
0.05 \\
0.10 \\
0.20 \\
0.50 \\
1.00 \\
1.50 \\
2.00 \\
2.50 \\
3.00 \\
3.50\end{array}$ & $\begin{array}{l}-0.00181 \\
-0.00362 \\
-0.00906 \\
-0.01809 \\
-0.03598 \\
-0.08668 \\
-0.15264 \\
-0.18822 \\
-0.19705 \\
-0.18931 \\
-0.17361 \\
-0.15347\end{array}$ & $\begin{array}{l}-0.00000 \\
-0.00000 \\
-0.00000 \\
-0.00001 \\
-0.00012 \\
-0.00180 \\
-0.01400 \\
-0.04433 \\
-0.09488 \\
-0.16036 \\
-0.23148 \\
-0.29980\end{array}$ \\
\hline $\begin{array}{l}0.125 \pi \\
0.250 \pi \\
0.375 \pi \\
0.500 \pi \\
0.625 \pi \\
0.750 \pi \\
0.875 \pi \\
1.000 \pi \\
1.125 \pi \\
1.250 \pi\end{array}$ & $\begin{array}{l}-0.06924 \\
-0.12782 \\
-0.16885 \\
-0.19089 \\
-0.19709 \\
-0.19264 \\
-0.18215 \\
-0.16832 \\
-0.15193 \\
-0.13273\end{array}$ & $\begin{array}{l}-0.00088 \\
-0.00689 \\
-0.02248 \\
-0.05035 \\
-0.09059 \\
-0.14051 \\
-0.19566 \\
-0.25139 \\
-0.30424 \\
-0.35250\end{array}$ \\
\hline
\end{tabular}

$\begin{array}{ll}-0.00001 & 0.00000 \\ -0.00004 & 0.0000 \\ -0.00023 & 0.00009 \\ -0.00090 & 0.00036 \\ -0.00360 & 0.00144 \\ -0.02192 & 0.00884 \\ -0.07981 & 0.03273 \\ -0.15559 & 0.06388 \\ -0.23240 & 0.0912 \\ -0.30209 & 0.10504 \\ -0.36476 & 0.10133 \\ -0.42347 & 0.08228 \\ -0.01369 & 0.0055 \\ -0.05161 & 0.02103 \\ -0.10576 & 0.04354 \\ -0.16670 & 0.06824 \\ -0.22699 & 0.08958 \\ -0.28288 & 0.1028 \\ -0.33398 & 0.10537 \\ -0.38166 & 0.09729 \\ -0.42742 & 0.08055 \\ -0.47192 & 0.05794\end{array}$

\begin{tabular}{|c|c|c|c|}
\hline $\begin{array}{l}-0.00181 \\
-0.00362 \\
-0.00906 \\
-0.01811 \\
-0.03618 \\
-0.08973 \\
-0.17424 \\
-0.024834 \\
-0.30751 \\
-0.34887 \\
-0.37203 \\
-0.37913\end{array}$ & $\begin{array}{l}0.00000 \\
0.00000 \\
0.00000 \\
0.00001 \\
0.00008 \\
0.00118 \\
0.00885 \\
0.02700 \\
0.05583 \\
0.09209 \\
0.13059 \\
0.16603\end{array}$ & $\begin{array}{r}-0.00181 \\
-0.00362 \\
-0.00905 \\
-0.01807 \\
-0.03582 \\
-0.08427 \\
-0.13673 \\
-0.14716 \\
-0.12016 \\
-0.05940 \\
0.03018 \\
0.13273\end{array}$ & $\begin{array}{r}0.00000 \\
0.00000 \\
0.00000 \\
0.00001 \\
0.00011 \\
0.00142 \\
0.00551 \\
0.00329 \\
-0.00786 \\
-0.01943 \\
-0.02141 \\
-0.00479\end{array}$ \\
\hline $\begin{array}{l}-c .07073 \\
-c .13894 \\
-c .20204 \\
-c .25772 \\
-c .30377 \\
-0.33887 \\
-0.36260 \\
-0.37553 \\
-0.37913\end{array}$ & $\begin{array}{l}0.00057 \\
0.00442 \\
0.01402 \\
0.03048 \\
0.05342 \\
0.08118 \\
0.11132 \\
0.14115 \\
0.16823\end{array}$ & $\begin{array}{r}-0.06803 \\
-0.11933 \\
-0.14513 \\
-0.14548 \\
-0.12330 \\
-0.08019 \\
-0.01774 \\
0.05891 \\
0.13960\end{array}$ & $\begin{array}{r}0.00075 \\
0.00391 \\
0.00590 \\
0.00214 \\
-0.00690 \\
-0.01668 \\
-0.02217 \\
-0.01899 \\
-0.00274\end{array}$ \\
\hline
\end{tabular}

$\begin{array}{lr}-0.00001 & 0.00000 \\ -0.00004 & 0.00001 \\ -0.00023 & 0.00009 \\ -0.00090 & 0.00036 \\ -0.00358 & 0.00146 \\ -0.02098 & 0.00937 \\ -0.06751 & 0.03737 \\ -0.11056 & 0.07084 \\ -0.13656 & 0.08484 \\ -0.14456 & 0.06468 \\ -0.13050 & 0.01215 \\ -0.08551 & -0.06038 \\ -0.01332 & 0.00572 \\ -0.04642 & 0.02344 \\ -0.08440 & 0.04994 \\ -0.11535 & 0.07454 \\ -0.13527 & 0.08490 \\ -0.14420 & 0.07412 \\ -0.14084 & 0.04200 \\ -0.12128 & -0.00710 \\ -0.08110 & -0.06561 \\ -0.01805 & -0.12297\end{array}$

$\begin{array}{ll}-0.00181 & 0.00000 \\ -0.00362 & 0.00000 \\ -0.00906 & 0.00000 \\ -0.01809 & 0.00004 \\ -0.03602 & 0.00030 \\ -0.08712 & 0.00442 \\ -0.15375 & 0.02791 \\ -0.18602 & 0.06530 \\ -0.18709 & 0.09718 \\ -0.17359 & 0.11181 \\ -0.16132 & 0.11160 \\ -0.15664 & 0.10570 \\ -0.06947 & 0.00221 \\ -0.12886 & 0.01524 \\ -0.16944 & 0.04059 \\ -0.18776 & 0.07057 \\ -0.18774 & 0.09539 \\ -0.17789 & 0.10945 \\ -0.16669 & 0.11302 \\ -0.15920 & 0.11011 \\ -0.15658 & 0.10529 \\ -0.15740 & 0.10164\end{array}$


$\frac{a}{\lambda}=0.5 \quad \frac{\alpha}{\beta}=0.70$ $\beta \mathrm{h}$

$\mathrm{C}(\mathrm{h}, 0)$

$\begin{array}{lll}0.01 & -0.00071 & -0.00000 \\ 0.02 & -0.00141 & -0.00000 \\ 0.05 & -0.00353 & -0.00000 \\ 0.10 & -0.00705 & -0.00001 \\ 0.20 & -0.01404 & -0.00010 \\ 0.50 & -0.03411 & -0.00155 \\ 1.00 & -0.06188 & -0.01139 \\ 1.50 & -0.08004 & -0.03376 \\ 2.00 & -0.08853 & -0.06766 \\ 2.50 & -0.08863 & -0.10893 \\ 3.00 & -0.08151 & -0.15305 \\ 3.50 & -0.06804 & -0.19689 \\ 0.125 \pi & -0.02714 & -0.00076 \\ 0.250 \pi & -0.05104 & -0.00576 \\ 0.375 \pi & -0.06951 & -0.01788 \\ 0.500 \pi & -0.08181 & -0.03794 \\ 0.625 \pi & -0.08821 & -0.06488 \\ 0.750 \pi & -0.08939 & -0.09659 \\ 0.875 \pi & -0.08593 & -0.13077 \\ 1.000 \pi & -0.07830 & -0.16559 \\ 1.125 \pi & -0.06691 & -0.19984 \\ 1.250 \pi & -0.05221 & -0.23286\end{array}$

$S(h, 0)$

$\begin{array}{ll}-0.00000 & 0.00000 \\ -0.00001 & 0.00001 \\ -0.00009 & 0.00006 \\ -0.00035 & 0.00025 \\ -0.00141 & 0.00098 \\ -0.00862 & 0.00591 \\ -0.03226 & 0.02074 \\ -0.06600 & 0.03766 \\ -0.10529 & 0.04983 \\ -0.14729 & 0.05339 \\ -0.19037 & 0.04780 \\ -0.23327 & 0.03446 \\ & \\ -0.00535 & 0.00371 \\ -0.02055 & 0.01368 \\ -0.04339 & 0.02692 \\ -0.07131 & 0.03980 \\ -0.10231 & 0.04921 \\ -0.13504 & 0.05333 \\ -0.16868 & 0.05169 \\ -0.20259 & 0.04473 \\ -0.23617 & 0.03331 \\ -0.26890 & 0.01836\end{array}$

$E(h, 0)$

$\begin{array}{ll}-0.00071 & 0.00000 \\ -0.00141 & 0.00000 \\ -0.00353 & 0.00000 \\ -0.00706 & 0.00000 \\ -0.01409 & 0.00003 \\ -0.03483 & 0.00046 \\ -0.06688 & 0.00335 \\ -0.09375 & 0.00991 \\ -0.11390 & 0.01969 \\ -0.12691 & 0.03105 \\ -0.13357 & 0.04206 \\ -0.13543 & 0.05120 \\ -0.02750 & 0.00022 \\ -0.05363 & 0.00169 \\ -0.07715 & 0.00526 \\ -0.09704 & 0.01113 \\ -0.11267 & 0.01890 \\ -0.12387 & 0.02774 \\ -0.13094 & 0.03668 \\ -0.13451 & 0.04488 \\ -0.13543 & 0.05174 \\ -0.13460 & 0.05696\end{array}$

$\frac{a}{\lambda}=0$.
C $(h, h)$

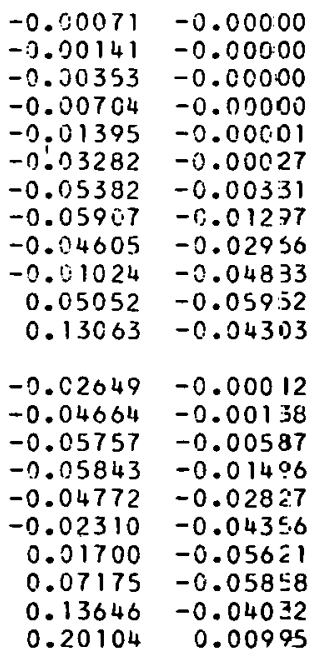

$0.13646-0.04032$
$\mathrm{S}(\mathrm{h}, \mathrm{h})$

$\begin{array}{lr}-0.00000 & 0.00000 \\ -0.00001 & 0.00001 \\ -0.00009 & 0.00006 \\ -0.00035 & 0.00025 \\ -0.00139 & 0.00098 \\ -0.00808 & 0.00598 \\ -0.02590 & 0.02073 \\ -0.04517 & 0.03384 \\ -0.06426 & 0.03266 \\ -0.08098 & 0.00825 \\ -0.08573 & -0.04317 \\ -0.06107 & -0.11730 \\ & \\ -0.00515 & 0.00374 \\ -0.01775 & 0.01384 \\ -0.03279 & 0.02629 \\ -0.04788 & 0.03480 \\ -0.06289 & 0.03345 \\ -0.07682 & 0.01798 \\ -0.08577 & -0.01399 \\ -0.08264 & -0.06232 \\ -0.05776 & -0.12287 \\ -0.00071 & -0.18570\end{array}$

$E(h, h)$

$\begin{array}{ll}-0.00071 & 0.00000 \\ -0.00141 & 0.00000 \\ -0.00353 & 0.00000 \\ -0.00704 & 0.00001 \\ -0.01400 & 0.00012 \\ -0.03344 & 0.00168 \\ -0.05695 & 0.00985 \\ -0.06679 & 0.02103 \\ -0.06716 & 0.02888 \\ -0.06457 & 0.03177 \\ -0.06271 & 0.03178 \\ -0.06215 & 0.03110 \\ -0.02681 & 0.00085 \\ -0.04852 & 0.00557 \\ -0.06194 & 0.01387 \\ -0.06725 & 0.02244 \\ -0.06730 & 0.02848 \\ -0.06533 & 0.03135 \\ -0.06347 & 0.03197 \\ -0.06244 & 0.03159 \\ -0.06215 & 0.03106 \\ -0.06222 & 0.03073\end{array}$

$\begin{array}{lll}0.01 & -0.00028 & -0.00000 \\ 0.02 & -0.00055 & -0.00000 \\ 0.05 & -0.00138 & -0.00000 \\ 0.10 & -0.00275 & -0.00001 \\ 0.20 & -0.00549 & -0.00006 \\ 0.50 & -0.01352 & -0.00093 \\ 1.00 & -0.02563 & -0.00686 \\ 1.50 & -0.03502 & -0.02027 \\ 2.00 & -0.04056 & -0.04076 \\ 2.50 & -0.04138 & -0.06635 \\ 3.00 & -0.03712 & -0.09484 \\ 3.50 & -0.02794 & -0.12450 \\ 0.125 \pi & -0.01069 & -0.00046 \\ 0.250 \pi & -0.02069 & -0.00347 \\ 0.375 \pi & -0.02935 & -0.01074 \\ 0.500 \pi & -0.03607 & -0.02277 \\ 0.625 \pi & -0.04031 & -0.03906 \\ 0.750 \pi & -0.04166 & -0.05860 \\ 0.875 \pi & -0.03989 & -0.08029 \\ 1.000 \pi & -0.03500 & -0.10318 \\ 1.125 \pi & -0.02714 & -0.12655 \\ 1.250 \pi & -0.01664 & -0.14986\end{array}$

$\begin{array}{llll}-0.00000 & 0.00000 & -0.00028 & 0.00000 \\ -0.00001 & 0.00001 & -0.00055 & 0.00000 \\ -0.00003 & 0.00003 & -0.00138 & 0.00000 \\ -0.00014 & 0.00014 & -0.00275 & 0.00000 \\ -0.00055 & 0.00055 & -0.00549 & 0.00001 \\ -0.00342 & 0.00328 & -0.01352 & 0.00018 \\ -0.01352 & 0.01140 & -0.02567 & 0.00127 \\ -0.02979 & 0.02045 & -0.03542 & 0.00364 \\ -0.05139 & 0.02672 & -0.04228 & 0.00696 \\ -0.07711 & 0.02810 & -0.04638 & 0.01052 \\ -0.10547 & 0.02403 & -0.04830 & 0.01367 \\ -0.13504 & 0.01487 & -0.04879 & 0.01603 \\ & & & \\ -0.00212 & 0.00206 & -0.01069 & 0.00009 \\ -0.00839 & 0.00755 & -0.02070 & 0.00065 \\ -0.01864 & 0.01473 & -0.02946 & 0.00197 \\ -0.03255 & 0.02157 & -0.03657 & 0.00407 \\ -0.04966 & 0.02641 & -0.04188 & 0.00670 \\ -0.06938 & 0.02826 & -0.04546 & 0.00952 \\ -0.09099 & 0.02675 & -0.04756 & 0.01217 \\ -0.11378 & 0.02193 & -0.04855 & 0.01443 \\ -0.13708 & 0.01407 & -0.04879 & 0.01616 \\ -0.16037 & 0.00355 & -0.04860 & 0.01738\end{array}$$$
\frac{\alpha}{\beta}=1.00
$$

$\begin{array}{rr}-0.00028 & -0.00000 \\ -0.00055 & -0.00000 \\ -0.00137 & -0.00000 \\ -0.00274 & -0.00000 \\ -0.00544 & -0.00003 \\ -0.01287 & -0.00042 \\ -0.02164 & -0.00338 \\ -0.02431 & -0.01118 \\ -0.01718 & -0.02516 \\ 0.00656 & -0.04317 \\ 0.05492 & -0.05526 \\ 0.13204 & -0.03840 \\ & \\ -0.01036 & -0.00020 \\ -0.01851 & -0.00164 \\ -0.02341 & -0.00550 \\ -0.02402 & -0.01276 \\ -0.01816 & -0.02388 \\ -0.00245 & -0.03793 \\ 0.02711 & -0.05103 \\ 0.07391 & -0.05480 \\ 0.13825 & -0.03532 \\ 0.21318 & 0.02684\end{array}$

$\begin{array}{rr}-0.00000 & 0.00000 \\ -0.00001 & 0.00001 \\ -0.00003 & 0.00003 \\ -0.00014 & 0.00014 \\ -0.00054 & 0.00054 \\ -0.00315 & 0.00323 \\ -0.01045 & 0.01055 \\ -0.02046 & 0.01606 \\ -0.03453 & 0.012 .66 \\ -0.05141 & -0.00774 \\ -0.06151 & -0.05370 \\ -0.04240 & -0.12957 \\ & \\ -0.00200 & 0.00204 \\ -0.00699 & 0.00724 \\ -0.01365 & 0.01308 \\ -0.02217 & 0.01630 \\ -0.03336 & 0.01338 \\ -0.04663 & 0.00039 \\ -0.05834 & -0.02696 \\ -0.06040 & -0.07222 \\ -0.03916 & -0.13574 \\ 0.02457 & -0.21045\end{array}$

$\begin{array}{ll}-0.00028 & 0.00000 \\ -0.00055 & 0.00000 \\ -0.00137 & 0.00000 \\ -0.00274 & 0.00001 \\ -0.00544 & 0.00005 \\ -0.01284 & 0.00064 \\ -0.02114 & 0.00348 \\ -0.02415 & 0.00683 \\ -0.02427 & 0.00877 \\ -0.02377 & 0.00935 \\ -0.02349 & 0.00936 \\ -0.02342 & 0.00928 \\ & \\ -0.01035 & 0.00032 \\ -0.01829 & 0.00203 \\ -0.02273 & 0.00476 \\ -0.02427 & 0.00721 \\ -0.02430 & 0.00869 \\ -0.02390 & 0.00928 \\ -0.02359 & 0.00938 \\ -0.02345 & 0.00933 \\ -0.02342 & 0.00927 \\ -0.02342 & 0.00924\end{array}$


$\beta \mathrm{h}$

$C(h, 0)$

$\operatorname{Sin}, 0)$

$-0.00002$

-0.00009
-0.00054

$-0.002$

$-0.00853 \quad 0.00623$

C. 03886

$-0.18460 \quad 0.15287$

$-0.46668 \quad 0.52625$

$-0.466680 .52625$

$\begin{array}{ll}-0.52350 & 0.69203 \\ -0.52965 & 0.77300\end{array}$

$\begin{array}{ll}-0.52965 & 0.77300 \\ -0.53771 & 0.75356\end{array}$

$-0.03234$

0.02400

0.09523

0.35550

0.51212

0.65118

0.74507

0.77709

0.74915

0.68226 $\frac{\mathrm{a}}{\lambda}=0.6 \quad \frac{\alpha}{\beta}=0.00$

$E(h, 0)$

$\mathrm{C}(\mathrm{h}, \mathrm{h})$

$S(h, h)$

$E(h, h)$

$-0.00429 \quad 0.00312$

$\begin{array}{ll}-0.00858 & 0.00624\end{array}$

$\begin{array}{lll}-0.02146 & 0.01559\end{array}$

$-0.04290 \quad 0.03120$

0.085690 .0624

0.15780

$-0.41025 \quad 0.32514$

$\begin{array}{ll}-0.70604 & 0.71505\end{array}$

$\begin{array}{ll}-0.77783 & 0.93154\end{array}$

$-0.78768 \quad 1.14484$

$-0.73540$

1.33793

$-0.16740 \quad 0.12337$

$-0.32793 \quad 0.25203$

$-0.474590 .39014$

$-0.600370 .53986$

$\begin{array}{ll}-0.69853 & 0.70064 \\ -0.70333 & 0.86908\end{array}$

$\begin{array}{ll}-0.70333 & 0.86908 \\ -0.79068 & 1.03905\end{array}$

$-0.77899 \quad 1.2024$

$-0.72969 \quad 1.3499$

0.00312

$0.00858 \quad 0.00624$

$-0.042770 .01519$

$-0.08467 \quad 0.06243$

$-0.19676 \quad 0.15631$

$\begin{array}{ll}-0.29941 & 0.30065\end{array}$

$-0.27586 \quad 0.38427$

$-0.055290 .37700$

$\begin{array}{ll}-0.05529 & 0.32333 \\ 0.09550 & 0.28288\end{array}$

0.310610 .236 .38

$-0.15981$

0.12278

$-0.27099$

$-0.30434$

$-0.26455$

$-0.18370$

$-0.09110$

0.14987

0.32716

0.51214

0.24269

0.34029

0.38855

0.33908

0.30043

0.27337

0.13851
$-0.00002$

0.00006

0.00156

$-0.00849 \quad 0.00627$

0.00827

$-0.158290 .04015$

$-0.230280 .16565$

$0.20400 \quad 0.50676$

$-0.11065 \cdot 0.55064$

$-0.01369 \quad 0.49424$

0.40134

$-0.03167$

$-0.11030$

$-0.19304$

0.23283

0.20904

0.14005

0.01271

0.09720

0.21525

.02450

0.10140

0.22838

0.37752

0.55071

0.53094

0.46957

0.39439
0.30259 $\begin{array}{ll}-0.00429 & 0.00312 \\ -0.00858 & 0.00624\end{array}$

$0.02145 \quad 0.01560$

$-0.04284 \quad 0.03124$

$0.08524 \quad 0.06285$

0.16307

$0.35302 \quad 0.35803$

$-0.314560 .74597$

$-0.16253 \quad 0.81354$

$0.02693 \quad 0.75967$

0.12601

$-0.30018 \quad 0.26993$

$-0.38166 \quad 0.43454$

$-0.38949 \quad 0.60121$

$\begin{array}{ll}-0.32373 & 0.73627\end{array}$

$\begin{array}{ll}-0.20878 & 0.80722\end{array}$

0.087210002

0.003260 .72842

$\begin{array}{rr}0.01587 & 0.02974 \\ -0.02839 & 0.54899\end{array}$

$\begin{array}{lllll}0.01 & -0.00421 & 0.00306 & -0.00002 & 0.00002 \\ 0.02 & -0.00842 & 0.00612 & -0.00008 & 0.00006 \\ 0.05 & -0.02105 & 0.01530 & -0.00052 & 0.00039 \\ 0.10 & -0.04203 & 0.03056 & -0.00210 & 0.00154 \\ 0.20 & -0.08353 & 0.06091 & -0.00834 & 0.00616 \\ 0.50 & -0.19984 & 0.14833 & -0.05053 & 0.03837 \\ 1.00 & -0.34152 & 0.26698 & -0.18048 & 0.15072 \\ 1.50 & -0.39578 & 0.32214 & -0.33556 & 0.32239 \\ 2.00 & -0.37760 & 0.28520 & -0.45691 & 0.51671 \\ 2.50 & -0.33762 & 0.15336 & -0.51400 & 0.67844 \\ 3.00 & -0.33086 & -0.03719 & -0.52264 & 0.75747 \\ 3.50 & -0.38045 & -0.22278 & -0.53350 & 0.73933 \\ & & & & \\ 0.12 \pi & -0.16014 & 0.11792 & -0.03162 & 0.02371 \\ 0.25 \pi & -0.29065 & 0.22180 & -0.11798 & 0.09396 \\ 0.37 \pi & -0.37107 & 0.29569 & -0.23614 & 0.20644 \\ 0.50 \pi & -0.39671 & 0.32294 & -0.35594 & 0.34967 \\ 0.62 \pi & -0.38040 & 0.29129 & -0.45011 & 0.50291 \\ 0.75 \pi & -0.34781 & 0.19961 & -0.50412 & 0.63863 \\ 0.87 \pi & -0.32757 & 0.06218 & -0.52167 & 0.73014 \\ 1.00 \pi & -0.33938 & -0.09318 & -0.52296 & 0.76159 \\ 1.12 \pi & -0.38551 & -0.23371 & -0.53573 & 0.73513 \\ 1.25 \pi & -0.44997 & -0.33373 & -0.58256 & 0.67121\end{array}$

$\begin{array}{ll}-0.00421 & 0.00306 \\ -0.00842 & 0.00612 \\ -0.02106 & 0.01530 \\ -0.04210 & 0.03062 \\ -0.08409 & 0.06132 \\ -0.20827 & 0.15484 \\ -0.40250 & 0.31998 \\ -0.56847 & 0.50217 \\ -0.69237 & 0.70203 \\ -0.76261 & 0.91284 \\ -0.77224 & 1.12122 \\ -0.72124 & 1.30956 \\ -0.16427 & 0.12107 \\ -0.32176 & 0.24729 \\ -0.46560 & 0.38273 \\ -0.58887 & 0.52947 \\ -0.68502 & 0.68695 \\ -0.74842 & 0.85176 \\ -0.77516 & 1.01791 \\ -0.76375 & 1.177417 \\ -0.71568 & 1.32127 \\ -0.03561 & 1.44067\end{array}$

$\frac{\Sigma}{\lambda}=0.6 \quad \frac{\alpha}{\beta}=0.005$

$\begin{array}{llll}1.25 \pi & -0.44997 & -0.33373\end{array}$

$\begin{array}{ll}-0.00421 & 0.00306 \\ -0.00842 & 0.00612 \\ -0.02104 & 0.01530 \\ -0.04197 & 0.03061 \\ -0.08309 & 0.06126 \\ -0.19311 & 0.15327 \\ -0.29415 & 0.29427 \\ -0.27204 & 0.37538 \\ -0.17462 & 0.36784 \\ -0.05704 & 0.31520 \\ 0.09164 & 0.27470 \\ 0.30242 & 0.22777 \\ & \\ -0.15683 & 0.12043 \\ -0.26607 & 0.23775 \\ -0.29925 & 0.33283 \\ -0.26112 & 0.37948 \\ -0.18277 & 0.37080 \\ -0.09227 & 0.33068 \\ 0.01026 & 0.29248 \\ 0.14510 & 0.26495 \\ 0.31856 & 0.22252 \\ 0.49859 & 0.13232\end{array}$

(1)

$\begin{array}{ll}-0.00002 & 0.00002 \\ -0.00008 & 0.00006 \\ -0.00052 & 0.00039 \\ -0.00209 & 0.00154 \\ -0.00830 & 0.00619 \\ -0.04880 & 0.03959 \\ -0.15455 & 0.16304 \\ -0.22477 & 0.34455 \\ -0.19973 & 0.49607 \\ -0.10999 & 0.53836 \\ -0.01621 & 0.48272 \\ 0.08374 & 0.39018 \\ -0.03096 & 0.02419 \\ -0.10774 & 0.09993 \\ -0.18842 & 0.22453 \\ -0.22728 & 0.37029 \\ -0.20458 & 0.48819 \\ -0.13823 & 0.53856 \\ -0.06199 & 0.51891 \\ 0.00953 & 0.45829 \\ 0.09205 & 0.38320 \\ 0.20673 & 0.29117\end{array}$

$-0.00421$ $-0.00842$

$-0.02105$

$-0.04204$

$-0.08364$

$-0.20125$

$-0.34618$

$-0.38612$

$-0.30888$

$-0.16126$

$-0.03009$

0.01222

$-0.16088$

$-0.29444$

$-0.37421$

$-0.38188$

$-0.31780$

$-0.20612$

$-0.00726$

$-0.03125$

0.00306 0.00612 0.01531 0.06168 0.15999 0.35102 0.56061 0.72977 0.74336 0.62644 0.12364 0.26474 0.58870 0.72034 0.78929 0.78234 0.61825 0.61825
0.54079 
Bh

$\mathrm{C}(\mathrm{h}, 0)$

0.0
0.02
0.05
0.10
0.20
0.50
1.00
1.50
2.00
2.50
3.00
3.50

$\begin{array}{lll}0.125 \pi & -0.15717 & 0.11567\end{array}$

$0.375 \pi \quad-0.36484 \quad 0.28923$

$0.500 \pi \quad-0.39096 \quad 0.31514$

$\begin{array}{lll}0.625 \pi & -0.37630 & 0.31514\end{array}$

$0.750 \pi-0.34584 \quad 0.19306$

$\begin{array}{lll}0.875 \pi & -0.34719 & 0.19306 \\ 0.000 \pi & -0.33916 & 0.05850\end{array}$

$1.000 \pi-0.33916-0.09322$

$1.125 \pi-0.33874-0.09322$

$\begin{array}{lll}1.125 \pi & -0.38374 & -0.23049 \\ 1.250 \pi & -0.44533 & -0.32877\end{array}$
$S(h, 0)$

$-0.00002$ -0.00008
-0.00051 $-0.00205$ $-0.00816$ $-0.04940$ $-0.17645$ $-0.32816$ $-0.44736$ $-0.50469$ $-0.51570$

$-0.52922$

$-0.03092$ $-0.11534$ $-0.34812$ $-0.44064$ $-0.49448$ $-0.51335$ $-0.51685$ $-0.53158$ $-0.57900$

$$
\frac{\mathrm{a}}{\lambda}=0.6 \quad \frac{\alpha}{\beta}=0.01
$$

0.00002
0.00006
0.00038
0.00152
0.00608
0.03789
0.14860
0.31721
0.50738
0.06517
0.74231
0.72543
0.02342
0.09271
0.20340
0.34395
0.49389
0.02635
0.71558
0.74647
0.72142
0.06033

$E(h, 0)$

$\begin{array}{ll}-0.00413 & 0.00300 \\ -0.00827 & 0.00601 \\ -0.02066 & 0.015 C 2 \\ -0.04131 & 0.03004 \\ -0.08252 & 0.06018 \\ -0.20437 & 0.15194 \\ -0.39490 & 0.31393 \\ -0.55761 & 0.49254 \\ -0.67896 & 0.68829 \\ -0.74768 & 0.89451 \\ -0.75709 & 1.09809 \\ -0.70736 & 1.28181 \\ -0.16120 & 0.11880 \\ -0.31571 & 0.24264 \\ -0.45677 & 0.37547 \\ -0.57760 & 0.51929 \\ -0.67177 & 0.67352 \\ -0.73381 & 0.83479 \\ -0.75995 & 0.99720 \\ -0.74881 & 1.15293 \\ -0.70194 & 1.29322 \\ -0.62397 & 1.40950\end{array}$

$\mathrm{C}(\mathrm{h}, \mathrm{h})$

$\begin{array}{ll}-0.00413 & 0.00300 \\ -0.00627 & 0.00601 \\ -0.02065 & 0.01502 \\ -0.04119 & 0.03004 \\ -0.08154 & 0.06011 \\ -0.18953 & 0.15030 \\ -0.28898 & 0.28803 \\ -0.26825 & 0.36670 \\ -0.17387 & 0.35889 \\ -0.05870 & 0.30723 \\ 0.08790 & 0.26669 \\ 0.29446 & 0.21940 \\ -0.15392 & 0.11813 \\ -0.26123 & 0.23291 \\ -0.29425 & 0.32553 \\ -0.25772 & 0.37061 \\ -0.18179 & 0.36180 \\ -0.09335 & 0.32245 \\ 0.00769 & 0.28469 \\ 0.14046 & 0.25673 \\ 0.31021 & 0.21421 \\ 0.48548 & 0.12633\end{array}$

$S(h, h)$

$\begin{array}{ll}-0.00002 & 0.00002 \\ -0.00008 & 0.00006 \\ -0.00051 & 0.00038 \\ -0.00205 & 0.00152 \\ -0.00811 & 0.00612 \\ -0.04770 & 0.03907 \\ -0.15089 & 0.16046 \\ -0.21939 & 0.33809 \\ -0.19557 & 0.48563 \\ -0.10930 & 0.52637 \\ -0.01862 & 0.47145 \\ 0.07891 & 0.37928 \\ -0.03026 & 0.02389 \\ -0.10523 & 0.09848 \\ -0.18392 & 0.22075 \\ -0.22186 & 0.36321 \\ -0.20021 & 0.47797 \\ -0.13647 & 0.52671 \\ -0.06301 & 0.50715 \\ 0.00645 & 0.44726 \\ 0.08701 & 0.37228 \\ 0.19841 & 0.28010\end{array}$

$E(h, h)$

$\begin{array}{ll}-0.00413 & 0.00300\end{array}$ $-0.00827 \quad 0.006 c 1$ $-0.04126 \quad 0.03009$ $-0.08208 \quad 0.06052$ $-0.19745 \quad 0.15696$ $-0.33948 \quad 0.34414$ $\begin{array}{ll}-0.37855 & 0.54904\end{array}$ $-0.30330 \quad 0.71393$ $\begin{array}{ll}-0.15996 & 0.77772\end{array}$ $\begin{array}{ll}-0.03308 & 0.72740\end{array}$ $\begin{array}{rr}-0.00771 & 0.61479\end{array}$

\section{$\begin{array}{ll}-0.15786 & 0.12132\end{array}$} $-0.28880 \quad 0.25965$ $\begin{array}{ll}-0.36690 & 0.41739 \\ -0.37441 & 0.57646\end{array}$ $-0.31198 \quad 0.70475$ $-0.203460 .77177$ $-0.089350 .76505$ $-0.001105 \quad 0.76535$ 0.006710 .69693 $\begin{array}{rr}0.0067 & 0.60693 \\ -0.03397 & 0.53262\end{array}$

$$
\frac{a}{\lambda}=0.6 \quad \frac{\alpha}{\beta}=0.02
$$

$\begin{array}{llllll}-0.00002 & 0.00001 & -0.00398 & 0.00289 & -0.00398 & 0.00289 \\ -0.00008 & 0.00006 & -0.00796 & 0.00578 & -0.00796 & 0.00578 \\ -0.00049 & 0.00037 & -0.01990 & 0.01446 & -0.01988 & 0.01446 \\ -0.00196 & 0.00149 & -0.03978 & 0.02893 & -0.03967 & 0.02892 \\ -0.00780 & 0.00594 & -0.07946 & 0.05795 & -0.07853 & 0.05787 \\ -0.04722 & 0.03694 & -0.19678 & 0.14630 & -0.18257 & 0.14452 \\ -0.16866 & 0.14445 & -0.38013 & 0.30218 & -0.27892 & 0.27596 \\ -0.31386 & 0.30711 & -0.53650 & 0.47383 & -0.26081 & 0.34994 \\ -0.42890 & 0.48927 & -0.65292 & 0.66160 & -0.17221 & 0.34160 \\ -0.48661 & 0.63953 & -0.71870 & 0.85896 & -0.06171 & 0.29176 \\ -0.50203 & 0.71308 & -0.72770 & 1.05326 & 0.08076 & 0.25117 \\ -0.52048 & 0.69854 & -0.68040 & 1.22806 & 0.27918 & 0.20334 \\ & & & & & \\ -0.02955 & 0.02284 & -0.15523 & 0.11440 & -0.14824 & 0.11365 \\ -0.11025 & 0.09025 & -0.30395 & 0.23359 & -0.25182 & 0.22353 \\ -0.22070 & 0.19744 & -0.43962 & 0.36135 & -0.28450 & 0.31142 \\ -0.33302 & 0.33281 & -0.55570 & 0.49951 & -0.25102 & 0.35350 \\ -0.42235 & 0.47640 & -0.64603 & 0.64745 & -0.17971 & 0.34441 \\ -0.47579 & 0.60263 & -0.70544 & 0.80186 & -0.09522 & 0.30650 \\ -0.49713 & 0.68747 & -0.73042 & 0.95703 & 0.00284 & 0.26955 \\ -0.50472 & 0.71729 & -0.71981 & 1.10549 & 0.13156 & 0.24084 \\ -0.52309 & 0.69489 & -0.67525 & 1.23890 & 0.29419 & 0.19828 \\ -0.57154 & 0.03910 & -0.60131 & 1.34918 & 0.46050 & 0.11489\end{array}$

$\begin{array}{ll}-0.00002 & 0.00001 \\ -0.00008 & 0.00006 \\ -0.00049 & 0.00037 \\ -0.00196 & 0.00149 \\ -0.00775 & 0.00597 \\ -0.04555 & 0.03805 \\ -0.14384 & 0.15544 \\ -0.20901 & 0.32557 \\ -0.18751 & 0.465 .46 \\ -0.10788 & 0.50324 \\ -0.02316 & 0.44966 \\ 0.06960 & 0.35827 \\ -0.02891 & 0.02328 \\ -0.10040 & 0.09564 \\ -0.17522 & 0.21337 \\ -0.21144 & 0.34948 \\ -0.19179 & 0.45825 \\ -0.13301 & 0.50383 \\ -0.06486 & 0.48445 \\ 0.00061 & 0.42592 \\ 0.07729 & 0.35125 \\ 0.18240 & 0.25897\end{array}$
$-0.00796$ $-0.01989$ $-0.03973$ $-0.07903$ $-0.19007$ $-0.32646$ 0.36385 $-0.29243$ $-0.03855$ $-0.00065$

$-0.15198$ $-0.27785$ $-0.35272$ $-0.35991$ $-0.30065$ $-0.19819$ $-0.09110$ $-0.01805$ $-0.00157$ $-0.03896$
0.00289 0.00578 0.01447 0.05828 0.15109 0.33080 0.52663 0.68328 0.74351 0.69649 0.59206

0.11680 0.24976 0.40093 0.55275 0.67459 0.73791 0.73164 0.66943 0.58479 $0.51643 \ldots$

$\begin{array}{lll}1.000 \pi & -0.33830 & -0.09338 \\ 1.2501 \pi & -0.37990 & -0.22446 \\ & -0.43618 & -0.31943\end{array}$ 
$\beta \mathrm{h}$

$\mathrm{C}(\mathrm{h}, 0)$

\begin{tabular}{|c|c|c|}
\hline $\begin{array}{l}0.01 \\
0.02 \\
0.05 \\
0.10 \\
0.20 \\
0.50 \\
1.00 \\
1.50 \\
2.00 \\
2.50 \\
3.00 \\
3.50\end{array}$ & $\begin{array}{l}-0.00383 \\
-0.00767 \\
-0.01916 \\
-0.03825 \\
-0.07604 \\
-0.18211 \\
-0.31282 \\
-0.36689 \\
-0.35817 \\
-0.33039 \\
-0.32908 \\
-0.37160\end{array}$ & $\begin{array}{r}0.00278 \\
0.00557 \\
0.01392 \\
0.02781 \\
0.05540 \\
0.13457 \\
0.24011 \\
0.28571 \\
0.24769 \\
0.12659 \\
-0.04380 \\
-0.20910\end{array}$ \\
\hline $\begin{array}{l}0.125 \pi \\
0.250 \pi \\
0.375 \pi \\
0.500 \pi \\
0.625 \pi \\
0.750 \pi \\
0.875 \pi \\
1.000 \pi \\
1.125 \pi \\
1.250 \pi\end{array}$ & $\begin{array}{l}-0.14585 \\
-0.26548 \\
-0.34099 \\
-0.36869 \\
-0.36008 \\
-0.33736 \\
-0.32442 \\
-0.33693 \\
-0.37578 \\
-0.42721\end{array}$ & $\begin{array}{r}0.10711 \\
0.20035 \\
0.26477 \\
0.28574 \\
0.25348 \\
0.16855 \\
0.04469 \\
-0.09361 \\
-0.21892 \\
-0.31078\end{array}$ \\
\hline
\end{tabular}

$S(h, 0)$

$\begin{array}{ll}-0.00502 & 0.00001\end{array}$

$\begin{array}{ll}-0.00047 & 0.00036\end{array}$

$-0.00187 \quad 0.00145$

$\begin{array}{lll}-0.16121 & 0.1404\end{array}$

$\begin{array}{ll}-0.30021 & 0.29736\end{array}$

$\begin{array}{lll}-0.41125 & 0.47190\end{array}$

$-0.46924$

$\begin{array}{ll}-0.48865 & 0.68523 \\ -0.51155 & 0.67282\end{array}$

$-0.02825$

$-0.10538$

$-0.31860$

$-0.40486$

$-0.45788$

$-0.49274$

$-0.51436$ $\begin{array}{lll}-0.00207 & 0.00006\end{array}$

$-0.00745 \quad 0.00579$

$\begin{array}{lll}-0.04514 & 0.0360\end{array}$ $\frac{a}{\lambda}=0.6 \quad \frac{\alpha}{\beta}=0.03$

$E(h, 0)$

$\mathrm{C}(\mathrm{h}, \mathrm{h})$

$S(h, h)$

$E(h, h)$

$\begin{array}{ll}-0.00383 & 0.00278 \\ -0.00767 & 0.00557 \\ -0.01916 & 0.01393 \\ -0.03831 & 0.02786 \\ -0.07652 & 0.05580 \\ -0.13948 & 0.14088 \\ -0.36591 & 0.29087 \\ -0.51620 & 0.45583 \\ -0.62798 & 0.63595 \\ -0.69084 & 0.82483 \\ -0.69945 & 1.01027 \\ -0.65446 & 1.17659 \\ -0.14947 & 0.11016 \\ -0.29263 & 0.22489 \\ -0.42311 & 0.34776 \\ -0.53462 & 0.48049 \\ -0.62127 & 0.62239 \\ -0.67816 & 0.77023 \\ -0.70204 & 0.91850 \\ -0.69193 & 1.06002 \\ -0.64957 & 1.18688 \\ -0.57945 & 1.29 .147\end{array}$

$\begin{array}{ll}-0.00383 & 0.00278 \\ -0.00766 & 0.00557 \\ -0.01915 & 0.01392 \\ -0.03820 & 0.02785 \\ -0.07562 & 0.05571 \\ -0.17536 & 0.13897 \\ -0.26921 & 0.26439 \\ -0.25353 & 0.33394 \\ -0.17037 & 0.32569 \\ -0.06434 & 0.27690 \\ 0.07403 & 0.23631 \\ 0.26474 & 0.18816 \\ -0.14278 & 0.10934 \\ -0.24276 & 0.21453 \\ -0.27506 & 0.29793 \\ -0.24444 & 0.33718 \\ -0.17747 & 0.32781 \\ -0.09676 & 0.29121 \\ -0.00162 & 0.25500 \\ 0.12313 & 0.22567 \\ 0.27906 & 0.18323 \\ 0.43710 & 0.10413\end{array}$

(1)

-0.00002
-0.00007
-0.00047
-0.00187
-0.00741
-0.04351
-0.13712
-0.19915
-0.17982
-0.10639
-0.02735
0.06073
-0.02762
-0.09579
-0.16694
-0.20151
-0.18375
-0.12961
-0.06646
-0.00486
0.06803
0.16718

0.00001
0.00006
0.00036
0.00145
0.00582
0.03705
0.15057
0.31354
0.44622
0.48120
0.42883
0.33826
0.02269
0.09287
0.20625
0.33632
0.43942
0.48204
0.46278
0.40551
0.33123
0.23911
0.02227

0.08785

0.32205

0.45960

0.66067

0.66950
0.01856

$\begin{array}{ll}-0.00383 & 0.00278 \\ -0.00767 & 0.00557 \\ -0.01916 & 0.01393 \\ -0.03826 & 0.02790 \\ -0.07611 & 0.05612 \\ -0.18296 & 0.14543 \\ -0.31394 & 0.31797 \\ -0.34972 & 0.50513 \\ -0.28194 & 0.65396 \\ -0.15452 & 0.71082 \\ -0.04341 & 0.66690 \\ -0.00819 & 0.57004 \\ -0.14632 & 0.11244 \\ -0.26731 & 0.24024 \\ -0.33908 & 0.38511 \\ -0.34597 & 0.53001 \\ -0.28972 & 0.64573 \\ -0.19299 & 0.70556 \\ -0.09247 & 0.69971 \\ -0.02434 & 0.64172 \\ -0.00904 & 0.56333 \\ -0.04341 & 0.50043\end{array}$

$$
\frac{a}{\lambda}=0.6 \quad \frac{\alpha}{\beta}=0.05
$$

$\begin{array}{ll}-0.00002 & 0.00001 \\ -0.00007 & 0.00006 \\ -0.00043 & 0.00035 \\ -0.00171 & 0.00138 \\ -0.00681 & 0.00551 \\ -0.04123 & 0.03421 \\ -0.14729 & 0.13264 \\ -0.27471 & 0.27882 \\ -0.37826 & 0.43922 \\ -0.43649 & 0.56935 \\ -0.46277 & 0.63337 \\ -0.49323 & 0.62468 \\ -0.02581 & 0.02117 \\ -0.09626 & 0.08322 \\ -0.19281 & 0.18060 \\ -0.27167 & 0.30165 \\ -0.37218 & 0.42799 \\ -0.42423 & 0.53753 \\ -0.45156 & 0.61075 \\ -0.46926 & 0.63767 \\ -0.49640 & 0.62194 \\ -0.54702 & 0.57946\end{array}$

$\begin{array}{ll}-0.00355 & 0.00258 \\ -0.00711 & 0.00517 \\ -0.01776 & 0.01281 \\ -0.03543 & 0.02533 \\ -0.07014 & 0.05154 \\ -0.16319 & 0.12850 \\ -0.25080 & 0.24270 \\ -0.23949 & 0.30409 \\ -0.16624 & 0.29426 \\ -0.06862 & 0.24899 \\ 0.06179 & 0.20847 \\ 0.23820 & 0.16021 \\ -0.13245 & 0.10121 \\ -0.22560 & 0.19761 \\ -0.25712 & 0.27268 \\ -0.23165 & 0.30673 \\ -0.17261 & 0.29684 \\ -0.09892 & 0.26253 \\ -0.00946 & 0.22783 \\ 0.10767 & 0.19737 \\ 0.25128 & 0.15556 \\ 0.39462 & 0.08449\end{array}$

$\begin{array}{ll}-0.00002 & 0.00001 \\ -0.00007 & 0.00006 \\ -0.00043 & 0.00035 \\ -0.00171 & 0.00138 \\ -0.00677 & 0.00554 \\ -0.03968 & 0.03512 \\ -0.12459 & 0.14129 \\ -0.18082 & 0.29091 \\ -0.16545 & 0.41032 \\ -0.10329 & 0.44017 \\ -0.03476 & 0.38991 \\ 0.04429 & 0.30109 \\ -0.02521 & 0.02154 \\ -0.08718 & 0.08756 \\ -0.15154 & 0.19275 \\ -0.18309 & 0.31159 \\ -0.16876 & 0.40429 \\ -0.12302 & 0.44148 \\ -0.06901 & 0.42238 \\ -0.01472 & 0.36738 \\ 0.05084 & 0.29409 \\ 0.13905 & 0.20289\end{array}$

$\begin{array}{ll}-0.00355 & 0.00258 \\ -0.00711 & 0.00517 \\ -0.01776 & 0.01292 \\ -0.03548 & 0.02587 \\ -0.07057 & 0.05203 \\ -0.16953 & 0.13475 \\ -0.29032 & 0.29380 \\ -0.32310 & 0.46475 \\ -0.26204 & 0.59909 \\ -0.14877 & 0.04977 \\ -0.05147 & 0.61144 \\ -0.02105 & 0.52811 \\ -0.13562 & 0.10422 \\ -0.24742 & 0.22229 \\ -0.31337 & 0.35534 \\ -0.31969 & 0.48732 \\ -0.26902 & 0.59171 \\ -0.18278 & 0.04511 \\ -0.09423 & 0.64002 \\ -0.03495 & 0.58964 \\ -0.02178 & 0.52239 \\ -0.05081 & 0.46915\end{array}$


$\beta h$

$C(h, 0)$

$0.0 .1 \quad-0.00330$

0.01

0.05

0.10

0.20

0.50

1.00

1.50

3.00

3.50

$0.125 \pi$

$0.250 \pi$

$0.375 \pi$

$0.500 \pi$

$0.750 \pi$

$0.875 \pi$

$1.125 \pi$

$1.250 \pi$

\subsection{9} 0.006590 .00479 0.01197 0.02391 0.0654204761 0.156970 .11518 $\begin{array}{lll}-0.27189 & 0.20267\end{array}$ $0.32485 \quad 0.23572$ $\begin{array}{ll}0.32782 & 0.19697\end{array}$ $\begin{array}{ll}0.31516 & 0.09069\end{array}$ $\begin{array}{ll}-0.32034 & -0.05332 \\ -0.35403 & -0.19237\end{array}$ 0.22970 $-0.32767$ $-0.32860$ 0.31816 $-0.31414$ $-0.32728$
$S(h, 0)$

$\begin{array}{ll}-0.00002 & 0.00001 \\ -0.00006 & 0.00005 \\ -0.00039 & 0.00033 \\ -0.00156 & 0.00131 \\ -0.00622 & 0.00524 \\ -0.03766 & 0.03248 \\ -0.13456 & 0.12527 \\ -0.25144 & 0.26151 \\ -0.34810 & 0.40910 \\ -0.40623 & 0.52762 \\ -0.43806 & 0.58619 \\ -0.47457 & 0.58058 \\ -0.02357 & 0.02012 \\ -0.08793 & 0.07880 \\ -0.17623 & 0.17017 \\ -0.26710 & 0.28263 \\ -0.34231 & 0.39882 \\ -0.39328 & 0.49871 \\ -0.42361 & 0.56528 \\ -0.44650 & 0.59051 \\ -0.47801 & 0.57833 \\ -0.52942 & 0.54291\end{array}$

$\frac{a}{\lambda}=0.6 \quad \frac{\alpha}{\beta}=0.07$

\author{
$E(h, 0)$
}

$\begin{array}{ll}-0.00330 & 0.00240 \\ -0.00659 & 0.00479 \\ -0.01648 & 0.01198 \\ -0.03295 & 0.02396 \\ -0.06581 & 0.04799 \\ -0.16289 & 0.12110 \\ -0.31417 & 0.24970 \\ -0.44237 & 0.39041 \\ -0.53697 & 0.54292 \\ -0.58983 & 0.70137 \\ -0.59701 & 0.85523 \\ -0.56019 & 0.99155 \\ -0.12851 & 0.09471 \\ -0.25140 & 0.19320 \\ -0.36306 & 0.29833 \\ -0.45803 & 0.41136 \\ -0.53140 & 0.53148 \\ -0.57923 & 0.65573 \\ -0.59915 & 0.77932 \\ -0.59083 & 0.89619 \\ -0.55621 & 0.95992 \\ -0.49952 & 1.08453\end{array}$

C (h, h)

$\mathrm{S}(\mathrm{h}, \mathrm{h})$

$E(h, h)$

$\begin{array}{ll}-0.00330 & 0.09240 \\ -0.00659 & 0.00479 \\ -0.01647 & 0.01197 \\ -0.03285 & 0.02395 \\ -0.06505 & 0.04787 \\ -0.15143 & 0.11882 \\ -0.23366 & 0.22281 \\ -0.22610 & 0.27690 \\ -0.16162 & 0.26617 \\ -0.07172 & 0.22333 \\ 0.05104 & 0.18298 \\ 0.21455 & 0.13518 \\ -0.12287 & 0.09369 \\ -0.20967 & 0.18203 \\ -0.24033 & 0.24959 \\ -0.21937 & 0.27901 \\ -0.16734 & 0.26862 \\ -0.10002 & 0.23623 \\ -0.01601 & 0.20245 \\ 0.09392 & 0.17161 \\ 0.22654 & 0.13081 \\ 0.35729 & 0.06706\end{array}$

-0.00002
-0.00006
-0.00039
-0.00156
-0.00618
-0.03618
-0.11320
-0.16423
-0.15233
-0.10006
-0.04102
0.02949
-0.02300
-0.07933
-0.13757
-0.16641
-0.15509
-0.11673
-0.07083
-0.02329
0.03536
0.11379

0.00001
0.00005
0.00033
0.00131
0.00527
0.03327
0.13258
0.27004
0.37760
0.40288
0.35441
0.26745
0.02044
0.08253
0.18016
0.28882
0.37224
0.40462
0.38558
0.33258
0.26051
0.17083
$-0.00330$ $-0.00659 \quad 0.00479$ $-0.01647 \quad 0.01198$ $-0.03290 \quad 0.02399$ $0.06543 \quad 0.04825$ $-0.15708 \quad 0.12485$ $-0.268490 .27146$ $-0.24351 \quad 0.54888$ $-0.142810 .59405$ $-0.05760 \quad 0.56059$ $-0.03133 \quad 0.48891$

$\begin{array}{ll}-0.12570 & 0.09660\end{array}$ $\begin{array}{ll}-0.12570 & 0.09660 \\ -0.28961 & 0.30568\end{array}$ $-0.295410 .32787$ $-0.24976 \quad 0.54227$ $-0.17289 \quad 0.58993$ $-0.09488 \quad 0.58549$ $-0.04330 \quad 0.54173$ $-0.031950 .48404$ $\begin{array}{ll}-0.05648 & 0.43897\end{array}$

$\begin{array}{llr}0.01 & -0.00294 & 0.00214 \\ 0.02 & -0.00589 & 0.00428 \\ 0.05 & -0.01471 & 0.01069 \\ 0.10 & -0.02938 & 0.02135 \\ 0.20 & -0.05844 & 0.04249 \\ 0.50 & -0.14044 & 0.10250 \\ 1.00 & -0.24478 & 0.17849 \\ 1.50 & -0.29642 & 0.20398 \\ 2.00 & -0.30589 & 0.16531 \\ 2.50 & -0.30173 & 0.06857 \\ 3.00 & -0.31037 & -0.05931 \\ 3.50 & -0.33898 & -0.18275 \\ 0.125 \pi & -0.11230 & 0.08184 \\ 0.250 \pi & -0.20610 & 0.15078 \\ 0.375 \pi & -0.26923 & 0.19440 \\ 0.500 \pi & -0.29978 & 0.20251 \\ 0.625 \pi & -0.30604 & 0.17031 \\ 0.750 \pi & -0.30252 & 0.10109 \\ 0.875 \pi & -0.30342 & 0.00657 \\ 1.000 \pi & -0.31673 & -0.09616 \\ 1.125 \pi & -0.34141 & -0.19029 \\ 1.250 \pi & -0.36879 & -0.26489\end{array}$

$\begin{array}{ll}-0.00001 & 0.00001 \\ -0.00005 & 0.00005 \\ -0.00034 & 0.00030 \\ -0.00136 & 0.00122 \\ -0.00543 & 0.00486 \\ -0.03286 & 0.03002 \\ -0.11751 & 0.11495 \\ -0.22030 & 0.23765 \\ -0.30764 & 0.36821 \\ -0.36508 & 0.47162 \\ -0.40320 & 0.52315 \\ -0.44645 & 0.52118 \\ -0.02057 & 0.01861 \\ -0.07675 & 0.07256 \\ -0.15401 & 0.15563 \\ -0.23421 & 0.25647 \\ -0.30225 & 0.35919 \\ -0.35142 & 0.44647 \\ -0.38505 & 0.50447 \\ -0.41385 & 0.52745 \\ -0.45018 & 0.51952 \\ -0.50203 & 0.49256\end{array}$

$\begin{array}{ll}-0.00294 & 0.00214 \\ -0.00589 & 0.00428 \\ -0.01472 & 0.01070 \\ -0.02943 & 0.02140 \\ -0.05877 & 0.04285 \\ -0.14542 & 0.10812 \\ -0.28022 & 0.22270 \\ -0.39403 & 0.34759 \\ -0.47755 & 0.48221 \\ -0.52390 & 0.02109 \\ -0.53018 & 0.75486 \\ -0.49849 & 0.87228 \\ -0.11475 & 0.08457 \\ -0.22434 & 0.17239 \\ -0.32368 & 0.26592 \\ -0.40789 & 0.36613 \\ -0.47264 & 0.47214 \\ -0.51464 & 0.58120 \\ -0.53203 & 0.68901 \\ -0.52483 & 0.79026 \\ -0.49508 & 0.87945 \\ -0.44674 & 0.95163\end{array}$

$\begin{array}{cc}-0.00294 & 0.00214 \\ -0.00589 & 0.00428 \\ -0.01471 & 0.01069 \\ -0.02934 & 0.02138 \\ -0.05810 & 0.04272 \\ -0.13537 & 0.10565 \\ -0.21013 & 0.19601 \\ -0.20723 & 0.24058 \\ -0.15403 & 0.22865 \\ -0.07454 & 0.18876 \\ 0.03741 & 0.14884 \\ 0.18390 & 0.10243 \\ -0.10979 & 0.08344 \\ -0.18788 & 0.16095 \\ -0.21717 & 0.21859 \\ -0.20190 & 0.24201 \\ -0.15891 & 0.23095 \\ -0.10006 & 0.20090 \\ -0.02372 & 0.16851 \\ 0.07620 & 0.13730 \\ 0.19450 & 0.09848 \\ 0.30962 & 0.04446\end{array}$

$\begin{array}{ll}-0.00001 & 0.00001 \\ -0.00005 & 0.00005 \\ -0.00034 & 0.00030 \\ -0.00136 & 0.00122 \\ -0.00539 & 0.00488 \\ -0.03149 & 0.03066 \\ -0.09803 & 0.12051 \\ -0.14224 & 0.24170 \\ -0.13478 & 0.33382 \\ -0.09514 & 0.35317 \\ -0.04861 & 0.30694 \\ 0.01012 & 0.22292 \\ -0.02004 & 0.01887 \\ -0.06884 & 0.07550 \\ -0.11899 & 0.16284 \\ -0.14430 & 0.25799 \\ -0.13683 & 0.32932 \\ -0.10787 & 0.35549 \\ -0.07245 & 0.33648 \\ -0.03405 & 0.28609 \\ 0.01506 & 0.21615 \\ 0.08082 & 0.12947\end{array}$

$\begin{array}{ll}-0.00294 & 0.00214 \\ -0.00589 & 0.00428 \\ -0.01471 & 0.01070 \\ -0.02938 & 0.02143 \\ -0.05842 & 0.04308 \\ -0.14011 & 0.11135 \\ -0.23877 & 0.24110 \\ -0.26509 & 0.37743 \\ -0.21806 & 0.48143 \\ -0.13366 . & 0.51944 \\ -0.06382 & 0.49216 \\ -0.04274 & 0.43496 \\ -0.11217 & 0.08620 \\ -0.20394 & 0.18307 \\ -0.25732 & 0.29060 \\ -0.26242 & 0.39513 \\ -0.22337 & 0.47582 \\ -0.15867 & 0.51601 \\ -0.09417 & 0.5124 .1 \\ -0.05230 & 0.47696 \\ -0.04323 & 0.43113 \\ -0.06228 & 0.39602\end{array}$

$\frac{\mathrm{a}}{\lambda}=0.6 \quad \frac{\alpha}{\beta}=0.10$

$-0.06228$

0.39602 
$\frac{\mathrm{a}}{\lambda}=0.6 \quad \frac{\alpha}{\beta}=0.20$

$\beta \mathrm{h}$

$$
\mathrm{C}(\mathrm{h}, 0)
$$

0.0

0.02

0.05

0.120

0.20
0.50

1.00

2.00

2.50

3.00
3.50

$0.125 \pi$

$0.250 \pi$

$0.500 \pi$

$0.625 \pi$

$0.750 \pi$

$1.000 \pi$

$1.125 \pi$

$1.250 \pi$
$\mathrm{S}(\mathrm{h}, \mathrm{c})$

$-0.00001$

$-0.00003$

$-0.00022$

$-0.00343$

$-0.02090$

$-0.07481$

$-0.14245$

$-0.20564$

$-0.30507$

$-0.35751$

$-0.01301$

$-0.04870$

$-0.09844$

$-0.15197$

$-0.20140$

$-0.24381$

$-0.31900$

$-0.36149$

$-0.41086$
$E(h, 0)$

0.00001

0.00004

0.00023

0.00373

0.02292

0.17338

0.26188

0.32967

0.36482
0.36938

0.01425

0.05485

0.11551
0.18640

0.25590

0.31325

C. 36881

0.36885

0.35802
$-0.00202$

$-0.01010 \quad 0.00293$

$-0.02018 \quad 0.00734$

$-0.04030 \quad 0.02939$

$-0.09964 \quad 0.07408$

$-0.19140 \quad 0.15207$

0.267910 .23599

$0.32306 \quad 0.32481$

$\begin{array}{ll}-0.35699 & 0.49822\end{array}$

$-0.337790 .56963$

$\begin{array}{ll}-0.07866 & 0.05797\end{array}$

$-0.15347 \quad 0.11792$

$\begin{array}{ll}-0.22076 & 0.18126\end{array}$

$\begin{array}{ll}0.27714 & 0.24834\end{array}$

$0.31986 \quad 0.31823$

0.38885

$-0.353700 .45723$

$-0.33575 \quad 0.57391$

$-0.30733 \quad 0.61641$
$C(h, h)$

$\begin{array}{ll}-0.00202 \quad 0.00147 \\ -0.00404 & 0.00293\end{array}$

0.00293

0.00733

$-0.02013 \quad 0.01466$

$-0.09320 \quad 0.07146$

$-0.14763 \quad 0.1280$

0.15036

$-0.126410 .13578$

$\begin{array}{rr}-0.07331 & 0.10210 \\ 0.00879 & 0.06448\end{array}$

$0.11404 \quad 0.02518$

$\begin{array}{ll}-0.07547 & 0.05674\end{array}$

$\begin{array}{ll}-0.13043 & 0.10690 \\ -0.15487 & 0.1406\end{array}$

$-0.154870 .14061$

$-0.12929 \quad 0.13776$

$-0.09137 \quad 0.11258$

$-0.03618 \quad 0.08352$

$0.12154 \quad 0.02243$

$\begin{array}{rr}0.12154 & 0.02243 \\ 0.20339 & -0.00797\end{array}$
$S(h, h)$

$\begin{array}{ll}-0.00001 & 0.00001 \\ -0.00003 & 0.00004\end{array}$

$-0.00003$

$-0.00022$

$-0.00086$

$-0.01975$

$-0.06060$

$-0.08856$

$-0.07935$

$-0.03434$

$-0.01260$

$-0.04280$

$-0.07340$

$-0.09137$

$-0.08325$

-0.07186
-0.05635

$-0.05635$

-0.03172
0.00508
0.00023

0.00374

0.02319

0.08765
0.16818

0.16818

0.22395

0.22998

0.11538

0.01436 0.05594 0.11655 0.22145 0.23357 0.21472 0.17121 0.10938
0.03455
$E(h, h)$

$-0.00202$

$-0.01009 \quad 0.00734$

$-0.02015 \quad 0.01469$

$-0.09570 \quad 0.07603$

$-0.16153 \quad 0.16240$

$-0.17849 \quad 0.24911$

$-0.15059 \quad 0.31145$

$-0.103710 .33285$

$-0.06772 \quad 0.31902$

$-0.05759-0.29206$

$-0.07674 \quad 0.05896$

$-0.13857 \quad 0.12417$

$-0.17354 \quad 0.19443$

$-0.15367 \quad 0.26000$

$-0.15367 \quad 0.30820$

$-0.11724 \quad 0.33098$

$-0.062120 .31163$

$-0.05781 \quad 0.31163$

$\begin{array}{ll}-0.06602 & 0.27506\end{array}$

$\begin{array}{llr}0.01 & -0.00095 & 0.00069 \\ 0.02 & -0.00190 & 0.00138 \\ 0.05 & -0.00475 & 0.00345 \\ 0.10 & -0.00949 & 0.00688 \\ 0.20 & -0.01892 & 0.01364 \\ 0.50 & -0.04630 & 0.03204 \\ 1.00 & -0.08634 & 0.05044 \\ 1.50 & -0.11751 & 0.04676 \\ 2.00 & -0.14117 & 0.02038 \\ 2.50 & -0.15980 & -0.02279 \\ 3.00 & -0.17427 & -0.07434 \\ 3.50 & -0.18340 & -0.12740 \\ & & \\ 0.125 \pi & -0.03672 & 0.02590 \\ 0.250 \pi & -0.07020 & 0.04493 \\ 0.375 \pi & -0.09845 & 0.05186 \\ 0.506 \pi & -0.12125 & 0.04432 \\ 0.625 \pi & -0.13964 & 0.02297 \\ 0.750 \pi & -0.15486 & -0.00915 \\ 0.875 \pi & -0.16756 & -0.04790 \\ 1.000 \pi & -0.17747 & -0.08943 \\ 1.125 \pi & -0.18379 & -0.13098 \\ 1.250 \pi & -0.18571 & -0.17119\end{array}$

$\begin{array}{ll}-0.00000 & 0.00001 \\ -0.00001 & 0.00002 \\ -0.00008 & 0.00013 \\ -0.00034 & 0.00053 \\ -0.00134 & 0.00213 \\ -0.00819 & 0.01300 \\ -0.03032 & 0.04777 \\ -0.06101 & 0.09353 \\ -0.09596 & 0.13774 \\ -0.13396 & 0.17118 \\ -0.17583 & 0.19046 \\ -0.22201 & 0.19711 \\ & \\ -0.00511 & 0.00811 \\ -0.01944 & 0.03077 \\ -0.04055 & 0.06348 \\ -0.06577 & 0.10015 \\ -0.09331 & 0.13480 \\ -0.12268 & 0.16298 \\ -0.15425 & 0.18254 \\ -0.18849 & 0.19348 \\ -0.22531 & 0.19720 \\ -0.26397 & 0.19551\end{array}$

$$
\frac{\mathrm{a}}{\lambda}=0.6 \quad \frac{\alpha}{\beta}=0.40
$$

$\begin{array}{lllr}-0.00095 & 0.00069 & -0.00095 & 0.00069 \\ -0.00190 & 0.00138 & -0.00190 & 0.00138 \\ -0.00475 & 0.00345 & -0.00475 & 0.00345 \\ -0.00949 & 0.00690 & -0.00947 & 0.00689 \\ -0.01895 & 0.01382 & -0.01878 & 0.01370 \\ -0.04678 & 0.03478 & -0.04427 & 0.03277 \\ -0.08931 & 0.07091 & -0.07311 & 0.05485 \\ -0.12389 & 0.10881 & -0.08337 & 0.05812 \\ -0.14793 & 0.14747 & -0.07722 & 0.04303 \\ -0.16041 & 0.18467 & -0.05253 & 0.01608 \\ -0.16203 & 0.211767 & -0.00631 & -0.01551 \\ -0.15498 & 0.24409 & 0.05787 & -0.04249 \\ & & & \\ -0.03696 & 0.02724 & -0.03571 & 0.02627 \\ -0.07183 & 0.05518 & -0.06311 & 0.04735 \\ -0.10270 & 0.08423 & -0.07875 & 0.05836 \\ -0.12797 & 0.11428 & -0.08349 & 0.05698 \\ -0.14656 & 0.14466 & -0.07825 & 0.04468 \\ -0.15800 & 0.17428 & -0.06178 & 0.02463 \\ -0.16247 & 0.20179 & -0.03219 & 0.00045 \\ -0.16079 & 0.22591 & 0.01040 & -0.02413 \\ -0.15425 & 0.24562 & 0.06268 & -0.04388 \\ -0.14443 & 0.26035 & 0.11849 & -0.05207\end{array}$

$\begin{array}{ll}-0.00000 & 0.00001 \\ -0.00001 & 0.00002 \\ -0.00008 & 0.00013 \\ -0.00034 & 0.00053 \\ -0.00133 & 0.00213 \\ -0.00761 & 0.01296 \\ -0.02301 & 0.04633 \\ -0.03548 & 0.08367 \\ -0.04468 & 0.10586 \\ -0.05511 & 0.10212 \\ -0.06530 & 0.07038 \\ -0.06686 & 0.01362 \\ -0.00488 & 0.00810 \\ -0.01630 & 0.03035 \\ -0.02805 & 0.06026 \\ -0.03686 & 0.08810 \\ -0.04400 & 0.10505 \\ -0.05191 & 0.10609 \\ -0.06062 & 0.08974 \\ -0.06707 & 0.05660 \\ -0.06639 & 0.00899 \\ -0.05350 & -0.04826\end{array}$

-0.00095
-0.00190
-0.00475
-0.00948
-0.01882
-0.04465
-0.07397
-0.08102
-0.07119
-0.05670
-0.04713
-0.04479
-0.03591
-0.06399
-0.07900
-0.08040
-0.07221
-0.06066
-0.05100
-0.04580
-0.04483
-0.04635

0.00069

0.00138

0.00345

0.00691

0.01387

0.03545

0.07373

0.10884

0.13126

0.13805

0.13449

0.12849

0.02759

0.05714

0.08714

0.11295

0.13017

0.13750

0.13705

0.13274

0.12525 
$S(h, 0)$

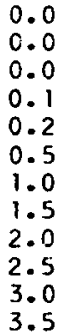
$0.375 \pi$ 0.01382 $0.625 \pi-0.06005-0.00815$ $0.750 \pi \quad-0.07037 \quad-0.01941$ $0.875 \pi \quad-0.07880-0.03914$ $1.000 \pi-0.08474-0.06132$ $1.125 \pi \quad-0.08784-0.08506$ $1.250 \pi-0.08803-0.10971$ $\frac{\mathrm{a}}{\lambda}=0.6 \quad \frac{\alpha}{\beta}=0.70$

$E(h, 0)$

$\mathrm{C}(\mathrm{h}, \mathrm{h})$

$S(h, h)$

$E(h, h)$

$\begin{array}{ll}-0.00031 & 0.00022 \\ -0.00061 & 0.00045 \\ -0.00153 & 0.00111 \\ -0.00306 & 0.00223 \\ -0.00611 & 0.00446 \\ -0.01505 & 0.01119 \\ -0.02847 & 0.02258 \\ -0.03897 & 0.03408 \\ -0.04590 & 0.04519 \\ -0.04926 & 0.05516 \\ -0.04968 & 0.06331 \\ -0.04811 & 0.06926 \\ -0.01190 & 0.00877 \\ -0.02300 & 0.01766 \\ -0.03259 & 0.02668 \\ -0.04018 & 0.03570 \\ -0.04552 & 0.04441 \\ -0.04863 & 0.05245 \\ -0.04978 & 0.05947 \\ -0.04939 & 0.0 .6522 \\ -0.04795 & 0.06958 \\ -0.04596 & 0.07259\end{array}$

$-0.00000$ $-0.00000 \quad 0.0000$ $-0.00002 \quad 0.00005$ $-0.00030 \quad 0.00022$ 0.001690 .00519 $0.00169 \quad 0.00519$ $-0.010130 .01778$ 0.01010 .03094 $-0.033450 .03360$ $-0.03345 \quad 0.03360$ $-0.06420-0.02873$

$\begin{array}{ll}-0.00108 & 0.00327\end{array}$ $\begin{array}{ll}-0.00363 & 0.01187\end{array}$ $-0.006770 .02278$ $-0.01105 \quad 0.03244$ $-0.01803 \quad 0.03783$ $-0.028690 .03635$ $-0.04236 \quad 0.02551$ $-0.056110 .00304$ $-0.06454-0.03233$ $-0.05989-0.07954$
$-0.00031$ $0.00153-0.00111$ $-0.00306 \quad 0.00223$ $-0.00606 \quad 0.00223$ $-0.014230 .01128$ $-0.022950 .02260$ 0.024440 .03165 0.024840 .03651 0.020280 .03772 $-0.018960 .03726$ $-0.01870 \quad 0.03662$ $-0.01150 \quad 0.00883$ $-0.02009 \quad 0.01785$ $-0.02432 \quad 0.02623$ $-0.02470 \quad 0.03261$ $\begin{array}{ll}-0.02298 & 0.03629\end{array}$ $-0.02091 \quad 0.03763$ $-0.01946 \quad 0.03758$ $-0.018810 .03705$ $-0.018710 .03659$ $-0.01883 \quad 0.03635$

$$
\frac{\mathrm{a}}{\lambda}=0.6 \quad \frac{\alpha}{\beta}=1.00
$$

\begin{tabular}{|c|c|c|c|c|}
\hline $\begin{array}{l}0.01 \\
0.02 \\
0.05 \\
0.10 \\
0.20 \\
0.50 \\
1.00 \\
1.50 \\
2.00 \\
2.50 \\
3.00 \\
3.50\end{array}$ & $\begin{array}{l}-0.00010 \\
-0.00020 \\
-0.00049 \\
-0.00099 \\
-0.00199 \\
-0.00513 \\
-0.01119 \\
-0.01838 \\
-0.02607 \\
-0.03311 \\
-0.03839 \\
-0.04117\end{array}$ & $\begin{array}{r}0.00007 \\
0.00014 \\
0.00036 \\
0.00072 \\
0.00111 \\
0.00319 \\
0.00415 \\
0.00135 \\
-0.00580 \\
-0.01710 \\
-0.03196 \\
-0.04959\end{array}$ & $\begin{array}{l}-0.00000 \\
-0.00000 \\
-0.00000 \\
-0.00001 \\
-0.00006 \\
-0.00038 \\
-0.00202 \\
-0.00604 \\
-0.01345 \\
-0.02466 \\
-0.03941 \\
-0.05699\end{array}$ & $\begin{array}{l}0.00000 \\
0.00000 \\
0.00002 \\
0.00009 \\
0.00034 \\
0.00208 \\
0.00775 \\
0.01557 \\
0.02386 \\
0.03118 \\
0.03652 \\
0.03929\end{array}$ \\
\hline $\begin{array}{l}0.125 \pi \\
0.250 \pi \\
0.375 \pi \\
0.500 \pi \\
0.625 \pi \\
0.750 \pi \\
0.875 \pi \\
1.000 \pi \\
1.125 \pi \\
1.250 \pi\end{array}$ & $\begin{array}{l}-0.00398 \\
-0.00844 \\
-0.01364 \\
-0.01947 \\
-0.02552 \\
-0.03122 \\
-0.03601 \\
-0.03945 \\
-0.04126 \\
-0.04130\end{array}$ & $\begin{array}{r}0.00263 \\
0.00414 \\
0.00364 \\
0.00060 \\
-0.00513 \\
-0.01345 \\
-0.02410 \\
-0.03671 \\
-0.05088 \\
-0.06622\end{array}$ & $\begin{array}{l}-0.00023 \\
-0.00110 \\
-0.00312 \\
-0.00686 \\
-0.01278 \\
-0.02105 \\
-0.03160 \\
-0.04414 \\
-0.05827 \\
-0.07361\end{array}$ & $\begin{array}{l}0.00130 \\
0.00496 \\
0.01038 \\
0.01675 \\
0.02327 \\
0.02924 \\
0.03413 \\
0.03758 \\
0.03937 \\
0.03940\end{array}$ \\
\hline
\end{tabular}

$\begin{array}{ll}-0.00010 & 0.00007 \\ -0.00020 & 0.00014 \\ -0.00049 & 0.00036 \\ -0.00099 & 0.00072 \\ -0.00197 & 0.00144 \\ -0.00484 & 0.00360 \\ -0.00907 & 0.00711 \\ -0.01227 & 0.01068 \\ -0.01426 & 0.01388 \\ -0.01517 & 0.01655 \\ -0.01528 & 0.01856 \\ -0.01493 & 0.01990 \\ -0.00383 & 0.00282 \\ -0.00736 & 0.00565 \\ -0.01035 & 0.00846 \\ -0.01262 & 0.01116 \\ -0.01416 & 0.01366 \\ -0.01501 & 0.01584 \\ -0.01530 & 0.01764 \\ -0.01521 & 0.01901 \\ -0.01489 & 0.01997 \\ -0.01449 & 0.02059\end{array}$

$\begin{array}{rr}-0.00010 & 0.00007 \\ -0.00020 & 0.00014 \\ -0.00049 & 0.00036 \\ -0.00099 & 0.00072 \\ -0.00197 & 0.00141 \\ -0.00483 & 0.00322 \\ -0.00916 & 0.00445 \\ -0.01274 & 0.00239 \\ -0.01430 & -0.00435 \\ -0.01060 & -0.01697 \\ 0.00404 & -0.03418 \\ 0.03718 & -0.04891 \\ -0.00382 & 0.00264 \\ -0.00737 & 0.00426 \\ -0.01055 & 0.00416 \\ -0.01313 & 0.00176 \\ -0.01431 & -0.00367 \\ -0.01249 & -0.01274 \\ -0.00513 & -0.02522 \\ 0.01121 & -0.03912 \\ 0.04031 & -0.04948 \\ 0.08485 & -0.04733\end{array}$

$\begin{array}{lr}-0.00000 & 0.00000 \\ -0.00000 & 0.00000 \\ -0.00000 & 0.00002 \\ -0.00001 & 0.00009 \\ -0.00005 & 0.00034 \\ -0.00031 & 0.00201 \\ -0.001117 & 0.00681 \\ -0.00347 & 0.011196 \\ -0.00941 & 0.01493 \\ -0.02080 & 0.01222 \\ -0.03666 & -0.00193 \\ -0.05017 & -0.03506 \\ & \\ -0.00020 & 0.00127 \\ -0.00071 & 0.00456 \\ -0.00173 & 0.00873 \\ -0.00404 & 0.01258 \\ -0.00881 & 0.01486 \\ -0.01694 & 0.01387 \\ -0.02838 & 0.00705 \\ -0.04123 & -0.00905 \\ -0.05066 & -0.03821 \\ -0.04778 & -0.08301\end{array}$

$\begin{array}{ll}-0.00010 & 0.00007 \\ -0.00020 & 0.00014 \\ -0.00049 & 0.00036 \\ -0.00099 & 0.00072 \\ -0.00195 & 0.00144 \\ -0.00454 & 0.00360 \\ -0.00713 & 0.00694 \\ -0.00764 & 0.00928 \\ -0.00721 & 0.01033 \\ -0.00677 & 0.01055 \\ -0.00659 & 0.01049 \\ -0.00656 & 0.01042 \\ -0.00368 & 0.00283 \\ -0.00631 & 0.00558 \\ -0.00750 & 0.00792 \\ -0.00761 & 0.00950 \\ -0.00724 & 0.01029 \\ -0.00687 & 0.01054 \\ -0.00665 & 0.01053 \\ -0.00657 & 0.01047 \\ -0.00656 & 0.01042 \\ -0.00657 & 0.01040\end{array}$


$\frac{a}{\lambda}=0 . \varepsilon$

$E(h, 0)$

0.00001

$0.00015 \quad 0.00047$

0.0015400 .00754

$0.06474 \quad 0.04602$

$0.25096 \quad 0.48055$

$\begin{array}{ll}0.35010 & 0.57902 \\ 0.40545 & 0.61507\end{array}$

$0.40545 \quad 0.61507$
$0.00062 \quad 0.00189$

$0.14624 \quad 0.33078$
$0.00956 \quad 0.02869$

0.03908

0.08976

0.24306

0.32437

0.38542

0.40858

0.38410

0.31513

0.02869

0.10904

0.35382

0.47100

0.55727

0.61641

0.61111

0.12806

$\begin{array}{ll}0.00123 & 0.0037 .8 \\ 0.00246 & 0.00757 \\ 0.00615 & 0.01892 \\ 0.01231 & 0.03783 \\ 0.02463 & 0.07563 \\ 0.06293 & 0.18338 \\ 0.13435 & 0.37159 \\ 0.22153 & 0.54366 \\ 0.32723 & 0.09729 \\ 0.45369 & 0.82412 \\ 0.00684 & 0.91556 \\ 0.76508 & 0.96435 \\ 0.04899 & 0.14920 \\ 0.10215 & 0.29395 \\ 0.16329 & 0.43451 \\ 0.23546 & 0.56672 \\ 0.32062 & 0.08688 \\ 0.41932 & 0.79090 \\ 0.53047 & 0.87457 \\ 0.65125 & 0.93396 \\ 0.77714 & 0.96600 \\ 0.90229 & 0.96895\end{array}$

0.90229 $\frac{\alpha}{\beta}=0.00$
$C(r . h)$

$\begin{array}{lr}0.00123 & 0.00378 \\ 0.00246 & 0.00757 \\ 0.00615 & 0.01871 \\ 0.01232 & 0.03775 \\ 0.02480 & 0.07497 \\ 0.06437 & 0.17818 \\ 0.13753 & 0.29447 \\ 0.19873 & 0.31316 \\ 0.21573 & 0.25133 \\ 0.19552 & 0.16302 \\ 0.18956 & 0.07501 \\ 0.22592 & -0.04115 \\ 0.04976 & 0.14323 \\ 0.10563 & 0.25542 \\ 0.16263 & 0.31270 \\ 0.20429 & 0.30820 \\ 0.21619 & 0.25741 \\ 0.20245 & 0.18840 \\ 0.18747 & 0.12000 \\ 0.19618 & 0.04687 \\ 0.22920 & -0.05109 \\ 0.25833 & -0.18158\end{array}$

$S(h, h)$

$0.00001 \quad 0.00002$ $\begin{array}{ll}0.00002 & 0.00008\end{array}$ $0.00015 \quad 0.0004$ 0.000620 .0018 0.04538 0.077350 .1584 0.324640 .32417 0.413760 .32417 0.477220 .19220 0.427220 .19220

\section{$0.00997 \quad 0.02845$} 0.112350 .20367 0.210090 .2874 0.32378 0.395820 .30304 0.429040 .24144 0.420960 .16300 $\begin{array}{ll}0.39595 & 0.07541 \\ 0.37028 & -0.03491\end{array}$
$E(h, h)$

$0.00123 \quad 0.00378$ $0.00246 \quad 0.00757$ $0.00615 \quad 0.01892$ $0.01234 \quad 0.03782$ 0.02496 .0 .07547 0.164610 .18580 0.303420 .34865 0.462470 .48313 $0.59280 \quad 0.41835$ 0.644730 .47835 0.603130 .18672

$\begin{array}{lll}0.05108 & 0.14697\end{array}$ $\begin{array}{ll}0.11773 & 0.28336\end{array}$ 0.39545 0.325620 .46698 0.531530 .44500 0.630560 .36164 0.047810 .26231 $0.51955 \quad 0.14527$ 0.561530 .48447

$\begin{array}{lrlll}0.01 & 0.00120 & 0.00369 & 0.00001 & 0.00002 \\ 0.02 & 0.00240 & 0.00738 & 0.00002 & 0.00 .007 \\ 0.05 & 0.00599 & 0.01844 & 0.00015 & 0.00046 \\ 0.10 & 0.01198 & 0.03683 & 0.00061 & 0.00184 \\ 0.20 & 0.02391 & 0.07326 & 0.00244 & 0.00734 \\ 0.50 & 0.05872 & 0.17619 & 0.01541 & 0.04480 \\ 1.00 & 0.10834 & 0.30593 & 0.06322 & 0.16487 \\ 1.50 & 0.13314 & 0.35932 & 0.14354 & 0.32200 \\ 2.00 & 0.11251 & 0.33472 & 0.24526 & 0.46803 \\ 2.50 & 0.03249 & 0.26007 & 0.34086 & 0.56473 \\ 3.00 & -0.09992 & 0.18011 & 0.39356 & 0.60159 \\ 3.50 & -0.25190 & 0.13304 & 0.37603 & 0.60081 \\ & & & & \\ 0.125 \pi & 0.04651 & 0.14085 & 0.00946 & 0.02793 \\ 0.250 \pi & 0.08898 & 0.25841 & 0.03858 & 0.10615 \\ 0.375 \pi & 0.12106 & 0.33446 & 0.08834 & 0.21906 \\ 0.500 \pi & 0.13345 & 0.36022 & 0.15710 & 0.34444 \\ 0.625 \pi & 0.11595 & 0.33869 & 0.23762 & 0.45870 \\ 0.750 \pi & 0.06168 & 0.28415 & 0.31613 & 0.54323 \\ 0.875 \pi & -0.02837 & 0.21810 & 0.37464 & 0.58987 \\ 1.000 \pi & -0.14292 & 0.16236 & 0.39633 & 0.60355 \\ 1.125 \pi & -0.26197 & 0.13148 & 0.37207 & 0.60040 \\ 1.250 \pi & -0.36215 & 0.12734 & 0.30516 & 0.60146\end{array}$

$$
\frac{a}{\lambda}=0.8 \quad \frac{\alpha}{\beta}=0.005
$$

$\begin{array}{ll}0.00120 & 0.00369 \\ 0.00240 & 0.00738 \\ 0.00600 & 0.01845 \\ 0.01200 & 0.03690 \\ 0.02407 & 0.07375 \\ 0.06137 & 0.18369 \\ 0.13100 & 0.36231 \\ 0.21594 & 0.52998 \\ 0.32081 & 0.67958 \\ 0.44676 & 0.80296 \\ 0.59072 & 0.89182 \\ 0.74526 & 0.93917 \\ 0.04777 & 0.14452 \\ 0.09961 & 0.28662 \\ 0.15920 & 0.42363 \\ 0.22951 & 0.55244 \\ 0.31243 & 0.06944 \\ 0.40847 & 0.77066 \\ 0.51653 & 0.85200 \\ 0.63383 & 0.90969 \\ 0.75598 & 0.94078 \\ 0.87728 & 0.94364\end{array}$

(1)

$\begin{array}{llll}0.00001 & 0.00002 & 0.00120 & 0.00369 \\ 0.00002 & 0.00007 & 0.00240 & 0.00738 \\ 0.00015 & 0.00046 & 0.00600 & 0.01845 \\ 0.00061 & 0.00184 & 0.01204 & 0.03688 \\ 0.00247 & 0.00732 & 0.02434 & 0.07360 \\ 0.01641 & 0.04417 & 0.06550 & 0.18116 \\ 0.07601 & 0.15407 & 0.16040 & 0.33979 \\ 0.18672 & 0.26740 & 0.29536 & 0.44591 \\ 0.31607 & 0.31532 & 0.44961 & 0.47052 \\ 0.40175 & 0.27700 & 0.57563 & 0.40791 \\ 0.41416 & 0.18882 & 0.62568 & 0.29178 \\ 0.38513 & 0.08318 & 0.58579 & 0.18515 \\ & & & \\ 0.00985 & 0.02769 & 0.04980 & 0.14331 \\ 0.04404 & 0.10213 & 0.11475 & 0.27622 \\ 0.11018 & 0.19803 & 0.20423 & 0.38533 \\ 0.20525 & 0.27937 . & 0.31692 & 0.45484 \\ 0.30737 & 0.31490 & 0.43864 & 0.47182 \\ 0.38457 & 0.29535 & 0.54544 & 0.43365 \\ 0.41622 & 0.23631 & 0.61204 & 0.35322 \\ 0.40790 & 0.16051 & 0.62288 & 0.25767 \\ 0.38282 & 0.07509 & 0.58038 & 0.17982 \\ 0.35629 & -0.03267 & 0.50588 & 0.14551\end{array}$


$\beta h$

$\mathrm{C}(\mathrm{h}, 0)$.

0.0

0.02

0.05

0.20

0.50

1.00

1.50

3.00

0.125

$0.125 \pi$
$0.250 \pi$

$0.375 \pi$

$0.500 \pi$

$0.625 \pi$

$0.875 \pi$

$1.125 \pi \cdot-0.14284$

$1.250 \pi$ $\frac{a}{\lambda}=0.8$

$E(h, 0)$

$0.00117 \quad 0.00360$

$0.00234 \quad 0.00720$

$.0 .00585 \quad 0.01799$

$0.01170 \quad 0.03598$

$0.02347 \quad 5.07192$

$\begin{array}{ll}0.05984 & 3.17913 \\ 0.12773 & 0.35326\end{array}$

$0.21049 \quad 3.51664$

$.0 .31260 \quad 0.66231$

$\begin{array}{ll}0.43513 & 0.78235 \\ 0.57502 & 0.86870\end{array}$

0.725010 .91466

0.046590 .14093

$0.09713 \quad 0.27948$

$0.15521 \quad 0.41302$

0.223710 .53852

$0.30445,0.65244$

$\begin{array}{ll}0.39789 & 0.75093 \\ 0.50295 & 0.83001\end{array}$

$0.61689 \cdot 0.88605$

$\begin{array}{ll}0.61689 & 0.88605 \\ 0.73541 & 0.91622\end{array}$

$0.85296 \quad 0.91899$ $\frac{\alpha}{\beta}=0.01$

\author{
$\mathrm{C}(\mathrm{h}, \mathrm{h})$
}

$S(h, h)$

$E(h, h)$
$S(h, 0)$

$\begin{array}{ll}0.00001 & 0.00002 \\ 0.00002 & 0.00007 \\ 0.00015 & 0.00045 \\ 0.00060 & 0.00179 \\ 0.00242 & 0.00714 \\ 0.01524 & 0.04362 \\ 0.06231 & 0.16050 \\ 0.14088 & 0.31346 \\ 0.23971 & 0.45585 \\ 0.33190 & 0.55082 \\ 0.38205 & 0.58843 \\ 0.36431 & 0.59067 \\ & \\ 0.00936 & 0.02719 \\ 0.03808 & 0.10333 \\ 0.08694 & 0.21324 \\ 0.15410 & 0.33531 \\ 0.23231 & 0.44673 \\ 0.30813 & 0.52957 \\ 0.36420 & 0.57604 \\ 0.38447 & 0.59098 \\ 0.36044 & 0.58984 \\ 0.29552 & 0.59267\end{array}$

0.00001 0.00002 0.00015 0.00244 0.01620 0.07469 0.18253 0.30775 0.39010

0.37242

0.00974

0.0433

0.10806

0.20052

0.37366

0.40380

0.39524

0.37008

0.34276
0.00002 0.00007 0.00045 0.00713 0.04299 0.14985 0.25993 0.27034 0.18548

0.02696 0.09937 0.19255
0.27157 0.27157 0.30627 0.23129 0.23128 0.182330 .04641 $0.21259-0.04804$ 0.12655
$0.00117 \quad 0.00360$ $\begin{array}{ll}0.00234 & 0.00720 \\ 0.00585 & 0.01799\end{array}$ $0.01174 \quad 0.03596$ $0.02374 \quad 0.07177$ $0.06386 \quad 0.17663$ $0.15630 \quad 0.33116$ $0.28751 \quad 0.43435$ $0.43710 \quad 0.45824$ $0.55896 \quad 0.39773$ 0.607210 .28585 $0.56894 \quad 0.18350$

$0.04856 \quad 0.13974$ $0.11185 \quad 0.269 .26$ $0.19895 \quad 0.37547$ 0.308440 .44302 $0.42648 \quad 0.45949$ $0.52980 \quad 0.42258$ $0.59407 \cdot 0.34499$ $0.60451 \quad 0.25307$ 0.563770 .25307 0.492550 .14558

$\begin{array}{lrl}0.01 & 0.00111 & 0.00342 \\ 0.02 & 0.00222 & 0.00684 \\ 0.05 & .0 .00556 & 0.01710 \\ 0.10 & 0.01111 & 0.03416 \\ 0.20 & 0.02216 & 0.06795 \\ 0.50 & 0.05425 & 0.16345 \\ 1.00 & 0.09893 & 0.28819 \\ 1.50 & 0.11916 & 0.33502 \\ 2.00 & 0.09659 & 0.31478 \\ 2.50 & 0.01992 & 0.24898 \\ 3.00 & -0.10304 & 0.17750 \\ 3.50 & -0.24209 & 0.13358 \\ & & \\ 0.125 \pi & 0.04302 & 0.13065 \\ 0.250 \pi & 0.08173 & 0.23986 \\ 0.375 \pi & 0.10989 & 0.31099 \\ 0.500 \pi & 0.11896 & 0.33614 \\ 0.625 \pi & 0.10002 & 0.31824 \\ 0.750 \pi & 0.04750 & 0.27031 \\ 0.875 \pi & -0.03693 & 0.21162 \\ 1.000 \pi & -0.14252 & 0.16134 \\ 1.125 \pi & -0.25117 & 0.13196 \\ 1.250 \pi & -0.34268 & 0.12476\end{array}$

$\begin{array}{ll}0.00001 & 0.00002 \\ 0.00002 & 0.00007 \\ 0.00015 & 0.00042 \\ 0.00059 & 0.00170 \\ 0.00236 & 0.00677 \\ 0.01489 & 0.04134 \\ 0.06050 & 0.15209 \\ 0.13570 & 0.29708 \\ 0.22901 & 0.43248 \\ 0.31475 & 0.52410 \\ 0.36014 & 0.56300 \\ 0.34205 & 0.56907 \\ 0.00915 & 0.02577 \\ 0.03709 & 0.09793 \\ 0.08419 & 0.20207 \\ 0.14826 & 0.31781 \\ 0.22207 & 0.42378 \\ 0.29280 & 0.50333 \\ 0.34428 & 0.54942 \\ 0.36193 & 0.56662 \\ 0.33836 & 0.56917 \\ 0.27716 & 0.57523\end{array}$

$$
\frac{\mathrm{a}}{\lambda}=0.8 \quad \frac{\alpha}{\beta}=0.02
$$

$\begin{array}{ll}0.00111 & 0.00342 \\ 0.00222 & 0.00684 \\ 0.00556 & 0.01711 \\ 0.01113 & 0.03422 \\ 0.02232 & 0.06839 \\ 0.05690 & 0.17033 \\ 0.12142 & 0.33584 \\ 0.20001 & 0.49096 \\ 0.29682 & 0.62909 \\ 0.41278 & 0.74269 \\ 0.54487 & 0.82424 \\ 0.68614 & 0.86754 \\ 0.04430 & 0.13401 \\ 0.09235 & 0.26572 \\ 0.14753 & 0.39260 \\ 0.21255 & 0.51172 \\ 0.28909 & 0.61974 \\ 0.37756 & 0.71298 \\ 0.47686 & 0.78773 \\ 0.58434 & 0.84060 \\ 0.69592 & 0.86900 \\ 0.80635 & 0.87161\end{array}$

$\begin{array}{lr}0.00111 & 0.00342 \\ 0.00222 & 0.00684 \\ 0.00556 & 0.01710 \\ 0.01114 & 0.03414 \\ 0.02241 & 0.06780 \\ 0.05790 & 0.16114 \\ 0.12216 & 0.26663 \\ 0.17400 & 0.28528 \\ 0.18664 & 0.23265 \\ 0.16838 & 0.15455 \\ 0.16356 & 0.07246 \\ 0.19427 & -0.03613 \\ 0.04485 & 0.12953 \\ 0.04485 & 0.23107 \\ 0.09439 & 0.28351 \\ 0.14371 & 0.2835 \\ 0.17852 & 0.28121 \\ 0.18716 & 0.23790 \\ 0.17441 & 0.17740 \\ 0.16160 & 0.11491 \\ 0.16923 & 0.04582 \\ 0.19701 & -0.04522 \\ 0.22179 & -0.16211\end{array}$

.

$\begin{array}{lr}0.00001 & 0.00002 \\ 0.00002 & 0.00007 \\ 0.00015 & 0.00042 \\ 0.00059 & 0.00170 \\ 0.00239 & 0.00675 \\ 0.01580 & 0.04072 \\ 0.07209 & 0.14175 \\ 0.17446 & 0.24562 \\ 0.29179 & 0.29025 \\ 0.36786 & 0.25750 \\ 0.37729 & 0.17894 \\ 0.34810 & 0.08156 \\ & . \\ 0.00951 & 0.02554 \\ 0.04205 & 0.09406 \\ 0.10393 & 0.18206 \\ 0.19140 & 0.25662 \\ 0.28397 & 0.28976 \\ 0.35282 & 0.27 .351 \\ 0.38008 & 0.22155 \\ 0.37103 & 0.15317 \\ 0.34570 & 0.07402 \\ 0.31700 & -0.02630\end{array}$

.




$$
\mathrm{C}(\mathrm{h}, 0)
$$

0.01
0.02
0.05

0.02

0.05

0.10

0.50

1.00

1.50

2.00

3.50

$0.125 \pi$

$0.250 \pi$

$0.375 \pi$

0.500

0.625

$0.875 \pi$

1.000

1.125
$0.00106 \quad 0.00325$ $0.00211 \quad 0.00651$ $0.00529 \quad 0.01626$ $0.01056 \quad 0.03249$ $0.02106 \quad 0.06462$ $0.05145 \quad 0.15547$ 0.093120 .27057 $0.11060 \quad 0.31975$ $0.08697 \quad 0.30212$ $\begin{array}{ll}0.01247 & 0.24165 \\ -0.10467 & 0.17528\end{array}$ $-0.10467 \quad 0.17528$

0.040850 .12426 . $0.07723 \quad 0.22525$ $\begin{array}{lll}0.10301 & 0.29627\end{array}$ $0.11011 \quad 0.32099$ $0.09038 \quad 0.30526$ 0.20706 $-0.24445 \quad 0.13166$ $-0.33068 \quad 0.12273$

$$
\frac{a}{\lambda}=0.8 \quad \frac{\alpha}{\beta}=0.03
$$

$S(h, 0)$

$\begin{array}{ll}0.00001 & 0.00002 \\ 0.00002 & 0.00006 \\ 0.00014 & 0.00040 \\ 0.00058 & 0.00161 \\ 0.00231 & 0.00642 \\ 0.01453 & 0.03918 \\ 0.05872 & 0.14413 \\ 0.13070 & 0.28158 \\ 0.21883 & 0.41038 \\ 0.29858 & 0.49877 \\ 0.33963 & 0.53874 \\ 0.32125 & 0.54871 \\ & \\ 0.00894 & 0.02443 \\ 0.03610 & 0.09281 \\ 0.08150 & 0.19149 \\ 0.14263 & 0.30126 \\ 0.21232 & 0.40207 \\ 0.27831 & 0.47849 \\ 0.32557 & 0.52412 \\ 0.34084 & 0.54329 \\ 0.31773 & 0.54911 \\ 0.25996 & 0.55801\end{array}$

$E(h, 0)$

C: $(h, h)$

$\begin{array}{ll}0.00106 & 0.00325 \\ 0.00211 & 0.00651 \\ 0.00529 & 0.01627 \\ 0.01058 & 0.03254 \\ 0.02123 & 0.06504 \\ 0.05411 & 0.16197 \\ 0.11543 & 0.31927 \\ 0.19004 & 0.46656 \\ 0.28184 & 0.59753 \\ 0.39157 & 0.70505 \\ 0.51631 & 0.78207 \\ 0.64937 & 0.82286 \\ 0.04213 & 0.12744 \\ 0.08780 & 0.25265 \\ 0.14022 & 0.37319 \\ 0.20194 & 0.48626 \\ 0.27452 & 0.58867 \\ 0.35827 & 0.67695 \\ 0.45212 & 0.74761 \\ 0.55352 & 0.79749 \\ 0.65857 & 0.82423 \\ 0.76229 & 0.82669\end{array}$

$S(h, h)$

\begin{tabular}{lr}
0.00106 & \multicolumn{1}{c}{$c .00325$} \\
0.00212 & 0.00651 \\
0.00529 & 0.01626 \\
0.01059 & 0.03247 \\
0.02130 & 0.06447 \\
0.05492 & 0.15324 \\
0.11513 & 0.25372 \\
0.16278 & 0.27228 \\
0.17349 & 0.22373 \\
0.15605 & 0.15023 \\
0.15162 & 0.07100 \\
0.17987 & -0.03393 \\
0.04258 & 0.12318 \\
0.08922 & 0.21979 \\
0.13509 & 0.26996 \\
0.16684 & 0.26360 \\
0.17404 & 0.22862 \\
0.16170 & 0.17192 \\
0.14977 & 0.11217 \\
0.15686 & 0.04510 \\
0.18239 & -0.04262 \\
0.20539 & -0.15340
\end{tabular}

0.00001 0.02002 0.00014 0.05058 2.01539 0.06958 16675 0.27671 0.34695 0.35453 0.32520

0.00928 0.00928 0.04076
0.09994 0.18272 0.20943 0.33320 0.35778 0.35778 0.32275 0.29289 0.0006 0.00161 0.00640 0.03858 0.13410 0.23213 0.27472 0.24530 0.0804

0.02420 0.08904 0.17214 0.24253 0.27418 0.2599 0.14838 0.14838 $0.20539-0.15340$
$E(h, h)$

$0.00106 \quad 0.00325$ 0.002120 .0065 $0.01061 \quad 0.03252$ $0.02147 \quad 0.06490$ 0.057720 .15963 $0.14092 \quad 0.29876$ $0.25816 \quad 0.39103$ $0.39049 \quad 0.41224$ $0.49701 \quad 0.35944$ $0.53867 \quad 0.26309$ 0.506290 .17620

\section{$0.04390 \quad 0.12632$} $0.10097 \quad 0.24313$ $0.17914 \quad 0.33848$ $0.27676 \quad 0.39874$ 0.381150 .41334 $0.47166 \quad 0.38105$ 0.31384 $0.53638 \quad 0.23511$ $0.50194 \quad 0.17190$ 0.14445

$\begin{array}{lrl}0.01 & 0.00096 & 0.00294 \\ 0.02 & 0.00191 & 0.00589 \\ 0.05 & 0.00478 & 0.01471 \\ 0.10 & 0.00955 & 0.02938 \\ 0.20 & 0.01903 & 0.05844 \\ 0.50 & 0.04629 & 0.14067 \\ 1.00 & 0.08247 & 0.24528 \\ 1.50 & 0.09514 & 0.29128 \\ 2.00 & 0.06986 & 0.27821 \\ 2.50 & -0.00046 & 0.22720 \\ 3.00 & -0.10699 & 0.16990 \\ 3.50 & -0.22421 & 0.13146 \\ & & \\ 0.125 \pi & 0.03682 & 0.11241 \\ 0.250 \pi & 0.06896 & 0.20069 \\ 0.375 \pi & 0.09047 & 0.26892 \\ 0.500 \pi & 0.09415 & 0.29273 \\ 0.625 \pi & 0.07322 & 0.28081 \\ 0.750 \pi & 0.02422 & 0.24394 \\ 0.875 \pi & -0.05023 & 0.19754 \\ 1.000 \pi & -0.14049 & 0.15644 \\ 1.125 \pi & -0.23182 & 0.12979 \\ 1.250 \pi & -0.30872 & 0.11806\end{array}$

$\begin{array}{ll}0.00001 & 0.00001 \\ 0.00002 & 0.00006 \\ 0.00014 & 0.00036 \\ 0.00055 & 0.00145 \\ 0.00221 & 0.00576 \\ 0.01383 & 0.03520 \\ 0.05526 & 0.12945 \\ 0.12123 & 0.25304 \\ 0.19990 & 0.36969 \\ 0.26896 & 0.45199 \\ 0.30238 & 0.49347 \\ 0.28367 & 0.50991 \\ 0.00852 & 0.02194 \\ 0.03410 & 0.08336 \\ 0.07632 & 0.17200 \\ 0.13201 & 0.27078 \\ 0.19417 & 0.36210 \\ 0.25166 & 0.43268 \\ 0.29147 & 0.47720 \\ 0.30264 & 0.49956 \\ 0.28044 & 0.51079 \\ 0.22876 & 0.52433\end{array}$

$\frac{a}{\lambda}=0.8$

$$
\frac{\alpha}{\beta}=0.05
$$

$\begin{array}{lr}0.0009 E & 0.00294 \\ 0.00191 & 0.00589 \\ 0.0047 \varepsilon & 0.01471 \\ 0.00958 & 0.02936 \\ 0.01924 & 0.05331 \\ 0.0494 & 0.13859 \\ 0.10225 & 0.22976 \\ 0.14236 & 0.24803 \\ 0.1497 & 0.20672 \\ 0.13365 & 0.14153 \\ 0.12973 & 0.06776 \\ 0.15367 & -0.03007 \\ 0.03838 & 0.11140 \\ 0.07972 & 0.19886 \\ 0.11933 & 0.24480 \\ 0.14564 & 0.24503 \\ 0.15031 & 0.21096 \\ 0.13865 & 0.16109 \\ 0.12815 & 0.10644 \\ 0.13410 & 0.04338 \\ 0.15580 & -0.03804 \\ 0.17587 & -0.13783\end{array}$

$\begin{array}{lr}0.00001 & 0.00001 \\ 0.00002 & 0.00006 \\ 0.00014 & 0.00036 \\ 0.00055 & 0.00145 \\ 0.00223 & 0.00575 \\ 0.01458 & 0.03462 \\ 0.06477 & 0.12002 \\ 0.15237 & 0.20740 \\ 0.24899 & 0.24623 \\ 0.30879 & 0.22269 \\ 0.31298 & 0.16042 \\ 0.28336 & 0.07798 \\ 0.00882 & 0.02173 \\ 0.03825 & 0.07978 \\ 0.09240 & 0.15393 \\ 0.16656 & 0.21669 \\ 0.24268 & 0.24563 \\ 0.29736 & 0.23480 \\ 0.31712 & 0.19471 \\ 0.30660 & 0.13910 \\ 0.28083 & 0.07144 \\ 0.24922 & -0.01511\end{array}$

10

0.00294 0.00589 0.01471 0.05868 0.14427 0.35204 0.37086 0.32480 0.2418 0.16805 0.11420 0.21954 0.30513 0.35890 0.37184 0.34359 0.28538 0.21794 0.14146 
0.12827

\section{$0.03319 \quad 0.10169$} $0.06156 \quad 0.18718$ $0.08028 \quad 0.24473$ $0.05856 \quad 0.25823$ 0.011890 .22738 $-0.05672 \quad 0.18768$ $-0.13836 \quad 0.15160$ $\begin{array}{ll}-0.22014 & 0.12659 \\ -0.28908 & 0.11279\end{array}$

$$
\frac{a}{\lambda}=0.8
$$

$E(h, 0)$

$0.00001 \quad 0.00001$ $0.00013 \quad 0.00033$ $0.00053 \quad 0.00130$ $0.00210 \quad 0.00518$ $0.01312 \quad 0.03162$ $0.05193 \quad 0.11629$ 0.112410 .1127 0.182730 .33324 $0.24258 \quad 0.40992$ $0.26964 \quad 0.4522$ 0.250820 .47359

$0.00810 \quad 0.01971$ $0.03225 \quad 0.07488$ $0.07140 \quad 0.15453$ 0.122160 .24348 0.177670 .3263 0.227820 .39155 0.261310 .43479 $\begin{array}{ll}0.26913 & 0.45947 \\ 0.24786 & 0.47485\end{array}$ $0.20137 \quad 0.49188$ $\frac{\alpha}{\beta}=0.07$

$\mathrm{C}(\mathrm{h}, \mathrm{h})$

$S(h, h)$

$E(h, h)$

$\begin{array}{ll}0.00086 & 0.00266 \\ 0.00173 & 0.00532 \\ 0.00433 & 0.01331 \\ 0.00866 & 0.02661 \\ 0.01736 & 0.05319 \\ 0.04424 & 0.13242 \\ 0.09427 & 0.26078 \\ 0.15491 & 0.38051 \\ 0.22909 & 0.48636 \\ 0.31712 & 0.57263 \\ 0.41629 & 0.63389 \\ 0.52101 & 0.66602 \\ 0.03445 & 0.10421 \\ 0.07175 & 0.20646 \\ 0.11446 & 0.30467 \\ 0.16456 & 0.39647 \\ 0.22319 & 0.47922 \\ 0.29048 & 0.55016 \\ 0.36538 & 0.00655 \\ 0.44569 & 0.64607 \\ 0.52821 & 0.66710 \\ 0.60896 & 0.66903\end{array}$

$\begin{array}{lr}0.00086 & 0.00266 \\ 0.00173 & 0.00532 \\ 0.00433 & 0.01330 \\ 0.00865 & 0.02655 \\ 0.01739 & 0.05273 \\ 0.04444 & 0.12534 \\ 0.09080 & 0.20808 \\ 0.12448 & 0.22592 \\ 0.12893 & 0.19081 \\ 0.11393 & 0.13285 \\ 0.11029 & 0.06424 \\ 0.13061 & -0.02691 \\ 0.03459 & 0.10075 \\ 0.07122 & 0.17993 \\ 0.10539 & 0.22200 \\ 0.12703 & 0.22350 \\ 0.12958 & 0.19448 \\ 0.11847 & 0.15053 \\ 0.10903 & 0.10050 \\ 0.11405 & 0.04132 \\ 0.13244 & -0.03421 \\ 0.15024 & -0.12444\end{array}$

0.00001 0.00002

0.00013

0.00053

0.01379

0.00024

0.13926

0.22422

0.27621

0.24632

0.00836

0.03585

0.08540

0.21875

0.26558

0.26973

0.24375

0.21103
0.00001

0.00005

0.00032

0.00517

0.03707

0.18538

0.22085

0.14902

0.14902
0.07540

0.01951 0.07150 0.13768 0.22019 0.21227 0.17866 0.13025 0.06949
-0.00878
0.00086 0.00173 0.00433 0.00868 0.01755 0.04714 0.20815 0.31172 0.39312 0.42418 0.03587 0.08228 0.14524 0.22285 0.30448 0.41584 0.42251 0.35643
0.00266 0.00532 0.01331 0.02660 0.13039 0.24319 0.31695 0.33366 0.33366 0.29346 0.15936 0.10323 0.19823 0.27508 0.32304 0.33451 0.30980
0.25937 0.25937 0.20161 0.15632
0.13710

$$
\frac{\mathrm{a}}{\lambda}=0.8 \quad \frac{\alpha}{\beta}=0.10
$$

\begin{tabular}{|c|c|c|}
\hline $\begin{array}{l}0.01 \\
0.02 \\
0.05 \\
0.10 \\
0.20 \\
0.50 \\
1.00 \\
1.50 \\
2.00 \\
2.50 \\
3.00 \\
3.50\end{array}$ & $\begin{array}{r}0.00074 \\
0.00149 \\
0.00372 \\
0.00742 \\
0.01477 \\
0.03554 \\
0.06080 \\
0.06463 \\
0.03741 \\
-0.02328 \\
-0.10825 \\
-0.19828\end{array}$ & $\begin{array}{l}0.00229 \\
0.00458 \\
0.01144 \\
0.02285 \\
0.04546 \\
0.10955 \\
0.19200 \\
0.23081 \\
0.22606 \\
0.19297 \\
0.15292 \\
0.12170\end{array}$ \\
\hline $\begin{array}{l}0.125 \pi \\
0.250 \pi \\
0.375 \pi \\
0.500 \pi \\
0.625 \pi \\
0.750 \pi \\
0.875 \pi \\
1.000 \pi \\
1.125 \pi \\
1.250 \pi\end{array}$ & $\begin{array}{r}0.02841 \\
0.05192 \\
0.06520 \\
0.06282 \\
0.04056 \\
-0.00270 \\
-0.06358 \\
-0.13421 \\
-0.20408 \\
-0.26317\end{array}$ & $\begin{array}{l}0.0875 \\
0.1613 \\
0.2112 \\
0.2325 \\
0.2270 \\
0.2041 \\
0.1726 \\
0.1428 \\
0.1200 \\
0.104\end{array}$ \\
\hline
\end{tabular}

$\begin{array}{llll}0.00000 & 0.00001 & 0.00074 & 0.00229 \\ 0.00002 & 0.00004 & 0.00149 & 0.00458 \\ 0.00012 & 0.00028 & 0.00372 & 0.01144 \\ 0.00049 & 0.00111 & 0.00744 & 0.02289 \\ 0.00194 & 0.00441 & 0.01493 & 0.04574 \\ 0.01209 & 0.02692 & 0.03804 & 0.11386 \\ 0.04720 & 0.09903 & 0.08099 & 0.22406 \\ 0.10032 & 0.19404 & 0.13290 & 0.32655 \\ 0.15988 & 0.28554 & 0.19612 & 0.41678 \\ 0.20826 & 0.35454 & 0.27073 & 0.48992 \\ 0.22770 & 0.39706 & 0.35424 & 0.54152 \\ 0.20906 & 0.42359 & 0.44174 & 0.56839 \\ & & & \\ 0.00747 & 0.01678 & 0.02962 & 0.08961 \\ 0.02950 & 0.06376 & 0.06166 & 0.17746 \\ 0.06450 & 0.13165 & 0.09829 & 0.26167 \\ 0.10872 & 0.20778 & 0.14114 & 0.34018 \\ 0.15568 & 0.27947 & 0.19111 & 0.41072 \\ 0.19662 & 0.33754 & 0.24820 & 0.47091 \\ 0.22242 & 0.37863 & 0.31144 & 0.51854 \\ 0.22634 & 0.40550 & 0.37888 & 0.55173 \\ 0.20643 & 0.42524 & 0.44773 & 0.56928 \\ 0.16640 & 0.44584 & 0.51465 & 0.57089\end{array}$

$\begin{array}{lr}0.00074 & 0.00229 \\ 0.00149 & 0.00458 \\ 0.00372 & 0.01144 \\ 0.00745 & 0.02284 \\ 0.01493 & 0.04535 \\ 0.03791 & 0.10782 \\ 0.07596 & 0.17937 \\ 0.10160 & 0.19639 \\ 0.10262 & 0.16890 \\ 0.08884 & 0.12012 \\ 0.08524 & 0.05863 \\ 0.10116 & -0.02326 \\ 0.02960 & 0.08665 \\ 0.06015 & 0.15489 \\ 0.08743 & 0.19175 \\ 0.10331 & 0.19467 \\ 0.10331 & 0.17188 \\ 0.09283 & 0.13537 \\ 0.08451 & 0.09145 \\ 0.08812 & 0.03783 \\ 0.10264 & -0.02970 \\ 0.11794 & -0.10780\end{array}$

$\begin{array}{lr}0.00000 & 0.00001 \\ 0.00002 & 0.00004 \\ 0.00012 & 0.00028 \\ 0.00049 & 0.00111 \\ 0.00196 & 0.00440 \\ 0.01264 & 0.02641 \\ 0.05397 & 0.09102 \\ 0.12173 & 0.15678 \\ 0.19186 & 0.18786 \\ 0.23144 & 0.17530 \\ 0.22887 & 0.13333 \\ 0.19871 & 0.07140 \\ & \\ 0.00769 & 0.01659 \\ 0.03245 & 0.06067 \\ 0.07583 & 0.11651 \\ 0.13232 & 0.16386 \\ 0.18744 & 0.18715 \\ 0.22448 & 0.18271 \\ 0.23490 & 0.15709 \\ 0.22224 & 0.11786 \\ 0.19609 & 0.06632 \\ 0.16263 & -0.00085\end{array}$

0.00074 0.00149 0.00372 0.00747 0.01509 0.04050 0.09806 0.17712 0.26330 0.32983 0.35475 0.03083 0.07057 0.12410 0.25733

0.31428

0.3428

0.34812

0.35344

$0.16263-0.00085$

0.00229 0.00458 0.01144
0.02287 0.04563 0.11203 0.20839 0.27076 0.2847 .4 0.25198 0.19483 0.08873 0.17009 0.23546
0.27587 0.27587 0.28544 0.26522
0.22457 0.024878 0.17878 0.12880 


$$
\frac{a}{\lambda}=0.8 \quad \frac{\alpha}{\beta}=0.20
$$

$E(h, 0)$

$\begin{array}{ll}0.00000 & 0.00001 \\ 0.00001 & 0.00003 \\ 0.00009 & 0.00016 \\ 0.00036 & 0.00055 \\ 0.00145 & 0.00258 \\ 0.00892 & 0.01575 \\ 0.03373 & 0.05814 \\ 0.06840 & 0.11495 \\ 0.10333 & 0.17241 \\ 0.12757 & 0.22122 \\ 0.13269 & 0.25949 \\ 0.11628 & 0.29120 \\ 0 & \\ 0.00554 & 0.00982 \\ 0.02143 & 0.03736 \\ 0.04538 & 0.07747 \\ 0.07358 & 0.12334 \\ 0.10102 & 0.16844 \\ 0.12228 & 0.20832 \\ 0.13283 & 0.24143 \\ 0.13015 & 0.26889 \\ 0.11444 & 0.29327 \\ 0.08830 & 0.31710\end{array}$

$\begin{array}{ll}0.00045 & 0.00138 \\ 0.00090 & 0.00277 \\ 0.00225 & 0.00692 \\ 0.00450 & 0.01384 \\ 0.00903 & 0.02767 \\ 0.02299 & 0.06882 \\ 0.04882 & 0.13510 \\ 0.07973 & 0.19615 \\ 0.11685 & 0.24915 \\ 0.15985 & 0.29133 \\ 0.20692 & 0.32045 \\ 0.25501 & 0.33524 \\ 0.01791 & 0.05418 \\ 0.03722 & 0.10713 \\ 0.05916 & 0.15759 \\ 0.08460 . & 0.20421 \\ 0.11393 & 0.24561 \\ 0.14696 & 0.28045 \\ 0.18294 & 0.30756 \\ 0.22059 & 0.32611 \\ 0.25825 & 0.33573 \\ 0.29404 & 0.33660\end{array}$

$C(h, h)$

$S(h, h)$

$\begin{array}{lr}0.00045 & 0.00133 \\ 0.00090 & 0.00277 \\ 0.00225 & 0.00692 \\ 0.00450 & 0.01381 \\ 0.00899 & 0.002744 \\ 0.02234 & 0.00529 \\ 0.04181 & 0.10950 \\ 0.05089 & 0.12310 \\ 0.04563 & 0.11112 \\ 0.03417 & 0.08264 \\ 0.02956 & 0.03991 \\ 0.03661 & -0.01767 \\ & \\ 0.01761 & 0.05245 \\ 0.03422 & 0.09409 \\ 0.04665 & 0.11785 \\ 0.05097 & 0.12272 \\ 0.04639 & 0.11263 \\ 0.03727 & 0.09224 \\ 0.03043 & 0.06327 \\ 0.03046 & 0.02500 \\ 0.03743 & -0.02204 \\ 0.04848 & -0.07307\end{array}$

$E(h, h)$

$\begin{array}{llll}0.00000 & 0.00001 & 0.00045 & 0.00138 \\ 0.00001 & 0.00003 & 0.00090 & 0.00277 \\ 0.00009 & 0.00016 & 0.00225 & 0.00692 \\ 0.00036 & 0.00065 & 0.00452 & 0.01383 \\ 0.00146 & 0.00257 & 0.00913 & 0.02759 \\ 0.00921 & 0.01537 & 0.02441 & 0.06755 \\ 0.03704 & 0.05250 & 0.05842 & 0.12457 \\ 0.07797 & 0.09034 & 0.10346 & 0.16022 \\ 0.11536 & 0.11090 & 0.15017 & 0.16793 \\ 0.13150 & 0.11009 & 0.18414 & 0.15136 \\ 0.12142 & 0.09212 & 0.19611 & 0.12425 \\ 0.09195 & 0.05805 & 0.18828 & 0.10265 \\ 0.00566 & 0.00967 & 0.01861 . & 0.05357 \\ 0.02291 & 0.03513 & 0.04230 & 0.10211 \\ 0.05077 & 0.06707 & 0.07348 & 0.14023 \\ 0.08396 & 0.09456 & 0.11029 & 0.16306 \\ 0.11319 & 0.11016 & 0.14702 & 0.16830 \\ 0.12963 & 0.11228 & 0.17641 & 0.15794 \\ 0.12949 & 0.10308 & 0.19300 & 0.13811 \\ 0.11463 & 0.08420 & 0.19552 & 0.11700 \\ 0.08945 & 0.05506 & 0.18728 & 0.10166 \\ 0.05786 & 0.01471 & 0.17441 & 0.09563\end{array}$

$\frac{a}{\lambda}=0.8 \quad \frac{\alpha}{\beta}=0.40$

$\begin{array}{ll}0.00000 & 0.00000 \\ 0.00001 & 0.00001 \\ 0.00005 & 0.00006 \\ 0.00018 & 0.00022 \\ 0.00073 & 0.00088 \\ 0.00446 & 0.00540 \\ 0.01627 & 0.02030 \\ 0.03133 & 0.04158 \\ 0.04448 & 0.06584 \\ 0.05128 & 0.09070 \\ 0.04939 & 0.11513 \\ 0.03882 & 0.13876 \\ 0.00278 & 0.00335 \\ 0.01052 & 0.01291 \\ 0.02152 & 0.02734 \\ 0.03343 & 0.04491 \\ 0.04369 & 0.06403 \\ 0.05010 & 0.08357 \\ 0.05148 & 0.10294 \\ 0.04723 & 0.12191 \\ 0.03781 & 0.14035 \\ 0.02412 & 0.15802\end{array}$

$\begin{array}{ll}0.00016 & 0.00051 \\ 0.00033 & 0.00101 \\ 0.00082 & 0.00253 \\ 0.00165 & 0.00507 \\ 0.00330 & 0.01012 \\ 0.00840 & 0.02514 \\ 0.01774 & 0.04912 \\ 0.02870 & 0.07078 \\ 0.04149 & 0.08907 \\ 0.05578 & 0.103 .10 \\ 0.07074 & 0.11237 \\ 0.08526 & 0.11686 \\ 0.00655 & 0.01980 \\ 0.01356 & 0.03905 \\ 0.02144 & 0.05716 \\ 0.03041 & 0.07360 \\ 0.04050 & 0.08787 \\ 0.05156 & 0.09953 \\ 0.06321 & 0.10832 \\ 0.07495 & 0.11411 \\ 0.08621 & 0.11700 \\ 0.09645 & 0.11726\end{array}$

$\begin{array}{rr}0.00016 & 0.00051 \\ 0.00033 & 0.00101 \\ 0.00082 & 0.00253 \\ 0.00164 & 0.00506 \\ 0.00326 & 0.01005 \\ 0.00776 & 0.02400 \\ 0.01248 & 0.04109 \\ 0.011139 & 0.04842 \\ 0.00464 & 0.04642 \\ -0.00399 & 0.03510 \\ -0.00977 & 0.01347 \\ -0.00893 & -0.01728 \\ 0.00624 & 0.01924 \\ 0.01104 & 0.03489 \\ 0.01284 & 0.04483 \\ 0.01073 & 0.04859 \\ 0.00526 & 0.04637 \\ -0.00160 & 0.03937 \\ -0.00748 & 0.025 .54 \\ -0.01034 & 0.00554 \\ -0.00855 & -0.01951 \\ -0.00070 & -0.04657\end{array}$

0.00000 0.00001 0.00005 0.00018
0.00073 0.00073 0.00451 0.01685 0.03233 0.04346 0.04398 0.03179 0.00854

0.00280 0.01080 0.02236 0.03435 0.04295 0.04512 0.03947 0.02618 $-0.01653$
0.00000 0.00001 0.00006 0.00022
0.00087 0.00521 0.01770 0.03110 0.04106 0.04635 0.04576 0.03641

0.00328 0.01185 0.02267 i. 04048 0.04535 0.04694 0.04415 0.03536 0.01916

$\begin{array}{ll}0.00016 & 0.00051 \\ 0.00033 & 0.00101 \\ 0.00082 & 0.00253 \\ 0.00165 & .0 .00506 \\ 0.00334 & 0.01008 \\ 0.00887 & 0.02456 \\ 0.02075 & 0.04453 \\ 0.03537 & 0.05619 \\ 0.04910 & 0.05853 \\ 0.05797 & 0.05428 \\ 0.06073 & 0.04818 \\ 0.05925 & 0.04397 \\ 0.00678 & 0.01952 \\ 0.01520 & 0.03680 \\ 0.02578 & 0.04977 \\ 0.03747 & 0.05706 \\ 0.04822 & 0.05863 \\ 0.05606 & 0.05591 \\ 0.06005 & 0.05119 \\ 0.06061 & 0.04669 \\ 0.05908 & 0.04379 \\ 0.05695 & 0.04278\end{array}$


Bh

$\mathrm{C}(\mathrm{h}, 0)$

0.01
0.02
0.05
0.10
0.20
0.50
1.00
1.50
2.00
2.50
3.00
3.50

3.50

$\begin{array}{ll}0.125 \pi & 0.00127 \\ 0.250 \pi & 0.00163\end{array}$

$0.375 \pi \quad 0.00038$

$0.500 \pi-0.00285$

$0.625 \pi \quad-0.00805$

$0.750 \pi \quad-0.01492$

$0.875 \pi \quad-0.02300$

$1.000 \pi \quad-0.03176$

$1.125 \pi$

$-0.03176$

$-0.04951$
$S(h, 0)$

$\begin{array}{ll}0.00000 & 0.00000 \\ 0.00000 & 0.00000 \\ 0.00001 & 0.00001 \\ 0.00005 & 0.00004 \\ 0.00023 & 0.00017 \\ 0.00139 & 0.00108 \\ 0.00501 & 0.00434 \\ 0.00946 & 0.00974 \\ 0.01310 & 0.01720 \\ 0.01453 & 0.02644 \\ 0.01296 & 0.03698 \\ 0.00813 & 0.04812 \\ & \\ 0.00087 & 0.00067 \\ 0.00326 & 0.00267 \\ 0.00659 & 0.00602 \\ 0.01006 & 0.01068 \\ 0.01289 & 0.01659 \\ 0.01440 & 0.02363 \\ 0.01415 & 0.03156 \\ 0.01192 & 0.04010 \\ 0.00768 & 0.04889 \\ 0.00151 & 0.05757\end{array}$

$\frac{a}{\lambda}=0.8 \quad \frac{\alpha}{\beta}=0.70$

$E(h, 0)$

$\begin{array}{ll}0.00004 & 0.00011 \\ 0.00007 & 0.00022 \\ 0.00018 & 0.00056 \\ 0.00036 & 0.00112 \\ 0.00073 & 0.00224 \\ 0.00185 & 0.00555 \\ 0.00389 & 0.01077 \\ 0.00620 & 0.01535 \\ 0.00879 & 0.01905 \\ 0.01153 & 0.02175 \\ 0.01421 & 0.02341 \\ 0.01662 & 0.02416 \\ 0.00145 & 0.00438 \\ 0.00298 & 0.00859 \\ 0.00468 & 0.01249 \\ 0.00655 & 0.01593 \\ 0.00859 & 0.01882 \\ 0.01073 & 0.02108 \\ 0.01288 & 0.02270 \\ 0.01493 & 0.02371 \\ 0.01677 & 0.02419 \\ 0.01834 & 0.02423\end{array}$

$\mathrm{C}(\mathrm{h}, \mathrm{h})$

$S(h, h)$

$\begin{array}{rr}0.00004 & 0.00011 \\ 0.00007 & 0.00022 \\ 0.00018 & 0.00056 \\ 0.00036 & 0.00112 \\ 0.00071 & 0.00223 \\ 0.00159 & 0.00538 \\ 0.00191 & 0.00960 \\ 0.09022 & 0.01207 \\ -0.00342 & 0.01211 \\ -0.00821 & 0.00838 \\ -0.01252 & -0.00058 \\ -0.01337 & -0.01525 \\ 0.00132 & 0.00429 \\ 0.00200 & 0.00798 \\ 0.00155 & 0.01070 \\ -0.00019 & 0.01224 \\ -0.00310 & 0.01221 \\ -0.00679 & 0.00993 \\ -0.01057 & 0.00464 \\ -0.01328 & -0.00418 \\ -0.01320 & -0.01643\end{array}$

C. 00000 0.00000 0.00006 0.00023 0.00138 0.00489 0.00877 0.01079 0.00884 0.00130

$-0.01238$

0.00087 0.00322 0.00634 0.00922 0.01075 0.00991 0.00585 $-0.00197$

$-0.01352$ -0.01352
-0.02776
0.00000 0.00000 0.0000 0.00017 0.00103 0.00359 0.00359 0.00691
0.01074. 0.01485 0.01485 0.01745

0.00065 0.00236 0.00471 0.00742 0.01368 0.01669 0.01841 0.01717 0.01081
$E(h, h)$

$0.00004 \quad 0.00011$ $0.00007 \quad 0.00022$ 0.000180 .00056 $0.00074 \quad 0.00223$ $0.00194 \quad 0.00538$ $0.00440 \quad 0.00953$ $0.00710 \quad 0.01171$ $0.00930 \quad 0.01210$ $0.01 .048 \quad 0.01155$ $\begin{array}{ll}0.01 .048 & 0.01155 \\ 0.01079 & 0.01089\end{array}$ $0.01067 \quad 0.01053$

$0.00149 \quad 0.00430$ $0.00328,0.00797$ $0.00537 \quad 0.01054$ $0.00746 \quad 0.01186$ $\begin{array}{ll}0.00917 & 0.01211\end{array}$ $0.01025 \quad 0.01175$ 0.01072 . 0.01120 0.010780 .01076 $\begin{array}{ll}0.01065 & 0.01052 \\ 0.01051 & 0.01045\end{array}$

$\begin{array}{lrr}0.01 & 0.00001 & 0.00002 \\ 0.02 & 0.00002 & 0.00005 \\ 0.05 & 0.00004 & 0.00012 \\ 0.10 & 0.00008 & 0.00025 \\ 0.20 & 0.00016 & 0.00050 \\ 0.50 & 0.00031 & 0.00126 \\ 1.00 & 0.00007 & 0.00258 \\ 1.50 & -0.00112 & 0.00389 \\ 2.00 & -0.00346 & 0.00492 \\ 2.50 & -0.00692 & 0.00527 \\ 3.00 & -0.01129 & 0.00452 \\ 3.50 & -0.01626 & 0.00238 \\ & & \\ 0.125 \pi & 0.00027 & 0.00098 \\ 0.250 \pi & 0.00027 & 0.00201 \\ 0.375 \pi & -0.00023 & 0.00306 \\ 0.500 \pi & -0.00138 & 0.00406 \\ 0.625 \pi & -0.00325 & 0.00486 \\ 0.750 \pi & -0.00582 & 0.00526 \\ 0.875 \pi & -0.00900 & 0.00506 \\ 1.000 \pi & -0.01266 & 0.00407 \\ 1.125 \pi & -0.01662 & 0.00217 \\ 1.250 \pi & -0.02070 & -0.00070\end{array}$

0.00000

0.00000

0.00002

0.00007

0.00040

0.00146

0.00280

0.00394

0.00436

0.00365

0.00025

0.00094

0.00193

0.00299

0.00388

0.00434

0.00417

0.00320

0.00130
-0.00157
0.00000 0.00000 0.00000 0.00001 0.0003 0.00022 0.00097 0.00247 0.00492 0.00839 0.01275
0.01770

0.00013 0.00057 0.00140 0.00275 0.00471 0.00730 0.01047 0.01410 0.01805
0.02214

$$
\frac{a}{\lambda}=0.8 \quad \frac{\alpha}{\beta}=1.00
$$

$\begin{array}{ll}0.00001 & 0.00002 \\ 0.00002 & 0.00005 \\ 0.00004 & 0.00012 \\ 0.00008 & 0.00025 \\ 0.00016 & 0.00050 \\ 0.00041 & 0.00123 \\ 0.00085 & 0.00236 \\ 0.00134 & 0.00333 \\ 0.00186 & 0.00408 \\ 0.00239 & 0.00460 \\ 0.00287 & 0.00490 \\ 0.00327 & 0.00502 \\ 0.00032 & 0.00097 \\ 0.00066 & 0.00189 \\ 0.00102 & 0.00273 \\ 0.00141 & 0.00345 \\ 0.00183 & 0.00403 \\ 0.00224 & 0.00447 \\ 0.00264 & 0.00477 \\ 0.00299 & 0.00495 \\ 0.00329 & 0.00503 \\ 0.00353 & 0.00503\end{array}$

$\begin{array}{rr}0.00001 & 0.00002 \\ 0.00002 & 0.00005 \\ 0.00004 & 0.00012 \\ 0.00008 & 0.00025 \\ 0.00016 & 0.00049 \\ 0.00033 & 0.00121 \\ 0.00025 & 0.00229 \\ -0.00050 & 0.00306 \\ -0.00205 & 0.00315 \\ -0.00438 & 0.00172 \\ -0.00689 & -0.00242 \\ -0.00769 & -0.01041 \\ 0.00028 & 0.00096 \\ 0.00035 & 0.00185 \\ 0.00007 & 0.00261 \\ -0.00067 & 0.00313 \\ -0.00191 & 0.00318 \\ -0.00365 & 0.00235 \\ -0.00569 & 0.00008 \\ -0.00742 & -0.00425 \\ -0.00760 & -0.01112 \\ -0.00420 & -0.02048\end{array}$

$\begin{array}{ll}0.00000 & 0.00000 \\ 0.00000 & 0.00000 \\ 0.00000 & 0.00000 \\ 0.00002 & 0.00001 \\ 0.00007 & 0.00003 \\ 0.00039 & 0.00020 \\ 0.00136 & 0.00077 \\ 0.00241 & 0.00172 \\ 0.00282 & 0.00324 \\ 0.00168 & 0.00542 \\ -0.00223 & 0.00772 \\ -0.01009 & 0.00828 \\ & \\ 0.00025 & 0.00013 \\ 0.00090 & 0.00048 \\ 0.00176 & 0.00105 \\ 0.00252 & 0.00189 \\ 0.00283 & 0.00310 \\ 0.00224 & 0.00474 \\ 0.00016 & 0.00663 \\ -0.00402 & 0.00818 \\ -0.01079 & 0.00817 \\ -0.02011 & 0.00459\end{array}$
0.00001 $0.00008 \quad 0.00025$ $0.00016 \quad 0.00049$ $0.00043 \quad 0.00118$ $0.00093 \quad 0.00204$ 0.001430 .00245 0.001790 .00251 $0.00194 \quad 0.00244$ $\begin{array}{ll}0.00198 & 0.00237 \\ 0.00197 & 0.00234\end{array}$ $0.00033 \cdot 0.00095$ $0.00071 \quad 0.00172$ $0.00112 \quad 0.00224$ $0.00150 \quad 0.00247$ $0.00177 \quad 0.00252$ 0.001920 .00247 $0.00197 \quad 0.00240$ $0.00198 \quad 0.00236$ $\begin{array}{ll}0.00197 & 0.00234 \\ 0.00196 & 0.00233\end{array}$ 


$$
\frac{a}{\lambda}=1.0 \quad \frac{\alpha}{\beta}=0.00
$$

$\beta h$

$\begin{array}{lrr}0.01 & 0.00318 & -0.00000 \\ 0.02 & 0.00637 & -0.00060 \\ 0.05 & 0.01591 & -0.00000 \\ 0.10 & 0.03173 & -0.00001 \\ 0.20 & 0.06323 & -0.00007 \\ 0.50 & 0.15245 & -0.00097 \\ 1.00 & 0.26676 & -0.00599 \\ 1.50 & 0.31523 & -0.01146 \\ 2.00 & 0.28931 & -0.00424 \\ 2.50 & 0.20376 & 0.03198 \\ 3.00 & 0.09244 & 0.10486 \\ 3.50 & -0.00522 & 0.20347 \\ 0.125 \pi & 0.12173 & -0.00049 \\ 0.250 \pi & 0.22450 & -0.00334 \\ 0.375 \pi & 0.29250 & -0.00839 \\ 0.500 \pi & 0.31599 & -0.01160 \\ 0.625 \pi & 0.29354 & -0.00557 \\ 0.756 \pi & 0.23275 & 0.01785 \\ 0.875 \pi & 0.14903 & 0.06386 \\ 1.000 \pi & 0.06185 & 0.13118 \\ 1.125 \pi & -0.01060 & 0.21051 \\ 1.250 \pi & -0.05703 & 0.28608\end{array}$

$S(1,0)$

$\begin{array}{ll}0.00002 & -0.00000 \\ 0.00006 & -0.00000 \\ 0.00040 & -0.00000 \\ 0.00159 & -0.00000 \\ 0.00634 & -0.00001 \\ 0.03890 & -0.00038 \\ 0.14529 & -0.00558 \\ 0.29061 & -0.02398 \\ 0.43553 & -0.05935 \\ 0.54237 & -0.10277 \\ 0.53921 & -0.13187 \\ 0.57968 & -0.12033 \\ 0.02421 & -0.00015 \\ 0.09284 & -0.00223 \\ 0.19450 & -0.01023 \\ 0.31211 & -0.02800 \\ 0.42584 & -0.05630 \\ 0.51725 & -0.09057 \\ 0.57361 & -0.12077 \\ 0.59140 & -0.13372 \\ 0.57755 & -0.11756 \\ 0.54731 & -0.06672\end{array}$

$E(h, 0)$

$0.00318-0.00000$ $0.00637-0.00000$ $0.01592-0.00000$ $0.03183-0.00001$ $0.06365-0.00007$ $0.15898-0.00105$ $0.31678-0.00834$ $0.47157-0.02773$ $0.62034-0.06420$ $0.75864-0.12133$ $0.88068-0.20063$ $0.97982-0.30095$

$0.12492-0.00051$ $0.24929-0.00406$ $0.37239-0.01358$ $0.49309-0.03175$ $0.60977-0.06087$ $0.72026-0.10265$ $0.82183-0.15804$ $0.91138-0.22703$ $\begin{array}{ll}0.98561 & -0.30851 \\ 1.04138 & -0.40018\end{array}$
$\mathrm{C}(\mathrm{h}, \mathrm{h})$

$0.00318-0.00000$ $0.00637-0.00000$ $0.01591-0.00000$ $0.03177-0.00003$ $\begin{array}{lll}0.06319 & -0.00027\end{array}$ $0.15188-0.00397$ $0.26140-0.02312$ $0.29714-0.06206$ $0.25915-0.08543$ $0.18623-0.08503$ $0.12240-0.08270$ $0.12147-0.00197$ $0.22207-0.01400$ $0.28348-0.03842$ $0.29570-0.06686$ $0.26374-0.08556$ $0.20774-0.08736$ $0.15187-0.08115$ $0.10764-0.08809$ $0.06634-0.12436$ $\begin{array}{ll}0.06634 & -0.12436 \\ 0.00966 & -0.18429\end{array}$
$S(h, h)$

$0.00002-0.00000$ $0.00040-0.00000$ $0.00634-0.00003$ $0.03875-0.00114$ $0.14191-0.01627$ $0.26862-0.06670$ $0.36011-0.15301$ $0.37482-0.24140$ $\begin{array}{ll}0.37482 & -0.24140 \\ 0.31976 & -0.29378\end{array}$ $0.23138-0.31229$

$0.02415-0.00044$ $0.09172-0.00658$ $0.18726-0.02941$ $0.28507-0.07719$ $0.35578-0.14608$ $0.37901-0.21846$ $0.35391-0.27309$ $0.29674-0.30112$ $.22468-0.31 .323$ $0.13963-0.32863$ $0.00006-0.00000$
$E(h, h)$

$\begin{array}{ll}0.00318 . & -0.00000 \\ 0.00637 & -0.00000 \\ 0.01591 & -0.00000 \\ 0.03183 & -0.00003 \\ 0.06362 & -0.00027 \\ 0.15839 & -0.00417 \\ 0.31017 & -0.03210 \\ 0.44034 & -0.10032 \\ 0.52474 & -0.20904 \\ 0.54074 & -0.33562 \\ 0.48488 & -0.43914 \\ 0.38461 & -0.48000 \\ 0.12465 & -0.00203 \\ 0.24655 & -0.01588 \\ 0.36013 & -0.05132 \\ 0.45569 & -0.11352 \\ 0.52069 & -0.20009 \\ 0.54388 & -0.29958 \\ 0.52095 & -0.39284 \\ 0.45905 & -0.45832 \\ 0.37734 & -0.47995 \\ 0.30223 & -0.45442\end{array}$

\begin{tabular}{|c|c|c|c|c|}
\hline $\begin{array}{l}0.01 \\
0.02 \\
0.05 \\
0.10 \\
0.20 \\
0.50 \\
1.00 \\
1.50 \\
2.00 \\
2.50 \\
3.00 \\
3.50\end{array}$ & $\begin{array}{r}0.00308 \\
0.00617 \\
0.01542 \\
0.03079 \\
0.06127 \\
0.14773 \\
0.25851 \\
0.30557 \\
0.28079 \\
0.19851 \\
0.09131 \\
-0.00316\end{array}$ & $\begin{array}{r}-0.00000 \\
-0.00000 \\
-0.00000 \\
-0.00001 \\
-0.00006 \\
-0.00088 \\
-0.00534 \\
-0.00976 \\
-0.00155 \\
0.03463 \\
0.10568 \\
0.20071\end{array}$ & $\begin{array}{l}0.00002 \\
0.00006 \\
0.00039 \\
0.00154 \\
0.00615 \\
0.03770 \\
0.14075 \\
0.28145 \\
0.42180 \\
0.52554 \\
0.57179 \\
0.56414\end{array}$ & $\begin{array}{l}-0.00000 \\
-0.00000 \\
-0.00000 \\
-0.00001 \\
-0.00004 \\
-0.00055 \\
-0.00599 \\
-0.02409 \\
-0.05809 \\
-0.09915 \\
-0.12580 \\
-0.11313\end{array}$ \\
\hline $\begin{array}{l}0.125 \pi \\
0.250 \pi \\
0.375 \pi \\
0.500 \pi \\
0.625 \pi \\
0.750 \pi \\
0.875 \pi \\
1.000 \pi \\
1.125 \pi \\
1.250 \pi\end{array}$ & $\begin{array}{r}0.11797 \\
0.21755 \\
0.28346 \\
0.30633 \\
0.28484 \\
0.22640 \\
0.14583 \\
0.06180 \\
-0.00840 \\
-0.05409\end{array}$ & $\begin{array}{r}-0.00044 \\
-0.00300 \\
-0.00741 \\
-0.00973 \\
-0.00293 \\
0.02068 \\
0.06584 \\
0.13112 \\
0.20746 \\
0.27979\end{array}$ & $\begin{array}{l}0.02346 \\
0.08995 \\
0.18841 \\
0.30227 \\
0.41241 \\
0.50109 \\
0.55614 \\
0.57429 \\
0.56221 \\
0.53440\end{array}$ & $\begin{array}{l}-0.00026 \\
-0.00256 \\
-0.01063 \\
-0.02799 \\
-0.05518 \\
-0.08769 \\
-0.11585 \\
-0.12713 \\
-0.11036 \\
-0.06051\end{array}$ \\
\hline
\end{tabular}

$$
\frac{a}{\lambda}=1.0 \quad \frac{\alpha}{\beta}=0.005
$$

$\begin{array}{ll}0.00308 & -0.00000 \\ 0.00617 & -0.00000 \\ 0.01542 & -0.00000 \\ 0.03085 & -0.00001 \\ 0.06168 & -0.00007 \\ 0.15406 & -0.00102 \\ 0.30695 & -0.00808 \\ 0.45685 & -0.02686 \\ 0.60085 & -0.06216 \\ 0.73461 & -0.11741 \\ 0.85254 & -0.19403 \\ 0.94824 & -0.29087 \\ 0.12105 & -0.00049 \\ 0.24156 & -0.00394 \\ 0.36081 & -0.01316 \\ 0.47769 & -0.03075 \\ 0.59062 & -0.05893 \\ 0.69750 & -0.09935 \\ 0.79568 & -0.15289 \\ 0.88218 & -0.21953 \\ 0.95382 & -0.29816 \\ 1.00759 & -0.38654\end{array}$

$0.00002-0.00000$ $0.00006-0.00000$ $\begin{array}{ll}0.000006 & -0.000000 \\ 0.00039 & -0.00000\end{array}$ $0.00154-0.00001$ $0.00154-0.00006$ $\begin{array}{ll}0.00614 & -0.00006 \\ 0.03754 & -0.00129\end{array}$ $\begin{array}{ll}0.03754 & -0.00129 \\ 0.13738 & -0.01632\end{array}$ $0.13738-0.0052$ $\begin{array}{ll}0.25984 & -0.06529 \\ 0.34824 & -0.14828\end{array}$ $\begin{array}{ll}0.34824 & -0.14828 \\ 0.36282 & -0.23271\end{array}$ $0.36282-0.23271$

$\begin{array}{lll}0.02340 & -0.00054\end{array}$ $0.08883-0.00677$ $0.27572-0.0754$ $0.34404-0.14164$ $0.36671-0.2108$ $0.34294-0.26282$ $0.21831-0.30072$ $0.13529-0.31495$ $\begin{array}{ll}0.31024 & -0.28244 \\ 0.22483 & -0.29985\end{array}$ $0.18122-0.02916$ $0.28810-0.28937$
$0.00308-0.00000$ $0.00617-0.00000$ $0.01542-0.00000$ $0.03084-0.00003$ $0.06165-0.00026$ $0.15347-0.00404$ $0.30043-0.03108$ $0.42627-0.09702$ $0.50770-0.20190$ $0.52311-0.32367$ $0.46955-0.42297$ $0.12078-0.00197$ $0.23884-0.01538$ $0.34875-0.04967$ $0.44109-0.10976$ $0.50379-0.19327$ $0.44483-0.44133$ $0.36678-0.46200$ $0.52613-0.28904$ $0.50412-0.37800$ 
0.01

0.02

0.05

0.10

0.20

1.00

1.50

2.00

2.50

3.50

$0.125 \pi$

$0.250 \pi$

$0.375 \pi$

$0.625 \pi$

$0.750 \pi$

$0.875 \pi$

$0.875 \pi$

$1.125 \pi$

$1.250 \pi$
$\mathrm{C}(\mathrm{h}, 0)$

$0.00299-0.00000$ $0.00598-0.00000$ $0.01494-0.00000$ $0.02984-0.00001$ $\begin{array}{lll}0.05938 & -0.00005\end{array}$ $0.14315-0.00079$ $0.25051-0.00473$ $0.29620-0.00815$ $0.19338 \quad 0.00093$ $\begin{array}{ll}0.19338 & 0.03707 \\ 0.09013 & 0.10634\end{array}$ $-0.00128 \quad 0.19796$

$0.11432-0.00040$ $0.21081-0.00268$ $0.27470-0.00648$ 0.00797
0.0097 $0.27640-0.00045$ 0.220210 .02331 $0.14267 \quad 0.06765$ $-0.00638$ $\begin{array}{ll}-0.000638 & 0.20444 \\ -0.05135 & 0.27369\end{array}$
$S(h, 0)$

$0.00001-0.00050$ $0.00006-0.00060$ $0.00037-0.00000$ $0.00149-0.00002$ $\begin{array}{lll}0.00596 & -0.00007\end{array}$ $\begin{array}{ll}0.03653 & -0.00071\end{array}$ $\begin{array}{lll}0.13635 & -0.00637\end{array}$ $0.40852-0.05685$ $0.50926-0.05685$ $0.5026-0.09568$ $0.54902-0.10629$

$0.02273-0.00036$ $\begin{array}{ll}0.08715 & -0.00288 \\ 0.18250 & -0.01099\end{array}$ $0.29275-0.02795$ $0.39942-0.05407$ $0.48546-0.08491$ $0.53924-0.11114$ $0.55771-0.12084$ $0.54728-0.10354$ $0.52176-0.05465$

$$
\frac{a}{\lambda}=1.5 \quad \frac{\alpha}{\beta}=0.01
$$

$E(h, 0)$

$0.00299-[.00000$ $0.00598-C .00000$ $0.01495-0.00000$ $0.02989-0.00001$ $0.05977-0.00006$ $0.14929-0.00099$ $0.29741-0.00783$ $0.44260-0.02601$ $0.58197-0.06018$ $0.71134-0.11362$ $0.82529-0.18760$ $0.91767-0.28112$

$\begin{array}{lll}0.11730 & -0.00048\end{array}$ $0.23407-0.00381$ $0.34959-0.01274$ $0.46277-0.02978$ $0.57208-0.05706$ $0.67546-0.09615$ $0.77037-0.14791$ $0.85392-0.21227$ $0.92306-0.28816$ $0.97489-0.37337$
$C(h, h)$

$0.00299-9.00000$ $0.00598-0.00000$ $0.01494-0.00000$ $0.02984-0.00003$ $0.05934-0.00024$ $0.14260-0.00360$ $0.24535-0.02361$ $0.27912-0.05571$ $0.24740-0.07686$ $0.17701-0.07507$ $0.06592-0.10948$

$0.11406-0.00179$ $0.20845-0.01269$ $0.26609-0.03466$ $0.27786-0.05994$ $0.24862-0.07613$ $0.19698-0.07722$ $0.14483-0.07180$ $0.10244-0.07918$ $\begin{array}{ll}0.00205 & -0.11358 \\ 0.00797 & -0.16941\end{array}$
$S(h, h)$

$0.00001-0.00000$ $0.00006-0.00000$ $0.00037-0.00000$ $0.00149-0.00002$ $0.00595-0.00009$ $0.03637-0.00142$ $0.13209-0.01636$ $0.25135-0.06390$ $0.33677-0.14371$ $0.35121-0.22433$ $0.30101-0.27152$ $0.21845-0.28786$

$0.02267-0.00064$ $0.08602-0.00694$ $\begin{array}{ll}539 & -0.02889\end{array}$ $0.26669-0.07368$ $0.33271-0.13735$ $0.35483-0.20351$ $0.33232-0.25295$ $0.27971-0.27806$ $0.21211-0.28867$ $0.13109-0.30178$
$E(h, h)$

$0.00299-0.00000$ $0.00598-0.00000$ $0.01495-0.00000$ $0.02989-0.00003$ $0.05974-0.00025$ $0.14871-0.00392$ $0.29099-0.03009$ $0.41265-0.09383$ 0.49121 -0.19499 $0.50605-0.31215$ $0.45470-0.40740$ $0.36311-0.44477$

$0.11704-0.00191$ $0.23138-0.01489$ $-0.04808$ $0.42696-0.10614$ $0.48745-0.18668$ $0.50895-0.27886$ $0.48783-0.36487$ $0.43104-0.42497$ $0.28836-0.42159$

$$
\frac{a}{\lambda}=1.0 \quad \frac{\alpha}{\beta}=0.02
$$

$\begin{array}{rr}0.00281 & -0.00000 \\ 0.00561 & -0.00000 \\ 0.01403 & -0.00000 \\ 0.02802 & -0.00001 \\ 0.05576 & -0.00004 \\ 0.13443 & -0.00063 \\ 0.23525 & -0.00361 \\ 0.27833 & -0.00521 \\ 0.25667 & 0.00556 \\ 0.18346 & 0.04145 \\ 0.08764 & 0.10728 \\ 0.00199 & 0.19249 \\ & \\ 0.10735 & -0.00032 \\ 0.19796 & -0.00210 \\ 0.25800 & -0.00477 \\ 0.27910 & -0.00475 \\ 0.26027 & 0.00406 \\ 0.20830 & 0.02803 \\ 0.13645 & 0.07074 \\ 0.06109 & 0.13032 \\ -0.00286 & 0.19847 \\ -0.04639 & 0.26200\end{array}$

$0.00001-0.00000$ $0.00006-0.00000$ $0.00035-0.00001$ $0.00140-0.00003$ $0.00559-0.00012$ $0.03430-0.00100$ \begin{tabular}{ll}
-0.00705 \\
\hline
\end{tabular} $0.25573-0.02424$ $0.38325-0.05446$ $0.47829-0.08909$ $0.52272-0.10916$ $0.52002-0.09364$

$0.02134-0.00055$ $0.08181-0.00343$ $0.17126-0.01163$ $0.27462-0.02780$ $0.37470-0.05193$ $0.45571-0.07964$ $0.50705-0.10231$ $0.52604-0.10914$ $0.51861-0.09091$ $0.49728-0.04389$
$0.00281-0.00000$ 0.00000 $0.00561-0.00000$ $0.01404-0.00000$ $0.02807-0.00001$ $0.05613-0.00006$ $0.14019-0.00093$ $0.27923-0.00735$ $0.41540-0.02440$ $0.54597-0.05641$ $0.66699-0.10639$ $\begin{array}{ll}0.77339 & -0.17552 \\ 0.85946 & -0.26260\end{array}$

$0.11016-0.00045$ $0.21978-0.00359$ $0.32818-0.01196$ $0.43431-0.02794$ $0.53671-0.05349$ $0.63344-0.09007$ $0.72213-0.13843$ $0.86447-0.26915$ $0.91266-0.34835$ $0.80008-0.19847$
$0.00281-0.00000$ $0.00561-0.00000$ $0.01403-0.00000$ $0.02802-0.00003$ $0.05573-0.00022$ $0.13389-0.00327$ $0.23029-0.02132$ $0.26220-0.04992$ $0.23047-0.06815$ $0.16815-0.06601$ $0.11121-0.06565$ $0.06172-0.09972$

$0.10709-0.00162$ $0.19567-0.01148$ $0.24978-0.03122$ $0.26110-0.05364$ $0.23435-0.06756$ $0.18669-0.06800$ $0.13799-0.06325$ $0.09736-0.07095$ $0.05796-0.10357$ $0.00637-0.15569$

$\begin{array}{ll}0.00001 & -0.00000 \\ 0.00006 & -0.00000 \\ 0.00035 & -0.00001 \\ 0.00140 & -0.00003 \\ 0.00559 & -0.00014 \\ 0.03414 & -0.00166 \\ 0.12465 & -0.01637 \\ 0.23521 & -0.06120 \\ 0.31500 & -0.13501 \\ 0.32916 & -0.20851 \\ 0.28336 & -0.25094 \\ 0.20621 & -0.26521 \\ & \\ 0.02128 & -0.00080 \\ 0.08068 & -0.00724 \\ 0.16428 & -0.02831 \\ 0.24953 & -0.07031 \\ 0.31119 & -0.12916 \\ 0.33225 & -0.18962 \\ 0.31208 & -0.23433 \\ 0.26365 & -0.25673 \\ 0.26021 & -0.26589 \\ 0.12309 & -0.27688\end{array}$

$\begin{array}{ll}0.00281 & -0.00000 \\ 0.00561 & -0.00000 \\ 0.01404 & -0.00000 \\ 0.02807 & -0.00003 \\ 0.05610 & -0.00024 \\ 0.13961 & -0.00368 \\ 0.27299 & -0.02820 \\ 0.38670 & -0.08776 \\ 0.45982 & -0.18189 \\ 0.47359 & -0.29033 \\ 0.42639 & -0.37796 \\ 0.34272 & -0.41215 \\ & \\ 0.10989 & -0.00179 \\ 0.21715 & -0.01397 \\ 0.31672 & -0.04503 \\ 0.40004 & -0.09924 \\ 0.45633 & -0.17417 \\ 0.47627 & -0.25959 \\ 0.45680 & -0.33891 \\ 0.40472 & -0.39406 \\ 0.33670 & -0.41210 \\ 0.27489 & -0.39114\end{array}$




$$
\frac{\mathrm{a}}{\lambda}=1.0 \quad \frac{\alpha}{\beta}=0.03
$$

$S(h, 0)$

$C(h, h)$

0.0

0.02
0.05

0.10

0.20

1.00

1.50

2.00

2.50

3.00
3.50

$0.00264-0.00000$ $0.00527-0.00000$ $0.01318-0.00000$ $0.02632-0.30000$ $0.05236-0.00003$ $0.12624-0.00049$ $0.22092-0.00260$ $0.26154-0.00260$ $0.24176 \quad 0.00958$ 0.173990 .04514 $\begin{array}{ll}0.08499 & 0.10774 \\ 0.00466 & 0.18707\end{array}$

$\begin{array}{llll}0.125 \pi & 0.10081 & -0.00025\end{array}$ $\begin{array}{llll}0.250 \pi & 0.18590 & -0.00157\end{array}$ $\begin{array}{llll}0.375 \pi & 0.24232 & -0.00325\end{array}$ $0.500 \pi \quad 0.26232 \quad-0.00190$ $\begin{array}{llll}0.625 \pi & 0.24508 & 0.0080\end{array}$ $\begin{array}{llll}0.750 \pi & 0.19701 & 0.03207\end{array}$ $0.875 \pi \quad 0.13038 \quad 0.07320$ $\begin{array}{lll}1.000 \pi & 0.06022 & 0.12933\end{array}$ $\begin{array}{rrr}1.125 \pi & 0.00006 & 0.19259 \\ 1.250 \pi & -0.04208 & 0.25094\end{array}$

$\begin{array}{ll}0.00001 & -0.00000 \\ 0.00005 & -0.00000 \\ 0.00033 & -0.00001 \\ 0.00132 & -0.00004 \\ 0.00525 & -0.00016 \\ 0.03220 & -0.00124 \\ 0.120111 & -0.00761 \\ 0.23993 & -0.02422 \\ 0.35960 & -0.05215 \\ 0.44930 & -0.08300 \\ 0.49251 & -0.09929 \\ 0.49258 & -0.08225 \\ 0.02004 & -0.00071 \\ 0.07680 & -0.00391 \\ 0.16071 & -0.01214 \\ 0.25765 & -0.02757 \\ 0.35157 & -0.04985 \\ 0.42788 & -0.07473 \\ 0.47690 & -0.09420 \\ 0.49627 & -0.09851 \\ 0.49146 & -0.07954 \\ 0.47382 & -0.03431\end{array}$

$\begin{array}{ll}0.00264 & -0.00000 \\ 0.00527 & -0.00000 \\ 0.01318 & -0.00000 \\ 0.02636 & -0.00001 \\ 0.05271 & -0.00006 \\ 0.13164 & -0.00087 \\ 0.26215 & -0.00690 \\ 0.38987 & -0.02289 \\ 0.51220 & -0.05287 \\ 0.62541 & -0.09963 \\ 0.72476 & -0.16417 \\ 0.80495 & -0.24530 \\ & \\ 0.10344 & -0.00042 \\ 0.20637 & -0.00336 \\ 0.30808 & -0.01123 \\ 0.40760 & -0.02621 \\ 0.50353 & -0.05014 \\ 0.59405 & -0.08437 \\ 0.67692 & -0.12955 \\ 0.74964 & -0.18557 \\ 0.80961 & -0.25139 \\ 0.85441 & -0.32501\end{array}$

$0.00264-0.00000$ $0.00527-0.00000$ $0.01318-0.00000$ $0.02631-0.00003$ $0.05233-0.00020$ $0.12571-0.00296$ $0.21616-0.01923$ $0.24632-0.04463$ $0.21731-0.06024$ $0.15980-0.05774$ $0.10580-0.05814$ $0.05770-0.09067$

$0.10056-0.00147$ $0.18368-0.01038$ $0.23447-9.02808$ $0.24536-0.04789$ $0.22088-0.05978$ $0.17687-0.05962$ $0.13134-0.05545$ $0.09241-0.06335$ $0.00486-0.14303$ $0.05406-0.08428$
$\mathrm{S}(\mathrm{h}, \mathrm{h})$

$0.00001-0.00000$ $0.00005-0.00000$ $0.00033-0.00001$ $0.00132-0.00004$ $0.00525-0.00018$ $0.03204-0.0018$ $0.11683-0.01631$ $0.22013-0.05860$ $0.29469-0.12685$ $0.30854-0.19384$ $\begin{array}{ll}0.26675 & -0.23190 \\ 0.19462 & -0.24420\end{array}$ $0.01998-0.00095$ $0.07567-0.00747$ $0.15389-0.02770$ $0.23349-0.06708$ $0.29111-0.12748$ $0.31118-0.17672$ $0.29311-0.21710$ $0.24850-0.23699$ $0.18894-0.24476$ $0.11560-0.25379$
$E(h, h)$

$0.00264-0.00000$ $\begin{array}{lll}0.00527 & -0.00000\end{array}$ $0.01318-0.00000$ $0.02636-0.00003$ $0.05268-0.00022$ $0.13108-0.00345$ $0.25610-0.02644$ $0.36238-0.08209$ $0.43045-0.16967$ $0.44322-0.27004$ $\begin{array}{ll}0.39983 & -0.35067 \\ 0.32340 & -0.38194\end{array}$

$0.10318-0.00168$ $0.20380-0.01310$ $0.29702-0.04218$ $0.37482-0.09279$ $0.44570-0.24164$ $0.42776-0.31480$ $0.37998-0.36541$ $\begin{array}{ll}0.31793 & -0.38190 \\ 0.26185 & -0.36289\end{array}$ $0.42720-0.16251$

$\begin{array}{lrr}0.01 & 0.00232 & -0.00000 \\ 0.02 & 0.00465 & -0.00000 \\ 0.05 & 0.01162 & -0.00060 \\ 0.10 & 0.02321 & -0.00000 \\ 0.20 & 0.04618 & -0.00002 \\ 0.50 & 0.11133 & -0.00024 \\ 1.00 & 0.19486 & -0.00091 \\ 1.50 & 0.23098 & 0.00174 \\ 2.00 & 0.21448 & 0.01610 \\ 2.50 & 0.15634 & 0.05074 \\ 3.00 & 0.07940 & 0.10743 \\ 3.50 & 0.00853 & 0.17642 \\ & & \\ 0.125 \pi & 0.08890 & -0.00012 \\ 0.250 \pi & 0.16394 & -0.00068 \\ 0.375 \pi & 0.21378 & -0.00070 \\ 0.500 \pi & 0.23176 & 0.00284 \\ 0.625 \pi & 0.21732 & 0.01444 \\ 0.750 \pi & 0.17613 & 0.03840 \\ 0.875 \pi & 0.11874 & 0.07653 \\ 1.000 \pi & 0.05778 & 0.12644 \\ 1.125 \pi & 0.00437 & 0.18114 \\ 1.250 \pi & -0.03508 & 0.23056\end{array}$

$\begin{array}{ll}0.00001 & -0.00000 \\ 0.00005 & -0.00000 \\ 0.00029 & -0.00001 \\ 0.00116 & -0.00006 \\ 0.00463 & -0.00024 \\ 0.02839 & -0.00164 \\ 0.10583 & -0.00847 \\ 0.21127 & -0.02394 \\ 0.31675 & -0.04781 \\ 0.39675 & -0.07214 \\ 0.43752 & -0.08210 \\ 0.44207 & -0.06276 \\ 0.01767 & -0.00097 \\ 0.06768 & -0.00466 \\ 0.14156 & -0.01287 \\ 0.22686 & -0.02690 \\ 0.30965 & -0.04591 \\ 0.37746 & -0.06588 \\ 0.42216 & -0.07993 \\ 0.44194 & -0.08009 \\ 0.44143 & -0.06013 \\ 0.42991 & -0.01824\end{array}$

$$
\frac{\alpha}{\beta}=0.05
$$

$\begin{array}{ll}0.00001 & -0.00000 \\ 0.00005 & -0.00000 \\ 0.00029 & -0.00001 \\ 0.00116 & -0.00006 \\ 0.00463 & -0.00025 \\ 0.02823 & -0.00219 \\ 0.10265 & -0.01605 \\ 0.19288 & -0.05366 \\ 0.25805 & -0.11205 \\ 0.27124 & -0.16763 \\ 0.23643 & -0.19801 \\ 0.17331 & -0.20669 \\ & \\ 0.01761 & -0.00118 \\ 0.06657 & -0.00777 \\ 0.13507 & -0.02638 \\ 0.20453 & -0.06103 \\ 0.25489 & -0.10752 \\ 0.27310 & -0.15360 \\ 0.25865 & -0.18640 \\ 0.22073 & -0.20184 \\ 0.16823 & -0.20703 \\ 0.10200 & -0.21259\end{array}$

$\begin{array}{ll}0.00232 & -0.00000 \\ 0.00465 & -0.00000 \\ 0.01162 & -0.00000 \\ 0.02324 & -0.00002 \\ 0.04646 & -0.00020 \\ 0.11554 & -0.00304 \\ 0.22540 & -0.02323 \\ 0.31824 & -0.07181 \\ 0.37722 & -0.14764 \\ 0.38821 & -0.23363 \\ 0.35154 & -0.30189 \\ 0.28777 & -0.32804 \\ 0.09097 & -0.00148 \\ 0.17950 & -0.01153 \\ 0.26122 & -0.03701 \\ 0.32906 & -0.08112 \\ 0.37442 & -0.14146 \\ 0.39032 & -0.20940 \\ 0.37509 & -0.27164 \\ 0.33489 & -0.31426 \\ 0.28324 & -0.32801 \\ 0.23709 & -0.31240\end{array}$

$\begin{array}{ll}0.00232 & -0.00000 \\ 0.00465 & -0.00000 \\ 0.01162 & -0.00000 \\ 0.02325 & -0.00001 \\ 0.04649 & -0.00005 \\ 0.11608 & -0.00077 \\ 0.23108 & -0.00608 \\ 0.34343 & -0.02015 \\ 0.45080 & -0.04646 \\ 0.54986 & -0.08737 \\ 0.63648 & -0.14363 \\ 0.70610 & -0.21404 \\ 0.09122 & -0.00037 \\ 0.18194 & -0.00296 \\ 0.27150 & -0.00989 \\ 0.35900 & -0.02306 \\ 0.44320 & -0.04406 \\ 0.52245 & -0.07403 \\ 0.59481 & -0.11348 \\ 0.65811 & -0.16224 \\ 0.71013 & -0.21932 \\ 0.74884 & -0.28293\end{array}$

$0.00232-0.00000$ $0.00465-0.00000$ $0.01162-0.00000$ $0.02321-0.00002$ $0.04615-0.00016$ $0.11082-0.00242$ $0.19047-0.0155$ $\begin{array}{ll}0.19047 & -0.01555 \\ 0.19314 & -0.03544\end{array}$ $0.14362-0.0435$ $\begin{array}{ll}0.09544 & -0.04492 \\ 0.05019 & -0.07452\end{array}$

$0.08866-0.00121$ $0.16186-0.00845$ $0.20664-0.02259$ $0.15856-0.04514$ $0.08294-0.04989$ $0.04679-0.07772$ 


$$
\frac{a}{\lambda}=1.0
$$

$E(h, 0)$

$0.00001-0.00000$ $0.00004-0.00000$ $0.00026-0.00002$ $\begin{array}{ll}0.00026 & -0.00002 \\ 0.00102 & -0.00007 \\ 0.00409 & -0.00029\end{array}$ $0.00409-0.00029$ $0.02503-0.00193$ $0.18611-0.02338$ $0.27920-0.04381$ $0.35066-0.06280$ $0.38900-0.0678$

$0.01558-0.00116$ $\begin{array}{ll}0.01558 & -0.00116\end{array}$ $0.12472-0.01325$ $0.19985-0.02601$ $0.37405-0.0678$ $0.39387-0.06491$ $\begin{array}{ll}0.39660 & -0.04447 \\ 0.38980 & -0.00564\end{array}$
$0.125 \pi$ $0.250 \pi$ $0.375 \pi$ $0.500 \pi$ $0.625 \pi$ $0.750 \pi$

$0.875 \pi$

$1.000 \pi$

$1.250 \pi$ $0.09326-0.00902$ $0.27292-0.04224$ $0.33325-0.05817$

$\begin{array}{lrlll}0.01 & 0.00170 & 0.00000 & 0.00001 & -0.00000 \\ 0.02 & 0.00340 & 0.00000 & 0.00003 & -0.00000 \\ 0.05 & 0.00849 & 0.00000 & 0.00021 & -0.00002 \\ 0.10 & 0.01695 & 0.00000 & 0.00085 & -0.00009 \\ 0.20 & 0.03373 & 0.00001 & 0.00338 & -0.00034 \\ 0.50 & 0.08132 & 0.00017 & 0.02073 & -0.00219 \\ 1.00 & 0.14243 & 0.00186 & 0.07717 & -0.00940 \\ 1.50 & 0.16943 & 0.00859 & 0.15399 & -0.02220 \\ 2.00 & 0.15901 & 0.02563 & 0.23134 & -0.03838 \\ 2.50 & 0.11907 & 0.05700 & 0.29185 & -0.05118 \\ 3.00 & 0.06490 & 0.10154 & 0.32664 & -0.05078 \\ 3.50 & 0.01221 & 0.15110 & 0.33780 & -0.02894 \\ 0.125 \pi & 0.06494 & 0.00008 & 0.01290 & -0.00134 \\ 0.250 \pi & 0.11978 & 0.00078 & 0.04938 & -0.00562 \\ 0.375 \pi & 0.15638 & 0.00343 & 0.10320 & -0.01334 \\ 0.500 \pi & 0.17016 & 0.01025 & 0.16537 & -0.02439 \\ 0.025 \pi & 0.16093 & 0.02393 & 0.22609 & -0.03721 \\ 0.750 \pi & 0.13276 & 0.04645 & 0.27689 & -0.04840 \\ 0.875 \pi & 0.09282 & 0.07788 & 0.31250 & -0.05324 \\ 1.000 \pi & 0.04926 & 0.11558 & 0.33186 & -0.04700 \\ 1.125 \pi & 0.00894 & 0.15436 & 0.33794 & -0.02657 \\ 1.250 \pi & -0.02439 & 0.18775 & 0.33622 & 0.00812\end{array}$

$0.00205-6.00000$ $0.00410-c .00000$ $0.01025-C .00000$ $0.02050-c .00001$ $0.04100-C .00004$ $0.10236-C .00068$ $0.20368-0.00536$ $-0.01773$ $0.39676-0.04082$ $0.55896-0.12566$ $0.61940-0.18678$

$0.08044-0.00033$ $0.16040-0.00261$ $0.23927-0.00871$ $0.31621-0.02029$ $0.39009-0.03872$ $0.45949-0.06495$ $\begin{array}{ll}0.52267 & -0.099440 \\ 0.57777 & -0.14484\end{array}$ $0.57777-0.14184$ $\begin{array}{ll}0.62289 & -0.19135 \\ 0.65634 & -0.24630\end{array}$

$$
\frac{\mathrm{a}}{\lambda}=1.0 \quad \frac{\alpha}{\beta}=0.10
$$

$\begin{array}{ll}0.00170 & -0.00000 \\ 0.00340 & -0.00000 \\ 0.00849 & -0.00000 \\ 0.01698 & -0.00000 \\ 0.03395 & -0.00004 \\ 0.08476 & -0.00056 \\ 0.16856 & -0.00443 \\ 0.25011 & -0.01464 \\ 0.32760 & -0.03362 \\ 0.39855 & -0.06291 \\ 0.46003 & -0.10283 \\ 0.50892 & -0.15226 \\ 0.06661 & -0.00027 \\ 0.13278 & -0.00216 \\ 0.19795 & -0.00720 \\ 0.26138 & -0.01674 \\ 0.32213 & -0.03190 \\ 0.37898 & -0.05339 \\ 0.43054 & -0.08149 \\ 0.47528 & -0.11595 \\ 0.51174 & -0.15594 \\ 0.53861 & -0.20007\end{array}$

$\frac{\alpha}{\beta}=0.07$

$C(h, h)$

$S(h, h)$

$E(h, h)$

$\begin{array}{ll}0.00205 & -0.00000 \\ 0.00410 & -0.00000 \\ 0.01025 & -0.00002 \\ 0.12046 & -0.00002 \\ 0.04070 & -0.00013 \\ 0.09769 & -0.00197 \\ 0.16784 & -0.01247 \\ 0.19190 & -0.02783 \\ 0.17159 & -0.03532 \\ 0.12895 & -0.03181 \\ 0.08571 & -0.03383 \\ 0.04336 & -0.06072 \\ 0.07817 & -0.00093 \\ 0.14265 & -0.00682 \\ 0.18213 & -0.01802 \\ 0.19137 & -0.02964 \\ 0.17417 & -0.03523 \\ 0.14194 & -0.03328 \\ 0.10686 & -0.03062 \\ 0.07408 & -0.03851 \\ 0.04018 & -0.06350 \\ -0.00051 & -0.10150\end{array}$

$0.00001-0.00000$ $0.00004-0.00000$ $0.00026-0.00002$ $-0.00057$ $0.00408-0.00030$ $\begin{array}{ll}0.02487 & -0.0024\end{array}$ $0.09021-0.01562$ $0.16907-0.04908$ $0.22612-0.09904$ $0.23863-0.14506$ $0.20960-0.16902$ $0.15428-0.17450$

$0.01552-0.00134$ $\begin{array}{ll}0.05857-0.0078 \\ 0.11858 & -0.0249\end{array}$ $0.11858-0.02499$ $0.17925-0.05549$ $0.22334-0.0952$ $0.23987-0.1336$ $0.12808-0.17174$ $0.19608-0.17174$ $\begin{array}{ll}0.14974 & -0.17465 \\ 0.09004 & -0.17729\end{array}$
$0.00205-0.00000$ 0.00410-0.00000 0.01025 - 0.00000 $0.02050-0.00002$ $0.04097-0.00017$ $0.10184 \quad-0.00268$ $0.19838-0.0204$ $0.27948-0.06283$ $0.33058-0.12847$ $0.34004-0.20215$ $0.25584-0.28181$

$0.08020-0.00131$ $0.15810-0.01014$ $0.22974-0.03248$ $0.28889-0.07092$ $0.32817-0.12315$ $0.34184-0.18148$ $0.32891-0.23442$ $0.2908-0.27031$ $\begin{array}{ll}0.25209 & -0.28178 \\ 0.21411 & -0.26896\end{array}$

10.0


$\beta \mathrm{h}$
$\mathrm{C}(\mathrm{h}, 0)$

0.01
0.02

0.05

0.10

0.20
0.50

0.50

1.50

.00

2.50
3.00

3.50

$0.125 \pi$

0.250

$0.375 \pi$

0.625

0.750

$0.875 \pi$

$1.000 \pi$

$1.125 \pi$

$1.250 \pi$
0.000910 .00000

0.001810 .00000

$0.00453 \quad 0.00000$

$\begin{array}{ll}0.00904 & 0.00000 \\ 0.01800 & 0.00003\end{array}$

0.043410 .00046

$0.07626 \quad 0.00367$

$0.09146 \quad 0.01234$

$0.08744 \quad 0.0284$

0.067910 .0517

$0.03950 \quad 0.08002$

$0.00854 \quad 0.10753$

$0.03466 \quad 0.00022$

$0.06402 \quad 0.00177$

$\begin{array}{ll}0.08391 & 0.00601 \\ 0.09203 & 0.01414\end{array}$

$0.08833 \quad 0.02697$

$0.07475 \quad 0.04450$

$0.05444 \quad 0.0656$

$0.03077 \quad 0.08813$

$\begin{array}{rr}0.003077 & 0.08813 \\ -0.01715 & 0.12613\end{array}$
$S(h, j)$

$0.00000-0.00000$

$0.00002-0.00000$

$\begin{array}{ll}0.00011 & -0.00002 \\ 0.00045 & -0.00009\end{array}$

$\begin{array}{ll}0.00045 & -0.00009 \\ 0.00180 & -0.00036\end{array}$

$\begin{array}{ll}0.00180 & -0.00036 \\ 0.01106 & -0.00223\end{array}$

$\begin{array}{ll}0.01106 & -0.00223 \\ 0.04119 & -0.00844\end{array}$

$0.04119-0.00844$

$\begin{array}{ll}0.08244 & -0.01694 \\ 0.12494 & -0.02447\end{array}$

$0.12494-0.02447$

$0.16046-0.02650$

$\begin{array}{rr}0.18492 & -0.01870 \\ 0.19880 & 0.00121\end{array}$

$0.00688-0.00138$

$0.02635-0.00536$

$0.05512-0.01134$

$0.08861-0.01816$

$0.12200 \quad-0.02404$

$0.15129-0.02676$

$0.17408-0.02406$

$0.18981-0.01430$

$0.19944 \quad 0.00301$

$0.20468 \quad 0.02700$

$\frac{a}{\lambda}=1.0 \quad \frac{\alpha}{\beta}=0.20$

$E(i, 0)$

$\mathrm{C}(\mathrm{h}, \mathrm{h})$

$S(h, h)$

$E(h, h)$

$0.00091-0.00000$

$\begin{array}{ll}0.00091 & -0.00000 \\ 0.00181 & -0.00005\end{array}$

$\begin{array}{ll}0.00181 & -0.00001 \\ 0.00453 & -0.00000\end{array}$

$\begin{array}{ll}0.00453 & -0.00000 \\ 0.00904 & -0.00000\end{array}$

$0.01798-0.00003$

$0.04309-0.00039$

$0.07398-0.00206$

$0.08552-0.00278$

$\begin{array}{ll}0.07897 & 0.0003\end{array}$

$0.06149 \quad 0.00502$

$\begin{array}{lr}0.03891 & 0.00334 \\ 0.01266 & -0.01070\end{array}$

$0.17302-0.01761$

$0.20942-0.03263$

$0.26450-0.07707$

$0.03552-0.00015$

$0.07072-0.00115$

$0.10522-0.00381$

$0.13856-0.00883$

$0.17019-0.01672$

$0.18944-0.02777$

$0.22561-0.04203$

$0.24797-0.05924$

$\begin{array}{ll}0.26587 & -0.07886 \\ 0.27882 & -0.10011\end{array}$

$0.03450-0.00020$

$0.06285-0.00125$

$0.08043-0.00263$

$0.08556-0.00259$

$\begin{array}{ll}0.07992 & 0.00002\end{array}$

$0.06721 \quad 0.00394$

0.050720 .00549

$0.03184 \quad 0.0007$

$0.01073-0.01212$

$-0.01180-0.03151$

$\begin{array}{lrllr}0.01 & 0.00026 & 0.00000 & 0.00000 & -0.00000 \\ 0.02 & 0.00052 & 0.00000 & 0.00001 & -0.00000 \\ 0.05 & 0.00129 & 0.00000 & 0.00003 & -0.00001 \\ 0.10 & 0.00257 & 0.00000 & 0.00013 & -0.00005 \\ 0.20 & 0.00512 & 0.00002 & 0.00051 & -0.00020 \\ 0.50 & 0.01240 & 0.00034 & 0.00316 & -0.00124 \\ 1.00 & 0.02204 & 0.00257 & 0.01190 & -0.00437 \\ 1.50 & 0.02699 & 0.00796 & 0.02434 & -0.00783 \\ 2.00 & 0.02655 & 0.01670 & 0.03816 & -0.00956 \\ 2.50 & 0.02111 & 0.02785 & 0.05136 & -0.00774 \\ 3.00 & 0.01162 & 0.03965 & 0.06265 & -0.00127 \\ 3.50 & -0.00094 & 0.05013 & 0.07141 & 0.00987 \\ & & & & \\ 0.125 \pi & 0.00989 & 0.00017 & 0.00196 & -0.00078 \\ 0.250 \pi & 0.01839 & 0.00128 & 0.00757 & -0.00288 \\ 0.375 \pi & 0.02441 & 0.00409 & 0.01603 & -0.00567 \\ 0.500 \pi & 0.02726 & 0.00901 & 0.02627 & -0.00822 \\ 0.625 \pi & 0.02677 & 0.01597 & 0.03715 & -0.00954 \\ 0.750 \pi & 0.02314 & 0.02449 & 0.04772 & -0.00871 \\ 0.875 \pi & 0.01683 & 0.03377 & 0.05727 & -0.00513 \\ 1.000 \pi & 0.00834 & 0.04284 & 0.06540 & 0.00143 \\ 1.125 \pi & -0.00189 & 0.05076 & 0.07191 & 0.01078 \\ 1.250 \pi & -0.01351 & 0.05681 & 0.07667 & 0.02244\end{array}$

$\begin{array}{ll}0.00026 & -0.00000 \\ 0.00052 & -0.00000 \\ 0.00129 & -0.00000 \\ 0.00258 & -0.00000 \\ 0.00515 & -0.00001 \\ 0.01284 & -0.00008 \\ 0.02539 & -0.00066 \\ 0.03732 & -0.00215 \\ 0.04827 & -0.00483 \\ 0.05786 & -0.00878 \\ 0.06571 & -0.01387 \\ 0.07153 & -0.01978 \\ 0.01010 & -0.00004 \\ 0.02006 & -0.00033 \\ 0.02973 & -0.00107 \\ 0.03894 & -0.00246 \\ 0.04751 & -0.00459 \\ 0.05526 & -0.00752 \\ 0.06200 & -0.01119 \\ 0.06759 & -0.01548 \\ 0.07190 & -0.02021 \\ 0.07491 & -0.02514\end{array}$

$0.00091-0.00000$

$0.00181-0.00000$

$0.00453-0.00000$

$0.00906-0.00001$

$0.01809-0.00008$

$0.04484-0.00118$

$0.08651-0.00880$

$0.12019-0.02636$

$0.14033-0.05208$

$0.14389-0.07906$

$0.13351-0.09864$

$0.11710-0.10550$

$0.03536-0.00057$ $0.06928-0.00442$ $0.09972-0.01388$ $0.12398-0.02962$ $0.13941-0.05006$ $0.14453-0.07170$ $0.14006-0.09020$ $0.12905-0.10196$ $0.11600-0.10549$ $0.10529-0.10192$

$$
\frac{a}{\lambda}=1.0 \quad \frac{\alpha}{\beta}=0.40
$$

$\begin{array}{rrrr}0.00026 & 0.00000 & 0.00000 & -0.00000 \\ 0.00052 & 0.06000 & 0.00001 & -0.00000 \\ 0.00129 & 0.00000 & 0.00003 & -0.00001 \\ 0.00257 & 0.00000 & 0.00013 & -0.00005 \\ 0.00512 & 0.00001 & 0.00051 & -0.00021 \\ 0.01227 & 0.00010 & 0.00310 & -0.00128 \\ 0.02116 & 0.00088 & 0.01103 & -0.00496 \\ 0.02491 & 0.00319 & 0.02053 & -0.00995 \\ 0.02356 & 0.00695 & 0.02836 & -0.01384 \\ 0.01785 & 0.01033 & 0.03240 & -0.01412 \\ 0.00828 & 0.01054 & 0.03131 & -0.0097 .9 \\ -0.00429 & 0.00537 & 0.02379 & -0.00182 \\ & & & \\ 0.00982 & 0.00004 & 0.00194 & -0.00079 \\ 0.01792 & 0.00040 & 0.00722 & -0.00313 \\ 0.02312 & 0.00150 & 0.01442 & -0.00669 \\ 0.02501 & 0.01366 & 0.02181 & -0.01063 \\ 0.02381 & 0.00685 & 0.02790 & -0.01365 \\ 0.01991 & 0.00956 & 0.03170 & -0.01450 \\ 0.01354 & 0.01100 & 0.03258 & -0.01252 \\ 0.00495 & 0.00969 & 0.02989 & -0.00782 \\ -0.00521 & 0.00479 & 0.02301 & -0.00120 \\ -0.01562 & -0.00395 & 0.01158 & 0.00606\end{array}$

$\begin{array}{ll}0.00026 & -0.00000 \\ 0.00052 & -0.00000 \\ 0.00129 & -0.00000 \\ 0.00258 & -0.00000 \\ 0.00514 & -0.00002 \\ 0.01270 & -0.00033 \\ 0.02414 & -0.00242 \\ 0.03286 & -0.00694 \\ 0.03766 & -0.01303 \\ 0.03846 & -0.01878 \\ 0.03652 & -0.02249 \\ 0.03383 & -0.02364 \\ 0.01003 & -0.00016 \\ 0.01947 & -0.00123 \\ 0.02763 & -0.00376 \\ 0.03379 & -0.00774 \\ 0.03745 & -0.01257 \\ 0.03859 & -0.01728 \\ 0.03771 & -0.02095 \\ 0.03576 & -0.02306 \\ 0.03367 & -0.02364 \\ 0.03214 & -0.02314\end{array}$


0.0

\begin{tabular}{|c|c|c|}
\hline $\begin{array}{l}0.01 \\
0.02 \\
0.05 \\
0.10 \\
0.20 \\
0.50 \\
1.00 \\
1.50 \\
2.00 \\
2.50 \\
3.00 \\
3.50\end{array}$ & $\begin{array}{r}0.00004 \\
0.00008 \\
0.00020 \\
0.00039 \\
0.00078 \\
0.00190 \\
0.00349 \\
0.00446 \\
0.00452 \\
0.00342 \\
0.00100 \\
-0.00280\end{array}$ & $\begin{array}{l}0.00000 \\
0.00000 \\
0.00000 \\
0.00000 \\
0.00001 \\
0.00010 \\
0.00075 \\
0.00230 \\
0.00478 \\
0.00794 \\
0.01132 \\
0.01440\end{array}$ \\
\hline $\begin{array}{l}0.125 \pi \\
0.250 \pi \\
0.375 \pi \\
0.500 \pi \\
0.625 \pi \\
0.750 \pi \\
0.875 \pi \\
1.000 \pi \\
1.125 \pi \\
1.250 \pi\end{array}$ & $\begin{array}{r}0.00151 \\
0.00287 \\
0.00393 \\
0.00453 \\
0.00455 \\
0.00387 \\
0.00239 \\
0.00006 \\
-0.00311 \\
-0.00709\end{array}$ & $\begin{array}{l}0.00005 \\
0.00037 \\
0.00119 \\
0.00260 \\
0.00457 \\
0.00699 \\
0.00962 \\
0.01224 \\
0.01459 \\
0.01646\end{array}$ \\
\hline
\end{tabular}

$\begin{array}{ll}0.00000 & -0.00000 \\ 0.00000 & -0.00000 \\ 0.00000 & -0.00000 \\ 0.06002 & -0.00001 \\ 0.00008 & -0.00005 \\ 0.00049 & -0.00033 \\ 0.00191 & -0.00113 \\ 0.00417 & -0.00194 \\ 0.00709 & -0.00217 \\ 0.01039 & -0.00133 \\ 0.01374 & 0.00092 \\ 0.01675 & 0.00466 \\ & \\ 0.00030 & -0.00021 \\ 0.00119 & -0.00075 \\ 0.00263 & -0.00145 \\ 0.00455 & -0.00202 \\ 0.00686 & -0.00219 \\ 0.00942 & -0.00170 \\ 0.01207 & -0.00039 \\ 0.01464 & 0.00183 \\ 0.01693 & 0.00497 \\ 0.01875 & 0.00895\end{array}$

$E(h, 0)$

$0.00004-0.00000$ $0.00008-0.00000$ $0.00020-0.00000$ $\begin{array}{ll}0.00039 & -0.00000 \\ 0.00078 & -0.00000\end{array}$ $0.00195-0.00001$ $0.00383-0.00010$ $0.00557-0.00032$ $0.00712-0.00069$ $0.00841-0.00123$ $0.00942-0.00188$ $0.01012-0.00258$

$0.00153-0.00001$ $0.00303-0.00005$ $0.00447-0.00016$ $0.00580-0.00036$ $0.00701-0.00066$ $0.00807-0.00106$ $0.00895-0.00154$ $0.00965-0.00207$ $\begin{array}{ll}0.01016 & -0.00263 \\ 0.01050 & -0.00318\end{array}$

$$
\frac{a}{\lambda}=1.0 \quad \frac{\alpha}{\beta}=1.00
$$

$\begin{array}{lrl}0.01 & 0.00001 & 0.00000 \\ 0.02 & 0.00001 & 0.00000 \\ 0.05 & 0.00003 & 0.00000 \\ 0.10 & 0.00006 & 0.00000 \\ 0.20 & 0.00012 & 0.00000 \\ 0.50 & 0.00029 & 0.00002 \\ 1.00 & 0.00057 & 0.00017 \\ 1.50 & 0.00076 & 0.00054 \\ 2.00 & 0.00079 & 0.00117 \\ 2.50 & 0.00052 & 0.00202 \\ 3.00 & -0.00017 & 0.00301 \\ 3.50 & -0.00137 & 0.00397 \\ & & \\ 0.125 \pi & 0.00023 & 0.00001 \\ 0.250 \pi & 0.00046 & 0.00009 \\ 0.375 \pi & 0.00065 & 0.00028 \\ 0.500 \pi & 0.00078 & 0.00062 \\ 0.625 \pi & 0.00080 & 0.00112 \\ 0.750 \pi & 0.00063 & 0.00176 \\ 0.875 \pi & 0.00024 & 0.00250 \\ 1.000 \pi & -0.00046 & 0.00329 \\ 1.125 \pi & -0.00147 & 0.00404 \\ .1 .250 \pi & -0.00284 . & 0.00467\end{array}$

$\begin{array}{ll}0.00000 & -0.00000 \\ 0.00000 & -0.00000 \\ 0.00000 & -0.00000 \\ 0.00000 & -0.00000 \\ 0.00001 & -0.00001 \\ 0.00008 & -0.00007 \\ 0.00032 & -0.00025 \\ 0.00076 & -0.00043 \\ 0.00143 & -0.00047 \\ 0.00229 & -0.00022 \\ 0.00327 & 0.00046 \\ 0.00423 & 0.00166 \\ 0.00005 & -0.00004 \\ 0.00019 & -0.00016 \\ 0.00045 & -0.00032 \\ 0.00084 & -0.00045 \\ 0.00137 & -0.00047 \\ 0.00202 & -0.00033 \\ 0.00277 & 0.00006 \\ 0.00355 & 0.00075 \\ 0.00429 & 0.00176 \\ 0.00492 & 0.00313\end{array}$

$\begin{array}{ll}0.00001 & -0.00000 \\ 0.00001 & -0.00000 \\ 0.00003 & -0.00000 \\ 0.00006 & -0.00000 \\ 0.00012 & -0.00000 \\ 0.00029 & -0.00000 \\ 0.00058 & -0.00001 \\ 0.00083 & -0.00005 \\ 0.00105 & -0.00010 \\ 0.00123 & -0.00017 \\ 0.00135 & -0.00025 \\ 0.00144 & -0.00034 \\ & \\ 0.00023 & -0.00000 \\ 0.00046 & -0.00001 \\ 0.00067 & -0.00002 \\ 0.00087 & -0.00005 \\ 0.00104 & -0.00010 \\ 0.00118 & -0.00015 \\ 0.00130 & -0.00021 \\ 0.00138 & -0.00028 \\ 0.00144 & -0.00034 \\ 0.00148 & -0.00041\end{array}$

$\mathrm{C}(\mathrm{h}, \mathrm{h})$

$0.00004 \quad 0.00000$ $0.00020 \quad 0.00000$ $0.00039 \quad 0.00000$ $\begin{array}{ll}0.00187 & 0.00060 \\ 0.000330 & 0.00066\end{array}$ $0.00330 \quad 0.00046$ $0.00401 \quad 0.00140$ $0.00378 \quad 0.00275$ $0.00229 \quad 0.00408$ $-0.00080 \quad 0.00461$ $-0.00540$

$0.00150 \quad 0.00003$ $0.00277 \quad 0.00023$ $0.00365 \quad 0.00073$ $0.00404 \quad 0.00157$ $0.00383 \quad 0.00265$ $0.00287 \quad 0.00374$ $-0.00196 \quad 0.00447$ $-0.00575 \quad 0.00309$ $\begin{array}{rr}-0.000575 & 0.00309 \\ -0.00988 & -0.00023\end{array}$

$S(h, h)$

$E(h, h)$

$0.00000-0.00000$ $0.00000-0.00000$ $0.00000-0.00000$ $0.00002-0.0000$ $0.00008-0.00005$ $0.00047-0.00033$ $0.00171-0.00117$ $0.00334-0.00208$ $0.00502-0.00242$ $0.00636-0.00154$ $0.00636 \quad-0.00154$ 0.005110 .0009

$0.00030-0.00021$ $0.00111-0.00077$ $0.00171-0.00077$ $0.00359-0.00218$ $0.00490-0.00243$ $0.00604-0.00195$ $0.00672-0.00050$ 0.006520 .0001 $0.00490 \quad 0.00544$ $\begin{array}{ll}0.00490 & 0.00544 \\ 0.00127 & 0.00927\end{array}$
$0.00004-0.00000$ $0.00008-0.00000$ $0.00020-0.00000$ $0.00039-0.00000$ $0.00191-0.00000$ $0.00356-0.00035$ $0.00471-0.00094$ $0.00527-0.00164$ $0.00535-0.00221$ $0.00520-0.00252$ $0.00502-0.00260$

$0.00152-0.00002$ $0.00290-0.00018$ $0.00403-0.00053$ $0.00482-0.00104$ $0.00525-0.00159$ $0.00537-0.00207$ $0.00529-0.00240$ $0.00514-0.00256$ $\begin{array}{ll}0.00493 & -0.00257\end{array}$
0.00001 0.00000 $0.00006 \quad 0.00000$ $0.00012 \quad 0.00000$ $0.00029 \quad 0.00002$ $0.00053 \quad 0.00012$ $0.00066 \quad 0.00037$ $0.00060 \quad 0.00075$ $0.00016 \quad 0.00119$ $\begin{array}{ll}-0.00089 & 0.00146 \\ -0.00269 & 0.00105\end{array}$

\section{$0.00023 \quad 0.0000$}

$\begin{array}{ll}0.00043 & 0.00006 \\ 0.00059 & 0.00019\end{array}$ $0.00067 \quad 0.00041$

$0.00062 \quad 0.00072$

$0.00034 \quad 0.00107$ $\begin{array}{ll}-0.00027 & 0.00137\end{array}$ $\begin{array}{ll}-0.00132 & 0.00144 \\ -0.00284 & 0.00098\end{array}$ $-0.00468-0.00046$
$0.00000-0.00000$ $0.00000-0.00000$ $0.00000-0.00000$ $0.00000-0.00000$ $0.00001-0.00001$ $0.00007-0.00007$ $0.00028-0.00024$ $0.00059-0.0004$

$0.00100-0.00042$

$0.00143-0.00005$

$\begin{array}{ll}0.00167 & 0.00094 \\ 0.00123 & 0.00269\end{array}$

$0.00005-0.00004$ $0.00017-0.00016$ $0.00038-0.00031$ $0.00064-0.00043$ $0.00096-0.00043$ $0.00131-0.00021$ $0.00160 \quad 0.00035$ 0.001650 .00136 $0.00115 \quad 0.00283$ $\begin{array}{lll}-0.00031 & 0.00464\end{array}$
$0.00001-0.00000$ $0.00001-0.00000$ $0.00003-0.00000$ $0.00006-0.00000$ $0.00012-0.00000$ $0.00029-0.00001$

$0.00053-0.00005$

$0.00068-0.00013$

$0.00074-0.00021$

$0.00075-0.00026$

$\begin{array}{ll}0.00074 & -0.00029 \\ 0.00073 & -0.00030\end{array}$

$0.00023-0.00000$ $0.00043-0.00003$ $0.00059 \quad-0.00007$ $0.00069-0.00014$ $0.00074-0.00020$ $0.00075-0.00025$ $0.00075-0.00028$ $0.00073-0.00029$

$0.00073-0.00030$ $0.00072-0.00029 \ldots$ 
$\mathrm{C}(\mathrm{h}, \mathrm{h})$

$0.00000-0.00001$ $0.00010-0.00032$ $0.00041-0.00126$ $0.00163-0.00503$ $.00977-0.03093$ $0.03379-0.11600$ $0.07349-0.36167$ $0.07246-0.45949$ $0.06518-0.50603$

$0.00614 \quad-0.01922$ $0.02246-0.07415$ $0.04345-0.15684$ $0.06218-0.25489$ $0.07300-0.35310$ $0.07404-0.43580$ $0.06850-0.48999$ $0.06450-0.50840$ $0.09960-0.44822$

$\begin{array}{ll}0.00082 & -0.00252 \\ 0.00164 & -0.00505 \\ 0.00410 & -0.01261 \\ 0.00819 & -0.02523 \\ 0.01635 & -0.05046 \\ 0.04026 & -0.12627 \\ 0.07616 & -0.25323 \\ 0.10343 & -0.38114 \\ 0.11793 & -0.50943 \\ 0.11598 & -0.63638 \\ 0.09462 & -0.75897 \\ 0.05211 & -0.87289 \\ 0.03184 & -0.09913 \\ 0.06156 & -0.19862 \\ 0.08708 & -0.29869 \\ 0.10635 & -0.39931 \\ 0.11739 & -0.50008 \\ 0.11842 & -0.60016 \\ 0.10791 & -0.69819 \\ 0.08476 & -0.79232 \\ 0.04841 & -0.88025 \\ -0.00101 & -0.95932\end{array}$

$0.00032-0.00252$ $0.001 .54-0.00505$ $0.00409-0.0126$ $0.00816-0.02519$ $0.01610-0.05015$ $0.03654-0.12140$ $0.05031-0.21436$ $0.03581-0.25377$ $0.01002-0.23140$ $\begin{array}{lll}-0.00441 & -0.16974\end{array}$ $-0.00767-0.11156$ $-0.02842-0.08249$

$0.03000-0.09678$ $0.04809-0.17974$ $0.04703-0.23552$ $0.03225-0.25420$ $0.03225-0.25420$ $-0.00207-0.18905$ $-0.00606-0.13785$ $-0.01026-0.10029$ $-0.03125-0.08138$ $-0.07725-0.08138$
$S(h, h)$

$0.00000-0.00001$ $0.00002-0.00005$ $0.00010-0.00032$ $0.00041-0.00126$ $0.00924-0.0310$ $0.02593-0.11705$ $0.02506-0.23374$ $-0.07431-0.38994$ $-0.13542-0.37447$ $-0.17929-0.31652$

$0.00593-0.01926$ $0.01934-0.07448$ $0.02821-0.15698$ $-0.00739-0.33255$ $-0.05501-0.38230$ $-6.10669-0.38969$ $-0.14937-0.36082$ -C. $18199-0.31184$ $-0.11687-0.311470$
$E(h, h)$

$0.00082-0.00252$ $0.00164-0.00505$ $0.00410-0.01261$ $0.00817-0.02523$ $0.01621-0.05049$ $0.05897-0.25472$ $0.04731-0.37949$ $-0.00579-0.48641$ $-0.09592-0.55396$ $-0.20108-0.56370$ $-0.28629-0.51391$

$0.03078-0.09931$

$0.05317-0.19965$

$0.05921-0.30008$

$0.04238-0.39616$ $-0.00051-0.47966$ $-0.06716-0.53984$ 0.0.14840 -0.56668 $-0.22886-0.55511$ $-0.29049-0.50875$ $-0.31824-0.44111$

\begin{tabular}{|c|c|c|}
\hline $\begin{array}{l}0.01 \\
0.02 \\
0.05 \\
0.10 \\
0.20 \\
0.50 \\
1.00 \\
1.50 \\
2.00 \\
2.50 \\
3.00 \\
3.50\end{array}$ & $\begin{array}{l}0.00079 \\
0.00158 \\
0.00394 \\
0.00788 \\
0.01564 \\
0.03724 \\
0.06277 \\
0.07210 \\
0.07126 \\
0.07404 \\
0.09373 \\
0.13358\end{array}$ & $\begin{array}{l}-0.00243 \\
-0.00486 \\
-0.01214 \\
-0.02425 \\
-0.04827 \\
-0.11657 \\
-0.20497 \\
-0.24321 \\
-0.22133 \\
-0.14555 \\
-0.03801 \\
0.06971\end{array}$ \\
\hline $\begin{array}{l}0.125 \pi \\
0.250 \pi \\
0.375 \pi \\
0.500 \pi \\
0.625 \pi \\
0.750 \pi \\
0.875 \pi \\
1.000 \pi \\
1.125 \pi \\
1.250 \pi\end{array}$ & $\begin{array}{l}0.02991 \\
0.05377 \\
0.06787 \\
0.07233 \\
0.07139 \\
0.07198 \\
0.08119 \\
0.10319 \\
0.13684 \\
0.17499\end{array}$ & $\begin{array}{r}-0.09302 \\
-0.17210 \\
-0.22517 \\
-0.24380 \\
-0.22493 \\
-0.17183 \\
-0.09412 \\
-0.00610 \\
0.07630 \\
0.13962\end{array}$ \\
\hline
\end{tabular}

$\begin{array}{llll}0.00000 & -0.00001 & 0.00079 & -0.00243 \\ 0.00002 & -0.00005 & 0.00158 & -0.00486 \\ 0.00010 & -0.00030 & 0.00395 & -0.01215 \\ 0.00039 & -0.00122 & 0.00789 & -0.02430 \\ 0.00154 & -0.00485 & 0.01574 & -0.04860 \\ 0.00926 & -0.02983 & 0.03877 & -0.12159 \\ 0.03207 & -0.11241 & 0.07334 & -0.24383 \\ 0.05635 & -0.22809 & 0.09958 & -0.36695 \\ 0.07025 & -0.34826 & 0.11354 & -0.49037 \\ 0.07012 & -0.44234 & 0.11166 & -0.01242 \\ 0.06461 & -0.48733 & 0.09114 & -0.73019 \\ 0.07157 & -0.47672 & 0.05034 & -0.83952 \\ & & & \\ 0.00582 & -0.01854 & 0.03066 & -0.09546 \\ 0.02131 & -0.07150 & 0.05928 & -0.19126 \\ 0.04126 & -0.15116 & 0.08385 & -0.28760 \\ 0.05916 & -0.24556 & 0.10239 & -0.38443 \\ 0.06974 & -0.34002 & 0.11301 & -0.48138 \\ 0.07132 & -0.41954 & 0.11400 & -0.57760 \\ 0.06706 & -0.47174 & 0.10391 & -0.67181 \\ 0.06445 & -0.48974 & 0.08168 & -0.76221 \\ 0.07299 & -0.47418 & 0.04679 & -0.84659 \\ 0.10065 & -0.43332 & -0.00059 & -0.92240\end{array}$

$\begin{array}{ll}0.00079 & -0.00243 \\ 0.00158 & -0.00486 \\ 0.00394 & -0.01214 \\ 0.00786 & -0.02426 \\ 0.01551 & -0.04830 \\ 0.03523 & -0.11688 \\ 0.04881 & -0.20627 \\ 0.03555 & -0.24407 \\ 0.01161 & -0.22264 \\ 0.00179 & -0.16368 \\ 0.00530 & -0.10788 \\ 0.02607 & -0.07941 \\ & \\ 0.02892 & -0.09318 \\ 0.04649 & -0.17300 \\ 0.04672 & -0.22657 \\ 0.03225 & -0.24447 \\ 0.01317 & -0.22591 \\ 0.00040 & -0.18215 \\ -0.00345 & -0.13316 \\ 0.00801 & -0.09697 \\ 0.02885 & -0.07828 \\ -0.07386 & -0.06644\end{array}$

$0.00000-0.00001$ $0.00002-0.00005$ c. $00010-0.00030$ $0.00039-0.00122$ $c .00153-0.00485$ $0.00875-0.02990$ $0.02450-0.11277$ $0.02347-0.22494$ $-0.01110-0.32576$ $-0.01110-0.32576$ $0.072852-0.36028$ $-0.17020-0.30473$

$0.00562-0.01857$ $0.01830-0.07178$ $0.02731-0.15119$ $0.02073-0.24097$ $-10.00746-0.31976$ $-0.05258-0.36750$ $-10.10138-0.37474$ $-0.14173-0.34726$ $-17.17279-0.3002$

$-10.20622-0.24472$

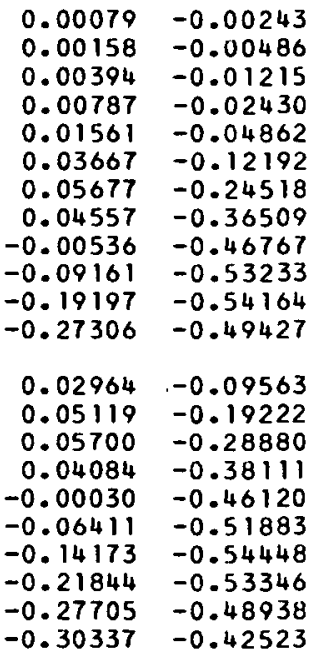


$\beta \mathrm{h}$

$C(h, 0)$

$S(h, 0)$

\begin{tabular}{|c|c|c|}
\hline $\begin{array}{l}0.01 \\
0.02 \\
0.05 \\
0.10 \\
0.20 \\
0.50 \\
1.00 \\
1.50 \\
2.00 \\
2.50 \\
3.00 \\
3.50\end{array}$ & $\begin{array}{l}0.00076 \\
0.00152 \\
0.00380 \\
0.00758 \\
0.01507 \\
0.03591 \\
0.06080 \\
0.07048 \\
0.07068 \\
0.07436 \\
0.09386 \\
0.13193\end{array}$ & $\begin{array}{r}-0.00234 \\
-0.00468 \\
-0.011169 \\
-0.02336 \\
-0.04648 \\
-0.11224 \\
-0.19727 \\
-0.23393 \\
-0.21277 \\
-0.13992 \\
-0.03667 \\
0.06684\end{array}$ \\
\hline $\begin{array}{l}0.125 \pi \\
0.250 \pi \\
0.375 \pi \\
0.500 \pi \\
0.625 \pi \\
0.750 \pi \\
0.875 \pi \\
1.000 \pi \\
1.125 \pi \\
1.250 .\end{array}$ & $\begin{array}{l}0.02882 \\
0.05196 \\
0.06591 \\
0.07083 \\
0.07072 \\
0.07212 \\
0.08160 \\
0.10298 \\
0.13502 \\
0.17087\end{array}$ & $\begin{array}{r}-0.08957 \\
-0.16567 \\
-0.21667 \\
-0.23448 \\
-0.21624 \\
-0.16517 \\
-0.09053 \\
-0.00603 \\
0.07319 \\
0.13441\end{array}$ \\
\hline
\end{tabular}

0.01

0.02

0.05

0.20

0.50

1.00

1.50

2.00

2.50

3.50

$0.125 \pi$

$0.250 \pi$

$0.375 \pi$

$0.500 \pi$

$0.750 \pi$

$0.875 \pi$

$1.125 \pi$

$$
\begin{array}{ll}
0.00070 & -0.00217 \\
0.00141 & -0.00434 \\
0.00352 & -0.01084 \\
0.00703 & -0.02166 \\
0.01398 & -0.04311 \\
0.03339 & -0.10406 \\
0.05704 & -0.18273 \\
0.06730 & -0.21643 \\
0.06935 & -0.19664 \\
0.07462 & -0.12927 \\
0.09370 & -0.03405 \\
0.12849 & 0.06161 \\
0.02677 & -0.08305 \\
0.04851 & -0.15353 \\
0.06214 & -0.20062 \\
0.06786 & -0.21691 \\
0.06925 & -0.19985 \\
0.07206 & -0.15259 \\
0.08200 & -0.08372 \\
0.10219 & -0.00579 \\
0.13125 & 0.06751 \\
0.16291 & 0.12478
\end{array}
$$

$\frac{\mathrm{a}}{\lambda}=1.2 . \quad \frac{\alpha}{\beta}=0.01$

$0.00000-0.00001$ $0.00001-0.00005$ $0.00009-6.00029$ $0.00037-0.00117$ $0.00146-0.00468$ $0.00878-0.0287$ $0.03043-0.10836$ $0.05359-0.21976$ $0.06716 \quad-0.33537$ $0.06786-0.42586$ $0.06399-0.46935$ $0.07234-0.45979$

$0.00552-0.01788$ $0.02021-0.06894$ $0.0391 .7-0.14570$ $0.05628-0.23658$ $0.06663-0.32745$ $0.06871-0.40392$ $0.06557-0.45420$ $0.06430-0.47181$ $0.07382-0.45742$ $0.10147-0.41892$

$\begin{array}{llll}0.00000 & -0.00001 & 0.00070 & -0.00217 \\ 0.00001 & -0.00004 & 0.00141 & -0.00434 \\ 0.00008 & -0.00027 & 0.00352 & -0.01085 \\ 0.00033 & -0.00109 & 0.00704 & -0.02170 \\ 0.00131 & -0.00435 & 0.01406 & -0.04340 \\ 0.00789 & -0.02676 & 0.03462 & -0.10858 \\ 0.02738 & -0.10071 & 0.06548 & -0.21769 \\ 0.04843 & -0.20403 & 0.08887 & -0.32746 \\ 0.06136 & -0.31104 & 0.10130 & -0.43735 \\ 0.06354 & -0.39479 & 0.09964 & -0.54582 \\ 0.06259 & -0.43544 & 0.08145 & -0.65022 \\ 0.07339 & -0.42779 & 0.04538 & -0.74689 \\ 0.00496 & -0.01663 & 0.02738 & -0.08525 \\ 0.01817 & -0.06410 & 0.05293 & -0.17077 \\ 0.03529 & -0.13536 & 0.07485 & -0.25672 \\ 0.05092 & -0.21960 & 0.09138 & -0.34304 \\ 0.06081 & -0.30371 & 0.10084 & -0.42935 \\ 0.06376 & -0.37447 & 0.10172 & -0.51490 \\ 0.06262 & -0.42114 & 0.09276 & -0.59850 \\ 0.06375 & -0.43797 & 0.07307 & -0.67857 \\ 0.07495 & -0.42571 & 0.04225 & -0.75313 \\ 0.10248 & -0.39152 & 0.00049 & -0.81995\end{array}$

$E(h, 0)$

$\begin{array}{ll}0.00076 & -0.00234 \\ 0.00152 & -0.00468 \\ 0.00380 & -0.01170 \\ 0.00760 & -0.02340 \\ 0.01516 & -0.04680 \\ 0.03733 & -0.71709 \\ 0.07062 & -0.23479 \\ 0.09587 & -0.35329 \\ 0.10930 & -0.77202 \\ 0.10750 & -0.58936 \\ 0.08778 & -0.70250 \\ 0.04863 & -0.30743 \\ 0.02952 & -0.09193 \\ 0.05709 & -0.18417 \\ 0.08074 & -0.27691 \\ 0.09858 & -0.37011 \\ 0.10880 & -0.46337 \\ 0.10975 & -0.55590 \\ 0.10005 & -0.04643 \\ 0.07870 & -0.73324 \\ 0.04523 & -0.81421 \\ 0.00020 & -0.88690\end{array}$

$C(h, h)$

$S(h, h)$

$E(h, h)$

$\begin{array}{rr}0.00070 & -0.00234 \\ 0.00152 & -0.00468 \\ 0.00380 & -0.01169 \\ 0.00757 & -0.02336 \\ 0.01494 & -0.04651 \\ 0.03397 & -0.11253 \\ -0.04734 & -0.19848 \\ 0.03526 & -0.23474 \\ 0.01307 & -0.21421 \\ 0.00063 & -0.15783 \\ -0.00309 & -0.10429 \\ -0.02384 & -0.07640 \\ 0.02787 & -0.08972 \\ 0.04494 & -0.16651 \\ 0.04553 & -0.21797 \\ 0.03220 & -0.23512 \\ 0.01453 & -0.21734 \\ 0.00268 & -0.17549 \\ -0.00105 & -0.12860 \\ -0.00592 & -0.09372 \\ -0.02656 & -0.07526 \\ -0.07018 & -0.06333\end{array}$

$0.00000-0.0000$ $0.00001-0.00005$ $0.00009-0.00029$

\begin{tabular}{|c|c|}
\hline $\begin{array}{r}0.00070 \\
0.00141 \\
0.00352 \\
0.00702 \\
0.01386 \\
0.03159 \\
0.04454 \\
0.03460 \\
0.01561 \\
0.00493 \\
0.00087 \\
-0.01973\end{array}$ & $\begin{array}{l}-0.00217 \\
-0.00434 \\
-0.01084 \\
-0.02166 \\
-0.04313 \\
-0.10431 \\
-0.18377 \\
-0.21714 \\
-0.19830 \\
-0.14670 \\
-0.09737 \\
-0.07064\end{array}$ \\
\hline $\begin{array}{r}0.02588 \\
0.04199 \\
0.04322 \\
0.03198 \\
0.01686 \\
0.00673 \\
0.00325 \\
-0.00212 \\
-0.02232 \\
-0.06332\end{array}$ & $\begin{array}{l}-0.08318 \\
-0.15425 \\
-0.20174 \\
-0.21749 \\
-0.20117 \\
-0.16286 \\
-0.11988 \\
-0.08745 \\
-0.06949 \\
-0.05745\end{array}$ \\
\hline
\end{tabular}
$0.00037-0.00117$ $0.00145-0.00468$ $0.00829-0.02883$ $0.02313-0.10864$ $0.02196-0.21648$ $-0.01098-0.31323$ $-0.06748-0.36039$ $-0.12196 .-0.34664$ $-0.16154-0.29337$

$0.00533-0.01791$ $0.01731-0.06919$ $0.02574-0.14560$ $0.01933-0.23188$ $\begin{array}{ll}-0.00752 & -0.30748\end{array}$ $-0.05025-0.35328$ $\begin{array}{ll}-0.09634 & -0.36037\end{array}$ $-0.13445-0.33421$ $-0.16402-0.28900$ $-0.19603-0.23512$ $\frac{\alpha}{\beta}=0.02$

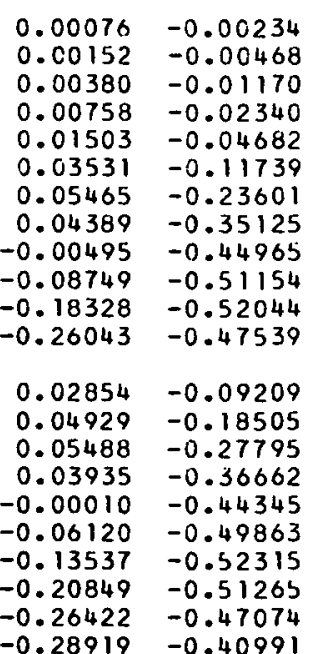

$-0.28919-0.40991$

$\begin{aligned} 0.00000 & -0.00001 \\ 0.00001 & -0.00004 \\ 0.00008 & -0.00027 \\ 0.00033 & -0.00109 \\ 0.00130 & -0.00436 \\ 0.00743 & -0.02681 \\ 0.02061 & -0.10085 \\ 0.01918 & -0.20052 \\ -0.01071 & -0.28964 \\ -0.06129 & -0.33314 \\ -0.10979 & -0.32091 \\ -0.14543 & -0.27186 \\ 0.00478 & -0.01665 \\ 0.01547 & -0.06428 \\ 0.02284 & -0.13506 \\ 0.01676 & -0.21472 \\ -0.00760 & -0.28434 \\ -0.04592 & -0.32654 \\ -0.08698 & -0.33332 \\ -0.12096 & -0.30957 \\ -0.14769 & -0.26779 \\ -0.17700 & -0.21699\end{aligned}$

$\begin{aligned} 0.00070 & -0.00217 \\ 0.00141 & -0.00434 \\ 0.00352 & -0.01085 \\ 0.00703 & -0.02170 \\ 0.01394 & -0.04342 \\ 0.03274 & -0.10884 \\ 0.05065 & -0.21868 \\ 0.04072 & -0.32511 \\ -0.00421 & -0.41567 \\ -0.07980 & -0.47237 \\ -0.16705 & -0.48050 \\ -0.23691 & -0.43975 \\ 0.02647 & -0.08539 \\ 0.04569 & -0.17152 \\ 0.05086 & -0.25745 \\ 0.03054 & -0.33928 \\ 0.00024 & -0.40998 \\ -0.05576 & -0.46057 \\ -0.12347 & -0.48297 \\ -0.18992 & -0.47344 \\ -0.24033 & -0.43555 \\ -0.26280 & -0.38085\end{aligned}$


$\beta \mathrm{h}$

$C(h, 0)$

$S(h, 0)$

$\mathrm{C}(\mathrm{h}, \mathrm{h})$

$S(h, h)$

$E(h, h)$

$\begin{array}{llrl}0.00065 & -0.00201 & 0.00065 & -0.00201 \\ 0.00131 & -0.00402 & 0.00131 & -0.00402 \\ 0.00327 & -0.01006 & 0.00327 & -0.01006 \\ 0.00653 & -0.02012 & 0.00651 & -0.02009 \\ 0.01304 & -0.04025 & 0.01286 & -0.03999 \\ 0.03210 & -0.10069 & 0.02937 & -0.09669 \\ 0.06071 & -0.20183 & 0.04189 & -0.17016 \\ 0.08239 & -0.30353 & 0.03383 & -0.20087 \\ 0.09389 & -0.40523 & 0.01768 & -0.18356 \\ 0.09235 & -0.50549 & 0.00858 & -0.13630 \\ 0.07557 & -0.60184 & 0.00429 & -0.09680 \\ 0.04235 & -0.69089 & -0.01604 & -0.06520 \\ & & & \\ 0.02539 & -0.07906 & 0.02404 & -0.07712 \\ 0.04908 & -0.15835 & 0.03923 & -0.14290 \\ 0.06940 & -0.23801 & 0.04101 & -0.18572 \\ 0.08470 & -0.31795 & 0.03162 & -0.20118 \\ 0.09346 & -0.39783 & 0.01875 & -0.18519 \\ 0.09427 & -0.47693 & 0.01014 & -0.15111 \\ 0.08600 & -0.55413 & 0.00691 & -0.11166 \\ 0.06785 & -0.62797 & 0.00117 & -0.08148 \\ 0.03946 & -0.69663 & -0.01852 & -0.06405 \\ 0.00107 & -0.75806 & -0.05708 & -0.05202\end{array}$

$0.00000-0.0000$ $0.00001-0.00004$ $0.00097-0.00025$ $0.00030-0.00102$ $0.00117-0.00405$ $0.00665-0.02493$ $0.01833-0.09362$ $0.01669-0.18576$ $\begin{array}{ll}-0.01042 & -0.26787\end{array}$ $-0.05567-0.30802$ $-0.13081-0.2519$

$0.00428-0.01549$ $0.01381-0.05972$ $0.02022-0.12528$ $0.01446-0.1988$ $-0.00762-0.26300$ $\begin{array}{lll}-0.04196 & -0.3018\end{array}$ $-0.07851-0.30835$ $-0.10875-0.2867$ $-0.13286-0.2481$ $-0.15964-0.20020$
$0.06291-0.40668$

$0.10275-0.36591$
$0.00065-0.00201$ $0.00131-0.00402$ $0.00327-0.01006$ $0.00652-0.02012$ $0.01293-0.04026$ $0.03035-0.10092$ $0.04694-0.20262$ $0.03778-0.30092$ $-0.00356-0.38426$ $-0.07278-0.43621$ $-0.21551-0.40677$

$0.02454-0.07917$ $0.04235-0.15898$ $0.04714-0.23846$ $0.03392-0.31399$ $0.00054-0.37903$ $-0.05080-0.4254$ $-0.11261-0.44588$ $-0.17301-0.43723$ $-0.21859-0.40299$ $-0.23881-0.35379$
0.0
0.0
0.0
0.1
0.2
0.5
1.0
1.5
2.0
2.5
3.0
3.5

$0.125 \pi$ $0.250 \pi$ $0.375 \pi$ $0.500 \pi$ $0.625 \pi$ $0.750 \pi$ $0.875 \pi$

$1.000 \pi$

$1.250 \pi$
$0.00056-0.00173$ $0.00112-0.00346$ $0.00281-0.00865$ $0.00561-0.01727$ $0.01116-0.03438$ $0.02684-0.08292$ $0.04705-0.14526$ $0.05831-0.17146$ $0.06439-0.15522$ $0.07299-0.10180$ $0.09063-0.02677$

$0.02146-0.06620$ $0.02146-0.06620$ $0.05202-0.15928$ $0.05933-0.17174$ $0.05396-0.15780$ $0.06396-0.15780$ 0.06590 $0.09748-0.00445$ 0.119310 .05396 $0.14107 \quad 0.10116$

$\begin{array}{llll}0.00000 & -0.00001 & 0.00056 & -0.06173 \\ 0.00001 & -0.00004 & 0.00112 & -0.00346 \\ 0.00006 & -0.00022 & 0.00281 & -0.00865 \\ 0.00024 & -0.00088 & 0.00562 & -0.01730 \\ 0.00094 & -0.00351 & 0.01121 & -0.03461 \\ 0.00562 & -0.02153 & 0.02761 & -0.08659 \\ 0.01984 & -0.08087 & 0.05219 & -0.17350 \\ 0.03564 & -0.16336 & 0.07079 & -0.28079 \\ 0.04678 & -0.24840 & 0.08065 & -0.34790 \\ 0.05214 & -0.31498 & 0.07934 & -0.43356 \\ 0.05758 & -0.34832 & 0.05505 & -0.51562 \\ 0.07334 & -0.34499 & 0.03687 & -0.59118 \\ 0.00357 & -0.01339 & 0.02183 & -0.06799 \\ 0.01312 & -0.05152 & 0.04220 & -0.13615 \\ 0.02567 & -0.10859 & 0.05965 & -0.20456 \\ 0.03759 & -0.17576 & 0.07278 & -0.27315 \\ 0.04619 & -0.24258 & 0.08028 & -0.34157 \\ 0.05097 & -0.29877 & 0.08098 & -0.40918 \\ 0.05424 & -0.33625 & 0.07393 & -0.47502 \\ 0.06054 & -0.35092 & 0.05849 & -0.53782 \\ 0.07505 & -0.34358 & 0.03442 & -0.59604 \\ 0.10155 & -0.31961 & 0.00198 & -0.64797\end{array}$

$\frac{\alpha}{\beta}=0.05$

$\begin{array}{cc}0.00056 & -0.00173 \\ 0.00112 & -0.00346 \\ 0.00281 & -0.00865 \\ 0.00560 & -0.01728 \\ 0.01106 & -0.03439 \\ 0.02539 & -0.08308 \\ 0.03703 & -0.14590 \\ 0.03209 & -0.17192 \\ 0.02068 & -0.15727 \\ 0.01422 & -0.11753 \\ 0.00969 & -0.07868 \\ -0.00981 & -0.05526 \\ 0.02074 & -0.06629 \\ 0.03424 & -0.12265 \\ 0.03687 & -0.15997 \\ 0.03054 & -0.17217 \\ 0.02143 & -0.15948 \\ 0.01538 & -0.12999 \\ 0.01263 & -0.09665 \\ 0.00646 & -0.07043 \\ -0.01207 & -0.05413 \\ -0.04627 & -0.04237\end{array}$

$\begin{array}{ll}0.00000 & -0.00001 \\ 0.00001 & -0.00004 \\ 0.00006 & -0.00022 \\ 0.00024 & -0.00088 \\ 0.00093 & -0.00351 \\ 0.00531 & -0.02155 \\ 0.01443 & -0.08069 \\ 0.01248 & -0.15947 \\ -0.00981 & -0.22924 \\ -0.04593 & -0.26347 \\ -0.07986 & -0.25479 \\ -0.10550 & -0.21621 \\ & \\ 0.00342 & -0.01340 \\ 0.01096 & -0.05155 \\ 0.01576 & -0.10783 \\ 0.01060 & -0.17062 \\ -0.00754 & -0.22510 \\ -0.03507 & -0.25817 \\ -0.06392 & -0.26402 \\ -0.08773 & -0.24610 \\ -0.10719 & -0.21289 \\ -0.12935 & -0.17030\end{array}$

$\begin{array}{ll}0.00056 & -0.00173 \\ 0.00112 & -0.00346 \\ 0.00281 & -0.00865 \\ 0.00561 & -0.01731 \\ 0.01112 & -0.03463 \\ 0.02609 & -0.08675 \\ 0.04032 & -0.17395 \\ 0.03252 & -0.25781 \\ -0.00246 & -0.32839 \\ -0.06051 & -0.37200 \\ -0.12646 & -0.37821 \\ -0.17832 & -0.34803 \\ 0.02110 & -0.06807 \\ 0.03639 & -0.13658 \\ 0.04049 & -0.20459 \\ 0.02924 & -0.26891 \\ 0.00099 & -0.32398 \\ -0.04215 & -0.36299 \\ -0.09366 & -0.38005 \\ -0.14354 & -0.37293 \\ -0.18083 & -0.34495 \\ -0.19720 & -0.30516\end{array}$


$\beta \mathrm{h}$

$C(h, 0)$

0.01
0.02

0.02
0.05

0.10

0.20

0.50

1.00

1.50

2.00

2.50
3.00

3.50

$0.125 \pi$

$0.250 \pi$

$0.375 \pi$

$0.625 \pi$

$0.750 \pi$

$0.875 \pi$

$1.000 \pi$

$1.125 \pi$

$1.250 \pi$

$0.00048-0.00149$ $0.00097-0.00298$ $0.00242-0.00744$ $0.00483-0.01486$ $0.00961-0.02956$ $0.02321-0.07127$ $0.04136-0.1246 .7$ $0.05281-0.14682$ $0.06057-0.13259$ $0.07046-0.08670$ $0.08704-0.02244$ $0.10953 \quad 0.04305$

$0.01851-0.05691$ $0.03440-0.10495$ $0.04615-0.13661$ $0.05401-0.14702$ $0.06706-0.10248$ $0.07777-0.05596$ $0.09301-0.00329$ $0.11113 \quad 0.04718$ $0.12806 \quad 0.08886$
$S(h, 0)$

$0.00000-0.00001$ $0.00001-0.00003$ $0.00005-0.00019$ $0.00019-0.00076$ $0.00075-0.00303$ $0.00454-0.01863$ $0.01592-0.06988$ $0.02895-0.14093$ $0.03902-0.21400$ $0.04568-0.27129$ $\begin{array}{ll}0.05385 & -0.30056 \\ 0.07143 & -0.29921\end{array}$

$0.00285-0.01158$ $0.01050-0.04455$ $0.02067-0.09378$ $0.03062-0.15160$ $0.03842-0.20900$ $0.04391-0.25730$ $0.04908-0.28977$ $0.05753-0.30313$ $0.07317-0.29814$ $0.09859-0.27920$

$$
\frac{a}{\lambda}=1.2
$$

$E(h, 0)$ $0.00097-0.00298$ $0.00242-0.00744$ $0.00483-0.01488$ $0.00964-0.02977$ $0.02374-0.07446$ $0.04486-0.14915$ $0.06083-0.22406$ $0.06928-0.29868$ $0.06816-0.37186$ $0.03208-0.50588$

$0.01878-C .05846$ $0.03628-C .11706$ $0.05127-G .17582$ $0.06254-0.23466$ $0.06896-0.29326$ $0.06956-0.35105$ $0.06355-0.40721$ $0.05042-0.46062$ $0.00260-0.55387$ $\frac{\alpha}{\beta}=0.07$

$$
\mathrm{C}(\mathrm{h}, \mathrm{h})
$$

$S(h, h)$

$E(h, h)$
$0.00000-0.00001$ $0.00001-0.000 \mathrm{C} 3$ $0.00005-0.00019$ $0.00019-0.00076$ $0.00074-0.00303$ $0.00422-0.01863$ $0.01126-0.06955$ $0.00915-0.13696$ $\begin{array}{lll}-0.00917 & -0.19632\end{array}$ $-0.03789-0.22555$ $-0.06439-0.21860$ $0.00272-0.01159$ $0.00864-0.04450$ $0.01215-0.09283$ $0.00755-0.14646$ $-0.00734-0.19281$ $-0.02933-0.22097$ $-0.05197-0.22623$ $-0.07055-0.21127$ $-0.08606-0.18260$ $-0.10423-0.14473$
$0.00048-0.00149$ $0.00097-0.00298$ $0.00242-0.00744$ $0.00482-0.01488$ $0.00956-0.02978$ $0.02243-0.07458$ $0.03464-0.14934$ $0.02800-0.22088$ $-0.00161-0.28065$ $-0.05030-0.31727$ $-0.10501-0.32244$ $-0.14753-0.29774$

$0.01814-0.05853$ $0.03127-0.11733$ $0.03478-0.17553$ $0.02521-0.23031$ $0.00130-0.27694$ $\begin{array}{ll}-0.03495 & -0.30973\end{array}$ $-0.11908-0.31810$ $-0.14957-0.29524$ $-0.16284 .-0.26306$

$\begin{array}{llr}0.01 & 0.00039 & -0.00119 \\ 0.02 & 0.00077 & -0.00237 \\ 0.05 & 0.00193 & -0.00593 \\ 0.10 & 0.00385 & -0.01185 \\ 0.20 & 0.00767 & -0.02357 \\ 0.50 & 0.01865 & -0.05680 \\ 1.00 & 0.03407 & -0.09914 \\ 1.50 & 0.04531 & -0.11639 \\ 2.00 & 0.05450 & -0.10468 \\ 2.50 & 0.06536 & -0.06801 \\ 3.00 & 0.08031 & -0.01680 \\ 3.50 & 0.09784 & 0.03599 \\ 0.125 \pi & 0.01484 & -0.04537 \\ 0.250 \pi & 0.02798 & -0.08355 \\ 0.375 \pi & 0.03850 & -0.10853 \\ 0.500 \pi & 0.04665 & -0.11648 \\ 0.625 \pi & 0.05382 & -0.10647 \\ 0.750 \pi & 0.06187 & -0.08059 \\ 0.875 \pi & 0.07228 & -0.04353 \\ 1.000 \pi & 0.0 .8519 & -0.00146 \\ 1.125 \pi & 0.09901 & 0.03937 \\ 1.250 \pi & 0.11058 & 0.07419\end{array}$

$\begin{array}{ll}0.00000 & -0.00001 \\ 0.00001 & -0.00002 \\ 0.00003 & -0.00015 \\ 0.00013 & -0.00061 \\ 0.00053 & -0.00244 \\ 0.00320 & -0.01499 \\ 0.01134 & -0.05615 \\ 0.02108 & -0.11301 \\ 0.02970 & -0.17134 \\ 0.03745 & -0.21722 \\ 0.04807 & -0.24140 \\ 0.06687 & -0.24207 \\ 0.00201 & -0.00932 \\ 0.00744 & -0.03582 \\ 0.01481 & -0.07530 \\ 0.02240 & -0.12153 \\ 0.02913 & -0.16735 \\ 0.03515 & -0.20597 \\ 0.04204 & -0.23227 \\ 0.05235 & -0.24386 \\ 0.06857 & -0.24136 \\ 0.09206 & -0.22803\end{array}$

$\begin{array}{ll}0.00039 & -0.00119 \\ 0.00077 & -0.00237 \\ 0.00193 & -0.00593 \\ 0.00385 & -0.01187 \\ 0.00769 & -0.02374 \\ 0.01893 & -0.05937 \\ 0.03576 & -0.11888 \\ 0.04846 & -0.17844 \\ 0.05515 & -0.23759 \\ 0.05427 & -0.29538 \\ 0.04471 & -0.35032 \\ 0.02603 & -0.40045 \\ & \\ 0.01497 & -0.04662 \\ 0.02893 & -0.09332 \\ 0.04086 & -0.14010 \\ 0.04981 & -0.18685 \\ 0.05490 & -0.23330 \\ 0.05538 & -0.27898 \\ 0.05064 & -0.32320 \\ 0.04035 & -0.36510 \\ 0.02442 & -0.40365 \\ 0.00312 & -0.43775\end{array}$

$$
\frac{a}{\lambda}=1.2 \quad \frac{\alpha}{\beta}=0.10
$$

$\begin{array}{ll}0.00039 & -0.00119 \\ 0.00077 & -0.00231 \\ 0.00193 & -0.00593 \\ 0.00384 & -0.01185 \\ 0.00760 & -0.02358 \\ 0.01765 & -0.05681 \\ 0.02713 & -0.09938 \\ 0.02709 & -0.11658 \\ 0.02339 & -0.10681 \\ 0.02128 & -0.08063 \\ 0.01702 & -0.05393 \\ 0.00041 & -0.03533 \\ 0.01434 & -0.04541 \\ 0.02435 & -0.08374 \\ 0.02801 & -0.10875 \\ 0.02661 & -0.11672 \\ 0.02363 & -0.10827 \\ 0.02174 & -0.08888 \\ 0.02004 & -0.06658 \\ 0.01397 & -0.04779 \\ 0.00137 & -0.03432 \\ 0.02700 & -0.02399\end{array}$

$\begin{array}{rlrl}0.00000 & -0.00001 & 0.00039 & -0.00119 \\ 0.00001 & -0.00002 & 0.00077 & -0.00237 \\ 0.00003 & -0.00015 & 0.00193 & -0.00593 \\ 0.00013 & -0.00061 & 0.00385 & -0.01187 \\ 0.00052 & -0.00244 & 0.00762 & -0.02375 \\ 0.00294 & -0.01498 & 0.01788 & -0.05944 \\ 0.00762 & -0.05569 & 0.02758 & -0.11879 \\ 0.00541 & -0.10909 & 0.02236 & -0.17516 \\ -0.00819 & -0.15579 & -0.00070 & -0.22175 \\ -0.02837 & -0.17893 & -0.03809 & -0.24991 \\ -0.04634 & -0.17388 & -0.07944 & -0.25386 \\ -0.06026 & -0.14737 & -0.11101 & -0.23557 \\ 0.00191 & -0.00932 & 0.01446 & -0.04666 \\ 0.00595 & -0.03570 & 0.02491 & -0.09343 \\ 0.00804 & -0.0748 & 0.02769 & -0.13949 \\ 0.00417 & -0.11659 & 0.02017 & -0.18255 \\ -0.00686 & -0.15303 & 0.00156 & -0.21887 \\ -0.02245 & -0.17524 & -0.02636 & -0.24415 \\ -0.03797 & -0.17968 & -0.05903 & -0.25500 \\ -0.05049 & -0.16815 & -0.08995 & -0.25062 \\ -0.06123 & -0.14498 & -0.11251 & -0.23373 \\ -0.07443 & -0.11318 & -0.12217 & -0.21033\end{array}$


$\beta \mathrm{h}$

C (h, 0 i

$S(h, 0)$

$\begin{array}{lll}0.01 & 0.00018 & -0.00056 \\ 0.02 & 0.00036 & -0.00112 \\ 0.05 & 0.00091 & -0.00279 \\ 0.10 & 0.00181 & -0.00557 \\ 0.20 & 0.00362 & -0.01109 \\ 0.50 & 0.00901 & -0.02607 \\ 1.00 & 0.01774 & -0.04628 \\ 1.50 & 0.02636 & -0.05381 \\ 2.00 & 0.03538 & -0.04763 \\ 2.50 & 0.04515 & -0.02972 \\ 3.00 & 0.05508 & -0.00456 \\ 3.50 & 0.06326 & 0.02266 \\ 0.125 \pi & 0.00709 & -0.02132 \\ 0.250 \pi & 0.01403 & -0.03912 \\ 0.375 \pi & 0.02080 & -0.05051 \\ 0.500 \pi & 0.02759 & -0.05376 \\ 0.625 \pi & 0.03469 & -0.04852 \\ 0.750 \pi & 0.04228 & -0.03584 \\ 0.875 \pi & 0.05017 & -0.01777 \\ 1.000 \pi & 0.05768 & 0.00315 \\ 1.125 \pi & 0.06369 & 0.02449 \\ 1.250 \pi & 0.06683 & 0.04443\end{array}$

\begin{tabular}{|c|c|c|}
\hline $\begin{array}{l}0.01 \\
0.02 \\
0.05 \\
0.10 \\
0.20 \\
0.50 \\
1.00 \\
1.50 \\
2.00 \\
2.50 \\
3.00 \\
3.50\end{array}$ & $\begin{array}{l}0.00004 \\
0.00008 \\
0.00020 \\
0.00040 \\
0.00081 \\
0.00210 \\
0.00472 \\
0.00815 \\
0.01231 \\
0.01671 \\
0.02052 \\
0.02282\end{array}$ & $\begin{array}{r}-0.00012 \\
-0.00025 \\
-0.00062 \\
-0.00123 \\
-0.00245 \\
-0.00589 \\
-0.01017 \\
-0.011165 \\
-0.00987 \\
-0.00505 \\
0.00212 \\
0.01076\end{array}$ \\
\hline $\begin{array}{l}0.125 \pi \\
0.250 \pi \\
0.375 \pi \\
0.500 \pi \\
0.625 \pi \\
0.750 \pi \\
0.875 \pi \\
1.000 \pi \\
1.125 \pi \\
1.250 \pi\end{array}$ & $\begin{array}{l}0.00162 \\
0.00351 \\
0.00584 \\
0.00870 \\
0.011199 \\
0.01547 \\
0.01874 \\
0.02136 \\
0.02290 \\
0.02300\end{array}$ & $\begin{array}{r}-0.00471 \\
-0.00862 \\
-0.011106 \\
-0.01160 \\
-0.01011 \\
-0.00671 \\
-0.00117 \\
0.00445 \\
0.011138 \\
0.01869\end{array}$ \\
\hline
\end{tabular}

$\begin{array}{ll}0.00000 & -0.00000 \\ 0.00000 & -0.00001 \\ 0.00001 & -0.00007 \\ 0.00003 & -0.00030 \\ 0.00014 & -0.00119 \\ 0.00085 & -0.00727 \\ 0.00323 & -0.02714 \\ 0.00685 & -0.05448 \\ 0.01189 & -0.08254 \\ 0.01933 & -0.10509 \\ 0.03067 & -0.11821 \\ 0.04706 & -0.12108 \\ 0.00053 & -0.00452 \\ 0.00205 & -0.01734 \\ 0.00438 & -0.03636 \\ 0.00746 & -0.05857 \\ 0.01146 & -0.08061 \\ 0.01686 & -0.09945 \\ 0.02440 & -0.11292 \\ 0.03478 & -0.12003 \\ 0.04837 & -0.12093 \\ 0.06493 & -0.11668\end{array}$

$\begin{array}{ll}-0.00000 & -0.00000 \\ -0.00000 & -0.00000 \\ -0.00000 & -0.00002 \\ -0.00000 & -0.00007 \\ -0.00002 & -0.00028 \\ -0.00010 & -0.00171 \\ -0.00020 & -0.00642 \\ 0.00022 & -0.01300 \\ 0.00185 & -0.02003 \\ 0.00530 & -0.02612 \\ 0.01086 & -0.03032 \\ 0.01840 & -0.03209 \\ & \\ -0.00007 & -0.00106 \\ -0.00018 & -0.00409 \\ -0.00014 & -0.00862 \\ 0.00036 & -0.01401 \\ 0.00168 & -0.01953 \\ 0.00410 & -0.02453 \\ 0.00780 & -0.02849 \\ 0.01281 & -0.03108 \\ 0.01897 & -0.03212 \\ 0.02598 & -0.03157\end{array}$

$E(h, 0)$

$\begin{array}{ll}0.00018 & -0.00056 \\ 0.00036 & -0.00112 \\ 0.00091 & -0.00279 \\ 0.00181 & -0.00558 \\ 0.00362 & -0.01117 \\ 0.00890 & -0.02792 \\ 0.01679 & -0.05581 \\ 0.02270 & -0.08354 \\ 0.02579 & -0.11081 \\ 0.02540 & -0.13713 \\ 0.02112 & -0.16175 \\ 0.01291 & -0.18380 \\ 0.00704 & -0.02193 \\ 0.01359 & -0.04385 \\ 0.01917 & -0.06571 \\ 0.02333 & -0.08744 \\ 0.02568 & -0.10885 \\ 0.02590 & -0.12970 \\ 0.02377 & -0.14965 \\ 0.01919 & -0.16829 \\ 0.01220 & -0.18520 \\ 0.00302 & -0.19990\end{array}$

$\mathrm{C}(\mathrm{h}, \mathrm{h})$

$S(h, h)$

$\begin{array}{ll}0.00018 & -0.06056 \\ 0.00036 & -0.00112 \\ 0.00091 & -0.00279 \\ 0.00181 & -0.00557 \\ 0.06359 & -0.01109 \\ 0.00852 & -0.02666 \\ 0.01440 & -0.04620 \\ 0.01745 & -0.05376 \\ 0.01941 & -0.04916 \\ 0.02001 & -0.03702 \\ 0.01792 & -0.02336 \\ 0.00723 & -0.01169 \\ 0.00685 & -0.02132 \\ 0.01226 & -0.03908 \\ 0.01573 & -0.05039 \\ 0.01775 & -0.05379 \\ 0.01928 & -0.04983 \\ 0.02047 & -0.04091 \\ 0.02004 & -0.03011 \\ 0.01583 & -0.01978 \\ 0.00616 & -0.01100 \\ -0.00884 & -0.00397\end{array}$

$0.00000-0.00000$ $0.00000-0.00001$ $0.00001-0.00007$ $0.00003-c .00030$ $0.05014-0.00118$ $0.00073-0.00724$ $\begin{array}{lll}0.00149 & -0.02661\end{array}$ $-0.00031-0.05147$ $-0.00520-0.07290$ $-0.01064-0.08381$ $-0.01410-0.03185$ $-0.01637-0.06835$

$0.00048-0.00451$ $0.00134-0.01715$ $0.00124-0.03529$ $-0.00085-0.05492$ $-0.00478-0.07163$ $-0.00921-0.08198$ $-0.01266-0.08441$ $-0.01474-0.07911$ $\begin{array}{ll}-0.01657 & -0.06705 \\ -0.02000 & -0.04905\end{array}$
E(h, h)

$0.00018-0.00056$ $0.00036-0.00112$ $0.00091-0.00279$ $\begin{array}{ll}0.00181 & -0.00550 \\ 0.00359 & -0.01117\end{array}$ $\begin{array}{ll}0.00359 & -0.01117 \\ 0.00840 & -0.02790\end{array}$ $0.00840-0.02790$ $0.01290-0.05541$ $0.01056-0.08087$ $0.00055-0.10118$ $-0.01497-0.11292$ $\begin{array}{ll}-0.03123 & -0.11452 \\ -0.04293 & -0.10780\end{array}$

$0.00680-0.02192$ $0.01167-0.04372$ $0.01295-0.06485$ $0.00959-0.08415$ $0.00151 \quad-0.09995$ $-0.01019-0.11057$ $-0.02332-0.11496$ $-0.03521-0.11329$ $\begin{array}{ll}-0.04346 & -0.10714 \\ -0.04683 & -0.09904\end{array}$

$$
\frac{a}{\lambda}=1.2 \quad \frac{\alpha}{\beta}=0.40
$$

$\begin{array}{ll}0.00004 & -0.00012 \\ 0.00008 & -0.00025 \\ 0.00020 & -0.00062 \\ 0.00040 & -0.00124 \\ 0.00080 & -0.00247 \\ 0.00197 & -0.00617 \\ 0.00370 & -0.01230 \\ 0.00498 & -0.01831 \\ 0.00564 & -0.02411 \\ 0.00556 & -0.02956 \\ 0.00471 & -0.03451 \\ 0.00312 & -0.03878 \\ 0.00156 & -0.00485 \\ 0.00300 & -0.00968 \\ 0.00422 & -0.01446 \\ 0.00512 & -0.01915 \\ 0.00562 & -0.02370 \\ 0.00566 & -0.02804 \\ 0.00523 & -0.03210 \\ 0.00433 & -0.03580 \\ 0.00299 & -0.03904 \\ 0.00128 & -0.04178\end{array}$

$0.00004-0.00012$ $0.00003-0.00025$ $0.00020-0.00062$ $0.00040-0.00123$ $0.00080-0.00245$ $0.00199-0.010588$ $0.00392-0.01007$ $0.00593-0.0115 t$ $0.00795-0.01032$ $0.00926-0.00705$ $0.00855-0.00252$ $0.00466 \quad 0.0022 C$

$0.00157-0.00471$ $0.00309-0.00856$ $0.00462-0.01095$ $0.00622-0.01155$ $0.000903-0.00815$ $0.00925-0.0049$ $0.00731-0.00129$ $0.00426 \quad 0.0025 \mathrm{i}$ $-0.00144 \quad 0.00558$
$-0.00000-0.00000$ $-0.00000-0.00000$ $-0.00000-0.00002$ $\begin{array}{ll}-0.00000 & -0.00007 \\ -0.00002 & -0.00028\end{array}$ $-0.00013-0.00169$ $-0.00057-0.00616$ $-0.00126-0.01181$ $-0.00168-0.01676$ $-0.00109-0.01951$ $0.00069-0.01907$ $-0.00008-0.00106$ $-0.00033-0.00399$ $-0.00580-0.00813$ $-0.00136-0.01259$ $-0.00167-0.01646$ $-0.00139-0.01901$ $-0.00033-0.01973$ $0.00135-0.01827$ $0.00315-0.01440$ $0.00439-0.00809$

$\begin{array}{rr}0.00004 & -0.00012 \\ 0.00008 & -0.00025 \\ 0.00020 & -0.00062 \\ 0.00040 & -0.00124 \\ 0.00079 & -0.00247 \\ 0.00185 & -0.00615 \\ 0.00282 & -0.01206 \\ 0.00235 & -0.01726 \\ 0.00046 & -0.02111 \\ -0.00221 & -0.02315 \\ -0.00473 & -0.02342 \\ -0.00634 & -0.02251 \\ & \\ 0.00150 & -0.00484 \\ 0.00256 & -0.00957 \\ 0.00283 & -0.01402 \\ 0.00216 & -0.01790 \\ 0.00064 & -0.02089 \\ -0.00141 & -0.02276 \\ -0.00354 & -0.02348 \\ -0.00530 & -0.02324 \\ -0.00640 & -0.02243 \\ -0.00681 & -0.02146\end{array}$


$\beta \mathrm{h}$

$\mathrm{C}(\mathrm{h}, 0)$

$\operatorname{Sin}, 0)$

$0.00000 .-0.00001$

$0.00001-0.00003$

$0.60002-0.00006$

$0.00004-0.00013$

0.00024 .0 .00062

$0.00063-0.00107$

$0.00126-0.00121$

$0.00210-0.0012$

$0.00303-0.00012$

$0.00303-0.00012$

$0.00430 \quad 0.00307$

3.50

$0.125 \pi$

$0.250 \pi$

$0.375 \pi$

0.500

$0.625 \pi$

$0.750 \pi$

i.

$1.000 \pi$

$1.125 \pi$
$1.250 \pi$

$0.00018-0.00049$

$0.00043-0.00090$

$0.00082-0.00116$

$0.00136-0.00120$

$0.00203-0.00096$

$0.00276-0.00040$

$0.00346 \quad 0.00048$

$0.00402 \quad 0.00170$

$0.00432 \quad 0.0032$

$0.00427 \quad 0.00498$ $\frac{a}{\lambda}=1.2 \quad \frac{\alpha}{\beta}=0.70$

$E(h, 0)$

$\mathrm{C}(\mathrm{h}, \mathrm{h})$

$S(h, h)$

$E(h, h)$

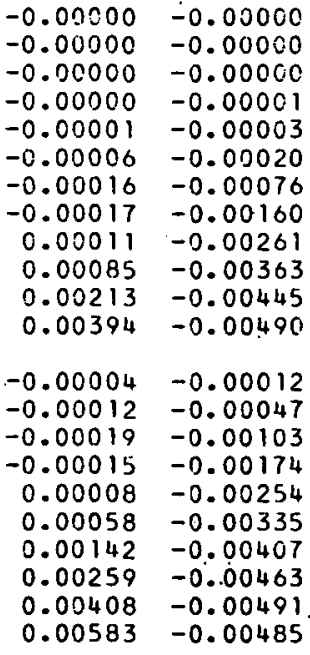

$0.00000-0.00001$

$0.00001-0.00003$

$0.00002-0.00006$

$0.00004,-0.00013$

$0.00008-0.00026$

$0.00020=0.00064$

$0.00038-0.00127$

$0.00051-0.00188$

$0.00058-0.00245$

$0.00057-0.00296$

$0.00049-0.00341$

$0.00036-0.00377$

$0.00016 \quad-0.00050$

$0.00031-0.00100$

$0.00044-0.00149$

$0.00053-0.00196$

$0.00058-0.00241$

$0.00058-0.00282$

$0.00054-0.00319$

$0.00046-0.00352$

$0.00035-0.00379$

$0.00021-0.00401$

$\begin{array}{rr}0.00000 & -0.00001 \\ 0.00001 & -0.00003 \\ 0.00002 & -0.00006 \\ 0.00004 & -0.00013 \\ 0.00008 & -0.00026 \\ 0.00022 & -0.00061 \\ 0.00053 & -0.00105 \\ 0.00096 & -0.00119 \\ 0.00145 & -0.00097 \\ 0.00181 & -0.00034 \\ 0.00173 & 0.00070 \\ 0.00085 & 0.00203 \\ & \\ 0.00017 & -0.00049 \\ 0.00038 & -0.00089 \\ 0.00067 & -0.00113 \\ 0.00103 & -0.00118 \\ 0.00141 & -0.00100 \\ 0.00174 & -0.00057 \\ 0.00185 & 0.00013 \\ 0.00158 & 0.00106 \\ 0.00075 & 0.00212 \\ -0.00080 & 0.00308\end{array}$

$-0.00000-0.00000$

$-0.00000-0.00000$

$-0.00000-0.00000$

$-0.00000-0.00001$

$-0.00001-0.00003$

$-0.00006-0.00019$

$-0.00020-0.00070$

$-0.00030-0.00138$

$-0.00022-0.00205$

$0.00021-0.00249$

$0.00106-0.00243$

$0.00221-0.00152$

$-0.00004-0.00012$

$-0.00013-0.00045$

$-0.00025-0.00094$

$-0.00031-0.00148$

$0.00024-0.00201$

$0.00005-0.00240$

$0.00059-0.00255$

$0.00137-0.00228$

$\begin{array}{rr}0.00229 & -0.00141 \\ 0.00312 & 0.00018\end{array}$
0.01

0.02

0.05
0.10

0.20

0.20

0.50
1.00

1.50

2.00

2.50

3.00
3.50

$0.125 \pi$ $0.250 \pi$

$0.500 \pi$

$0.625 \pi$

$0.750 \pi$

$0.875 \pi$

$1.000 \pi$

$1.125 \pi$
$1.250 \pi$

$0.00000-0.00000$

$0.00000-0.00000$

$0.00000-0.00001$

$0.00000-0.00001$

$0.00001-0.00003$

$0.00003 \cdot-0.00006$

$0.00008-0.00012$

$0.00018-0.00013$

$0.00033-0.00008$

$0.00051 \quad 0.00006$

$\begin{array}{ll}0.00068 & 0.00033 \\ 0.00078 & 0.00075\end{array}$

$0.00002-0.00005$

$0.00005-0.00010$

$0.00011-0.00013$

$0.00020-0.00013$

$0.00032-0.00009$

$0.00046 \quad 0.00001$

$0.00060 \quad 0.00018$

$\begin{array}{lll}0.00072 & 0.00044\end{array}$

$0.00078 \quad 0.00078$

$$
\frac{a}{\lambda}=1.2 \quad \frac{\alpha}{\beta}=1.00
$$

$\begin{array}{ll}-0.00000 & -0.000000 \\ -0.00000 & -0.00000 \\ -0.00000 & -0.00000 \\ -0.00000 & -0.00000 \\ -0.00000 & -0.00000 \\ -0.00001 & -0.00002 \\ -0.00003 & -0.00009 \\ -0.00004 & -0.00021 \\ 0.00001 & -0.00037 \\ 0.00015 & -0.00055 \\ 0.00042 & -0.00072 \\ 0.00083 & -0.00082 \\ & \\ -0.00001 & -0.00001 \\ -0.00002 & -0.00006 \\ -0.00004 & -0.00013 \\ -0.00004 & -0.00023 \\ 0.00000 & -0.00035 \\ 0.00010 & -0.00050 \\ 0.00027 & -0.00064 \\ 0.00052 & -0.00075 \\ 0.00087 & -0.00082 \\ 0.00130 & -0.00081\end{array}$

$0.00000-0.00000$

$0.00000-0.00000$

$0.00000-0.00001$

$0.00000-0.00001$

$0.00001-0.00003$

$0.00002-0.00007$

$0.00004 \cdot-0.00013$

$0.00005-0.00019$

$0.00006-0.00030$

$0.00005-0.00034$

$0.00004-0.00037$

$0.00002-0.00005$

$0.00003-0.00010$

$0.00005-0.00015$

$0.00005-0.00020$

$0.00006-0.00024$

$0.00006-0.00028$

$0.00006-0.00032$

$0.00005-0.00035$

$0.00004-0.00037$

$0.00003-0.00039$
$0.00000-0.00000$ $0.00000-0.00000$ $0.00000-0.0000$ $0.00000-0.0000$ $0.00001-0.00003$ $0.00003-0.00006$ $0.00007-0.0001$ $0.00014-0.00013$ $0.00023-0.00008$ $0.00031 \quad 0.00005$ $0.00030 \quad 0.00030$ $0.00008 \quad 0.00067$

$0.00002-0.00005$ $0.00005-0.00009$ $0.00009-0.00012$ $0.00015-0.00012$ $0.00023-0.00009$ $0.00023-0.00009$ $0.00033 \quad 0.00016$ $0.00033 \quad 0.00016$ $0.00027 \quad 0.00040$

$-0.00041 \quad 0.00101$
$-0.00000-0.00000$ $-0.00000-0.00000$ $-0.00000-0.00000$ $-0.00000-0.00000$ $-0.00000-0.00000$ $-0.00001-0.00002$ $-0.00003-0.00008$ $\begin{array}{ll}-0.00005 & -0.00017\end{array}$ $-0.00002-0.00028$ $0.00010-0.00036$ $0.00033-0.00035$ $0.00069-0.00013$

$-0.00001-0.00001$ $-0.00002-0.00005$ $-0.00004-0.00011$ $-0.00005-0.00019$ $-0.00002-0.00027$ $0.00005-0.00034$ $0.00020-0.00037$ $0.00042-0.00032$

$\begin{array}{ll}0.00102 & 0.00037\end{array}$
$0.00000-0.00001$ $0.00001-0.00003$ $\begin{array}{ll}0.00002-0.0006 \\ 0.00004 & -0.00013\end{array}$ 0.000013 0.00026
$0.00008-0.0006$ $0.00019-0.00064$ $0.00029-0.00122$ $0.00025-0.00170$ $0.00009-0.00202$ $-0.00025-0.00219$ $-0.00034-0.00214$

$0.00016-0.00050$ $0.00026-0.00098$ $0.00029-0.00141$ $0.00023-0.00176$ $-0.00005-0.00215$ $\begin{array}{ll}-0.00018 & -0.00219\end{array}$ $-0.00028-0.00218$. $-0.00034-0.00214$ $-0.00036-0.00210$

$\begin{aligned} 0.00000 & -0.00000 \\ 0.00000 & -0.00000 \\ 0.00000 & -0.00001 \\ 0.00000 & -0.00001 \\ 0.00001 & -0.00003 \\ 0.00002 & -0.00007 \\ 0.00003 & -0.00012 \\ 0.00003 & -0.00017 \\ 0.00001 & -0.00020 \\ -0.00000 & -0.00021 \\ -0.00001 & -0.00021 \\ -0.00001 & -0.00020 \\ & \\ 0.00002 & -0.00005 \\ 0.00003 & -0.00010 \\ 0.00003 & -0.00014 \\ 0.00002 & -0.00017 \\ 0.00001 & -0.00019 \\ 0.00000 & -0.00020 \\ -0.00001 & -0.00021 \\ -0.00001 & -0.00021 \\ -0.00001 & -0.00020 \\ -0.00002 & -0.00020\end{aligned}$




\begin{tabular}{|c|c|c|}
\hline $\begin{array}{l}0.01 \\
0.02 \\
0.05 \\
0.10 \\
0.20 \\
0.50 \\
1.00 \\
1.50 \\
2.00 \\
2.50 \\
3.00 \\
3.50\end{array}$ & $\begin{array}{r}-0.00184 \\
-0.00368 \\
-0.00919 \\
-0.01837 \\
-0.03656 \\
-0.08843 \\
-0.15627 \\
-0.18627 \\
-0.16846 \\
-0.10396 \\
-0.00691 \\
0.09775\end{array}$ & $\begin{array}{l}-0.00134 \\
-0.00267 \\
-0.00668 \\
-0.01334 \\
-0.02652 \\
-0.06363 \\
-0.10974 \\
-0.12804 \\
-0.11982 \\
-0.09716 \\
-0.07698 \\
-0.07203\end{array}$ \\
\hline $\begin{array}{l}0.125 \pi \\
0.250 \pi \\
0.375 \pi \\
0.500 \pi \\
0.625 \pi \\
0.750 \pi \\
0.875 \pi \\
1.000 \pi \\
1.125 \pi \\
1.250 \pi\end{array}$ & $\begin{array}{r}-0.07051 \\
-0.13090 \\
-0.17200 \\
-0.18676 \\
-0.17143 \\
-0.12671 \\
-0.05835 \\
0.02320 \\
0.10448 \\
0.17179\end{array}$ & $\begin{array}{l}-0.05092 \\
-0.09299 \\
-0.11964 \\
-0.12830 \\
-0.12110 \\
-0.10417 \\
-0.08580 \\
-0.07372 \\
-0.07236 \\
-0.08096\end{array}$ \\
\hline
\end{tabular}

$\begin{array}{ll}-0.00001 & -0.00001 \\ -0.00004 & -0.00003 \\ -0.00023 & -0.00017 \\ -0.00092 & -0.00007 \\ -0.00367 & -0.00266 \\ -0.02261 & -0.01619 \\ -0.08594 & -0.05889 \\ -0.17667 & -0.11302 \\ -0.27412 & -0.16078 \\ -0.35381 & -0.18972 \\ -0.39381 & -0.19878 \\ -0.38247 & -0.19901 \\ -0.01404 & -0.01011 \\ -0.05443 & -0.03813 \\ -0.11605 & -0.07781 \\ -0.19061 & -0.12055 \\ -0.26731 & -0.15783 \\ -0.33412 & -0.18363 \\ -0.37974 & -0.19627 \\ -0.39595 & -0.19903 \\ -0.37982 & -0.19914 \\ -0.33495 & -0.20537\end{array}$

$\begin{array}{ll}-0.00184 & -0.00134 \\ -0.00368 & -0.00267 \\ -0.00920 & -0.00668 \\ -0.01840 & -0.01336 \\ -0.03681 & -0.02670 \\ -0.09224 & -0.06635 \\ -0.18601 & -0.12985 \\ -0.28258 & -0.18758 \\ -0.38268 & -0.23655 \\ -0.48628 & -0.27368 \\ -0.59235 & -0.29603 \\ -0.09873 & -0.30097 \\ -0.07236 & -0.05225 \\ -0.14548 & -0.10313 \\ -0.22003 & -0.15123 \\ -0.29653 & -0.19512 \\ -0.37525 & -0.23334 \\ -0.45617 & -0.26439 \\ -0.53888 & -0.28683 \\ -0.62256 & -0.29930 \\ -0.70596 & -0.30062 \\ -0.78738 & -0.28994\end{array}$

$\begin{array}{ll}-0.00184 & -0.00134 \\ -0.00368 & -0.00267 \\ -0.00919 & -0.00668 \\ -0.01837 & -0.01333 \\ -0.03661 & -0.02643 \\ -0.08917 & -0.06227 \\ -0.16084 & -0.10001 \\ -0.19666 & -0.10186 \\ -0.18521 & -0.07717 \\ -0.13572 & -0.04941 \\ -0.08055 & -0.03440 \\ -0.05339 & -0.02248 \\ -0.07088 & -0.05025 \\ -0.13342 & -0.08798 \\ -0.17859 & -0.10476 \\ -0.19797 & -0.09951 \\ -0.18760 & -0.07938 \\ -0.15227 & -0.05638 \\ -0.10650 & -0.04030 \\ -0.06908 & -0.03175 \\ -0.05290 & -0.02114 \\ -0.05692 & 0.00361\end{array}$

$\begin{array}{ll}-0.00001 & -0.00001\end{array}$ $-0.00004-0.00003$ $-0.00023-0.00017$ $\begin{array}{ll}-0.00092 & -0.00067\end{array}$ $-0.00367-0.00265$ $-0.02280-0.01584$ $-0.08824 \quad-0.05354$ $-0.28249-0.08477$ $-0.34957-0.06466$ $\begin{array}{ll}-0.34957 & -0.06466 \\ -0.36388 & -0.01340\end{array}$ $-0.33523 \quad 0.03815$

$-0.01411-0.00997$ $-0.05543-0.03605$ $-0.11990-0.06777$ $-0.19836-0.09144$ $-0.27595-0.0955$ $-0.33526-0.07651$ $-0.36344-0.04028$ $-0.35896 \quad 0.00164$ $\begin{array}{ll}-0.33243 & 0.04155 \\ -0.29862 & 0.08254\end{array}$

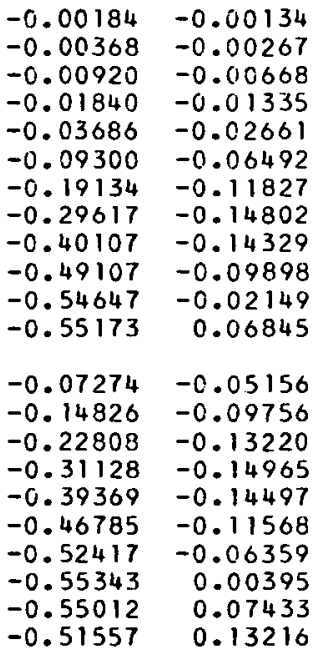

$$
\frac{\mathrm{a}}{\lambda}=1.4 \quad \frac{\alpha}{\beta}=0.005
$$

$\begin{array}{llll}-0.00001 & -0.00001 & -0.00176 & -0.00128 \\ -0.00004 & -0.00003 & -0.00352 & -0.00256 \\ -0.00022 & -0.00016 & -0.00880 & -0.00639 \\ -0.00088 & -0.00063 & -0.01760 & -0.01279 \\ -0.00352 & -0.00253 & -0.03522 & -0.02555 \\ -0.02171 & -0.01539 & -0.08826 & -0.06349 \\ -0.08246 & -0.05601 & -0.17798 & -0.12425 \\ -0.16936 & -0.10762 & -0.27036 & -0.17948 \\ -0.26247 & -0.15344 & -0.36608 & -0.22629 \\ -0.33838 & -0.18180 & -0.46508 & -0.26178 \\ -0.37632 & -0.19173 & -0.56636 & -0.28312 \\ -0.36543 & -0.19358 & -0.66787 & -0.28784 \\ & & & \\ -0.01348 & -0.00961 & -0.06925 & -0.05000 \\ -0.05225 & -0.03626 & -0.13921 & -0.09868 \\ -0.11132 & -0.07403 & -0.21053 & -0.14470 \\ -0.19269 & -0.11481 & -0.28370 & -0.18668 \\ -0.25597 & -0.15060 & -0.35897 & -0.22322 \\ -0.31965 & -0.17572 & -0.43631 & -0.25290 \\ -0.36301 & -0.18863 & -0.51531 & -0.27434 \\ -0.37831 & -0.19241 & -0.59529 & -0.28624 \\ -0.36290 & -0.19382 & -0.67477 & -0.28750 \\ -0.32028 & -0.20095 & -0.75239 & -0.27732\end{array}$

$-0.32028-0.20095$

$\begin{array}{ll}-0.00176 & -0.00123 . \\ -0.00352 & -0.00256 \\ -0.00880 & -0.00639 \\ -0.01758 & -0.01275 \\ -0.03503 & -0.02530 \\ -0.08530 & -0.05962 \\ -0.15370 & -0.09596 \\ -0.18766 & -0.09826 \\ -0.17651 & -0.07542 \\ -0.12933 & -0.04946 \\ -0.07690 & -0.03499 \\ -0.05093 & -0.02278 \\ -0.06782 & -0.04811 \\ -0.12756 & -0.08432 \\ -0.17057 & -0.10065 \\ -0.18887 & -0.09612 \\ -0.17880 & -0.07747 \\ -0.14507 & -0.05601 \\ -0.10155 & -0.04080 \\ -0.06598 & -0.03227 \\ -0.05045 & -0.02143 \\ -0.05397 & 0.00286\end{array}$

$\begin{array}{ll}-0.00001 & -0.00001 \\ -0.00004 & -0.00003 \\ -0.00022 & -0.00016 \\ -0.00088 & -0.00063 \\ -0.00353 & -0.00252 \\ -0.02189 & -0.01505 \\ -0.08462 & -0.05087 \\ -0.17598 & -0.08397 \\ -0.27006 & -0.09028 \\ -0.33384 & -0.06224 \\ -0.34742 & -0.01432 \\ -0.32014 & 0.03439 \\ -0.01355 & -0.00948 \\ -0.05319 & -0.03425 \\ -0.11492 & -0.06439 \\ -0.18989 & -0.08692 \\ -0.26384 & -0.09103 \\ -0.32025 & -0.07330 \\ -0.34701 & -0.03945 \\ -0.34275 & -0.00013 \\ -0.31746 & 0.03763 \\ -0.28491 & 0.07686\end{array}$

$-0.28491$

$\begin{array}{ll}-0.00176 & -0.00128 \\ -0.00352 & -0.00256 \\ -0.00880 & -0.00639 \\ -0.01761 & -0.01277 \\ -0.03527 & -0.02546 \\ -0.08899 & -0.06212 \\ -0.18304 & -0.11315 \\ -0.28318 & -0.14156 \\ -0.38322 & -0.13706 \\ -0.46889 & -0.09490 \\ -0.52150 & -0.02132 \\ -0.52649 & 0.06383 \\ -0.06961 & -0.04934 \\ -0.14185 & -0.09334 \\ -0.21815 & -0.12645 \\ -0.29760 & -0.14312 \\ -0.37619 & -0.13866 \\ -0.44681 & -0.11077 \\ -0.50034 & -0.06127 \\ -0.52810 & 0.00278 \\ -0.52497 & 0.06938 \\ -0.49236 & 0.12397 \\ -0.1230 & \end{array}$

$\begin{array}{lll}0.01 & -0.00176 & -0.00128 \\ 0.02 & -0.00352 & -0.00256 \\ 0.05 & -0.00880 & -0.00639 \\ 0.10 & -0.01758 & -0.01276 \\ 0.20 & -0.03499 & -0.02538 \\ 0.50 & -0.08460 & -0.06093 \\ 1.00 & -0.14935 & -0.10528 \\ 1.50 & -0.17773 & -0.12333 \\ 2.00 & -0.16030 & -0.11629 \\ 2.50 & -0.09835 & -0.09549 \\ 3.00 & -0.00556 & -0.07672 \\ 3.50 & 0.09433 & -0.07182 \\ 0.125 \pi & -0.06747 & -0.04875 \\ 0.250 \pi & -0.12517 & -0.08912 \\ 0.375 \pi & -0.16431 & -0.11491 \\ 0.500 \pi & -0.17814 & -0.12369 \\ 0.025 \pi & -0.16317 & -0.11745 \\ 0.750 \pi & -0.12016 & -0.10196 \\ 0.875 \pi & -0.05471 & -0.08496 \\ 1.000 \pi & 0.02319 & -0.07365 \\ 1.125 \pi & 0.10075 & -0.07208 \\ 1.250 \pi & 0.16505 & -0.07948\end{array}$


$C(h, 0)$

$S(h, 0)$

0.0

$\begin{array}{ll}-0.00168 & -0.00122 \\ -0.00337 & -0.000245\end{array}$

$\begin{array}{ll}-0.00842 & -0.00245\end{array}$

$\begin{array}{lll}0.20 & -0.03348 & -0.02429\end{array}$

$0.50 \quad-0.08093-0.05834$

$\begin{array}{lll}1.00 & -0.14274 & -0.10100 \\ 1.50 & -0.16957 & -0.11879\end{array}$

$2.00-0.152 \dot{5} 4-0.11284$

$\begin{array}{lll}3.00 & -0.00428 & -0.07634\end{array}$

3.50

$0.125 \pi$

$0.09107-0.07147$

$0.250 \pi \quad-0.11969-0.0854$

$0.625 \pi-0.15530-0.11388$

$0.750 \pi-0.11394-0.09973$

$0.875 \pi-0.05126-0.08402$

$1.000 \pi \quad 0.02317-0.07343$

$1.125 \pi \quad 0.09720-0.07169$

$0.09720-0.07169$
$-0.01682-0.01222$

$2.50-0.09303-0.09377$

$0.500 \pi-0.16992-0.11036$

$$
\frac{\mathrm{a}}{\lambda}=1.5 \quad \frac{\alpha}{\beta}=0.01
$$

$E(h, 0)$

$C(h, h)$

$S(h, h)$

$E(h, h)$

$\begin{array}{ll}-0.00001 & -0.00001 \\ -0.00003 & -0.00002 \\ -0.00021 & -0.00015 \\ -0.00085 & -0.00060 \\ -0.00338 & -0.00240 \\ -0.02085 & -0.01463 \\ -0.07913 & -0.05327 \\ -0.16235 & -0.10247 \\ -0.25132 & -0.14644 \\ -0.32364 & -0.17422 \\ -0.35964 & -0.18493 \\ -0.34917 & -0.18824 \\ -0.01295 & -0.00913 \\ -0.05015 & -0.03447 \\ -0.10679 & -0.07042 \\ -0.17511 & -0.10935 \\ -0.24512 & -0.14370 \\ -0.30582 & -0.16816 \\ -0.34703 & -0.18130 \\ -0.36149 & -0.18600 \\ -0.34677 & -0.18857 \\ -0.30626 & -0.19651\end{array}$

$-0.00168-0.00122$ $-0.00337-0.00245$ $-0.00842-0.00612$ $-0.01685-0.01224$ $-0.03371-0.02445$ $-0.17031-0.0678$ $-0.25867-0.17172$ $-0.35019-0.21643$ $-0.44479-0.25040$ $\begin{array}{ll}-0.44479 & -0.25040 \\ -0.54152 & -0.27078\end{array}$

$-0.03838-0.27528$

$-0.06627-0.04785$ $-0.13321-0.09443$ $-0.20145-0.13846$ $-0.27143-0.17861$ $-0.34340-0.21355$ $-0.41731-0.24192$ $-0.49278-0.26239$ $-0.56905-0.27375$ $-0.64495-0.2749$ $-0.71895-0.26525$

$$
\frac{\mathrm{a}}{\lambda}=1.4 \quad \frac{\alpha}{\beta}=0.02
$$

\begin{tabular}{|c|c|c|c|c|}
\hline $\begin{array}{l}0.01 \\
0.02 \\
0.05 \\
0.10 \\
0.20 \\
0.50 \\
1.00 \\
1.50 \\
2.00 \\
2.50 \\
3.00 \\
3.50\end{array}$ & $\begin{array}{r}-0.00154 \\
-0.00309 \\
-0.00771 \\
-0.01540 \\
-0.03066 \\
-0.07407 \\
-0.13038 \\
-0.15438 \\
-0.13810 \\
-0.08319 \\
-0.00196 \\
0.08500\end{array}$ & $\begin{array}{l}-0.00112 \\
-0.00224 \\
-0.00560 \\
-0.011119 \\
-0.02225 \\
-0.05349 \\
-0.09296 \\
-0.11020 \\
-0.10617 \\
-0.09023 \\
-0.07522 \\
-0.07046\end{array}$ & $\begin{array}{l}-0.00001 \\
-0.00003 \\
-0.00020 \\
-0.00078 \\
-0.00312 \\
-0.01922 \\
-0.07286 \\
-0.14921 \\
-0.23045 \\
-0.29612 \\
-0.32853 \\
-0.31886\end{array}$ & $\begin{array}{l}-0.00001 \\
-0.00002 \\
-0.00014 \\
-0.00054 \\
-0.00217 \\
-0.01321 \\
-0.04817 \\
-0.09291 \\
-0.13340 \\
-0.16002 \\
-0.17202 \\
-0.17785\end{array}$ \\
\hline $\begin{array}{l}0.125 \pi \\
0.250 \pi \\
0.375 \pi \\
0.500 \pi \\
0.625 \pi \\
0.750 \pi \\
0.875 \pi \\
1.000 \pi \\
1.125 \pi \\
1.250 \pi\end{array}$ & $\begin{array}{r}-0.05909 \\
-0.10943 \\
-0.14322 \\
-0.15460 \\
-0.14068 \\
-0.10241 \\
-0.04490 \\
0.02309 \\
0.09059 \\
0.14674\end{array}$ & $\begin{array}{l}-0.04277 \\
-0.07845 \\
-0.10180 \\
-0.11078 \\
-0.10702 \\
-0.09528 \\
-0.08190 \\
-0.07262 \\
-0.07057 \\
-0.07492\end{array}$ & $\begin{array}{l}-0.01194 \\
-0.04621 \\
-0.09827 \\
-0.16088 \\
-0.22480 \\
-0.27998 \\
-0.31724 \\
-0.33014 \\
-0.31668 \\
-0.28006\end{array}$ & $\begin{array}{l}-0.00825 \\
-0.03116 \\
-0.06374 \\
-0.09920 \\
-0.13084 \\
-0.15402 \\
-0.16747 \\
-0.17376 \\
-0.17835 \\
-0.18765\end{array}$ \\
\hline
\end{tabular}

(a)

$\begin{array}{ll}-0.00168 & -0.00122 \\ -0.00337 & -0.00245 \\ -0.00842 & -0.00612 \\ -0.01682 & -0.01221 \\ -0.03352 & -0.02421 \\ -0.08160 & -0.05708 \\ -0.14688 & -0.09206 \\ -0.17907 & -0.09479 \\ -0.16822 & -0.07367 \\ -0.12323 & -0.04940 \\ -0.07338 & -0.03546 \\ -0.04855 & -0.02301 \\ & \\ -0.06488 & -0.04605 \\ -0.12196 & -0.08080 \\ -0.16292 & -0.09670 \\ -0.18019 & -0.09283 \\ -0.17042 & -0.07558 \\ -0.13821 & -0.05555 \\ -0.09682 & -0.04117 \\ -0.06299 & -0.03268 \\ -0.04807 & -0.02166 \\ -0.05114 & 0.00216\end{array}$

$\begin{array}{ll}-0.00001 & -0.00001 \\ -0.00003 & -0.00002 \\ -0.00021 & -0.00015 \\ -0.00085 & -0.00060 \\ -0.00339 & -0.00239 \\ -0.02101 & -0.01430 \\ -0.08115 & -0.04833 \\ -0.16850 & -0.07981 \\ -0.25820 & -0.08606 \\ -0.31884 & -0.05991 \\ -0.33173 & -0.01507 \\ -0.30573 & 0.03090 \\ -0.01301 & -0.00901 \\ -0.05103 & -0.03254 \\ -0.11015 & -0.06117 \\ -0.18179 & -0.08261 \\ -0.25227 & -0.08669 \\ -0.30593 & -0.07023 \\ -0.33134 & -0.03861 \\ -0.32729 & -0.00174 \\ -0.30316 & 0.03398 \\ -0.27181 & 0.07152\end{array}$

$\begin{array}{ll}-0.00154 & -0.00112 \\ -0.00309 & -0.00224 \\ -0.00771 & -0.00560 \\ -0.01543 & -0.01120 \\ -0.03087 & -0.02239 \\ -0.07735 & -0.05564 \\ -0.15594 & -0.10886 \\ -0.23679 & -0.15720 \\ -0.32045 & -0.19812 \\ -0.40685 & -0.22909 \\ -0.49505 & -0.24768 \\ -0.58324 & -0.25178 \\ & \\ -0.06069 & -0.04382 \\ -0.12198 & -0.08647 \\ -0.18444 & -0.12677 \\ -0.24846 & -0.16350 \\ -0.31425 & -0.19544 \\ -0.38176 & -0.22135 \\ -0.45062 & -0.24003 \\ -0.52013 & -0.25039 \\ -0.58922 & -0.25149 \\ -0.65648 & -0.24267\end{array}$

18
17
33
74
18
20
01
06
30
20
21
26
56
85
44
56

$\begin{array}{ll}-0.00001 & -0.00001 \\ -0.00003 & -0.00002 \\ -0.00020 & -0.00014 \\ -0.00078 & -0.00054 \\ -0.00313 & -0.00216 \\ -0.01937 & -0.01291 \\ -0.07463 & -0.04362 \\ -0.15451 & -0.07208 \\ -0.23605 & -0.07806 \\ -0.29087 & -0.05549 \\ -0.30247 & -0.01628 \\ -0.27880 & 0.02463 \\ -0.01200 & -0.00813 \\ -0.04698 & -0.02937 \\ -0.10120 & -0.05521 \\ -0.16661 & -0.07464 \\ -0.23067 & -0.07863 \\ -0.27922 & -0.06447 \\ -0.30213 & -0.03692 \\ -0.29844 & -0.00453 \\ -0.27645 & 0.02741 \\ -0.24732 & 0.06175\end{array}$

$-0.00168-0.00122$ $-0.00337-0.00245$ $-0.00842-0.00612$ $\begin{array}{ll}-0.01685 & -0.01222\end{array}$ $\begin{array}{ll}-0.033716 & -0.02437 \\ -0.08516 & -0.05945\end{array}$ $-0.17509-0.10824$ $-0.27076-0.13539$ $-0.36618-0.13110$ $-0.44772-0.09098$ $-0.44772 \quad-0.09098$ $\begin{array}{rr}-0.49767 & -0.02113 \\ -0.50241 & 0.05949\end{array}$

\section{$-0.06661-0.04722$} $-0.13571-0.08931$ $-0.20865-0.12096$ $-0.28452-0.13688$ $-0.35948-0.13263$ $-0.42672 \quad-0.10608$ $-0.50393-0.00172$ $-0.50097 \quad 0.06474$ $\begin{array}{ll}-0.50097 & 0.06474 \\ -0.47020 & 0.11628\end{array}$

$\begin{array}{ll}-0.00154 & -0.00112 \\ -0.00309 & -0.00224 \\ -0.00771 & -0.00560 \\ -0.01543 & -0.01120 \\ -0.03091 & -0.02231 \\ -0.07797 & -0.05443 \\ -0.16023 & -0.09906 \\ -0.24753 & -0.12384 \\ -0.33432 & -0.11995 \\ -0.40820 & -0.08361 \\ -0.45325 & -0.02066 \\ -0.45751 & 0.05161 \\ & \\ -0.06099 & -0.04324 \\ -0.12423 & -0.08175 \\ -0.19088 & -0.11067 \\ -0.26007 & -0.12519 \\ -0.32824 & -0.12133 \\ -0.38920 & -0.09727 \\ -0.43517 & -0.05478 \\ -0.45887 & -0.00014 \\ -0.45623 & 0.05630 \\ -0.42882 & 0.10222\end{array}$




$\begin{array}{lll}0.01 & -0.00141 & -0.00103 \\ 0.02 & -0.00283 & -0.00205 \\ 0.05 & -0.00706 & -0.00513 \\ 0.10 & -0.01410 & -0.01025 \\ 0.20 & -0.02807 & -0.02038 \\ 0.50 & -0.06779 & -0.04904 \\ 1.00 & -0.11910 & -0.08555 \\ 1.50 & -0.14055 & -0.10221 \\ 2.00 & -0.12502 & -0.09983 \\ 2.50 & -0.07433 & -0.08061 \\ 3.00 & 0.00007 & -0.07371 \\ 3.50 & 0.07946 & -0.06907 \\ 0.125 \pi & -0.05409 & -0.03919 \\ 0.250 \pi & -0.10006 & -0.07206 \\ 0.375 \pi & -0.13069 & -0.09390 \\ 0.500 \pi & -0.14066 & -0.10291 \\ 0.625 \pi & -0.12743 & -0.10050 \\ 0.750 \pi & -0.09201 & -0.09686 \\ 0.875 \pi & -0.03920 & -0.07951 \\ 1.000 \pi & 0.02296 & -0.07138 \\ 1.125 \pi & 0.08456 & -0.06911 \\ 1.250 \pi & 0.13596 & -0.07181\end{array}$

$1.125 \pi \cdot 0.08456-0.06911$

$\begin{array}{ll}-0.00001 & -0.00000 \\ -0.00003 & -0.00002 \\ -0.00018 & -0.00012 \\ -0.00072 & -0.00049 \\ -0.00288 & -0.00196 \\ -0.01772 & -0.01193 \\ -0.06709 & -0.04356 \\ -0.13714 & -0.08424 \\ -0.21136 & -0.12154 \\ -0.27101 & -0.14699 \\ -0.30021 & -0.15999 \\ -0.29127 & -0.16787 \\ -0.01101 & -0.00745 \\ -0.04258 & -0.02816 \\ -0.09044 & -0.05768 \\ -0.14783 & -0.08999 \\ -0.20621 & -0.11915 \\ -0.25639 & -0.14109 \\ -0.29008 & -0.15472 \\ -0.30160 & -0.16228 \\ -0.28929 & -0.16851 \\ -0.25613 & -0.17886\end{array}$

$\begin{array}{ll}-0.28929 & -0.16851 \\ -0.25613 & -0.17886\end{array}$

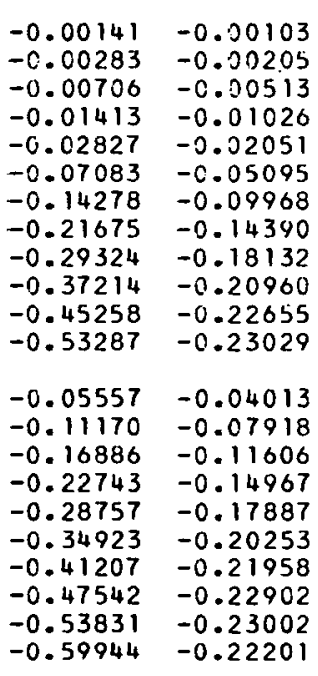

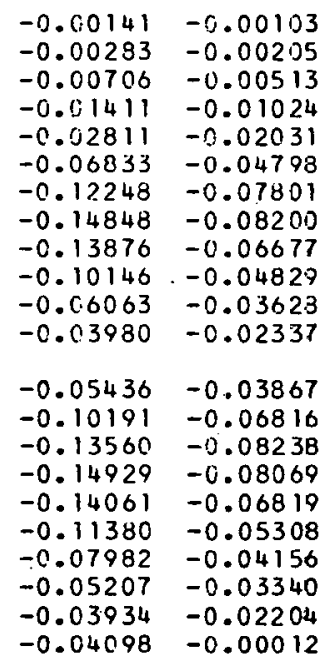

$\begin{array}{ll}-0.00001 & -0.00000 \\ -0.00003 & -0.00002 \\ -0.00018 & -0.00012 \\ -0.00072 & -0.00049 \\ -0.00288 & -0.00195 \\ -0.01785 & -0.01165 \\ -0.06864 & -0.03936 \\ -0.14169 & -0.06509 \\ -0.21583 & -0.07086 \\ -0.26542 & -0.05140 \\ -0.27583 & -0.01716 \\ -0.25422 & 0.01922 \\ -0.01106 & -0.00734 \\ -0.04326 & -0.02651 \\ -0.09298 & -0.04982 \\ -0.15272 & -0.06743 \\ -0.21096 & -0.07133 \\ -0.25490 & -0.05918 \\ -0.27556 & -0.03522 \\ -0.27216 & -0.00681 \\ -0.25206 & 0.02173 \\ -0.22497 & 0.05310\end{array}$

$-0.00141-0.00103$ $-0.00283-0.00205$ $-0.00706-0.00513$ $-0.01413-0.01025$ $\begin{array}{lll}-0.02831 & -0.02043\end{array}$ $-0.07139-0.04984$ $-0.14662-0.09066$ $-0.22629-0.11328$ $-0.30524-0.10975$ $-0.37217-0.07684$ $-0.41279-0.02010$ $-0.41664 \quad 0.04468$

$\begin{array}{ll}-0.05585 & -0.03959\end{array}$ $\begin{array}{ll}-0.11371 & -0.07483 \\ -0.17462 & -0.10127\end{array}$ $-0.23771-0.11451$ $-0.29972-0.11100$ $-0.35499-0.08919$ $-0.39652-0.05081$ $-0.39652-0.05081$ $-0.41784-0.00167$ $\begin{array}{ll}-0.41549 & 0.04887 \\ -0.39108 & 0.08980\end{array}$

$$
\frac{a}{\lambda}=i .4 \quad \frac{\alpha}{\beta}=0.05
$$

$\begin{array}{lrlll}0.01 & -0.00118 & -0.00086 & -0.00001 & -0.00000 \\ 0.02 & -0.00237 & -0.00172 & -0.00002 & -0.00002 \\ 0.05 & -0.00592 & -0.00430 & -0.00015 & -0.00010 \\ 0.10 & -0.01183 & -0.00859 & -0.00061 & -0.00040 \\ 0.20 & -0.02354 & -0.01710 & -0.00245 & -0.00160 \\ 0.50 & -0.05678 & -0.04123 & -0.01506 & -0.00973 \\ 1.00 & -0.09938 & -0.07247 & -0.05689 & -0.03561 \\ 1.50 & -0.11649 & -0.08790 & -0.11589 & -0.06925 \\ 2.00 & -0.10243 & -0.08808 & -0.17787 & -0.10093 \\ 2.50 & -0.05917 & -0.07927 & -0.22718 & -0.12410 \\ 3.00 & 0.00338 & -0.06983 & -0.25091 & -0.13834 \\ 3.50 & 0.06976 & -0.06547 & -0.24325 & -0.14917 \\ 0.125 \pi & -0.04533 & -0.03292 & -0.00936 & -0.00607 \\ 0.250 \pi & -0.08366 & -0.06079 & -0.03615 & -0.02298 \\ 0.375 \pi & -0.10884 & -0.07989 & -0.07660 & -0.04723 \\ 0.500 \pi & -0.11644 & -0.08875 & -0.12484 & -0.07406 \\ 0.625 \pi & -0.10452 & -0.08847 & -0.17359 & -0.09885 \\ 0.750 \pi & -0.07416 & -0.08222 & -0.21515 & -0.11846 \\ 0.875 \pi & -0.02956 & -0.07417 & -0.24276 & -0.13207 \\ 1.000 \pi & 0.02253 & -0.06797 & -0.25195 & -0.14141 \\ 1.125 \pi & 0.07403 & -0.06538 & -0.24161 & -0.14999 \\ 1.250 \pi & 0.11724 & -0.06556 & -0.21431 & -0.16173\end{array}$

$\begin{array}{ll}-0.00118 & -0.00086 \\ -0.00237 & -0.00172 \\ -0.00592 & -0.00430 \\ -0.01185 & -0.00861 \\ -0.02371 & -0.01720 \\ -0.05940 & -0.04273 \\ -0.11970 & -0.08356 \\ -0.18163 & -0.12059 \\ -0.24556 & -0.15186 \\ -0.31135 & -0.17545 \\ -0.37824 & -0.18955 \\ -0.44480 & -0.19265 \\ -0.04661 & -0.03365 \\ -0.09366 & -0.06639 \\ -0.14154 & -0.09729 \\ -0.19056 & -0.12541 \\ -0.24082 & -0.14982 \\ -0.29226 & -0.16956 \\ -0.34458 & -0.18376 \\ -0.39721 & -0.19160 \\ -0.44931 & -0.19243 \\ -0.49980 & -0.18581\end{array}$

$\begin{array}{ll}-0.00118 & -0.00086 \\ -0.00237 & -0.00172 \\ -0.00592 & -0.00430 \\ -0.01183 & -0.00859 \\ -0.02357 & -0.01704 \\ -0.05722 & -0.04032 \\ -0.10215 & -0.06609 \\ -0.12313 & -0.07088 \\ -0.11443 & -0.06013 \\ -0.08341 & -0.04616 \\ -0.04979 & -0.03582 \\ -0.03222 & -0.02301 \\ & \\ -0.04555 & -0.03247 \\ -0.08517 & -0.05749 \\ -0.11287 & -0.07017 \\ -0.12369 & -0.07004 \\ -0.11600 & -0.06117 \\ -0.09360 & -0.04987 \\ -0.06560 & -0.04064 \\ -0.04270 & -0.03297 \\ -0.03179 & -0.02173 \\ -0.03248 & -0.00170\end{array}$

$-0.00001-0.00000$ $\begin{array}{ll}-0.00001 & -0.00000 \\ -0.00002 & -0.00002\end{array}$ $\begin{array}{ll}-0.00002 & -0.00002 \\ -0.00015 & -0.00010\end{array}$ $-0.00061-0.00040$ $-0.00245-0.00159$ $-0.01516-0.00949$ $-0.05806-0.03204$ $-0.11919-0.05308$ $-0.18054-0.05841$ $-0.22114-0.04408$ $\begin{array}{rr}-0.22949 & -0.01811 \\ -0.21134 & 0.01056\end{array}$

$\begin{array}{lll}-0.00940 & -0.00598\end{array}$ $-0.03666-0.02158$ $-0.07851-0.04056$ $-0.12836-0.05503$ $-0.17653-0.05872$ $-0.21257-0.04990$ $-0.22936-0.03189$ $-0.22643-0.01010$ $\begin{array}{lll}-0.20950 & 0.0126\end{array}$ $\begin{array}{lll}-0.18595 & 0.03869\end{array}$
$-0.00118-0.00086$ $-0.00237-0.00172$ $-0.00592-0.00430$ $-0.01185-0.00860$ $-0.02374-0.01714$ $\begin{array}{ll}-0.05985 & -0.04178\end{array}$ $-0.12278-0.07593$ $-0.18912,-0.09477$ $-0.25446-0.09187$ $-0.30939-0.06488$ $-0.34242-0.01879$ $-0.34555 \quad 0.03326$

$-0.04683-0.03320$ $-0.09528-0.06271$ $-0.14613 \quad-0.08478$ $-0.19860-0.09580$ $-0.24990-0.09291$ $-0.29534-0.07498$ $\begin{array}{ll}-0.32923 & -0.04368\end{array}$ $-0.34650-0.00392$ $-0.34463 \quad 0.03661$ $-0.32526 \quad 0.069 \mathrm{U}$ 
$\beta \mathrm{h}$

$C(h, 0)$

$S(h, 0)$

0.01

0.02

0.05

0.10

0.20

1.00

2.00

2.50

3.00

$0.125 \pi \quad-0.03799-0.02765$

$\begin{array}{lll}0.250 \pi & -0.06995 & -0.05129\end{array}$

$0.375 \mathrm{~m}-0.09065-0.06797$

$0.500 \pi \quad-0.09640-0.07650$

$0.625 \pi-0.08570-0.07770$

$0.750 \pi \quad-0.05965-0.07398$

$0.875 \mathrm{~N}-0.02189-0.06843$

$1.000 \pi \quad 0.02191-0.06371$

$\begin{array}{lll}1.125 . \pi & 0.06515 & -0.06097 \\ 1.250 \pi & 0.10167 & -0.05943\end{array}$ $\frac{a}{\lambda}=1.7 \quad \frac{\alpha}{\beta}=0.07$

$E(h, 0)$

$\mathrm{C}(\mathrm{h}, \mathrm{h})$

$\mathrm{S}(\mathrm{h}, \mathrm{h})$

$E(h, h)$

$-0.00099-0.00072$

$-0.00199 .-0.00144$

$-0.00497-0.00361$

$-0.00994-0.00722$

$-0.01988-0.01442$

$-0.04981-0.03583$

$-0.10035 \quad-0.07006$

$-0.15220-0.10106$

$-0.20562-0.12719$

$-0.26049-0.14686$

$-0.31612-0.1585$

$-0.37130-0.16117$

$\begin{array}{ll}-0.03908 & -0.02822\end{array}$

$-0.07853-0.05567$

$-0.20167-0.12548$

$-0.24459-0.14196$

$-0.28815-0.15378$

$-0.33186-0.16029$

$\begin{array}{ll}-0.37503 & -0.16098 \\ -0.41674 & -0.15552\end{array}$

$-0.00099-0.00072$ $-0.00199-0.00141$

$-0.00992-0.00720$

$-0.04792-0.03388$

$-0.08520-0.05600$

$-0.09435-0.0538$

-0.06846 -0.0433

$-0.06846 \quad-0.04337$

$0.04064-0.0344$

$-0.03817-0.02727$

$-0.07118-0.04848$

$-0.09396-0.05976$

$\begin{array}{ll}-0.09396 & -0.05976 \\ -0.10250 & -0.06072\end{array}$

$-0.09568-0.0546$

$-0.07691-0.04625$

$-0.05372-0.03885$

$-0.03472-0.03174$

$\begin{array}{ll}-0.02534 & -0.02093 \\ -0.02539 & -0.00273\end{array}$ $-0.00013-0.00008$
$-0.11865-0.08155$

$-0.15966-0.10509$

$-0.00497-0.0036$

$-0.04792-0.0338$ $\begin{array}{ll}-0.00001 & -0.00000\end{array}$ $-0.00002-0.00001$ $-0.00052-0.00033$ $-0.01288-0.00772$ 0.02606
$0.012-0.04328$ $-0.10030-0.04328$ $-0.15113-0.04817$ $-0.17564 \quad 0.0042$

$-0.00799-0.00486$ $-0.03108-0.01755$ $-0.06630-0.03300$ $-0.10794-0.04490$ $-0.14783-0.04835$ $-0.17742-0.04210$ $-0.19107 \quad-0.02870$ $-0.18846-0.0121$ $\begin{array}{ll}-0.17407 & 0.00589 \\ -0.15351 & 0.02748\end{array}$ $-0.00208-0.00129$ $-0.18441-0.03780$

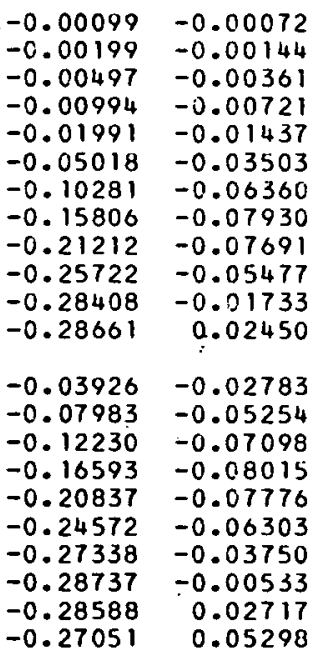

$-0.00099-0.00072$ 90.00144 $944-0.0072$ $-0.05018-0.03503$ $-0.10281-0.06360$ $-0.21212-0.07691$
-0.054 $-0.25722-0.0547$ $-0.28661 \quad 0.02450$

$-0.03926-0.02783$ $-0.07983-0.05254$ $-0.16593-0.08015$ $-0.20837-0.07776$ $-0.27338-0.03750$ $\begin{array}{ll}-0.28588 & -0.02717\end{array}$ $-0.20201-0.13308$

$$
\frac{a}{\lambda}=1.4 \quad \frac{\alpha}{\beta}=0.10
$$

$\begin{array}{ll}-0.00000 & -0.00000 \\ -0.00002 & -0.00001 \\ -0.00010 & -0.00006 \\ -0.00041 & -0.00024 \\ -0.00163 & -0.00095 \\ -0.01003 & -0.00582 \\ -0.03768 & -0.02148 \\ -0.07618 & -0.04244 \\ -0.11591 & -0.06358 \\ -0.14679 & -0.08152 \\ -0.16109 & -0.09605 \\ -0.15580 & -0.10968 \\ & \\ -0.00623 & -0.00363 \\ -0.02400 & -0.01380 \\ -0.05061 & -0.02861 \\ -0.08197 & -0.04553 \\ -0.11319 & -0.06212 \\ -0.13934 & -0.07676 \\ -0.15630 & -0.08907 \\ -0.16157 & -0.09985 \\ -0.15475 & -0.11067 \\ -0.13756 & -0.12310\end{array}$

$-0.00076-0.00055$ $-0.00153-0.0011$ $\begin{array}{ll}-0.00382 & -0.00277\end{array}$ $\begin{array}{ll}-0.00763 & -0.00554\end{array}$ $-0.01527-0.01108$ $-0.03825-0.0275$ $-0.07703-0.05378$ $-0.11674-0.07752$ $\begin{array}{ll}-0.15757 & -0.09749\end{array}$ $-0.19935-0.11248$ $-0.24154-0.12137$ $-0.28319-0.12332$

$\begin{array}{ll}-0.03002 & -0.02167\end{array}$ $-0.06029-0.04274$ $-0.09106-0.06259$ $-0.12246 \quad-0.0806$ $-0.15455-0.09619$ $-0.18726-0.10875$ $-0.22035-0.11773$ $-0.25344-0.12266$ $-0.31731-0.11908$
$-0.00076-0.00055$ $-0.00153-0.00111$ $\begin{array}{lll}-0.00381 & -0.00277\end{array}$ $\begin{array}{ll}-0.00762 & -0.00553\end{array}$ $-0.01517-0.01098$ $-0.03673-0.02611$ $-0.06491-0.04367$ $-0.07714-0.04903$ $-0.07060-0.04526$ $-0.05073-0.03857$ $-0.02959-0.0315$ $-0.01784-0.02022$

$-0.02928-0.02099$ $-0.05439-0.03757$ $-0.07139-0.04695$ $-0.07733-0.04891$ $-0.07165-0.0457$

$-0.05716 \quad-0.04052$

$-0.03955-0.03522$

$-0.02504-0.02901$

$-0.01749-0.01914$

$-0.01699-0.00349$

$\begin{array}{ll}-0.00000 & -0.00000 \\ -0.00002 & -0.00001 \\ -0.00010 & -0.00006 \\ -0.00041 & -0.00024 \\ -0.00163 & -0.00095 \\ -0.01007 & -0.00566 \\ -0.03823 & -0.01909 \\ -0.07749 & -0.03185 \\ -0.11590 & -0.03611 \\ -0.14066 & -0.03001 \\ -0.14532 & -0.01753 \\ -0.13304 & -0.00206 \\ & \\ -0.00625 & -0.00356 \\ -0.02425 & -0.01286 \\ -0.05147 & -0.02420 \\ -0.08329 & -0.03309 \\ -0.11342 & -0.03617 \\ -0.13550 & -0.03266 \\ -0.14550 & -0.02428 \\ -0.14324 & -0.01344 \\ -0.131 .77 & -0.00086 \\ -0.111486 & 0.01527\end{array}$

$-0.00076-0.00055$ $-0.00153-0.00111$ $-0.00382-0.00277$ $-0.00764-0.00554$ $-0.01529-0.01104$ $-0.03852-0.02689$ $-0.07878-0.04875$ $-0.12077-0.06069$ $-0.16147-0.05890$ $-0.19500-0.04246$ $\begin{array}{rr}-0.21470 & -0.01505 \\ -0.21655 & 0.01508\end{array}$ $-0.03015 \quad-0.02137$ $-0.06123-0.04030$ $-0.09363-0.05437$ $-0.18650-0.04858$ $-0.20689-0.02976$ $-0.21708-0.00635$ $-0.21603 \quad 0.01698$

$\begin{array}{ll}-0.20516 & 0.03526\end{array}$ $-0.12672-0.06133$ $-0.15865-0.05954$ 
$\frac{\mathrm{a}}{\lambda}=1.4 \quad \frac{\alpha}{\beta}=0.20$

$\beta \mathrm{h}$

$C(h, 0)$

$S(h, 0)$

$E(h, 0)$

$\mathrm{C}(\mathrm{h}, \mathrm{h})$

0.0

$-0.00032-0.00023$

$0.02-0.00063-0.00046$

$\begin{array}{lll}0.05 & -0.00158 & -0.00115\end{array}$

0.10

0.20

1.00

2.00

3.00

3.50

$0.125 \pi$ $0.250 \pi$ $0.500 \pi$ $0.625 \pi$ $0.750 \pi$ $0.875 \pi$

$1.125 \pi$ $1.250 \pi$ $\begin{array}{lll}-0.00316 & -0.00230\end{array}$ $-0.00628-0.00458$ $-0.01505-0.01122$ $-0.02566-0.02088$ $0.02260-0.03229$ $0.00885-0.03422$ $0.02929-0.03193$

$-0.01205-0.00890$ $-0.02190-0.01701$ $-0.02769-0.02372$ $\begin{array}{ll}-0.02826 & -0.02874\end{array}$ $-0.02332-0.03206$ $0.01345-0.03388$ $0.00002-0.03441$ $0.01532-0.03374$ $\begin{array}{ll}0.03058 & -0.03169 \\ 0.04419 & -0.02789\end{array}$

$\begin{array}{ll}-0.00000 & -0.00000 \\ -0.00001 & -0.00000 \\ -0.00005 & -0.00002 \\ -0.00018 & -0.00008 \\ -0.00072 & -0.00033 \\ -0.00443 & -0.00204 \\ -0.01654 & -0.00773 \\ -0.03312 & -0.01596 \\ -0.04982 & -0.02552 \\ -0.06239 & -0.03564 \\ -0.06785 & -0.04620 \\ -0.06521 & -0.05741 \\ -0.00276 & -0.00127 \\ -0.01057 & -0.00491 \\ -0.02215 & -0.01044 \\ -0.03558 & -0.01725 \\ -0.04869 & -0.02479 \\ -0.05940 & -0.03269 \\ -0.06611 & -0.04083 \\ -0.06792 & -0.04930 \\ -0.06475 & -0.05821 \\ -0.05721 & -0.06752\end{array}$

$\begin{array}{ll}-0.00032 & -0.00023 \\ -0.00063 & -0.00046 \\ -0.00158 & -0.00115 \\ -0.00317 & -0.00230 \\ -0.00634 & -0.00460 \\ -0.01586 & -0.01141 \\ -0.03190 & -0.02227 \\ -0.04823 & -0.03204 \\ -0.06488 & -0.04018 \\ -0.08173 & -0.04623 \\ -0.09852 & -0.04977 \\ -0.11482 & -0.05054 \\ -0.01245 & -0.00899 \\ -0.02499 & -0.01771 \\ -0.03768 & -0.02591 \\ -0.05057 & -0.03330 \\ -0.06366 & -0.03965 \\ -0.07688 & -0.04473 \\ -0.09012 & -0.04833 \\ -0.10320 & -0.05028 \\ -0.11591 & -0.05048 \\ -0.12795 & -0.04891\end{array}$

$\begin{array}{ll}-0.00032 & -0.00023 \\ -0.00063 & -0.00046 \\ -0.00158 & -0.00115 \\ -0.00316 & -0.00230 \\ -0.00629 & -0.00457 \\ -0.01514 & -0.01096 \\ -0.02626 & -0.01906 \\ -0.03053 & -0.02314 \\ -0.02673 & -0.02405 \\ -0.01806 & -0.02299 \\ -0.00892 & -0.019 .59 \\ -0.00315 & -0.01253 \\ -0.01210 & -0.00877 \\ -0.02221 & -0.01606 \\ -0.02862 & -0.02096 \\ -0.03026 & -0.02343 \\ -0.02721 & -0.02406 \\ -0.02082 & -0.02347 \\ -0.01328 & -0.02172 \\ -0.00683 & -0.01813 \\ -0.00293 & -0.01186 \\ -0.00205 & -0.00270\end{array}$

$S(h, h)$

$-0.00000-0.00000$ $-0.00001-0.00000$ $-0.00005-0.00002$ $-0.00072-0.00033$ $-0.00444-0.000197$ $-0.01660-0.0019$ $-0.03298-0.00669$ $-0.04837-0.01397$ $-0.05781-0.01397$ $\begin{array}{ll}-0.04837 & -0.01397 \\ -0.05897 & -0.01397\end{array}$

$-0.05256-0.00843$

$-0.00276-0.00124$ $\begin{array}{lll}-0.01061 & -0.00449\end{array}$ $-0.02220-0.00852$ $-0.03535-0.01195$ $-0.04739-0.01388$ $-0.05591-0.01419$ $-0.05944-0.01327$ $\begin{array}{ll}-0.05784 & -0.01137\end{array}$ $-0.05189-0.00806$ $-0.04266-0.00232$
$E(h, h)$

$\begin{array}{ll}-0.00032 & -0.00023 \\ -0.00063 & -0.00046 \\ -0.00158 & -0.00115 \\ -0.00317 & -0.00230 \\ -0.00634 & -0.00458 \\ -0.01595 & -0.01114 \\ -0.03244 & -0.02009 \\ -0.04926 & -0.02489 \\ -0.06505 & -0.02421 \\ -0.07754 & -0.01811 \\ -0.08455 & -0.00841 \\ -0.08520 & 0.00168 \\ & \\ -0.01249 & -0.00886 \\ -0.02529 & -0.01665 \\ -0.03844 & -0.02237 \\ -0.05160 & -0.02514 \\ -0.06398 & -0.02445 \\ -0.07443 & -0.02035 \\ -0.08182 & -0.01355 \\ -0.08536 & -0.00544 \\ -0.08503 & 0.00230 \\ -0.08161 & 0.00808\end{array}$

\begin{tabular}{|c|c|c|}
\hline $\begin{array}{l}.01 \\
.02 \\
.05 \\
0.10 \\
0.20 \\
.50 \\
.00 \\
1.50 \\
2.00 \\
2.50 \\
3.00 \\
3.50\end{array}$ & $\begin{array}{l}-0.00005 \\
-0.00011 \\
-0.00027 \\
-0.00054 \\
-0.00108 \\
-0.00257 \\
-0.00425 \\
-0.00441 \\
-0.00278 \\
0.00046 \\
0.00482 \\
0.00967\end{array}$ & $\begin{array}{l}-0.00004 \\
-0.00008 \\
-0.00020 \\
-0.00040 \\
-0.00079 \\
-0.00198 \\
-0.00398 \\
-0.00592 \\
-0.00768 \\
-0.00896 \\
-0.00944 \\
-0.00873\end{array}$ \\
\hline $\begin{array}{l}0.125 \pi \\
0.250 \pi \\
.375 \pi \\
0.500 \pi \\
0.625 \pi \\
0.750 \pi \\
0.875 \pi \\
1.000 \pi \\
1.125 \pi \\
1.250 \pi\end{array}$ & $\begin{array}{r}-0.00206 \\
-0.00369 \\
-0.00451 \\
-0.00428 \\
-0.00296 \\
-0.00062 \\
0.00252 \\
0.00617 \\
0.01001 \\
0.01373\end{array}$ & $\begin{array}{l}-0.00156 \\
-0.00312 \\
-0.00468 \\
-0.00619 \\
-0.00756 \\
-0.00866 \\
-0.00932 \\
-0.00937 \\
-0.00863 \\
-0.00699\end{array}$ \\
\hline
\end{tabular}

$\begin{array}{ll}-0.00000 & -0.00000 \\ -0.00000 & -0.00000 \\ -0.00001 & -0.00000 \\ -0.00004 & -0.00001 \\ -0.00014 & -0.00004 \\ -0.00086 & -0.00023 \\ -0.00320 & -0.00095 \\ -0.00639 & -0.00227 \\ -0.00959 & -0.00433 \\ -0.01199 & -0.00719 \\ -0.01298 & -0.01083 \\ -0.01224 & -0.01501 \\ & \\ -0.00053 & -0.00014 \\ -0.00205 & -0.00057 \\ -0.00428 & -0.00134 \\ -0.00687 & -0.00251 \\ -0.00938 & -0.00415 \\ -0.01143 & -0.00628 \\ -0.01269 & -0.00891 \\ -0.01295 & -0.01197 \\ -0.01213 & -0.01531 \\ -0.01023 & -0.01873\end{array}$

$$
\frac{\alpha}{\beta}=0.40
$$

$\begin{array}{ll}-0.00005 & -0.00004 \\ -0.00011 & -0.00008 \\ -0.00027 & -0.00020 \\ -0.00055 & -0.00040 \\ -0.00109 & -0.00079 \\ -0.00273 & -0.00196 \\ -0.00547 & -0.00382 \\ -0.00823 & -0.00547 \\ -0.01100 & -0.00683 \\ -0.01374 & -0.00781 \\ -0.01640 & -0.00837 \\ -0.01890 & -0.00849 \\ & \\ -0.00214 & -0.00155 \\ -0.00429 & -0.00304 \\ -0.00645 & -0.00444 \\ -0.00863 & -0.00568 \\ -0.01080 & -0.00674 \\ -0.01296 & -0.00757 \\ -0.01508 & -0.00815 \\ -0.01713 & -0.00845 \\ -0.01906 & -0.00848 \\ -0.02084 & -0.00825\end{array}$

$\begin{array}{cc}-0.00005 & -0.00004 \\ -0.00011 & -0.00008 \\ -0.00027 & -0.00020 \\ -0.00054 & -0.00040 \\ -0.00108 & -0.00079 \\ -0.00258 & -0.00193 \\ -0.00433 & -0.003 .03 \\ -0.00472 & -0.00497 \\ -0.00371 & -0.00593 \\ -0.00173 & -0.00626 \\ 0.00051 & -0.00548 \\ 0.00227 & -0.00314 \\ -0.00207 & -0.00153 \\ -0.00372 & -0.00294 \\ -0.00464 & -0.00415 \\ -0.00465 & -0.00514 \\ -0.00382 & -0.00588 \\ -0.00236 & -0.00625 \\ -0.00060 & -0.00604 \\ 0.00109 & -0.00499 \\ 0.00236 & -0.00292 \\ 0.00286 & 0.00014\end{array}$

$-0.00000-0.00000$ $-0.00000-0.00000$ $-0.00001-0.00000$ $-0.00004-0.0000$ $-0.00014-0.00004$ $-0.00086-0.0002$ $-0.00315 \quad-0.00075$ $-0.00613-0.0014$ $\begin{array}{lll}-0.00881 & -0.00223\end{array}$ $-0.01034-0.00317$ $-0.01016-0.0041$ $-0.00806-0.00469$

$\begin{array}{ll}-0.00053 & -0.00013\end{array}$ $-0.00203-0.0004$ $\begin{array}{ll}-0.00418 & -0.00099\end{array}$ $-0.00655-0.00155$ $-0.00865-0.00217$ $\begin{array}{ll}-0.01006 & -0.00288\end{array}$ $-0.01049-0.00367$ $-0.00976-0.00437$ $\begin{array}{ll}-0.00784 & -0.00470\end{array}$ $\begin{array}{ll}-0.00481 & -0.00427\end{array}$
$-0.00005-0.00004$ $-0.00011-0.00008$ $-0.00027-0.00020$ $-0.00055 \quad-0.00040$ $-0.00109-0.00079$ $-0.00274-0.00191$ $-0.00550 \quad-0.00341$ $-0.00820-0.00419$ $-0.01058-0.00409$ $-0.01231-0.00325$ $-0.01320-0.00203$ $-0.01328-0.00090$

$-0.00215 \quad-0.00152$ $-0.00431-0.00284$ $-0.00648-0.00378$ $-0.00856-0.00423$ $-0.01042-0.00413$ $-0.01190-0.00355$ $-0.01287-0.00266$ $-0.01330-0.00169$ $-0.01326-0.00084$ $\begin{array}{ll}-0.01292 & -0.00026\end{array}$ 


$\begin{array}{lll}0.01 & -0.00000 & -0.00000 \\ 0.02 & -0.00001 & -0.00001 \\ 0.05 & -0.00002 & -0.00001 \\ 0.10 & -0.00004 & -0.00003 \\ 0.20 & -0.00008 & -0.00006 \\ 0.50 & -0.00018 & -0.00015 \\ 1.00 & -0.00029 & -0.00033 \\ 1.50 & -0.00027 & -0.00056 \\ 2.00 & -0.00006 & -0.00082 \\ 2.50 & 0.00034 & -0.00103 \\ 3.00 & 0.00092 & -0.00113 \\ 3.50 & 0.00165 & -0.00101 \\ & -0 & \\ 0.125 \pi & -0.00015 & -0.00011 \\ 0.250 \pi & -0.00026 & -0.00025 \\ 0.375 \pi & -0.00030 & -0.00041 \\ 0.500 \pi & -0.00025 & -0.00060 \\ 0.025 \pi & -0.00009 & -0.00080 \\ 0.750 \pi & 0.00020 & -0.00096 \\ 0.875 \pi & 0.00061 & -0.00110 \\ 1.000 \pi & 0.00112 & -0.00112 \\ 1.125 \pi & 0.00170 & -0.00100 \\ 1.250 \pi & 0.00233 & -0.00069\end{array}$

0.01

0.02

0.05

0.10

0.20
0.50

0.50
1.00

1.50

2.00

2.50
3.00

3.00
3.50

$0.125 \pi$

$0.250 \pi$

$0.375 \pi$
$0.500 \pi$

$0.625 \pi$

0.750

$0.875 \pi$

$-1.000 \pi$

$1.250 \pi$

$\begin{array}{ll}-0.00000 & -0.00000 \\ -0.00000 & -0.00000 \\ -0.00000 & -0.00000 \\ -0.00000 & -0.00000 \\ -0.00001 & -0.00000 \\ -0.00007 & -0.00000 \\ -0.00027 & -0.00003 \\ -0.00056 & -0.00013 \\ -0.00088 & -0.00035 \\ -0.00113 & -0.00074 \\ -0.00124 & -0.00130 \\ -0.00113 & -0.00201 \\ & \\ -0.00004 & -0.00000 \\ -0.00017 & -0.00001 \\ -0.00037 & -0.00005 \\ -0.00061 & -0.00015 \\ -0.00086 & -0.00033 \\ -0.00107 & -0.00061 \\ -0.00121 & -0.00100 \\ -0.00124 & -0.00149 \\ -0.00111 & -0.00207 \\ -0.00080 & -0.00269\end{array}$

$-0.00080-0.00269$

$\begin{array}{ll}-0.00000 & -0.00000 \\ -0.00001 & -0.00001 \\ -0.00002 & -0.00001 \\ -0.00004 & -0.00003 \\ -0.00008 & -0.00006 \\ -0.00019 & -0.00014 \\ -0.00034 & -0.00027 \\ -0.00058 & -0.00034 \\ -0.00077 & -0.00048 \\ -0.00095 & -0.00054 \\ -0.00112 & -0.00058 \\ -0.00127 & -0.00059 \\ & \\ -0.00015 & -0.00011 \\ -0.00031 & -0.00022 \\ -0.00046 & -0.00031 \\ -0.00061 & -0.00040 \\ -0.00075 & -0.00047 \\ -0.00090 & -0.00053 \\ -0.00103 & -0.00056 \\ -0.00116 & -0.00058 \\ -0.00128 & -0.00059 \\ -0.00138 & -0.00057\end{array}$

$$
\frac{a}{\lambda}=1.4
$$$$
\frac{\alpha}{\beta}=1.00
$$

$\begin{array}{cc}-0.00000 & -0.00000 \\ -0.00001 & -0.00001 \\ -0.00002 & -0.00001 \\ -0.00004 & -0.00003 \\ -0.00008 & -0.00006 \\ -0.00018 & -0.00014 \\ -0.00030 & -0.00039 \\ -0.00029 & -0.00047 \\ -0.00016 & -0.00063 \\ 0.00011 & -0.00071 \\ 0.00046 & -0.00060 \\ 0.00082 & -0.00013 \\ -0.00015 & -0.00011 \\ -0.00026 & -0.00023 \\ -0.00031 & -0.00030 \\ -0.00028 & -0.00050 \\ -0.00017 & -0.00062 \\ 0.00002 & -0.00070 \\ 0.00028 & -0.00069 \\ 0.00057 & -0.00052 \\ 0.00084 & -0.00014 \\ 0.00098 & 0.00048\end{array}$

$-0.00000-0.00000$ $-0.00000-0.00060$ $-0.00000-0.00000$ $-0.00000-0.00000$ $-0.00001-0.00000$ $-0.00007-0.00000$ $-0.00026-0.00001$ $-0.00052-0.00006$ $-0.00075-0.00018$ $-0.00088-0.00039$ $-0.00079-0.00068$ $-0.00039-0.00098$

$-0.00004-0.00000$ $-0.00017-0.00001$ $-0.00035-0.00003$ $-0.00055-0.00007$ $-0.00074-0.00017$ $\begin{array}{ll}-0.00086 & -0.00032\end{array}$ $-0.00087-0.00053$ $-0.00072-0.00077$ $-0.00035-0.00099$ $0.00027-0.00109$

$\begin{array}{ll}-0.00000 & -0.00000 \\ -0.00000 & -0.00000 \\ -0.00000 & -0.00000 \\ -0.00000 & -0.00000 \\ -0.00001 & -0.00000 \\ -0.00001 & -0.00001 \\ -0.00002 & -0.00003 \\ -0.00002 & -0.00005 \\ 0.00001 & -0.00008 \\ 0.00006 & -0.00011 \\ 0.00014 & -0.00013 \\ 0.00026 & -0.00011 \\ & \\ -0.00001 & -0.00001 \\ -0.00002 & -0.00002 \\ -0.00002 & -0.00004 \\ -0.00001 & -0.00006 \\ 0.00001 & -0.00008 \\ 0.00004 & -0.00011 \\ 0.00010 & -0.00012 \\ 0.00017 & -0.00013 \\ 0.00027 & -0.00011 \\ 0.00038 & -0.00005\end{array}$

$\begin{array}{lr}-0.00000 & 0.00000 \\ -0.00000 & 0.00000 \\ -0.00000 & 0.00000 \\ -0.00000 & 0.00000 \\ -0.00000 & 0.00000 \\ -0.00001 & 0.00000 \\ -0.00002 & 0.00000 \\ -0.00005 & -0.00001 \\ -0.00009 & -0.00003 \\ -0.00012 & -0.00008 \\ -0.00013 & -0.00017 \\ -0.00011 & -0.00028 \\ & \\ -0.00000 & 0.00000 \\ -0.00001 & 0.00000 \\ -0.00003 & -0.00000 \\ -0.00006 & -0.00001 \\ -0.00008 & -0.00003 \\ -0.00011 & -0.00007 \\ -0.00013 & -0.00012 \\ -0.00013 & -0.00020 \\ -0.00011 & -0.00029 \\ -0.00005 & -0.00040\end{array}$

$\begin{array}{ll}-0.00000 & -0.00000 \\ -0.00000 & -0.00000 \\ -0.00000 & -0.00000 \\ -0.00000 & -0.00000 \\ -0.00001 & -0.00000 \\ -0.00001 & -0.00001 \\ -0.00003 & -0.00002 \\ -0.00004 & -0.00003 \\ -0.00005 & -0.00003 \\ -0.00007 & -0.00004 \\ -0.00008 & -0.00004 \\ -0.00009 & -0.00004 \\ & \\ -0.00001 & -0.00001 \\ -0.00002 & -0.00002 \\ -0.00003 & -0.00002 \\ -0.00004 & -0.00003 \\ -0.00005 & -0.00003 \\ -0.00006 & -0.00004 \\ -0.00007 & -0.00004 \\ -0.00008 & -0.00004 \\ -0.00009 & -0.00004 \\ -0.00009 & -0.00004\end{array}$

$\begin{array}{ll}-0.00000 & -0.00000 \\ -0.00000 & -0.00000 \\ -0.00000 & -0.00000 \\ -0.00000 & -0.00000 \\ -0.00001 & -0.00000 \\ -0.00001 & -0.00001 \\ -0.00002 & -0.00003 \\ -0.00002 & -0.00004 \\ -0.00000 & -0.00006 \\ 0.00004 & -0.00008 \\ 0.00010 & -0.00006 \\ 0.00017 & 0.00002 \\ -0.00001 & -0.00001 \\ -0.00002 & -0.00002 \\ -0.00002 & -0.00003 \\ -0.00002 & -0.00005 \\ -0.00000 & -0.00006 \\ 0.00002 & -0.00007 \\ 0.00006 & -0.00007 \\ 0.00012 & -0.00004 \\ 0.00017 & 0.00003 \\ 0.00021 & 0.00016\end{array}$

(1)

$E(h, h)$

$\begin{array}{ll}-0.00000 & -0.00000 \\ -0.00001 & -0.00001 \\ -0.00002 & -0.00001 \\ -0.00004 & -0.00003 \\ -0.00008 & -0.00006 \\ -0.00019 & -0.00014 \\ -0.00038 & -0.00024 \\ -0.00056 & -0.00029 \\ -0.00070 & -0.00028 \\ -0.00079 & -0.00024 \\ -0.00083 & -0.00019 \\ -0.00083 & -0.00014 \\ -0.00015 & -0.00011 \\ -0.00030 & -0.00020 \\ -0.00045 & -0.00026 \\ -0.00058 & -0.00029 \\ -0.00069 & -0.00029 \\ -0.00077 & -0.00026 \\ -0.00081 & -0.00021 \\ -0.00083 & -0.00017 \\ -0.00083 & -0.00014 \\ -0.00082 & -0.00012\end{array}$

$\begin{array}{ll}-0.00000 & -0.00000 \\ -0.00000 & -0.00000 \\ -0.00000 & -0.00000 \\ -0.00000 & -0.00000 \\ -0.00001 & -0.00000 \\ -0.00001 & -0.00001 \\ -0.00003 & -0.00002 \\ -0.00004 & -0.00002 \\ -0.00005 & -0.00002 \\ -0.00005 & -0.00002 \\ -0.00005 & -0.00002 \\ -0.00005 & -0.00001 \\ & \\ -0.00001 & -0.00001 \\ -0.00002 & -0.00001 \\ -0.00003 & -0.00002 \\ -0.00004 & -0.00002 \\ -0.00005 & -0.00002 \\ -0.00005 & -0.00002 \\ -0.00005 & -0.00002 \\ -0.00005 & -0.00001 \\ -0.00005 & -0.00001 \\ -0.00005 & -0.00001\end{array}$




\begin{tabular}{|c|c|c|}
\hline $\begin{array}{l}0.01 \\
0.02 \\
0.05 \\
0.10 \\
0.20 \\
0.50 \\
1.00 \\
1.50 \\
2.00 \\
2.50 \\
3.00 \\
3.50\end{array}$ & $\begin{array}{l}-0.00161 \\
-0.00322 \\
-0.00804 \\
-0.01607 \\
-0.03196 \\
-0.07691 \\
-0.13383 \\
-0.15743 \\
-0.14541 \\
-0.10717 \\
-0.05985 \\
-0.02106\end{array}$ & $\begin{array}{r}0.00117 \\
0.00234 \\
0.00584 \\
0.01168 \\
0.02325 \\
0.05635 \\
0.10015 \\
0.11998 \\
0.10760 \\
0.06101 \\
-0.01280 \\
-0.09735\end{array}$ \\
\hline $\begin{array}{l}0.125 \pi \\
0.250 \pi \\
0.375 \pi \\
0.500 \pi \\
0.625 \pi \\
0.750 \pi \\
0.875 \pi \\
1.000 \pi \\
1.125 \pi \\
1.250 \pi\end{array}$ & $\begin{array}{l}-0.06146 \\
-0.11292 \\
-0.14644 \\
-0.15779 \\
-0.14735 \\
-0.11993 \\
-0.08357 \\
-0.04737 \\
-0.01903 \\
-0.00260\end{array}$ & $\begin{array}{r}0.04489 \\
0.08366 \\
0.11048 \\
0.12031 \\
0.10968 \\
0.07769 \\
0.02684 \\
-0.03657 \\
-0.10299 \\
-0.16119\end{array}$ \\
\hline
\end{tabular}

$\begin{array}{ll}-0.00001 & 0.00001 \\ -0.00003 & 0.00002 \\ -0.00020 & 0.00015 \\ -0.00080 & 0.00058 \\ -0.00321 & 0.00233 \\ -0.01960 & 0.01443 \\ -0.07249 & 0.05538 \\ -0.14292 & 0.11562 \\ -0.21075 & 0.18209 \\ -0.25870 & 0.24001 \\ -0.27874 & 0.27023 \\ -0.27509 & 0.26083 \\ -0.01221 & 0.00894 \\ -0.04655 & 0.03496 \\ -0.09658 & 0.07516 \\ -0.15314 & 0.12505 \\ -0.20630 & 0.17791 \\ -0.24764 & 0.22558 \\ -0.27219 & 0.25949 \\ -0.27964 & 0.27191 \\ -0.27429 & 0.25860 \\ -0.26370 & 0.21964\end{array}$

$$
\frac{\mathrm{a}}{\lambda}=1.6 . \quad \frac{\alpha}{\beta}=0.00
$$

$E(h, 0)$

$C(h, h)$

$\begin{array}{ll}-0.00161 & 0.00117 \\ -0.00322 & 0.00234 \\ -0.00805 & 0.00585 \\ -0.01609 & 0.01170 \\ -0.03217 & 0.02341 \\ -0.08020 & 0.05878 \\ -0.15872 & 0.11937 \\ -0.23377 & 0.18345 \\ -0.30338 & 0.25237 \\ -0.36535 & 0.32702 \\ -0.41727 & 0.40761 \\ -0.45664 & 0.49357 \\ -0.06307 & 0.04607 \\ -0.12533 & 0.09303 \\ -0.18594 & 0.14172 \\ -0.24400 & 0.19289 \\ -0.29853 & 0.24716 \\ -0.34844 & 0.30494 \\ -0.39261 & 0.36541 \\ -0.42980 & 0.43145 \\ -0.45882 & 0.49963 \\ -0.47850 & 0.57012\end{array}$

$S(h, h)$

$E(h, h)$

$$
\frac{a}{\lambda}=1.6 \quad \frac{\alpha}{\beta}=0.005
$$

\begin{tabular}{|c|c|c|}
\hline $\begin{array}{l}0.01 \\
0.02 \\
0.05 \\
0.10 \\
0.20 \\
0.50 \\
1.00 \\
1.50 \\
2.00 \\
2.50 \\
3.00 \\
3.50\end{array}$ & $\begin{array}{l}-0.00153 \\
-0.00306 \\
-0.00765 \\
-0.01528 \\
-0.03039 \\
-0.07316 \\
-0.12744 \\
-0.15023 \\
-0.13937 \\
-0.10366 \\
-0.05912 \\
-0.02217\end{array}$ & $\begin{array}{r}0.00111 \\
0.00222 \\
0.00556 \\
0.011110 \\
0.02211 \\
0.05355 \\
0.09501 \\
0.11344 \\
0.10112 \\
0.05635 \\
-0.01397 \\
-0.09412\end{array}$ \\
\hline $\begin{array}{l}0.125 \pi \\
0.250 \pi \\
0.375 \pi \\
0.500 \pi \\
0.625 \pi \\
0.750 \pi \\
0.875 \pi \\
1.000 \pi \\
1.125 \pi \\
1.250 \pi\end{array}$ & $\begin{array}{l}-0.05846 \\
-0.10747 \\
-0.13952 \\
-0.15064 \\
-0.14116 \\
-0.11561 \\
-0.08148 \\
-0.04730 \\
-0.02021 \\
-0.00394\end{array}$ & $\begin{array}{r}0.04268 \\
0.07944 \\
0.10471 \\
0.11368 \\
0.10314 \\
0.07233 \\
0.02375 \\
-0.03654 \\
-0.09945 \\
-0.15444\end{array}$ \\
\hline
\end{tabular}

$\begin{array}{ll}-0.00001 & 0.00001 \\ -0.00003 & 0.00002 \\ -0.00019 & 0.00014 \\ -0.00076 & 0.00056 \\ -0.00304 & 0.00223 \\ -0.01857 & 0.01381 \\ -0.06871 & 0.05295 \\ -0.13555 & 0.11034 \\ -0.20012 & 0.17398 \\ -0.24617 & 0.22801 \\ -0.26616 & 0.25608 \\ -0.26401 & 0.24657 \\ -0.01157 & 0.00856 \\ -0.04411 & 0.03339 \\ -0.09156 & 0.07182 \\ -0.14526 & 0.11931 \\ -0.19587 & 0.16945 \\ -0.23547 & 0.21445 \\ -0.25940 & 0.24613 \\ -0.26736 & 0.25749 \\ -0.26334 & 0.24443 \\ -0.25433 & 0.20721\end{array}$

$\begin{array}{ll}-0.00153 & 0.00111 \\ -0.00306 & 0.00222 \\ -0.00765 & 0.00556 \\ -0.01530 & 0.01112 \\ -0.03059 & 0.02226 \\ -0.07626 & 0.05589 \\ -6.15092 & 0.11351 \\ -0.22227 & 0.17443 \\ -0.28841 & 0.23992 \\ -0.34727 & 0.31081 \\ -0.39656 & 0.38731 \\ -0.43391 & 0.46885 \\ -0.05998 & 0.04381 \\ -0.11918 & 0.08846 \\ -0.17681 & 0.13476 \\ -0.23199 & 0.18340 \\ -0.28381 & 0.23496 \\ -0.33122 & 0.28984 \\ -0.37315 & 0.34821 \\ -0.40845 & 0.40994 \\ -0.43597 & 0.47460 \\ -0.45462 & 0.54141\end{array}$

$\begin{array}{ll}-0.00153 & 0.00111 \\ -0.00306 & 0.00222 \\ -0.00765 & 0.00556 \\ -0.01527 & 0.01111 \\ -0.03034 & 0.02216 \\ -0.07239 & 0.05438 \\ -0.12183 & 0.10049 \\ -0.13472 & 0.12708 \\ -0.11414 & 0.12338 \\ -0.07917 & 0.08953 \\ -0.05165 & 0.04410 \\ -0.03782 & 0.01593 \\ & \\ -0.05809 & 0.04309 \\ -0.10461 & 0.08237 \\ -0.13085 & 0.11285 \\ -0.13354 & 0.12856 \\ -0.11643 & 0.12478 \\ -0.08927 & 0.10161 \\ -0.06362 & 0.06657 \\ -0.04665 & 0.03322 \\ -0.03710 & 0.01520 \\ -0.02684 & 0.01840\end{array}$

$\begin{array}{ll}-0.00001 & 0.00001 \\ -0.00003 & 0.00002 \\ -0.00019 & 0.00014 \\ -0.00076 & 0.00056 \\ -0.00303 & 0.00224 \\ -0.01838 & 0.01402 \\ -0.06556 & 0.05582 \\ -0.12026 & 0.12146 \\ -0.15654 & 0.19735 \\ -0.15783 & 0.26031 \\ -0.12723 & 0.28974 \\ -0.08215 & 0.28449 \\ & \\ -0.01150 & 0.00864 \\ -0.04291 & 0.03457 \\ -0.08555 & 0.07692 \\ -0.12707 & 0.13204 \\ -0.15498 & 0.19194 \\ -0.16126 & 0.24497 \\ -0.14565 & 0.27983 \\ -0.11520 & 0.29118 \\ -0.07890 & 0.28330 \\ -0.04061 & 0.26781\end{array}$

$$
\begin{aligned}
& -0 \\
& -0 \\
& -0 \\
& -0 \\
& -0 \\
& -0 \\
& -0 \\
& -0 \\
& -0 \\
& -0 \\
& -0 \\
& -0 \\
& -0 \\
& -0 \\
& -0 \\
& -0 \\
& -0.1 \\
& -0 \\
& -0 \\
& -0 \\
& -0.2 \\
& -0.1 \\
& -0.1
\end{aligned}
$$
$-0.00306$ $-0.00765$ $-0.01530$ $-0.03054$ $-0.07546$ $-0.19828$ $-0.22852$ $-0.22676$ -0.19019
-0.12582

-0.05959 .
$-0.11600$ $-0.16561$ $-0.20423$ $-0.22731$ $-0.23086$ $-0.21275$ $-0.12079$

$-0.06246$

0.00111 0.00222 0.00556 0.01113 0.02232 0.05675 0.11996 0.19366 0.27699 0.36229 0.43524
0.47878 0.04423 0.09170 0.14492 0.20497 0.33829 0.40122 0.45126 0.48032 
Bh

$\mathrm{C}(\mathrm{h}, 0)$

0.01

0.02
0.05

0.05
0.10
0.20

0.20
0.50

1.00

1.50

2.00

3.00

3.50

$0.125 \pi$

$0.250 \pi$

$0.375 \pi$

$0.625 \pi$

0.750
0.875

-8
$1.000 \pi$

$.000 \pi$

$1.250 \pi$

$\begin{array}{lr}-0.00146 & 0.00106 \\ -0.00291 & 0.00211 \\ -0.00727 & 0.00529 \\ -0.01453 & 0.01056 \\ -0.02890 & 0.02102 \\ -0.06959 & 0.05090 \\ -0.12135 & 0.09013 \\ -0.14336 & 0.10725 \\ -0.13357 & 0.09501 \\ -0.10022 & 0.05201 \\ -0.05831 & -0.01501 \\ -0.02309 & -0.09101 \\ -0.05561 & 0.04057 \\ -0.10228 & 0.07544 \\ -0.13294 & 0.09923 \\ -0.14382 & 0.10741 \\ -0.13523 & 0.09697 \\ -0.11142 & 0.06730 \\ -0.07939 & 0.02088 \\ -0.04710 & -0.03644 \\ -0.02120 & -0.09605 \\ -0.00511 & -0.14802\end{array}$

$\mathrm{S}(\mathrm{h}, 0)$

$\begin{array}{ll}-0.00001 & 0.00001 \\ -0.00003 & 0.00002 \\ -0.00018 & 0.00013 \\ -0.00072 & 0.00054 \\ -0.00288 & 0.00214 \\ -0.01760 & 0.01322 \\ -0.06513 & 0.05062 \\ -0.12856 & 0.10531 \\ -0.19003 & 0.16568 \\ -0.23425 & 0.21662 \\ -0.25416 & 0.24269 \\ -0.25335 & 0.23310 \\ -0.01097 & 0.00820 \\ -0.04181 & 0.03194 \\ -0.08680 & 0.06862 \\ -0.13779 & 0.11383 \\ -0.18598 & 0.16140 \\ -0.22391 & 0.20388 \\ -0.24723 & 0.23355 \\ -0.25563 & 0.24386 \\ -0.25281 & 0.23104 \\ -0.24524 & 0.19547\end{array}$

$\frac{a}{\lambda}=1.5 \quad \frac{\alpha}{\beta}=0.01$

$E(h, 0)$

$\mathrm{C}(\mathrm{h}, \mathrm{h})$

$S(h, h)$

$E(h, h)$
$-0.00146$

$-0.00291$

$-0.01452 \quad 0.00528$

$\begin{array}{ll}-0.01452 & 0.01057 \\ -0.02886 & 0.02108\end{array}$

$\begin{array}{ll}-0.06886 & 0.05168\end{array}$

$-0.11599 \quad 0.09533$

$-0.12860 \quad 0.12022$

- $0.10959 \quad 0.11633$

$\begin{array}{ll}-0.07689 & 0.08405\end{array}$

$-0.03713$

0.04114
0.01468

$-0.05525$

0.04096

$\begin{array}{lll}-0.09955 & 0.07821\end{array}$

$-0.12467$

$-0.11172$

$-0.08637$

$-0.06224$

$-0.04598$

-0.03638
-0.02598

0.10696
0.12157

0.11767

0.0955

0.06233

0.03091

0.01400
0.01707
$-0.00001$

$-0.00003$

$-0.00018$

$-0.00287$

$-0.0174$

$-0.01741$

$-0.11394$

$-0.14848$

$-0.15018$

$-0.12196$

$-0.07972$

$-0.01089$

$-0.04065$

$-0.08105$

$-0.14098$

$-0.14698$

$-0.15325$

$-0.11070$

$-0.07666$

$-0.04029$
0.00001
0.00002

0.00013

0.00054

0.00214

0.01341
0.05332

0.11576

0.18763

0.24698

0.27455

0.00827

0.03305

0.07342

0.12580

0.18252

0.23255

0.27586

0.26832

0.25355
$-0.00291$

0.01058

$-0.02905$

$\begin{array}{ll}-0.07176 & 0.05397\end{array}$

0.11404

$-0.21711$

$-0.215460 .26302$

$-0.100930 .41265$

$-0.18093 \quad 0.41265$

\section{$\begin{array}{ll}-0.05667 & 0.04206\end{array}$}

$\begin{array}{lll}-0.11029 & 0.08718\end{array}$

$-0.15743 \quad 0.13775$

$-0.191508 \quad 0.19475$

$-0.219330 .25707$

0.219210 .32105

$-0.16584 \quad 0.42775$

$\begin{array}{ll}-0.11557 & 0.45511 \\ -0.06079 & 0.45770\end{array}$

$\begin{array}{llr}0.01 & -0.00132 & 0.00096 \\ 0.02 & -0.00263 & 0.00191 \\ 0.05 & -0.00658 & 0.00478 \\ 0.10 & -0.01314 & 0.00955 \\ 0.20 & -0.02614 & 0.01901 \\ 0.50 & -0.06297 & 0.04598 \\ 1.00 & -0.11003 & 0.08112 \\ 1.50 & -0.13054 & 0.09587 \\ 2.00 & -0.12266 & 0.08385 \\ 2.50 & -0.09360 & 0.04416 \\ 3.00 & -0.05645 & -0.01673 \\ 3.50 & -0.02445 & -0.08511 \\ 0.125 \pi & -0.05031 & 0.03666 \\ 0.250 \pi & -0.09264 & 0.06802 \\ 0.375 \pi & -0.12068 & 0.08913 \\ 0.500 \pi & -0.13108 & 0.09589 \\ 0.625 \pi & -0.12408 & 0.08569 \\ 0.750 \pi & -0.10342 & 0.05818 \\ 0.875 \pi & -0.07522 & 0.01579 \\ 1.000 \pi & -0.04639 & -0.03607 \\ 1.125 \pi & -0.02268 & -0.08963 \\ 1.250 \pi & -0.00702 & -0.13609\end{array}$

$\begin{array}{llll}-0.00001 & 0.00000 & -0.00132 & 0.00096 \\ -0.00003 & 0.00002 & -0.00263 & 0.00191 \\ -0.00016 & 0.00012 & -0.00658 & 0.00478 \\ -0.00065 & 0.00049 & -0.01316 & 0.00957 \\ -0.00258 & 0.00196 & -0.02631 & 0.01914 \\ -0.01580 & 0.01211 & -0.06559 & 0.04807 \\ -0.05851 & 0.04626 & -0.12976 & 0.09760 \\ -0.11565 & 0.09592 & -0.19105 & 0.14992 \\ -0.17138 & 0.15028 & -0.24781 & 0.20612 \\ -0.21217 & 0.19556 & -0.29824 & 0.26686 \\ -0.23178 & 0.21802 & -0.34040 & 0.33229 \\ -0.23327 & 0.20837 & -0.37227 & 0.40187 \\ -0.00985 & 0.00751 & -0.05158 & 0.03768 \\ -0.03755 & 0.02922 & -0.10248 & 0.07607 \\ -0.07802 & 0.06265 & -0.15201 & 0.11585 \\ -0.12399 & 0.10363 & -0.19940 & 0.15762 \\ -0.16768 & 0.14644 & -0.24386 & 0.20187 \\ -0.20250 & 0.18432 & -0.28450 & 0.24891 \\ -0.22460 & 0.21034 & -0.32039 & 0.29886 \\ -0.23369 & 0.21877 & -0.35055 & 0.35161 \\ -0.23295 & 0.20646 & -0.37403 & 0.40677 \\ -0.22788 & 0.17395 & -0.38991 & 0.46365\end{array}$

$$
\frac{a}{\lambda}=1.0 \quad \frac{\alpha}{\beta}=0.02
$$

$\begin{array}{ll}-0.00132 & 0.00096 \\ -0.00263 & 0.00191 \\ -0.00658 & 0.00478 \\ -0.01313 & 0.00955 \\ -0.02610 & 0.01906 \\ -0.06230 & 0.04668 \\ -0.10515 & 0.08579 \\ -0.11717 & 0.10760 \\ -0.10100 & 0.10338 \\ -0.07242 & 0.07403 \\ -0.04903 & 0.03572 \\ -0.03564 & 0.01237 \\ -0.04998 & 0.03701 \\ -0.09015 & 0.07051 \\ -0.11317 & 0.09609 \\ -0.11634 & 0.10871 \\ -0.10284 & 0.10464 \\ -0.08075 & 0.08438 \\ -0.05940 & 0.05457 \\ -0.04447 & 0.02665 \\ -0.03487 & 0.01177 \\ -0.02431 & 0.01460\end{array}$

$-0.0000$

$-0.00000$

$-0.00016$

$-0.00065$

$-0.0025$

-0.01563
-0.05574

$-0.0557$

-0.10229
-0.13360

$-0.13360$

$-0.11195$

$-0.074 .97$

$-0.00978$

-0.03648
-0.07274

$-0.07274$

$-0.13222$

$-0.13843$

$-0.10220$

$-0.07224$

0.00000

0.00002

0.00012

0.00049

0.00197

0.01228

0.04866

0.10516

0.16963

0.22236

0.24654
0.24171

0.00758

0.0302

0.06690

0.11420

0.16506

0.16506
0.20959

0.23849

0.24761

0.24069

0.22720
$-0.00132$

$-0.00263$

$-0.00658$

$-0.01316$

$-0.02627$

$-0.12391$

$-0.17020$

$-0.19598$

$-0.1945$

-0.16373
-0.10995

$-0.05124$

$-0.09970$

$-0.14225$

$-0.17528$

$-0.19496$

$-0.19798$

$-0.18269$

$-0.10577$

$-0.05743$
0.00096 0.00191 0.00478 0.00957 0.01919 0.04880 0.10306 0.16614 0.23717 0.30948 0.37092
0.40733 0.03803 0.07881 0.12445 0.17581 0.28918 0.34232 0.38434 0.40860 


\begin{tabular}{|c|c|c|}
\hline $\begin{array}{l}0.01 \\
0.02 \\
0.05 \\
0.10 \\
0.20 \\
0.50 \\
1.00 \\
1.50 \\
2.00 \\
2.50 \\
3.00 \\
3.50 .\end{array}$ & $\begin{array}{l}-0.00119 \\
-0.00238 \\
-0.00595 \\
-0.01188 \\
-0.02364 \\
-0.05699 \\
-0.09977 \\
-0.11887 \\
-0.111261 \\
-0.08730 \\
-0.05437 \\
-0.02527\end{array}$ & $\begin{array}{r}0.00086 \\
0.00173 \\
0.00432 \\
0.00864 \\
0.01719 \\
0.04153 \\
0.07300 \\
0.08569 \\
0.07396 \\
0.03733 \\
-0.01803 \\
-0.07963\end{array}$ \\
\hline $\begin{array}{l}0.125 \pi \\
0.250 \pi \\
0.375 \pi \\
0.500 \pi \\
0.625 \pi \\
0.750 \pi \\
0.875 \pi \\
1.000 \pi \\
1.125 \pi \\
1.250 \pi\end{array}$ & $\begin{array}{l}-0.04551 \\
-0.08391 \\
-0.10956 \\
-0.111946 \\
-0.11383 \\
-0.09591 \\
-0.07108 \\
-0.04533 \\
-0.02362 \\
-0.00844\end{array}$ & $\begin{array}{r}0.03313 \\
0.06133 \\
0.08005 \\
0.08559 \\
0.07569 \\
0.05018 \\
0.01146 \\
-0.03550 \\
-0.08369 \\
-0.012528\end{array}$ \\
\hline
\end{tabular}

$\begin{array}{ll}-0.00001 & 0.00000 \\ -0.00002 & 0.00002 \\ -0.00015 & 0.00011 \\ -0.00058 & 0.00045 \\ -0.00232 & 0.00180 \\ -0.01419 & 0.01109 \\ -0.05257 & 0.04228 \\ -0.10405 & 0.08738 \\ -0.15458 & 0.13634 \\ -0.19220 & 0.17660 \\ -0.21139 & 0.19593 \\ -0.21474 & 0.18631 \\ -0.00884 & 0.00688 \\ -0.03373 & 0.02673 \\ -0.07012 & 0.05720 \\ -0.11159 & 0.09435 \\ -0.15121 & 0.13290 \\ -0.18318 & 0.16667 \\ -0.20408 & 0.18949 \\ -0.21365 & 0.19632 \\ -0.21459 & 0.18453 \\ -0.21159 & 0.15479\end{array}$

$\begin{array}{llll}-0.00119 & 0.00086 & -0.00119 & 0.00086 \\ -0.00238 & 0.00173 & -0.00238 & 0.00173 \\ -0.00595 & 0.00432 & -0.00595 & 0.00432 \\ -0.01190 & 0.00865 & -0.01188 & 0.00864 \\ -0.02380 & 0.01731 & -0.02360 & 0.01723 \\ -0.05931 & 0.04347 & -0.05637 & 0.04216 \\ -0.11733 & 0.08825 & -0.09533 & 0.07721 \\ -0.17272 & 0.13553 & -0.10675 & 0.07629 \\ -0.22397 & 0.18628 & -0.06303 & 0.07135 \\ -0.26946 & 0.24107 & -0.06806 & 0.05514 \\ -0.30745 & 0.30002 & -0.04706 & 0.03039 \\ -0.33613 & 0.36263 & -0.03406 & 0.01025 \\ & & & \\ -0.04665 & 0.03407 & -0.04521 & 0.03344 \\ -0.09267 & 0.06879 & -0.08164 & 0.06357 \\ -0.13744 & 0.10475 & -0.10273 & 0.08632 \\ -0.18026 & 0.14249 & -0.10611 & 0.09720 \\ -0.22040 & 0.12244 & -0.09463 & 0.09302 \\ -0.25707 & 0.22488 & -0.07538 & 0.07448 \\ -0.28942 & 0.26991 & -0.05649 & 0.04769 \\ -0.31659 & 0.31742 & -0.04278 & 0.02285 \\ -0.33771 & 0.36703 & -0.03328 & 0.00973 \\ -0.35197 & 0.41813 & -0.02270 & 0.01235\end{array}$

$\begin{array}{ll}-0.00001 & 0.00000 \\ -0.00002 & 0.00002 \\ -0.00015 & 0.00011 \\ -0.00058 & 0.00045 \\ -0.00232 & 0.00180 \\ -0.01403 & 0.01124 \\ -0.05002 & 0.04440 \\ -0.09184 & 0.09553 \\ -0.12023 & 0.15338 \\ -0.12316 & 0.20025 \\ -0.10274 & 0.22143 \\ -0.07037 & 0.21682 \\ & \\ -0.00878 & 0.00694 \\ -0.03274 & 0.02761 \\ -0.06528 & 0.06095 \\ -0.09708 & 0.10363 \\ -0.11895 & 0.14930 \\ -0.12507 & 0.18895 \\ -0.11527 & 0.21444 \\ -0.09429 & 0.22229 \\ -0.06794 & 0.21589 \\ -0.03834 & 0.20352\end{array}$

$-0.00119$

$-0.00238-0.00086$ $-0.00595 \quad 0.00433$ $-0.01190 \quad 0.00866$ $\begin{array}{ll}-0.02375 & 0.01736 \\ -0.05867 & 0.04412\end{array}$ $\begin{array}{ll}-0.05867 & 0.04412 \\ -0.11198 & 0.09314\end{array}$ $\begin{array}{ll}-0.11198 & 0.09314 \\ -0.15372 & 0.15001\end{array}$ $-0.15372 \quad 0.15001$ $\begin{array}{ll}-0.17691 & 0.21386 \\ -0.17560 & 0.27863\end{array}$ $\begin{array}{ll}-0.17560 & 0.27863 \\ -0.14816 & 0.33342\end{array}$ $\begin{array}{ll}-0.14816 & 0.33342 \\ -0.10046 & 0.36573\end{array}$

$\begin{array}{ll}-0.04634 & 0.03439\end{array}$ $-0.09013 \quad 0.07124$ $-0.12853 \quad 0.11244$ $\begin{array}{ll}-0.15829 & 0.15871\end{array}$ $\begin{array}{ll}-0.17599 & 0.20907\end{array}$ $\begin{array}{ll}-0.17870 & 0.26047 \\ -0.16505 & 0.30795\end{array}$ $\begin{array}{ll}-0.16505 & 0.30795 \\ -0.13624 & 0.34535\end{array}$ $-0.09676 \quad 0.36686$ $\begin{array}{lll}-0.05411 & 0.36890\end{array}$

$$
\frac{\mathrm{a}}{\lambda}=1.6 \quad \frac{\alpha}{\beta}=0.05
$$

$\begin{array}{llr}0.01 & -0.00097 & 0.00071 \\ 0.02 & -0.00195 & 0.00141 \\ 0.05 & -0.00487 & 0.00354 \\ 0.10 & -0.00972 & 0.00706 \\ 0.20 & -0.01934 & 0.01405 \\ 0.50 & -0.04666 & 0.03389 \\ 1.00 & -0.08203 & 0.05913 \\ 1.50 & -0.09857 & 0.06844 \\ 2.00 & -0.09486 & 0.05744 \\ 2.50 & -0.07571 & 0.02623 \\ 3.00 & -0.04977 & -0.01962 \\ 3.50 & -0.02564 & -0.06976 \\ & & \\ 0.125 \pi & -0.03725 & 0.02706 \\ 0.250 \pi & -0.06884 & 0.04987 \\ 0.375 \pi & -0.09030 & 0.06458 \\ 0.500 \pi & -0.09922 & 0.06817 \\ 0.625 \pi & -0.09574 & 0.05895 \\ 0.750 \pi & -0.08233 & 0.03705 \\ 0.875 \pi & -0.06306 & 0.00468 \\ 1.000 \pi & -0.04246 & -0.03392 \\ 1.125 \pi & -0.02420 & -0.07304 \\ 1.250 \pi & -0.01012 & -0.10652\end{array}$

$\begin{array}{ll}-0.00000 & 0.00000 \\ -0.00002 & 0.00002 \\ -0.00012 & 0.00009 \\ -0.00047 & 0.00038 \\ -0.00187 & 0.00151 \\ -0.01144 & 0.00929 \\ -0.04244 & 0.03530 \\ -0.08425 & 0.07251 \\ -0.12582 & 0.11226 \\ -0.15783 & 0.14413 \\ -0.17592 & 0.15837 \\ -0.18188 & 0.14905 \\ -0.00712 & 0.00577 \\ -0.02720 & 0.02237 \\ -0.05665 & 0.04767 \\ -0.09040 & 0.07822 \\ -0.12302 & 0.10950 \\ -0.14997 & 0.13639 \\ -0.16860 & 0.15392 \\ -0.17862 & 0.15824 \\ -0.18199 & 0.14751 \\ -0.18206 & 0.12254\end{array}$

$\begin{array}{ll}-0.00097 & 0.00071 \\ -0.00195 & 0.00141 \\ -0.00487 & 0.00354 \\ -0.00973 & 0.00708 \\ -0.01946 & 0.01416 \\ -0.04850 & 0.03555 \\ -0.09593 & 0.07215 \\ -0.14116 & 0.11076 \\ -0.18295 & 0.15214 \\ -0.21997 & 0.19673 \\ -0.25081 & 0.24458 \\ -0.27403 & 0.29527 \\ -0.03815 & 0.02787 \\ -0.07578 & 0.05625 \\ -0.11235 & 0.08563 \\ -0.14731 & 0.11644 \\ -0.18004 & 0.14901 \\ -0.20989 & 0.18356 \\ -0.23618 & 0.22016 \\ -0.25822 & 0.25868 \\ -0.27530 & 0.29882 \\ -0.28682 & 0.34006\end{array}$

$\begin{array}{ll}-0.00097 & 0.00071 \\ -0.00195 & 0.00141 \\ -0.00487 & 0.00354 \\ -0.00972 & 0.00707 \\ -0.01931 & 0.01409 \\ -0.04615 & 0.03439 \\ -0.07835 & 0.06253 \\ -0.08860 & 0.07712 \\ -0.07884 & 0.07246 \\ -0.05978 & 0.05029 \\ -0.04281 & 0.02282 \\ -0.03076 & 0.00662 \\ -0.03700 & 0.02731 \\ -0.06695 & 0.05167 \\ -0.08465 & 0.05966 \\ -0.08825 & 0.07770 \\ -0.08003 & 0.07347 \\ -0.06545 & 0.05792 \\ -0.05063 & 0.03626 \\ -0.03906 & 0.01046 \\ -0.02997 & 0.00623 \\ -0.01967 & 0.00850\end{array}$

$\begin{array}{ll}-0.00000 & 0.00000 \\ -0.00002 & 0.00002 \\ -0.00012 & 0.00009 \\ -0.00047 & 0.00038 \\ -0.00187 & 0.00151 \\ -0.01130 & 0.00942 \\ -0.04029 & 0.03697 \\ -0.07405 & 0.07886 \\ -0.09742 & 0.12547 \\ -0.10107 & 0.16251 \\ -0.08648 & 0.17872 \\ -0.06170 & 0.17445 \\ -0.00707 & 0.00582 \\ -0.02637 & 0.02305 \\ -0.05258 & 0.05060 \\ -0.07831 & 0.08548 \\ -0.09632 & 0.12221 \\ -0.10215 & 0.15366 \\ -0.09562 & 0.17349 \\ -0.08016 & 0.17923 \\ -0.05979 & 0.17367 \\ -0.03572 & 0.16314\end{array}$

$\begin{array}{ll}-0.00097 & 0.00071 \\ -0.00195 & 0.00141 \\ -0.00487 & 0.00354 \\ -0.00973 & 0.00708 \\ -0.01943 & 0.01419 \\ -0.04797 & 0.03607 \\ -0.09147 & 0.07607 \\ -0.12540 & 0.12229 \\ -0.14415 & 0.17389 \\ -0.14312 & 0.22585 \\ -0.12132 & 0.26943 \\ -0.08378 & 0.29488 \\ -0.03789 & 0.02812 \\ -0.07366 & 0.05822 \\ -0.10495 & 0.09178 \\ -0.12911 & 0.12934 \\ -0.14341 & 0.17003 \\ -0.14559 & 0.21133 \\ -0.13471 & 0.24923 \\ -0.11190 & 0.27886 \\ -0.08089 & 0.29577 \\ -0.04768 & 0.29736\end{array}$


$\mathrm{C}(\mathrm{h}, 0)$

0.0
0.0
0.0
0.1
0.20
0.50
1.00
1.50
2.00
2.50
3.00
3.50

$\begin{array}{ll}-0.00080 & 0.00058 \\ -0.00159 & 0.00116\end{array}$

$\begin{array}{ll}-0.00398 & 0.00289\end{array}$

$\begin{array}{lll}-0.00795 & 0.00578\end{array}$

$-0.01582 \quad 0.01148$

$-0.06746 \quad 0.04790$

$-0.08173 \quad 0.05465$

$-0.07984 \quad 0.04449$

$-0.065390 .01790$

$\begin{array}{ll}-0.02484 & -0.02017\end{array}$

$\begin{array}{lll}0.125 \pi & -0.03049 \quad 0.02210\end{array}$

$0.250 \pi \quad-0.05649 \quad 0.04055$

$\begin{array}{lll}0.375 \pi & -0.07443-0.05210\end{array}$

$\begin{array}{llll}0.500 \pi & -0.08241 & 0.05427\end{array}$

$\begin{array}{llll}0.625 \pi & -0.08047 & 0.04582\end{array}$

$\begin{array}{lll}0.750 \pi & -0.07048 & 0.02702\end{array}$

$0.875 \pi-0.05552-0.00008$

$1.000 \pi \quad-0.03898-0.03192$

$\begin{array}{lll}1.125 \pi & -0.02359-0.06382\end{array}$

$1.250 \pi-0.01068-0.09091$
$S(h, 0)$

$\begin{array}{ll}-0.00000 & 0.00000 \\ -0.00002 & 0.00001 \\ -0.00009 & 0.00008 \\ -0.00038 & 0.00032 \\ -0.00151 & 0.00126 \\ -0.00922 & 0.00779 \\ -0.03426 & 0.02947 \\ -0.06823 & 0.06019 \\ -0.10248 & 0.09250 \\ -0.12971 & 0.11775 \\ -0.14648 & 0.12818 \\ -0.15395 & 0.11935 \\ -0.00574 & 0.00484 \\ -0.02194 & 0.01871 \\ -0.04578 & 0.03972 \\ -0.07326 & 0.06486 \\ -0.10015 & 0.09028 \\ -0.12289 & 0.11171 \\ -0.13939 & 0.12518 \\ -0.14938 & 0.12771 \\ -0.15423 & 0.11802 \\ -0.15630 & 0.09697\end{array}$

$\frac{a}{\lambda}=1.6 \quad \frac{\alpha}{\beta}=0.07$

$E(h, 0)$

$\begin{array}{ll}-0.00080 & 0.00058 \\ -0.00159 & 0.00116 \\ -0.00398 & 0.00289 \\ -0.00796 & 0.00579 \\ -0.01592 & 0.01158 \\ -0.03967 & 0.02907 \\ -0.07843 & 0.05899 \\ -0.11536 & 0.09052 \\ -0.14944 & 0.12425 \\ -0.17957 & 0.16054 \\ -0.20460 & 0.19939 \\ -0.22340 & 0.24042 \\ -0.03120 & 0.02279 \\ -0.06196 & 0.04599 \\ -0.09185 & 0.07000 \\ -0.12038 & 0.09515 \\ -0.14707 & 0.12171 \\ -0.17137 & 0.14984 \\ -0.19274 & 0.17957 \\ -0.21061 & 0.21082 \\ -0.22444 & 0.24329 \\ -0.23373 & 0.27657\end{array}$

$C(h, h)$

0.00058 $-0.00398 \quad 0.00116$ $\begin{array}{ll}-0.00795 & 0.00578\end{array}$ $-0.01579 \quad 0.01152$ $-0.03778 \quad 0.02806$ $-0.06440 \quad 0.05065$ $-0.07352 \quad 0.06175$ $\begin{array}{lll}-0.06671 & 0.05709\end{array}$ $-0.052180 .03864$ $-0.02741 \quad 0.00372$

$-0.03028 \quad 0.02230$ $\begin{array}{lll}-0.05491 & 0.04201\end{array}$ $\begin{array}{lll}-0.06976 & 0.05622\end{array}$ $-0.07339-0.06209$ $\begin{array}{ll}-0.06759 & 0.05797\end{array}$ $-0.05657-0.04490$ $-0.04492 \quad 0.02723$ $-0.03515$ $\begin{array}{ll}-0.02666 & 0.00342 \\ -0.01689 & 0.00540\end{array}$
$S(h, h)$ $-0.00002$ $-0.00009$ $-0.00038$ $-0.00150$ $-0.03245$ $-0.05972$ $-0.07898$ $-0.08300$

$-0.05382$

$-0.00570$

$-0.02124$

$-0.04236$

$-0.06318$

$-0.07805$

$-0.08350$

$-0.07934$

$-0.06804$

-0.068230
-0.03276
0.00000 0.00001
0.00008 0.00032 0.00127 0.00788 0.03077 0.0651 0.10271 0.13201 0.14435 0.14034

0.00487

0.01924

0.04201
0.07050

0.10011 0.1001 0.12507 0.14049 0.14459 0.13058
$E(h, h)$

$\begin{array}{ll}-0.00080 & 0.00058 \\ -0.00159 & 0.00116 \\ -0.00398 & 0.00289 \\ -0.00796 & 0.00579 \\ -0.01589 & 0.01161 \\ -0.03922 & 0.02949 \\ -0.07472 & 0.06213 \\ -0.10230 & 0.09970 \\ -0.11746 & 0.14140 \\ -0.11664 & 0.18308 \\ -0.09932 & 0.21774 \\ -0.06979 & 0.23779 \\ -0.03098 & 0.02300 \\ -0.06019 & 0.04757 \\ -0.08569 & 0.07492 \\ -0.10530 & 0.10541 \\ -0.11686 & 0.13828 \\ -0.11862 & 0.17147 \\ -0.10994 & 0.20171 \\ -0.09188 & 0.22519 \\ -0.06752 & 0.23848 \\ -0.04167 & 0.23973\end{array}$

$\begin{array}{llr}0.01 & -0.00059 & 0.00043 \\ 0.02 & -0.00118 & 0.00086 \\ 0.05 & -0.00294 & 0.00214 \\ 0.10 & -0.00588 & 0.00427 \\ 0.20 & -0.01171 & 0.00849 \\ 0.50 & -0.02832 & 0.02038 \\ 1.00 & -0.05031 & 0.03492 \\ 1.50 & -0.06170 & 0.03896 \\ 2.00 & -0.06156 & 0.03015 \\ 2.50 & -0.05217 & 0.00925 \\ 3.00 & -0.03777 & -0.01970 \\ 3.50 & -0.02242 & -0.05021 \\ 0.125 \pi & -0.02258 & 0.01631 \\ 0.250 \pi & -0.04199 & 0.02974 \\ 0.375 \pi & -0.05571 & 0.03774 \\ 0.500 \pi & -0.06236 & 0.03851 \\ 0.625 \pi & -0.06192 & 0.03123 \\ 0.750 \pi & -0.05559 & 0.01632 \\ 0.875 \pi & -0.04535 & -0.00452 \\ 1.000 \pi & -0.03338 & -0.02850 \\ 1.125 \pi & -0.02141 & -0.05218 \\ 1.250 \pi & -0.01027 & -0.07211\end{array}$

$\begin{array}{ll}-0.00000 & 0.00000 \\ -0.00001 & 0.00001 \\ -0.00007 & 0.00006 \\ -0.00027 & 0.00024 \\ -0.00109 & 0.00097 \\ -0.00667 & 0.00597 \\ -0.02485 & 0.02247 \\ -0.04976 & 0.04553 \\ -0.07542 & 0.06927 \\ -0.09679 & 0.08712 \\ -0.11141 & 0.09354 \\ -0.11979 & 0.08566 \\ -0.00415 & 0.00371 \\ -0.01589 & 0.01430 \\ -0.03326 & 0.03021 \\ -0.05349 & 0.04900 \\ -0.07365 & 0.06766 \\ -0.09129 & 0.08295 \\ -0.10494 & 0.09201 \\ -0.11433 & 0.09279 \\ -0.12020 & 0.08459 \\ -0.12391 & 0.06819\end{array}$

$$
\frac{\mathrm{a}}{\lambda}=1.6 \quad \frac{\alpha}{\beta}=0.10
$$

$\begin{array}{ll}-0.00059 & 0.00043 \\ -0.00118 & 0.00086 \\ -0.00294 & 0.00214 \\ -0.00589 & 0.00428 \\ -0.01177 & 0.00856 \\ -0.02933 & 0.02150 \\ -0.05798 & 0.04361 \\ -0.08523 & 0.06687 \\ -0.11032 & 0.09171 \\ -0.13244 & 0.11835 \\ -0.15076 & 0.14677 \\ -0.16445 & 0.17665 \\ -0.02307 & 0.01685 \\ -0.04582 & 0.03401 \\ -0.06789 & 0.05174 \\ -0.08894 & 0.07029 \\ -0.10858 & 0.08984 \\ -0.12644 & 0.11050 \\ -0.14209 & 0.13229 \\ -0.15514 & 0.15510 \\ -0.16520 & 0.17874 \\ -0.17194 & 0.20286\end{array}$

$\begin{array}{ll}-0.00000 & 0.00000 \\ -0.00001 & 0.00001 \\ -0.00007 & 0.00006 \\ -0.00027 & 0.00024 \\ -0.00109 & 0.00097 \\ -0.00658 & 0.00603 \\ -0.02345 & 0.02336 \\ -0.04328 & 0.04889 \\ -0.05774 & 0.07617 \\ -0.06185 & 0.09680 \\ -0.05607 & 0.10492 \\ -0.04351 & 0.10122 \\ & \\ -0.00412 & 0.00373 \\ -0.01535 & 0.01467 \\ -0.03063 & 0.03177 \\ -0.04583 & 0.05283 \\ -0.05700 & 0.07430 \\ -0.06179 & 0.09199 \\ -0.06002 & 0.10254 \\ -0.05309 & 0.10487 \\ -0.04244 & 0.10068 \\ -0.02815 & 0.09325\end{array}$

(1)

$\begin{array}{ll}-0.00059 & 0.00043 \\ -0.00118 & 0.00086 \\ -0.00294 & 0.00214 \\ -0.00589 & 0.00428 \\ -0.01175 & 0.00858 \\ -0.02899 & 0.02180 \\ -0.05516 & 0.04586 \\ -0.07538 & 0.07338 \\ -0.08640 & 0.10368 \\ -0.08583 & 0.13364 \\ -0.07356 & 0.15822 \\ -0.05294 & 0.17223 \\ -0.02291 & 0.01700 \\ -0.04447 & 0.03514 \\ -0.06322 & 0.05525 \\ -0.07757 & 0.07755 \\ -0.08597 & 0.10143 \\ -0.08724 & 0.12533 \\ -0.08106 & 0.14689 \\ -0.06833 & 0.16345 \\ -0.05138 & 0.17271 \\ -0.03362 & 0.17358\end{array}$


Bh

$\mathrm{C}(\mathrm{h}, 0)$

$\begin{array}{llr}0.01 & -0.00022 & 0.00016 \\ 0.02 & -0.00043 & 0.00031 \\ 0.05 & -0.00108 & 0.00078 \\ 0.10 & -0.00215 & 0.00156 \\ 0.20 & -0.00429 & 0.00310 \\ 0.50 & -0.01043 & 0.00738 \\ 1.00 & -0.01895 & 0.01219 \\ 1.50 & -0.02417 & 0.01252 \\ 2.00 & -0.02562 & 0.00768 \\ 2.50 & -0.02362 & -0.00169 \\ 3.00 & -0.01896 & -0.01372 \\ 3.50 & -0.01241 & -0.02585 \\ & & \\ 0.125 \pi & -0.00830 & 0.00593 \\ 0.250 \pi & -0.01563 & 0.01058 \\ 0.375 \pi & -0.02123 & 0.01288 \\ 0.500 \pi & -0.02461 & 0.01215 \\ 0.625 \pi & -0.02564 & 0.00820 \\ 0.750 \pi & -0.02450 & 0.00137 \\ 0.875 \pi & -0.02158 & -0.00750 \\ 1.000 \pi & -0.01727 & -0.01726 \\ 1.125 \pi & -0.01191 & -0.02662 \\ 1.250 \pi & -0.00568 & -0.03440\end{array}$

$S(h, 0)$

$-0.00000$

$-0.00000$

$-0.0000$

$-0.00037$

-0.00037
-0.00226

$-0.00853$

$-0.01748$

$-0.02745$

$-0.04518$

$-0.05175$

$-0.00140$

$-0.00542$

$-0.01149$

$-0.01887$

$-0.02672$

$-0.03435$

-0.04125
-0.04720

$-0.05214$

$-0.05610$ $\frac{a}{\lambda}=1.6 \quad \frac{\alpha}{\beta}=0.20$

$E(h, 0)$

$C(h, h)$

$S(h, h)$

$E(h, h)$

0.00000 0.00000 0.00002

0.00010

0.00040

0.00907

0.01801

0.02667

0.03243

0.03336
0.02871

0.00152

0.00580

0.01211

0.02610

0.03120

0.03358

0.03261

0.02820
0.02073 $\begin{array}{ll}-0.00022 & 0.00016\end{array}$

$-0.00043 \quad 0.00031$

$-0.00108 \quad 0.00078$

$-0.00215 \quad 0.00157$

$-0.00431 \quad 0.00313$

$-0.01073 \quad 0.00786$

$\begin{array}{lll}-0.02118 & 0.01593\end{array}$

$-0.03108 \quad 0.02438$

$-0.04012 \quad 0.0333$

$\begin{array}{ll}-0.04802 & 0.04284 \\ -0.05448 & 0.05286\end{array}$

$\begin{array}{ll}-0.05448 & 0.05286 \\ -0.05924 & 0.06324\end{array}$

$-0.00844 \quad 0.00617$

$-0.01675 \quad 0.0124$

$-0.024790 .01889$

$-0.032420 .0256$

$-0.04588 \quad 0.04005$

$\begin{array}{ll}-0.05950 & 0.06396 \\ -0.06180 & 0.07222\end{array}$ $\begin{array}{ll}-0.05143 & 0.0477\end{array}$

$-0.056010 .05577$ $\begin{array}{ll}-0.00022 & 0.00015\end{array}$

$-0.00043 \quad 0.00031$

$-0.00215 \quad 0.00155$

$-0.00428 \quad 0.00311$

$-0.01030 \quad 0.00749$

$-0.0183040 .00749$

$-0.02181 \quad 0.0128$

$-0.02187 \quad 0.01171$

$-0.01948-0.00579$

$-0.01567-0.00032$

$-0.01053-0.00355$

$\begin{array}{ll}-0.00823 & 0.00598\end{array}$ $-0.01516 \quad 0.01094$ $-0.01986 \quad 0.01396$ $-0.02196 \quad 0.01204$ 0.017450 .00256 $-0.01437-0.00163$ $\begin{array}{ll}-0.01013 & -0.00361 \\ -0.00497 & -0.00275\end{array}$ $-0.02202 \quad 0.01436$ $-0.02034 \quad 0.00760$
$-0.00000$ $-0.00000$ $-0.00009$ $-0.00222$ -0.00222
-0.00795 $-0.01488$ $-0.01488$ $-0.02351$ 0.02351

$-0.02055$

\section{$-0.00139$} $-0.00519$ $-0.01042$ -0.01581 . $-0.02022$ $-0.02297$ $-0.02387$ $-0.02298$ -0.02022
-0.01521
0.00000 0.00000 0.00002 0.00010 0.00040 0.00246 0.01888 0.02841 0.03490 0.03662 0.00152 0.00591 0.01253 0.02030 0.02778 0.03349 0.03624 0.03360 0.02946
$-0.00022$ $-0.00043$ $-0.00108$ -0.00215
-0.00430 -0.00430
-0.01059 $-0.02006$ $-0.02724$ $-0.03105$ $-0.03105$ 0.03087 -0.030879
-0.02077

$-0.00837$ $-0.01621$ $-0.02294$ $-0.03090$ $-0.03090$ $-0.03134$ $-0.02538$ $-0.02538$ -0.02031
-0.01523
0.00016 0.00031 0.00157 0.00314 0.00314
0.00797 0.01666 0.02643 0.03687 0.05466 0.05891 0.00622 0.01281 0.02002 0.02788 0.04411 0.05110 0.05627 0.05905
0.05930

$$
\frac{a}{\lambda}=1.6 \quad \frac{\alpha}{\beta}=0.40
$$

$\begin{array}{llr}0.01 & -0.00003 & 0.00002 \\ 0.02 & -0.00006 & 0.00004 \\ 0.05 & -0.00014 & 0.00010 \\ 0.10 & -0.00029 & 0.00021 \\ 0.20 & -0.00058 & 0.00041 \\ 0.50 & -0.00142 & 0.00097 \\ 1.00 & -0.00270 & 0.00149 \\ 1.50 & -0.00371 & 0.00123 \\ 2.00 & -0.00431 & 0.00010 \\ 2.50 & -0.00437 & -0.00178 \\ 3.00 & -0.00376 & -0.00410 \\ 3.50 & -0.00240 & -0.00644 \\ & & \\ 0.125 \pi & -0.00112 & 0.00078 \\ 0.250 \pi & -0.00218 & 0.00134 \\ 0.375 \pi & -0.00310 & 0.00149 \\ 0.500 \pi & -0.00383 & 0.00112 \\ 0.625 \pi & -0.00428 & 0.00021 \\ 0.750 \pi & -0.00441 & -0.00118 \\ 0.875 \pi & -0.00415 & -0.00291 \\ 1.000 \pi & -0.00345 & -0.00478 \\ 1.125 \pi & -0.00227 & -0.00659 \\ 1.2501 \pi & -0.00061 & -0.00814\end{array}$

更

$-0.00000$

$-0.00000$

0.00000

0.00000

$-0.00000 \quad 0.00000$

$-0.00004$

$\begin{array}{ll}-0.00025 & 0.00040 \\ -0.00101 & 0.00146\end{array}$

$\begin{array}{ll}-0.00222 & 0.0028\end{array}$

$\begin{array}{ll}-0.00383 & 0.00410\end{array}$

$\begin{array}{ll}-0.00572 & 0.00478\end{array}$

$\begin{array}{ll}-0.00777 & 0.00458 \\ -0.00976 & 0.00334\end{array}$

$-0.00016$

$-0.00063$

$\begin{array}{lll}-0.00139 & 0.00193\end{array}$

$\begin{array}{ll}-0.00242 & 0.00304\end{array}$

$-0.00370$

$-0.00516$

$-0.00673$

$-0.00988$

$-0.01123$

$\begin{array}{ll}-0.00003 & 0.00002 \\ -0.00006 & 0.00004 \\ -0.00014 & 0.00010 \\ -0.00029 & 0.00021 \\ -0.00058 & 0.00042 \\ -0.00144 & 0.00105 \\ -0.00283 & 0.00213 \\ -0.00413 & 0.00324 \\ -0.00531 & 0.00440 \\ -0.00631 & 0.00561 \\ -0.00712 & 0.00686 \\ -0.00769 & 0.00811 \\ -0.00113 & 0.00083 \\ -0.00224 & 0.00166 \\ -0.00330 & 0.00252 \\ -0.00431 & 0.00340 \\ -0.00523 & 0.00432 \\ -0.00604 & 0.00526 \\ -0.00674 & 0.00623 \\ -0.00730 & 0.00721 \\ -0.00772 & 0.00820 \\ -0.00799 & 0.00917\end{array}$

$\begin{array}{rr}-0.00003 & 0.00002 \\ -0.00006 & 0.00004 \\ -0.00014 & 0.00010 \\ -0.00029 & 0.00021 \\ -0.00057 & 0.00041 \\ -0.00140 & 0.00098 \\ -0.00256 & 0.00157 \\ -0.00335 & 0.00152 \\ -0.00371 & 0.00079 \\ -0.00359 & -0.00036 \\ -0.00291 & -0.00149 \\ -0.00156 & -0.00212 \\ -0.00111 & 0.00079 \\ -0.00210 & 0.00139 \\ -0.00289 & 0.00164 \\ -0.00342 & 0.00115 \\ -0.00369 & 0.00087 \\ -0.00368 & -0.00001 \\ -0.00333 & -0.00095 \\ -0.00260 & -0.00174 \\ -0.00145 & -0.00213 \\ 0.00005 & -0.00194\end{array}$

-0.00000
-0.00000
-0.00000
-0.00001
-0.00004
-0.00025
-0.00091
-0.00181
-0.00276
-0.00362
-0.00423
-0.00430
-0.00015
-0.00059
-0.00122
-0.00194
-0.00269
-0.00339
-0.00397
-0.00432
-0.00427
-0.00365

0.00000

0.00000

0.00000

0.00002

0.00006

0.00040
0.00146

0.00286

0.00412

0.00480

0.00463
0.00360

$-0.00003$

$-0.00006$

$-0.00014$

$-0.00058$

$-0.00141$

$-0.00265$

$-0.00356$

$-0.00401$

$-0.00400$

$-0.00361$

0.00025

0.00094

0.00195

0.00306

0.00404

0.00468

0.00483

0.00442

0.00350

0.00216

0.00002

0.00004

0.00010

0.00042

0.00106

0.00220

$0.00343^{\circ}$

0.00467

0.00657

0.00083

$-0.00302$

$-0.00365$

$-0.00400$

$-0.00405$

$-0.00384$

$-0.00345$

$-0.00300$

0.00170

0.00361

0.00458

0.00548

0.00621
0.00672

0.00697

0.00699 
$\beta \mathrm{h}$

$C(h, 0)$

$0.01-0.00000 \quad 0.00000$

0.02

0.05

0.10

0.50

1.50

2.00

2. 50

3.00
3.50

$0.125 \pi$

$0.250 \pi$

$0.375 \pi$

0.500

$0.875 \pi$

$1.000 \pi$

$1.250 \pi$
$S(h, 0)$

$\begin{array}{lr}-0.00000 & 0.00000 \\ -0.00000 & 0.00000 \\ -0.00000 & 0.00000 \\ -0.00000 & 0.00001 \\ -0.00000 & 0.00000 \\ -0.00001 & 0.00002 \\ -0.00004 & 0.00009 \\ -0.00011 & 0.00018 \\ -0.00022 & 0.00026 \\ -0.00039 & 0.00030 \\ -0.00061 & 0.00027 \\ -0.00085 & 0.00012 \\ & \\ -0.00001 & 0.00002 \\ -0.00002 & 0.00006 \\ -0.00096 & 0.00012 \\ -0.00012 & 0.00019 \\ -0.00021 & 0.00026 \\ -0.00034 & 0.00030 \\ -0.00050 & 0.00030 \\ -0.00068 & 0.00024 \\ -0.00086 & 0.00011 \\ -0.00104 & -0.00010\end{array}$

$\frac{a}{\lambda}=1.6 \quad \frac{\alpha}{\beta}=0.70$

$E(h, 0)$

$\mathrm{C}(\mathrm{h}, \mathrm{h})$

$S(h, h)$

$-0.00000 \quad 0.00000$

$-0.00000 \quad 0.00000$

$-0.00001 \quad 0.00001$

$\begin{array}{ll}-0.000003 & 0.000002\end{array}$

$-0.00007 \quad 0.00005$

$-0.00014 \quad 0.0000$

$-0.00020 \quad 0.00005$

$-0.00024-0.00002$

$\begin{array}{lll}-0.00025 & -0.00013\end{array}$

$\begin{array}{rr}-0.00017 & -0.00025 \\ 0.00000 & -0.00033\end{array}$

$-0.00030 \quad 0.00027$

$-0.00034 \quad 0.00032$

$\begin{array}{ll}-0.00036 & 0.00037\end{array}$

$\begin{array}{ll}-0.00006 & 0.00004\end{array}$

$-0.000110 .00008$

$-0.00016 \quad 0.00012$

$\begin{array}{ll}-0.00021 & 0.00016\end{array}$

$\begin{array}{ll}-0.00025 & 0.00021\end{array}$

$\begin{array}{ll}-0.00029 & 0.00025 \\ -0.00032 & 0.00029\end{array}$

$\begin{array}{ll}-0.00034 & 0.00034\end{array}$

$-0.00036 \quad 0.00038$
$-0.00000$

$-0.00000$

-0.00000
$-0.000 n 0$

$-0.00000$

$-0.0000$

$-0.00004$

$-0.00008$

$-0.00016$

$-0.00036$

$-0.00042$

$-0.00001$

$-0.00002$

$-0.00005$

$-0.00015$

$-0.00022$

$-0.00031$

$-0.00042$

$-0.00039$

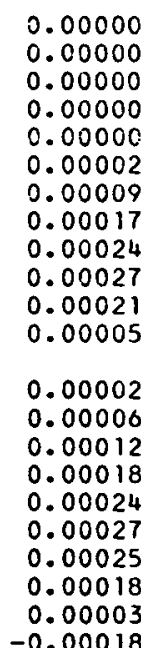

$E(h, h)$

$\begin{array}{ll}-0.00000 & 0.00000 \\ -0.00000 & 0.00000 \\ -0.00001 & 0.00001 \\ -0.00001 & 0.00001 \\ -0.00003 & 0.00002 \\ -0.00007 & 0.00005 \\ -0.00013 & 0.00011 \\ -0.00017 & 0.00016 \\ -0.00019 & 0.0002 .1 \\ -0.00019 & 0.00025 \\ -0.00017 & 0.00028 \\ -0.00016 & 0.00029 \\ & \\ -0.00005 & 0.00004 \\ -0.00010 & 0.00008 \\ -0.00014 & 0.00013 \\ -0.00017 & 0.00017 \\ -0.00019 & 0.00021 \\ -0.00019 & 0.00024 \\ -0.00018 & 0.00027 \\ -0.00017 & 0.00028 \\ -0.00016 & 0.00029 \\ -0.00015 & 0.00029\end{array}$

$$
\frac{a}{\lambda}=1.6 \quad \frac{\alpha}{\beta}=1.00
$$

$\begin{array}{lr}-0.00000 & 0.00000 \\ -0.00000 & 0.00000 \\ -0.00000 & 0.00000 \\ -0.00000 & 0.00000 \\ -0.00000 & 0.00000 \\ -0.00000 & 0.00000 \\ -0.00000 & 0.00001 \\ -0.00001 & 0.00001 \\ -0.00001 & 0.00002 \\ -0.00003 & 0.00002 \\ -0.00005 & 0.00002 \\ -0.00008 & 0.00000 \\ -0.00000 & 0.00000 \\ -0.00000 & 0.00000 \\ -0.00000 & 0.00001 \\ -0.00001 & 0.00001 \\ -0.00001 & 0.00002 \\ -0.00002 & 0.00002 \\ -0.00004 & 0.00002 \\ -0.00006 & 0.00001 \\ -0.00008 & -0.00000 \\ -0.00011 & -0.00003\end{array}$

$\begin{array}{ll}-0.00000 & 0.00000 \\ -0.00000 & 0.00000 \\ -0.00000 & 0.00000 \\ -0.00000 & 0.00000 \\ -0.00000 & 0.00000 \\ -0.00000 & 0.00000 \\ -0.00001 & 0.00001 \\ -0.00001 & 0.00001 \\ -0.00001 & 0.00001 \\ -0.00001 & 0.00001 \\ -0.00002 & 0.00002 \\ -0.00002 & 0.00002 \\ -0.00000 & 0.00000 \\ -0.00001 & 0.00000 \\ -0.00001 & 0.00001 \\ -0.00001 & 0.00001 \\ -0.00001 & 0.00001 \\ -0.00001 & 0.00001 \\ -0.00002 & 0.00001 \\ -0.00002 & 0.00002 \\ -0.00002 & 0.00002 \\ -0.00002 & 0.00002\end{array}$

$\begin{array}{rr}-0.00000 & 0.00000 \\ -0.00000 & 0.00000 \\ -0.00000 & 0.00000 \\ -0.00000 & 0.00000 \\ -0.00000 & 0.00000 \\ -0.00000 & 0.00000 \\ -0.00001 & 0.00000 \\ -0.00001 & 0.00000 \\ -0.00002 & -0.00001 \\ -0.00002 & -0.00002 \\ -0.00001 & -0.00003 \\ 0.00002 & -0.00004 \\ -0.00000 & 0.00000 \\ -0.00001 & 0.00000 \\ -0.00001 & 0.00000 \\ -0.00001 & 0.00000 \\ -0.00002 & -0.00000 \\ -0.00002 & -0.00001 \\ -0.00001 & -0.00002 \\ -0.00000 & -0.00003 \\ 0.00002 & -0.00004 \\ 0.00005 & -0.00004\end{array}$

$\begin{array}{lr}-0.00000 & 0.00000 \\ -0.00000 & 0.00000 \\ -0.00000 & 0.00000 \\ -0.00000 & 0.00000 \\ -0.00000 & 0.00000 \\ -0.00000 & 0.00000 \\ -0.00000 & 0.00001 \\ -0.00000 & 0.00001 \\ -0.00001 & 0.00002 \\ -0.00002 & 0.00002 \\ -0.00003 & 0.00001 \\ -0.00004 & -0.00001 \\ -0.00000 & 0.00000 \\ -0.00000 & 0.00000 \\ -0.00000 & 0.00001 \\ -0.00000 & 0.00001 \\ -0.00001 & 0.00002 \\ -0.00002 & 0.00002 \\ -0.00003 & 0.00001 \\ -0.00004 & 0.00000 \\ -0.00004 & -0.00002 \\ -0.00004 & -0.00005\end{array}$

a

$-0.00000$

$-0.00000$

$-0.00000$

$-0.00000$

$-0.00000$

$-0.00001$

0.0001

$-0.00001$

$-0.00001$

$-0.00001$

$-0.00000$

$-0.00001$

$-0.00001$

$-0.00001$

0.00001

$-0.00001$

$-0.00001$

$-0.00001$

$-0.00001$

0.00000 0.00000 0.00000 0.00000 0.00000 0.00000 0.00001 0.00001 0.00001 0.00001 0.000001 0.00000 0.00000 0.00001 0.00001 0.00001 0.00001 0.00001 0.00001 0.00001 0.00001 
$\beta \mathrm{h}$

$C(h, 0)$

$\begin{array}{lrr}0.01 & 0.00055 & 0.00168 \\ 0.02 & 0.00109 & 0.00336 \\ 0.05 & 0.00273 & 0.00841 \\ 0.10 & 0.00546 & 0.01679 \\ 0.20 & 0.01088 & 0.03340 \\ 0.50 & 0.02648 & 0.08051 \\ 1.00 & 0.04770 & 0.14077 \\ 1.50 & 0.05780 & 0.16628 \\ 2.00 & 0.05087 & 0.15254 \\ 2.50 & 0.02310 & 0.10639 \\ 3.00 & -0.02420 & 0.04371 \\ 3.50 & -0.08247 & -0.01615 \\ 0.125 \pi & 0.02105 & 0.06430 \\ 0.250 \pi & 0.03959 & 0.11851 \\ 0.375 \pi & 0.05289 & 0.15431 \\ 0.500 \pi & 0.05797 & 0.16668 \\ 0.625 \pi & 0.05205 & 0.15478 \\ 0.750 \pi & 0.03327 & 0.12218 \\ 0.875 \pi & 0.00164 & 0.07604 \\ 1.000 \pi & -0.04015 & 0.02566 \\ 1.125 \pi & -0.08651 & -0.01971 \\ 1.250 \pi & -0.12955 & -0.05308\end{array}$

$S(h, 0)$

$\begin{array}{ll}0.00000 & 0.00001 \\ 0.00001 & 0.00003 \\ 0.00007 & 0.00021 \\ 0.00027 & 0.00084 \\ 0.00109 & 0.00335 \\ 0.00680 & 0.02054 \\ 0.02672 & 0.07661 \\ 0.05768 & 0.15309 \\ 0.09461 & 0.22972 \\ 0.12855 & 0.28721 \\ 0.14774 & 0.31340 \\ 0.14108 & 0.30726 \\ 0.00420 & 0.01278 \\ 0.01664 & 0.04898 \\ 0.03667 & 0.10251 \\ 0.06271 & 0.16442 \\ 0.09188 & 0.22457 \\ 0.11975 & 0.27354 \\ 0.14071 & 0.30451 \\ 0.14886 & 0.31469 \\ 0.13948 & 0.30585 \\ 0.11055 & 0.28382\end{array}$

$\frac{\mathrm{a}}{\lambda}=1.8 \quad \frac{\alpha}{\beta}=0.00$

E!h, 0;

$C(h, h)$

$S(h, h)$

$0.00000 \quad 0.00001$ $0.00001 \quad 0.00003$ $0.00007 \quad 0.0002$ 0.000270 .00084 $0.00110 \quad 0.00335$ $0.00702 \quad 0.02044$ $0.07087 \quad 0.14432$ 0.126220 .20248 $\begin{array}{ll}0.12622 & 0.20248\end{array}$ $\begin{array}{ll}0.18185 & 0.22750 \\ 0.21917 & 0.21389\end{array}$ $\begin{array}{ll}0.22977 & 0.17489\end{array}$

$0.00429 \quad 0.01275$ $0.01790 \quad 0.04836$ $0.04238 \quad 0.09924$ $0.07805 \quad 0.15385$ $0.12194 \quad 0.19916$ $0.16696 \quad 0.22442$ $\begin{array}{lll}0.20367 & 0.22508\end{array}$ $0.22476 \quad 0.20454$ $\begin{array}{ll}0.22970 & 0.17179 \\ 0.22627 & 0.13528\end{array}$

$\begin{array}{ll}0.00864 & 0.04650 \\ -0.01181 & 0.03192\end{array}$
$E(h, h)$

$0.00055 \quad 0.00168$ $0.00109 \quad 0.00336$ $0.00273 \quad 0.0084$ 0.011010 .01681 0.028520 .03357 0.023950 .08357 $0.11190 \quad 0.23520$ 0.174890 .23520 0.250360 .29099 $\begin{array}{ll}0.25036 & 0.32222 \\ 0.32923 & 0.32133\end{array}$ $0.39056 \quad 0.28637$

$0.02204 \quad 0.06579$ $0.04749 \quad 0.12997$ $0.07938 \quad 0.19043$ $0.11990 \quad 0.24426$ $0.16980 \quad 0.28764$ 0.227780 .31626 0.290020 .32610 $0.35021 \quad 0.31476$ 0.400320 .28285 $0.43221 \quad 0.23499$

$\begin{array}{lrr}0.01 & 0.00052 & 0.00159 \\ 0.02 & 0.00103 & 0.00318 \\ 0.05 & 0.00258 & 0.00794 \\ 0.10 & 0.00516 & 0.01587 \\ 0.20 & 0.01028 & 0.03157 \\ 0.50 & 0.02499 & 0.07610 \\ 1.00 & 0.04484 & 0.13310 \\ 1.50 & 0.05392 & 0.15739 \\ 2.00 & 0.04675 & 0.14472 \\ 2.50 & 0.01993 & 0.10151 \\ 3.00 & -0.02503 & 0.04262 \\ 3.50 & -0.07988 & -0.01394 \\ & & \\ 0.125 \pi & 0.01988 & 0.06077 \\ 0.250 \pi & 0.03730 & 0.11203 \\ 0.375 \pi & 0.04961 & 0.14595 \\ 0.500 \pi & 0.05401 & 0.15780 \\ 0.625 \pi & 0.04792 & 0.14682 \\ 0.750 \pi & 0.02968 & 0.11631 \\ 0.875 \pi & -0.00053 & 0.07303 \\ 1.000 \pi & -0.04009 & 0.02561 \\ 1.125 \pi & -0.08367 & -0.01732 \\ 1.250 \pi & -0.12384 & -0.04928\end{array}$

$\begin{array}{ll}0.00000 & 0.00001 \\ 0.00001 & 0.00003 \\ 0.00007 & 0.00020 \\ 0.00026 & 0.00079 \\ 0.00105 & 0.00316 \\ 0.00652 & 0.01938 \\ 0.02555 & 0.07228 \\ 0.05494 & 0.14448 \\ 0.08969 & 0.21691 \\ 0.12124 & 0.27149 \\ 0.13853 & 0.29681 \\ 0.13134 & 0.29192 \\ & \\ 0.00403 & 0.01206 \\ 0.01593 & 0.04622 \\ 0.03502 & 0.09673 \\ 0.05970 & 0.15518 \\ 0.08714 & 0.21204 \\ 0.11312 & 0.25846 \\ 0.13234 & 0.28808 \\ 0.13933 & 0.29826 \\ 0.12978 & 0.29066 \\ 0.10193 & 0.27066\end{array}$

$\begin{array}{ll}0.00052 & 0.00159 \\ 0.00103 & 0.00318 \\ 0.00258 & 0.00795 \\ 0.00517 & 0.01589 \\ 0.01035 & 0.03178 \\ 0.02610 & 0.07935 \\ 0.05389 & 0.15793 \\ 0.08498 & 0.23489 \\ 0.12084 & 0.30919 \\ 0.16266 & 0.37951 \\ 0.21133 & 0.44427 \\ 0.26720 & 0.50160 \\ 0.02042 & 0.06235 \\ 0.04165 & 0.12435 \\ 0.06451 & 0.18558 \\ 0.08974 & 0.24560 \\ 0.11803 & 0.30388 \\ 0.14996 & 0.35978 \\ 0.18600 & 0.41256 \\ 0.22642 & 0.46135 \\ 0.27130 & 0.50521 \\ 0.32047 & 0.54313\end{array}$

$$
\frac{a}{\lambda}=1.8 \quad \frac{\alpha}{\beta}=0.005
$$

$\begin{array}{rlll}0.00052 & 0.00159 & 0.00000 & 0.00001 \\ 0.00103 & 0.00318 & 0.00001 & 0.00003 \\ 0.00258 & 0.00794 & 0.00007 & 0.00020 \\ 0.00516 & 0.01586 & 0.00026 & 0.00079 \\ 0.01033 & 0.03155 & 0.00105 & 0.00316 \\ 0.02580 & 0.07573 & 0.00672 & 0.01929 \\ 0.05035 & 0.13034 & 0.02850 & 0.07068 \\ 0.06813 & 0.14942 & 0.06733 & 0.13609 \\ 0.06991 & 0.13180 & 0.11938 & 0.19100 \\ 0.05021 & 0.09205 & 0.17135 & 0.21489 \\ 0.01642 & 0.05338 & 0.20596 & 0.20264 \\ -0.01086 & 0.03197 & 0.21563 & 0.16645 \\ 0.02028 & 0.06059 & 0.00411 & 0.01203 \\ 0.04020 & 0.11065 & 0.01712 & 0.04562 \\ 0.05791 & 0.14161 & 0.04039 & 0.09359 \\ 0.06957 & 0.14896 & 0.07411 & 0.14508 \\ 0.07050 & 0.13409 & 0.11537 & 0.18786 \\ 0.05795 & 0.10441 & 0.15747 & 0.21187 \\ 0.03407 & 0.07129 & 0.19162 & 0.21287 \\ 0.00699 & 0.04529 & 0.21109 & 0.19402 \\ -0.01192 & 0.03119 & 0.21557 & 0.16356 \\ -0.01391 & 0.02596 & 0.21233 & 0.12920\end{array}$

$\begin{array}{ll}0.00052 & 0.00159 \\ 0.00103 & 0.00318 \\ 0.00258 & 0.00795 \\ 0.00517 & 0.01589 \\ 0.01040 & 0.03176 \\ 0.02695 & 0.07896 \\ 0.06042 & 0.15459 \\ 0.10566 & 0.22214 \\ 0.16503 & 0.27472 \\ 0.23604 & 0.30410 \\ 0.31010 & 0.30328 \\ 0.37319 & 0.27053 \\ 0.02083 & 0.06217 \\ 0.04487 & 0.12280 \\ 0.07498 & 0.17989 \\ 0.11321 & 0.23067 \\ 0.16024 & 0.27156 \\ 0.21481 & 0.29850 \\ 0.27330 & 0.30775 \\ 0.32978 & 0.29712 \\ 0.37670 & 0.26724 \\ 0.40651 & 0.22251\end{array}$


$\beta h$

$C(h, 0)$

0.01

0.02

0.05

0.10

0.20

1.00

1.50

2.00

3.00

3.50

$0.125 \pi$

$0.250 \pi$
$0.375 \pi$

$0.500 \pi$

$0.625 \pi$

$0.750 \pi$
$0.875 \pi$

$1.000 \pi$

1.125

$1.250 \pi$
$S(h, 0)$

0.00000 0.00001

0.00006

0.00101

0.00625

0.02443

0.05233

0.08504

0.12991

0.12226

0.00386 0.01525

0.03344

0.05682

0.10686

0.12448

0.13042

0.12073

0.09393

0.02547

$\begin{array}{ll}-0.08090 & -0.01517 \\ -0.11840 & -0.04578\end{array}$ $\frac{a}{\lambda}=1.8 \quad \frac{\alpha}{\beta}=0.01$

$E(h, 0)$

$\mathrm{C}(\mathrm{h}, \mathrm{h})$

$S(h, h)$

$E(h, h)$

$\begin{array}{ll}0.00049 & 0.00150 \\ 0.00098 & 0.00300 \\ 0.00244 & 0.00751 \\ 0.00488 & 0.01502 \\ 0.00978 & 0.03003 \\ 0.02467 & 0.07498 \\ 0.05093 & 0.14924 \\ 0.08030 & 0.22194 \\ 0.11415 & 0.29211 \\ 0.15364 & 0.35849 \\ 0.19955 & 0.41959 \\ 0.25223 & 0.47364 \\ 0.01929 & 0.05893 \\ 0.03936 & 0.11750 \\ 0.06096 & 0.17536 \\ 0.08479 & 0.23206 \\ 0.11150 & 0.28710 \\ 0.14165 & 0.33987 \\ 0.17566 & 0.38967 \\ 0.21378 & 0.43589 \\ 0.25609 & 0.47735 \\ 0.30243 & 0.51277\end{array}$

$0.00049 \quad 0.00150$ $0.00088 \quad 0.00300$ $0.00244 \quad 0.0075$ 0.01499 $0.00976 \quad 0.0298$ 0.02435 $0.04735 \quad 0.1232$ $0.06373 \quad 0.14143$ $0.04603 \quad 0.08804$ $\begin{array}{rr}0.01426 & 0.05172 \\ -0.01104 & 0.03120\end{array}$

\subsection{5} 0.03787 0.05437 0.06502 0.06550 0.05338 0.03081 0.00547 $-0.01201$ $-0.01360$

\subsection{7} 0.10459 0.13392 0.14106 0.12728 0.09959 0.06859 0.04406 0.03042 0.28270 0.27622
0.25808 0.064920 .12515
$0.00000 \quad 0.00001$ 0.00003 $0.00006 \quad 0.00019$ $0.00025 \quad 0.00075$ $\begin{array}{lll}0.00101 & 0.00298\end{array}$ $0.00644 \quad 0.01819$ $\begin{array}{ll}0.02720 & 0.06667\end{array}$ 0.12834
0.18017 0.112920 .1801 $\begin{array}{ll}0.16146 & 0.20299 \\ 0.19355 & 0.19199\end{array}$

0.202370 .15841

\subsection{4} 0.01637 0.03848 0.07036 c. 10916 0.14853 0.18029 0.19827 0.20230 0.19922
0.01135 0.04303 0.08827 0.13682 0.17720 0.20002 0.20134 0.18404 0.15570 0.12336
$0.00049 \quad 0.00150$ $0.00098 \quad 0.00300$ $0.00244 \quad 0.0075$ $0.00489 \quad 0.01502$ $0.00983 \quad 0.0300$ $0.02546 \quad 0.07462$ $0.05708 \quad 0.14605$ 0.095730 .20979 0.155730 .25936 $0.29209 \quad 0.28624$ $\begin{array}{ll}0.29209 & 0.28624 \\ 0.35119 & 0.25557\end{array}$

\section{$0.01968 \quad 0.05875$} $0.04239 \quad 0.11603$ $0.07083 \quad 0.16993$ 0.106890 .21785 0.151210 .25639 $0.225755 \quad 0.28174$ $0.31054 \quad 0.28047$ $\begin{array}{ll}0.35448 & 0.25249 \\ 0.38234 & 0.21069\end{array}$

$\begin{array}{lrr}0.01 & 0.00044 & 0.00134 \\ 0.02 & 0.00087 & 0.00268 \\ 0.05 & 0.00218 & 0.00670 \\ 0.10 & 0.00435 & 0.01339 \\ 0.20 & 0.00867 & 0.02664 \\ 0.50 & 0.02101 & 0.06425 \\ 1.00 & 0.03724 & 0.11253 \\ 1.50 & 0.04375 & 0.13348 \\ 2.00 & 0.03611 & 0.12358 \\ 2.50 & 0.01200 & 0.08811 \\ 3.00 & -0.02665 & 0.03919 \\ 3.50 & -0.07246 & -0.00858 \\ & & \\ 0.125 \pi & 0.01674 & 0.05130 \\ 0.250 \pi & 0.03117 & 0.09465 \\ 0.375 \pi & 0.04092 & 0.12350 \\ 0.500 \pi & 0.04362 & 0.13392 \\ 0.625 \pi & 0.03722 & 0.12528 \\ 0.750 \pi & 0.02061 & 0.10031 \\ 0.875 \pi & -0.00575 & 0.06452 \\ 1.000 \pi & -0.03935 & 0.02495 \\ 1.125 \pi & -0.07558 & -0.01148 \\ 1.250 \pi & -0.10829 & -0.03957\end{array}$

$\begin{array}{ll}0.00000 & 0.00001 \\ 0.00001 & 0.00003 \\ 0.00006 & 0.00017 \\ 0.00023 & 0.00067 \\ 0.00092 & 0.00266 \\ 0.00574 & 0.01628 \\ 0.02232 & 0.06073 \\ 0.04747 & 0.12147 \\ 0.07645 & 0.18267 \\ 0.10176 & 0.22937 \\ 0.11425 & 0.25223 \\ 0.10591 & 0.25036 \\ 0.00355 & 0.01013 \\ 0.01397 & 0.03882 \\ 0.03048 & 0.08128 \\ 0.05148 & 0.13048 \\ 0.07435 & 0.17853 \\ 0.09538 & 0.21812 \\ 0.11015 & 0.24400 \\ 0.11427 & 0.25402 \\ 0.10445 & 0.24948 \\ 0.07964 & 0.23460\end{array}$

$$
\frac{a}{\lambda}=1.8 \quad \frac{\alpha}{\beta}=0.02
$$

$\begin{array}{llrl}0.00044 & 0.00134 & 0.00044 & 0.00134 \\ 0.00087 & 0.00268 & 0.00087 & 0.00268 \\ 0.00218 & 0.00671 & 0.00218 & 0.00670 \\ 0.00436 & 0.01341 & 0.00436 & 0.01339 \\ 0.00873 & 0.02682 & 0.00871 & 0.02662 \\ 0.02203 & 0.06696 & 0.02169 & 0.06393 \\ 0.04547 & 0.13326 & 0.04188 & 0.11015 \\ 0.07169 & 0.19815 & 0.05575 & 0.12672 \\ 0.10188 & 0.26072 & 0.05592 & 0.11281 \\ 0.13706 & 0.31987 & 0.03857 & 0.08046 \\ 0.17793 & 0.37426 & 0.01048 & 0.04842 \\ 0.22476 & 0.42231 & -0.01132 & 0.02957 \\ & & & \\ 0.01723 & 0.052 .62 & 0.01707 & 0.05115 \\ 0.03515 & 0.10493 & 0.03361 & 0.09345 \\ 0.05443 & 0.15657 & 0.04791 & 0.11978 \\ 0.07569 & 0.207 .17 & 0.05677 & 0.12646 \\ 0.09952 & 0.25625 & 0.05651 & 0.11466 \\ 0.12639 & 0.30329 & 0.04520 & 0.09057 \\ 0.15667 & 0.34763 & 0.02501 & 0.06338 \\ 0.19059 & 0.38858 & 0.00283 & 0.04153 \\ 0.22819 & 0.42534 & -0.01213 & 0.02880 \\ 0.26932 & 0.45705 & -0.01303 & 0.02296\end{array}$

$\begin{array}{llll}0.00000 & 0.00001 & 0.00044 & 0.00134 \\ 0.00001 & 0.00003 & 0.00087 & 0.00268 \\ 0.00006 & 0.00017 & 0.00218 & 0.00671 \\ 0.00023 & 0.00067 & 0.00437 & 0.01341 \\ 0.00093 & 0.00265 & 0.00878 & 0.02680 \\ 0.00591 & 0.01619 & 0.02274 & 0.06663 \\ 0.02477 & 0.05931 & 0.05094 & 0.13036 \\ 0.05774 & 0.11415 & 0.08897 & 0.18713 \\ 0.10104 & 0.16036 & 0.13866 & 0.23116 \\ 0.14340 & 0.18116 & 0.19782 & 0.25565 \\ 0.17095 & 0.17235 & 0.25915 & 0.25498 \\ 0.17823 & 0.14343 & 0.31103 & 0.22807 \\ & & & \\ 0.00362 & 0.01010 & 0.01757 & 0.05246 \\ 0.01496 & 0.03829 & 0.03785 & 0.10358 \\ 0.03494 & 0.07851 & 0.06319 & 0.15164 \\ 0.06343 & 0.12169 & 0.09529 & 0.19429 \\ 0.09774 & 0.15769 & 0.13466 & 0.22853 \\ 0.13218 & 0.17832 & 0.18017 & 0.25099 \\ 0.15963 & 0.18013 & 0.22873 & 0.25868 \\ 0.17493 & 0.16559 & 0.27537 & 0.24991 \\ 0.17815 & 0.14107 & 0.31391 & 0.22537 \\ 0.17534 & 0.11241 & 0.33824 & 0.18888\end{array}$


$\frac{\mathrm{a}}{\lambda}=1.8 \quad \frac{\alpha}{\beta}=0.03$

$\beta \mathrm{h}$

$\mathrm{C}(\mathrm{h}, 0)$

0.0

0.05

0.10

0.20
0.50

1.00

2.00

2.50

3.00
3.50

$0.125 \pi$

$0.250 \pi$

$0.375 \pi$
$0.500 \pi$

$0.625 \pi$

$0.750 \pi$

$0.875 \pi$

$1.125 \pi$

$-0.07054-0.0085$

$\begin{array}{rr}0.00039 & 0.00120 \\ 0.00078 & 0.00240 \\ 0.00195 & 0.00599 \\ 0.00389 & 0.01196 \\ 0.00774 & 0.02380 \\ 0.01871 & 0.05739 \\ 0.03290 & 0.10062 \\ 0.03803 & 0.11960 \\ 0.03026 & 0.11122 \\ 0.00785 & 0.08011 \\ -0.02712 & 0.03686 \\ -0.06780 & -0.00588 \\ 0.01492 & 0.04582 \\ 0.02766 & 0.08458 \\ 0.03599 & 0.11049 \\ 0.03779 & 0.12005 \\ 0.03132 & 0.11270 \\ 0.01576 & 0.09035 \\ -0.00830 & 0.05930 \\ -0.03847 & 0.02418 \\ -0.07054 & -0.00851 \\ -0.09911 & -0.03429\end{array}$

$0.00000 \quad 0.00001$

0.000010 .00002

$0.00005 \quad 0.00015$

$0.00021 \quad 0.00059$

0.000850 .00236

$0.00526 \quad 0.01449$

0.0233850 .05407

$0.04374 \quad 0.16293$

$0.09058 \quad 0.20505$

$0.10050 \quad 0.22635$

$0.00326 \quad 0.00902$

$0.01279 \quad 0.03456$

$\begin{array}{ll}0.02777 & 0.07238\end{array}$

0.046640 .11626

$0.06690=0.15922$

$0.08516 \quad 0.19483$

$0.09750 \quad 0.21849$

$0.10013 \quad 0.22830$

$\begin{array}{ll}0.09032 & 0.22533 \\ 0.06735 & 0.21320\end{array}$
$E(h, D)$

Cih, h)

$\begin{array}{llrl}0.00039 & 0.00120 & 0.00039 & 0.00120 \\ 0.00078 & 0.00240 & 0.00078 & 0.00240 \\ 0.00195 & 0.00599 & 0.00195 & 0.00599 \\ 0.00389 & 0.01198 & 0.00389 & 0.01196 \\ 0.00780 & 0.02395 & 0.00778 & 0.02378 \\ 0.01967 & 0.05980 & 0.01932 & 0.05710 \\ 0.04061 & 0.11899 & 0.03703 & 0.09847 \\ 0.06400 & 0.17690 & 0.04875 & 0.11354 \\ 0.09093 & 0.23271 & 0.04812 & 0.10168 \\ 0.12228 & 0.28542 & 0.03217 & 0.07347 \\ 0.15865 & 0.33383 & 0.00731 & 0.04518 \\ 0.20029 & 0.37655 & -0.01148 & 0.02787 \\ & & & \\ 0.01539 & 0.04700 & 0.01522 & 0.04568 \\ 0.03139 & 0.09370 & 0.02983 & 0.08350 \\ 0.04860 & 0.13980 & 0.04221 & 0.10713 \\ 0.06757 & 0.13495 & 0.04955 & 0.11337 \\ 0.08882 & 0.22872 & 0.04869 & 0.10329 \\ 0.11277 & 0.27064 & 0.03816 & 0.08231 \\ 0.13973 & 0.31014 & 0.02009 & 0.05845 \\ 0.16991 & 0.34657 & 0.00064 & 0.03897 \\ 0.20333 & 0.37923 & -0.01215 & 0.02712 \\ 0.23984 & 0.40739 & -0.01250 & 0.02107\end{array}$

$S(h, h)$ 0.00001 0.00005 0.00021 0.00085 0.00541 0.02255 0.09043 0.12738

0.15102

0.15696

0.00332

0.01366

0.03172

0.05718

0.08752

0.11765

0.14138

0.15425
0.00001 0.00002 0.00015 0.00236 0.01441 0.05276 0.14274 0.16171 0.15474
0.12983

0.00899

0.03407 0.06984 0.10824
0.14036 0.15900 0.16118 0.14898 0.12776
0.10235
$E(h, h)$

0.00039 0.00078 0.0039 0.00390 0.02030 0.07933 0.12347 0.17585 0.22993

0.27547

0.01569

0.03379

0.05638

0.08496

0.11992

0.20313

列

0.27799

0.2992

0.00120 0.00240 0.00599 0.02393 0.05950 0.1635 0.20603 0.22772 0.20353

0.04685 0.09247 0.13532 0.20369 0.22360 0.23039 0.22268 0.16930

$\begin{array}{lrr}0.01 & 0.00031 & 0.00096 \\ 0.02 & 0.00062 & 0.00191 \\ 0.05 & 0.00155 & 0.00478 \\ 0.10 & 0.00310 & 0.00954 \\ 0.20 & 0.00617 & 0.01898 \\ 0.50 & 0.01485 & 0.04580 \\ 1.00 & 0.02567 & 0.08045 \\ 1.50 & 0.02868 & 0.09603 \\ 2.00 & 0.02098 & 0.09008 \\ 2.50 & 0.00167 & 0.06613 \\ 3.00 & -0.02699 & 0.03223 \\ 3.50 & -0.05918 & -0.00209 \\ 0.125 \pi & 0.01186 & 0.03656 \\ 0.250 \pi & 0.02177 & 0.06756 \\ 0.375 \pi & 0.02781 & 0.08844 \\ 0.500 \pi & 0.02829 & 0.09647 \\ 0.625 \pi & 0.02194 & 0.09120 \\ 0.750 \pi & 0.00835 & 0.07445 \\ 0.875 \pi & -0.01171 & 0.04989 \\ 1.000 \pi & -0.03608 & 0.02216 \\ 1.125 \pi & -0.06131 & -0.00424 \\ 1.250 \pi & -0.08317 & -0.02597\end{array}$

$$
\frac{a}{\lambda}=1.8 \quad \frac{\alpha}{\beta}=0.05
$$

$\begin{array}{llllll}0.00000 & 0.00000 & 0.00031 & 0.00096 & 0.00031 & 0.00096 \\ 0.00001 & 0.00002 & 0.00062 & 0.00191 & 0.00062 & 0.00191 \\ 0.00004 & 0.00012 & 0.00155 & 0.00478 & 0.00155 & 0.00477 \\ 0.00018 & 0.00047 & 0.00311 & 0.00955 & 0.00310 & 0.00954 \\ 0.00071 & 0.00187 & 0.00622 & 0.01910 & 0.00620 & 0.01897 \\ 0.00442 & 0.01148 & 0.01569 & 0.04769 & 0.01533 & 0.04556 \\ 0.01698 & 0.04287 & 0.03238 & 0.09487 & 0.02895 & 0.07869 \\ 0.03540 & 0.08591 & 0.05101 & 0.14100 & 0.03724 & 0.09116 \\ 0.05559 & 0.12969 & 0.07243 & 0.18539 & 0.03549 & 0.08258 \\ 0.07183 & 0.16398 & 0.09732 & 0.22724 & 0.02203 & 0.06111 \\ 0.07782 & 0.18242 & 0.12614 & 0.28560 & 0.00247 & 0.03898 \\ 0.06866 & 0.18425 & 0.15904 & 0.29936 & -0.01152 & 0.02439 \\ 0.00274 & 0.00715 & 0.01227 & 0.03748 & 0.01210 & 0.03644 \\ 0.01070 & 0.02739 & 0.02503 & 0.07472 & 0.02349 & 0.06666 \\ 0.02304 & 0.05741 & 0.03874 & 0.11146 & 0.03276 & 0.08571 \\ 0.03827 & 0.09233 & 0.05385 & 0.14740 & 0.03770 & 0.09112 \\ 0.05417 & 0.12670 & 0.07075 & 0.18222 & 0.03603 & 0.08378 \\ 0.06794 & 0.15556 & 0.08977 & 0.21552 & 0.02693 & 0.06788 \\ 0.07645 & 0.17532 & 0.11116 & 0.24684 & 0.01240 & 0.04948 \\ 0.07690 & 0.18451 & 0.13504 & 0.27567 & -0.00260 & 0.03395 \\ 0.06742 & 0.18385 & 0.16144 & 0.30148 & -0.01198 & 0.02370 \\ 0.04775 & 0.17594 & 0.19021 & 0.32366 & -0.01153 & 0.01758\end{array}$

$\begin{array}{ll}0.00000 & 0.00000 \\ 0.00001 & 0.00002 \\ 0.00004 & 0.00012 \\ 0.00018 & 0.00047 \\ 0.00072 & 0.00187 \\ 0.00454 & 0.01141 \\ 0.01867 & 0.04177 \\ 0.04246 & 0.08035 \\ 0.07246 & 0.11316 \\ 0.10059 & 0.128 .92 \\ 0.11792 & 0.12476 \\ 0.12171 & 0.10629 \\ 0.00279 & 0.00712 \\ 0.01138 & 0.02698 \\ 0.02611 & 0.05527 \\ 0.04647 & 0.08567 \\ 0.07022 & 0.11125 \\ 0.09327 & 0.12650 \\ 0.11096 & 0.12913 \\ 0.12024 & 0.12062 \\ 0.12160 & 0.10470 \\ 0.11924 & 0.08468\end{array}$

0.00031 0.00062 0.00155 0.00311 0.00625 0.03621 0.03621 0.06307 0.09790

0.18101

0.21610

0.01251

0.02693

0.02693

0.0448

0.06752

0.0951

0.12675

0.16021

0.19203

0.23425

0.00096 0.00191 0.00478 0.00955 0.04744 0.04744 0.13280 0.13280 0.16367 0.18025 0.16207

0.03736 0.07370 0.13784 0.16183 0.17746 0.18277 0.16026 0.13597 
Bh

$C(h, 0)$

0.01

0.02

0.05

0.10

0.50

1.00

1.50

2.00

3.00

3.50

$0.125 \pi$

$0.250 \pi$

$0.375 \pi$

$0.500 \pi$

$0.625 \pi$

$0.750 \pi$
$0.875 \pi$

$1.250 \pi$

$0.00025 \quad 0.00076$ 0.00050

0.00124

0.00247

0.00491

0.01178

0.02002

0.01421

$-0.00235$

$-0.02588$

0.00943

0.01713

0.02148

0.02109
0.01507 .

0.00328

$-0.01344$

$-0.05312-0.00160$

$-0.06993-0.01993$
$S(h, 0)$

0.00000

0.00001

0.00004

0.00015

0.00060

0.00370

0.01472

0.04498

0.05702
0.06030

0.05129

0.00230

0.00893

0.03139

0.04389

0.05425

0.06002

0.05018

0.03337

0.00001

0.00009

0.00148

0.00910

0.03400

0.10330

0.13125

0.15025

0.00566

0.02172

0.04555

0.10089

0.12430

0.14924

0.15005

0.14509 $\frac{\mathrm{a}}{\lambda}=1.3 \quad \frac{\alpha}{\beta}=0.07$

$E(h, 0)$

$\mathrm{C}(\mathrm{h}, \mathrm{h})$

$S(h, h)$

$E(h, h)$

$\begin{array}{ll}0.00025 & 0.00076 \\ 0.00050 & 0.00152 \\ 0.00124 & 0.00381 \\ 0.00248 & 0.00762 \\ 0.00496 & 0.01524 \\ 0.01251 & 0.03803 \\ 0.02581 & 0.07565 \\ 0.04065 & 0.11238 \\ 0.05769 & 0.14769 \\ 0.07745 & 0.18092 \\ 0.10029 & 0.21131 \\ 0.12629 & 0.23799 \\ 0.00979 & 0.02989 \\ 0.01996 & 0.05958 \\ 0.019089 & 0.08886 \\ 0.04292 & 0.11748 \\ 0.05636 & 0.14517 \\ 0.07147 & 0.17162 \\ 0.08842 & 0.19646 \\ 0.10733 & 0.21928 \\ 0.12818 & 0.23967 \\ 0.15086 & 0.25715\end{array}$

0.00025

0.00152

0.00247

$0.00494 \quad 0.01513$

$0.01216 \quad 0.03635$

$0.02263 \quad 0.06289$

$0.02840 \quad 0.07320$

0.026010 .06703

$0.01467 \quad 0.05067$

$-0.00079 \quad 0.03330$

$\begin{array}{ll}-0.01124 & 0.02101\end{array}$

$0.00962 \quad 0.02907$

0.01850

0.06858

$0.02863 \quad 0.07324$

$0.02651 \quad 0.06793$

$0.01868-0.05587$

$0.00697 \quad 0.04163$

$-0.00467$

$\begin{array}{ll}-0.01155 & 0.02038 \\ -0.01062 & 0.01448\end{array}$

0.00000

0.00001

0.00004

0.00015

0.00380

0.01544

0.01544

0.03459

0.07951

0.09214

0.09434

0.00233

0.00946

0.02148

0.03777

0.07401

0.08717

0.09370

0.09422

0.09200

0.00001

0.00009

0.00037

0.00148

0.00904

0.03362

0.06362

0.0898

0.10287

0.10063

0.00564

0.02136

0.04375

0.06784

0.08823

0.10072

0.10353

0.09766

0.0857

0.06990

$\begin{array}{lrr}0.01 & 0.00018 & 0.00054 \\ 0.02 & 0.00035 & 0.00109 \\ 0.05 & 0.00088 & 0.0027 \\ 0.10 & 0.00176 & 0.00542 \\ 0.20 & 0.00350 & 0.01079 \\ 0.50 & 0.00833 & 0.02606 \\ 1.00 & 0.01378 & 0.04601 \\ 1.50 & 0.01396 & 0.05550 \\ 2.00 & 0.00744 & 0.05313 \\ 2.50 & -0.00563 & 0.04061 \\ 3.00 & -0.02320 & 0.02194 \\ 3.50 & -0.04146 & 0.00178 \\ & & \\ 0.125 \pi & 0.00669 & 0.02079 \\ 0.250 \pi & 0.01196 & 0.03853 \\ 0.375 \pi & 0.01455 & 0.05073 \\ 0.500 \pi & 0.01346 & 0.05588 \\ 0.625 \pi & 0.00815 & 0.05368 \\ 0.750 \pi & -0.00128 & 0.04505 \\ 0.875 \pi & -0.01402 & 0.03179 \\ 1.000 \pi & -0.02850 & 0.01619 \\ 1.125 \pi & -0.04262 & 0.00045 \\ 1.250 \pi & -0.05405 & -0.01378\end{array}$

$\frac{\alpha}{\beta}=0.10$

$\begin{array}{rl}0.00018 & 0.00054 \\ 0.00035 & 0.00109 \\ 0.00088 & 0.00271 \\ 0.00176 & 0.00542 \\ 0.00351 & 0.01078 \\ 0.00859 & 0.02591 \\ 0.01563 & 0.04494 \\ 0.01886 & 0.05267 \\ 0.01610 & 0.04898 \\ 0.00733 & 0.03807 \\ -0.00362 & 0.02591 \\ -0.01042 & 0.01641 \\ & \\ 0.00682 & 0.02071 \\ 0.01293 & 0.03798 \\ 0.01735 & 0.04910 \\ 0.01887 & 0.05279 \\ 0.01652 & 0.04956 \\ 0.01032 & 0.04159 \\ 0.00178 & 0.03184 \\ -0.00624 & 0.02287 \\ -0.01059 & 0.01587 \\ -0.00933 & 0.01059\end{array}$

10.
0.00003

0.00000 0.0000

$0.00012 \quad 0.00026$ $\begin{array}{ll}0.00046 & 0.00105\end{array}$ $\begin{array}{ll}0.00289 & 0.00037\end{array}$ $\begin{array}{ll}0.01159 & 0.02330\end{array}$ $\begin{array}{ll}0.02542 & 0.04485\end{array}$ $0.04177 \quad 0.06352$ $\begin{array}{ll}0.05597 & 0.07344\end{array}$ $0.06371 \quad 0.07296$

0.064320 .06423

$0.00178 \quad 0.00398$ $0.00716 \quad 0.01505$ $0.01601 \quad 0.03083$ $0.02767 \quad 0.04784$ $0.04058 \quad 0.04784$ $\begin{array}{ll}0.04058 & 0.06240 \\ 0.05240 & 0.07167\end{array}$ 0.060790 .07443

$0.06451 \quad 0.07118$

$0.06419 \quad 0.07119$

0.062110 .05225
0.00025 $0.00050 \quad 0.00152$ $0.00248 \quad 0.00762$ $0.00499 \quad 0.01522$ 0.012910 .03782 0.028840 .07384 0.077630 .13002 0.078090 .1302 0.109800 .14337 0.142510 .14304 $0.00998 \quad 0.02979$ $0.02146 \quad 0.05874$ 0.035730 .0858 $0.05367-0.1096$ $0.07543 \quad 0.12858$ 0.100260 .14085 $\begin{array}{ll}0.12637 & 0.14499\end{array}$ $0.15103 \quad 0.14037$ 0.171020 .12766 $0.18340 \quad 0.10913$

$\begin{array}{ll}0.00018 & 0.00054 \\ 0.00035 & 0.00109 \\ 0.00088 & 0.00271 \\ 0.00177 & 0.00543 \\ 0.00355 & 0.01084 \\ 0.00919 & 0.02693 \\ 0.02050 & 0.05251 \\ 0.03555 & 0.07498 \\ 0.05481 & 0.09207 \\ 0.07714 & 0.10134 \\ 0.09957 & 0.10112 \\ 0.11785 & 0.09167 \\ & \\ 0.00711 & 0.02121 \\ 0.01527 & 0.04179 \\ 0.02538 & 0.06098 \\ 0.03803 & 0.07779 \\ 0.05328 & 0.09106 \\ 0.07055 & 0.09959 \\ 0.08854 & 0.10246 \\ 0.10536 & 0.09931 \\ 0.11885 & 0.09074 \\ 0.12709 & 0.07841\end{array}$


$\beta \mathrm{h}$

$\mathrm{C}(\mathrm{h}, 0)$

0.01

0.02

0.0

0.10

0.20
0.50

1.00

2.50

3.50

$0.125 \pi$

$0.250 \pi$

$0.375 \pi$
$0.500 \pi$

$0.625 \pi$

$0.750 \pi$

0.875 म

$1.000 \%$

$1.250 \pi$

0.00028

0.00057

.003020 .0

0.00620

$-0.01930$
$S(h, 0)$

\section{$0.00006 \quad 0.00018$}

0.001120 .00348

$0.00262 \quad 0.00844$

$\begin{array}{ll}0.00394 & 0.01508\end{array}$

$-0.01299 \quad 0.00893$

0.00893

0.00672

0.01255

0.01673

0.01879

0.01857

0.01624

0.01218

$\begin{array}{ll}-0.00953 & 0.01218 \\ -0.01489 & 0.00693 \\ -0.01967 & 0.00100\end{array}$

$\begin{array}{rr}-0.01967 & 0.00100 \\ -0.02314 & -0.00518\end{array}$

0.00070 $\frac{a}{\lambda}=1.8 \quad \frac{\alpha}{\beta}=0.20$

$E(h, 0)$

$\mathrm{C}(\mathrm{h}, \mathrm{h})$

$S(h, h)$

$E(h, h)$

$0.00000 \quad 0.00000$

$0.00000 \quad 0.00000$

$0.00001 \quad 0.00002$

$0.00005 \quad 0.00008$

$\begin{array}{ll}0.00018 & 0.00033\end{array}$

0.001120 .00201

0.004730 .00756

$\begin{array}{ll}0.01148 & 0.02394\end{array}$

$0.01305 \quad 0.0315$

0.03718

0.04032

c. 00125

$0.00265 \quad 0.0048$

$0.00548 \quad 0.01018$

$0.00858 \quad 0.01662$

$0.01128 \quad 0.02333$

$0.01286-0.02953$

$0.01277-0.03465$

$0.00649 \quad 0.04044$

$\begin{array}{ll}0.00649 & 0.04044 \\ 0.00052 & 0.04105\end{array}$

$\begin{array}{llll}0.00006 & 0.00018 & 0.00006 & 0.00018 \\ 0.00011 & 0.00035 & 0.00011 & 0.00035 \\ 0.00028 & 0.00188 & 0.00028 & 0.00088 \\ 0.00057 & 0.00175 & 0.00057 & 0.00175 \\ 0.00114 & 0.00350 & 0.00113 & 0.00348 \\ 0.00287 & 0.00374 & 0.00270 & 0.00338 \\ 0.00592 & 0.01736 & 0.00453 & 0.01409 \\ 0.00930 & 0.02572 & 0.00465 & 0.01762 \\ 0.01315 & 0.03370 & 0.00262 & 0.01711 \\ 0.01756 & 0.04112 & -0.00106 & 0.01421 \\ 0.02259 & 0.04781 & -0.00478 & 0.01025 \\ 0.02822 & 0.05359 & -0.00654 & 0.00620 \\ 0.00225 & 0.00687 & 0.00217 & 0.00670 \\ 0.00458 & 0.01368 & 0.00391 & 0.01235 \\ 0.00708 & 0.02037 & 0.00480 & 0.01615 \\ 0.00982 & 0.02688 & 0.00449 & 0.01774 \\ 0.01285 & 0.03313 & 0.00283 & 0.01725 \\ 0.01623 & 0.03905 & 0.00009 & 0.01521 \\ 0.01999 & 0.04455 & -0.00303 & 0.01230 \\ 0.02412 & 0.04955 & -0.00555 & 0.00909 \\ 0.02863 & 0.05395 & -0.00654 & 0.00593 \\ 0.03345 & 0.05767 & -0.00547 & 0.00297\end{array}$

$\frac{a}{\lambda}=1.8$

$\frac{\alpha}{\beta}=0.40$
0.00000 0.00000 0.00001 0.00005 0.00018 0.00114 0.00438 0.01402 0.01761 0.01878 0.01771

0.00070 0.00276 0.00594 0.00981 0.01369 0.01680 0.01852 0.01867

0.01600 0.00000 0.00002 0.00008 0.00199 0.00727 0.07409 0.02422 0.02522 C. 02332 0.00124 0.00470 0.00964 0.01505 0.01989 0.02337 0.02509 0.02497 0.02308 0.01944

$\begin{array}{lrr}0.01 & 0.00001 & 0.00002 \\ 0.02 & 0.00001 & 0.00004 \\ 0.05 & 0.00003 & 0.00009 \\ 0.10 & 0.00006 & 0.00018 \\ 0.20 & 0.00012 & 0.00036 \\ 0.50 & 0.00026 & 0.00089 \\ 1.00 & 0.00031 & 0.00163 \\ 1.50 & 0.00000 & 0.00210 \\ 2.00 & -0.00068 & 0.00221 \\ 2.50 & -0.00166 & 0.00190 \\ 3.00 & -0.00274 & 0.00114 \\ 3.50 & -0.00368 & -0.00003 \\ & & \\ 0.125 \pi & 0.00021 & 0.00070 \\ 0.250 \pi & 0.00032 & 0.00134 \\ 0.375 \pi & 0.00024 & 0.00184 \\ 0.500 \pi & -0.00007 & 0.00214 \\ 0.625 \pi & -0.00062 & 0.00222 \\ 0.750 \pi & -0.00136 & 0.00203 \\ 0.875 \pi & -0.00220 & 0.00157 \\ 1.000 \pi & -0.00303 & 0.00085 \\ 1.125 \pi & -0.00373 & -0.00013 \\ 1.250 \pi & -0.00419 & -0.00132\end{array}$

$\begin{array}{rlllrr}0.00000 & 0.00000 & 0.00001 & 0.00002 & 0.00001 & 0.00002 \\ 0.00000 & 0.00000 & 0.00001 & 0.00004 & 0.00001 & 0.00004 \\ 0.00000 & 0.00000 & 0.00003 & 0.00009 & 0.00003 & 0.00009 \\ 0.00001 & 0.00001 & 0.00006 & 0.00018 & 0.00006 & 0.00018 \\ 0.00003 & 0.00003 & 0.00012 & 0.00036 & 0.00012 & 0.00036 \\ 0.00016 & 0.00020 & 0.00030 & 0.00091 & 0.00027 & 0.00088 \\ 0.00058 & 0.00076 & 0.00061 & 0.00180 & 0.00037 & 0.00158 \\ 0.00108 & 0.00162 & 0.00096 & 0.00266 & 0.00019 & 0.00198 \\ 0.00144 & 0.00267 & 0.00135 & 0.00347 & -0.00027 & 0.00205 \\ 0.00145 & 0.00377 & 0.00179 & 0.00421 & -0.00089 & 0.00181 \\ 0.00095 & 0.00478 & 0.00228 & 0.00486 & -0.00143 & 0.00127 \\ -0.00009 & 0.00556 & 0.00282 & 0.00541 & -0.00161 & 0.00049 \\ & & & & & \\ 0.00010 & 0.00012 & 0.00023 & 0.00072 & 0.00022 & 0.00070 \\ 0.00037 & 0.00048 & 0.00043 & 0.00142 & 0.00036 & 0.00131 \\ 0.00078 & 0.00104 & 0.00073 & 0.00211 & 0.00034 & 0.00176 \\ 0.00114 & 0.00176 & 0.00101 & 0.00278 & 0.00014 & 0.00201 \\ 0.00142 & 0.00259 & 0.00132 & 0.00341 & -0.00023 & 0.00206 \\ 0.00148 & 0.00345 & 0.00166 & 0.00400 & -0.00071 & 0.00191 \\ 0.00127 & 0.00429 & 0.00203 & 0.00455 & -0.00118 & 0.00157 \\ 0.00071 & 0.00503 & 0.00243 & 0.00503 & -0.00153 & 0.001107 \\ -0.00018 & 0.00560 & 0.00285 & 0.00545 & -0.00160 & 0.00043 \\ -0.00136 & 0.00594 & 0.00330 & 0.00579 & -0.00132 & -0.00028\end{array}$

0.00006 $0.00011 \quad 0.00018$ $0.00028 \quad 0.00088$ $0.00057 \quad 0.00175$ 0.001150 .00350 0.002960 .00868 0.006570 .01685 0.017290 .02391 $0.02379 \cdot 0.03189$ $0.03017 \quad 0.03184$ 0.03514

0.00229 0.00491 0.00812 0.01206 0.01672 0.02187 0.03753 0.02929

0.00684 0.01344 0.01953 0.02478 0.03138 0.03221 0.03134 0.02904 0.02586

$\begin{array}{llll}0.00000 & 0.00000 & 0.00001 & 0.00002 \\ 0.00000 & 0.00000 & 0.00001 & 0.00004 \\ 0.00000 & 0.00000 & 0.00003 & 0.00009 \\ 0.00001 & 0.00001 & 0.00006 & 0.00018 \\ 0.00003 & 0.00003 & 0.00012 & 0.00036 \\ 0.00016 & 0.00019 & 0.00031 & 0.00090 \\ 0.00059 & 0.00072 & 0.00068 & 0.00174 \\ 0.00115 & 0.00143 & 0.00114 & 0.00243 \\ 0.00163 & 0.00218 & 0.00169 & 0.00292 \\ 0.00184 & 0.00280 & 0.00227 & 0.00316 \\ 0.00165 & 0.00317 & 0.00279 & 0.00316 \\ 0.00112 & 0.00312 & 0.00315 & 0.00298 \\ 0.00010 & 0.00012 & 0.00024 & 0.00071 \\ 0.00038 & 0.00046 & 0.00051 & 0.00139 \\ 0.00079 & 0.00096 & 0.00083 & 0.00200 \\ 0.00123 & 0.00154 & 0.00121 & 0.00251 \\ 0.00161 & 0.00213 & 0.00165 & 0.00289 \\ 0.00181 & 0.00264 & 0.00211 & 0.00312 \\ 0.00179 & 0.00303 & 0.00254 & 0.00319 \\ 0.00153 & 0.00321 & 0.00291 & 0.00312 \\ 0.00107 & 0.00310 & 0.00317 & 0.00296 \\ 0.00051 & 0.00263 & 0.00331 & 0.00275\end{array}$


0.01

0.02

0.05

0.10

0.20

0.50
1.00

1.00

2.00

50

3.00

$0.125 \pi$

$0.250 \pi$

$0.375 \pi$

$0.625 \pi$

$0.750 \pi$

$.000 \pi$

$1.125 \pi$
$1.250 \pi$
$0.00000 \quad 0.00000$

$0.00000 \quad c .00000$

$0.00000 \quad 0.00000$

$0.00000 \quad 0.00001$

$0.00001 \quad 0.00003$

$0.00001 \quad 0.00006$

$\begin{array}{ll}-0.00006 & 0.00009\end{array}$

$-0.00020 \quad 0.00003$

$-0.00026-0.00005$

$0.00001 \quad 0.00002$

$0.00001 \quad 0.00005$

$0.00000 \quad 0.00007$

$-0.00006 \quad 0.00009$

$0.00010 \quad 0.00009$

$-0.00016 \quad 0.00006$

$-0.00027-0.00006$

$\begin{array}{ll}-0.00030 & -0.000016\end{array}$
Sịn, 0)

$0.00000 \quad 0.00000$

$0.00000 \quad 0.00000$

$0.00000 \quad 0.00000$

$0.00000 \quad 0.00000$

$0.00000 \quad 0.00000$

$0.00001 \quad 0.00001$

$0.00003 \quad 0.00003$

$0.00007 \quad 0.00011$

$0.00006 \quad 0.00018$

$\begin{array}{rr}0.000002 & 0.00025 \\ -0.00007 & 0.00031\end{array}$

0.00000

$0.00002 \quad 0.00002$

$0.00004 \quad 0.00004$

$0.00007 \quad 0.00011$

$0.00006 \quad 0.00016$

$0.00004 \quad 0.00021$

$-0.00008 \quad 0.00032$

$-0.00018$

$\frac{a}{\lambda}=1.8 \quad \frac{\alpha}{\beta}=0.70$

$E(h, 0)$

$\mathrm{C}(\mathrm{h}, \mathrm{h})$

$S(h, h)$

$E(h, h)$

$0.00000 \quad 0.00000$

$0.00000 \quad 0.00000$

$0.00000 \quad 0.00000$

$0.00000 \quad c .00001$

$0.00000 \quad 0.00001$

$0.00001 \quad 0.00003$

$0.00003 \quad 0.00000$

$0.00004 \quad 0.00011$

$0.00006 \quad 0.00014$

$0.00007 \quad 0.00016$

$0.00001 \quad 0.00002$

$0.00002 \quad 0.00005$

$0.00002 \quad 0.00007$

$0.00003 \quad 0.00009$

$0.00005 \quad 0.00013$

$0.00007 \quad 0.00015$

$0.00008 \quad 0.00016$

$\begin{array}{ll}0.00009 & 0.00017 \\ 0.00010 & 0.00018\end{array}$

$0.00000 \quad c .00000$

$0.00000 \quad 0.00000$

$0.00000 \quad 0.00000$

$0.00000 \quad 0.0000$

$0.00000 \quad 0.0000$

$0.00001 \quad 0.00003$

$0.00001 \quad 0.00006$

$-0.00001 \quad 0.00008$

0.00000

$\begin{array}{ll}-0.00008 & 0.00007 \\ -0.00012 & 0.00003\end{array}$

$-0.00013-0.00004$

$0.00001 \quad 0.00002$

$0.00001 \quad 0.00005$

$0.00000 \quad 0.0000$

$\begin{array}{ll}0.00001 & 0.00008\end{array}$

$-0.00004 \quad 0.00008$

$-0.00007 \quad 0.00008$

0.00006

$-0.00013-0.00004$

$\begin{array}{ll}-0.00013 & -0.00004 \\ -0.00010 & -0.00012\end{array}$

\subsection{0}

$0.00000 \quad 0.00000$

$0.00000 \quad 0.0000$

$0.00001 \quad 0.00001$

$0.00003 \quad 0.00002$

$0.00005 \quad 0.00005$

$0.00007 \quad 0.00009$

$\begin{array}{ll}0.00007 & 0.00013\end{array}$

$-0.00003 \quad 0.00016$

0.00000

0.00000

0.00002

0.00004

0.00005

0.00007

0.00007

0.00006

0.00002

-0.00003
-0.00011
0.00017

0.00000

0.00001

0.00003

0.00008

0.00012

0.00015

0.00017

0.00017
0.00013
0.00000

0.000000 .00000

0.0000

0.000050 .0000

$0.00007 \quad 0.00010$

$0.00008-0.00010$

$0.00009 \quad 0.00010$

$0.00001 \quad 0.00002$

$0.00002 \quad 0.00005$

$0.00003 \quad 0.00007$

0.000040 .00008

0.00007

$0.00008 \quad 0.00010$

$0.00009 \quad 0.00010$

$\begin{array}{ll}0.00009 & 0.00010 \\ 0.00009 & 0.00009\end{array}$ $\frac{a}{\lambda}=1.8 \quad \frac{\alpha}{\beta}=1.00$

$\begin{array}{lrr}0.01 & 0.00000 & 0.00000 \\ 0.02 & 0.00000 & 0.00000 \\ 0.05 & 0.00000 & 0.00000 \\ 0.10 & 0.00000 & 0.00000 \\ 0.20 & 0.00000 & 0.00000 \\ 0.50 & 0.00000 & 0.00000 \\ 1.00 & 0.00000 & 0.00000 \\ 1.50 & -0.00000 & 0.00000 \\ 2.00 & -0.00000 & 0.00000 \\ 2.50 & -0.00001 & 0.00000 \\ 3.00 & -0.00001 & 0.00000 \\ 3.50 & -0.00002 & -0.00001 \\ & & \\ 0.125 \pi & 0.00000 & 0.000000 \\ 0.250 \pi & 0.00000 & 0.00000 \\ 0.375 \pi & -0.00000 & 0.00000 \\ 0.500 \pi & -0.00000 & 0.00000 \\ 0.625 \pi & -0.00000 & 0.00000 \\ 0.750 \pi & -0.00001 & 0.00000 \\ 0.875 \pi & -0.00001 & 0.00000 \\ 1.000 \pi & -0.00001 & -0.00000 \\ 1.125 \pi & -0.00002 & -0.00001 \\ 1.250 \pi & -0.00002 & -0.00002\end{array}$

$\begin{array}{rr}0.00000 & 0.00000 \\ 0.00000 & 0.00000 \\ 0.00000 & 0.00000 \\ 0.00000 & 0.00000 \\ 0.00000 & 0.00000 \\ 0.00000 & 0.00000 \\ 0.00000 & 0.00000 \\ 0.00000 & 0.00000 \\ 0.00000 & 0.00001 \\ 0.00000 & 0.00001 \\ -0.00000 & 0.00001 \\ -0.00001 & 0.00002 \\ & \\ 0.00000 & 0.00000 \\ 0.00000 & 0.00000 \\ 0.00000 & 0.00000 \\ 0.00000 & 0.00000 \\ 0.00000 & 0.00000 \\ 0.00000 & 0.00001 \\ 0.00000 & 0.00001 \\ -0.00000 & 0.00002 \\ -0.00001 & 0.00002 \\ -0.00002 & 0.00002\end{array}$

$\begin{array}{ll}0.00000 & 0.00000 \\ 0.00000 & 0.00000 \\ 0.00000 & 0.00000 \\ 0.00000 & 0.00000 \\ 0.00000 & 0.00000 \\ 0.00000 & 0.00000 \\ 0.00000 & 0.00000 \\ 0.00000 & 0.00000 \\ 0.00000 & 0.00000 \\ 0.00000 & 0.00000 \\ 0.00000 & 0.00001 \\ 0.00000 & 0.00001 \\ 0.00000 & 0.00000 \\ 0.00000 & 0.00000 \\ 0.00000 & 0.00000 \\ 0.00000 & 0.00000 \\ 0.00000 & 0.00000 \\ 0.00000 & 0.00000 \\ 0.00000 & 0.00000 \\ 0.00000 & 0.00001 \\ 0.00000 & 0.00001 \\ 0.00000 & 0.00001\end{array}$

$\begin{array}{rr}0.00000 & 0.00000 \\ 0.00000 & 0.00000 \\ 0.00000 & 0.00000 \\ 0.00000 & 0.00000 \\ 0.00000 & 0.00000 \\ 0.00000 & 0.00000 \\ 0.00000 & 0.00000 \\ -0.00000 & 0.00000 \\ -0.00000 & 0.00000 \\ -0.00001 & 0.00000 \\ -0.00001 & -0.00000 \\ -0.00001 & -0.00001 \\ 0.00000 & 0.00000 \\ 0.00000 & 0.00000 \\ -0.00000 & 0.00000 \\ -0.00000 & 0.00000 \\ -0.00000 & 0.00000 \\ -0.00000 & 0.00000 \\ -0.00001 & 0.00000 \\ -0.00001 & -0.00000 \\ -0.00001 & -0.00001 \\ -0.00001 & -0.00002\end{array}$

(1)

0.00000

0.00000 0.00000 0.00000 0.00000 0.00000 0.00000 0.00000 0.00000 0.00000 $-0.00000$

$-0.00001$ 0.00000
0.00000 0.00000 0.00000 0.00000 0.00000 0.00000 $-0.00000$ $-0.00001$

$-0.00002$
0.00000 0.00000 0.00000 0.00000 0.00000 0.00000 0.00000 0.00000 0.00000 0.0000 0.0000 0.00001

0.00000 0.00000 0.00000 0.00000 0.00000 0.00001 0.00001 0.00001 0.00001
0.00000 0.00000 0.00000 0.00000 0.00000 0.00000 0.00000 0.00000 0.00000 0.00000 0.00000 0.00000

\subsection{0} 0.00000 0.00000 0.00000 0.00000 0.00000 0.00000 0.00000

0.00000
0.00000 0.00000 0.00000 0.00000 0.00000 0.00000 0.00000 0.00000 0.00000

\subsection{0} 0.00000 0.00000
0.00000 0.00000 0.00000
0.00000 0.00000
0.00000 0.00000 0.00000 
$\beta \mathrm{h}$

$\mathrm{C}(\mathrm{h}, 0)$

$S(h, 0)$

0.01
0.02
0.05

0.02
0.05
0.10

0.20

0.50

.50

1.50
2.00
2.50
3.00

3.50

$0.125 \pi$ $0.250 \pi$

$0.500 \pi$

$0.625 \pi$

$0.750 \pi$
$0.875 \pi$

$0.875 \pi$
$-\quad 1.000 \pi$

$1.125 \pi$

$1.250 \pi$
$0.00159-0.00000$

$0.00318-0.00000$

$0.00795-0.00000$

$0.01589-0.00000$

$0.03162-0.00002$

$0.07628-0.00024$

$0.13379-0.00151$

$0.15847-0.00291$

$0.14472-0.00100$

$0.09708 \quad 0.00891$

$\begin{array}{rr}0.02919 & 0.02991 \\ -0.04017 & 0.06035\end{array}$

$0.06090-0.00012$ $0.11247-0.00084$

$0.14684-0.00212$

$0.15886-0.00294$

$0.14698-0.00135$

$\begin{array}{ll}0.14698 & 0.00135 \\ 0.06470 & 0.00500\end{array}$

$0.00885 \quad 0.03779$

$-0.04450 \quad 0.03762$

0.06262 $\frac{a}{\lambda}=2.0 \quad \frac{\alpha}{\beta}=0.00$

$E(h, 0)$

$C(h, h)$

$S(h, h)$

$E(h, h)$

$0.00001-0.00000$ $0.00003-0.00000$ $0.00020-0.00000$ $0.00080-0.00000$ $0.00317-0.00000$ $0.07303-0.0014$ $0.14723-0.00613$ $0.22340-0.01543$ $0.28253-0.02727$ $\begin{array}{ll}0.31070 & -0.03559 \\ 0.30334 & -0.03192\end{array}$

$\begin{array}{lll}0.01211 & -0.00004\end{array}$ $0.04657-0.00056$ $0.09800-0.00259$ $0.15836-0.00717$ $0.21820-0.0146$ $0.26823-0.02389$ $0.30097-0.03236$ $0.31215-0.03614$ $0.30164-0.03103$ $0.27343-0.01381$
$0.001 .59-0.00000$ $0.00318-0.00000$ $0.00796-0.00000$ $0.01592-0.00000$ $0.03183-0.00002$ $0.07956-0.00026$ $0.15896-0.00210$ $0.23798-0.00707$ $0.31619-0.01667$ $0.39292-0.03227$ $0.46719-0.05509$ $0.53767-0.08607$

$0.06249-0.00013$ $0.12491-0.00102$ $0.18717-0.00344$ $0.24912-0.00812$ $0.31052-0.01578$ $0.37106-0.02709$ $0.48760,-0.00300$ $0.59361-0.11942$

$\begin{array}{ll}0.00159 & -0.00000 \\ 0.00318 & -0.00000 \\ 0.60795 & -0.00000 \\ 0.01589 & -0.00001 \\ 0.03161 & -0.00007 \\ 0.07621 & -0.00100 \\ 0.13309 & -0.00676 \\ 0.15599 & -0.01669 \\ 0.14067 & -0.02339 \\ 0.09747 & -0.01753 \\ 0.04879 & 0.00270 \\ 0.01693 & 0.02583 \\ 0.06086 & -0.00049 \\ 0.11215 & -0.00358 \\ 0.14564 & -0.01008 \\ 0.15607 & -0.01807 \\ 0.14294 & -0.02323 \\ 0.11157 & -0.02085 \\ 0.07233 & -0.00885 \\ 0.03736 & 0.00975 \\ 0.01567 & 0.02705 \\ 0.00915 & 0.03390\end{array}$

$0.00001-0.00000$ $0.00003-0.00000$ $0.00020-0.00000$ $0.00080-0.00000$ $\begin{array}{lll}0.00317 & -0.00001\end{array}$ $0.01946-0.00028$ $0.07258-0.00426$ $0.14409-0.01864$ $0.21138-0.04742$ $0.25216-0.08581$ $\begin{array}{ll}0.25532 & -0.12075 \\ 0.22776 & -0.14082\end{array}$ $0.01211-0.00011$ $0.04642-0.00169$ $0.09701-0.00785$ $0.15444-0.02184$ $0.20714-0.04488$ $0.24412-0.07446$ $0.25843-0.10455$ $0.22521-0.14160$ $0.19394-0.14757$ $0.01948-0.00010$ $0.54233-0.08851$

$$
\frac{a}{\lambda}=2.0 \quad \frac{\alpha}{\beta}=0.005
$$

$\begin{array}{ll}0.00001 & -0.00000 \\ 0.00003 & -0.00000 \\ 0.00019 & -0.00000 \\ 0.00075 & -0.00000 \\ 0.00298 & -0.00002 \\ 0.01829 & -0.00018 \\ 0.06857 & -0.00161 \\ 0.13823 & -0.00617 \\ 0.20973 & -0.01478 \\ 0.26532 & -0.02538 \\ 0.29201 & -0.03231 \\ 0.28558 & -0.02782 \\ 0.01137 & -0.00009 \\ 0.04373 & -c .00072 \\ 0.09201 & -c .00278 \\ 0.14867 & -c .00716 \\ 0.20485 & -c .01404 \\ 0.25186 & -0.02240 \\ 0.28272 & -0.02976 \\ 0.29348 & -0.03253 \\ 0.28402 & -0.02691 \\ 0.25804 & -0.01005\end{array}$

$\begin{array}{ll}0.00149 & -0.00000 \\ 0.00299 & -0.00000 \\ 0.00747 & -0.00000 \\ 0.01495 & -0.00000 \\ 0.02989 & -0.00002 \\ 0.07471 & -0.00025 \\ 0.14927 & -0.00198 \\ 0.22345 & -0.00664 \\ 0.29686 & -0.01564 \\ 0.36884 & -0.03028 \\ 0.43849 & -0.05168 \\ 0.50454 & -0.08071 \\ 0.05868 & -0.00012 \\ 0.11730 & -0.00096 \\ 0.17576 & -0.00323 \\ 0.23391 & -0.00762 \\ 0.29154 & -0.01481 \\ 0.34833 & -0.02542 \\ 0.40387 & -0.04003 \\ 0.45762 & -0.05909 \\ 0.50890 & -0.08300 \\ 0.55693 & -0.11195\end{array}$

$\begin{array}{ll}0.00149 & -0.00000 \\ 0.00299 & -0.00000 \\ 0.00747 & -0.00000 \\ 0.01492 & -0.00001 \\ 0.02989 & -0.0000 .5 \\ 0.07157 & -0.00091 \\ 0.12497 & -0.00612 \\ 0.14651 & -0.01502 \\ 0.13228 & -0.02074 \\ 0.09204 & -0.01482 \\ 0.04662 & 0.00414 \\ 0.01666 & 0.02535 \\ 0.05715 & -0.00045 \\ 0.10531 & -0.00324 \\ 0.13676 & -0.00911 \\ 0.14660 & -0.01623 \\ 0.13440 & -0.02063 \\ 0.10518 & -0.01802 \\ 0.06861 & -0.00662 \\ 0.03592 & 0.01065 \\ 0.01545 & 0.02645 \\ 0.00895 & 0.03239\end{array}$

$\begin{array}{ll}0.00159 & -0.00000 \\ 0.00318 & -0.00000 \\ 0.00796 & -0.00000 \\ 0.01591 & -0.00001 \\ 0.03183 & -0.00007 \\ 0.07948 & -0.00105 \\ 0.15810 & -0.00833 \\ 0.23360 & -0.02754 \\ 0.30135 & -0.06288 \\ 0.35417 & -0.11550 \\ 0.38358 & -0.18166 \\ 0.38275 & -0.25171 \\ & \\ 0.06246 & -0.00051 \\ 0.12456 & -0.00406 \\ 0.18553 & -0.01355 \\ 0.24380 & -0.03150 \\ 0.29681 & -0.05971 \\ 0.34100 & -0.09871 \\ 0.37224 & -0.14720 \\ 0.38661 & -0.20167 \\ 0.38151 & -0.25631 \\ 0.35697 & -0.30371\end{array}$

$\begin{array}{lrr}0.01 & 0.00149 & -0.00000 \\ 0.02 & 0.00299 & -0.00000 \\ 0.05 & 0.00747 & -0.00000 \\ 0.10 & 0.01492 & -0.00000 \\ 0.20 & 0.02969 & -0.00001 \\ 0.50 & 0.07164 & -0.00020 \\ 1.00 & 0.12564 & -0.00119 \\ 1.50 & 0.14884 & -0.00207 \\ 2.00 & 0.13601 & 0.00035 \\ 2.50 & 0.09144 & 0.011027 \\ 3.00 & 0.02788 & 0.03031 \\ 3.50 & -0.03719 & 0.05872 \\ 0.125 \pi & 0.05719 & -0.00010 \\ 0.250 \pi & 0.10562 & -0.00067 \\ 0.375 \pi & 0.13790 & -0.00164 \\ 0.500 \pi & 0.14921 & -0.00202 \\ 0.625 \pi & 0.13813 & -0.00003 \\ 0.750 \pi & 0.10689 & 0.00643 \\ 0.875 \pi & 0.06113 & 0.01896 \\ 1.000 \pi & 0.00882 & 0.03772 \\ 1.125 \pi & -0.04126 & 0.06082 \\ 1.250 \pi & -0.08132 & 0.08416\end{array}$

$0.00001-0.00000$ $0.00003-0.00000$ $0.00019-0.00000$ $0.00075-0.00000$ $0.00298-0.00002$ $0.01827-0.00036$ $0.06813-0.00428$ $0.13518-c .01790$ $0.19826-C .04474$ $0.23658-C .08021$ $0.23988-C .11226$ $0.21451-C .13056$

$0.01137-0.00016$ $0.04358-0.00178$ $0.09104-0.0077$ $0.14488-0.02090$ $0.19428-0.04237$ $0.22900-0.06975$ $0.24260-0.09743$ $0.23495-0.11904$ $0.21274-0.13128$

$\begin{array}{ll}0.00149 & -0.00000 \\ 0.00299 & -0.00000 \\ 0.00747 & -0.00000 \\ 0.01495 & -0.00001 \\ 0.02989 & -0.00006 \\ 0.07464 & -0.00099 \\ 0.14843 & -0.00782 \\ 0.21924 & -0.02584 \\ 0.28272 & -0.05895 \\ 0.33213 & -0.10816 \\ 0.35960 & -0.16994 \\ 0.35883 & -0.23520 \\ 0.05865 & -0.00048 \\ 0.11695 & -0.00381 \\ 0.17417 & -0.01271 \\ 0.22881 & -0.02955 \\ 0.27847 & -0.05597 \\ 0.31982 & -0.09246 \\ 0.34902 & -0.13778 \\ 0.36242 & -0.18859 \\ 0.35767 & -0.23948 \\ 0.33486 & -0.28355\end{array}$


Bh

$C(h, 0)$

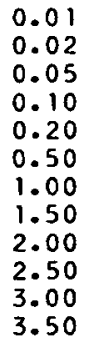

$0.125 \pi$

$0.250 \pi$

$0.375 \pi$

$0.500 \pi$

0.625

$0.750 \pi$
$0.875 \pi$

$1.000 \pi$

1.125 $\begin{array}{ll}0.00140 & -0.00000 \\ 0.00281 & -0.00000\end{array}$

$0.00702-0.00000$

$0.01401-0.00000$

$0.02788-0.00001$

$0.06727-0.00016$

$0.11799-0.00091$

$0.13979-0.00132$

$0.12783 \quad 0.00153$

$0.08612 \quad 0.011142$

0.026610 .03056

$0.05370-0.00008$

$0.09918-0.00053$

$0.12950-0.00121$

$0.14016-0.00120$

$\begin{array}{lll}0.12981 & 0.00113\end{array}$

0.100590 .00767

$\begin{array}{ll}0.05775 & 0.01979\end{array}$

$0.00874 \quad 0.03753$

$-0.03828 \quad 0.05902$

0.08042

$$
\frac{a}{\lambda}=2.0
$$

$\frac{\alpha}{\beta}=$

$E(h, 0)$

$0.00001-0.00000$ $0.00003-0.00000$ $0.00018-0.00000$ $0.00070-0.000 \mathrm{Cl}$ $0.00280-0.00003$ $0.01717-0.00025$ $0.06439-0.00178$ $0.12977-0.00619$ $0.19691 \quad-0.01416$ $0.24917-0.02363$ $0.26887-0.02411$

$0.01068-0.00014$ $0.04106-0.00086$ $0.08639-0.00294$ $0.13958-0.00712$ $0.19232-0.01348$ $0.23650-0.02101$ $0.26559-0.02736$ $0.27594-0.02924$ $26744-0.02319$ $0.24352-0.00669$

$\begin{array}{ll}0.00140 & -0.00000 \\ 0.00281 & -0.00000 \\ 0.00702 & -0.00000 \\ 0.01404 & -0.00000 \\ 0.02807 & -0.00001 \\ 0.07016 & -0.00023 \\ 0.14017 & -0.00186 \\ 0.20981 & -0.00624 \\ 0.27871 & -0.01469 \\ 0.34624 & -0.02842 \\ 0.41155 & -0.04848 \\ 0.47345 & -0.07569 \\ 0.05511 & -0.00011 \\ 0.11015 & -0.00090 \\ 0.16504 & -0.00303 \\ 0.21963 & -0.00716 \\ 0.27371 & -0.01390 \\ 0.32700 & -0.02386 \\ 0.37909 & -0.03755 \\ 0.42948 & -0.05543 \\ 0.47753 & -0.07783 \\ 0.52252 & -0.10494\end{array}$

$C(h, h)$

$0.00140-0.00000$ $0.00281-0.00000$ $0.00702-0.00000$ $0.01401-0.00001$ $0.02788-0.00000$ $.06720-0.0008$ $0.13760-0.00554$ $0.12440-0.01832$ $0.08600-0.01238$ 0.044520 .00540 0.044520 .00540

$0.05367-0.0004$ i $0.09889-0.00291$ $0.12841-0.00822$ $0.13769-0.01455$ $0.12637-0.01826$ $0.09915-0.01546$ $0.06505-0.00463$ $0.03450 \quad 0.01142$ $0.01517 \quad 0.02583$ $0.00873 \quad 0.03096$
$S(h, h)$

$0.00001-0.00000$ $0.00003-0.00000$ $0.00018-0.00000$ $0.00070-0.0000$ $0.00280-0.00003$ $0.01715-0.00042$ $\begin{array}{ll}0.06395 & -0.00428\end{array}$ $\begin{array}{ll}0.12682 & -0.01718\end{array}$ $0.18597-0.04221$ $0.22199-0.07498$ $0.22538-0.10437$ $0.20201-0.12104$

$0.01067-0.00020$ $0.04091-0.00186$ $0.08544-0.00756$ $0.18223-0.04001$ $0.18223-0.0400$ $0.21483-0.06534$ $0.22775-0.09080$ $0.22088-0.11055$ $0.17264-0.12682$
$E(h, h)$

$0.00140-0.00000$ $0.00281-0.00000$ $0.00702-0.00000$ $0.01404-0.00001$ $0.02807-0.00006$ $0.13935-0.00734$ $0.13935-0.00734$ $0.20577-0.02424$ $0.31146-0.10129$ $0.33711-0.10129$ $0.33640-0.21978$

$0.05508-0.00045$ $0.10981-0.00358$ $0.16350-0.01193$ $0.21474-0.02772$ $0.26126-0.05247$ $0.29995-0.08661$ $0.32724-0.12896$ $0.33974-0.17636$ $\begin{array}{ll}0.33533 & -0.22376 \\ 0.31413 & -0.26472\end{array}$

$$
\frac{a}{\lambda}=2.0 \quad \frac{\alpha}{\beta}=0.02
$$

$0.00001-0.00000$ $0.00002-0.00000$ $0.00015-0.00000$ $0.00062-0.00001$ $0.00247-0.00005$ $0.01514-0.00037$ $\begin{array}{lll}0.05677 & -0.00204\end{array}$ $0.11440-0.00616$ $0.17359-0.01298$ $0.21980-0.02048$ $0.24251-0.02403$ $0.23836-0.01772$

$0.00942-0.00021$ $0.03620-0.00109$ $0.07616-0.00318$ $0.12304-0.00698$ $0.16954-0.01242$ $0.20856-0.01849$ $0.23444-0.02311$ $0.24400-0.02349$ $0.23716-0.01680$ $0.21686-0.00109$

$\begin{array}{ll}0.00124 & -0.00000 \\ 0.00248 & -0.00000 \\ 0.00619 & -0.00000 \\ 0.01238 & -0.00000 \\ 0.02476 & -0.00001 \\ 0.06187 & -0.00021 \\ 0.12360 & -0.00164 \\ 0.18498 & -0.00550 \\ 0.24566 & -0.01294 \\ 0.30511 & -0.02502 \\ 0.36253 & -0.04266 \\ 0.41689 & -0.06656 \\ 0.04860 & -0.00010 \\ 0.09714 & -0.00079 \\ 0.14552 & -0.00267 \\ 0.19363 & -0.00631 \\ 0.24127 & -0.01225 \\ 0.28818 & -0.02102 \\ 0.33400 & -0.03306 \\ 0.37828 & -0.04877 \\ 0.42048 & -0.06844 \\ 0.45994 & -0.09222\end{array}$

$0.00124-0.00000$ $0.00248-0.00000$ $0.00619-0.00000$ $0.01236-0.0000$ $0.02459-0.00005$ $0.05926-0.00068$ $0.10346-0.00451$ $0.12138-0.01080$ $0.11001-0.01414$ $0.07745-0.00821$

$\begin{array}{ll}0.04051 & 0.00743 \\ 0.01553 & 0.02378\end{array}$

$0.04733-0.00034$ $0.08719-0.00240$ $0.11323-0.00667$ $0.12148-0.01162$ $0.11172-0.01415$ $0.08809-0.01106$ $0.05843-0.00129$ $\begin{array}{lll}0.03171 & 0.01257\end{array}$ $0.01448 \quad 0.02458$ $0.00820 \quad 0.02831$
$0.00001-0.00000$ $0.00002-0.00000$ $0.00015-0.00000$ $0.00062-0.00001$ $0.00247-0.00005$ $\begin{array}{lll}0.01512 & -0.00051\end{array}$ $0.05634-0.00424$ $0.11164-0.01580$ $0.16363-0.03757$ $0.19547-0.06553$ $0.19899-0.09021$

$0.17916-0.10401$

$\begin{array}{lll}0.00941 & -0.00027\end{array}$ $0.03606-0.00196$ $0.07525-0.00724$ $0.11964-0.01828$ $0.16035-0.03568$ $0.18909-0.05736$ $0.20075-0.07886$ $0.19523-0.09534$ $0.17727-0.10455$ $0.15362-0.10891$

$\begin{array}{ll}0.00124 & -0.00000 \\ 0.00248 & -0.00000 \\ 0.00619 & -0.00000 \\ 0.01238 & -0.00001 \\ 0.02475 & -0.00005 \\ 0.06180 & -0.00082 \\ 0.12283 & -0.00647 \\ 0.18126 & -0.02133 \\ 0.23346 & -0.04855 \\ 0.27391 & -0.08883 \\ 0.29628 & -0.13911 \\ 0.29567 & -0.19190 \\ & \\ 0.04857 & -0.00040 \\ 0.09681 & -0.00315 \\ 0.14409 & -0.01051 \\ 0.18914 & -0.02439 \\ 0.22997 & -0.04611 \\ 0.26385 & -0.07600 \\ 0.28768 & -0.11297 \\ 0.29857 & -0.15423 \\ 0.29474 & -0.19534 \\ 0.27643 & -0.23074\end{array}$


$0.00001-0.00000$ $0.00002-0.00000$ $0.00014-0.00000$ $0.00055-0.00002$ $0.00218-0.00007$ $0.01336-0.00045$ $0.05005-0.00221$ $0.10085-0.00605$ $0.15305-0.01189$ $0.19393-0.01776$ $\begin{array}{ll}0.21434 & -0.01962 \\ 0.21135 & -0.01254\end{array}$ $0.00831-0.00027$ $0.03192-0.00124$ $0.06715-0.00331$ $0.10847-0.00677$ $0.14949-0.01142$ $0.18397-0.01627$ $0.20700-0.01950$ $0.21582-0.01872$ $\begin{array}{rr}0.21036 & -0.01162 \\ 0.19313 & 0.00327\end{array}$

$\begin{array}{lll}0.875 \pi & 0.04590 & 0.021\end{array}$

$1.000 \pi \quad 0.00806 \quad 0.03575$

$\begin{array}{lll}1.125 \pi & -0.02859 & 0.05191 \\ 1.250 \pi & -0.05873 & 0.06704\end{array}$

$\begin{array}{ll}0.00109 & -0.00000 \\ 0.00218 & -0.00000 \\ 0.00546 & -0.00000 \\ 0.01092 & -0.00000 \\ 0.02183 & -0.00001 \\ 0.05456 & -0.00018 \\ 0.10399 & -0.00144 \\ 0.16309 & -0.00484 \\ 0.21654 & -0.01140 \\ 0.26386 & -0.02204 \\ 0.31935 & -0.03755 \\ 0.36710 & -0.05853 \\ 0.04286 & -0.00009 \\ 0.08566 & -0.00070 \\ 0.12831 & -0.00236 \\ 0.17071 & -0.00556 \\ 0.21267 & -0.01079 \\ 0.25396 & -0.01851 \\ 0.29427 & -0.02910 \\ 0.33319 & -0.04291 \\ 0.37024 & -0.06018 \\ 0.40485 & -0.08104\end{array}$

$0.00109-0.00010$ $0.00218-0.000 .00$ $0.00546-0.000 .00$ $0.01090-0.00000$ $0.02168-f, .00004$ $0.05226-0.00055$ $0.09122-0.00365$ $0.09728-0.01069$ $0.06899-0.00486$ $0.03676 \quad 0.00392$ $0.01462 \quad 0.02266$

$0.04174-0.00027$ $0.07688-0.00195$ $0.09984-0.00537$ $0.10719-0.00918$ $0.09876-0.01076$ $0.07825-0.0075$ $0.05243 \quad 0.0013$ $0.02904 \quad 0.0133$ $0.01366 \quad 0.0233$ $0.10707-0.00857$ $0.00760 \quad 0.02591$
$0.00001-0.00000$ $0.00002-0.00000$ $0.00014-0.00000$ $0.00055-0.00002$ $0.00218-0.00007$ $0.01333-0.00058$ $0.04964-0.00415$ $0.09829-0.01451$ $0.14401-0.03344$ $0.17216-0.05729$ $0.15889-0.08934$ $0.00830-0.00032$ $0.03178-0.00201$ $\begin{array}{ll}0.03178 & -0.00201 \\ 0.06628 & -0.00688\end{array}$ $0.00628-0.001688$ $0.14111-0.03181$ $0.16648-0.05037$ $\begin{array}{ll}0.16648 & -0.05037 \\ 0.17699 & -0.06851\end{array}$ $0.176990-0.06851$ $0.17259-0.08222$ $0.13665-0.09346$
$E(h, h)$

$0.00109-0.00000$ $0.00218 \quad-0.00000$ $0.00546-0.00000$ $0.01092-0.00001$ $0.02183-0.00005$ $0.05450-0.00072$ $0.10827-0.00570$ $0.15967-0.01877$ $0.20549-0.04260$ $0.24088-0.07790$ 12173 $0.04283-0.00035$ $\begin{array}{ll}0.08535 & -0.00278 \\ 0.12698 & -0.00925\end{array}$ $0.16660-0.02146$ $0.20243-0.04052$ $0.23243-0.006669$ $0.25290-0.09897$ $0.26238-0.13488$ $0.25907-0.17054$ $0.24325-0.20112$

$$
\frac{a}{\lambda}=2.0 \quad \frac{\alpha}{\beta}=0.05
$$

$0.00085-0.00000$ $0.00170-0.00000$ $0.00425-0.00000$ $0.00849-0.00000$ $0.01698-0.00001$ $0.04243-0.00014$ $0.08475-0.00112$ $0.12677-0.00376$ $0.16824-0.00885$ $0.20877-0.01709$ $0.24781-0.02908$ $0.28464-0.04527$

$0.03333-0.00007$ $0.06661-0.00054$ $0.09976-0.00183$ $\begin{array}{ll}0.13268 & -0.00432 \\ 0.16524 & -0.00838\end{array}$ $0.19724-0.01436$ $0.22843-0.02256$ $0.25849-0.03322$ $0.28706-0.04654$ $0.31369-0.06259$

$\begin{array}{llll}0.00085 & -0.00000 & 0.00000 & -0.00000 \\ 0.00170 & -0.00000 & 0.00002 & -0.00000 \\ 0.00424 & -0.00000 & 0.00011 & -0.00001 \\ 0.00848 & -0.00000 & 0.00042 & -0.00002 \\ 0.01686 & -0.00002 & 0.00169 & -0.00009 \\ 0.04064 & -0.00035 & 0.01037 & -0.00065 \\ 0.07093 & -0.00233 & 0.03854 & -0.00386 \\ 0.08333 & -0.00513 & 0.07621 & -0.01218 \\ 0.07606 & -0.00557 & 0.11159 & -0.02650 \\ 0.05467 & -0.00003 & 0.13363 & -0.04382 \\ 0.03008 & 0.01064 & 0.13710 & -0.05825 \\ 0.01282 & 0.02035 & 0.12496 & -0.06582 \\ & & & \\ 0.03246 & -0.00088 & 0.00645 & -0.00037 \\ 0.05978 & -0.06126 & 0.02469 & -0.00201 \\ 0.07764 & -0.00338 & 0.05144 & -0.00613 \\ 0.08345 & -0.00549 & 0.08164 & -0.01387 \\ 0.07718 & -0.00571 & 0.10935 & -0.02530 \\ 0.06169 & -0.00253 & 0.12912 & -0.03886 \\ 0.04209 & 0.00488 & 0.13767 & -0.05173 \\ 0.02410 & 0.01386 & 0.13494 & -0.06112 \\ 0.01182 & 0.02076 & 0.12374 & -0.06611 \\ 0.00631 & 0.02175 & 0.10802 & -0.06864\end{array}$

$\begin{array}{ll}0.00085 & -0.00000 \\ 0.00170 & -0.00000 \\ 0.00425 & -0.00000 \\ 0.00849 & -0.00000 \\ 0.01698 & -0.00004 \\ 0.04237 & -0.00056 \\ 0.08412 & -0.00442 \\ 0.12390 & -0.01454 \\ 0.15919 & -0.03294 \\ 0.18630 & -0.05991 \\ 0.20114 & -0.09323 \\ 0.20076 & -0.12776 \\ 0.03331 & -0.00027 \\ 0.06634 & -0.00216 \\ 0.09862 & -0.00718 \\ 0.12925 & -0.01661 \\ 0.15685 & -0.03129 \\ 0.17959 & -0.05135 \\ 0.19546 & -0.07596 \\ 0.20264 & -0.10317 \\ 0.20016 & -0.12999 \\ 0.18835 & -0.15283\end{array}$ $\begin{array}{ll}0.16897 & -0.01149 \\ 0.16558 & -0.00412\end{array}$

$0.15316 \quad 0.00909$ 
$C(h, 0)$

0.01

0.02

0.05

0.20

0.20
0.50

1.00

1.50

2.00

3.00

3.50

$0.125 \pi$

$0.250 \pi$

0.375

$0.500 \pi$
$0.625 \pi$

$0.750 \pi$

$0.875 \pi$

1.000

$1.125 \pi$

$1.250 \pi$
$0.00066 \quad 0.00000$ $0.00132 \quad 0.00000$ $0.000659 \quad 0.00000$ $0.01312 \quad 0.00000$ $0.01312 \quad 0.06001$ $0.01430 \quad 0.02628$ $-0.01474$

$0.02527 \quad 0.00004$ $0.04667 \quad 0.00035$ $0.06099 \quad 0.00130$ $0.06616 \quad 0.00336$ $0.06162 \quad 0.00707$ 0.028740 .02053 0.005940 .02967 $\begin{array}{ll}-0.03598 & 0.04644\end{array}$ $0.05554 \quad 0.00009$ $0.05595 \quad 0.00076$ $0.06073 \quad 0.00288$ $0.04176 \quad 0.01541$ 0.028440 .02053
$S(h, 0)$

$0.00000-0.00000$ $0.00001-0.00000$ $0.00008-0.00001$ $0.00033-0.00002$ $0.00132-0.00009$ $0.00808-0.00058$ $0.03027-0.00234$ $0.06099-0.00515$ $0.09266-0.00827$ $0.11781-0.01005$ $0.13114-0.00814$ $\begin{array}{ll}0.13114 & -0.00814 \\ 0.13092 & -0.00022\end{array}$ $0.00502-0.00036$ $\begin{array}{ll}0.01931 & -0.00144 \\ 0.04060 & -0.00325\end{array}$ $0.09049-0.00806$ $0.11162-0.00981$ $0.12613-0.00972$ 0.13243 .0 .00659 $\begin{array}{ll}0.12147 & 0.01216\end{array}$ $0.06560-0.00560$

$$
\frac{a}{\lambda}=2.0 \quad \frac{\alpha}{\beta}=0.07
$$

$E(h, 0)$

$0.00132-0.00000$

$0.00330-0.00000$

$0.00660-0.00000$

$0.01321-0.00001$

$0.03300-0.00011$

$0.06590-0.00087$

$0.09854-0.00292$

$0.13072-0.00687$

$0.02593-0.00005$

$0.05180-0.00042$

$\begin{array}{ll}0.05180 & -0.00042 \\ 0.07756 & -0.00142\end{array}$

$0.10313-0.00335$

$0.12839-0.00651$

$0.15318-0.01114$

$\begin{array}{lll}0.17732 & -0.01748\end{array}$

$0.20054-0.02572$

$0.22257-0.03599$

$0.24306-0.04833$
$0.00066 \quad-0.00000$

$0.19229-0.02252$

$\begin{array}{ll}0.19229 & -0.02252 \\ 0.22070 & -0.03501\end{array}$
$\mathrm{C}(\mathrm{h}, \mathrm{h})$ $0.00132-0.00000$ $0.00330-0.00000$ $0.00659-0.00000$ $0.01312-0.00002$ $0.03160-0.00023$ $0.05515-0.00142$ $0.06487-0.0028$ $0.05947-0.0022$ 0.024430 .01118 $0.01060 \quad 0.01803$ $\begin{array}{ll}0.04648 & -0.00078\end{array}$ $0.06038-0.00201$ $0.06498-0.00299$
$0.06031-0.00239$ $0.06031-0.00239$ 0.048590 .00089 $\begin{array}{lll}0.03366 & 0.00678\end{array}$ $0.01977 \quad 0.01355$ $0.00994 \quad 0.01829$ $0.00503 \quad 0.01827$
$0.00066-0.00000$ $0.04326 \quad 0.00280$

$0.02524-0.00011$
$S(h, h)$ $0.00001-0.00000$ $0.00008-0.00001$ $0.00033-0.00002$ $0.00132-0.0000$ $0.00806-0.00066$ $0.02993-0.00350$ $0.05911-0.01017$ $0.08653-0.02101$ $0.10382-0.03355$ $0.10704-0.0435$ $0.09827-0.04840$

$0.00502-0.00039$ $0.01918-0.00190$ \begin{tabular}{ll}
$0.03993-0.0053$ \\
\hline
\end{tabular} $0.06332-0.01148$ $0.08479-0.02011$ $0.10023-0.03002$ $0.10718-0.03908$ $0.09736-0.04858$ $0.08529-0.05021$
$0.00000-0.00000$
$E(h, h)$

$0.00066-0.00000$ $0.00132-0.00000$ $0.00330-0.00000$ $0.01320-0.0000$ $0.03295-0.00044$ $0.06536-0.00344$ $0.09615-0.01126$ $0.12333-0.02543$ $0.14409-0.04608$ $0.15510-0.09742$

$0.02590-0.00021$ $0.05157-0.00168$ $0.07659-0.00557$ $0.12153-0.01286$ $0.13897-0.03954$ $0.15107-0.05830$ 0.15652 -0.0789 $0.15465-0.09909$ $0.14584-0.11615$ $0.00660-0.00000$

$$
\frac{a}{\lambda}=2.0 \quad \frac{\alpha}{\beta}=0.10
$$

\begin{tabular}{|c|c|c|c|c|}
\hline $\begin{array}{l}0.01 \\
0.02 \\
0.05 \\
0.10 \\
0.20 \\
0.50 \\
1.00 \\
1.50 \\
2.00 \\
2.50 \\
3.00 \\
3.50\end{array}$ & $\begin{array}{r}0.00045 \\
0.00091 \\
0.00226 \\
0.00452 \\
0.00900 \\
0.02171 \\
0.038 .12 \\
0.04533 \\
0.04186 \\
0.02898 \\
0.01011 \\
-0.01015\end{array}$ & $\begin{array}{l}0.00000 \\
0.00000 \\
0.00000 \\
0.00000 \\
0.00001 \\
0.00011 \\
0.00093 \\
0.00317 \\
0.00748 \\
0.01403 \\
0.02218 \\
0.03023\end{array}$ & $\begin{array}{l}0.00000 \\
0.00001 \\
0.00006 \\
0.00023 \\
0.00090 \\
0.00554 \\
0.02077 \\
0.04187 \\
0.06372 \\
0.08127 \\
0.09097 \\
0.09161\end{array}$ & $\begin{array}{r}-0.00000 \\
-0.00000 \\
-0.00001 \\
-0.00002 \\
-0.00009 \\
-0.00056 \\
-0.00213 \\
-0.00430 \\
-0.00623 \\
-0.00657 \\
-0.00372 \\
0.00359\end{array}$ \\
\hline $\begin{array}{l}0.125 \pi \\
0.250 \pi \\
0.375 \pi \\
0.500 \pi \\
0.625 \pi \\
0.750 \pi \\
0.875 \pi \\
1.000 \pi \\
1.125 \pi \\
1.250 \pi\end{array}$ & $\begin{array}{r}0.01733 \\
0.03203 \\
0.04188 \\
0.04548 \\
0.04247 \\
0.03348 \\
0.02008 \\
0.00435 \\
-0.01149 \\
-0.0 .2552\end{array}$ & $\begin{array}{l}0.00006 \\
0.00045 \\
0.00153 \\
0.00365 \\
0.00709 \\
0.011193 \\
0.01797 \\
0.02456 \\
0.03072 \\
0.03514\end{array}$ & $\begin{array}{l}0.00345 \\
0.01324 \\
0.02786 \\
0.04504 \\
0.06221 \\
0.07691 \\
0.08722 \\
0.09206 \\
0.09134 \\
0.08582\end{array}$ & $\begin{array}{r}-0.00035 \\
-0.00135 \\
-0.00286 \\
-0.00462 \\
-0.00613 \\
-0.00673 \\
-0.00565 \\
-0.00214 \\
0.00427 \\
0.01359\end{array}$ \\
\hline
\end{tabular}

$\begin{array}{ll}0.00045 & -0.00000 \\ 0.00091 & -0.00000 \\ 0.00226 & -0.00000 \\ 0.00453 & -0.00000 \\ 0.00906 & -0.00000 \\ 0.02263 & -0.00007 \\ 0.04518 & -0.00060 \\ 0.06753 & -0.00200 \\ 0.08952 & -0.00470 \\ 0.11093 & -0.00905 \\ 0.13144 & -0.01535 \\ 0.15069 & -0.02381 \\ 0.01778 & -0.00004 \\ 0.03552 & -0.00029 \\ 0.05317 & -0.00098 \\ 0.07067 & -0.00230 \\ 0.08793 & -0.00445 \\ 0.10485 & -0.00761 \\ 0.12127 & -0.01193 \\ 0.13704 & -0.01752 \\ 0.15195 & -0.02447 \\ 0.16578 & -0.03280\end{array}$

$\begin{array}{ll}0.00045 & -0.00000 \\ 0.00091 & -0.00000 \\ 0.00226 & -0.00000 \\ 0.00452 & -0.00000 \\ 0.00900 & -0.00001 \\ 0.02167 & -0.00010 \\ 0.03782 & -0.00057 \\ 0.04456 & -0.00081 \\ 0.04111 & 0.00066 \\ 0.03037 & 0.00486 \\ 0.01765 & 0.01070 \\ 0.00784 & 0.01475 \\ 0.01731 & -0.00005 \\ 0.03188 & -0.00033 \\ 0.04142 & -0.00074 \\ 0.04466 & -0.00074 \\ 0.04166 & 0.00046 \\ 0.03391 & 0.00338 \\ 0.02393 & 0.00773 \\ 0.01443 & 0.01220 \\ 0.00734 & 0.01486 \\ 0.00333 & 0.01407\end{array}$

(1)

$\begin{array}{ll}0.00000 & -0.00000 \\ 0.00001 & -0.00000 \\ 0.00006 & -0.00001 \\ 0.00023 & -0.00002 \\ 0.00090 & -0.00009 \\ 0.00552 & -0.00061 \\ 0.02049 & -0.00291 \\ 0.04041 & -0.00769 \\ 0.05917 & -0.01483 \\ 0.07121 & -0.02251 \\ 0.07396 & -0.02807 \\ 0.06853 & -0.03036 \\ 0.00344 & -0.00037 \\ 0.01314 & -0.00166 \\ 0.02732 & -0.00430 \\ 0.04329 & -0.00859 \\ 0.05797 & -0.01426 \\ 0.06866 & -0.02041 \\ 0.07375 & -0.02568 \\ 0.07313 & -0.02903 \\ 0.06794 & -0.03043 \\ 0.05975 & -0.03113\end{array}$

$0.00045-0.00000$ $0.00091-0.00000$ $0.00226-0.00000$ $0.00453-0.00000$ $0.00906-0.00002$ $0.02259-0.00030$ $0.04476-0.00235$ $0.06572-0.00768$ $0.08411-0.01725$ $0.09802-0.03109$ $0.10550-0.04786$ $0.10533-0.06488$

$0.01776-0.00015$ $0.03534-0.00115$ $0.05242-0.00380$ $0.06852-0.00876$ $0.08289-0.01640$ $0.09460-0.02672$ $0.10625-0.05280$ $0.10504-0.06597$ $0.09936-0.07697$ 
$0.00000-0.00000$ $0.00000-0.00000$ $0.00002-0.00000$ $0.00006-0.00001$ $0.00026-0.00005$ $0.00158-0.00031$ $0.00593-0.00110$ $0.01203-0.00196$ $0.01848-0.00233$ $0.02392-0.00160$ $0.02733 \quad 0.00067$

$\begin{array}{ll}0.00098 & -0.00019\end{array}$ $0.00378-0.00072$ $0.00797-0.00142$ $0.01296-0.00206$ $0.01803-0.00233$ $0.02592-0.00195$ $0.02785 \quad-0.00016$ 0.0278510 .00162 $0.02707 \quad 0.00906$

$\begin{array}{ll}0.00013 & -0.00000 \\ 0.00026 & -0.00000 \\ 0.00064 & -0.00000 \\ 0.00129 & -0.00000 \\ 0.00258 & -0.00000 \\ 0.00644 & -0.00002 \\ 0.01284 & -0.00017 \\ 0.01916 & -0.00057 \\ 0.02535 & -0.00132 \\ 0.03132 & -0.00254 \\ 0.03699 & -0.00428 \\ 0.04224 & -0.00659 \\ 0.00506 & -0.00001 \\ 0.01010 & -0.00068 \\ 0.01511 & -0.00028 \\ 0.02005 & -0.00065 \\ 0.02490 & -0.00126 \\ 0.02963 & -0.00214 \\ 0.03418 & -0.00334 \\ 0.03852 & -0.00487 \\ 0.04258 & -0.00677 \\ 0.04631 & -0.00901\end{array}$

$0.00013 \quad 0.00000$ $0.00026 \quad 2.00000$ $0.00064 \quad 0.00000$ $0.00129 \quad 0.00000$ $0.00256 \quad 0.00000$ 0.006170 .00002 $0.01078 \quad 0.00022$ $0.01278 \quad 0.00087$ $0.01200 \quad 0.00222$ $0.00915 \quad 0.00417$ $0.00219 \quad 0.00670$ $\begin{array}{lll}0.00493 & 0.00001\end{array}$ $0.00908 \quad 0.00010$ $0.01182 \quad 0.00038$ $0.01283 \quad 0.00102$ $0.01214 \quad 0.00210$ $0.01011 \quad 0.00358$ $\begin{array}{ll}0.00736 & 0.00517 \\ 0.00449 & 0.00638\end{array}$ $0.00449 \quad 0.00638$ $\begin{array}{ll}0.00018 & 0.00571\end{array}$
$0.00000-0.00000$ $0.00000-0.00000$ $0.00002-0.00000$ $0.00006-0.00001$ $0.00026-0.00005$ $0.00157-0.00032$ $0.00531-0.00131$ $0.01146-0.00288$ $0.01685-0.00466$ $0.02056-0.00602$ $\begin{array}{ll}0.02184 & -0.00643 \\ 0.02089 & -0.00598\end{array}$

$0.00098-0.00020$ $0.00373-0.00081$ $0.00775-0.00181$ $0.01227-0.00313$ $\begin{array}{ll}0.01650 & -0.00453 \\ 0.01972 & -0.00571\end{array}$ $0.02151-0.00636$ $\begin{array}{ll}0.02151 & -0.00636 \\ 0.02174 & -0.00637\end{array}$ $0.02053-0.00593$ $0.01807-0.00552$
$E(h, h)$

$\begin{array}{ll}0.00013 & -0.00000 \\ 0.00026 & -0.00000 \\ 0.00064 & -0.00000 \\ 0.00129 & -0.00000 \\ 0.00258 & -0.00001 \\ 0.00642 & -0.00008 \\ 0.01267 & -0.00066 \\ 0.01849 & -0.00214 \\ 0.02348 & -0.00473 \\ 0.02715 & -0.00837 \\ 0.02905 & -0.01262 \\ 0.02902 & -0.01676 \\ & \\ 0.00505 & -0.00004 \\ 0.01002 & -0.00032 \\ 0.01481 & -0.00107 \\ 0.01926 & -0.00244 \\ 0.02316 & -0.00451 \\ 0.02626 & -0.00724 \\ 0.02833 & -0.01045 \\ 0.02924 & -0.01384 \\ 0.02895 & -0.01702 \\ 0.02763 & -0.01957\end{array}$

$$
\frac{a}{\lambda}=2.0 \quad \frac{\alpha}{\beta}=0.40
$$

$\begin{array}{ll}0.00000 & -0.00000 \\ 0.00000 & -0.00000 \\ 0.00000 & -0.00000 \\ 0.00001 & -0.00000 \\ 0.00002 & -0.00001 \\ 0.00013 & -0.00005 \\ 0.00049 & -0.00017 \\ 0.00102 & -0.00028 \\ 0.00163 & -0.00029 \\ 0.00221 & -0.00009 \\ 0.00264 & 0.00036 \\ 0.00285 & 0.00106 \\ 0.00008 & -0.00003 \\ 0.00031 & -0.00011 \\ 0.00067 & -0.00022 \\ 0.00111 & -0.00029 \\ 0.00159 & -0.00029 \\ 0.00205 & -0.00017 \\ 0.00245 & 0.00010 \\ 0.00273 & 0.00054 \\ 0.00285 & 0.00111 \\ 0.00278 & 0.00180\end{array}$

$\begin{array}{ll}0.00001 & -0.00000 \\ 0.00002 & -0.00000 \\ 0.00005 & -0.00000 \\ 0.00010 & -0.00000 \\ 0.00021 & -0.00000 \\ 0.00052 & -0.00000 \\ 0.00104 & -0.00001 \\ 0.00154 & -0.00005 \\ 0.00203 & -0.00011 \\ 0.00250 & -0.00020 \\ 0.00293 & -0.00033 \\ 0.00332 & -0.00050 \\ 0.00041 & -0.00000 \\ 0.00082 & -0.00001 \\ 0.00122 & -0.00002 \\ 0.00161 & -0.00005 \\ 0.00200 & -0.00010 \\ 0.00237 & -0.00017 \\ 0.00272 & -0.00026 \\ 0.00304 & -0.00038 \\ 0.00335 & -0.00052 \\ 0.00362 & -0.00068\end{array}$

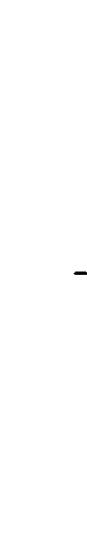

0.00000 $0.00002 \quad 0.00000$ $0.00005 \quad 0.00000$ $0.00010 \quad 0.00000$ $0.00021 \quad 0.00000$ $0.00050 \quad 0.00001$ $0.00088 \quad 0.00008$ $0.00106 \quad 0.00025$ $\begin{array}{ll}0.00102 & 0.00051 \\ 0.00078 & 0.00081\end{array}$ $\begin{array}{ll}0.00078 & 0.00081 \\ 0.00039 & 0.00103\end{array}$ $-0.00006 \quad 0.00103$ $0.00040 \quad 0.00001$ $0.00074 \quad 0.00004$ $0.00097 \quad 0.00013$ $0.00107 \quad 0.00028$ $0.00103 \quad 0.00049$ $0.00086 \quad 0.00073$ $0.00060 \quad 0.00094$ $0.00026 \quad 0.00106$ $-0.00009 \quad 0.00102$ $\begin{array}{ll}-0.00042 & 0.00079\end{array}$ $\begin{array}{ll}0.00001 & -0.00000 \\ 0.00002 & -0.00000 \\ 0.00005 & -0.00000 \\ 0.00010 & -0.00000 \\ 0.00021 & -0.00000 \\ 0.00052 & -0.00001 \\ 0.00102 & -0.00005 \\ 0.00146 & -0.00017 \\ 0.00183 & -0.00036 \\ 0.00209 & -0.00061 \\ 0.00221 & -0.00088 \\ 0.00221 & -0.00113 \\ 0.00041 & -0.00000 \\ 0.00081 & -0.00003 \\ 0.00118 & -0.00008 \\ 0.00152 & -0.00019 \\ 0.00181 & -0.00034 \\ 0.00203 & -0.00053 \\ 0.00216 & -0.00074 \\ 0.00222 & -0.00096 \\ 0.00220 & -0.00114 \\ 0.00213 & -0.00128\end{array}$

$\begin{array}{llll}0.00000 & -0.00000 & 0.00001 & -0.00000 \\ 0.00000 & -0.00000 & 0.00002 & -0.00000 \\ 0.00000 & -0.00000 & 0.00005 & -0.00000 \\ 0.00001 & -0.00000 & 0.00010 & -0.00000 \\ 0.00002 & -0.00001 & 0.00021 & -0.00000 \\ 0.00013 & -0.00005 & 0.00052 & -0.00001 \\ 0.00047 & -0.00018 & 0.00102 & -0.00005 \\ 0.00095 & -0.00035 & 0.00146 & -0.00017 \\ 0.00144 & -0.00046 & 0.00183 & -0.00036 \\ 0.00182 & -0.00044 & 0.00209 & -0.00061 \\ 0.00202 & -0.00027 & 0.00221 & -0.00088 \\ 0.00194 & 0.00002 & 0.00221 & -0.00113 \\ 0.00008 & -0.00003 & 0.00041 & -0.00000 \\ 0.00030 & -0.00012 & 0.00081 & -0.00003 \\ 0.00064 & -0.00024 & 0.00118 & -0.00008 \\ 0.00102 & -0.00037 & 0.00152 & -0.00019 \\ 0.00140 & -0.00045 & 0.00181 & -0.00034 \\ 0.00173 & -0.00046 & 0.00203 & -0.00053 \\ 0.00195 & -0.00038 & 0.00216 & -0.00074 \\ 0.00203 & -0.00020 & 0.00222 & -0.00096 \\ 0.00193 & 0.00004 & 0.00220 & -0.00114 \\ 0.00162 & 0.00026 & 0.00213 & -0.00128\end{array}$




$\begin{array}{lrllr}0.01 & 0.00000 & 0.00000 & 0.00000 & -0.00000 \\ 0.02 & 0.00000 & 0.00030 & 0.00000 & -0.00000 \\ 0.05 & 0.00000 & 0.00000 & 0.00000 & -0.00000 \\ 0.10 & 0.00000 & 0.00000 & 0.00000 & -0.00000 \\ 0.20 & 2.00000 & 0.00000 & 0.00000 & -0.00000 \\ 0.50 & 0.00001 & 0.00000 & 0.00000 & -0.00000 \\ 1.00 & 0.00002 & 0.00000 & 0.00001 & -0.00001 \\ 1.50 & 0.00003 & 0.00002 & 0.00003 & -0.00001 \\ 2.00 & 0.00003 & 0.00003 & 0.00005 & -0.00001 \\ 2.50 & 0.00001 & 0.00005 & 0.00007 & 0.00000 \\ 3.00 & -0.00001 & 0.00007 & 0.00009 & 0.00003 \\ 3.50 & -0.00005 & 0.00009 & 0.00010 & 0.00007 \\ & & & & \\ 0.125 \pi & 0.00001 & 0.00000 & 0.00000 & -0.00000 \\ 0.250 \pi & 0.00002 & 0.00000 & 0.00001 & -0.00000 \\ 0.375 \pi & 0.00002 & 0.00001 & 0.00002 & -0.00001 \\ 0.500 \pi & 0.00003 & 0.00002 & 0.00003 & -0.00001 \\ 0.625 \pi & 0.00003 & 0.00003 & 0.00005 & -0.00001 \\ 0.750 \pi & 0.00002 & 0.00005 & 0.00006 & -0.00000 \\ 0.875 \pi & 0.00000 & 0.00006 & 0.00008 & 0.00001 \\ 1.000 \pi & -0.00002 & 0.00008 & 0.00009 & 0.00003 \\ 1.125 \pi & -0.00006 & 0.00009 & 0.00010 & 0.00007 \\ 1.250 \pi & -0.00010 & 0.00009 & 0.00010 & 0.00011\end{array}$

$$
\frac{a}{\lambda}=2.0 \quad \frac{\alpha}{\beta}=0.70
$$

$E(h, 0)$

$\begin{array}{ll}0.00000 & -0.00000 \\ 0.00000 & -0.00000 \\ 0.00000 & -0.00000 \\ 0.00000 & -0.00000 \\ 0.00000 & -0.00000 \\ 0.00001 & -0.00000 \\ 0.00002 & -0.00000 \\ 0.00004 & -0.00000 \\ 0.00005 & -0.00000 \\ 0.00006 & -0.00000 \\ 0.00007 & -0.00001 \\ 0.00007 & -0.00001 \\ 0.00001 & -0.00000 \\ 0.00002 & -0.00000 \\ 0.00003 & -0.00000 \\ 0.00004 & -0.00000 \\ 0.00005 & -0.00000 \\ 0.00005 & -0.00000 \\ 0.00006 & -0.00001 \\ 0.00007 & -0.00001 \\ 0.00007 & -0.00001 \\ 0.00008 & -0.00001\end{array}$

$\mathrm{C}(\mathrm{h}, \mathrm{h})$

$\cdot S(h, h)$

$\begin{array}{rr}0.00000 & 0.00000 \\ 0.00000 & 0.00000 \\ 0.00000 & 0.00000 \\ 0.00000 & 0.00000 \\ 0.00000 & 0.00000 \\ 0.00001 & 0.00000 \\ 0.00002 & 0.00000 \\ 0.00003 & 0.00001 \\ 0.00003 & 0.00002 \\ 0.00002 & 0.00004 \\ -0.00000 & 0.00005 \\ -0.00003 & 0.00005 \\ & \\ 0.00001 & 0.00000 \\ 0.00002 & 0.00000 \\ 0.00002 & 0.00001 \\ 0.00003 & 0.00001 \\ 0.00003 & 0.00002 \\ 0.00002 & 0.00004 \\ 0.00001 & 0.00004 \\ -0.00001 & 0.00005 \\ -0.00003 & 0.00005 \\ -0.00005 & 0.00003\end{array}$

$0.00000-0.00000$ $0.00000-0.00000$ $0.00000-0.00000$ $0.00000-0.00000$ $0.00000-0.00000$ $0.00001-0.0000$ $0.00002-0.0000$ $0.00004-0.0000$ $0.00005-0.0000$ $0.00006 \quad 0.00003$ $0.00000-0.00000$ $0.00001-0.00000$ $0.00002-0.0000$ $0.00003-0.0000$ $0.00004-0.0000$ $0.00005-0.0000$ $0.00006-0.00000$ $\begin{array}{ll}0.00007 & 0.0000\end{array}$ $0.00006 \quad 0.0000$ $0.00004 \quad 0.00006$ $0.00000-0.00000$
$E(h, h)$

$\begin{array}{ll}0.00000 & -0.00000 \\ 0.00000 & -0.00000 \\ 0.00000 & -0.00000 \\ 0.00000 & -0.00000 \\ 0.00000 & -0.00000 \\ 0.00001 & -0.00000 \\ 0.00002 & -0.00000 \\ 0.00003 & -0.00000 \\ 0.00004 & -0.00001 \\ 0.00004 & -0.00001 \\ 0.00005 & -0.00002 \\ 0.00005 & -0.00002 \\ 0.00001 & -0.00000 \\ 0.00002 & -0.00000 \\ 0.00003 & -0.00000 \\ 0.00003 & -0.00000 \\ 0.00004 & -0.00001 \\ 0.00004 & -0.00001 \\ 0.00005 & -0.00001 \\ 0.00005 & -0.00002 \\ 0.00005 & -0.00002 \\ 0.00005 & -0.00002\end{array}$

$$
\frac{a}{\lambda}=2.0 \quad \frac{\alpha}{\beta}=1.00
$$

$0.00000-0.00000$ $0.00000-0.00000$ $0.00000-0.00000$ $0.00000-0.00000$ $0.00000-0.00000$ $0.00000-0.00000$ $0.00000-0.00000$ $0.00000-0.00000$

$0.00000-0.00000$

$0.00000-0.00000$

$0.00000-0.00000$

$0.00000-0.00000$

$0.00000-0.00000$ $0.00000-0.00000$ $0.00000-0.00000$ $0.00000-0.00000$ $0.00000-0.00000$ $0.00000-0.00000$ $0.00000-0.00000$ $0.00000-0.00000$ $0.00000-0.00000$ $0.00000-0.00000$

$\begin{array}{rrrr}0.00000 & 0.00000 & 0.00000 & -0.00000 \\ 0.00000 & 0.00000 & 0.00000 & -0.00000 \\ 0.00000 & 0.00000 & 0.00000 & -0.00000 \\ 0.00000 & 0.00000 & 0.00000 & -0.00000 \\ 0.00000 & 0.00000 & 0.00000 & -0.00000 \\ 0.00000 & 0.00000 & 0.00000 & -0.00000 \\ 0.00000 & 0.00000 & 0.00000 & -0.00000 \\ 0.00000 & 0.00000 & 0.00000 & -0.00000 \\ 0.00000 & 0.00000 & 0.00000 & -0.00000 \\ 0.00000 & 0.00000 & 0.00000 & -0.00000 \\ -0.00000 & 0.00000 & 0.00000 & 0.00000 \\ -0.00000 & 0.00000 & 0.00000 & 0.00000 \\ & & & \\ 0.00000 & 0.00000 & 0.00000 & -0.00000 \\ 0.00000 & 0.00000 & 0.00000 & -0.00000 \\ 0.00000 & 0.00000 & 0.00000 & -0.00000 \\ 0.00000 & 0.00000 & 0.00000 & -0.00000 \\ 0.00000 & 0.00000 & 0.00000 & -0.00000 \\ 0.00000 & 0.00000 & 0.00000 & -0.00000 \\ -0.00000 & 0.00000 & 0.00000 & 0.00000 \\ -0.00000 & 0.00000 & 0.00000 & 0.00000 \\ -0.00000 & 0.00000 & 0.00000 & 0.00000 \\ -0.00000 & 0.00000 & 0.00000 & 0.00000\end{array}$

$\begin{array}{ll}0.00000 & -0.00000 \\ 0.00000 & -0.00000 \\ 0.00000 & -0.00000 \\ 0.00000 & -0.00000 \\ 0.00000 & -0.00000 \\ 0.00000 & -0.00000 \\ 0.00000 & -0.00000 \\ 0.00000 & -0.00000 \\ 0.00000 & -0.00000 \\ 0.00000 & -0.00000 \\ 0.00000 & -0.00000 \\ 0.00000 & -0.00000 \\ 0.00000 & -0.00000 \\ 0.00000 & -0.00000 \\ 0.00000 & -0.00000 \\ 0.00000 & -0.00000 \\ 0.00000 & -0.00000 \\ 0.00000 & -0.00000 \\ 0.00000 & -0.00000 \\ 0.00000 & -0.00000 \\ 0.00000 & -0.00000 \\ 0.00000 & -0.00000\end{array}$

0.00000

0.00000
0.00000 
$\beta \mathrm{h}$

$\mathrm{C}(\mathrm{h}, 0)$

0.01
0.02
0.05
0.10
0.20
0.50
1.00
1.50
2.00
2.50
3.00
3.50

$0.125 \pi$

0.250

0.375

$\begin{array}{ll}-0.11753 & 0.00136\end{array}$

$\begin{array}{lll}0.375 \pi & -0.11753 & 0.00136 \\ 0.625 \pi & -0.12717 & 0.00189 \\ 0.0250 & -0.11760 & 0.00086\end{array}$

$\begin{array}{lll}0.750 \pi & -0.09058 & -0.00324\end{array}$

$0.875 \pi \quad-0.05069-0.01165$

$1.000 \pi \quad-0.00461-0.02463$

$\begin{array}{lll}1.125 \pi & 0.04014 & -0.04100 \\ 1.250 \pi & 0.07657 & -0.05794\end{array}$
$S(h, 0)$

$\begin{array}{ll}-0.00001 & 0.00000 \\ -0.00003 & 0.00000 \\ -0.00016 & 0.00000 \\ -0.00064 & 0.00000 \\ -0.00254 & 0.00000 \\ -0.01558 & 0.00006 \\ -0.05846 & 0.00090 \\ -0.11798 & 0.00393 \\ -0.17929 & 0.00992 \\ -0.22720 & 0.01758 \\ -0.25026 & 0.02360 \\ -0.24406 & 0.02058 \\ -0.00969 & 0.00002 \\ -0.03727 & 0.00036 \\ -0.07847 & 0.00166 \\ -0.12692 & 0.00460 \\ -0.17509 & 0.00940 \\ -0.21557 & 0.01539 \\ -0.24225 & 0.02089 \\ -0.25146 & 0.02336 \\ -0.24261 & 0.01999 \\ -0.21835 & 0.00851\end{array}$

$E(h, 0)$

$\begin{array}{ll}-0.00127 & 0.00000 \\ -0.00255 & 0.00000 \\ -0.00637 & 0.00000 \\ -0.01273 & 0.00000 \\ -0.02546 & 0.00001 \\ -0.06365 & 0.00017 \\ -0.12723 & 0.00135 \\ -0.19060 & 0.00454 \\ -0.25356 & 0.01072 \\ -0.31575 & 0.02081 \\ -0.37664 & 0.03568 \\ -0.43553 & 0.05607 \\ -0.04999 & 0.00008 \\ -0.09995 & 0.00065 \\ -0.14983 & 0.00220 \\ -0.19955 & 0.00521 \\ -0.24898 & 0.01014 \\ -0.29797 & 0.01746 \\ -0.34626 & 0.02757 \\ -0.39356 & 0.04097 \\ -0.43947 & 0.05768 \\ -0.48352 & 0.07828\end{array}$

$\mathrm{C}(\mathrm{h}, \mathrm{h})$

$\begin{array}{rr}-0.00127 & 0.00000 \\ -0.00255 & 0.00000 \\ -0.00636 & 0.00000 \\ -0.01271 & 0.00001 \\ -0.02527 & 0.00004 \\ -0.06100 & 0.00064 \\ -0.10671 & 0.00435 \\ -0.12558 & 0.01078 \\ -0.11369 & 0.01511 \\ -0.07762 & 0.01061 \\ -0.03337 & -0.00564 \\ 0.00056 & -0.02808 \\ -0.04870 & 0.00032 \\ -0.08983 & 0.00229 \\ -0.11691 & 0.00649 \\ -0.12573 & 0.01168 \\ -0.11552 & 0.01502 \\ -0.08960 & 0.01312 \\ -0.05540 & 0.00387 \\ -0.02202 & -0.01183 \\ 0.00216 & -0.02955 \\ 0.01291 & -0.04274\end{array}$

$S(h, h)$

$\begin{array}{ll}-0.00001 & 0.00000\end{array}$

$-0.00003 \quad 0.00000$

$\begin{array}{ll}-0.00016 & 0.00000 \\ -0.00064 & 0.00000\end{array}$

$\begin{array}{ll}-0.00064 & 0.00000 \\ -0.00254 & 0.00000\end{array}$

$\begin{array}{ll}-0.05823 & 0.00274\end{array}$

$\begin{array}{ll}-0.17293 & 0.03124\end{array}$

$\begin{array}{lll}-0.21081 & 0.05773\end{array}$

$\begin{array}{ll}-0.21975 & 0.08323 \\ -0.20203 & 0.09852\end{array}$

$-0.00969 \quad 0.00007$

$\begin{array}{ll}-0.03719 & 0.00109\end{array}$

$-0.07796 \quad 0.00506$

$-0.12487 \quad 0.01420$

$\begin{array}{ll}-0.16925 & 0.02953\end{array}$

$\begin{array}{ll}-0.20264 & 0.04977\end{array}$

$\begin{array}{ll}-0.21905 & 0.07120\end{array}$

$-0.21700 \quad 0.08888$

$\begin{array}{ll}-0.20014 & 0.09907 \\ -0.17577 & 0.10177\end{array}$ $\begin{array}{ll}-0.01557 & 0.00019\end{array}$

$-0.11634 \quad 0.01210$

$\mathrm{E}(\mathrm{h}, \mathrm{h})$

$\begin{array}{ll}-0.00127 & 0.00000 \\ -0.00255 & 0.00000 \\ -0.00637 & 0.00000 \\ -0.01273 & 0.00001 \\ -0.02546 & 0.00004 \\ -0.06361 & 0.00067 \\ -0.12678 & 0.00536 \\ -0.18832 & 0.01784 \\ -0.24573 & 0.04127 \\ -0.29488 & 0.07739 \\ -0.33032 & 0.12558 \\ -0.34634 & 0.18180 \\ & \\ -0.04998 & 0.00033 \\ -0.09977 & 0.00261 \\ -0.14898 & 0.00873 \\ -0.19678 & 0.02043 \\ -0.24176 & 0.03914 \\ -0.28190 & 0.06568 \\ -0.31461 & 0.10003 \\ -0.33705 & 0.14095 \\ -0.34661 & 0.18577 \\ -0.34159 & 0.23044\end{array}$

$$
\frac{a}{\lambda}=2.5 \quad \frac{\alpha}{\beta}=0.005
$$

$\begin{array}{lrr}0.01 & -0.00118 & 0.00000 \\ 0.02 & -0.00235 & 0.00000 \\ 0.05 & -0.00588 & 0.00000 \\ 0.10 & -0.01175 & 0.00000 \\ 0.20 & -0.02338 & 0.00001 \\ 0.50 & -0.05642 & 0.00012 \\ 1.00 & -0.09898 & 0.00072 \\ 1.50 & -0.11729 & 0.00120 \\ 2.00 & -0.10710 & -0.00043 \\ 2.50 & -0.07149 & -0.00685 \\ 3.00 & -0.02015 & -0.01978 \\ 3.50 & 0.03338 & -0.03816 \\ 0.125 \pi & -0.04504 & 0.00006 \\ 0.250 \pi & -0.08320 & 0.00041 \\ 0.375 \pi & -0.10865 & 0.00098 \\ 0.500 \pi & -0.11759 & 0.00116 \\ 0.625 \pi & -0.10878 & -0.00018 \\ 0.750 \pi & -0.08387 & -0.00438 \\ 0.875 \pi & -0.04710 & -0.01246 \\ 1.000 \pi & -0.00459 & -0.02456 \\ 1.125 \pi & 0.03677 & -0.03953 \\ 1.250 \pi & 0.07058 & -0.05474\end{array}$

$\begin{array}{llll}-0.00001 & 0.00000 & -0.00118 & 0.00000 \\ -0.00002 & 0.00000 & -0.00235 & 0.00000 \\ -0.00015 & 0.00000 & -0.00589 & 0.00000 \\ -0.00059 & 0.00000 & -0.01177 & 0.00000 \\ -0.00235 & 0.00001 & -0.02354 & 0.00001 \\ -0.01441 & 0.00013 & -0.05884 & 0.00016 \\ -0.05404 & 0.00106 & -0.11761 & 0.00125 \\ -0.10904 & 0.00397 & -0.17618 & 0.00420 \\ -0.16570 & 0.00941 & -0.23436 & 0.00990 \\ -0.21003 & 0.01608 & -0.29180 & 0.01923 \\ -0.23151 & 0.02037 & -0.34803 & 0.03296 \\ -0.22608 & 0.01727 & -0.40238 & 0.05177 \\ & & & \\ -0.00896 & 0.00007 & -0.04622 & 0.00008 \\ -0.03445 & 0.00049 & -0.09240 & 0.00060 \\ -0.07253 & 0.00181 & -0.13850 & 0.00204 \\ -0.11730 & 0.00459 & -0.18445 & 0.00482 \\ -0.16183 & 0.00894 & -0.23013 & 0.00937 \\ -0.19926 & 0.01420 & -0.27538 & 0.01613 \\ -0.22401 & 0.01881 & -0.31998 & 0.02547 \\ -0.23268 & 0.02047 & -0.36365 & 0.03775 \\ -0.22477 & 0.01666 & -0.40601 & 0.05326 \\ -0.20268 & 0.00545 & -0.44665 & 0.07226\end{array}$

$\begin{array}{ll}-0.00001 & 0.00000 \\ -0.00002 & 0.00000 \\ -0.00015 & 0.00000 \\ -0.00059 & 0.00000 \\ -0.00235 & 0.00002 \\ -0.01439 & 0.00024 \\ -0.05381 & 0.00276 \\ -0.10746 & 0.01151 \\ -0.15971 & 0.02907 \\ -0.19474 & 0.05311 \\ -0.20322 & 0.07604 \\ -0.18726 & 0.08962 \\ -0.00895 & 0.00011 \\ -0.03437 & 0.00116 \\ -0.07203 & 0.00495 \\ -0.11534 & 0.01345 \\ -0.15631 & 0.02750 \\ -0.18716 & 0.04591 \\ -0.20244 & 0.06525 \\ -0.20079 & 0.08108 \\ -0.18554 & 0.09011 \\ -0.16330 & 0.09245\end{array}$

$\begin{array}{ll}-0.00118 & 0.00000 \\ -0.00235 & 0.00000 \\ -0.00589 & 0.00000 \\ -0.01177 & 0.00000 \\ -0.02354 & 0.00004 \\ -0.05880 & 0.00062 \\ -0.11718 & 0.00495 \\ -0.17402 & 0.01648 \\ -0.22699 & 0.03809 \\ -0.27228 & 0.07138 \\ -0.30489 & 0.11572 \\ -0.31961 & 0.16736 \\ -0.04620 & 0.00030 \\ -0.09222 & 0.00241 \\ -0.13769 & 0.00806 \\ -0.18182 & 0.01887 \\ -0.22333 & 0.03613 \\ -0.26032 & 0.06060 \\ -0.29044 & 0.09223 \\ -0.31107 & 0.12985 \\ -0.31986 & 0.17100 \\ -0.31526 & 0.21195\end{array}$




$\begin{array}{lrrrr}0.01 & -0.00109 & 0.00000 & -0.00001 & 0.00000 \\ 0.02 & -0.00218 & 0.00000 & -0.00002 & 0.00000 \\ 0.05 & -0.00544 & 0.00000 & -0.00014 & 0.00000 \\ 0.10 & -0.01086 & 0.00000 & -0.00054 & 0.00001 \\ 0.20 & -0.02162 & 0.00001 & -0.00217 & 0.00002 \\ 0.50 & -0.05216 & 0.00009 & -0.01332 & 0.00018 \\ 1.00 & -0.09150 & 0.00050 & -0.04995 & 0.00119 \\ 1.50 & -0.10844 & 0.00063 & -0.10078 & 0.00397 \\ 2.00 & -0.09907 & -0.00134 & -0.15316 & 0.00891 \\ 2.50 & -0.06625 & -0.00773 & -0.19418 & 0.01470 \\ 3.00 & -0.01889 & -0.01993 & -0.21417 & 0.01801 \\ 3.50 & 0.03056 & -0.03681 & -0.20944 & 0.01435 \\ & & & & \\ 0.125 \pi & -0.04164 & 0.00005 & -0.00828 & 0.00010 \\ 0.250 \pi & -0.07691 & 0.00029 & -0.03184 & 0.00059 \\ 0.375 \pi & -0.10045 & 0.00065 & -0.06704 & 0.00193 \\ 0.500 \pi & -0.10872 & 0.00053 & -0.10842 & 0.00455 \\ 0.625 \pi & -0.10062 & -0.00107 & -0.14957 & 0.00850 \\ 0.750 \pi & -0.07767 & -0.00532 & -0.18420 & 0.01311 \\ 0.875 \pi & -0.04376 & -0.01307 & -0.20715 & 0.01693 \\ 1.000 \pi & -0.00452 & -0.02436 & -0.21533 & 0.01788 \\ 1.125 \pi & 0.03371 & -0.03805 & -0.20825 & 0.01373 \\ 1.250 \pi & 0.06510 & -0.05170 & -0.18814 & 0.00281\end{array}$

$E(h, 0)$

$\begin{array}{ll}-0.00109 & 0.00000 \\ -0.00218 & 0.00000 \\ -0.00544 & 0.00000 \\ -0.01088 & 0.00000 \\ -0.02176 & 0.00001 \\ -0.05440 & 0.00014 \\ -0.10872 & 0.00115 \\ -0.16285 & 0.00388 \\ -0.21661 & 0.00915 \\ -0.26967 & 0.01777 \\ -0.32159 & 0.03044 \\ -0.37175 & 0.04781 \\ -0.04273 & 0.00007 \\ -0.08542 & 0.00056 \\ -0.12803 & 0.00188 \\ -0.17049 & 0.00445 \\ -0.21270 & 0.00866 \\ -0.25450 & 0.01490 \\ -0.29569 & 0.02353 \\ -0.33601 & 0.03486 \\ -0.37511 & 0.04918 \\ -0.41259 & 0.06671\end{array}$

C (h, h)

$\begin{array}{lr}-0.00109 & 0.00000 \\ -0.00218 & 0.00000 \\ -0.00544 & 0.00000 \\ -0.01086 & 0.00000 \\ -0.02162 & 0.00003 \\ -0.05212 & 0.00050 \\ -0.09113 & 0.00339 \\ -0.10734 & 0.00825 \\ -0.09738 & 0.01109 \\ -0.06698 & 0.00654 \\ -0.02961 & -0.00745 \\ -0.00069 & -0.02598 \\ -0.04162 & 0.00025 \\ -0.07676 & 0.00130 \\ -0.09990 & 0.00503 \\ -0.10749 & 0.00891 \\ -0.09892 & 0.01108 \\ -0.07713 & 0.00884 \\ -0.04823 & 0.00063 \\ -0.01999 & -0.01264 \\ 0.00070 & -0.02716 \\ 0.01030 & -0.03759\end{array}$

S $(h, h)$

$\begin{array}{ll}-0.00001 & 0.00000 \\ -0.00002 & 0.00000 \\ -0.00014 & 0.00000 \\ -0.00054 & 0.00001 \\ -0.00217 & 0.00003 \\ -0.01331 & 0.00029 \\ -0.04973 & 0.00275 \\ -0.09927 & 0.01093 \\ -0.14750 & 0.02705 \\ -0.17990 & 0.04887 \\ -0.18795 & 0.06947 \\ -0.17356 & 0.08152 \\ -0.00828 & 0.00014 \\ -0.03177 & 0.00121 \\ -0.06656 & 0.00483 \\ -0.10654 & 0.01273 \\ -0.14436 & 0.02562 \\ -0.17288 & 0.04235 \\ -0.18709 & 0.05980 \\ -0.18579 & 0.07397 \\ -0.17200 & 0.08195 \\ -0.15170 & 0.08397\end{array}$

$E(h, h)$

$\begin{array}{ll}-0.00109 & 0.00000 \\ -0.00218 & 0.00000 \\ -0.00544 & 0.00000 \\ -0.01088 & 0.00000 \\ -0.02176 & 0.00004 \\ -0.05436 & 0.00058 \\ -0.10330 & 0.00458 \\ -0.16080 & 0.01522 \\ -0.20967 & 0.03516 \\ -0.25142 & 0.06584 \\ -0.28142 & 0.10663 \\ -0.29494 & 0.15406 \\ -0.04271 & 0.00028 \\ -0.08525 & 0.00223 \\ -0.12725 & 0.00745 \\ -0.16800 & 0.01743 \\ -0.20630 & 0.03335 \\ -0.24040 & 0.05591 \\ -0.26813 & 0.08503 \\ -0.28710 & 0.11962 \\ -0.29517 & 0.15741 \\ -0.29096 & 0.19495\end{array}$

$\frac{\mathrm{a}}{\lambda}=2.5 \quad \frac{\alpha}{\beta}=0.02$

$\begin{array}{lr}-0.00000 & 0.00000 \\ -0.00002 & 0.00000 \\ -0.00012 & 0.00000 \\ -0.00046 & 0.00001 \\ -0.00185 & 0.00004 \\ -0.01138 & 0.00026 \\ -0.04268 & 0.00137 \\ -0.08611 & 0.00392 \\ -0.13086 & 0.00799 \\ -0.16599 & 0.01229 \\ -0.18334 & 0.01400 \\ -0.17978 & 0.00951 \\ -0.00708 & 0.00016 \\ -0.02721 & 0.00075 \\ -0.05728 & 0.00209 \\ -0.09263 & 0.00442 \\ -0.12780 & 0.00766 \\ -0.15743 & 0.01117 \\ -0.17718 & 0.01369 \\ -0.18444 & 0.01351 \\ -0.17880 & 0.00889 \\ -0.16210 & -0.00140\end{array}$

$\begin{array}{ll}-0.00093 & 0.00000 \\ -0.00186 & 0.00000 \\ -0.00465 & 0.00000 \\ -0.00930 & 0.00000 \\ -0.01860 & 0.00001 \\ -0.04649 & 0.00012 \\ -0.09291 & 0.00098 \\ -0.13915 & 0.00331 \\ -0.18504 & 0.00782 \\ -0.23032 & 0.015117 \\ -0.27459 & 0.02598 \\ -0.31731 & 0.04076 \\ -0.03652 & 0.00006 \\ -0.07300 & 0.00048 \\ -0.10940 & 0.00161 \\ -0.14567 & 0.00380 \\ -0.18171 & 0.00740 \\ -0.21738 & 0.01272 \\ -0.25251 & 0.02008 \\ -0.28687 & 0.02974 \\ -0.32017 & 0.04193 \\ -0.35206 & 0.05684\end{array}$

$\begin{array}{rr}-0.00093 & 0.00000 \\ -0.00186 & 0.00000 \\ -0.00465 & 0.00000 \\ -0.00928 & 0.00000 \\ -0.01847 & 0.00003 \\ -0.04454 & 0.00039 \\ -0.07791 & 0.00261 \\ -0.09175 & 0.00623 \\ -0.08342 & 0.00793 \\ -0.05777 & 0.00344 \\ -0.02618 & -0.00863 \\ -0.00151 & -0.02392 \\ -0.03557 & 0.00019 \\ -0.06559 & 0.00139 \\ -0.08536 & 0.00386 \\ -0.09189 & 0.00669 \\ -0.08471 & 0.00797 \\ -0.06634 & 0.00554 \\ -0.04194 & -0.00175 \\ -0.01802 & -0.01296 \\ -0.00031 & -0.02488 \\ 0.00825 & -0.03310\end{array}$

$\begin{array}{ll}-0.00000 & 0.00000 \\ -0.00002 & 0.00000 \\ -0.00012 & 0.00000 \\ -0.00046 & 0.00001 \\ -0.00185 & 0.00004 \\ -0.01137 & 0.00035 \\ -0.04246 & 0.00270 \\ -0.08472 & 0.00985 \\ -0.12583 & 0.02341 \\ -0.15356 & 0.04138 \\ -0.16078 & 0.05798 \\ -0.14911 & 0.06741 \\ -0.00707 & 0.00019 \\ -0.027114 & 0.00128 \\ -0.05682 & 0.00456 \\ -0.09091 & 0.01138 \\ -0.12315 & 0.02222 \\ -0.1475 .2 & 0.03605 \\ -0.15984 & 0.05024 \\ -0.15910 & 0.06154 \\ -0.14782 & 0.06774 \\ -0.13089 & 0.06920\end{array}$

$\begin{array}{ll}-0.00093 & 0.00000 \\ -0.00186 & 0.00000 \\ -0.00465 & 0.00000 \\ -0.00930 & 0.00000 \\ -0.01860 & 0.00003 \\ -0.04645 & 0.00049 \\ -0.09252 & 0.00391 \\ -0.13729 & 0.01299 \\ -0.17890 & 0.02996 \\ -0.21436 & 0.05601 \\ -0.23976 & 0.09055 \\ -0.25118 & 0.13056 \\ -0.03650 & 0.00024 \\ -0.07284 & 0.00190 \\ -0.10869 & 0.00636 \\ -0.14343 & 0.01487 \\ -0.17603 & 0.02842 \\ -0.20501 & 0.04758 \\ -0.22852 & 0.07227 \\ -0.24457 & 0.10152 \\ -0.25137 & 0.13338 \\ -0.24783 & 0.16493\end{array}$

$\begin{array}{lrr}0.01 & -0.00093 & 0.00000 \\ 0.02 & -0.00186 & 0.00000 \\ 0.05 & -0.00465 & 0.00000 \\ 0.10 & -0.00928 & 0.00000 \\ 0.20 & -0.01848 & 0.00000 \\ 0.50 & -0.04458 & 0.00004 \\ 1.00 & -0.07820 & 0.00015 \\ 1.50 & -0.09271 & -0.00029 \\ 2.00 & -0.08478 & -0.00275 \\ 2.50 & -0.05688 & -0.00898 \\ 3.00 & -0.01656 & -0.01985 \\ 3.50 & 0.02569 & -0.03410 \\ & & \\ 0.125 \pi & -0.03558 & 0.00002 \\ 0.250 \pi & -0.06573 & 0.00011 \\ 0.375 \pi & -0.08585 & 0.00011 \\ 0.500 \pi & -0.09295 & -0.00047 \\ 0.625 \pi & -0.08609 & -0.00246 \\ 0.750 \pi & -0.06659 & -0.00672 \\ 0.875 \pi & -0.03774 & -0.01383 \\ 1.000 \pi & -0.00431 & -0.02366 \\ 1.125 \pi & 0.02839 & -0.03512 \\ 1.250 \pi & 0.05546 & -0.04610\end{array}$


$\beta \mathrm{h}$

$C(h, 0)$

$S(h, 0)$ $-0.00159-0.00000$ $-0.00397 \quad 0.00000$ $\begin{array}{ll}-0.00793 & 0.00000 \\ -0.01579 & 0.00000\end{array}$ $-0.015790 .00000$ $-0.03810 \quad 0.00000$ $-0.06684-0.00011$ $-0.07926-0.00095$ $-0.07255-0.00372$ $-0.04882-0.00971$ $\begin{array}{rr}-0.01447 & -0.01938 \\ 0.02167 & -0.03142\end{array}$ 3.00
3.50

$\begin{array}{llll}0.125 \pi & -0.03041 & 0.00000\end{array}$ $\begin{array}{llll}0.375 \pi & -0.07338 & -0.00028\end{array}$ $0.500 \pi \quad-0.07947 \quad-0.00119$ $\begin{array}{llll}0.625 \pi & -0.07367 & -0.00342\end{array}$ $\begin{array}{lll}0.750 \pi & -0.05708 & -0.00760\end{array}$ $1.000 \pi \quad-0.00401-0.02265$

$\begin{array}{lll}1.125 \pi & 0.02398 & -0.03225 \\ 1.250 \pi & 0.04737 & -0.04107\end{array}$ $0.250 \pi \quad-0.05618-0.00003$ $0.875 \pi \quad-0.03252-0.01409$

$$
\frac{a}{\lambda}=2.5 \quad \frac{\alpha}{\beta}=0.03
$$

$E(h, c)$

$C(h, h)$

$S\left(h, r_{-}\right)$

$E(h, h)$

$\begin{array}{llll}-0.00000 & 0.00000 & -0.100079 & 0.00000 \\ -0.00002 & 0.00000 & -0.00159 & 0.00000 \\ -0.00010 & 0.00000 & -0.00397 & 0.00000 \\ -0.00040 & 0.00001 & -0.00795 & 0.00000 \\ -0.00158 & 0.00005 & -0.01590 & 0.00001 \\ -0.00973 & 0.00032 & -0.03973 & 0.00011 \\ -0.03647 & 0.00147 & -0.07939 & 0.00084 \\ -0.07357 & 0.00380 & -0.11889 & 0.00283 \\ -0.11183 & 0.00715 & -0.15808 & 0.00667 \\ -0.14193 & 0.01028 & -0.19671 & 0.01295 \\ -0.15699 & 0.01079 & -0.23445 & 0.02216 \\ -0.15435 & 0.00579 & -0.27084 & 0.03476 \\ -0.00605 & 0.00019 & -0.03121 & 0.00005 \\ -0.02325 & 0.00085 & -0.06238 & 0.00041 \\ -0.04895 & 0.00216 & -0.09349 & 0.00137 \\ -0.07914 & 0.00423 & -0.12446 & 0.00325 \\ -0.10921 & 0.00689 & -0.15523 & 0.00632 \\ -0.13458 & 0.00952 & -0.18567 & 0.01086 \\ -0.15159 & 0.01104 & -0.21564 & 0.01714 \\ -0.15802 & 0.01005 & -0.24492 & 0.02537 \\ -0.15355 & 0.00519 & -0.27328 & 0.03576 \\ -0.13968 & -0.00443 & -0.30042 & 0.04844\end{array}$

$\begin{array}{lr}-0.00079 & 0.00000 \\ -0.00159 & 0.00000 \\ -0.00397 & 0.00000 \\ -0.00793 & 0.00000 \\ -0.01579 & 0.00002 \\ -0.03307 & 0.00030 \\ -0.06557 & 0.00199 \\ -0.07344 & 0.00463 \\ -0.07145 & 0.00545 \\ -0.04931 & 0.00110 \\ -0.02309 & -0.00931 \\ -0.00201 & -0.02194 \\ & \\ -0.03040 & 0.00015 \\ -0.05605 & 0.00107 \\ -0.07295 & 0.00292 \\ -0.07857 & 0.00494 \\ -0.07255 & 0.00553 \\ -0.05704 & 0.00302 \\ -0.05643 & -0.00345 \\ -0.01615 & -0.01294 \\ -0.00097 & -0.02272 \\ 0.00665 & -0.02916\end{array}$

$-0.00000$

$-0.00002 \quad 0.00000$

$0.00010 \quad 0.0000$

0.0000

$\begin{array}{ll}-0.00158 & 0.00005\end{array}$

0.009710 .00039

$-0.036270 .00261$

$-0.07231 \quad 0.00884$

$-0.10736 \quad 0.02027$

$-0.13110 \quad 0.0350$

$-0.12811$

0.0483

0.05571

$\begin{array}{ll}-0.00604 & 0.00022\end{array}$

$\begin{array}{ll}-0.02318 & 0.00130\end{array}$

$-0.04852 \cdot 0.00426$

$-0.07759 \quad 0.01015$

$\begin{array}{ll}-0.10508 & 0.01928 \\ -0.12591 & 0.03069\end{array}$

$-0.13658 \quad 0.0422$

$\begin{array}{ll}-0.13627 & 0.05119\end{array}$

$\begin{array}{ll}-0.12704 & 0.05595 \\ -0.11289 & 0.05696\end{array}$

$$
\frac{\mathrm{a}}{\lambda}=2.5 \quad \frac{\alpha}{\beta}=0.05
$$

$\begin{array}{lll}0.01 & -0.00058 & 0.00000 \\ 0.02 & -0.00116 & -0.00000 \\ 0.05 & -0.00290 & -0.00000 \\ 0.10 & -0.00580 & -0.00000 \\ 0.20 & -0.01153 & -0.00000 \\ 0.50 & -0.02783 & -0.00005 \\ 1.00 & -0.04883 & -0.00043 \\ 1.50 & -0.05793 & -0.00172 \\ 2.00 & -0.05313 & -0.00472 \\ 2.50 & -0.03594 & -0.01006 \\ 3.00 & -0.01095 & -0.01769 \\ 3.50 & 0.01556 & -0.02631 \\ 0.125 \pi & -0.02221 & -0.00002 \\ 0.250 \pi & -0.04103 & -0.00020 \\ 0.375 \pi & -0.05361 & -0.00075 \\ 0.500 \pi & -0.05810 & -0.00203 \\ 0.625 \pi & -0.05394 & -0.00442 \\ 0.750 \pi & -0.04193 & -0.00827 \\ 0.875 \pi & -0.02410 & -0.01362 \\ 1.000 \pi & -0.00331 & -0.02012 \\ 1.125 \pi & 0.01728 & -0.02688 \\ 1.250 \pi & 0.03478 & -0.03253\end{array}$

$\begin{array}{ll}-0.00058 & 0.00000 \\ -0.00116 & 0.00000 \\ -0.00290 & 0.00000 \\ -0.00581 & 0.00000 \\ -0.01161 & 0.00000 \\ -0.02902 & 0.00008 \\ -0.05798 & 0.00061 \\ -0.08680 & 0.00207 \\ -0.11536 & 0.00487 \\ -0.14349 & 0.00943 \\ -0.17093 & 0.01613 \\ -0.19733 & 0.02527 \\ -0.02279 & 0.00004 \\ -0.04556 & 0.00030 \\ -0.06826 & 0.00100 \\ -0.09086 & 0.00237 \\ -0.11329 & 0.00461 \\ -0.13546 & 0.00792 \\ -0.15725 & 0.01248 \\ -0.17852 & 0.01846 \\ -0.19909 & 0.02599 \\ -0.21874 & 0.03518\end{array}$

$\begin{array}{rr}-0.00058 & 0.00000 \\ -0.00116 & 0.00000 \\ -0.00290 & 0.00000 \\ -0.07580 & 0.00000 \\ -0.01153 & 0.00001 \\ -0.02780 & 0.00017 \\ -0.04861 & 0.00111 \\ -0.05732 & 0.00236 \\ -0.05242 & 0.00206 \\ -0.03698 & -0.00187 \\ -0.01781 & -0.06964 \\ -0.00238 & -0.01827 \\ & \\ -0.02220 & 0.00008 \\ -0.04093 & 0.00061 \\ -0.05328 & 0.00158 \\ -0.05744 & 0.00247 \\ -0.05320 & 0.00219 \\ -0.04215 & -0.00030 \\ -0.02741 & -0.06537 \\ -0.01278 & -0.01219 \\ -0.00160 & -0.01877 \\ 0.00442 & -0.0226 ?\end{array}$

-

$\begin{array}{ll}-0.00000 & 0.00000 \\ -0.00001 & 0.00000 \\ -0.00007 & 0.00000 \\ -0.00029 & 0.00001 \\ -0.00116 & 0.00006 \\ -0.00709 & 0.00042 \\ -0.02645 & 0.00234 \\ -0.05269 & 0.00709 \\ -0.07820 & 0.01519 \\ -0.09563 & 0.02515 \\ -0.10079 & 0.03368 \\ -0.09457 & 0.03795 \\ -0.00441 & 0.00025 \\ -0.01692 & 0.00125 \\ -0.03538 & 0.00365 \\ -0.05653 & 0.00804 \\ -0.07654 & 0.01450 \\ -0.09178 & 0.02227 \\ -0.09980 & 0.02980 \\ -0.10002 & 0.03539 \\ -0.09384 & 0.03807 \\ -0.08392 & 0.03843\end{array}$

$-0.00079$

$-0.00159$

$-0.00397$

$-0.01589$

$-0.03970$

$-0.11723$

$-0.15265$

$-0.15265$

$-0.18276$

-0.20427
-0.21391

$-0.03119$

$-0.06223$

$-0.09284$

$-0.12246$

$-0.15021$

$-0.17483$

$-0.19476$

$-0.20833$

$-0.21407$

$-0.21110$
0.00000

0.00000

0.00000

0.00000

0.00003

0.0042

0.01108

0.02553

0.04765

0.07689
0.111065

0.00020 0.00162 0.00543 0.02422 0.04050 0.06143 0.08616 0.11302

0.13953

$\begin{array}{ll}-0.00058 & 0.00000 \\ -0.00116 & 0.00000 \\ -0.00290 & 0.00000 \\ -0.00580 & 0.00000 \\ -0.01161 & 0.00002 \\ -0.02899 & 0.00031 \\ -0.05768 & 0.00243 \\ -0.08546 & 0.00807 \\ -0.11114 & 0.01854 \\ -0.13285 & 0.03449 \\ -0.14828 & 0.05544 \\ -0.15514 & 0.07947 \\ & \\ -0.02278 & 0.00015 \\ -0.04543 & 0.00118 \\ -0.06773 & 0.00396 \\ -0.08926 & 0.00923 \\ -0.10937 & 0.01759 \\ -0.12715 & 0.02934 \\ -0.14147 & 0.04438 \\ -0.15118 & 0.06206 \\ -0.15526 & 0.08115 \\ -0.15316 & 0.09987\end{array}$


$\beta \mathrm{h}$

$C(h, 0)$

0.01

0.02

0.05

0.10

0.50

1.00

1.50

2.50
3.00

3.50

$0.125 \pi$

$0.250 \pi$

$0.375 \pi$

$0.625 \pi$

$0.750 \pi$

$0.875 \pi$

1.000

$1.125 \pi$
$1.250 \pi$
$-0.00042-0.00000$

- $0.00085-0.00000$

$-0.00423-0.00600$

$-0.00423-1.00000$

$-0.02033-0.00007$

$\begin{array}{ll}-0.00842 & -0.00000 \\ -0.03567 & -0.00007\end{array}$

$-0.04236-0.00201$

$-0.0389 .1-0.00490$

$-0.02643-0.00952$

$0.00820-0.01552$

$0.01134-0.02172$

$-0.01 b 22-c .00003$

$\begin{array}{ll}-0.02948 & -0.00027\end{array}$

$-0.03918-0.00095$

$-0.04249-0.00232$

$-0.03949-0.00464$

$-0.03079-0.00802$

$-0.01781-0.01239$

$0.00259-0.01733$

$\begin{array}{ll}0.02576 & -0.02568\end{array}$
$S(h, 0)$

$\begin{array}{ll}-0.00030 & 0.00000\end{array}$

0.00500

$-0.00005 \quad 0.00000$

$-0.00021 \quad 0.00001$

$-0.00085 \quad 0.00006$

$\begin{array}{ll}-0.00519 & 0.00037\end{array}$

- $0.01946 \quad 0.00143$

$\begin{array}{ll}-0.03926 & 0.0024 \\ -0.05974 & 0.00450\end{array}$

$-0.07605 \quad 0.00450$

$\begin{array}{ll}-0.08462 & 0.00329\end{array}$

$-0.08405-0.00184$

$-0.00323$

$-0.01240$

$-0.02611$

$-0.05833$

$-0.07203$

$-0.08143$

-0.08539
-0.08367

$-0.07703$

0.00023

0.00090

0.00145

0.0032

0.0044

0.00505

0.00452

0.00222

$-0.00926$

$$
\frac{a}{\lambda}=2.5
$$

$E(h, \jmath)$
$C(h, h)$

$\begin{array}{lr}-0.00042 & 0.00000 \\ -0.00085 & 0.00000 \\ -0.00212 & 0.00000 \\ -0.00423 & 0.00000 \\ -0.00842 & 0.00001 \\ -0.02030 & 0.00009 \\ -0.03550 & 0.00056 \\ -0.04190 & 0.00098 \\ -0.03846 & 0.00611 \\ -0.02742 & -0.00331 \\ -0.01362 & -0.00912 \\ -0.00227 & -0.01504 \\ -0.01621 & 0.00005 \\ -0.02989 & 0.00031 \\ -0.03891 & 0.00076 \\ -0.04200 & 0.00097 \\ -0.03902 & 0.00025 \\ -0.03112 & -10.00204 \\ -0.02055 & -0.00600 \\ -0.00996 & -0.01093 \\ -0.00163 & -0.01536 \\ 0.00306 & -0.01764\end{array}$

$S(h, h)$

$-0.00000 \quad 0.00000$
-0.000010 .00000

$-0.00001 \quad 0.00000$

$\begin{array}{ll}-0.00005 & 0.00000\end{array}$

$\begin{array}{lll}-0.00021 & 0.00001\end{array}$

$\begin{array}{ll}-0.00084 & 0.00006\end{array}$

$\begin{array}{ll}-0.00518 & 0.00041 \\ -0.01930 & 0.00203\end{array}$

$\begin{array}{lll}-0.03841 & 0.00564\end{array}$

$-0.05700 \quad 0.01138$

$0.06982 \quad 0.01807$

$-0.07390 \quad 0.02343$

$-0.05322 \quad 0.00024$

$-0.01235$

$-0.01235$

$-0.04121$

$-0.05578$

$-0.06696$

$-0.07299$

$-0.06932$

$-0.06233$
0.00114
0.00306

0.00633

0.01091

0.02105

0.02443

0.02581
0.02575
$E(h, h)$

$\begin{array}{lll}-0.00042 & 0.000 \mathrm{CO}\end{array}$ $\begin{array}{ll}-0.00085 & 0.0000 c\end{array}$ $0.00212 \quad 0.80000$ 0.00000 0.004170 .0001 $-0.042100 .00178$ $-0.062310 .00587$ 0.08310 .00587 $-0.096580 .02490$ $-0.10764 \quad 0.03098$ $-0.11253 \quad 0.05768$

$-0.01664 \quad 0.00011$ $-0.03317 \quad 0.00086$ $-0.049410 .00289$ $-0.06506 \quad 0.00672$ $-0.07964 \quad 0.01277$ $-0.102770 .02126$ 0.100710 .03206 $-0.112610 .04470$ $-0.111130 .07150$

$$
\frac{a}{\lambda}=2.5 \quad \frac{\alpha}{\beta}=0.10
$$

$\begin{array}{lll}0.01 & -0.00026 & -0.00000 \\ 0.02 & -0.00053 & -0.00000 \\ 0.05 & -0.00132 & -0.00000 \\ 0.10 & -0.00264 & -0.00000 \\ 0.20 & -0.00526 & -0.00000 \\ 0.50 & -0.01269 & -0.00007 \\ 1.00 & -0.02228 & -0.00059 \\ 1.50 & -0.02649 & -0.00196 \\ 2.00 & -0.02439 & -0.00443 \\ 2.50 & -0.01664 & -0.00798 \\ 3.00 & -0.00521 & -0.01215 \\ 3.50 & 0.00723 & -0.01595 \\ 0.125 \pi & -0.01013 & -0.00004 \\ 0.250 \pi & -0.01872 & -0.00029 \\ 0.375 \pi & -0.02448 & -0.00097 \\ 0.500 \pi & -0.02658 & -0.00224 \\ 0.625 \pi & -0.02475 & -0.00421 \\ 0.750 \pi & -0.01935 & -0.00687 \\ 0.875 \pi & -0.01125 & -0.01003 \\ 1.000 \pi & -0.00167 & -0.01331 \\ 1.125 \pi & 0.00805 & -0.01617 \\ 1.250 \pi & 0.01669 & -0.01793\end{array}$

$-0.00000$ -0.00001
-0.00003 -0.000003
-0.00013 $-0.00053$

$-0.00324$

$-0.0121$

$-0.02453$

$-0.03740$

-0.04775
-0.05339

$-0.05339$

$-0.00201$

$-0.00774$

$-0.0163$

$-0.03651 \quad 0.0031$

$\begin{array}{ll}-0.04518 & 0.00315\end{array}$

$\begin{array}{ll}-0.05124 & 0.0022\end{array}$

$-0.05397-0.00006$

$-0.05321-0.00383$

$-0.04932-0.00904$

$\begin{array}{ll}-0.00026 & 0.00000 \\ -0.00053 & 0.00000 \\ -0.00132 & 0.00000 \\ -0.00265 & 0.00000 \\ -0.00529 & 0.00000 \\ -0.01323 & 0.00004 \\ -0.02642 & 0.00028 \\ -0.03953 & 0.00094 \\ -0.05249 & 0.00221 \\ -0.06521 & 0.00428 \\ -0.07757 & 0.00729 \\ -0.08941 & 0.01139 \\ & \\ -0.01039 & 0.00002 \\ -0.02076 & 0.00014 \\ -0.03110 & 0.00046 \\ -0.04137 & 0.00108 \\ -0.05155 & 0.00209 \\ -0.06158 & 0.00359 \\ -0.07142 & 0.00565 \\ -0.08098 & 0.00834 \\ -0.09020 & 0.01171 \\ -0.09896 & 0.01581\end{array}$

更

-0.00000
-0.00001
-0.00003
-0.00013
-0.00053
-0.00323
-0.01203
-0.02392
-0.03552
-0.04363
-0.04648
-0.04433
-0.00201
-0.00770
-0.01608
-0.02567
-0.03476
-0.04179
-0.04573
-0.04632
-0.04404
-0.03985

0.00000

0.00000

0.00000

0.00001

0.00005

0.00035

0.00157

0.00395

0.00738

0.01102

0.01357
0.01428

$-0.00026$

$-0.00053$

$-0.00132$

$-0.00265$

$-0.00529$

$-0.01321$

$-0.02624$

$-0.03878$

$-0.05986$

$-0.06658$

$-0.06952$

0.00000

0.00000

0.00000

0.00000

0.00001

0.00111

0.00365

0.01537

0.02448

0.00021

$-0.01038$

0.03475

0.00092

0.00227

0.00438

0.00711

0.01003

0.01250

0.01396

$-0.00137-0.001120$

$-0.03985$

0.01390

$-0.02069$

0.00007

0.00180

$-0.03079 \quad 0.00180$

$\begin{array}{lll}-0.04948 & 0.00791\end{array}$

$\begin{array}{ll}-0.05735 & 0.01311\end{array}$

$\begin{array}{ll}-0.06363 & 0.01969\end{array}$

$\begin{array}{ll}-0.06782 & 0.02733\end{array}$

$-0.06957$

$-0.06869$

0.03546
0.04331 


$$
\frac{a}{\lambda}=2.5 \quad \frac{\alpha}{\beta}=0.20
$$

$\beta \mathrm{h}$

$\mathrm{C}(\mathrm{h}, 0)$

$S(h, 0)$

$E(h, 0)$

\begin{tabular}{|c|c|c|}
\hline $\begin{array}{l}0.01 \\
0.02 \\
0.05 \\
0.10 \\
0.20 \\
0.50 \\
1.00 \\
1.50 \\
2.00 \\
2.50 \\
3.00 \\
3.50\end{array}$ & $\begin{array}{r}-0.00006 \\
-0.00011 \\
-0.00027 \\
-0.00055 \\
-0.00109 \\
-0.00264 \\
-0.00465 \\
-0.00556 \\
-0.00515 \\
-0.00351 \\
-0.00099 \\
0.00194\end{array}$ & $\begin{array}{l}-0.00000 \\
-0.00000 \\
-0.00000 \\
-0.00000 \\
-0.00000 \\
-0.00004 \\
-0.00029 \\
-0.00090 \\
-0.00188 \\
-0.00310 \\
-0.00429 \\
-0.00509\end{array}$ \\
\hline $\begin{array}{l}0.125 \pi \\
0.250 \pi \\
0.375 \pi \\
0.500 \pi \\
0.625 \pi \\
0.750 \pi \\
0.875 \pi \\
1.000 \pi \\
1.125 \pi\end{array}$ & $\begin{array}{l}-0.00211 \\
-0.00390 \\
-0.00512 \\
-0.00558 \\
-0.00522 \\
-0.00409 \\
-0.00234 \\
-0.00018 \\
0.00214\end{array}$ & $\begin{array}{l}-0.00002 \\
-0.00014 \\
-0.00046 \\
-0.00101 \\
-0.00180 \\
-0.00274 \\
-0.00372 \\
-0.00457 \\
-0.00513\end{array}$ \\
\hline
\end{tabular}

$0.01 \quad-0.00000 \quad-0.00000$

$\begin{array}{lll}0.02 & -0.00000 & -0.00000\end{array}$

$0.05-0.00001-0.00000$

$0.10-0.00002-0.00000$

$\begin{array}{lll}0.10 & -0.00005 & -0.00000 \\ 0.50 & -0.00011 & -0.00000\end{array}$

$\begin{array}{ll}0.50 & -0.000120-0.00000\end{array}$

$1.50-0.00025-0.00008$

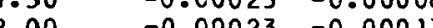

$\begin{array}{lll}2.00-0.00023 & -0.00017\end{array}$

$\begin{array}{lll}2.50 & -0.00015 & -0.00027 \\ 3.00 & -0.00000 & -0.00036\end{array}$

$\begin{array}{lll}3.50 & 0.00019 & -0.00041\end{array}$

$0.125 \pi$

$-0.00009-0.00000$

$-0.00017-0.00001$

$-0.00023-0.00004$

$0.500 \pi \quad-0.00025-0.00009$

$0.625 \pi \quad-0.00023 \quad-0.00016$

$0.750 \pi-0.00018-0.00024$

$0.875 \pi \quad-0.00008-0.00032$

$\begin{aligned} & 1.000 \pi \\ & 1.125 \pi\end{aligned} \quad 0.00005-0.00038$

$\begin{array}{lll}1.125 \pi & 0.00021 & -0.00041 \\ 1.250 \pi & 0.00038 & -0.00039\end{array}$

$\begin{array}{rr}-0.00000 & 0.00000 \\ -0.00000 & 0.00000 \\ -0.00001 & 0.00000 \\ -0.00003 & 0.00001 \\ -0.00011 & 0.00002 \\ -0.00067 & 0.00013 \\ -0.00254 & 0.00046 \\ -0.00516 & 0.00080 \\ -0.00793 & 0.00089 \\ -0.01027 & 0.00048 \\ -0.01169 & -0.00059 \\ -0.01195 & -0.00234 \\ & \\ -0.00042 & 0.00008 \\ -0.00161 & 0.00030 \\ -0.00341 & 0.00059 \\ -0.00555 & 0.00083 \\ -0.00774 & 0.00089 \\ -0.00968 & 0.00066 \\ -0.01112 & 0.00003 \\ -0.01189 & -0.00102 \\ -0.01192 & -0.00249 \\ -0.01123 & -0.00425\end{array}$

$-0.00006 \quad 0.00000$

$-0.00055 \quad 0.00000$

$-0.00110 \quad 0.0000$

$\begin{array}{ll}-0.00275 & 0.0000\end{array}$

$-0.00549 \quad 0.00006$

$-0.01087 .0 .00046$

$\begin{array}{lll}-0.01347 & 0.00088\end{array}$

$\begin{array}{ll}-0.01598 & 0.00149\end{array}$

$-0.00216 \quad 0.00000$

$\begin{array}{lll}-0.00431 & 0.00003\end{array}$

$-0.00646 \quad 0.00009$

$-0.01067 \quad 0.00022$

$-0.01273 \quad 0.00074$

$\begin{array}{ll}-0.01667 & 0.00170\end{array}$

$-0.02026 \quad 0.00318$ $\begin{array}{lll}-0.00011 & 0.00000\end{array}$

$-0.00028 \quad 0.00000$

$\begin{array}{ll}-0.00820 & 0.00019\end{array}$

$-0.01836 .0 .00231$

$-0.01473 \quad 0.00116$ $\frac{a}{\lambda}=2.5 \quad \frac{\alpha}{\beta}=0.40$

$\begin{array}{rrrr}-0.00000 & 0.00000 & -0.00000 & 0.00000 \\ -0.00000 & 0.00000 & -0.00000 & 0.00000 \\ -0.00000 & 0.00000 & -0.00001 & 0.00000 \\ -0.00000 & 0.00000 & -0.00002 & 0.00000 \\ -0.00000 & 0.00000 & -0.00005 & 0.00000 \\ -0.00003 & 0.00001 & -0.00012 & 0.00000 \\ -0.00017 & 0.00004 & -0.00024 & 0.00000 \\ -0.00023 & 0.00006 & -0.00035 & 0.00001 \\ -0.00037 & 0.00006 & -0.00047 & 0.00002 \\ -0.00051 & 0.00001 & -0.00057 & 0.00004 \\ -0.00060 & -0.00010 & -0.00068 & 0.00006 \\ -0.00064 & -0.00027 & -0.00077 & 0.00010 \\ -0.00002 & 0.00001 & -0.00009 & 0.00000 \\ -0.00007 & 0.00003 & -0.00018 & 0.00000 \\ -0.00015 & 0.00005 & -0.00028 & 0.00000 \\ -0.00025 & 0.00006 & -0.00037 & 0.00001 \\ -0.00036 & 0.00006 & -0.00046 & 0.00002 \\ -0.00047 & 0.00003 & -0.00054 & 0.00003 \\ -0.00056 & -0.00004 & -0.00063 & 0.00005 \\ -0.00062 & -0.00015 & -0.00077 & 0.00007 \\ -0.00064 & -0.00029 & -0.00078 & 0.00010 \\ -0.00060 & -0.00045 & -0.00085 & 0.00013\end{array}$

$C(h, h)$

$S(h, h)$

$\begin{array}{ll}-0.00016 & -0.00000 \\ -0.00011 & -0.00000 \\ -0.00027 & -0.00000 \\ -0.00055 & -0.00000 \\ -0.00109 & -0.00000 \\ -0.00264 & -0.00002 \\ -0.00462 & -0.00014 \\ -0.00549 & -0.00049 \\ -0.00514 & -0.00115 \\ -0.00382 & -0.00207 \\ -0.00203 & -0.00300 \\ -0.00033 & -0.00354 \\ -0.00210 & -0.00001 \\ -0.00388 & -0.000 .07 \\ -0.00507 & -0.00023 \\ -0.00551 & -0.00057 \\ -0.00520 & -0.00109 \\ -0.00427 & -0.00179 \\ -0.00295 & -0.00256 \\ -0.00152 & -0.00321 \\ -0.00023 & -0.00355 \\ 0.00074 & -0.00342\end{array}$

$E(h, h)$

$\begin{array}{llll}-0.00000 & 0.00000 & -0.00006 & 0.00000 \\ -0.00000 & 0.00000 & -0.00011 & 0.00000 \\ -0.00001 & 0.00000 & -0.00028 & 0.00000 \\ -0.00003 & 0.00001 & -0.00055 & 0.00000 \\ -0.00011 & 0.00002 & -0.00110 & 0.00000 \\ -10.00067 & 0.00014 & -0.00274 & 0.00003 \\ -0.00250 & 0.00053 & -0.00543 & 0.00023 \\ -0.00497 & 0.00112 & -0.00799 & 0.00075 \\ -0.00742 & 0.00174 & -0.01029 & 0.00168 \\ -0.00923 & 0.00214 & -0.01216 & 0.00305 \\ -0.01005 & 0.00214 & -0.01343 & 0.00478 \\ -0.00981 & 0.00174 & -0.01397 & 0.00665 \\ & & & \\ -0.00042 & 0.00008 & -0.00216 & 0.00001 \\ -0.00160 & 0.00033 & -0.00429 & 0.00011 \\ -0.00334 & 0.00073 & -0.00636 & 0.00037 \\ -0.00534 & 0.00121 & -0.00833 & 0.00085 \\ -0.00726 & 0.00170 & -0.01013 & 0.00160 \\ -0.00881 & 0.00206 & -0.01167 & 0.00262 \\ -0.00977 & 0.00220 & -0.01288 & 0.00388 \\ -0.01008 & 0.00206 & -0.01366 & 0.00530 \\ -0.00976 & 0.00170 & -0.01398 & 0.00678 \\ -0.00891 & 0.00132 & -0.01383 & 0.00816\end{array}$

$\begin{array}{ll}-0.00000 & -C .00000 \\ -0.00000 & -0.00000 \\ -0.00001 & -0.00000 \\ -0.00002 & -0.00000 \\ -0.00005 & -0.00000 \\ -0.00011 & -0.00000 \\ -0.00020 & -0.00002 \\ -0.00024 & -0.00006 \\ -0.00023 & -0.00013 \\ -0.00017 & -0.00021 \\ -0.00007 & -0.00027 \\ 0.00004 & -0.00028 \\ -0.00008 & -0.00000 \\ -0.00017 & -0.00001 \\ -0.06022 & -0.00003 \\ -0.00025 & -0.00007 \\ -0.00023 & -0.00012 \\ -0.00019 & -0.00018 \\ -0.00012 & -0.00024 \\ -0.00064 & -0.00028 \\ 0.00005 & -0.00028 \\ 0.00014 & -0.00024\end{array}$

$\begin{array}{lr}-0.00000 & 0.00000 \\ -0.00000 & 0.00000 \\ -0.00000 & 0.00000 \\ -0.00000 & 0.00000 \\ -0.00000 & 0.00000 \\ -0.00003 & 0.00001 \\ -0.00011 & 0.00004 \\ -0.00022 & 0.00008 \\ -0.00034 & 0.00009 \\ -0.00044 & 0.00008 \\ -0.00050 & 0.00003 \\ -0.00050 & -0.00005 \\ -0.00002 & 0.00001 \\ -0.00007 & 0.00003 \\ -0.00015 & 0.00005 \\ -0.00024 & 0.00008 \\ -0.00033 & 0.00009 \\ -0.00042 & 0.00009 \\ -0.00048 & 0.00006 \\ -0.00051 & 0.00001 \\ -0.00050 & -0.00005 \\ -0.00044 & -0.00012\end{array}$

$\begin{array}{ll}-0.00000 & 0.00000 \\ -0.00000 & 0.00000 \\ -0.00001 & 0.00000 \\ -0.00002 & 0.00000 \\ -0.00005 & 0.00000 \\ -0.00012 & 0.00000 \\ -0.00023 & 0.00001 \\ -0.00034 & 0.00003 \\ -0.00043 & 0.00007 \\ -0.00050 & 0.00012 \\ -0.00055 & 0.00018 \\ -0.00057 & 0.00024 \\ -0.00009 & 0.00000 \\ -0.00018 & 0.00000 \\ -0.00027 & 0.00002 \\ -0.00035 & 0.00004 \\ -0.00042 & 0.00007 \\ -0.00048 & 0.00010 \\ -0.00053 & 0.00015 \\ -0.00056 & 0.00020 \\ -0.00057 & 0.00025 \\ -0.00056 & 0.00029\end{array}$


Bh $C(h, 0)$

0.0

$0.02-0.00000-0.00000$

$0.05 \quad-0.00000-0.00000$

$0.10 \quad-0.00000-0.00000$

$\begin{array}{lll}0.20 & -0.00000 & -0.00000 \\ 0.50 & -0.00000 & -0.00000\end{array}$

$\begin{array}{lll}0.50 & -0.00000 & -0.00000 \\ 1.00 & -0.00000 & -0.00000\end{array}$

$1.50-0.00000-0.05000$

$2.00-0.00000-0.00000$

$2.50-0.02000-0.00000$

$\begin{array}{lll}3.00 & 0.00000 & -0.00001 \\ 3.50 & 0.00001 & -0.00001\end{array}$

$0.125 \pi \quad-0.00000 \quad-0.00000$

$0.250 \pi \quad-0.00000-0.00000$

$0.375 \pi-0.00000-0.00000$

$\begin{array}{lll}0.500 \pi & -0.00000 & -0.00000 \\ 0.625 \pi & -0.00000 & -0.00000\end{array}$

$0.750 \pi \quad-0.00000-0.00000$

$\begin{array}{llll}0.875 \pi & 0.00000 & -0.00001\end{array}$

$\begin{array}{lll}1.000 \pi & 0.00000 & -0.0000\end{array}$

$\begin{array}{lll}1.125 \pi & 0.00001 & -0.00001 \\ 1.250 \pi & 0.00001 & -0.00001\end{array}$ $\frac{a}{\lambda}=2.5 \quad \frac{\alpha}{\beta}=0.70$

$E(h, 0)$

$\mathrm{C}(\mathrm{h}, \mathrm{h})$

$S(h, h)$

$E(h, h)$

$-0.00000-0.00000$

$\begin{array}{ll}-0.00000 & 0.00000\end{array}$

$-0.00000 \quad 0.00000$

$-0.00000 \quad 0.00000$

$-0.00000 \quad 0.00000$

$-0.00000 \quad 0.00000$

$-0.00000 \quad 0.00050$

$-0.00001-0.00000$

$-0.00001-0.00000$

$0.00001-0.00001$

$-0.00000 \quad 0.00000$

$-0.00000 \quad 0.00000$

$-0.00000 \quad 0.00000$

$\begin{array}{ll}-0.00000 & 0.00000 \\ -0.00000 & 0.00000\end{array}$

$-0.00001-0.00000$

$-0.00001-0.00000$

$\begin{array}{ll}-0.00001 & -0.00001 \\ -0.00001 & -0.00001\end{array}$

$-0.00000$

0.0000

$-0.00000 \quad 0.00000$

$-0.00500 \quad c .00000$

$\begin{array}{ll}-0.000000 & 0.00000 \\ -0.00001 & 0.00000\end{array}$

$\begin{array}{ll}-0.00001 & 0.00000 \\ -0.00001 & 0.00000\end{array}$

$-0.000010 .00000$

$-0.00000 \quad 0.00000$

$-0.00000 \quad 0.00000$

$-0.00000 \quad 0.00000$

$\begin{array}{ll}-0.00001 & 0.00000\end{array}$

$-0.00001 \quad 0.00000$

$\begin{array}{ll}-0.00001 & 0.00000 \\ -0.00001 & 0.00000\end{array}$



$-0.00000 \quad 0.00000$

$\begin{array}{lll}-0.00000 & 0.00000\end{array}$

$\begin{array}{ll}-0.00001 & 0.00000\end{array}$

$-0.00000 \quad 0.00000$

$-0.00000 \quad 0.00000$

$-0.00000 \quad 0.00000$

$\begin{array}{ll}-9.00000 & 0.00000\end{array}$

$$
\frac{a}{\lambda}=2.5 \quad \frac{\alpha}{\beta}=1.00
$$

$\begin{array}{ll}-0.00000 & 0.00000 \\ -0.00000 & 0.00000 \\ -0.00000 & 0.00000 \\ -0.00000 & 0.00000 \\ -0.00000 & 0.00000 \\ -0.00000 & 0.00000 \\ -0.00000 & 0.00000 \\ -0.00000 & 0.00000 \\ -0.00000 & 0.00000 \\ -0.00000 & -0.00000 \\ -0.00000 & -0.00000 \\ -0.00000 & -0.00000 \\ -0.00000 & 0.00000 \\ -0.00000 & 0.00000 \\ -0.00000 & 0.00000 \\ -0.00000 & 0.00000 \\ -0.00000 & 0.00000 \\ -0.00000 & -0.00000 \\ -0.00000 & -0.00000 \\ -0.00000 & -0.00000 \\ -0.00000 & -0.00000 \\ -0.00000 & -0.00000\end{array}$

0
0
0
0
0
0
0
0
0
0
0
0
0
0
0
0
0

$\begin{array}{llll}-0.00000 & 0.00000 & -0.00000 & -0.00000 \\ -0.00000 & 0.00000 & -0.00000 & -0.00000 \\ -0.00000 & 0.00000 & -0.00000 & -0.00000 \\ -0.00000 & 0.00000 & -0.00000 & -0.00000 \\ -0.00000 & 0.00000 & -0.00000 & -0.00000 \\ -0.00000 & 0.00000 & -0.00000 & -0.00000 \\ -0.00000 & 0.00000 & -0.00000 & -0.00000 \\ -0.00000 & 0.00000 & -0.00000 & -0.00000 \\ -0.00000 & 0.00000 & -0.00000 & -0.00000 \\ -0.00000 & 0.00000 & -0.00000 & -0.00000 \\ -0.00000 & 0.00000 & 0.00000 & -0.00000 \\ -0.00000 & 0.00000 & 0.00000 & -0.00000 \\ & & & \\ -0.00000 & 0.00000 & -0.00000 & -0.00000 \\ -0.00000 & 0.00000 & -0.00000 & -0.00000 \\ -0.00000 & 0.00000 & -0.00000 & -0.00000 \\ -0.00000 & 0.00000 & -0.00000 & -0.00000 \\ -0.00000 & 0.00000 & -0.00000 & -0.00000 \\ -0.00000 & 0.00000 & -0.00000 & -0.00000 \\ -0.00000 & 0.00000 & 0.00000 & -0.00000 \\ -0.00000 & 0.00000 & 0.00000 & -0.00000 \\ -0.00000 & 0.00000 & 0.00000 & -0.00000 \\ -0.00000 & 0.00000 & 0.00000 & -0.00000\end{array}$

$-0.00000$

0.00000

\begin{tabular}{|c|c|c|}
\hline $\begin{array}{l}0.01 \\
0.02 \\
0.05 \\
0.10 \\
0.20 \\
0.50 \\
1.00 \\
1.50\end{array}$ & $\begin{array}{r}-0.000000 \\
-0.00000 \\
-0.00000 \\
-0.00000 \\
-0.00000 \\
-0.00000 \\
-0.00000 \\
-0.00000 \\
-0.00000 \\
-0.00000 \\
0.00000 \\
0.00000\end{array}$ & $\begin{array}{l}-0.000 \\
-0.000 \\
-0.000 \\
-0.000 \\
-0.000 \\
-0.000 \\
-0.000 \\
-0.000 \\
-0.000 \\
-0.000 \\
-0.000 \\
-0.000\end{array}$ \\
\hline $\begin{array}{l}125 \pi \\
250 \pi \\
375 \pi \\
500 \pi \\
625 \pi \\
750 \pi \\
875 \pi \\
000 \pi\end{array}$ & $\begin{array}{r}-0.00000 \\
-0.00000 \\
-0.00000 \\
-0.00000 \\
-0.00000 \\
-0.00000 \\
0.00000 \\
0.00000 \\
0.00000 \\
0.00000\end{array}$ & $\begin{array}{l}-0.00 \\
-0.00 \\
-0.00 \\
-0.00 \\
-0.00 \\
-0.00 \\
-0.00 \\
-0.00 \\
-0.00 \\
-0.00\end{array}$ \\
\hline
\end{tabular}

$\begin{array}{rrrr}-0.00000 & 0.00000 & -0.00000 & 0.00000 \\ -0.00000 & 0.00000 & -0.00000 & 0.00000 \\ -0.00000 & 0.00000 & -0.00000 & 0.00000 \\ -0.00000 & 0.00000 & -0.00000 & 0.00000 \\ -0.00000 & 0.00000 & -0.00000 & 0.00000 \\ -0.00000 & 0.00000 & -0.00000 & 0.00000 \\ -0.00000 & 0.00000 & -0.00900 & 0.00000 \\ -0.00000 & 0.00000 & -0.00000 & 0.00000 \\ -0.00000 & 0.00000 & -0.00000 & 0.00000 \\ -0.00001 & 0.00000 & -0.00000 & 0.00000 \\ -0.00001 & -0.00000 & -0.00000 & 0.00000 \\ -0.00001 & -0.00000 & -0.00000 & 0.00000 \\ -0.00000 & 0.00000 & -0.00000 & 0.00000 \\ -0.00000 & 0.00000 & -0.00000 & 0.00000 \\ -0.00000 & 0.00000 & -0.00000 & 0.00000 \\ -0.00000 & 0.00000 & -1.00000 & 0.00000 \\ -0.00000 & 0.00000 & -0.00000 & 0.00000 \\ -0.00000 & 0.00000 & -0.00000 & 0.00000 \\ -0.00001 & -0.00000 & -0.00000 & 0.00000 \\ -0.00001 & -0.00000 & -0.00000 & 0.00000 \\ -0.00001 & -0.00000 & -0.00000 & 0.00000 \\ -0.00000 & -0.00001 & -0.00000 & 0.00000\end{array}$

$\begin{array}{rrrr}-0.00000 & 0.00000 & -0.00000 & 0.00000 \\ -0.00000 & 0.00000 & -0.00000 & 0.00000 \\ -0.00000 & 0.00000 & -0.00000 & 0.00000 \\ -0.00000 & 0.00000 & -0.00000 & 0.00000 \\ -0.00000 & 0.00000 & -0.00000 & 0.00000 \\ -0.00000 & 0.00000 & -0.00000 & 0.00000 \\ -0.00000 & 0.00000 & -0.00000 & 0.00000 \\ -0.00000 & 0.00000 & -0.00000 & 0.00000 \\ -0.00000 & 0.00000 & -0.00000 & 0.00000 \\ -0.00000 & -0.00000 & -0.00000 & 0.00000 \\ -0.00000 & -0.00000 & -0.00000 & 0.00000 \\ -0.00000 & -0.00000 & -0.00000 & 0.00000 \\ & & & \\ -0.00000 & 0.00000 & -0.00000 & 0.00000 \\ -0.09000 & 0.00000 & -0.00000 & 0.00000 \\ -0.00000 & 0.00000 & -0.00000 & 0.00000 \\ -0.00000 & 0.00000 & -0.00000 & 0.00000 \\ -0.00000 & 0.00000 & -0.00000 & 0.00000 \\ -0.00000 & 0.00000 & -0.00000 & 0.00000 \\ -0.00000 & -0.00000 & -0.00000 & 0.00000 \\ -0.00000 & -0.00000 & -0.00000 & 0.00000 \\ -0.00000 & -0.00000 & -0.00000 & 0.00000 \\ -0.00000 & -0.00000 & -0.00000 & 0.00000\end{array}$



$0.05 \quad 0.00530-0.00000$ $0.01059-0.00000$ $0.02108-0.00001$ $\begin{array}{lll}0.05086 & -0.00011\end{array}$ $0.08924-0.00067$ $0.10575-0.00130$ $0.09648-0.00044$ $0.06405 \quad 0.00405$ $\begin{array}{lll}3.00 & 0.01702 & 0.01364\end{array}$

$\begin{array}{llll}0.125 \pi & 0.04060 & -0.00005\end{array}$ $0.250 \pi \quad 0.07500-0.00037$ $\begin{array}{lll}0.375 \pi & 0.09797 & -0.00094\end{array}$ $\begin{array}{lll}0.375 \pi & 0.09797 & -0.00094 \\ 0.500 \pi & 0.10602 & -0.00131\end{array}$ $0.625 \pi \quad 0.09801-0.00060$ $0.750 \pi \quad 0.07534 \quad 0.00227$ $\begin{array}{lll}0.875 \pi & 0.04175 & 0.00816\end{array}$ $0.075 \pi \quad 0.041750 .00816$ $.125 \pi=0.0026900 .01728$ $\begin{array}{lll}1.125 \pi & -0.03558 & 0.02883 \\ 1.250 \pi & -0.06712 & 0.04086\end{array}$

$$
\frac{\mathrm{a}}{\lambda}=3.0 \quad \frac{\alpha}{\beta}=0.00
$$

$E(h, 0)$

$\begin{array}{ll}0.00001 & -0.00000 \\ 0.00002 & -0.00000 \\ 0.00013 & -0.00000 \\ 0.00053 & -0.00000 \\ 0.00211 & -0.00000 \\ 0.01299 & -0.00004 \\ 0.04874 & -0.00063 \\ 0.09840 & -0.00273 \\ 0.14966 & -0.00691 \\ 0.18987 & -0.01226 \\ 0.20933 & -0.01606 \\ 0.20401 & -0.01435 \\ 0.00808 & -0.00002 \\ 0.03106 & -0.00025 \\ 0.06542 & -0.00115 \\ 0.10587 & -0.00320 \\ 0.14615 & -0.00654 \\ 0.18009 & -0.01073 \\ 0.20256 & -0.01458 \\ 0.21035 & -0.01631 \\ 0.20277 & -0.01393 \\ 0.18174 & -0.00578\end{array}$

$0.00001-0.00000$ $-0.00000$ $0.00211-0.00000$ $-0.00004$ $0.09840-0.00273$ $0.14966-0.0069$ $0.20933-0.01606$

$0.00808-0.00002$ $0.03106-0.00025$ $0.06542-0.00115$ $0.14615-0.00654$ $0.18174-0.00578$

$\begin{array}{ll}0.00106 & -0.00000 \\ 0.00212 & -0.00000 \\ 0.00531 & -0.00000 \\ 0.01061 & -0.00000 \\ 0.02122 & -0.00001 \\ 0.05305 & -0.00012 \\ 0.10605 & -0.00094 \\ 0.15893 & -0.00316 \\ 0.21157 & -0.00746 \\ 0.26377 & -0.01452 \\ 0.31520 & -0.02495 \\ 0.36545 & -0.03932 \\ & \\ 0.04166 & -0.00006 \\ 0.08331 & -0.00045 \\ 0.12490 & -0.00153 \\ 0.16640 & -0.00362 \\ 0.20774 & -0.00706 \\ 0.24882 & -0.01217 \\ 0.28949 & -0.01925 \\ 0.32958 & -0.02859 \\ 0.36884 & -0.04046 \\ 0.40700 & -0.05508\end{array}$

$C(h, h)$

$S(h, h)$

$0.00106-0.00000$ $0.00212-0.00000$ $0.00530-0.00000$ $0.01059-0.00000$ $0.02108-0.00003$ $0.05084-0.00045$ $0.08903-0.00302$ $0.10501-0.00753$ $0.09527-0.01055$ $0.06442-0.00711$ $0.02458 \quad 0.00564$ $-0.00913 \quad 0.02487$

$0.04059-0.00022$ $0.07491-0.00160$ $0.09761-0.00452$ $\begin{array}{ll}0.09761 & -0.00452 \\ 0.10517 & -0.00816\end{array}$ $0.09680-0.01049$

$0.07486-0.00901$

$0.04476-0.00191$ $\begin{array}{lll}0.01379 & 0.01071\end{array}$ $-0.01089 \quad 0.02623$
$0.00001-0.00000$ $0.00002-0.00000$ $0.00013-0.00000$ $0.00053-0.00000$ $0.00211-0.00000$ $0.01298-0.00013$ $0.04860-0.00191$ $0.09744-0.00847$ $0.14592-0.02205$ $0.18010-0.04123$ $0.19092-0.06028$ $0.17853-0.07196$

$0.00807-0.00005$ $0.03102-0.00076$ $0.06513-0.00353$ $0.10467-0.00995$ $0.14271-0.02082$ $0.17241-0.03541$ $0.18865-0.05120$ $0.18950-0.06460$ $0.17703-0.07237$ $0.15659-0.07349$
$E(h, h)$

$0.00106-0.00000$ $0.00212-0.00000$ $0.00531-0.00000$ $0.01061-0.00000$ $0.05302-0.00047$ $0.05302-0.00047$ $0.15760-0.01247$ $0.20697-0.02900$ $0.25135-0.05515$ $0.28716-0.09112$ $0.28716-0.09112$

$0.04165-0.00023$ $0.08320-0.00181$ $0.12441-0.00608$ $0.16479-0.01430$ $0.20350-0.02754$ $0.23929-0.04662$ $0.29517-0.10295$ $0.29517-0.10295$ $0.31689-0.17685$

$$
\frac{a}{\lambda}=3.0 \quad \frac{\alpha}{\beta}=0.005
$$

$\begin{array}{lrr}0.01 & 0.00097 & -0.00000 \\ 0.02 & 0.00193 & -0.00000 \\ 0.05 & 0.00483 & -0.00000 \\ 0.10 & 0.00964 & -0.00000 \\ 0.20 & 0.01918 & -0.00001 \\ 0.50 & 0.04629 & -0.00008 \\ 1.00 & 0.08122 & -0.00047 \\ 1.50 & 0.09625 & -0.00075 \\ 2.00 & 0.08785 & 0.00044 \\ 2.50 & 0.05841 & 0.00492 \\ 3.00 & 0.01569 & 0.01389 \\ 3.50 & -0.02927 & 0.02664 \\ & & \\ 0.125 \pi & 0.03695 & -0.00004 \\ 0.250 \pi & 0.06826 & -0.00027 \\ 0.375 \pi & 0.08916 & -0.00063 \\ 0.500 \pi & 0.09649 & -0.00071 \\ 0.625 \pi & 0.08924 & 0.00026 \\ 0.750 \pi & 0.06866 & 0.00320 \\ 0.875 \pi & 0.03816 & 0.00881 \\ 1.000 \pi & 0.00267 & 0.01721 \\ 1.125 \pi & -0.03214 & 0.02759 \\ 1.250 \pi & -0.06094 & 0.03816\end{array}$

$\begin{array}{ll}0.00000 & -0.00000 \\ 0.00002 & -0.00000 \\ 0.00012 & -0.00000 \\ 0.00048 & -0.00000 \\ 0.00192 & -0.00001 \\ 0.01182 & -0.00010 \\ 0.04435 & -0.00076 \\ 0.08953 & -0.00276 \\ 0.13618 & -0.00648 \\ 0.17279 & -0.01101 \\ 0.19061 & -0.01388 \\ 0.18598 & -0.01159 \\ 0.00735 & -0.00005 \\ 0.02827 & -0.00035 \\ 0.05953 & -0.00128 \\ 0.09633 & -0.00319 \\ 0.13298 & -0.00616 \\ 0.16388 & -0.00974 \\ 0.18438 & -0.01285 \\ 0.19158 & -0.01391 \\ 0.18487 & -0.01116 \\ 0.16597 & -0.00322\end{array}$
$0.01931-0.0000$ $0.23998-0.01320$ $0.22638-0.01107$
$0.00097-0.00000$ $0.00193-0.00000$ $0.00483-0.00000$ $0.00966-0.00000$ $0.04827-0.0001$ $0.09650-0.00085$ $0.14462-0.0028$ $0.19251-0.00679$ $0.33241-0.03575$

$0.03792-0.00005$ $0.07581-0.00041$ $0.11366-0.00139$ $0.15142-0.00330$ $0.18903-0.0064$ $0.26337-0.01751$ $0.29980-0.02600$ $0.33548-0.03679$ $0.37015-0.05007$
$0.00097-0.00000$ $0.00193-0.00000$ $0.00483-0.00000$ $0.00964-0.00000$ $0.01918-0.00003$ $0.04627-0.00039$ $0.08102-0.00261$

$0.09557-0.00642$

$\begin{array}{lll}0.08678 & -0.00878\end{array}$

$\begin{array}{rr}0.02281 & 0.00630 \\ -0.00779 & 0.02363\end{array}$

$0.03694-0.00019$ $0.06817-0.00138$ $0.09573-0.00694$ $0.08817-0.00876$ $0.06832-0.00712$ $0.04109-0.00049$ $0.01304 \quad 0.01097$ $-0.00940 \quad 0.02484$
$0.05888-0.00531$ $0.08882-0.00389$
$0.00000-0.00000$ $0.00002-0.00000$ $0.00012-0.00000$ $0.00048-0.00000$ $0.00192-0.00001$ $0.01181-0.00017$

$0.04422-0.00192$

$0.08862-0.00797$

$0.13268-0.02023$

$0.16380-0.03733$

$\begin{array}{ll}0.17380 & -0.05411 \\ 0.16285 & -0.06422\end{array}$

$0.00735-0.00008$ $0.02822-0.00081$ $0.05924-0.00344$ $0.09519-0.00932$ $0.12977-0.01913$ $0.15679-0.03216$ $0.17163-0.04615$ $0.17259-0.05788$ $0.16151-0.06456$ $0.14320-0.06537$ $\begin{array}{ll}0.00097 & -0.00000 \\ 0.00193 & -0.00000 \\ 0.00483 & -0.00000 \\ 0.00966 & -0.00000 \\ 0.01931 & -0.00003 \\ 0.04825 & -0.00043 \\ 0.09626 & -0.00339 \\ 0.14337 & -0.01134 \\ 0.18823 & -0.02641 \\ 0.22851 & -0.05010 \\ 0.26097 & -0.08270 \\ 0.28185 & -0.12278 \\ & \\ 0.03791 & -0.00021 \\ 0.07571 & -0.00165 \\ 0.11319 & -0.00553 \\ 0.14990 & -0.01300 \\ 0.18507 & -0.02503 \\ 0.21757 & -0.04236 \\ 0.24588 & -0.06525 \\ 0.26822 & -0.09341 \\ 0.28276 & -0.12572 \\ 0.28787 & -0.16023\end{array}$ 
0.01

0.02

0.10

0.20

1.00

1.50

2.00

2.50

3.50

$0.125 \pi$

0.250

$0.375 \pi$

$0.500 \pi$

$0.625 \pi$

$0.750 \pi$

$1.000 \pi$

1.125

$1.250 \pi$
$0.00088-0.00000$ $0.00176-0.00000$ $0.00439-0.00000$ $0.00877-0.00000$ $0.01746-0.00000$ $0.04212-0.00005$ $0.07391-0.00029$ $0.08760-0.00029$ $\begin{array}{ll}0.07999 & 0.00116\end{array}$ $0.05326 \quad 0.00561$ $-0.02645 \quad 0.02550$ $0.06212-0.00018$ $\begin{array}{lll}0.08114 & -0.00037\end{array}$ $\begin{array}{lll}0.08783 & -0.00021\end{array}$ $0.08125 \quad 0.00097$ $0.06257 \quad 0.00395$ $0.03487 \quad 0.00929$ $0.00262 \quad 0.01701$ $\begin{array}{ll}0.000262 & 0.01701 \\ -0.05537 & 0.03562\end{array}$
$0.03363-0.00003$
$S(h, 0)$

$0.00000-0.00000$ $0.00002-0.00000$ $0.00011-0.00000$ $0.00044-0.00000$ $0.00175-0.00002$ $0.04036-0.00085$ $-0.00276$ $\begin{array}{lll}0.12391 & -0.00607\end{array}$ $0.15726-0.00989$ $\begin{array}{ll}0.17357 & -0.01196 \\ 0.16956 & -0.00922\end{array}$ $0.00669-0.00008$ $0.02572-0.00044$ $0.05417-0.00137$ $0.08765-0.00315$ $0.12100-0.00579$ $0.16784-0.01132$ $0.16856-0.00878$ $0.15157-0.00108$

$$
\frac{a}{\lambda}=3.0 \quad \frac{\alpha}{\beta}=0.01
$$
$0.01075-0.00014$ $0.14913-0.00885$
$E(h, 0)$

$0.00088-0.00000$ $0.00176-1.00000$ $0.00439-3.00000$ $0.01757-0.00001$ $0.04393-0.00010$ $0.08782-0.00078$ $0.13160-0.00261$ $0.17516-0.00618$ $0.21833-0.01201$ $0.30235-0.03250$

$0.03451-0.00005$ $0.06899-0.00038$ $0.10343-0.00127$ $0.13779-0.00300$ $0.20597-0.00585$ $0.20597-0.01007$ $0.27272-0.02364$ $0.30515-0.03345$ $0.33663-0.04551$ $0.00879-2.00000$

$C(h, h)$

$0.00088-0.00000$ $0.00176-0.00000$ $0.00439-0.00000$ $0.00877-0.00000$ $0.01746-0.00002$ $0.04210-0.00033$ $0.07372-0.00224$ $0.08698-0.00545$ $0.07905-0.00725$ $0.05381-0.00378$ $0.02115 \quad 0.00695$ $\begin{array}{lll}-0.00664 & 0.02242\end{array}$ $0.03362-0.00016$ $0.06203-0.00179$ $0.08083-0.00333$ $0.08713-0.00588$ $0.08535-0.0075$ $0.06235-0.00550$ $0.03770 \quad 0.00069$ 0.01229 $\begin{array}{ll}-0.00810 & 0.02349 \\ -0.01970 & 0.03421\end{array}$

$$
\frac{a}{\lambda}=3.0 \quad \frac{\alpha}{\beta}=0.02
$$

$0.00000-0.00000$ $0.00001-0.00000$ $0.00009-0.00000$ $0.00036-0.00001$ $0.00145-0.00003$ $0.00891-0.00020$ $0.03342-0.00099$ $0.06745-0.00270$ $0.10260-0.00532$ $0.13028-0.00798$ $0.14396-0.00880$ $0.14095-0.00544$

$0.00554 \quad-0.00012$ $0.02130-0.00055$ $0.04486-0.00148$ $0.07257-0.00302$ $0.10019-0.00511$ $0.12352-0.00730$ $0.13911-0.00876$ $0.14481-0.00838$
$0.14015-0.00500$

$0.12641 \quad 0.00216$

$\begin{array}{ll}0.25245 & -0.02765 \\ 0.27843 & -0.03760\end{array}$
$0.00073-0.00000$ $0.00146-0.00000$ $0.00364-0.00000$ $0.01446-0.00002$ $0.03487-0.00025$ $0.06105-0.00164$ $0.07205-0.00387$ $0.06560-0.00477$ $0.04492-0.00139$ $0.01814 \quad 0.00766$ $-0.00480 \quad 0.02010$

$0.02784-0.00012$ $0.05137-0.00087$ $0.06693-0.0024$ $0.07218-0.00415$ $0.06662-0.00482$ $0.05192-0.00294$ $0.01086 \quad 0.01103$ $\begin{array}{ll}-0.01585 & 0.02925\end{array}$ $0.00727-0.00000$ $0.03173 \quad 0.00245$
$0.00073-0.00000$ $0.00146-0.00000$ $0.00364-0.00000$ $0.00728-0.00000$ $0.01456-0.00001$ $0.03638-0.00008$ $0.07273-0.00064$ $0.10897-0.00216$ $0.14502-0.00511$ $0.18073-0.00994$ $0.21587-0.01706$ $0.25014-0.02687$

$0.02858-0.00004$ $0.05714 \quad-0.00031$ $0.08565-0.00105$ $0.11409-0.00248$ $\begin{array}{ll}0.14240 & -0.00484 \\ 0.17050 & -0.00833\end{array}$ $\begin{array}{ll}0.17050 & -0.00833 \\ 0.19831 & -0.01317\end{array}$

$0.19831-0.01317$

$0.22568-0.01955$
$S(h, h)$

$0.00000-0.00000$ $0.00002-0.00000$ $0.00011-0.00000$ $0.00175-0.00002$ $\begin{array}{ll}0.01075-0.00021 \\ 0.040225 & -0.00191\end{array}$ $0.04022-0.00191$ 0.08060 -0.00750 $0.12065-0.01856$ $0.14898-0.03380$ $0.14855-0.05730$

$0.00669-0.00011$ $0.02568-0.00086$ $0.05389-0.00333$ $0.08657-0.00872$ $0.11800-0.01758$ $0.14258-0.02921$ $0.15615-0.0415$ $0.14736-0.05758$ $\begin{array}{ll}0.14736 & -0.05758 \\ 0.13094 & -0.05813\end{array}$ $0.00044-0.00000$
$E(h, h)$.

$\begin{array}{ll}0.00088 & -0.00000 \\ 0.00176 & -0.00000 \\ 0.00439 & -0.00000 \\ 0.00879 & -0.00000 \\ 0.01757 & -0.00002 \\ 0.04391 & -0.00039 \\ 0.08758 & -0.00309 \\ 0.13042 & -0.01032 \\ 0.17118 & -0.02401 \\ 0.20774 & -0.04550 \\ 0.23717 & -0.07506 \\ 0.25607 & -0.11133 \\ 0.03450 & -0.00019 \\ 0.06889 & -0.00150 \\ 0.10299 & -0.00503 \\ 0.13636 & -0.01182 \\ 0.16832 & -0.02276 \\ 0.19781 & -0.03848 \\ 0.22349 & -0.05925 \\ 0.24374 & -0.08475 \\ 0.25689 & -0.11400 \\ 0.26151 & -0.14518\end{array}$

$\begin{array}{lrl}1.125 \pi & -0.00245 & 0.01633\end{array}$

0.02387
0.03101

$\begin{array}{llll}0.00000 & -0.00000 & 0.00073 & -0.00000 \\ 0.00001 & -0.00000 & 0.00146 & -0.00000 \\ 0.00009 & -0.00000 & 0.00364 & -0.00000 \\ 0.00036 & -0.00001 & 0.00728 & -0.00000 \\ 0.00145 & -0.00003 & 0.01455 & -0.00002 \\ 0.00890 & -0.00026 & 0.03636 & -0.00032 \\ 0.03329 & -0.00186 & 0.07251 & -0.00256 \\ 0.06667 & -0.00661 & 0.10793 & -0.00853 \\ 0.09977 & -0.01563 & 0.14158 & -0.01983 \\ 0.12326 & -0.02772 & 0.17170 & -0.03754 \\ 0.13116 & -0.03913 & 0.19588 & -0.06182 \\ 0.12362 & -0.04557 & 0.21136 & -0.09154 \\ & & & \\ 0.00554 & -0.00014 & 0.02857 & -0.00016 \\ 0.02126 & -0.00090 & 0.05705 & -0.00124 \\ 0.04459 & -0.00310 & 0.08525 & -0.00417 \\ 0.07161 & -0.00762 & 0.11284 & -0.00978 \\ 0.09758 & -0.01483 & 0.13922 & -0.01880 \\ 0.11794 & -0.02411 & 0.16353 & -0.03176 \\ 0.12929 & -0.03378 & 0.18465 & -0.04884 \\ 0.13042 & -0.04161 & 0.20127 & -0.06978 \\ 0.12267 & -0.04576 & 0.21204 & -0.09372 \\ 0.10945 & -0.04589 & 0.21581 & -0.11918 \ldots\end{array}$




$$
\frac{a}{\lambda}=3.0 \quad \frac{\alpha}{\beta}=0.03
$$

$\beta \mathrm{h}$

$C(h, 0)$

Síh, 0)

0.01

0.02

0.05

0.20

0.50

1.00

1.50

2.00

3.00

3.50

$0.125 \pi$

0.250

$0.500 . \pi$

$0.625 \pi$

$0.750 \pi$

$0.875 \pi$

$1.000 \pi$

$1.250 \pi$

$0.00060-0.00000$ $0.00121-0.00000$ $0.00301 \quad 0.00000$ $0.00602 \quad 0.00000$ 0.028890 .00000

$0.05070 \quad 0.00016$

$0.05070 \quad 0.00016$

0.054290 .0028

$0.03680 \quad 0.00695$

0.036800 .00695

$-0.01776 \quad 0.02096$

$0.02306 \quad 0.00001$

$0.04261 \quad 0.00006$

$0.05566 \quad 0.00032$

$0.06028 \quad 0.00106$

$0.05584 \quad 0.00267$

0.043140 .00554

0.024270 .01539

$0.00223 \quad 0.01539$

$\begin{array}{ll}-0.03792 & 0.02696\end{array}$
$0.00000-0.00000$ $0.00001-0.00000$ $0.00008-0.00020$ $0.00030-0.00001$ $0.00120-0.00004$ $0.00738-0.00024$ $0.02767-0.00105$ $0.05586-0.00258$ $0.08498-0.00465$ $0.10795-0.00643$ $0.11943-0.00638$

$0.11720-0.00270$

$0.00459-0.00014$ $0.01764-0.00062$ $0.03714-0.00151$ $0.06010-0.00285$ $0.08298-0.00448$ $0.10234-0.00603$ $0.11533-0.00675$ $0.12020-0.00578$ $0.11656-0.00227$
$E(h, 0)$

$0.00060-0.00000$ $0.00121-0.00000$ $0.00301-0.00000$ $0.00603-0.0000 \mathrm{C}$ $0.01205-0.0000 \mathrm{C}$ $0.03013-0.000 \mathrm{C7}$ $0.06023-0.00053$ $0.09023-0.00179$ $0.12006-0.00423$ $0.14960-0.00822$ $0.17864-0.01411$ $0.20695-0.02221$

$\begin{array}{lll}0.02367 & -0.00003\end{array}$ $0.04732-0.00026$ $0.07093-0.00087$ $0.09447-0.00206$

$0.11789-0.00400$

$0.14114-0.00689$

$0.16413-0.01090$

$0.18675-0.01617$

$0.20886-0.02285$ $0.23030-0.03107$
$C(h, h)$

$\begin{array}{rr}0.06060 & -0.00000 \\ 0.00121 & -0.00000 \\ 0.00301 & -0.00000 \\ 0.00602 & -0.00000 \\ 0.01197 & -0.00001 \\ 0.02888 & -0.00018 \\ 0.05056 & -0.00117 \\ 0.05968 & -0.00268 \\ 0.05443 & -0.00294 \\ 0.03749 & 0.00029 \\ 0.01551 & 0.00791 \\ -0.00345 & 0.01793 \\ 0.02306 & -0.00009 \\ 0.04254 & -0.00063 \\ 0.05543 & -0.00171 \\ 0.05980 & -0.00284 \\ 0.05527 & -0.00301 \\ 0.04323 & -0.00109 \\ 0.02667 & 0.00358 \\ 0.00951 & 0.01067 \\ -0.00447 & 0.01860 \\ -0.01280 & 0.02501\end{array}$

$S(h, h)$

$0.00000-0.00000$ $0.00001-0.00000$ $0.00008-0.00000$ $0.00030,-0.00001$ $0.00120-0.00004$ $0.02737-0.00028$ $0.02756-0.00177$ $0.08253-0.01315$ $0.10200-0.02273$ $0.10875-0.03152$ $0.10288-0.03620$

$0.00459-0.00016$ -0.00090 $0.01760-0.00090$ $0.03691-0.00285$ $0.05924-0.00664$ $0.09757-0.01990$ $0.09757-0.01990$ $0.10707-0.02743$ $0.10212-0.03632$ $0.09148-0.03616$
$E(h, h)$

$0.00060-0.00000$ $0.00121-0.00000$ $0.00301-0.00000$ $0.00603-0.00000$ $0.01205-0.00002$ .00027 $0.06003-0.00212$ $0.11710-0.01639$ $0.14191-0.03097$ $0.14197-0.03097$ $0.16178-0.05092$

$0.02360-0.00013$ $0.04723-0.00103$ $0.07057-0.00345$ $0.09337-0.00808$ $0.13518-0.02621$ $0.13518-0.02621$ $0.15256-0.04026$ $0.17502-0.05745$ $0.17810-0.09783$

\begin{tabular}{|c|c|c|}
\hline $\begin{array}{l}0.01 \\
0.02 \\
0.05 \\
0.10 \\
0.20 \\
0.50 \\
1.00 \\
1.50 \\
2.00 \\
2.50 \\
3.00 \\
3.50\end{array}$ & $\begin{array}{r}0.00041 \\
0.00083 \\
0.00207 \\
0.00413 \\
0.00821 \\
0.01982 \\
0.03478 \\
0.04127 \\
0.03780 \\
0.02540 \\
0.00728 \\
-0.01208\end{array}$ & $\begin{array}{l}0.00000 \\
0.00000 \\
0.00000 \\
0.00000 \\
0.00000 \\
0.00004 \\
0.00036 \\
0.00133 \\
0.00340 \\
0.00689 \\
0.01165 \\
0.01679\end{array}$ \\
\hline $\begin{array}{l}0.125 \pi \\
0.250 \pi \\
0.375 \pi \\
0.500 \pi \\
0.625 \pi \\
0.750 \pi \\
0.875 \pi \\
1.000 \pi \\
1.125 \pi \\
1.250 \pi\end{array}$ & $\begin{array}{r}0.01582 \\
0.02923 \\
0.03819 \\
0.04139 \\
0.03838 \\
0.02973 \\
0.01684 \\
0.00172 \\
-0.01334 \\
-0.02620\end{array}$ & $\begin{array}{l}0.00002 \\
0.00017 \\
0.00061 \\
0.00155 \\
0.00321 \\
0.00574 \\
0.00914 \\
0.01312 \\
0.01712 \\
0.02029\end{array}$ \\
\hline
\end{tabular}

$\begin{array}{ll}0.00000 & -0.00000 \\ 0.00001 & -0.00000 \\ 0.00005 & -0.00000 \\ 0.00021 & -0.00001 \\ 0.00082 & -0.00004 \\ 0.00506 & -0.00026 \\ 0.01893 & -0.00103 \\ 0.03832 & -0.00223 \\ 0.05832 & -0.00353 \\ 0.07418 & -0.00418 \\ 0.08227 & -0.00315 \\ 0.08109 & 0.00062 \\ 0.00315 & -0.00016 \\ 0.01210 & -0.00064 \\ 0.02548 & -0.00142 \\ 0.04122 & -0.00242 \\ 0.05694 & -0.00344 \\ 0.07029 & -0.00411 \\ 0.07933 & -0.00395 \\ 0.08288 & -0.00240 \\ 0.08068 & 0.00099 \\ 0.07335 & 0.00642\end{array}$

$$
\frac{a}{\lambda}=3.0 \quad \frac{\alpha}{\beta}=0.05
$$

$\begin{array}{ll}0.00041 & -0.00000 \\ 0.00083 & -0.00000 \\ 0.00207 & -0.00000 \\ 0.00413 & -0.00000 \\ 0.00827 & -0.00000 \\ 0.02067 & -0.00005 \\ 0.04130 & -0.00036 \\ 0.06187 & -0.00123 \\ 0.08230 & -0.00290 \\ 0.10250 & -0.00563 \\ 0.12234 & -0.00965 \\ 0.14165 & -0.01517 \\ 0.01623 & -0.00002 \\ 0.03245 & -0.00018 \\ 0.04864 & -0.00060 \\ 0.06477 & -0.00141 \\ 0.08081 & -0.00274 \\ 0.09672 & -0.00472 \\ 0.11243 & -0.00746 \\ 0.12787 & -0.01106 \\ 0.14295 & -0.01561 \\ 0.15755 & -0.02121\end{array}$

$0.00000-0.00000$ $0.00001-0.00000$ $0.00005-0.00000$ $0.00021-0.00001$ $0.00082-0.00004$ $0.00505-0.00029$ $0.01888-0.00153$ $0.03776-0.00444$ $0.05649-0.00931$ $0.06991-0.01530$ $0.07481-0.02042$ $0.07128-0.02275$

$0.00314-0.00017$ $0.01206-0.00083$ $0.02528-0.00234$ $0.04055-0.00501$ $0.05524-0.00890$ $0.06683-0.01357$ $0.07349-0.01810$ $0.07458-0.02142$ $0.07079-0.02279$ $0.06386-0.02227$

$\begin{array}{ll}0.00041 & -0.00000 \\ 0.00083 & -0.00000 \\ 0.00207 & -0.00000 \\ 0.00413 & -0.00000 \\ 0.00827 & -0.00001 \\ 0.02065 & -0.00018 \\ 0.04115 & -0.00145 \\ 0.06117 & -0.00483 \\ 0.08010 & -0.01118 \\ 0.09694 & -0.02108 \\ 0.11036 & -0.03455 \\ 0.111888 & -0.05089 \\ 0.01623 & -0.00009 \\ 0.03239 & -0.00070 \\ 0.04836 & -0.00236 \\ 0.06394 & -0.00553 \\ 0.07877 & -0.01060 \\ 0.09238 & -0.01786 \\ 0.10414 & -0.02736 \\ 0.11333 & -0.03894 \\ 0.11925 & -0.05208 \\ 0.12131 & -0.06593\end{array}$


. $\beta \mathrm{h}$

$\mathrm{C}(\mathrm{h}, 0)$

$\begin{array}{lrl}0.01 & 0.00028 & 0.00000 \\ 0.02 & 0.00057 & 0.00000 \\ 0.05 & 0.00142 & 0.00000 \\ 0.10 & 0.00283 & 0.00000 \\ 0.20 & 0.00563 & 0.00000 \\ 0.50 & 0.01360 & 0.00005 \\ 1.00 & 0.02387 & 0.00042 \\ 1.50 & 0.02833 & 0.00142 \\ 2.00 & 0.02599 & 0.00332 \\ 2.50 & 0.01752 & 0.00619 \\ 3.00 & 0.00508 & 0.00974 \\ 3.50 & -0.00832 & 0.01320 \\ 0.125 \pi & 0.01085 & 0.00002 \\ 0.250 \pi & 0.02005 & 0.00020 \\ 0.375 \pi & 0.02621 & 0.00068 \\ 0.500 \pi & 0.02842 & 0.00163 \\ 0.625 \pi & 0.02638 & 0.00314 \\ 0.750 \pi & 0.02048 & 0.00527 \\ 0.875 \pi & 0.01165 & 0.00791 \\ 1.000 \pi & 0.00125 & 0.01077 \\ 1.125 \pi & -0.00920 & 0.01341 \\ 1.250 \pi & -0.01826 & 0.01519\end{array}$

0.0

0.0

0.05

0.10

0.50

1.00

1.50

2.00

2.50

3.50

$0.125 \pi$
$0.250 \pi$

$0.250 \pi$
$0.375 \pi$

$0.500 \pi$

0.625

$0.750 \pi$

$0.875 \pi$

$1.125 \pi$

$1.250 \pi$

\begin{abstract}
0.00016
\end{abstract} 0.00032

0.00081

0.00161

0.00320

0.00772

0.01357

0.01612

0.01482

0.01001

0.00290

$-0.00488$

0.00617

0.01140

0.01490
0.01617

0.01504

0.01170
0.00667

0.00667

0.00069
-0.00539

$-0.01079$

0.00000

0.00000

0.00000

0.00000

0.00005

0.00038

0.00123

0.00272

0.00704

0.00896

0.00002

0.00062

0.00140

0.00140
0.00259

0.00413

0.00413

0.00590
0.00765

0.00765
0.00906

0.00977
$S(h, 0)$

$0.00000-0.00000$ $0.00001-0.00000$ $0.00004-0.00000$ $0.00014-0.00001$ $0.00057-0.00004$ $0.00347-0.00024$ $0.01302-0.00092$ $0.02629-0.00185$ $0.04005-0.00265$ $0.05102-0.0027$ $0.05674-0.00132$ $0.05616 \quad 0.00211$

$0.00216-0.00015$ $0.00830-0.00059$ $0.01748-0.00124$ $0.02829-0.00199$ $0.03910-0.00261$ $0.04832-0.00281$ $0.05462-0.00225$ $0.05722-0.00058$ $\begin{array}{ll}0.05589 & 0.00243 \\ 0.05105 & 0.00679\end{array}$

$$
\frac{a}{\lambda}=3.0 \quad \frac{\alpha}{\beta}=0.07
$$

$E(h, 0)$

$0.00028-0.00000$ $0.00057-0.00000$ $0.00142-0.00000$ $0.00284-0.00000$ $0.00567-0.00000$ $0.01418-0.00003$ $0.02833-0.00025$ -0.0008
0.0042 $0.05641-0.00199$ $0.07023-0.00385$ $0.08379-0.00660$ $0.09696-0.01037$

$0.01113-0.00002$ $0.02226-0.00012$ $0.03335-0.00041$ $0.05539-0.00188$ $0.05539-0.00188$ $0.06628-0.00323$ $0.07702-0.005 .10$ $0.08756-0.00756$ $\begin{array}{ll}0.09784 & -0.01067 \\ 0.10778 & -0.01448\end{array}$

$$
\mathrm{C}(\mathrm{h}, \mathrm{h})
$$

$0.00028-0.00000$ $0.00057-0.00000$ $0.00142-0.00000$ $0.00283-0.00000$ $0.00563-0.00000$ $0.01358-0.00004$ $0.02378-0.00021$ $0.02812-0.00026$ $\begin{array}{lll}0.02581 & 0.00052\end{array}$ $0.01813 \quad 0.00284$ $\begin{array}{rr}0.0008087 & 0.00670\end{array}$ $0.01085-0.00002$ $0.02001-0.00013$ $0.02608-0.00027$ $0.02819-0.00022$ 0.026190 .00041 0.020740 .00199 $0.01320 \quad 0.00461$ $-0.00137 \quad 0.01120$ $\begin{array}{ll}0.000137 & 0.01120 \\ -0.00568 & 0.01341\end{array}$
$S(h, h)$

$0.00000-0.00000$ $0.00001-0.00000$ $0.00004-0.00000$ $0.00014-0.00001$ $0.00057-0.00004$ $0.00347-0.00027$ $0.01294-0.00126$ $0.02587-0.00335$ $0.03869-0.00659$ $0.04795-0.01031$ $0.05152-0.01321$ $0.04940-0.01420$

$0.00216-0.00016$ $0.00827-0.00072$ $0.01732-0.00187$ $0.02778-0.00375$ $0.01584-0.00633$ $0.04582-0.00926$ $0.05049-0.01194$ $0.05144-0.01371$ $\begin{array}{ll}0.04909 & -0.01419 \\ 0.04455 & -0.01355\end{array}$
$E(h, h)$

$0.00028-0.00000$ $0.00057-0.00000$ $0.00142-0.00000$ $0.00284-0.00000$ $0.00567-0.00001$ $0.01416-0.00013$ $0.02820-0.00099$ $0.04189-0.00330$ $0.05479-0.00763$ $0.06622-0.01435$ $0.07529-0.02344$ $0.01113-0.00006$ $0.02220-0.00048$ $0.03314-0.00162$ $0.04378-0.00378$ $0.05389-0.00724$ $0.06314-0.01216$ $0.07109-0.01860$ $0.07729-0.02639$ $0.08262-0.04444$

$$
\frac{a}{\lambda}=3.0 \quad \frac{\alpha}{\beta}=0.10
$$
$\begin{array}{ll}0.00000 & -0.00000 \\ 0.00000 & -0.00000\end{array}$ $0.00002-0.0000$ $0.00008-0.0000$ $0.00032-0.00003$ $0.00197-0.00020$ $0.00740-0.0007$ $0.01496-0.00133$ $\begin{array}{lll}0.02282 & -0.00170\end{array}$ $0.02915-0.00141$ $0.03256 \quad-0.00004$

$0.00123-0.00012$ $0.00472-0.00046$ $0.00994-0.00093$ $0.01610-0.00141$ $0.02228-0.00169$ $0.02758-0.00159$ $0.03127-0.00089$ $0.03289 \quad 0.00058$

$0.03228 \quad 0.00287$

$0.02966 \quad 0.00590$

$\begin{array}{llrr}0.00016 & -0.00000 & 0.00016 & -0.00000 \\ 0.00032 & -0.00000 & 0.00032 & -0.00000 \\ 0.00081 & -0.00000 & 0.00081 & -0.00000 \\ 0.00161 & -0.00000 & 0.00161 & -0.00000 \\ 0.00322 & -0.00000 & 0.00320 & -0.00000 \\ 0.00805 & -0.00002 & 0.00772 & -0.00000 \\ 0.01609 & -0.00014 & 0.01351 & 0.00002 \\ 0.02408 & -0.00048 & 0.01600 & 0.00027 \\ 0.03201 & -0.00113 & 0.01475 & 0.00110 \\ 0.03983 & -0.00218 & 0.01048 & 0.00276 \\ 0.04749 & -0.00373 & 0.00483 & 0.00509 \\ 0.05491 & -0.00586 & -0.00032 & 0.00733 \\ & & & \\ 0.00633 & -0.00001 & 0.00616 & -0.00000 \\ 0.01264 & -0.00007 & 0.01137 & 0.00000 \\ 0.01894 & -0.00023 & 0.01482 & 0.00007 \\ 0.02521 & -0.00055 & 0.01604 & 0.00035 \\ 0.03144 & -0.00107 & 0.01496 & 0.00101 \\ 0.03760 & -0.00183 & 0.01193 & 0.00219 \\ 0.04367 & -0.00289 & 0.00772 & 0.00387 \\ 0.04961 & -0.00427 & 0.00325 & 0.00578 \\ 0.05540 & -0.00602 & -0.000 & 0.00746 \\ 0.06099 & -0.00816 & -0.00325 & 0.00839\end{array}$

$\begin{array}{llll}0.00000 & -0.00000 & 0.00016 & -0.00000 \\ 0.00000 & -0.00000 & 0.00032 & -0.00000 \\ 0.00002 & -0.00000 & 0.00081 & -0.00000 \\ 0.00008 & -0.00001 & 0.00161 & -0.00000 \\ 0.00032 & -0.00003 & 0.00322 & -0.00000 \\ 0.00197 & -0.00021 & 0.00804 & -0.00007 \\ 0.00735 & -0.00090 & 0.01601 & -0.00056 \\ 0.01467 & -0.00217 & 0.02374 & -0.00187 \\ 0.02196 & -0.00392 & 0.03100 & -0.00430 \\ 0.02729 & -0.00571 & 0.03739 & -0.00806 \\ 0.02949 & -0.00685 & 0.04242 & -0.01310 \\ 0.02853 & -0.00690 & 0.04557 & -0.01913 \\ 0.00122 & -0.00013 & 0.00632 & -0.00003 \\ 0.00122 & -0.00054 & 0.01261 & -0.00027 \\ 0.00983 & -0.00128 & 0.01880 & -0.00092 \\ 0.01576 & -0.00240 & 0.02481 & -0.00214 \\ 0.02148 & -0.00379 & 0.03049 & -0.00408 \\ 0.02605 & -0.00523 & 0.03567 & -0.00684 \\ 0.02881 & -0.00640 & 0.04010 & -0.01042 \\ 0.02951 & -0.00698 & 0.04352 & -0.01473 \\ 0.02837 & -0.00686 & 0.04570 & -0.01956 \\ 0.02593 & -0.00623 & 0.04645 & -0.02459\end{array}$


$\beta \mathrm{h}$

$\mathrm{C}(\mathrm{h}, 0)$

0.01

0.02

0.10

0.20

0.50

1.00

2.00

2.50

2.50
3.00

0.125

$0.250 \pi$

$0.375 \pi$

$0.500 \pi$

$0.625 \pi$

$0.750 \pi$
$0.875 \pi$

$1.000 \pi$

$1.125 \pi$

$1.250 \pi$
$0.00002 \quad 0.00000$

$\begin{array}{lll}0.00005 & 0.00000\end{array}$

$\begin{array}{lll}0.00012 & 0.00000\end{array}$

$0.00024 \quad 0.00000$

$0.00049 \quad 0.00000$

$0.00117 \quad 0.00002$

$\begin{array}{ll}0.00207 & 0.00013 \\ 0.00247 & 0.0004\end{array}$

$0.00228 \quad 0.0008$

$0.00153 \quad 0.00137$

$\begin{array}{rr}0.000037 & 0.00186 \\ -0.00097 & 0.00215\end{array}$

0.00094

0.00173

0.00228

0.00248

0.00231

0.000099

0.00000

0.00000
-0.00106

$-0.00208$ 0.00210 0.00212

0.00000

0.01

0.02 0.00000

0.10

0.20

0.50

1.00

1.50
2.00

2.00

3.00

3.50

0.00001

$0.00005 \quad 0.00001$

$0.00006 \quad 0.00002$

$0.00005 \quad 0.00004$

$0.00003 \quad 0.00006$

$\begin{array}{ll}-0.00005 & 0.00009\end{array}$

$0.125 \pi$

0.00002

0.00004

$0.250 \pi$

0.00005

$0.500 \pi \quad 0.00006$

$0.625 \pi \quad 0.00006$

$0.750 \pi \quad 0.00004$

$0.875 \pi \quad 0.00002$

$1.000 \pi-0.00002$

$1.125 \pi-0.00005$

0.00000

0.000 .00

0.00001

0.00002

0.00004

0.00006

0.00008

0.00009

0.00009

0.00009

$$
\frac{\mathrm{a}}{\lambda}=3.0 \quad \frac{\alpha}{\beta}=0.20
$$

$\mathrm{S}(\mathrm{h}, 0)$

$0.00000-0.00000$ $0.00000-0.00000$ $0.00001-0.00000$ $0.00005-0.0000$ $0.00030-0.00006$ $0.00113-0.00020$ $0.00230-0.00034$ $0.00354-0.00036$ $0.00458-0.00015$ $\begin{array}{ll}0.00520 & 0.00036 \\ 0.00527 & 0.00115\end{array}$

$0.00019-0.00004$ $0.00072-0.00013$ $0.00152-0.00026$ $0.00248-0.00035$ $0.00345-0.00037$ $0.00432-0.00024$ $0.00495 \quad 0.00006$ $0.00528 \quad 0.00055$ $0.00528 \quad 0.00055$ $\begin{array}{ll}0.00526 & 0.00122 \\ 0.00489 & 0.00199\end{array}$ $0.00000-0.00000$
$E(h, 0)$

$0.00002-0.00000$

$0.00005-0.00000$

$0.00012-0.00000$

$0.00024-0.00000$

$0.00049-0.00000$

$0.00122-0.00000$

$0.00244-0.00002$

$0.00365-0.00007$

$0.00484-0.00017$

$0.00601-0.00033$

$\begin{array}{ll}0.00715 & -0.00056 \\ 0.00825 & -0.00087\end{array}$

$0.00096-0.00000$

$0.00192-0.00001$

$0.00287-0.00004$

$0.00382-0.00008$

$0.00476-0.00016$

$0.00568-0.00028$

$0.00659-0.00043$

$0.00747-0.00064$

$0.00832-0.00090$

$0.00914-0.00121$
$\mathrm{C}(\mathrm{h}, \mathrm{h})$

$S(h, h)$

$E(h, h)$

$\begin{array}{rr}0.00002 & 0.00000 \\ 0.00005 & 0.00000 \\ 0.00012 & 0.00000 \\ 0.00024 & 0.00000 \\ 0.00049 & 0.00000 \\ 0.00117 & 0.00001 \\ 0.00206 & 0.00008 \\ 0.00245 & 0.00025 \\ 0.00228 & 0.00057 \\ 0.00166 & 0.00100 \\ 0.00079 & 0.00144 \\ -0.00009 & 0.00172 \\ & \\ 0.00094 & 0.00000 \\ 0.00173 & 0.00004 \\ 0.00226 & 0.00013 \\ 0.00246 & 0.00029 \\ 0.00231 & 0.00054 \\ 0.00187 & 0.00087 \\ 0.00124 & 0.00123 \\ 0.00053 & 0.00154 \\ -0.00014 & 0.00173 \\ -0.00067 & 0.00173\end{array}$

$$
\frac{a}{\lambda}=3.0 \quad \frac{\alpha}{\beta}=0.40
$$

$\begin{array}{ll}0.00000 & -0.00000 \\ 0.00000 & -0.00000 \\ 0.00000 & -0.00000 \\ 0.00000 & -0.00000 \\ 0.00000 & -0.00000 \\ 0.00001 & -0.00000 \\ 0.00003 & -0.00001 \\ 0.00006 & -0.00001 \\ 0.00009 & -0.00001 \\ 0.00012 & -0.00000 \\ 0.00014 & 0.00003 \\ 0.00015 & 0.00007 \\ & \\ 0.00000 & -0.00000 \\ 0.00002 & -0.00001 \\ 0.00004 & -0.00001 \\ 0.00006 & -0.00001 \\ 0.00009 & -0.00001 \\ 0.00011 & -0.00001 \\ 0.00013 & 0.00001 \\ 0.00015 & 0.00004 \\ 0.00015 & 0.00007 \\ 0.00014 & 0.00011\end{array}$

(1)
$0.00000-0.00000$ $0.00000-0.00000$ $0.00001-0.00000$ $0.00005-0.00001$ $0.00030-0.00006$ $0.00223-0.00047$ $0.00423-0.00080$ $0.00466-0.00072$

$0.00019-0.00004$ $0.00071-0.00015$ $0.00149-0.00031$ $0.00240-0.00050$ $0.00329-0.00068$ $0.00402-0.00079$ $0.00470-0.00067$ $0.00460-0.00044$

$\begin{array}{ll}0.00460 & -0.00044 \\ 0.00425 & -0.00018\end{array}$
$0.00000-0.00000$ $0.00112-0.00023$ $0.00336-0.00069$

$\begin{array}{llllll}0.00000 & -0.00000 & 0.00000 & 0.00000 & 0.00000 & -0.00000 \\ 0.00000 & -0.00000 & 0.00000 & 0.00000 & 0.00000 & -0.00000 \\ 0.00000 & -0.00000 & 0.00000 & 0.00000 & 0.00000 & -0.00000 \\ 0.00001 & -0.00000 & 0.00001 & 0.00000 & 0.00000 & -0.00000 \\ 0.00001 & -0.00000 & 0.00001 & 0.00000 & 0.00000 & -0.00000 \\ 0.00003 & -0.00000 & 0.00003 & 0.000100 & 0.00001 & -0.00000 \\ 0.00006 & -0.00000 & 0.00005 & 0.00011 & 0.00003 & -0.00001 \\ 0.00008 & -0.00000 & 0.00006 & 0.00002 & 0.00005 & -0.00002 \\ 0.00011 & -0.00000 & 0.00005 & 0.00003 & 0.00008 & -0.00002 \\ 0.00014 & -0.00001 & 0.00004 & 0.00005 & 0.00011 & -0.00002 \\ 0.00016 & -0.00001 & 0.00001 & 0.00007 & 0.00012 & -0.00000 \\ 0.00019 & -0.00002 & -0.00002 & 0.00007 & 0.00013 & 0.00002 \\ & & & & & \\ 0.00002 & -0.00000 & 0.00002 & 0.00000 & 0.00000 & -0.00000 \\ 0.00004 & -0.00000 & 0.00004 & 0.00000 & 0.00002 & -0.00001 \\ 0.00007 & -0.00000 & 0.00005 & 0.00001 & 0.00004 & -0.00001 \\ 0.00009 & -0.00000 & 0.00006 & 0.00002 & 0.00006 & -0.00002 \\ 0.00011 & -0.00000 & 0.00006 & 0.00003 & 0.00008 & -0.00002 \\ 0.00013 & -0.00001 & 0.00004 & 0.00005 & 0.000110 & -0.00002 \\ 0.00015 & -0.00001 & 0.00003 & 0.00006 & 0.00012 & -0.00001 \\ 0.00017 & -0.00001 & 0.00000 & 0.00007 & 0.00013 & 0.00000 \\ 0.00019 & -0.00002 & -0.00002 & 0.00007 & 0.00013 & 0.00002 \\ 0.00021 & -0.00003 & -0.00004 & 0.00007 & 0.00011 & 0.00004\end{array}$

$0.00002-0.00000$ $0.00005-0.00000$ $0.00012-0.00000$ $0.00024-0.00000$ $0.00049-0.00000$ $0.00122-0.00001$ $0.00242-0.00008$ $0.00358-0.00028$ $0.00464-0.00064$ $0.00556-0.00118$ $\begin{array}{ll}0.00627 & -0.00189 \\ 0.00670 & -0.00271\end{array}$ $0.00096-0.00001$ $0.00191-0.00004$ $0.00373-0.00032$ $0.00457-0.00061$ $0.00532-0.00100$ $\begin{array}{ll}0.00532 & -0.00100 \\ 0.00595 & -0.00151\end{array}$

$0.00642-0.00211$

$0.00672-0.00276$

$0.00682-0.00343$

$\begin{array}{ll}0.00000 & -0.00000 \\ 0.00000 & -0.00000 \\ 0.00000 & -0.00000 \\ 0.00001 & -0.00000 \\ 0.00001 & -0.00000 \\ 0.00003 & -0.00000 \\ 0.00006 & -0.00000 \\ 0.00008 & -0.00001 \\ 0.00010 & -0.00001 \\ 0.00012 & -0.00003 \\ 0.00014 & -0.00004 \\ 0.00015 & -0.00005 \\ 0.00002 & -0.00000 \\ 0.00004 & -0.00000 \\ 0.00006 & -0.00000 \\ 0.00008 & -0.00001 \\ 0.00010 & -0.00001 \\ 0.00012 & -0.00002 \\ 0.00013 & -0.0 .0003 \\ 0.00014 & -0.00004 \\ 0.00015 & -0.00006 \\ 0.00015 & -0.00007\end{array}$


$\beta \mathrm{h}$

$\mathrm{C}(\mathrm{h}, 0)$

0.01
0.02
0.05
0.10

0.01
0.02
0.10

0.10

0.20

1.50

1.00

5

3.00
3.50

$0.125 \pi$

$0.250 \pi$
$0.375 \pi$

$0.500 \pi$

$0.625 \pi$

$0.750 \pi$

$1.000 \pi$

1. $125 \pi$

$1.250 \pi$

0.00000

0.00000

0.00000

0.00000

0.00000

$-0.00000$

$-0.00000$
$\mathrm{S}(\mathrm{h}, 0)$

$0.00000 \quad 0.00000$

$0.00000 \quad 0.00000$

$0.00000 \quad 0.00000$

$\begin{array}{ll}0.00000 & 0.00000 \\ 0.00000 & 0.00000\end{array}$

$0.00000 \quad 0.00000$

0.000000 .00000

0.00000

$\begin{array}{ll}-0.00000 & 0.00000 \\ -0.00000 & 0.00000\end{array}$
0.00000

0.00000

0.00000

0.00000

0.00000

0.00000

0.00000

0.00000

0.00000
$E(h, 0)$

$0.00000-0.00000$

$0.00000-0.00000$

$0.00000-0.00000$

$0.00000-0.00000$

$0.00000-0.00000$

$0.00000-0.00000$

$0.00000-0.00000$

$0.00000-0.00000$

$0.00000-0.00000$

$0.00000 \quad 0.00000$

$0.00000 \quad 0.00000$

$0.00000-0.00000$

$0.00000-0.00000$

$0.00000-0.00000$

$0.00000-0.00000$

$0.00000-0.00000$

$0.00000-0.00000$

$0.00000 \quad 0.00000$

$0.00000 \quad 0.00000$

$0.00000 \quad 0.00000$

$0.00000 \quad 0.00000$
$0.00000-0.00000$

$0.00000-0.00000$

$0.00000-0.60000$

$\begin{array}{ll}0.00000 & -0.00000 \\ 0.00000 & -0.00000\end{array}$

$0.00000-0.60000$

$0.00000-0.00000$

$0.00000-0.00000$

$0.00000-0.00000$

$0.00000-0.00000$

$0.00000-0.00000$

$0.00000-0.00000$

$0.00000-0.00000$

$0.00000-0.00000$

$0.00000-0.00000$

$0.00000-0.00000$

$0.00000-0.00000$

$0.00000-0.00000$

$0.00000-0.00000$

$0.00000-0.00000$
$\mathrm{C}(\mathrm{h}, \mathrm{h})$

$\mathrm{S}(\mathrm{h}, \mathrm{h})$

$0.00000 \quad 0.00000$

$0.00000 \quad 0.00000$

$0.00000 \quad 0.00000$

$\begin{array}{ll}0.00000 & 0.00000 \\ 0.00000 & 0.00000\end{array}$

$0.00000 \quad 0.00000$

$0.00000 \quad 0.00000$

$\begin{array}{ll}-0.00000 & 0.00000 \\ -0.00000 & 0.00000\end{array}$

$0.00000 \quad 0.00000$

$0.00000 \quad 0.00000$

$0.00000 \quad 0.00000$

$0.00000 \quad 0.00000$

$0.00000 \quad 0.00000$

$0.00000 \quad 0.00000$

$-0.00000 \quad 0.00000$

$\begin{array}{ll}-0.000000 & 0.00000\end{array}$
$0.00000 \quad 0.00000$

$0.00000 \quad 0.00000$

$\begin{array}{ll}0.00000 & -0.00000 \\ 0.00000 & -0.00000 \\ 0.00000 & -0.00000 \\ 0.00000 & -0.00000 \\ 0.00000 & -0.00000 \\ 0.00000 & -0.00000 \\ 0.00000 & -0.00000 \\ 0.00000 & -0.00000 \\ 0.00000 & -0.00000 \\ 0.00000 & -0.00000 \\ 0.00000 & 0.00000 \\ 0.00000 & 0.00000 \\ & \\ 0.00000 & -0.00000 \\ 0.00000 & -0.00000 \\ 0.00000 & -0.00000 \\ 0.00000 & -0.00000 \\ 0.00000 & -0.00000 \\ 0.00000 & -0.00000 \\ 0.00000 & 0.00000 \\ 0.00000 & 0.00000 \\ 0.00000 & 0.00000 \\ 0.00000 & 0.00000\end{array}$

$$
\frac{a}{\lambda}=3.0 \quad \frac{\alpha}{\beta}=1.00
$$

$\begin{array}{rr}0.00000 & -0.00000 \\ 0.00000 & -0.00000 \\ 0.00000 & -0.00000 \\ 0.00000 & -0.00000 \\ 0.00000 & -0.00000 \\ 0.00000 & -0.00000 \\ 0.00000 & -0.00000 \\ 0.00000 & -0.00000 \\ 0.00000 & 0.00000 \\ 0.00000 & 0.00000 \\ 0.00000 & 0.00000 \\ 0.00000 & 0.00000 \\ 0.00000 & -0.00000 \\ 0.00000 & -0.00000 \\ 0.00000 & -0.00000 \\ 0.00000 & -0.00000 \\ 0.00000 & 0.00000 \\ 0.00000 & 0.00000 \\ 0.00000 & 0.00000 \\ 0.00000 & 0.00000 \\ 0.00000 & 0.00000 \\ 0.00000 & 0.00000\end{array}$

$\begin{array}{rr}0.00000 & -0.00000 \\ 0.00000 & -0.00000 \\ 0.00000 & -0.00000 \\ 0.00000 & -0.00000 \\ 0.00000 & -0.00000 \\ 0.00000 & -0.00000 \\ 0.00000 & -0.00000 \\ 0.00000 & -0.00000 \\ 0.00000 & 0.00000 \\ 0.00000 & 0.00000 \\ 0.00000 & 0.00000 \\ 0.00000 & 0.00000 \\ & \\ 0.00000 & -0.00000 \\ 0.00000 & -0.00000 \\ 0.00000 & -0.00000 \\ 0.00000 & -0.00000 \\ 0.00000 & -0.00000 \\ 0.00000 & 0.00000 \\ 0.00000 & 0.00000 \\ 0.00000 & 0.00000 \\ 0.00000 & 0.00000 \\ -0.00000 & 0.00000\end{array}$

$\begin{array}{llll}0.00000 & -0.00000 & 0.00000 & 0.00000 \\ 0.00000 & -0.00000 & 0.00000 & 0.00000 \\ 0.00000 & -0.00000 & 0.00000 & 0.00000 \\ 0.00000 & -0.00000 & 0.00000 & 0.00000 \\ 0.00000 & -0.00000 & 0.00000 & 0.00000 \\ 0.00000 & -0.00000 & 0.00000 & 0.00000 \\ 0.00000 & -0.00000 & 0.00000 & 0.00000 \\ 0.00000 & -0.00000 & 0.00000 & 0.00000 \\ 0.00000 & -0.00900 & -0.00000 & 0.00000 \\ 0.00000 & -0.00000 & -0.00000 & 0.00000 \\ 0.00000 & -0.00000 & -0.00000 & 0.00000 \\ 0.00000 & -0.00000 & -0.00000 & 0.00000 \\ & & & \\ 0.00000 & -0.00000 & 0.00000 & 0.00000 \\ 0.00000 & -0.00000 & 0.00000 & 0.00000 \\ 0.00000 & -0.00000 & 0.00000 & 0.00000 \\ 0.00000 & -0.00000 & 0.00000 & 0.00000 \\ 0.00000 & -0.00000 & -0.00000 & 0.00000 \\ 0.00000 & -0.00000 & -0.00000 & 0.00000 \\ 0.00000 & -0.00000 & -0.00000 & 0.00000 \\ 0.00000 & -0.00000 & -0.00000 & 0.00000 \\ 0.00000 & -0.00000 & -0.00000 & 0.00000 \\ 0.00000 & -0.00000 & -0.00000 & 0.00000\end{array}$

$-0.00000$
$E(h, h)$

$\begin{array}{ll}0.00000 & -0.00000 \\ 0.00000 & -0.00000 \\ 0.00000 & -0.00000 \\ 0.00000 & -0.00000 \\ 0.00000 & -0.00000 \\ 0.00000 & -0.00000 \\ 0.00000 & -0.00000 \\ 0.00000 & -0.00000 \\ 0.00000 & -0.00000 \\ 0.00000 & -0.00000 \\ 0.00000 & -0.00000 \\ 0.00000 & -0.00000 \\ 0.00000 & -0.00000 \\ 0.00000 & -0.00000 \\ 0.00000 & -0.00000 \\ 0.00000 & -0.00000 \\ 0.00000 & -0.00000 \\ 0.00000 & -0.00000 \\ 0.00000 & -0.00000 \\ 0.00000 & -0.00000 \\ 0.00000 & -0.00000 \\ 0.00000 & -0.00000\end{array}$

$\begin{array}{ll}0.00000 & -0.00000 \\ 0.00000 & -0.00000 \\ 0.00000 & -0.00000 \\ 0.00000 & -0.00000 \\ 0.00000 & -0.00000 \\ 0.00000 & -0.00000 \\ 0.00000 & -0.00000 \\ 0.00000 & -0.00000 \\ 0.00000 & -0.00000 \\ 0.00000 & -0.00000 \\ 0.00000 & -0.00000 \\ 0.00000 & -0.00000 \\ & \\ 0.00000 & -0.00000 \\ 0.00000 & -0.00000 \\ 0.00000 & -0.00000 \\ 0.00000 & -0.00000 \\ 0.00000 & -0.00000 \\ 0.00000 & -0.00000 \\ 0.00000 & -0.00000 \\ 0.00000 & -0.00000 \\ 0.00000 & -0.00000 \\ 0.00000 & -0.00000\end{array}$

$\begin{array}{lrl}0.01 & 0.00000 & 0.00000 \\ 0.02 & 0.00000 & 0.00000 \\ 0.05 & 0.00000 & 0.00000 \\ 0.10 & 0.00000 & 0.00000 \\ 0.20 & 0.00000 & 0.00000 \\ 0.50 & 0.00000 & 0.00000 \\ 1.00 & 0.00000 & 0.00000 \\ 1.50 & 0.00000 & 0.00000 \\ 2.00 & -0.00000 & 0.00000 \\ 2.50 & -0.00000 & 0.00000 \\ 3.00 & -0.00000 & 0.00000 \\ 3.50 & -0.00000 & 0.00000 \\ 0.125 \pi & 0.00000 & 0.00000 \\ 0.250 \pi & 0.00000 & 0.00000 \\ 0.375 \pi & 0.00000 & 0.00000 \\ 0.500 \pi & 0.00000 & 0.00000 \\ 0.625 \pi & -0.00000 & 0.00000 \\ 0.750 \pi & -0.00000 & 0.00000 \\ 0.875 \pi & -0.00000 & 0.00000 \\ 1.0001 \pi & -0.00000 & 0.00000 \\ 1.1251 \pi & -0.00000 & 0.00000 \\ 1.2501 \pi & -0.00000 & 0.00000\end{array}$


$\frac{a}{\lambda}=3 . j \quad \frac{\alpha}{\beta}=0.00$

$E(h, 0)$

$C(h, h)$

$S(h, h)$

$E(h, h)$

$\beta \mathrm{h}$

C (h, 0)

$S(x, 0)$

$\begin{array}{ll}-0.00000 & 0.00000 \\ -0.00002 & 0.00000\end{array}$

$-0.00002 \quad 0.00000$

$-0.00045 \quad 0.00000$

$\begin{array}{lll}-0.08439 & 0.00201\end{array}$

$-0.12842 \quad 0.00508$

$-0.16302 \quad 0.00903$

$\begin{array}{ll}-0.17984 & 0.01184 \\ -0.17520 & 0.01057\end{array}$

$\begin{array}{ll}-0.00692 \quad 0.00001 \\ -0.002663 & 0.00018\end{array}$

$-0.02663 \quad 0.00018$

$\begin{array}{lll}-0.05609 & 0.00085\end{array}$

$-0.09080 \quad 0.00235$

$-0.12540 \quad 0.00481$

$-0.15460 \quad 0.00790$

$\begin{array}{ll}-0.17397 & 0.01074\end{array}$

$\begin{array}{ll}-0.18072 & 0.01202\end{array}$

$-0.17411 \quad 0.01025$

$-0.15565$ $\begin{array}{lll}-0.00181 & 0.00000\end{array}$

$-0.011113 \quad 0.00003$

$-0.04178 \quad 0.00046$

$\begin{array}{ll}-0.00091 & -0.00000 \\ -0.00182 & 0.00000 \\ -0.00455 & 0.00000 \\ -0.00909 & 0.00000 \\ -0.01819 & 0.00001 \\ -0.04547 & 0.00009 \\ -0.09091 & 0.00069 \\ -0.13628 & 0.00232 \\ -0.18149 & 0.00549 \\ -0.22642 & 0.01069 \\ -0.27087 & 0.01840 \\ -0.31456 & 0.02906 \\ & \\ -0.03571 & 0.00004 \\ -0.07141 & 0.00033 \\ -0.10708 & 0.00113 \\ -0.14269 & 0.00266 \\ -0.17820 & 0.00520 \\ -0.21354 & 0.00896 \\ -0.24862 & 0.01419 \\ -0.28334 & 0.02110 \\ -0.31752 & 0.02991 \\ -0.35100 & 0.04080\end{array}$

$-0.00091 \quad 0.00000$ $-0.00182 \quad 0.00000$ $\begin{array}{lll}-0.00455 & 0.000 C 0\end{array}$ $\begin{array}{ll}-0.00908 & 0.00000\end{array}$ $\begin{array}{ll}-0.018 .07 & 0.000 \mathrm{C2}\end{array}$ $-0.04358 \quad 0.00033$ $\begin{array}{ll}-0.07637 & 0.00223 \\ -0.09019 & 0.00555\end{array}$ $\begin{array}{ll}-0.08173 & 0.00777\end{array}$ $\begin{array}{ll}-0.05505 & 0.00510\end{array}$ $-0.01917-0.00498$ $0.01313-0.02095$

$-0.03480 \quad 0.00016$ $\begin{array}{ll}-0.06424 & 0.00117 \\ -0.08376 & 0.00333\end{array}$ $\begin{array}{ll}-0.08376 & 0.00333\end{array}$ $\begin{array}{ll}-0.09036 & 0.00601 \\ -0.08325 & 0.00773\end{array}$ $\begin{array}{ll}-0.08325 & 0.00773\end{array}$ $\begin{array}{ll}-0.06422 & 0.00857\end{array}$ $\begin{array}{ll}-0.03754 & 0.00103\end{array}$ $\begin{array}{ll}-0.00912 & -0.00909\end{array}$ $\begin{array}{ll}0.01492 & -0.02213 \\ 0.03002 & -0.03479\end{array}$
$-0.00000$ 0.00000 $-0.011130 .0000$ $-0.04170 \quad 0.00140$ $0.12603 \quad 0.01636$ $-0.15676 \quad 0.03082$ $\begin{array}{ll}-0.15676 & 0.03082 \\ -0.15868 & 0.04545\end{array}$

$-0.00692 \quad 0.0000$
-0.02660 $-0.02660 \quad 0.00056$ $-0.05591 \quad 0.00260$ $\begin{array}{ll}-0.09004 & 0.00735\end{array}$ $\begin{array}{lll}-0.12321 & 0.01544\end{array}$ $\begin{array}{lll}-0.14969 & 0.02641\end{array}$ $\begin{array}{ll}-0.16502 & 0.0384\end{array}$ $-0.15743 \quad 0.05486$ 0.00000 $-0.00045 \quad 0.00000$ $-0.00181 \quad 0.00000$ $-0.126030 .00625$ $-0.16722 \quad 0.0488$

$-0.00091$ $-0.00455 \quad 0.0000 \mathrm{C}$ $-0.00909 \quad 0.00000$ $-0.01819 \quad 0.00002$ $-0.04545 \quad 0.00034$ $-0.090750 .00275$ $-0.13544 \quad 0.00920$ $\begin{array}{ll}-0.17857 & 0.02153\end{array}$ $-0.21847 \quad 0.04117$ $-0.25271 \quad 0.06880$
-0.278280 .10394 $\begin{array}{ll}-0.27828 & 0.10394\end{array}$

$\begin{array}{ll}-0.03571 & 0.00017\end{array}$ $-0.07135 \quad 0.00133$ $-0.106770 .00448$ $-0.14167 \quad 0.01055$ $-0.17550 \quad 0.02040$ $-0.20745 \quad 0.03472$ $-0.23640 \quad 0.05392$ $-0.26098 \quad 0.07805$
-0.27963 $\begin{array}{ll}-0.27963 & 0.10659 \\ -0.29084 & 0.13840\end{array}$

$$
\frac{a}{\lambda}=3.5 \quad \frac{\alpha}{\beta}=0.005
$$

$\begin{array}{lrr}0.01 & -0.00081 & -0.00000 \\ 0.02 & -0.00163 & 0.00000 \\ 0.05 & -0.00407 & 0.00000 \\ 0.10 & -0.00813 & 0.00000 \\ 0.20 & -0.01619 & 0.00000 \\ 0.50 & -0.03906 & 0.00005 \\ 1.00 & -0.06854 & 0.00032 \\ 1.50 & -0.08123 & 0.00049 \\ 2.00 & -0.07412 & -0.00042 \\ 2.50 & -0.04916 & -0.00373 \\ 3.00 & -0.01281 & -0.01028 \\ 3.50 & 0.02569 & -0.01958 \\ 0.125 \pi & -0.03118 & 0.00003 \\ 0.125 \pi & -0.05760 & 0.00018 \\ 0.250 \pi & -0.0570 \\ 0.375 \pi & -0.07524 & 0.00043 \\ 0.500 \pi & -0.08144 & 0.00046 \\ 0.625 \pi & -0.07529 & -0.00029 \\ 0.750 \pi & -0.05786 & -0.00246 \\ 0.875 \pi & -0.03195 & -0.00657 \\ 1.000 \pi & -0.00169 & -0.01270 \\ 1.125 \pi & 0.02815 & -0.02027 \\ 1.250 \pi & 0.05301 & -0.02797\end{array}$

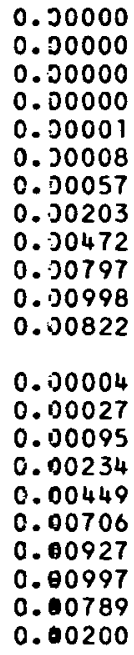

$\begin{array}{llrr}-0.00081 & -0.00000 & -0.00081 & 0.00000 \\ -0.00163 & 0.00000 & -0.00163 & 0.00000 \\ -0.00407 & 0.00000 & -0.00407 & 0.00000 \\ -0.00815 & 0.00000 & -0.00813 & 0.00000 \\ -0.01629 & 0.00000 & -0.01619 & 0.00002 \\ -0.04073 & 0.00008 & -0.03905 & 0.00028 \\ -0.08144 & 0.00062 & -0.06841 & 0.00187 \\ -0.12208 & 0.00208 & -0.08081 & 0.00461 \\ -0.16257 & 0.00492 & -0.07346 & 0.00627 \\ -0.20280 & 0.00957 & -0.04950 & 0.00357 \\ -0.24259 & 0.01648 & -0.01751 & -0.00557 \\ -0.28168 & 0.02601 & 0.01135 & -0.01969 \\ -0.03199 & 0.00004 & -0.03117 & 0.00014 \\ -0.06397 & 0.00030 & -0.05754 & 0.00099 \\ -0.09593 & 0.00101 & -0.07503 & 0.00279 \\ -0.12782 & 0.00239 & -0.08096 & 0.00498 \\ -0.15962 & 0.00465 & -0.07463 & 0.00626 \\ -0.19126 & 0.00802 & -0.05768 & 0.00496 \\ -0.22267 & 0.01270 & -0.03390 & -0.00016 \\ -0.25374 & 0.01889 & -0.00854 & -0.00923 \\ -0.28433 & 0.02677 & 0.01286 & -0.02072 \\ -0.31427 & 0.03651 & 0.02658 & -0.03172\end{array}$

$\begin{array}{ll}-0.00000 & 0.00000 \\ -0.00002 & 0.00000 \\ -0.00010 & 0.00000 \\ -0.00041 & 0.00000 \\ -0.00162 & 0.00001 \\ -0.00997 & 0.00013 \\ -0.03735 & 0.00141 \\ -0.07501 & 0.00583 \\ -0.11283 & 0.01480 \\ -0.14036 & 0.02746 \\ -0.15049 & 0.04009 \\ -0.14245 & 0.04775 \\ -0.00620 & 0.00006 \\ -0.02383 & 0.00060 \\ -0.05007 & 0.00252 \\ -0.08061 & 0.00681 \\ -0.11030 & 0.01399 \\ -0.13401 & 0.02361 \\ -0.14780 & 0.03406 \\ -0.14991 & 0.04296 \\ -0.14136 & 0.04800 \\ -0.12568 & 0.04810\end{array}$

$\begin{array}{ll}-0.00081 & 0.00000 \\ -0.00163 & 0.00000 \\ -0.00407 & 0.00000 \\ -0.00815 & 0.00000 \\ -0.01629 & 0.00002 \\ -0.04072 & 0.00031 \\ -0.08128 & 0.00246 \\ -0.12129 & 0.00824 \\ -0.15988 & 0.01927 \\ -0.19554 & 0.03682 \\ -0.22612 & 0.06149 \\ -0.24892 & 0.09283 \\ -0.03199 & 0.00015 \\ -0.06391 & 0.00119 \\ -0.09563 & 0.00401 \\ -0.12687 & 0.00945 \\ -0.15714 & 0.01826 \\ -0.18570 & 0.03105 \\ -0.21156 & 0.04821 \\ -0.23349 & 0.06974 \\ -0.25012 & 0.09518 \\ -0.26011 & 0.1235 .1\end{array}$


$\frac{a}{\lambda}=3.5 \quad \frac{\alpha}{\beta}=0.01$

$\beta h$

$\mathrm{C}(\mathrm{h}, 0)$

0.01

$\begin{array}{ll}-0.00365 & 0.00000\end{array}$

$\begin{array}{lll}0.10 & -0.00729 & 0.00000\end{array}$

$\begin{array}{lll}0.50 & -0.03499 & 0.00003\end{array}$

$\begin{array}{lll}1.00 & -0.06140 & 0.00018\end{array}$

$\begin{array}{lll}1.50 & -0.07278 & 0.00012\end{array}$

$\begin{array}{lll}2.00 & -0.06643 & -0.00101\end{array}$

$2.50 \quad-0.04412-0.00428$

$\begin{array}{lll}3.00 & -0.01160 & -0.01033\end{array}$

$0.125 \pi$

$0.250 \pi$

$0.375 \pi$

0.625

$0.750 \pi$

$0.875 \pi$

1.000

$1.125 \pi$

$1.250 \pi$

\section{$-0.02793 \quad 0.00002$} $\begin{array}{ll}-0.05160 & 0.00011\end{array}$ $\begin{array}{ll}-0.06741 & 0.00021\end{array}$ $\begin{array}{ll}-0.06748 & 0.00005\end{array}$ $-0.05190-0.00306$ $-0.02872-0.00694$ $-0.00165-0.01251$ $0.02509-0.01919$ $0.04743-0.02581$
$\mathrm{S}(\mathrm{h}, 0)$

$\begin{array}{ll}-0.00000 & 0.00000 \\ -0.00001 & 0.00000 \\ -0.00009 & 0.00000 \\ -0.00036 & 0.00000 \\ -0.00145 & 0.00002 \\ -0.00893 & 0.00011 \\ -0.03353 & 0.00065 \\ -0.06771 & 0.00203 \\ -0.10304 & 0.00437 \\ -0.13085 & 0.00703 \\ -0.14448 & 0.00838 \\ -0.14104 & 0.00624 \\ & \\ -0.00556 & 0.00006 \\ -0.02137 & 0.00034 \\ -0.04501 & 0.00102 \\ -0.07285 & 0.00231 \\ -0.10061 & 0.00418 \\ -0.12407 & 0.00631 \\ -0.13969 & 0.00799 \\ -0.14525 & 0.00822 \\ -0.14019 & 0.00592 \\ -0.12568 & 0.00023\end{array}$

$E(h, 0)$

$\begin{array}{rr}-0.00073 & -(1.00000 \\ -0.00146 & 0.00000 \\ -0.00365 & 0.00000 \\ -0.00730 & 0.00000 \\ -0.01460 & 0.00000 \\ -0.03649 & 0.00007 \\ -0.07296 & 0.00055 \\ -0.10936 & 0.00186 \\ -0.14562 & 0.00440 \\ -0.18164 & 0.00857 \\ -0.21725 & 0.01475 \\ -0.25224 & 0.02329 \\ & \\ -0.02866 & 0.00003 \\ -0.05731 & 0.00027 \\ -0.08593 & 0.00090 \\ -0.11450 & 0.00214 \\ -0.14298 & 0.00417 \\ -0.17131 & 0.00719 \\ -0.19943 & 0.01138 \\ -0.22723 & 0.01691 \\ -0.25460 & 0.02397 \\ -0.28139 & 0.03268\end{array}$

$C(h, h)$

$-0.00073 \quad 0.00000$ $\begin{array}{ll}-0.00146 \quad 0.00060 \\ -0.00365 & 0.00000\end{array}$ $\begin{array}{ll}-0.00365 & 0.00060\end{array}$ $-0.00729 \quad 0.00000$ $\begin{array}{ll}-0.01450 & 0.00002\end{array}$ $\begin{array}{ll}-0.03498 & 0.00023 \\ -0.06129 & 0.00157\end{array}$ $-0.07240 \quad 0.00380$ $-0.06587 \quad 0.00499$ $\begin{array}{lll}-0.04451 & 0.00230\end{array}$ $-0.01598-0.00598$ $0.00982-0.01847$

$\begin{array}{ll}-0.02793 \quad 0.00012 \\ -0.05755 & 0.00083\end{array}$ $\begin{array}{ll}-0.05155 & 0.00083\end{array}$ $\begin{array}{ll}-0.06722 & 0.00233\end{array}$ $\begin{array}{ll}-0.07254 & 0.00410\end{array}$ $\begin{array}{lll}-0.06691 & 0.00500\end{array}$ $-0.05180 \quad 0.00352$
-0.03060 $-0.03060-0.00112$ $\begin{array}{ll}-0.00798 & -0.00924\end{array}$ $0.01126-0.01937$ $0.02355-0.02892$
$S(h, h)$

$-0.00000$ $-0.00001$ $-0.00009$ $-0.00145$ $-0.00893$ $-0.03345$ $-0.06717$ $-0.10101$ $-0.12568$

$-0.12789$

$-0.00555$ $-0.02134$ $-0.04484$ $-0.09875$ $-0.11998$ $-0.13238$ 13440 $-0.12693$ $-0.11310$

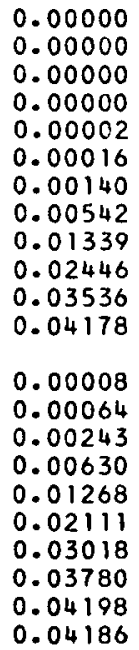
0.00000 0.00000 0.00002 0.00016 0.00140 0.00542 0.01339 0.02446 0.02446 0.04178

0.00008 0.00064 0.00243 0.00630 0.01268 0.02111 0.03018 . 0.04198 0.04186

$E(h, h)$

$-0.00073 \quad 0.00000$ $-0.00146 \quad 0.00000$ $-0.00365 \quad 0.00000$ $-0.01460 \quad 0.00000$ $-0.03648 \quad 0.00028$ $-0.07281 \quad 0.00220$ $-0.10863 \quad 0.00738$ $-0.14315 \quad 0.01725$ $-0.17502 \quad 0.03293$ 0.05496

$-0.02866 \quad 0.00013$ $\begin{array}{ll}-0.05725 & 0.00107\end{array}$ $\begin{array}{ll}-0.08566 & 0.00359\end{array}$ $\begin{array}{lll}-0.11362 & 0.00846\end{array}$ $-0.14069 \quad 0.01634$ $-0.16623 \quad 0.02778$ 0.04310 0.0623 $\begin{array}{ll}-0.22373 & 0.08500 \\ -0.23262 & 0.11021\end{array}$

$$
\frac{a}{\lambda}=3.5 \quad \frac{\alpha}{\beta}=0.02
$$

$\begin{array}{lr}-0.00000 & 0.00000 \\ -0.00001 & 0.00000 \\ -0.00007 & 0.00000 \\ -0.00029 & 0.00001 \\ -0.00117 & 0.00002 \\ -0.00717 & 0.00016 \\ -0.02691 & 0.00074 \\ -0.05433 & 0.00196 \\ -0.08269 & 0.00374 \\ -0.10505 & 0.00547 \\ -0.11611 & 0.00583 \\ -0.11357 & 0.00322 \\ & \\ -0.00446 & 0.00009 \\ -0.01715 & 0.00043 \\ -0.03612 & 0.00110 \\ -0.05846 & 0.00218 \\ -0.08074 & 0.00360 \\ -0.09959 & 0.00504 \\ -0.11219 & 0.00592 \\ -0.11678 & 0.00546 \\ -0.11291 & 0.00290 \\ -0.10149 & -0.00232\end{array}$

(1)
0.00000 $-0.00293 \quad 0.00000$ $-0.011640 .00001$ $-0.02807 \quad 0.00010$ $-0.04918 \quad 0.00108$ $-0.05811 \quad 0.00253$ $\begin{array}{ll}-0.05295 & 0.0030\end{array}$ $\begin{array}{ll}-0.03598 & 0.00042\end{array}$ $-0.01328-0.006 .40$ $0.00735-0.01616$

$\begin{array}{lll}-0.02241 & 0.00008\end{array}$

$-0.04137 \quad 0.00058$ $-0.05394 \quad 0.00159$ $\begin{array}{ll}-0.05823 & 0.0027\end{array}$ $\begin{array}{lll}-0.05378 & 0.00306\end{array}$ $-0.04177 \quad 0.00158$ $-0.02491-0.00246$ $-0.00690-0.00899$ $0.00851-0.01686$ $0.01852-0.02405$
$-0.00000$ $-0.00001$ $-0.00007$ $-0.00029$ $-0.00117$ $-0.00717$ $-0.02683$ $-0.05385$ $-0.08097$ . $-0.10309$

$-0.00446$

$-0.01712$

$-0.03596$

$-0.07915$

$-0.09620$

$-0.10623$

$-0.10804$

$-0.10235$

0.00000 0.00000 0.00000 0.00001 0.00002 0.00020 0.00135 0.00468 0.01096 0.01942 0.02749 0.03195

0.00011 0.00066 0.00222 0.00538 0.01041 0.01689 0.02370 0.02924 0.03207 0.03163
$-0.00050$ $-0.00117$ $-0.00293$ $-0.00586$ $-0.01172$ $-0.02928$ $-0.05842$ $-0.08712$ $-0.11475$ $-0.14022$ $-0.16199$ $-0.17815$

$-0.02300$ $-0.04594$ $-0.06872$ $-0.09112$

$-0.11279$

-0.112720
-0.15163

$-0.16722$

-0.17900
-0.18605
0.00000 0.00000 0.00000 . 0.00001 0.00177 0.00591 0.01381 0.02634 0.06611

0.00011 0.00086 0.00288 0.00678 0.01308 0.02223 0.03446 0.04976 0.06778 0.08777 


\begin{tabular}{|c|c|c|}
\hline $\begin{array}{l}0.01 \\
0.02 \\
0.05 \\
0.10 \\
0.20 \\
0.50 \\
1.00 \\
1.50 \\
2.00 \\
2.50 \\
3.00 \\
3.50\end{array}$ & $\begin{array}{r}-0.00047 \\
-0.00094 \\
-0.00235 \\
-0.00469 \\
-0.00934 \\
-0.02254 \\
-0.03955 \\
-0.04690 \\
-0.04287 \\
-0.02859 \\
-0.00773 \\
0.01451\end{array}$ & $\begin{array}{l}-0.00000 \\
-0.00000 \\
-0.00000 \\
-0.00000 \\
-0.00000 \\
-0.00002 \\
-0.00017 \\
-0.00076 \\
-0.00228 \\
-0.00518 \\
-0.00954 \\
-0.01470\end{array}$ \\
\hline $\begin{array}{l}0.125 \pi \\
0.250 \pi \\
0.375 \pi \\
0.500 \pi \\
0.625 \pi \\
0.750 \pi \\
0.875 \pi \\
1.000 \pi \\
1.125 \pi \\
1.250 \pi\end{array}$ & $\begin{array}{r}-0.01799 \\
-0.03324 \\
-0.04343 \\
-0.04703 \\
-0.04354 \\
-0.03358 \\
-0.01873 \\
-0.00133 \\
0.01594 \\
0.03055\end{array}$ & $\begin{array}{l}-0.00001 \\
-0.00007 \\
-0.00031 \\
-0.00091 \\
-0.00213 \\
-0.00419 \\
-0.00719 \\
-0.01097 \\
-0.01505 \\
-0.01860\end{array}$ \\
\hline
\end{tabular}

$\begin{array}{lll}0.01 & -0.00030 & -0.00000 \\ 0.02 & -0.00061 & -0.00000 \\ 0.05 & -0.00151 & -0.00000 \\ 0.10 & -0.00302 & -0.00000 \\ 0.20 & -0.00602 & -0.00000 \\ 0.50 & -0.01452 & -0.00003 \\ 1.00 & -0.02548 & -0.00029 \\ 1.50 & -0.03023 & -0.00103 \\ 2.00 & -0.02767 & -0.00252 \\ 2.50 & -0.01851 & -0.00490 \\ 3.00 & -0.00509 & -0.00802 \\ 3.50 & 0.00931 & -0.01124 \\ 0.125 \pi & -0.01159 & -0.00002 \\ 0.250 \pi & -0.02141 & -0.00014 \\ 0.375 \pi & -0.02798 & -0.00048 \\ 0.500 \pi & -0.03032 & -0.00119 \\ 0.625 \pi & -0.02810 & -0.00238 \\ 0.750 \pi & -0.02171 & -0.00412 \\ 0.875 \pi & -0.01217 & -0.00639 \\ 1.000 \pi & -0.00096 & -0.00896 \\ 1.125 \pi & 0.01025 & -0.01145 \\ 1.250 \pi & 0.01986 & -0.01329\end{array}$

$S\left(h_{1}, C_{1}\right)$

0.00000 0.00000 $-0.00023 \quad 0.00001$ $-0.00094 \quad 0.00003$ $\begin{array}{ll}-0.00575 & 0.00018\end{array}$ $\begin{array}{lll}-0.02159 & 0.00078\end{array}$ $\begin{array}{ll}-0.04360 & 0.00184\end{array}$ $\begin{array}{ll}-0.06637 & 0.00320\end{array}$

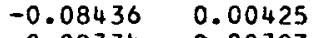
$\begin{array}{ll}-0.09334 & 0.00397 \\ -0.09147 & 0.00116\end{array}$

$\begin{array}{ll}-0.00358 & 0.00011\end{array}$ $\begin{array}{lll}-0.01376 & 0.00046\end{array}$ $\begin{array}{lll}-0.02899 & 0.00110\end{array}$ $\begin{array}{lll}-0.04691 & 0.00202\end{array}$ $\begin{array}{lll}-0.06481 & 0.00310\end{array}$ $\begin{array}{ll}-0.07996 & 0.00403\end{array}$ $-0.09013 \quad 0.00435$ $\begin{array}{ll}-0.09392 & 0.00348\end{array}$ $\begin{array}{lr}-0.09095 & 0.00085 \\ -0.08196 & -0.00386\end{array}$
$E(h, 0)$

$-0.00047-0.00000$ $-0.00094 \quad 0.00000$ $-0.00235 \quad 0.00000$
-0.00470 $-0.00940 \quad 0.00000$ $\begin{array}{ll}-0.02351 & 0.00004\end{array}$ $\begin{array}{lll}-0.04699 & 0.00036\end{array}$ $-0.07042 \quad 0.00120$ $\begin{array}{lll}-0.09374 & 0.00283\end{array}$ $\begin{array}{ll}-0.11689 & 0.00551 \\ -0.13975 & 0.00948\end{array}$ $\begin{array}{ll}-0.13975 & 0.00948 \\ -0.16218 & 0.01495\end{array}$

$\begin{array}{ll}-0.01846 \quad 0.00002 \\ -0.03691 & 0.00017\end{array}$ $\begin{array}{ll}-0.03691 & 0.00017\end{array}$ $\begin{array}{ll}-0.05534 & 0.00058 \\ -0.07373 & 0.00138\end{array}$ $\begin{array}{ll}-0.07373 & 0.00138\end{array}$ $\begin{array}{ll}-0.09205 & 0.00268\end{array}$ $-0.11026 \quad 0.00462$ $\begin{array}{ll}-0.12832 & 0.00731\end{array}$ $-0.14616 \quad 0.01087$ $\begin{array}{ll}-0.14616 & 0.01087 \\ -0.13084 & 0.01539\end{array}$
$C(h, h)$

$\begin{array}{lr}-0.00047 & 0.00000 \\ -0.00094 & 0.00000 \\ -0.00235 & 0.00000 \\ -0.00469 & 0.00000 \\ -0.00934 & 0.00001 \\ -0.02253 & 0.00011 \\ -0.03947 & 0.00073 \\ -0.04665 & 0.00162 \\ -0.04257 & 0.00162 \\ -0.02907 & -0.00081 \\ -0.01099 & -0.00642 \\ 0.00552 & -0.01406 \\ -0.01799 & 0.00006 \\ -0.03320 & 0.00039 \\ -0.04329 & 0.00105 \\ -0.04675 & 0.00171 \\ -0.04323 & 0.00168 \\ -0.03368 & 0.00021 \\ -0.02027 & -0.00322 \\ -0.00590 & -0.00848 \\ 0.00646 & -0.01459 \\ 0.01462 & -0.01999\end{array}$

$S(h, h)$ $-0.00001$ $-0.00006$ $-0.00023$ $-0.00094$ 0.00575 $-0.02152$ 0.04318 -0.08704
-0.08311

\section{$-0.00358$} $-0.01374$ $-0.02884$ $-0.04640$ $-0.06346$ $-0.07714$ $-0.08526$ $-0.08687$ $-0.08254$ $-0.07414$
0.00000
0.00000
0.00000
0.00001
0.00003
0.00021
0.00126
0.00401
0.00897
0.01542
0.02136
0.02439
0.00012
0.00066
0.00200
0.00458
0.00854
0.01351
0.01860
0.02260
0.02445
0.02381
$E(h, h)$

$\begin{array}{ll}-0.00047 & 0.00000 \\ -0.00094 & 0.00000 \\ -0.00235 & 0.00000 \\ -0.00470 & 0.00000 \\ -0.00940 & 0.00001 \\ -0.02349 & 0.00018 \\ -0.04687 & 0.00142 \\ -0.06988 & 0.00474 \\ -0.09199 & 0.01106 \\ -0.11234 & 0.02107 \\ -0.12969 & 0.03507 \\ -0.14254 & 0.05273 \\ -0.01846 & 0.00009 \\ -0.03686 & 0.00069 \\ -0.05513 & 0.00231 \\ -0.07308 & 0.00543 \\ -0.09042 & 0.01048 \\ -0.10673 & 0.01779 \\ -0.12144 & 0.02754 \\ -0.13385 & 0.03973 \\ -0.14322 & 0.05405 \\ -0.14881 & 0.06990\end{array}$

$\begin{array}{llll}-0.00000 & 0.00000 & -0.00030 & -0.00000 \\ -0.00001 & 0.00000, & -0.00061 & 0.00000 \\ -0.00004 & 0.00000 & -0.00151 & 0.00000 \\ -0.00015 & 0.00001 & -0.00303 & 0.00000 \\ -0.00060 & 0.00003 & -0.00606 & 0.00000 \\ -0.00371 & 0.00019 & -0.01514 & 0.00003 \\ -0.01391 & 0.00073 & -0.03026 & 0.00023 \\ -0.02809 & 0.00153 & -0.04534 & 0.00077 \\ -0.04278 & 0.00230 & -0.06035 & 0.00182 \\ -0.05444 & 0.00256 & -0.07523 & 0.00355 \\ -0.06036 & 0.00164 & -0.08990 & 0.00609 \\ -0.05938 & -0.00109 & -0.10428 & 0.00960 \\ & & & \\ -0.00231 & 0.00012 & -0.01189 & 0.00001 \\ -0.00887 & 0.00046 & -0.02378 & 0.00011 \\ -0.01867 & 0.00100 & -0.03564 & 0.00037 \\ -0.03023 & 0.00165 & -0.04747 & 0.00089 \\ -0.04177 & 0.00226 & -0.05926 & 0.00173 \\ -0.05157 & 0.00258 & -0.07096 & 0.00297 \\ -0.05822 & 0.00230 & -0.08256 & 0.00470 \\ -0.06080 & 0.00108 & -0.09401 & 0.00698 \\ -0.05906 & -0.00135 & -0.10525 & 0.00988 \\ -0.05346 & -0.00507 & -0.11623 & 0.01345\end{array}$

$\begin{array}{ll}-0.00030 & 0.00000 \\ -0.00061 & 0.00000 \\ -0.00151 & 0.00000 \\ -0.00302 & 0.00000 \\ -0.00602 & 0.00000 \\ -0.01451 & 0.00005 \\ -0.02542 & 0.00029 \\ -0.03007 & 0.00051 \\ -0.02752 & 0.00002 \\ -0.01897 & -0.00200 \\ -0.00747 & -0.00579 \\ 0.00315 & -0.01048 \\ -0.01159 & 0.00002 \\ -0.02138 & 0.00016 \\ -0.02789 & 0.00039 \\ -0.03014 & 0.00050 \\ -0.02793 & 0.00010 \\ -0.02189 & -0.00123 \\ -0.01337 & -0.00369 \\ -0.00421 & -0.00709 \\ 0.00376 & -0.01080 \\ 0.00919 & -0.01382\end{array}$

$\begin{array}{ll}-0.00000 & 0.00000 \\ -0.00001 & 0.00000 \\ -0.00004 & 0.00000 \\ -0.00015 & 0.00001 \\ -0.00060 & 0.00003 \\ -0.00370 & 0.00021 \\ -0.01385 & 0.00104 \\ -0.02778 & 0.00292 \\ -0.04175 & 0.00600 \\ -0.05205 & 0.00973 \\ -0.05623 & 0.01288 \\ -0.05404 & 0.01411 \\ -0.00230 & 0.00012 \\ -0.00884 & 0.00058 \\ -0.01856 & 0.00157 \\ -0.02984 & 0.00329 \\ -0.04081 & 0.00574 \\ -0.04964 & 0.00866 \\ -0.05497 & 0.01146 \\ -0.05621 & 0.01346 \\ -0.05369 & 0.01411 \\ -0.04857 & 0.01333\end{array}$

$\begin{array}{ll}-0.00030 & 0.00000 \\ -0.00061 & 0.00000 \\ -0.00151 & 0.00000 \\ -0.00303 & 0.00000 \\ -0.00606 & 0.00001 \\ -0.01513 & 0.00011 \\ -0.03017 & 0.00091 \\ -0.04495 & 0.00305 \\ -0.05912 & 0.00709 \\ -0.07210 & 0.01348 \\ -0.08313 & 0.02237 \\ -0.09126 & 0.03354 \\ -0.01189 & 0.00006 \\ -0.02374 & 0.00044 \\ -0.03548 & 0.00149 \\ -0.04700 & 0.00349 \\ -0.05811 & 0.00672 \\ -0.06853 & 0.01139 \\ -0.07790 & 0.01760 \\ -0.08577 & 0.02533 \\ -0.09168 & 0.03437 \\ -0.09520 & 0.04433\end{array}$


$S(h, 0)$

$\begin{array}{rr}-0.00000 & 0.00000 \\ -0.00000 & 0.00000 \\ -0.00002 & 0.00000 \\ -0.00010 & 0.00001 \\ -0.00039 & 0.00003 \\ -0.00239 & 0.00017 \\ -0.00896 & 0.00062 \\ -0.01810 & 0.00120 \\ -0.02759 & 0.00164 \\ -0.03516 & 0.00154 \\ -0.03908 & 0.00048 \\ -0.03859 & -0.00188 \\ & \\ -0.00148 & 0.00010 \\ -0.00571 & 0.00040 \\ -0.01203 & 0.00082 \\ -0.01948 & 0.00128 \\ -0.02694 & 0.00162 \\ -0.03330 & 0.00165 \\ -0.03764 & 0.00116 \\ -0.03940 & -0.00005 \\ -0.03839 & -0.00209 \\ -0.03488 & -0.00494\end{array}$

$\frac{\mathrm{a}}{\lambda}=3.5 \quad \frac{\alpha}{\beta}=0.07$

$E(h, 0)$

$C(h, h)$

$S(h, h)$

$E(h, h)$
$-0.00020 \quad 0.00000$ $-0.00039 \quad 0.00000$ $-0.00098 \quad 0.00000$ $-0.00195 \quad 0.00000$ $-0.00935 \quad 0.0000$ $\begin{array}{lll}-0.01638 & 0.00007\end{array}$ $-0.01938-0.00002$ $-0.01778-0.00065$ $-0.01236-0.00224$ $\begin{array}{rr}-0.00503 & -0.00480 \\ 0.00183 & -0.00769\end{array}$

$\begin{array}{ll}-0.00746 \quad 0.00001 \\ -0.01377 & 0.00004\end{array}$ $\begin{array}{ll}-0.01377 & 0.00004\end{array}$ $\begin{array}{ll}-0.01797 & 0.00007\end{array}$ $-0.01943-0.00006$ $-0.01805-0.00057$ $-0.01422-0.00167$ $-0.00880-0.0034$ $-0.00294-0.00563$ $\begin{array}{ll}0.00223 & -0.00788 \\ 0.00587 & -0.00955\end{array}$
$-0.00000$ $-0.00000$ $-0.00002$ $-0.00010$ $-0.00039$ $-0.00239$ $-0.00892$ $-0.01787$ $-0.02687$ $-0.03354$ $-0.03636$ $-0.03516$

$-0.00148$ $-0.00569$ $-0.01195$ ( $-0.02626$ $-0.03547$ $-0.03640$ $-0.03495$ $-0.0318$
0.00000 0.00000 0.00000 0.00001
0.00003 0.00018 0.00082 0.00210 0.00401 0.00615 0.00774 0.00808

0.00011 0.00048 0.00119 0.00233 0.00386 0.00555 0.00706 0.00798 0.00805 0.00730

$\begin{array}{ll}-0.00020 & 0.00000 \\ -0.00039 & 0.00000 \\ -0.00098 & 0.00000 \\ -0.00195 & 0.00000 \\ -0.00390 & 0.00000 \\ -0.00975 & 0.00007 \\ -0.01943 & 0.00059 \\ -0.02892 & 0.00196 \\ -0.03799 & 0.00455 \\ -0.04628 & 0.00863 \\ -0.05329 & 0.01428 \\ -0.05843 & 0.02133 \\ & \\ -0.00766 & 0.00004 \\ -0.01528 & 0.00029 \\ -0.02284 & 0.00096 \\ -0.03023 & 0.00224 \\ -0.03735 & 0.00431 \\ -0.04400 & 0.00729 \\ -0.04996 & 0.01125 \\ -0.05496 & 0.01615 \\ -0.05869 & 0.02186 \\ -0.06090 & 0.02812\end{array}$

$\begin{array}{lrlllll}0.01 & -0.00010 & -0.00000 & -0.00000 & 0.00000 & -0.00010 & -0.00000 \\ 0.02 & -0.00020 & -0.00000 & -0.00000 & 0.00000 & -0.00020 & 0.00000 \\ 0.05 & -0.00050 & -0.00000 & -0.00001 & 0.00000 & -0.00050 & 0.00000 \\ 0.10 & -0.00101 & -0.00000 & -0.00005 & 0.00001 & -0.00101 & 0.00000 \\ 0.20 & -0.00200 & -0.00000 & -0.00020 & 0.00002 & -0.00202 & 0.00000 \\ 0.50 & -0.00484 & -0.00003 & -0.00123 & 0.00012 & -0.00504 & 0.00001 \\ 1.00 & -0.00849 & -0.00025 & -0.00464 & 0.00044 & -0.01007 & 0.00008 \\ 1.50 & -0.01009 & -0.00079 & -0.00938 & 0.00079 & -0.01509 & 0.00026 \\ 2.00 & -0.00926 & -0.00171 & -0.01431 & 0.00097 & -0.02007 & 0.00061 \\ 2.50 & -0.00622 & -0.00294 & -0.01829 & 0.00072 & -0.02499 & 0.00118 \\ 3.00 & -0.00170 & -0.00424 & -0.02040 & -0.00020 & -0.02984 & 0.00202 \\ 3.50 & 0.00325 & -0.00526 & -0.02025 & -0.00189 & -0.03457 & 0.00317 \\ 0.125 \pi & -0.00386 & -0.00002 & -0.00077 & 0.00008 & -0.00396 & 0.00000 \\ 0.250 \pi & -0.00714 & -0.00012 & -0.00295 & 0.00028 & -0.00792 & 0.00004 \\ 0.375 \pi & -0.00933 & -0.00040 & -0.00623 & 0.00057 & -0.01186 & 0.00012 \\ 0.500 \pi & -0.01012 & -0.00090 & -0.01009 & 0.00084 & -0.01580 & 0.00029 \\ 0.625 \pi & -0.00940 & -0.00163 & -0.01397 & 0.00097 & -0.01971 & 0.00057 \\ 0.750 \pi & -0.00729 & -0.00257 & -0.01730 & 0.00085 & -0.02358 & 0.00099 \\ 0.875 \pi & -0.00410 & -0.00360 & -0.01961 & 0.00035 & -0.02742 & 0.00156 \\ 1.000 \pi & -0.00030 & -0.00458 & -0.02060 & -0.00060 & -0.03119 & 0.00231 \\ 1.125 \pi & 0.00358 & -0.00531 & -0.02016 & -0.00203 & -0.03489 & 0.00326 \\ 1.250 \pi & 0.00701 & -0.00558 & -0.01840 & -0.00386 & -0.03849 & 0.00443\end{array}$
$\frac{\alpha}{\beta}=0.10$

$-0.00010 \quad 0.00000$ $-0.00020 \quad 0.00000$ $\begin{array}{ll}-0.00050 & -0.00000 \\ -0.00101 & -0.00000\end{array}$ $-0.00200-0.00000$ $-0.00483-0.00000$ $-0.00847-0.00006$ $-0.01003-0.00027$ $-0.00924-0.00084$ $-0.00649-0.00189$ $-0.00273-0.00332$ $0.00086-0.00432$

$-0.00386-0.00000$ $-0.00712-0.00002$ $-0.00929-0.00011$ $-0.01006-0.00033$ $-0.00938-0.00079$ $-0.00743-0.00154$ $-0.00467-0.00257$ $-0.00164-0.00374$ $-0.00108-0.00481$ $0.00307-0.00547$

$\begin{array}{llll}-0.00000 & 0.00000 & -0.00010 & 0.00000 \\ -0.00000 & 0.00000 & -0.00020 & 0.00000 \\ -0.00001 & 0.00000 & -0.00050 & 0.00000 \\ -0.00005 & 0.00001 & -0.00101 & 0.00000 \\ -0.00020 & 0.00002 & -0.00202 & 0.00000 \\ -0.00123 & 0.00013 & -0.00504 & 0.00004 \\ -0.00461 & 0.00054 & -0.01003 & 0.00030 \\ -0.00923 & 0.00125 & -0.01492 & 0.00101 \\ -0.01389 & 0.00219 & -0.01957 & 0.00234 \\ -0.01738 & 0.00309 & -0.02380 & 0.00442 \\ -0.01894 & 0.00358 & -0.02735 & 0.00728 \\ -0.01847 & 0.00340 & -0.02993 & 0.01082 \\ -0.00077 & 0.00008 & -0.00396 & 0.00002 \\ -0.00294 & 0.00033 & -0.00790 & 0.00015 \\ -0.00617 & 0.00076 & -0.01179 & 0.00049 \\ -0.00992 & 0.00138 & -0.01560 & 0.00115 \\ -0.01357 & 0.00212 & -0.01924 & 0.00222 \\ -0.01655 & 0.00285 & -0.02264 & 0.00374 \\ -0.01842 & 0.00341 & -0.02567 & 0.00575 \\ -0.01900 & 0.00360 & -0.02819 & 0.00822 \\ -0.01837 & 0.00337 & -0.03007 & 0.01109 \\ -0.01684 & 0.00278 & -0.03117 & 0.01420\end{array}$


$C(h, 0)$

$S(h, 0)$ $\frac{a}{\lambda}=3.5 \quad \frac{\alpha}{\beta}=0.20$

$E(h, 0)$

$\mathrm{C}(\mathrm{h}, \mathrm{h})$

$\mathrm{S}(\mathrm{h}, \mathrm{h})$

$E(h, h)$
$0.01-0.00001-0.00000$ $0.02-0.00002-0.00000$ $-0.00006-0.00000$ $0.10-0.00011-0.00000$ $0.20-0.00022-0.00000$ $0.50-0.01054-0.00001$ $-0.00113-0.00006$ $-0.00104-0.00039$ $-0.00104-0.00039$ $3.00-0.05069-0.00062$ $\begin{array}{rrr}3.50 & 0.00047 & -0.00094\end{array}$

$0.125 \pi \quad-0.00043-0.00000$ $0.250 \pi \quad-0.00079-0.00003$ $0.375 \pi \quad-0.00104 \quad-0.00010$ $0.500 \pi \quad-0.00113 \quad-0.00021$ $\begin{array}{lll}0.625 \pi & -0.00105 & -0.00037\end{array}$ $\begin{array}{llll}0.750 \pi & -0.00081 & -0.00055\end{array}$ $0.875 \pi \quad-0.00044 \quad-0.00074$ $\begin{array}{lll}1.000 \pi & 0.00002 & -0.00088\end{array}$ $\begin{array}{lll}1.125 \pi & 0.00052 & -0.00094 \\ 1.250 \pi & 0.00099 & -0.00090\end{array}$

$\begin{array}{ll}-0.00001 & -0.00000 \\ -0.00002 & 0.00000 \\ -0.00006 & 0.00000 \\ -0.000111 & 0.00000 \\ -0.00022 & 0.00000 \\ -0.00056 & 0.00000 \\ -0.00112 & 0.00001 \\ -0.00167 & 0.00003 \\ -0.00222 & 0.00007 \\ -0.00276 & 0.00013 \\ -0.00329 & 0.00022 \\ -0.00380 & 0.00035 \\ -0.00044 & 0.00000 \\ -0.00088 & 0.00000 \\ -0.00131 & 0.00001 \\ -0.00175 & 0.00003 \\ -0.00218 & 0.00006 \\ -0.00260 & 0.00011 \\ -0.00302 & 0.00017 \\ -0.00343 & 0.00025 \\ -0.00383 & 0.00036 \\ -0.00422 & 0.00048\end{array}$

$\begin{array}{ll}-0.00001 & -1.000000 \\ -0.00002 & -10.00000 \\ -0.00006 & -0.00000 \\ -0.00011 & -0.00000 \\ -0.00022 & -0.00010 \\ -0.00054 & -0.00001 \\ -0.00094 & -0.00004 \\ -0.00112 & -0.00013 \\ -0.00104 & -0.00028 \\ -0.00074 & -0.00048 \\ -0.00032 & -0.00069 \\ 0.00012 & -0.00082 \\ & \\ -0.00043 & -0.00001 \\ -0.00079 & -0.00002 \\ -0.00103 & -0.00005 \\ -0.00113 & -0.00015 \\ -0.00106 & -0.00027 \\ -0.00085 & -0.00042 \\ -0.00054 & -0.00059 \\ -0.00019 & -0.00074 \\ 0.00015 & -0.00083 \\ 0.00043 & -0.00083\end{array}$
$-0.00000$ $-0.00000$ $-0.00001$ $-0.00002$ $-0.00014$ $-0.00051$ $-0.00103$ $-0.00156$ $-0.00197$ $-0.00218$

$-0.00009$ $-0.00033$ $-0.00069$ $-0.00111$ $-0.00152$ $-0.00187$ 0.00211 $-0.00221$ $-0.00202$
0.00000 0.00000 0.00000 0.00000 0.0000 0.00003 0.00010 0.00020 0.00029 0.00031 0.00025 0.00002 .00007 0.00014 0.00022 0.00028 0.00031 0.00029 0.00021 0.00008
-0.00007
$-0.00001$ $-0.00002$ $-0.00006$ $-0.00011$ $-0.00022$ $-0.00056$ $-0.00111$ 0.00215 0.00259 $-0.00322$ $-0.00044$ $-0.00087$ $-0.00130$ $-0.00172$ $-0.00211$ $-0.00247$ $-0.00279$ $-0.00305$

$-0.00324$

$-0.00334$
0.00000 0.00000 0.00000 0.00000 0.00000 0.00000 0.00011 0.00025 0.00047 0.000113 0.00000 0.00002 0.00005 0.00013 0.00024 0.00040 0.00061 0.00087 0.00115
0.00146

$\begin{array}{lll}0.01 & -0.00000 & -0.00000 \\ 0.02 & -0.00000 & -0.00000 \\ 0.05 & -0.00000 & -0.00000 \\ 0.10 & -0.00000 & -0.00000 \\ 0.20 & -0.00000 & -0.00000 \\ 0.50 & -0.00001 & -0.00000 \\ 1.00 & -0.00001 & -0.00000 \\ 1.50 & -0.00001 & -0.00000 \\ 2.00 & -0.00001 & -0.00001 \\ 2.50 & -0.00001 & -0.00002 \\ 3.00 & 0.00000 & -0.00002 \\ 3.50 & 0.00001 & -0.00002 \\ 0.125 \pi & -0.00001 & -0.00000 \\ 0.250 \pi & -0.00001 & -0.00000 \\ 0.375 \pi & -0.00001 & -0.00000 \\ 0.500 \pi & -0.00001 & -0.00001 \\ 0.625 \pi & -0.00001 & -0.00001 \\ 0.750 \pi & -0.00001 & -0.00001 \\ 0.875 \pi & -0.00000 & -0.00002 \\ 1.000 \pi & 0.00000 & -0.00002 \\ 1.1 .25 \pi & 0.00001 & -0.00002 \\ 1.250 \pi & .0 .00002 & -0.00002\end{array}$

$\begin{array}{rr}-0.00000 & 0.00000 \\ -0.00000 & 0.00000 \\ -0.00000 & 0.00000 \\ -0.00000 & 0.00000 \\ -0.00000 & 0.00000 \\ -0.00000 & 0.00000 \\ -0.00001 & 0.00000 \\ -0.00001 & 0.00000 \\ -0.00002 & 0.00000 \\ -0.00003 & -0.00000 \\ -0.00003 & -0.00001 \\ -0.00004 & -0.00002 \\ & \\ -0.00000 & 0.00000 \\ -0.00000 & 0.00000 \\ -0.00001 & 0.00000 \\ -0.00001 & 0.00000 \\ -0.00002 & 0.00000 \\ -0.00003 & 0.00000 \\ -0.00003 & -0.00000 \\ -0.00004 & -0.00001 \\ -0.00004 & -0.00002 \\ -0.00003 & -0.00003\end{array}$

$$
\frac{\mathrm{a}}{\lambda}=3.5 \quad \frac{\alpha}{\beta}=0.40
$$

$\begin{array}{lr}-0.00000 & -0.00000 \\ -0.00000 & 0.00000 \\ -0.00000 & 0.00000 \\ -0.00000 & 0.00000 \\ -0.00000 & 0.00000 \\ -0.00001 & 0.00000 \\ -0.00001 & 0.00000 \\ -0.00002 & 0.00000 \\ -0.00003 & 0.00000 \\ -0.00003 & 0.00000 \\ -0.00004 & 0.00000 \\ -0.00005 & 0.00000 \\ -0.00001 & 0.00000 \\ -0.00001 & 0.00000 \\ -0.00002 & 0.00000 \\ -0.00002 & 0.00000 \\ -0.00003 & 0.00000 \\ -0.00003 & 0.000 .00 \\ -0.00004 & 0.00000 \\ -0.00004 & 0.00000 \\ -0.00005 & 0.00000 \\ -0.00005 & 0.00001\end{array}$

$\begin{array}{ll}-0.00000 & -0.00000 \\ -0.00000 & -0.00000 \\ -0.00000 & -0.00000 \\ -0.00000 & -0.00000 \\ -0.00000 & -0.00000 \\ -0.00001 & -0.00000 \\ -0.00001 & -0.00000 \\ -0.00001 & -0.00000 \\ -0.00001 & -0.00001 \\ -0.00001 & -0.00001 \\ -0.00000 & -0.00002 \\ 0.00001 & -0.00002 \\ -0.00001 & -0.00000 \\ -0.00001 & -0.00000 \\ -0.00001 & -0.00000 \\ -0.00001 & -0.00000 \\ -0.00001 & -0.00001 \\ -0.00001 & -1.00001 \\ -0.00001 & -0.00002 \\ -0.00000 & -0.00002 \\ 0.00001 & -0.00002 \\ 0.00001 & -0.00002\end{array}$

$\begin{array}{rrrr}-0.00000 & 0.00000 & -0.00000 & 0.00000 \\ -0.00000 & 0.00000 & -0.00000 & 0.00000 \\ -0.00000 & 0.00000 & -0.00000 & 0.00000 \\ -0.00000 & 0.00000 & -0.00000 & 0.00000 \\ -0.00000 & 0.00000 & -0.00000 & 0.00000 \\ -0.00000 & 0.00000 & -0.00001 & 0.00000 \\ -0.00001 & 0.00000 & -0.00001 & 0.00000 \\ -0.00001 & 0.00000 & -0.00002 & 0.00000 \\ -0.00002 & 0.00000 & -0.00003 & 0.00000 \\ -0.00003 & 0.00000 & -0.00003 & 0.00001 \\ -0.00003 & -0.00000 & -0.00003 & 0.00001 \\ -0.00003 & -0.00001 & -0.00004 & 0.00001 \\ -0.00000 & 0.00000 & -0.00001 & 0.00000 \\ -0.00000 & 0.00000 & -0.00001 & 0.00000 \\ -0.00001 & 0.00000 & -0.00002 & 0.00000 \\ -0.00001 & 0.00000 & -0.00002 & 0.00000 \\ -0.00002 & 0.00000 & -0.00003 & 0.00000 \\ -0.00003 & 0.00000 & -0.00003 & 0.00000 \\ -0.00003 & 0.00000 & -0.00003 & 0.00001 \\ -0.00003 & -0.00000 & -0.00004 & 0.00001 \\ -0.00003 & -0.00001 & -0.00004 & 0.00001 \\ -0.00003 & -0.00001 & -0.00004 & 0.00002\end{array}$

$\begin{array}{rrrr}-0.00000 & 0.00000 & -0.00000 & 0.00000 \\ -0.00000 & 0.00000 & -0.00000 & 0.00000 \\ -0.00000 & 0.00000 & -0.00000 & 0.00000 \\ -0.00000 & 0.00000 & -0.00000 & 0.00000 \\ -0.00000 & 0.00000 & -0.00000 & 0.00000 \\ -0.00000 & 0.00000 & -0.00001 & 0.00000 \\ -0.00001 & 0.00000 & -0.00001 & 0.00000 \\ -0.00001 & 0.00000 & -0.00002 & 0.00000 \\ -0.00002 & 0.00000 & -0.00003 & 0.00000 \\ -0.00003 & 0.00000 & -0.00003 & 0.00001 \\ -0.00003 & -0.00000 & -0.00003 & 0.00001 \\ -0.00003 & -0.00001 & -0.00004 & 0.00001 \\ -0.00000 & 0.00000 & -0.00001 & 0.00000 \\ -0.00000 & 0.00000 & -0.00001 & 0.00000 \\ -0.00001 & 0.00000 & -0.00002 & 0.00000 \\ -0.00001 & 0.00000 & -0.00002 & 0.00000 \\ -0.00002 & 0.00000 & -0.00003 & 0.00000 \\ -0.00003 & 0.00000 & -0.00003 & 0.00000 \\ -0.00003 & 0.00000 & -0.00003 & 0.00001 \\ -0.00003 & -0.00000 & -0.00004 & 0.00001 \\ -0.00003 & -0.00001 & -0.00004 & 0.00001 \\ -0.00003 & -0.00001 & -0.00004 & 0.00002\end{array}$

0.00000 0.00000 0.00000
0.00000 0.00000 0.00000 0.00000 0.00000 0.00000 0.00001 0.00001 0.00000 0.00000 0.00000 0.00000 0.00000 0.00000 0.00001 0.00001 0.00001 0.00002 
$\mathrm{C}(\mathrm{h}, 0)$

$\begin{array}{lll}0.01 & -0.00000 & -0.00000\end{array}$ $0.02-0.00000-0.00000$ $0.05 \quad-0.00000-0.00000$ $0.10-0.00000-0.00000$ $0.50-0.00000-0.00000$ $\begin{array}{lll}1.00 & -0.00000 & -0.00000\end{array}$ $1.50 \quad-0.00000-0.00000$ $2.00-0.00000 \quad-0.00000$ $2.50-0.00000-0.00000$ $3.00 \quad 0.00000-0.00000$ $\begin{array}{lll}3.50 & 0.00000 & -0.00000\end{array}$

$0.125 \pi:-0.00000-0.00000$ $0.250 \pi-0.00000-0.00000$ $\begin{array}{lll}0.375 \pi & -0.00000 & -0.00000\end{array}$ $0.500 \pi \quad-0.00000-0.00000$ $\begin{array}{llll}0.625 \pi & 0.00000 & -0.00000\end{array}$ $0.750 \pi \quad-0.00000-0.00000$ $0.875 \pi \quad 0.00000-0.00000$ $\begin{array}{lll}1.000 \% \pi & 0.00000 & -0.00000\end{array}$ $\begin{array}{lll}1.125 \pi . & 0.00000 & -0.00000\end{array}$ $\begin{array}{lll}1.2501 \pi & 0.00000 & -0.00000\end{array}$
$S(h, 0)$

$\begin{array}{rr}-0.00000 & 0.00000 \\ -0.00000 & 0.00000 \\ -0.00000 & 0.00000 \\ -0.00000 & 0.00000 \\ -0.00000 & 0.00000 \\ -0.00000 & 0.00000 \\ -0.00000 & 0.00000 \\ -0.00000 & 0.00000 \\ -0.00000 & 0.00000 \\ -0.00000 & -0.00000 \\ -0.00000 & -0.00000 \\ -0.00000 & -0.00000 \\ & \\ -0.00000 & 0.00000 \\ -0.00000 & 0.00000 \\ -0.00000 & 0.00000 \\ -0.00000 & 0.00000 \\ -0.00000 & -0.00000 \\ -0.00000 & 0.00000 \\ -0.00000 & -0.00000 \\ -0.00000 & -0.00000 \\ -0.00000 & -0.00000 \\ -0.00000 & -0.00000\end{array}$

$\frac{\mathrm{a}}{\lambda}=3.5 \quad \frac{\alpha}{\beta}=0.70$

$E(h, 0)$

$\mathrm{C}(\mathrm{h}, \mathrm{h})$

$S(h, h)$

$\begin{array}{rr}-0.00000 & -0.00000 \\ -0.00000 & -0.00000 \\ -0.00000 & -0.00000 \\ -0.00000 & -0.00000 \\ -0.00000 & -0.00000 \\ -0.00000 & -0.00000 \\ -0.00000 & -0.00000 \\ -0.00000 & -0.00000 \\ 0.00000 & -0.00000 \\ -0.00000 & -0.00000 \\ 0.00000 & -0.00000 \\ 0.00000 & -0.00000 \\ & \\ -0.00000 & -0.00000 \\ -0.00000 & -0.00000 \\ -0.00000 & -0.00000 \\ -0.00000 & -0.00000 \\ 0.00000 & -0.00000 \\ -0.00000 & -0.00000 \\ -0.00000 & -0.00000 \\ 0.00000 & -0.00000 \\ 0.00000 & -0.00000 \\ 0.00000 & -0.00000\end{array}$

$\begin{array}{rr}-0.00000 & 0.00000 \\ -0.00000 & 0.00000 \\ -0.00000 & 0.00000 \\ -0.00000 & 0.00000 \\ -0.00000 & 0.00000 \\ -0.00000 & 0.00000 \\ -0.00000 & 0.00000 \\ -0.00000 & 0.00000 \\ -0.00000 & -0.00000 \\ -0.00000 & 0.00000 \\ -0.00000 & -0.00000 \\ -0.00000 & -0.00000 \\ & \\ -0.00000 & 0.00000 \\ -0.00000 & 0.00000 \\ -0.00000 & 0.00000 \\ -0.00000 & 0.00000 \\ -0.00000 & -0.00000 \\ -0.00000 & 0.00000 \\ -0.00000 & -0.00000 \\ -0.00000 & -0.00000 \\ -0.00000 & -0.00000 \\ -0.00000 & -0.00000\end{array}$

$E(h, h)$ $-0.00000$ $-0.00000$ $-0.00000$ $-0.00000$ $-0.00000$ $-0.00000$ $-0.00000$ $-0.00000$ $-0.00000$ $-0.00000$ $-0.00000$

$-0.00000$ $-0.00000$ $-0.00000$ $-0.00000$ $-0.00000$ $-0.00000$ $-0.00000$ $-0.00000$ 0.00000 0.00000 0.00000 .00000 0.00000 0.00000 0.00000 0.00000 0.00000 0.00000 0.00000 0.00000

0.000000000

$\begin{array}{ll}-0.00000 & 0.00000 \\ -0.00000 & 0.000000\end{array}$

$-0.00000 \quad 0.00000$

$-0.00000 \quad 0.00000$

$$
\frac{a}{\lambda}=3.5 \quad \frac{\alpha}{\beta}=1.00
$$

$\begin{array}{ll}-0.00000 & -0.00000 \\ -0.00000 & -0.00000 \\ -0.00000 & -0.00000 \\ -0.00000 & -0.00000 \\ -0.00000 & -0.00000 \\ -0.00000 & -0.00000 \\ -0.00000 & -0.00000 \\ -0.00000 & -0.00000 \\ 0.00000 & -0.00000 \\ 0.00000 & -0.00000 \\ 0.00000 & -0.00000 \\ 0.00000 & -0.00000 \\ -0.00000 & -0.00000 \\ -0.00000 & -0.00000 \\ -0.00000 & -0.00000 \\ -0.000 .00 & -0.00000 \\ 0.00000 & -0.00000 \\ 0.00000 & -0.00000 \\ 0.00000 & -0.00000 \\ 0.00000 & -0.00000 \\ 0.00000 & -0.00000 \\ 0.00000 & -0.00000\end{array}$

$\begin{array}{rr}-0.00000 & 0.00000 \\ -0.00000 & 0.00000 \\ -0.00000 & 0.00000 \\ -0.00000 & 0.00000 \\ -0.00000 & 0.00000 \\ -0.00000 & 0.00000 \\ -0.00000 & 0.00000 \\ -0.00000 & 0.00000 \\ -0.00000 & -0.00000 \\ -0.00000 & -0.00000 \\ -0.00000 & -0.00000 \\ -0.00000 & -0.00000 \\ -0.00000 & 0.00000 \\ -0.00000 & 0.00000 \\ -0.00000 & 0.00000 \\ -0.00000 & 0.00000 \\ -0.00000 & -0.00000 \\ -0.00000 & -0.00000 \\ -0.00000 & -0.00000 \\ -0.00000 & -0.00000 \\ -0.00000 & -0.00000 \\ 0.00000 & -0.00000\end{array}$

0.00000

$\begin{array}{ll}-0.00000 & 0.00000 \\ -0.00000 & 0.00000 \\ -0.00000 & 0.00000 \\ -0.00000 & 0.00000 \\ -0.00000 & 0.00000 \\ -0.00000 & 0.00000 \\ -0.00000 & 0.00000 \\ -0.00000 & 0.00000 \\ -0.00000 & 0.00000 \\ -0.00000 & 0.00000 \\ -0.00000 & 0.00000 \\ -0.00000 & 0.00000 \\ -0.00000 & 0.00000 \\ -0.00000 & 0.00000 \\ -0.00000 & 0.00000 \\ -0.00000 & 0.00000 \\ -0.00000 & 0.00000 \\ -0.00000 & 0.00000 \\ -0.00000 & 0.00000 \\ -0.00000 & 0.00000 \\ -0.00000 & 0.00000 \\ -0.00000 & 0.00000\end{array}$

$\begin{array}{rrrr}-0.00000 & 0.00000 & -0.00000 & -0.00000 \\ -0.00000 & 0.00000 & -0.00000 & 0.00000 \\ -0.00000 & 0.00000 & -0.00000 & 0.00000 \\ -0.00000 & 0.00000 & -0.00000 & 0.00000 \\ -0.00000 & 0.00000 & -0.00000 & 0.00000 \\ -0.00000 & 0.00000 & -0.00000 & 0.00000 \\ -0.00000 & 0.00000 & -0.00000 & 0.00000 \\ -0.00000 & 0.00000 & -0.00000 & 0.00000 \\ -0.00000 & -0.00000 & -0.00000 & 0.00000 \\ -0.00000 & -0.00000 & -0.00000 & 0.00000 \\ -0.00000 & -0.00000 & -0.00000 & 0.00000 \\ -0.00000 & -0.00000 & -0.00000 & 0.00000 \\ & & & \\ -0.00000 & 0.00000 & -0.00000 & 0.00000 \\ -0.00000 & 0.00000 & -0.00000 & 0.00000 \\ -0.00000 & 0.00000 & -0.00000 & 0.00000 \\ -0.00000 & 0.00000 & -0.00000 & 0.00000 \\ -0.00000 & -0.00000 & -0.00000 & 0.00000 \\ -0.00000 & -0.00000 & -0.00000 & 0.00000 \\ -0.00000 & -0.00000 & -0.00000 & 0.00000 \\ -0.00000 & -0.00000 & -0.00000 & 0.00000 \\ -0.00000 & -0.00000 & -0.00000 & 0.00000 \\ 0.00000 & -0.00000 & -0.00000 & 0.00000\end{array}$

0.00000 0.00000 0.00000 0.00000 0.00000
0.00000 0.00000 0.00000 0.00000

$\begin{array}{lrr}0.01 & -0.00000 & -0.00000 \\ 0.02 & -0.00000 & -0.00000 \\ 0.05 & -0.00000 & -0.00000 \\ 0.10 & -0.00000 & -0.00000 \\ 0.20 & -0.00000 & -0.00000 \\ 0.50 & -0.00000 & -0.00000 \\ 1.00 & -0.00000 & -0.00000 \\ 1.50 & -0.00000 & -0.00000 \\ 2.00 & 0.00000 & -0.00000 \\ 2.50 & 0.00000 & -0.00000 \\ 3.00 & 0.00000 & -0.00000 \\ 3.50 & 0.00000 & -0.00000 \\ 0.125 \pi & -0.00000 & -0.00000 \\ 0.250 \pi & -0.00000 & -0.00000 \\ 0.375 \pi & -0.00000 & -0.00000 \\ 0.500 \pi & -0.00000 & -0.00000 \\ 0.625 \pi & 0.00000 & -0.00000 \\ 0.750 \pi & 0.00000 & -0.00000 \\ 0.875 \pi & 0.00000 & -0.00000 \\ 1.000 \pi & 0.00000 & -0.00000 \\ 1.125 \pi & 0.00000 & -0.00000 \\ 1.250 \pi & 0.00000 & -0.00000\end{array}$

$-0.00000$ 


$$
\frac{a}{\lambda}=4.0 \quad \frac{\alpha}{\beta}=0.00
$$

Bh

$$
C(h, 0)
$$

$0.00080 \quad 0.00000$ $0.00159-0.00000$ $0.00398-0.00000$ $0.00794-0.00000$ $0.01581-0.00000$ $0.06694-0.00038$ $0.07934-0.00073$ $0.07236-0.00025$ $0.04786 \quad 0.00229$ $\begin{array}{rr}0.01210 & 0.00775 \\ -0.02587 & 0.01581\end{array}$

$0.03045-0.00003$ $0.05626-0.00021$ $0.07349-0.00053$ $0.07954-0.00074$ $0.05640 \quad 0.00129$ $0.03094 \quad 0.00463$ $\begin{array}{ll}-0.02831 & 0.01642 \\ -0.05289 & 0.02334\end{array}$ $0.03815-0.00006$ $0.07351-0.00034$ $0.00115 \quad 0.00982$
$S(h, 0)$

$\begin{array}{ll}0.00000 & -0.00000 \\ 0.00002 & -0.00000 \\ 0.00010 & -0.00000 \\ 0.00040 & -0.00000 \\ 0.00159 & -c .00000 \\ 0.00974 & -0.00002 \\ 0.03657 & -0.00035 \\ 0.07386 & -0.00154 \\ 0.11244 & -0.00390 \\ 0.14281 & -0.00692 \\ 0.15759 & -0.00908 \\ 0.15348 & -0.00810 \\ 0.00606 & -0.00001 \\ 0.02330 & -0.00014 \\ 0.04909 & -0.00065 \\ 0.07948 & -0.00180 \\ 0.10980 & -0.00369 \\ 0.13541 & -0.00606 \\ 0.15243 & -0.00824 \\ 0.15837 & -0.00923 \\ 0.15252 & -0.00786 \\ 0.13612 & -0.00317\end{array}$

$E(h, 0)$

$\begin{array}{ll}0.00080 & 0.00000 \\ 0.00159 & -0.00000 \\ 0.00398 & -0.00000 \\ 0.00796 & -0.00000 \\ 0.01592 & -0.00000 \\ 0.03979 & -0.00007 \\ 0.07955 & -0.00053 \\ 0.11927 & -0.00178 \\ 0.15889 & -0.00421 \\ 0.19831 & -0.00820 \\ 0.23741 & -0.014113 \\ 0.27600 & -0.02234 \\ 0.03125 & -0.00003 \\ 0.06249 & -0.00026 \\ 0.09371 & -0.00086 \\ 0.12489 & -0.00204 \\ 0.15600 & -0.00398 \\ 0.18700 & -0.00687 \\ 0.21783 & -0.01089 \\ 0.24840 & -0.01620 \\ 0.27862 & -0.02299 \\ 0.30836 & -0.03140\end{array}$

$\mathrm{C}(\mathrm{h}, \mathrm{h})$

$$
\begin{array}{rr}
0.00080 & -0.00000 \\
0.00159 & -0.00000 \\
0.00398 & -0.00000 \\
0.00794 & -0.00000 \\
0.01581 & -0.00002 \\
0.03814 & -0.00025 \\
0.06686 & -0.00171 \\
0.07903 & -0.00426 \\
0.07185 & -0.00596 \\
0.04806 & -0.00384 \\
0.01560 & 0.00425 \\
-0.01484 & 0.01748 \\
& \\
0.03045 & -0.00012 \\
0.05622 & -0.00090 \\
0.07334 & -0.00255 \\
0.07918 & -0.00461 \\
0.07300 & -0.00593 \\
0.05622 & -0.00501 \\
0.03234 & -0.00059 \\
0.00631 & 0.00760 \\
-0.01659 & 0.01849 \\
-0.03211 & 0.02958
\end{array}
$$

$$
\frac{a}{\lambda}=4.0 \quad \frac{\alpha}{\beta}=0.005
$$

$\begin{array}{lrrrr}0.01 & 0.00070 & 0.00000 & 0.00000 & -0.00000 \\ 0.02 & 0.00140 & -0.00000 & 0.00001 & -0.00000 \\ 0.05 & 0.00351 & -0.00000 & 0.00009 & -0.00000 \\ 0.10 & 0.00701 & -0.00000 & 0.00035 & -0.00000 \\ 0.20 & 0.01394 & -0.00000 & 0.00140 & -0.00001 \\ 0.50 & 0.03364 & -0.00004 & 0.00859 & -0.00006 \\ 1.00 & 0.05904 & -0.00023 & 0.03224 & -0.00045 \\ 1.50 & 0.06998 & -0.00033 & 0.06513 & -0.00156 \\ 2.00 & 0.06384 & 0.00039 & 0.09915 & -0.00358 \\ 2.50 & 0.04227 & 0.00293 & 0.12594 & -0.00600 \\ 3.00 & 0.01078 & 0.00791 & 0.13904 & -0.00747 \\ 3.50 & -0.02268 & 0.01496 & 0.13554 & -0.00606 \\ 0.125 \pi & 0.02686 & -0.00002 & 0.00534 & -0.00003 \\ 0.250 \pi & 0.04962 & -0.00013 & 0.02055 & -0.00022 \\ 0.375 \pi & 0.06482 & -0.00030 & 0.04329 & -0.00074 \\ 0.500 \pi & 0.07015 & -0.00030 & 0.07008 & -0.00179 \\ 0.625 \pi & 0.06485 & 0.00029 & 0.09682 & -0.00340 \\ 0.750 \pi & 0.04980 & 0.00196 & 0.11941 & -0.00533 \\ 0.875 \pi & 0.02738 & 0.00510 & 0.13445 & -0.00696 \\ 1.000 \pi & 0.00113 & 0.00975 & 0.13975 & -0.00745 \\ 1.125 \pi & -0.02484 & 0.01548 & 0.13470 & -0.00581 \\ 1.250 \pi & -0.04657 & 0.02130 & 0.12037 & -0.00128\end{array}$

$\begin{array}{ll}0.00070 & 0.00000 \\ 0.00140 & -0.00000 \\ 0.00351 & -0.00000 \\ 0.00702 & -0.00000 \\ 0.01404 & -0.00000 \\ 0.03509 & -0.00006 \\ 0.07016 & -0.00047 \\ 0.10518 & -0.00157 \\ 0.14011 & -0.00371 \\ 0.17486 & -0.00723 \\ 0.20931 & -0.01245 \\ 0.24331 & -0.01968 \\ 0.02756 & -0.00003 \\ 0.05511 & -0.00023 \\ 0.08264 & -0.00076 \\ 0.11013 & -0.00180 \\ 0.13756 & -0.00351 \\ 0.16489 & -0.00606 \\ 0.19206 & -0.00960 \\ 0.21900 & -0.01428 \\ 0.24562 & -0.02026 \\ 0.27181 & -0.02767\end{array}$

S(h, h)

$0.00000-0.00000$ $0.00002-0.00000$ $0.00010-0.00000$ $0.00040-0.00000$ $0.00159-0.00000$ $0.00974-0.00057$ $0.03651-0.00108$ $0.07346-0.00480$ $0.11083-0.01261$ $0.13856-0.02387$ $0.14220-0.04264$

$0.00606-0.00003$ $0.02328-0.00043$ $\begin{array}{lll}0.04897 & -0.00200\end{array}$ $0.07897-0.00565$ $0.10832-0.01190$ $0.13208-0.0204$ $0.14635-0.02985$ $0.14916-0.03808$ $\begin{array}{ll}0.14114 & -0.04287 \\ 0.12535 & -0.04289\end{array}$
$E(h, h)$

$0.00080-0.00000$ $0.00159-0.00000$ $0.00398-0.00000$ $0.00796-0.00000$ $0.01591-0.00002$ $0.03978-0.00026$ $0.07944-0.00210$ $0.11571-0.00706$ $0.15691-0.01658$ $0.19292-0.03185$ $0.22502-0.05363$ $0.25100-0.08194$

$0.03124-0.00013$ $0.06244-0.00102$ $0.09350-0.00343$ $0.12420-0.00810$ $0.15418-0.01570$ $0.18288-0.02681$ $0.20952-0.04186$ $0.23311-0.06101$ $0.25250-0.08410$ $0.26646-0.11053$
$0.00000-0.00000$ $0.00001-0.00000$ $0.00009-0.00000$ $0.00035-0.00000$ $0.00140-0.00001$ $0.00859-0.00011$ $0.03219-0.00108$ $0.06475-0.00443$ $0.09768-0.01125$ $0.12214-0.02092$ $0.13186-0.03068$ $0.12565-0.0366$

$0.00534-0.00005$ $0.02053-0.00047$ $0.04317-0.00192$ $0.06961-0.00518$ $0.09547-0.01063$ $0.11642-0.01797$ $0.12903-0.02601$ $0.13163-0.03291$ $0.12472-0.03678$ $0.11101-0.03656$ $\begin{array}{ll}0.00070 & -0.00000 \\ 0.00140 & -0.00000 \\ 0.00351 & -0.00000 \\ 0.00702 & -0.00000 \\ 0.01404 & -0.00001 \\ 0.03508 & -0.00023 \\ 0.07005 & -0.00186 \\ 0.10466 & -0.00623 \\ 0.13831 & -0.01461 \\ 0.17000 & -0.02805 \\ 0.19823 & -0.04720 \\ 0.22105 & -0.07206 \\ 0.02755 & -0.00011 \\ 0.05507 & -0.00090 \\ 0.08244 & -0.00303 \\ 0.10950 & -0.00714 \\ 0.13591 & -0.01383 \\ 0.16117 & -0.02362 \\ 0.18460 & -0.03685 \\ 0.20533 & -0.05368 \\ 0.22236 & -0.07396 \\ 0.23460 & -0.09714\end{array}$ 
$\frac{\mathrm{a}}{\lambda}=4.0 \quad \frac{\alpha}{\bar{\beta}}=0.01$

$\beta \mathrm{h}$

$C(h, 0)$

$\begin{array}{lrr}0.01 & 0.00062 & 0.00000 \\ 0.02 & 0.00124 & -0.00000 \\ 0.05 & 0.00309 & -0.00000 \\ 0.10 & 0.00618 & -0.00000 \\ 0.20 & 0.01230 & -0.00000 \\ 0.50 & 0.02967 & -0.00002 \\ 1.00 & 0.05207 & -0.00011 \\ 1.50 & 0.06172 & -0.00002 \\ 2.00 & 0.05632 & 0.00088 \\ 2.50 & 0.03734 & 0.00338 \\ 3.00 & 0.00960 & 0.00793 \\ 3.50 & -0.01991 & 0.01408 \\ & & \\ 0.125 \pi & 0.02368 & -0.00001 \\ 0.250 \pi & 0.04376 & -0.00007 \\ 0.375 \pi & 0.05716 & -0.00012 \\ 0.500 \pi & 0.06188 & 0.00004 \\ 0.625 \pi & 0.05722 & 0.00077 \\ 0.750 \pi & 0.04396 & 0.00246 \\ 0.875 \pi & 0.02422 & 0.00539 \\ 1.000 \pi & 0.00110 & 0.00956 \\ 1.125 \pi & -0.02181 & 0.01453 \\ 1.250 \pi & -0.04102 & 0.01942\end{array}$
$S(h, 0)$

$0.00000-0.00000$ $0.00001-0.00000$ $0.00008-0.00000$ $0.00031-0.00000$ $0.00758-0.00009$ $0.00758-0.000 \mathrm{C}$ $0.02844-0.00155$ $0.05744-0.00155$ $0.08743-0.00328$ $0.11108-0.00520$ $0.11970-0.00439$

$0.00471-0.00005$ $0.01812-0.00027$ $0.03818-0.00080$ $0.06180-0.00176$ $0.08537-0.00314$ $0.10531-0.00469$ $0.11860-0.00588$ $0.12333-0.00596$ $\begin{array}{rr}0.11897 & -0.00414 \\ 0.10644 & 0.00021\end{array}$
$E(h, 0)$

$0.00062 \quad 0.00000$ $0.00124-0.00000$ $-0.00000$ $0.00619-0.00000$ $0.03094-0.00000$ $0.03094-0.00005$ $0.061875-0.00138$ $0.09275-0.00138$ $0.12354-0.00327$ $\begin{array}{ll}0.15418 & -0.00037 \\ 0.18454 & -0.01097\end{array}$ $0.21449-0.01735$

$0.02430-0.00002$ $0.04860-0.00020$ $0.07288-0.00067$ $0.09712-0.0015$ $0.12130-0.00310$ $0.14539-0.00534$ $0.16933-0.00846$ $0.19307-0.01259$ $\begin{array}{ll}0.21653 & -0.01786 \\ 0.23959 & -0.02438\end{array}$ $\frac{a}{\lambda}=4.0 \quad \frac{\alpha}{\beta}=0.02$

$\begin{array}{lrl}0.01 & 0.00048 & 0.00000 \\ 0.02 & 0.00096 & 0.00000 \\ 0.05 & 0.00241 & 0.00000 \\ 0.10 & 0.00481 & 0.00000 \\ 0.20 & 0.00956 & 0.00000 \\ 0.50 & 0.02308 & 0.00000 \\ 1.00 & 0.04050 & 0.00006 \\ 1.50 & 0.04801 & 0.00042 \\ 2.00 & 0.04384 & 0.00152 \\ 2.50 & 0.02912 & 0.00387 \\ 3.00 & 0.00759 & 0.00765 \\ 3.50 & -0.01537 & 0.01235 \\ 0.125 \pi & 0.01842 & 0.00000 \\ 0.250 \pi & 0.03403 & 0.00002 \\ 0.375 \pi & 0.04446 & 0.00013 \\ 0.500 \pi & 0.04814 & 0.00051 \\ 0.625 \pi & 0.04453 & 0.00140 \\ 0.750 \pi & 0.03426 & 0.00305 \\ 0.875 \pi & 0.01894 & 0.00559 \\ 1.000 \pi & 0.00098 & 0.00893 \\ 1.125 \pi & -0.01685 & 0.01268 \\ 1.250 \pi & -0.03189 & 0.01611\end{array}$

$\begin{array}{ll}0.00000 & -0.00000 \\ 0.00001 & -0.00000 \\ 0.00006 & -0.00000 \\ 0.00024 & -0.00000 \\ 0.00096 & -0.00002 \\ 0.00589 & -0.00013 \\ 0.02211 & -0.00058 \\ 0.04466 & -0.00148 \\ 0.06800 & -0.00274 \\ 0.08642 & -0.00390 \\ 0.09553 & -0.00401 \\ 0.09337 & -0.00194 \\ 0.00366 & -0.00008 \\ 0.01409 & -0.00034 \\ 0.02969 & -0.00085 \\ 0.04806 & -0.00164 \\ 0.06640 & -0.00265 \\ 0.08192 & -0.00363 \\ 0.09231 & -0.00416 \\ 0.09608 & -0.00369 \\ 0.09281 & -0.00169 \\ 0.08323 & 0.00224\end{array}$

$\mathrm{C}(\mathrm{h}, \mathrm{h})$

$\begin{array}{rr}0.00062 & -0.00006 \\ 0.00124 & -0.00006 \\ 0.00309 & -0.00000 \\ 0.00618 & -0.00000 \\ 0.01230 & -0.00001 \\ 0.02966 & -0.00017 \\ 0.05199 & -0.00114 \\ 0.06147 & -0.00276 \\ 0.05596 & -0.00358 \\ 0.03763 & -0.00145 \\ 0.01259 & 0.00504 \\ -0.01097 & 0.01508 \\ 0.02368 & -0.00008 \\ 0.04372 & -0.00061 \\ 0.05704 & -0.00169 \\ 0.06160 & -0.00297 \\ 0.05685 & -0.00359 \\ 0.04392 & -0.00248 \\ 0.02551 & 0.00122 \\ 0.00541 & 0.000763 \\ -0.01234 & 0.01583 \\ -0.02452 & 0.02394\end{array}$

$\mathrm{S}(\mathrm{h}, \mathrm{h})$

$0.00000-0.00000$ $0.00001-0.00000$ $0.00008-0.00000$ $0.00031-0.00000$ $0.00123-0.00001$ $0.00757-0.00013$ $0.02838-0.00107$ $-0.00408$ $0.08610-0.01003$ $0.10767-0.01634$ $\begin{array}{ll}0.11633 & -0.02658 \\ 0.11103 & -0.03142\end{array}$

$0.00471-0.00007$ $0.01810-0.00049$ $0.03806-0.00184$ $0.06136-0.00474$ $0.08414-0.00950$ $0.10262-0.01582$ $0.11616-0.02843$ $0.11023-0.03155$ $0.09831-0.03114$
$E(h, h)$

$0.00062-0.00000$ $0.00124-0.00000$ $0.00309-0.00000$ $0.00619-0.00000$ $0.01238-0.00001$ $0.03093-0.00021$ $0.06177-0.00164$ $0.07227-0.00549$ $0.12192-0.01287$ $0.14981-0.02470$ $0.19467-0.06337$

$0.02430-0.00010$ $0.04856-0.00079$ $0.07269-0.00267$ $0.09654-0.00629$ $0.11980-0.01219$ $0.14204-0.02080$ $0.16265-0.03244$ $0.18087-0.04723$ $\begin{array}{ll}0.19582 & -0.06504 \\ 0.20656 & -0.08537\end{array}$

$\begin{array}{ll}0.00048 & 0.00000 \\ 0.00096 & -0.00000 \\ 0.00241 & -0.00000 \\ 0.00481 & -0.00000 \\ 0.00963 & -0.00000 \\ 0.02407 & -0.00004 \\ 0.04812 & -0.00032 \\ 0.07213 & -0.00107 \\ 0.09606 & -0.00254 \\ 0.11986 & -0.00495 \\ 0.14345 & -0.00853 \\ 0.16669 & -0.01347 \\ 0.01890 & -0.00002 \\ 0.03780 & -0.00015 \\ 0.05668 & -0.00052 \\ 0.07552 & -0.00123 \\ 0.09432 & -0.00241 \\ 0.11304 & -0.00415 \\ 0.13164 & -0.00657 \\ 0.15007 & -0.00978 \\ 0.16827 & -0.01387 \\ 0.18616 & -0.01893\end{array}$

$0.00048-0.00000$ $0.00096-0.00000$ $0.00241-0.00000$ $0.00481-0.00000$ $0.00956-0.00001$ $0.02307-0.00011$ $0.04043-0.00074$ $0.04782-0.00172$ $0.04359-0.00196$ $0.02945 \quad 0.00007$ $\begin{array}{rr}0.01013 & 0.00527 \\ -0.00814 & 0.01290\end{array}$ $0.01842-0.00006$ $0.03400-0.00040$ $0.04436-0.00109$ $0.04792-0.00183$ $0.04428-0.00200$ $0.03430-0.00083$ $0.02010 \quad 0.00226$ $0.00458 \quad 0.00727$ $-0.00920 \quad 0.01345$ $\begin{array}{ll}-0.01878 & 0.01937\end{array}$
$0.00000-0.00000$ $0.00001-0.00000$ $0.00006-0.00000$ $0.00024-0.00000$ $0.00096-0.00002$ $0.00589-0.00016$ $0.02206-0.00102$ $0.04435-0.00344$ $0.06689-0.00798$ $0.08368-0.01410$ $0.09055-0.01994$ $0.08670-0.02309$

$0.00366-0.00009$ $0.01408-0.00051$ $0.02958-0.00166$ $\begin{array}{ll}0.04768 & -0.00395 \\ 0.06537 & -0.00758\end{array}$ $0.07974-0.01226$ $0: 08848-0.01719$ $0.09048-0.02120$ $0.07710-0.02250$

$\begin{array}{ll}0.00048 & -0.00000 \\ 0.00096 & -0.00000 \\ 0.00241 & -0.00000 \\ 0.00481 & -0.00000 \\ 0.00963 & -0.00001 \\ 0.02406 & -0.00016 \\ 0.04803 & -0.00127 \\ 0.07172 & -0.00426 \\ 0.09472 & -0.00999 \\ 0.11633 & -0.01915 \\ 0.13552 & -0.03217 \\ 0.15098 & -0.04901 \\ & \\ 0.01890 & -0.00008 \\ 0.03776 & -0.00062 \\ 0.05652 & -0.00208 \\ 0.07503 & -0.00489 \\ 0.09308 & -0.00946 \\ 0.11031 & -0.01614 \\ 0.12626 & -0.02514 \\ 0.14034 & -0.03657 \\ 0.15187 & -0.05029 \\ 0.16013 & -0.06594\end{array}$


$\beta \mathrm{h}$

$\mathrm{C}(\mathrm{h}, 0)$

0.01

0.02

0.05
0.10
0.20

0.20

0.50

1.50

2.00

2.50

3.50

$0.125 \pi$

$0.250 \pi$

$0.3 .75 \pi$

$0.500 \pi$

$0.625 \pi$

$0.750 \pi$

$0.875 \pi$

1.000

$1.125 \pi$
$0.00037 \quad 0.00000$ $0.00075 \quad 0.00000$

$0.00374 \quad 0.00000$

$0.00744 \quad 0.00000$

$0.03150 \quad 0.00016$

$0.03735 \quad 0.00066$

$0.03413 \quad 0.00183$

$\begin{array}{ll}0.02277 & 0.00398 \\ 0.00598 & 0.00711\end{array}$

$-0.01190$

0.01433

0.02647

0.03459

0.03745

0.03466

0.01480

$-0.01306$

$-0.02485$

0.00001 .00077 0.0032 0.01094
$0.01795 \quad 0.00002$

$0.02271 \quad 0.00398$
S(h, 0)

$0.00000-0.00000$ $0.00001-0.00000$ $0.00005-0.00060$ $0.00019-0.00001$ $0.00075-0.00002$ $0.01720-0.00059$ $0.03474-0.00136$ $0.05289-0.00229$ $0.06725-0.00292$ $0.07285-0.00036$ $0.01096-0.00036$ $0.03738-0.00149$ $0.05165-0.00222$ $0.06374-0.00222$ $0.07186-0.00282$ $0.07487-0.00215$ $0.07243-0.00012$ $\begin{array}{rr}0.07243 & -0.00012 \\ 0.06509 & 0.00336\end{array}$ $0.013309-0.00036$ $0.00458-0.00014$

$0.00285-0.00009$

$$
\frac{a}{\lambda}=4.0 \quad \frac{\alpha}{\beta}=0.03
$$

$\mathrm{E}(\mathrm{h}, 0)$

$\begin{array}{ll}0.00037 & 0.00000 \\ 0.00075 & -0.00000 \\ 0.00187 & -0.00000 \\ 0.00374 & -0.00000 \\ 0.00749 & -0.00000 \\ 0.01872 & -0.00003 \\ 0.03742 & -0.00025 \\ 0.05609 & -0.00084 \\ 0.07470 & -0.00198 \\ 0.09319 & -0.00385 \\ 0.11150 & -0.00662 \\ 0.12954 & -0.01046 \\ 0.01470 & -0.00002 \\ 0.02940 & -0.00012 \\ 0.04408 & -0.00041 \\ 0.05873 & -0.00096 \\ 0.07334 & -0.00187 \\ 0.08788 & -0.00323 \\ 0.10233 & -0.00511 \\ 0.11664 & -0.00760 \\ 0.13077 & -0.01077 \\ 0.14465 & -0.01469\end{array}$

$0.14465-0.01077$

$$
\frac{a}{\lambda}=4.0 \quad \frac{\alpha}{\beta}=0.05
$$

\begin{tabular}{|c|c|c|c|c|}
\hline $\begin{array}{l}.01 \\
.02 \\
.05 \\
.10 \\
.20 \\
.50 \\
.00 \\
.50 \\
.00 \\
.50 \\
.00\end{array}$ & $\begin{array}{r}0.00023 \\
0.00045 \\
0.00113 \\
0.00226 \\
0.00450 \\
0.01086 \\
0.01906 \\
0.02261 \\
0.02068 \\
0.01379 \\
0.00368 \\
-0.00720\end{array}$ & $\begin{array}{l}0.00000 \\
0.00000 \\
0.00000 \\
0.00000 \\
0.00000 \\
0.00003 \\
0.00023 \\
0.00080 \\
0.00189 \\
0.00358 \\
0.00570 \\
0.00780\end{array}$ & $\begin{array}{l}0.00000 \\
0.00000 \\
0.00003 \\
0.00011 \\
0.00045 \\
0.00277 \\
0.01040 \\
0.02102 \\
0.03202 \\
0.04075 \\
0.04518 \\
0.04438\end{array}$ & $\begin{array}{r}-0.00000 \\
-0.00000 \\
-0.00000 \\
-0.00001 \\
-0.00002 \\
-0.00014 \\
-0.00053 \\
-0.00108 \\
-0.00157 \\
-0.00164 \\
-0.00086 \\
0.00117\end{array}$ \\
\hline $\begin{array}{l}0.125 \pi \\
0.250 \pi \\
0.375 \pi \\
0.500 \pi \\
0.625 \pi \\
0.750 \pi \\
0.875 \pi \\
1.000 \pi \\
1.125 \pi \\
1.250 \pi\end{array}$ & $\begin{array}{r}0.00867 \\
0.01601 \\
0.02093 \\
0.02267 \\
0.02100 \\
0.01620 \\
0.00902 \\
0.00056 \\
-0.00791 \\
-0.01518\end{array}$ & $\begin{array}{l}0.00001 \\
0.00011 \\
0.00038 \\
0.00092 \\
0.00179 \\
0.00304 \\
0.00460 \\
0.00632 \\
0.00793 \\
0.00904\end{array}$ & $\begin{array}{l}0.00172 \\
0.00663 \\
0.01397 \\
0.02262 \\
0.03126 \\
0.03861 \\
0.04359 \\
0.04550 \\
0.04414 \\
0.03982\end{array}$ & $\begin{array}{r}-0.00009 \\
-0.00034 \\
-0.00072 \\
-0.00116 \\
-0.00154 \\
-0.00168 \\
-0.00138 \\
-0.00042 \\
0.00136 \\
0.00399\end{array}$ \\
\hline
\end{tabular}

$C(h,-2)$

$\begin{array}{rr}0.00037 & -0.00000 \\ 0.00075 & -0.00000 \\ 0.00187 & -0.00000 \\ 0.00374 & -0.00000 \\ 0.00744 & -0.00000 \\ 0.01794 & -0.00007 \\ 0.03145 & -0.00046 \\ 0.03720 & -0.00101 \\ 0.03395 & -0.00089 \\ 0.02305 & 0.00098 \\ 0.00812 & 0.00515 \\ -0.00605 & 0.01095 \\ 0.01432 & -0.00004 \\ 0.02644 & -0.00025 \\ 0.03450 & -0.00067 \\ 0.03728 & -0.00105 \\ 0.03448 & -0.00094 \\ 0.02679 & 0.00021 \\ 0.01583 & 0.00277 \\ 0.00382 & 0.00670 \\ -0.00688 & 0.01136 \\ -0.01442 & 0.01566\end{array}$

$\mathrm{S}(\mathrm{h}, \mathrm{h})$

$0.00000-0.00000$ $0.00001-0.00000$ $0.00005-0.00000$ $0.00019-0.00001$ $0.00075-0.00002$ $0.00458-0.00017$ $\begin{array}{ll}0.004715 & -0.00093 \\ 0.03447 & -0.00289\end{array}$ $0.05198-0.00635$ $\begin{array}{ll}0.06506 & -0.01084 \\ 0.07050 & -0.01495\end{array}$ $0.06771-0.01692$

$0.00285-0.00010$ $0.01094-0.00049$ $0.02300-0.00146$ $0.03705-0.00328$ $0.05080-0.00605$ $0.06198-0.00951$ $0.06883-0.01304$ $0.07049-0.01579$ $0.06726-0.01695$ $0.06046-0.01618$
$E(h, h)$

$\begin{array}{ll}0.00037 & -0.00000 \\ 0.00075 & -0.00000 \\ 0.00187 & -0.00000 \\ 0.00374 & -0.00000 \\ 0.00749 & -0.00001 \\ 0.01871 & -0.00012 \\ 0.03735 & -0.00099 \\ 0.05575 & -0.00331 \\ 0.07360 & -0.00776 \\ 0.09034 & -0.01485 \\ 0.10517 & -0.02492 \\ 0.11710 & -0.03791 \\ 0.01470 & -0.00006 \\ 0.02937 & -0.00048 \\ 0.04394 & -0.00161 \\ 0.05832 & -0.00380 \\ 0.07232 & -0.00735 \\ 0.08568 & -0.01252 \\ 0.09802 & -0.01949 \\ 0.10889 & -0.02831 \\ 0.11778 & -0.03890 \\ 0.12414 & -0.05093\end{array}$

$\begin{array}{llrr}0.00023 & 0.00000 & 0.00023 & -0.00000 \\ 0.00045 & -0.00000 & 0.00045 & -0.00000 \\ 0.00113 & -0.00000 & 0.00113 & -0.00000 \\ 0.00226 & -0.00000 & 0.00226 & -0.00000 \\ 0.00453 & -0.00000 & 0.00450 & -0.00000 \\ 0.01132 & -0.00002 & 0.01085 & -0.00003 \\ 0.02263 & -0.00015 & 0.01902 & -0.00014 \\ 0.03392 & -0.00051 & 0.02251 & -0.00021 \\ 0.04516 & -0.00119 & 0.02060 & 0.00024 \\ 0.05633 & -0.00233 & 0.01410 & 0.00171 \\ 0.06737 & -0.00400 & 0.00517 & 0.00439 \\ 0.07824 & -0.00631 & -0.00339 & 0.00775 \\ 0.00889 & -0.00001 & 0.00866 & -0.00001 \\ 0.01778 & -0.00007 & 0.01600 & -0.00008 \\ 0.02666 & -0.00025 & 0.02087 & -0.00019 \\ 0.03552 & -0.00058 & 0.02257 & -0.00019 \\ 0.04434 & -0.00113 & 0.02092 & 0.00017 \\ 0.05312 & -0.00195 & 0.01633 & 0.00116 \\ 0.06184 & -0.00308 & 0.00979 & 0.00291 \\ 0.07047 & -0.00458 & 0.00258 & 0.00532 \\ 0.07898 & -0.00650 & -0.00390 & 0.00797 \\ 0.08732 & -0.00886 & -0.00858 & 0.01024\end{array}$

$0.00000-0.00000$ $0.00000-0.00000$ $0.00003-0.00000$ $0.00011-0.00001$ $0.00045-0.00002$ $0.00277-0.00015$ $0.01037-0.00074$ $0.02083-0.00200$ $0.03140-0.00401$ $0.03935-0.00641$ $0.04277-0.00837$ $0.04132-0.00900$

$0.00172-0.00009$ $0.00662-0.00042$ $0.01390-0.00110$ $0.02239-0.00224$ $0.03069-0.00384$ $0.03747-0.00572$ $0.04168-0.00750$ $0.04282-0.00872$ $0.04107-0.00898$ $0.03716-0.00819$
$0.00023-0.00000$ $0.00045-0.00000$ $0.00113-0.00000$ $0.00226-0.00000$ $0.00453-0.00000$ $0.01132-0.00007$ $0.02258-0.00000$ $0.03369-0.00200$ $0.04443-0.00467$ $0.07044-0.01495$

$0.00889-0.00004$ $0.01776-0.00029$ $0.02656-0.00097$ $0.03523-0.00229$ $0.04366-0.00443$ $0.05168-0.00753$ $0.05907-0.01170$ $0.06556-0.01697$ $0.07084-0.02326$ $0.07461-0.03039$ 
Bh

$C(h, 0)$

0.0

0.02

0.05

0.20

1.00

1.50

3.00

3.50

$0.125 \pi$

$0.250 \pi$

$0.375 \pi$

$0.625 \pi$

$0.750 \pi$

$0.875 \pi$

$1.000 \pi$

$1.125 \pi$
$1.250 \pi$

$0.00027 \quad 0.00000$

$0.00137 \quad 0.00000$

0.002720 .00000

$0.00657 \quad 0.00003$

$0.0133 \quad 0.00022$

0.013690 .00073

0.012530 .00162

$0.00837 \quad 0.00288$

$-0.00441$

0.00430

$0.00524 \quad 0.00001$

$0.00968 \quad 0.00011$

$\begin{array}{ll}0.01267 & 0.00036 \\ 0.01373 & 0.00083\end{array}$

$0.01273 \quad 0.00154$

$0.00983 \quad 0.00249$

$0.00548 \quad 0.00358$

$0.00034 \quad 0.00469$

$\begin{array}{ll}-0.00485 & 0.00560 \\ -0.00935 & 0.00607\end{array}$
$S(h, 0)$

$0.00000-0.00000$

$0.00000-0.00000$

$0.00002-0.00000$

$0.00007-0.00000$

$0.00027-0.00002$

$0.00168-0.00012$

$0.0063 \mathrm{C}-0.00043$

$0.01272-0.00081$

$0.01940-0.00106$

$0.02472-0.00091$

$\begin{array}{rr}0.02747 & -0.00010 \\ 0.02707 & 0.00154\end{array}$

$0.00104-0.00007$

$0.00401-0.00028$

$0.00845-0.00056$

$0.01369-0.00086$

$0.01894-0.00105$

$0.02341-0.00101$

0.02646 - -0.00061

$0.02768 \quad 0.00027$

$\begin{array}{ll}0.02693 & 0.00169 \\ 0.02437 & 0.00360\end{array}$ $\frac{a}{\lambda}=4.0 \quad \frac{\alpha}{\beta}=0.07$

$E(h, 0)$

$\mathrm{C}(\mathrm{h}, \mathrm{h})$

$S(h, h)$

$E(h, h)$

$\begin{array}{rrrrrr}0.00014 & 0.00000 & 0.00014 & -0.00000 & 0.00000 & -0.00000 \\ 0.00027 & -0.00000 & 0.00027 & -0.00000 & 0.00000 & -0.00000\end{array}$

$\begin{array}{llll}0.00027 & -0.00000 & 0.00027 & -0.00000\end{array}$

$0.00069-0.00000 \quad 0.00068-0.00000$

$\begin{array}{lllll}0.00137 & -0.00000 & 0.00137 & -0.00000\end{array}$

$0.00274-0.00000 \quad 0.00272-0.00000$

$0.01369-0.00009$

$0.013651-0.00009$

$0.02730-0.00072$

$0.03404-0.00140$

$0.04071-0.00241$

$0.04725-0.00381$

$0.00538-0.00001$

$0.01076-0.00004$

$0.01612-0.00015$

$0.02148-0.00035$

$0.02681-0.00068$

$0.03211-0.00118$

$0.03737-0.00186$

$0.04257-0.00277$

$0.04770-0.00392$

$0.05272-0.00534$

$0.00657-0.00000$

$0.01151-0.0000$

0.013630 .0001

$0.01250 \quad 0.0006$

0.008610 .0017

$\begin{array}{ll}0.00194 & 0.00538\end{array}$

$0.00524-0.00000$

$0.00968-0.0000$

$0.01263 \quad 0.0000$

$0.01367 \quad 0.00016$

0.012690 .00055

$0.00995 \quad 0.00132$

$0.00603 \quad 0.0025$

0.0039

$\begin{array}{ll}-0.00225 & 0.00550 \\ -0.00517 & 0.00668\end{array}$ $\begin{array}{ll}0.00000 & -0.00000 \\ 0.00002 & -0.00000\end{array}$ $0.00007-0.00000$ $0.0007-0.00000$ $0.00027-0.00002$ $0.00168-0.00012$ $0.00627-0.00055$ $0.01899-0.00253$ $0.01899-0.00253$ $0.02382-0.09379$ $0.02523-0.00471$

$0.00104-0.00008$ $0.00400-0.00032$ $0.00840-0.0007$ $0.01353-0.0015$ $0.01855-0.00244$ $0.02267-0.00345$ $0.02526-0.00431$ $0.02604-0.00478$ $0.02509-0.00468$ $0.02284-0.00400$

$\begin{array}{lrlllll}0.01 & 0.00006 & 0.00000 & 0.00000 & -0.00000 & 0.00006 & 0.00000 \\ 0.02 & 0.00013 & 0.00000 & 0.00000 & -0.00000 & 0.00013 & -0.00000 \\ 0.05 & 0.00032 & 0.00000 & 0.00001 & -0.00000 & 0.00032 & -0.00000 \\ 0.10 & 0.00064 & 0.00000 & 0.00003 & -0.00000 & 0.00064 & -0.00000 \\ 0.20 & 0.00128 & 0.00000 & 0.00013 & -0.00001 & 0.00129 & -0.00000 \\ 0.50 & 0.00309 & 0.00002 & 0.00079 & -0.00008 & 0.00322 & -0.00001 \\ 1.00 & 0.00543 & 0.00016 & 0.00296 & -0.00027 & 0.00644 & -0.00004 \\ 1.50 & 0.00645 & 0.00051 & 0.00600 & -0.00049 & 0.00965 & -0.00014 \\ 2.00 & 0.00591 & 0.00110 & 0.00916 & -0.00058 & 0.01284 & -0.00034 \\ 2.50 & 0.00395 & 0.00186 & 0.01170 & -0.00038 & 0.01600 & -0.00066 \\ 3.00 & 0.00104 & 0.00263 & 0.01305 & 0.00024 & 0.01912 & -0.00113 \\ 3.50 & -0.00216 & 0.00319 & 0.01291 & 0.00132 & 0.02218 & -0.00178 \\ & & 0.0017 & & & & \\ 0.125 \pi & 0.00247 & 0.00001 & 0.00049 & -0.00005 & 0.00253 & -0.00000 \\ 0.250 \pi & 0.00456 & 0.00008 & 0.00189 & -0.00018 & 0.00506 & -0.00002 \\ 0.375 \pi & 0.00596 & 0.00026 & 0.00398 & -0.00036 & 0.00758 & -0.00007 \\ 0.500 \pi & 0.00647 & 0.00058 & 0.00645 & -0.00051 & 0.01010 & -0.00016 \\ 0.625 \pi & 0.00600 & 0.00105 & 0.00894 & -0.00058 & 0.01260 & -0.00032 \\ 0.750 \pi & 0.00464 & 0.00163 & 0.01107 & -0.00047 & 0.01509 & -0.00055 \\ 0.875 \pi & 0.00258 & 0.00225 & 0.01255 & -0.00013 & 0.01756 & -0.00087 \\ 1.000 \pi & 0.00013 & 0.00282 & 0.01316 & 0.00050 & 0.01999 & -0.00130 \\ 1.125 \pi & -0.00237 & 0.00321 & 0.01285 & 0.00141 & 0.02239 & -0.00184 \\ 1.250 \pi & -0.00459 & 0.00330 & 0.01168 & 0.00254 & 0.02473 & -0.00250\end{array}$

$\frac{\alpha}{\beta}=0.10$

$\begin{array}{rrrr}0.00006 & -0.00000 & 0.00000 & -0.00000 \\ 0.00013 & 0.00000 & 0.00000 & -0.00000 \\ 0.00032 & 0.00000 & 0.00001 & -0.00000 \\ 0.00064 & 0.00000 & 0.00003 & -0.00000 \\ 0.00128 & 0.00000 & 0.00013 & -0.00001 \\ 0.00309 & 0.00001 & 0.00079 & -0.00008 \\ 0.00542 & 0.00006 & 0.00295 & -0.00033 \\ 0.00642 & 0.00022 & 0.00592 & -0.00075 \\ 0.00591 & 0.00061 & 0.00894 & -0.00127 \\ 0.00411 & 0.00128 & 0.01124 & -0.00173 \\ 0.00160 & 0.00217 & 0.01231 & -0.00193 \\ -0.00087 & 0.00303 & 0.01205 & -0.00170 \\ & & & \\ 0.00247 & 0.00000 & 0.00049 & -0.00005 \\ 0.00455 & 0.00002 & 0.00188 & -0.00020 \\ 0.00594 & 0.00010 & 0.00395 & -0.00046 \\ 0.00644 & 0.00026 & 0.00636 & -0.00082 \\ 0.00599 & 0.00058 & 0.00873 & -0.00123 \\ 0.00473 & 0.00106 & 0.01069 & -0.00161 \\ 0.00290 & 0.00171 & 0.01194 & -0.00187 \\ 0.00086 & 0.00243 & 0.01237 & -0.00191 \\ -0.00102 & 0.00308 & 0.01199 & -0.00167 \\ -0.00247 & 0.00350 & 0.01100 & -0.00120\end{array}$

$0.01100-0.00120$
$0.00014-0.00000$ $0.00027-0.00000$ . 0.00000 $00137-0.00000$ $\begin{array}{ll}0.00274 & -0.00000\end{array}$ 0.00685 -0.00005 $0.01365-0.00036$ 0.0012 $0.02682-0.00282$ $0.03285-0.00537$ $0.04237-0.01356$

$0.00538-0.00002$ $0.01074-0.00018$ $0.01606-0.00059$ $0.02129-0.00138$ $0.02636-0.00267$ $0.03117-0.00453$ $0.03560-0.00703$ $0.03947-0.01017$ $0.04261-0.0139$ $0.04484-0.01813$

$\begin{array}{lll}1.250 \pi & -0.00459 & 0.00330\end{array}$

$\begin{array}{ll}0.01285 & 0.00141 \\ 0.01168 & 0.00254\end{array}$

$\begin{array}{ll}0.00006 & -0.00000 \\ 0.00013 & -0.00000 \\ 0.00032 & -0.00000 \\ 0.00064 & -0.00000 \\ 0.00129 & -0.00000 \\ 0.00322 & -0.00002 \\ 0.00642 & -0.00017 \\ 0.00956 & -0.00057 \\ 0.01258 & -0.00132 \\ 0.01538 & -0.00251 \\ 0.01783 & -0.00417 \\ 0.01977 & -0.00628 \\ 0.00253 & -0.00001 \\ 0.00505 & -0.00008 \\ 0.00755 & -0.00028 \\ 0.01000 & -0.00065 \\ 0.01236 & -0.00125 \\ 0.01461 & -0.00212 \\ 0.01666 & -0.00327 \\ 0.01844 & -0.00472 \\ 0.01988 & -0.00644 \\ 0.02090 & -0.00836\end{array}$


$\beta \mathrm{h}$

$C(h, 0)$

$S(h, 0)$

$\begin{array}{lrl}0.01 & 0.00001 & 0.00000 \\ 0.02 & 0.00001 & 0.00000 \\ 0.05 & 0.00003 & 0.00000 \\ 0.10 & 0.00005 & 0.00000 \\ 0.20 & 0.00010 & 0.00000 \\ 0.50 & 0.00025 & 0.00000 \\ 1.00 & 0.00044 & 0.00003 \\ 1.50 & 0.00053 & 0.00009 \\ 2.00 & 0.00048 & 0.00018 \\ 2.50 & 0.00032 & 0.00029 \\ 3.00 & 0.00006 & 0.00038 \\ 3.50 & -0.00023 & 0.00043\end{array}$

$\begin{array}{lll}0.125 \pi & 0.00020 & 0.00000\end{array}$ $0.250 \pi \quad 0.00037 \quad 0.00001$ $\begin{array}{lll}0.375 \pi & 0.00049 & 0.00005\end{array}$ $\begin{array}{lll}0.500 \pi & 0.00053 & 0.00010\end{array}$ $\begin{array}{lll}0.625 \pi & 0.00049 & 0.00017\end{array}$ $\begin{array}{lll}0.750 \pi & 0.00038 & 0.00026\end{array}$ $\begin{array}{lll}0.875 \pi & 0.00020 & 0.00034\end{array}$ $\begin{array}{lll}1.000 \pi & -0.00002 & 0.00040 \\ 1.125 \pi & -0.00025 & 0.00043\end{array}$ $\begin{array}{lll}1.250 \pi & -0.00047 & 0.00040\end{array}$

$0.00000-0.00000$ $0.00000-0.00000$ $0.00000-0.00000$ $0.00000-0.00000$ $0.00001-0.00000$ $0.00006-0.00001$ $0.00024-0.00004$ $0.00049-0.00007$ $0.00076-0.00007$ $0.00098-0.00002$ $\begin{array}{ll}0.00111 & 0.00010 \\ 0.00111 & 0.00027\end{array}$ $0.00004-0.00001$ $0.00015-0.00003$ $0.00032-0.00005$ $0.00053-0.00007$ $0.00074-0.00007$ $0.00093-0.00004$ $0.00106 \quad 0.00003$ $0.00112 \quad 0.00014$ 0.001010 .00045

$\begin{array}{lrr}0.01 & 0.00000 & 0.00000 \\ 0.02 & 0.00000 & 0.00000 \\ 0.05 & 0.00000 & 0.00000 \\ 0.10 & 0.00000 & 0.00000 \\ 0.20 & 0.00000 & 0.00000 \\ 0.50 & 0.00000 & 0.00000 \\ 1.00 & 0.00000 & 0.00000 \\ 1.50 & 0.00000 & 0.00000 \\ 2.00 & 0.00000 & 0.00000 \\ 2.50 & 0.00000 & 0.00000 \\ 3.00 & -0.00000 & 0.00001 \\ 3.50 & -0.00000 & 0.00001 \\ 0.125 \pi & 0.00000 & 0.00000 \\ 0.250 \pi & 0.00000 & 0.00000 \\ 0.375 \pi & 0.00000 & 0.00000 \\ 0.500 \pi & 0.00000 & 0.00000 \\ 0.625 \pi & 0.00000 & 0.00000 \\ 0.750 \pi & 0.00000 & 0.00000 \\ 0.875 \pi & 0.00000 & 0.00000 \\ 1.000 \pi & -0.00000 & 0.00001 \\ 1.125 \pi & -0.00000 & 0.00001 \\ 1.250 \pi & -0.00001 & 0.00000\end{array}$
$0.00001 \quad 0.00000$ $0.00001-0.00000$
$E(h, 0)$

$C(h, h)$

$\mathrm{S}(\mathrm{h}, \mathrm{h})$

$\begin{array}{ll}0.00001 & 0.00000 \\ 0.00001 & -0.00000 \\ 0.00003 & -0.00000 \\ 0.00005 & -0.00000 \\ 0.00010 & -0.00000 \\ 0.00026 & -0.00000 \\ 0.00052 & -0.00000 \\ 0.00078 & -0.00001 \\ 0.00104 & -0.00003 \\ 0.00129 & -0.00005 \\ 0.00154 & -0.00009 \\ 0.00178 & -0.00014 \\ & \\ 0.00020 & -0.00000 \\ 0.00041 & -0.00000 \\ 0.00061 & -0.00001 \\ 0.00082 & -0.00001 \\ 0.00102 & -0.00003 \\ 0.00122 & -0.00004 \\ 0.00142 & -0.00007 \\ 0.00161 & -0.00010 \\ 0.00180 & -0.00015 \\ 0.00198 & -0.00020\end{array}$

$0.00001-0.00000$ $0.00001 \quad 0.00000$ $0.00005 \quad 0.00000$ $0.00010 \quad 0.00000$ $0.00025 \quad 0.00000$ $0.00044 \quad 0.00002$ $0.00052 \quad 0.00006$ $0.00049 \quad 0.00014$ $0.00034 \quad 0.00023$

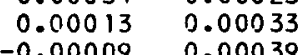

$0.00020 \quad 0.00000$ $0.00037 \quad 0.00001$ $0.00048 \quad 0.00003$ $0.00053 \quad 0.00007$ $0.00049 \quad 0.00013$ $0.00024 \quad 0.00028$ $0.00007 \quad 0.00035$ $\begin{array}{rr}0.000007 & 0.00035 \\ -0.00010 & 0.00039\end{array}$ $\begin{array}{ll}-0.000025 & 0.00039\end{array}$ $0.00003 \quad 0.00000$ $0.00039 \quad 0.00021$
$0.00000-0.00000$ $0.00000-0.00000$ $0.00000-0.00000$ $0.00000-0.00000$ $0.00001-0.00000$ $0.00006-0.0000$ $0.00024-0.00005$ $0.00048-0.00009$ $0.00073-0.00012$ $0.00093-0.00013$ $\begin{array}{rr}0.00104 & -0.00008 \\ 0.00104 & 0.00000\end{array}$

$0.00004 \quad-0.00001$ $0.00015-0.00003$ $0.00032-0.00006$ $0.00052-0.00010$ $0.00072-0.00012$ $0.00088-0.00013$ $0.00100-0.00011$ $0.00105-0.00006$ $0.00104 \quad 0.00001$ $0.00096 \quad 0.00009$
$E(h, h)$

$0.00001-0.00000$ $0.00001-0.00000$ $0.00003-0.00000$ $0.00005-0.00000$ $0.00010-0.00000$ $0.00026-0.00000$ $0.00052-0.00001$ $0.00077-0.00005$ $0.00101-0.00010$ $0.00123-0.00020$ $0.00141-0.00032$

$0.00020-0.00000$ $0.00041-0.00001$ $0.00061-0.00002$ $0.00080-0.00005$ $0.00099-0.00010$ $0.00117-0.00017$ $0.00132-0.00026$ $0.00146-0.00037$ $0.00146-0.00037$ $0.00164-0.00063$

$$
\frac{a}{\lambda}=4.0 \quad \frac{\alpha}{\beta}=0.40
$$

$E(H, 0)$

$0.00000-0.00000$ $0.00000-0.00000$ $0.00000-0.00000$ $0.00000-0.00000$ $0.00000-0.00000$ $0.00000-0.00000$ $0.00000-0.00000$ $0.00000-0.00000$ $0.00001-0.00000$ $\begin{array}{ll}0.00001 & 0.00000 \\ 0.00001 & 0.00000\end{array}$

\section{$0.00000-0.00000$} $0.00000-0.00000$ $0.00000-0.00000$ $0.00000-0.00000$ $0.00001-0.00000$ $0.00001 \quad 0.00000$ $0.00001 \quad 0.00000$ $0.00001 \quad 0.00000$ $0.00001 \quad 0.00001$

$\begin{array}{ll}0.00000 & 0.00000 \\ 0.00000 & -0.00000 \\ 0.00000 & -0.00000 \\ 0.00000 & -0.00000 \\ 0.00000 & -0.00000 \\ 0.00000 & -0.00000 \\ 0.00000 & -0.00000 \\ 0.00001 & -0.00000 \\ 0.00001 & -0.00000 \\ 0.00001 & -0.00000 \\ 0.00001 & -0.00000 \\ 0.00001 & -0.00000 \\ 0.00000 & -0.00000 \\ 0.00000 & -0.00000 \\ 0.00000 & -0.00000 \\ 0.00001 & -0.00000 \\ 0.00001 & -0.00000 \\ 0.00001 & -0.00000 \\ 0.00001 & -0.00000 \\ 0.00001 & -0.00000 \\ 0.00001 & -0.00000 \\ 0.00001 & -0.00000\end{array}$

1

$\begin{array}{rrrr}0.00000 & 0.00000 & 0.00000 & -0.00000 \\ 0.00000 & 0.00000 & 0.00000 & -0.00000 \\ 0.00000 & 0.00000 & 0.00000 & -0.00000 \\ 0.00000 & 0.00000 & 0.00000 & -0.00000 \\ 0.00000 & 0.00000 & 0.00000 & -0.00000 \\ 0.00000 & 0.00000 & 0.00000 & -0.00000 \\ 0.00000 & 0.00000 & 0.00000 & -0.00000 \\ 0.00000 & 0.00000 & 0.00000 & -0.00000 \\ 0.00000 & 0.00000 & 0.00001 & -0.00000 \\ 0.00000 & -0.00000 & 0.00001 & -0.00000 \\ 0.00000 & 0.00000 & 0.00001 & 0.00000 \\ 0.00000 & 0.00000 & 0.00001 & 0.00000 \\ & & & \\ 0.00000 & 0.00000 & 0.00000 & -0.00000 \\ 0.00000 & 0.00000 & 0.00000 & -0.00000 \\ 0.00000 & 0.00000 & 0.00000 & -0.00000 \\ 0.00000 & 0.00000 & 0.00000 & -0.00000 \\ 0.00000 & 0.00000 & 0.00001 & -0.00000 \\ 0.00000 & 0.00000 & 0.00001 & -0.00000 \\ 0.00000 & 0.00000 & 0.00001 & -0.00000 \\ -0.00000 & 0.00000 . & 0.00001 & 0.00000 \\ -0.00000 & 0.00000 & 0.00001 & 0.00000 \\ -0.00000 & 0.00000 & 0.00001 & 0.00000\end{array}$

$\begin{array}{ll}0.00000 & -0.00000 \\ 0.00000 & -0.00000 \\ 0.00000 & -0.00000 \\ 0.00000 & -0.00000 \\ 0.00000 & -0.00000 \\ 0.00000 & -0.00000 \\ 0.00000 & -0.00000 \\ 0.00000 & -0.00000 \\ 0.00001 & -0.00000 \\ 0.00001 & -0.00000 \\ 0.00001 & -0.00000 \\ 0.00001 & -0.00000 \\ & \\ 0.00000 & -0.00000 \\ 0.00000 & -0.00000 \\ 0.00000 & -0.00000 \\ 0.00001 & -0.00000 \\ 0.00001 & -0.00000 \\ 0.00001 & -0.00000 \\ 0.00001 & -0.00000 \\ 0.00001 & -0.00000 \\ 0.00001 & -0.00000 \\ 0.00001 & -0.00000\end{array}$




$$
\frac{\mathrm{a}}{\lambda}=4.0 \quad \frac{\alpha}{\beta}=0.70
$$

$E(h, 0)$

$\mathrm{C}(\mathrm{h}, \mathrm{h})$

$S(h, h)$

$E(h, h)$

$0.00000 \quad 0.00000$ $0.00000-0.00000$ $0.00000-0.00000$

$0.00000-0.00000$

$0.00000-0.00000$

$0.00000-0.00000$

$0.00000-0.00000$

$0.00000-0.00000$

$0.00000-0.00000$

$0.00000-0.00000$

$0.00000-0.00000$

$0.00000-0.00000$

$0.00000-0.00000$

$.00000-0.00000$

$0.00000-0.00000$

$0.00000-0.00000$

$0.00000-0.00000$

$0.00000-0.00000$

$\begin{array}{ll}0.00000 & -0.00000 \\ 0.00000 & -0.00000\end{array}$

$\begin{array}{rr}0.00000 & 0.00000 \\ 0.00000 & 0.00000 \\ 0.00000 & 0.00000 \\ 0.00000 & 0.00000 \\ 0.00000 & 0.00000 \\ 0.00000 & 0.00000 \\ 0.00000 & 0.00000 \\ 0.00000 & 0.00060 \\ -0.00000 & 0.00000 \\ -0.00060 & 0.00000 \\ -0.00000 & -0.000010 \\ -0.00000 & -0.00000 \\ 0.00000 & 0.00000 \\ 0.00000 & 0.00000 \\ 0.00000 & 0.00000 \\ 0.00000 & 0.00000 \\ -0.00000 & 0.00000 \\ -0.00000 & 0.00000 \\ -0.00000 & 0.00000 \\ -0.00000 & -0.00000 \\ -0.00000 & -0.00000 \\ -0.00000 & 0.00000\end{array}$

$0.00000-0.00000$

$0.00000-0.00000$

$0.00000-0.00000$

$c .00000-0.00000$

$0.00000-0.00000$

$0.00000-0.00000$

$0.00000-0.00000$

$0.00000-0.00000$

$0.00000 \quad 0.00000$

$-0.00000 \quad 0.00000$

$0.00000-0.00000$

$0.00000-0.00000$

$0.00000-0.00000$

$0.00000-0.00000$

$0.00000 \quad 0.00000$

$0.00000 \quad 0.00000$

$0.00000 \quad 0.00000$

$-0.00000 \quad 0.00000$

$-0.00000 \quad 0.00000$

$0.00000 \quad 0.00000$
$0.125 \pi$ $0.250 \pi$ $0.375 \pi$

$0.500 \pi$

$0.625 \pi$

$0.875 \pi$

$1.125 \pi$

$1.250 \pi$
$0.00000 \quad 0.00000$ $0.00000 \quad 0.00000$ $0.00000 \quad 0.00000$ $0.00000 \quad 0.00000$ $0.00000 \quad 0.00000$ $0.00000 \quad 0.00000$ $0.00000 \quad 0.00000$ $0.00000 \quad 0.00000$ $-0.00000 \quad 0.00000$ $-0.00000 \quad-0.00000$ $\begin{array}{ll}-0.00000 & 0.00000 \\ -0.000000 & -0.00000\end{array}$

\subsection{0} 0.00000 0.00000 $-0.00000$ $-0.00000$ $-0.00000$ $-0.00000-0.00000$ $-0.00000 \quad 0.00000$
$\mathrm{S}(\mathrm{h}, 0)$ $0.00000-0.00000$ $0.00000-0.00000$ $0.00000-0.00000$ $0.00000-0.00000$ $0.00000-0.00000$ $0.00000-0.00000$ $0.00000-0.00000$ $0.00000 \quad 0.00000$ $0.00000 \quad 0.00000$

$0.00000-0.00000$ $0.00000-0.00000$ $0.00000-0.00000$ $0.00000-0.00000$ $0.00000 \quad 0.00000$ 0.000000 .0000 $0.00000 \quad 0.00000$ $-0.00000 \quad 0.00000$ $\begin{array}{rr}-0.00000 & 0.00000 \\ 0.00000 & 0.00000\end{array}$ $0.00000 \quad 0.00000$ $0.00000 \quad 0.00000$

$0.00000-0.00000$

$\begin{array}{rr}0.000000 & 0.00000\end{array}$

$\begin{array}{ll}0.00000 & -0.00000 \\ 0.00000 & -0.00000 \\ 0.00000 & -0.00000 \\ 0.00000 & -0.00000 \\ 0.00000 & -0.00000 \\ 0.00000 & -0.00000 \\ 0.00000 & -0.00000 \\ 0.00000 & -0.00000 \\ 0.00000 & -0.00006 \\ 0.00000 & -0.00000 \\ 0.00000 & -0.00000 \\ 0.00000 & -0.00000 \\ 0.00000 & -0.00000 \\ 0.00000 & -0.00000 \\ 0.00000 & -0.00000 \\ 0.00000 & -0.00000 \\ 0.00000 & -0.00000 \\ 0.00000 & -0.00000 \\ 0.00000 & -0.00000 \\ 0.00000 & -0.00000 \\ 0.00000 & -0.00000 \\ 0.00000 & -0.00000\end{array}$

$$
\frac{a}{\lambda}=4.0 \quad \frac{\alpha}{\beta}=1.00
$$

$\begin{array}{lrrrr}0.01 & 0.00000 & 0.00000 & 0.00000 & -0.00000 \\ 0.02 & 0.00000 & 0.00000 & 0.00000 & -0.00000 \\ 0.05 & 0.00000 & 0.00000 & 0.00000 & -0.00000 \\ 0.10 & 0.00000 & 0.00000 & 0.00000 & -0.00000 \\ 0.20 & 0.00000 & 0.00000 & 0.00000 & -0.00000 \\ 0.50 & 0.00000 & 0.00000 & 0.00000 & -0.00000 \\ 1.00 & 0.00000 & 0.00000 & 0.00000 & -0.00000 \\ 1.50 & 0.00000 & 0.00000 & 0.00000 & -0.00000 \\ 2.00 & -0.00000 & 0.00000 & 0.00000 & 0.00000 \\ 2.50 & -0.00000 & 0.00000 & 0.00000 & 0.00000 \\ 3.00 & -0.00000 & 0.00000 & 0.00000 & 0.00000 \\ 3.50 & -0.00000 & 0.00000 & 0.00000 & 0.00000 \\ & & & & \\ 0.125 \pi & 0.00000 & 0.00000 & 0.00000 & -0.000000 \\ 0.250 \pi & 0.00000 & 0.00000 & 0.00000 & -0.00000 \\ 0.375 \pi & 0.00000 & 0.00000 & 0.00000 & -0.000000 \\ 0.500 \pi & 0.00000 & 0.00000 & 0.00000 & -0.00000 \\ 0.625 \pi & -0.00000 & 0.00000 & 0.00000 & 0.00000 \\ 0.750 \pi & -0.00000 & 0.00000 & 0.00000 & 0.00000 \\ 0.875 \pi & -0.00000 & 0.00000 & 0.00000 & 0.00000 \\ 1.000 \pi & -0.00000 & 0.00000 & 0.00000 & 0.00000 \\ 1.125 \pi & -0.00000 & 0.00000 & 0.00000 & 0.00000 \\ 1.250 \pi & -0.00000 & 0.00000 & -0.00000 & 0.00000\end{array}$

$\begin{array}{ll}0.00000 & -0.00000 \\ 0.00000 & -0.00000 \\ 0.00000 & -0.00000 \\ 0.00000 & -0.00000 \\ 0.00000 & -0.00000 \\ 0.00000 & -0.00000 \\ 0.00000 & -0.00000 \\ 0.00000 & -0.00000 \\ 0.00000 & -0.00000 \\ 0.00000 & -0.00000 \\ 0.00000 & -0.00000 \\ 0.00000 & -0.00000 \\ 0.00000 & -0.00000 \\ 0.00000 & -0.00000 \\ 0.00000 & -0.00000 \\ 0.00000 & -0.00000 \\ 0.00000 & -0.00000 \\ 0.00000 & -0.00000 \\ 0.00000 & -0.00000 \\ 0.00000 & -0.00000 \\ 0.00000 & -0.00000 \\ 0.00000 & -0.00000\end{array}$

$\begin{array}{llllll}0.00000 & 0.00000 & 0.00000 & 0.00000 & 0.00000 & -0.00000 \\ 0.00000 & -0.00000 & 0.00000 & 0.00000 & 0.00000 & -0.00000 \\ 0.00000 & -0.00000 & 0.00000 & 0.00000 & 0.00000 & -0.00000 \\ 0.00000 & -0.00000 & 0.00000 & 0.00000 & 0.00000 & -0.00000 \\ 0.00000 & -0.00000 & 0.00000 & 0.00000 & 0.00000 & -0.00000 \\ 0.00000 & -0.00000 & 0.00000 & 0.00000 & 0.00000 & -0.00000 \\ 0.00000 & -0.00000 & 0.00000 & 0.00000 & 0.00000 & -0.00000 \\ 0.00000 & -0.00000 & 0.00000 & 0.00000 & 0.00000 & -0.00000 \\ 0.00000 & -0.00000 & -0.00000 & 0.00000 & 0.00000 & 0.00000 \\ 0.00000 & -0.00000 & -0.00000 & 0.00000 & 0.00000 & 0.00000 \\ 0.00000 & -0.00000 & -0.00000 & 0.00000 & 0.00000 & 0.00000 \\ 0.00000 & -0.00000 & -0.00000 & 0.00000 & 0.00000 & 0.00000 \\ & & & & & \\ 0.00000 & -0.00000 & 0.00000 & 0.00000 & 0.00000 & -0.00000 \\ 0.00000 & -0.00000 & 0.00000 & 0.00000 & 0.00000 & -0.00000 \\ 0.00000 & -0.00000 & 0.00000 & 0.00000 & 0.00000 & -0.00000 \\ 0.00000 & -0.00000 & 0.00000 & 0.00000 & 0.00000 & -0.00000 \\ 0.00000 & -0.00000 & -0.00000 & 0.00000 & 0.00000 & 0.00000 \\ 0.00000 & -0.00000 & -0.00000 & 0.00000 & 0.00000 & 0.00000 \\ 0.00000 & -0.00000 & -0.00000 & 0.00000 & 0.00000 & 0.00000 \\ 0.00000 & -0.00000 & -0.00000 & 0.00000 & 0.00000 & 0.00000 \\ 0.00000 & -0.00000 & -0.00000 & 0.00000 & 0.00000 & 0.00000 \\ 0.00000 & -0.00000 & -0.00000 & 0.00000 & 0.00000 & 0.00000\end{array}$




$$
\frac{a}{\lambda}=4.5 \quad \frac{\alpha}{\beta}=0.00
$$

$C(h, 0)$

0.01

0.02

0.05

0.10

0.50

1.00

2.50

2.50

3.00

$0.125 \pi$

$0.125 \pi$
$0.250 \pi$

0.375

$0.625 \pi$

$0.750 \pi$

$0.875 \pi$
$1.000 \pi$

$1.125 \pi$

$1.250 \pi$
$S(h, 0)$

$\begin{array}{ll}-0.00071 & 0.00000 \\ -0.00141 & 0.00000 \\ -0.00354 & 0.00000 \\ -0.00706 & 0.00000 \\ -0.01405 & 0.00000 \\ -0.03391 & 0.00005 \\ -0.05951 & 0.00030 \\ -0.07053 & 0.00058 \\ -0.06432 & 0.00019 \\ -0.04250 & -0.00182 \\ -0.01059 & -0.00614 \\ 0.02337 & -0.01254 \\ -0.02707 & 0.00002 \\ -0.05001 & 0.00017 \\ -0.06533 & 0.00042 \\ -0.07071 & 0.00058 \\ -0.06535 & 0.00026 \\ -0.05011 & -0.00102 \\ -0.02741 & -0.00366 \\ -0.00081 & -0.00778 \\ 0.02556 & -0.01302 \\ 0.04763 & -0.01852\end{array}$

$E(r, 0)$

$-0.00000 \quad 0.00000$ $\begin{array}{ll}-0.00001 & 0.00000 \\ -0.00009 & 0.00000\end{array}$ $-0.00035 \quad 0.00000$ $-0.00141 \quad 0.00000$ $-0.00866 \quad 0.00002$ $-0.03251 \quad 0.00028$ $\begin{array}{ll}-0.006567 & 0.00028 \\ -0.06122\end{array}$ $-0.10000 \quad 0.00308$ $-0.12704 \quad 0.00548$ $\begin{array}{ll}-0.12704 & 0.00548 \\ -0.14022 & 0.00719\end{array}$ $-0.13654 \quad 0.00641$

$\begin{array}{ll}-0.00538 & 0.00001\end{array}$ $-0.02071$ $-0.04364$ $-0.07067$ $-0.12044$ 0.13562 $-0.13562$ $-0.14092$ $-0.12095$ 0.00011 0.00143 0.00292 0.00479 $0.0 \mathrm{C} 652$ 0.0072 0.00622
$\mathrm{C}(\mathrm{h}, \mathrm{h})$

$\begin{array}{ll}-0.00071 & 0.00000 \\ -0.00141 & 0.00000 \\ -0.00354 & 0.00000 \\ -0.00707 & 0.00000 \\ -0.01415 & 0.00000 \\ -0.03537 & 0.00005 \\ -0.07072 & 0.00042 \\ -0.10604 & 0.00141 \\ -0.11128 & 0.00333 \\ -0.17639 & 0.00649 \\ -0.21128 & 0.01118 \\ -0.24580 & 0.01770 \\ -0.02778 & 0.00003 \\ -0.05555 & 0.00020 \\ -0.08330 & 0.00068 \\ -0.11103 & 0.00161 \\ -0.13871 & 0.00315 \\ -0.16631 & 0.00543 \\ -0.19379 & 0.00861 \\ -0.22110 & 0.01283 \\ -0.24815 & 0.01822 \\ -0.27486 & 0.02480\end{array}$

$\begin{array}{lr}-0.00071 & 0.00003 \\ -0.00141 & 0.00000 \\ -0.00354 & 0.00000 \\ -0.00706 & 0.00000 \\ -0.01405 & 0.00001 \\ -0.03390 & 0.00020 \\ -0.05945 & 0.00135 \\ -0.07031 & 0.00337 \\ -0.06396 & 0.00472 \\ -0.04265 & 0.00300 \\ -0.01312 & -0.00360 \\ 0.01537 & -0.01464 \\ & \\ -0.02707 & 0.00010 \\ -0.04998 & 0.00071 \\ -0.06523 & 0.00202 \\ -0.07046 & 0.00365 \\ -0.06498 & 0.00469 \\ -0.04999 & 0.00394 \\ -0.02842 & 0.00036 \\ -0.00453 & -0.00637 \\ 0.01706 & -0.01549 \\ 0.03244 & -0.02509\end{array}$

$S(h, h)$

$-0.00000 \quad 0.00000$
-0.000010 .00000 $-0.00001 \quad 0.00000$ $-0.00009 \quad 0.00000$ $\begin{array}{ll}-0.00035 & 0.00000\end{array}$ $-0.00866 \quad 0.00006$ $-0.03247 \quad 0.00085$ $-0.06539 \quad 0.00380$ $-0.09886 \quad 0.01000$ $-0.12403 \quad 0.01900$ $-0.13446$ $\begin{array}{ll}-0.13446 & 0.02831 \\ -0.12852 & 0.03417\end{array}$

$-0.00538 \quad 0.00002$ $\begin{array}{ll}-0.02070 & 0.00034\end{array}$ $-0.04356 \quad 0.00158$ $\begin{array}{ll}0.0700447 \\ -0.09660 & 0.000944\end{array}$ $-0.09660 \quad 0.00944$ $-0.118090 .0162$ 0.13130 0.02382 0.134370 .03048 $\begin{array}{ll}-0.12758 & 0.03436 \\ -0.11336 & 0.03421\end{array}$
$E(h, h)$

$\begin{array}{ll}-0.00071 & 0.00000\end{array}$ $-0.00141$ $-0.00707 \quad 0.00000$ $-0.01415 \quad 0.00001$ $-0.03536 \quad 0.00021$ $-0.07064 \quad 0.00166$ $-0.10564 \quad 0.00558$ $-0.13989 \quad 0.01315$ $-0.17258 \quad 0.02535$ $-0.20246 \quad 0.04291$ $\begin{array}{ll}-0.20246 & 0.04291 \\ -0.22789 & 0.06607\end{array}$

\section{$\begin{array}{ll}-0.02777 & 0.00010\end{array}$} $\begin{array}{ll}-0.0275752 & 0.0008\end{array}$ $-0.08316 \quad 0.00272$ $-0.13743 \quad 0.00641$ $-0.16340 \quad 0.02132$ $-0.163400 .02132$ 0.0720 .0333 $-0.21920 \quad 0.04891$ $\begin{array}{ll}-0.22943 & 0.06786 \\ -0.24461 & 0.08995\end{array}$

$\begin{array}{lrr}0.01 & -0.00061 & 0.00000 \\ 0.02 & -0.00123 & 0.00000 \\ 0.05 & -0.00307 & 0.00000 \\ 0.10 & -0.00613 & 0.00000 \\ 0.20 & -0.01220 & 0.00000 \\ 0.50 & -0.02944 & 0.00003 \\ 1.00 & -0.05166 & 0.00017 \\ 1.50 & -0.06124 & 0.00023 \\ 2.00 & -0.05586 & -0.00037 \\ 2.50 & -0.03695 & -0.00237 \\ 3.00 & -0.00928 & -0.00627 \\ 3.50 & 0.02019 & -0.01177 \\ 0.125 \pi & -0.02350 & 0.00001 \\ 0.250 \pi & -0.04342 & 0.00010 \\ 0.375 \pi & -0.05672 & 0.00022 \\ 0.500 \pi & -0.06139 & 0.00020 \\ 0.625 \pi & -0.05675 & -0.00028 \\ 0.750 \pi & -0.04355 & -0.00161 \\ 0.875 \pi & -0.02387 & -0.00407 \\ 1.000 \pi & -0.00080 & -0.00771 \\ 1.125 \pi & 0.02209 & -0.01218 \\ 1.250 \pi & 0.04129 & -0.01671\end{array}$

$$
\frac{\mathrm{a}}{\lambda}=4.5 \quad \frac{\alpha}{\beta}=0.005
$$

$\begin{array}{ll}-0.00000 & 0.00000 \\ -0.00001 & 0.00000 \\ -0.00008 & 0.00000 \\ -0.00031 & 0.00000 \\ -0.00122 & 0.00001 \\ -0.00752 & 0.00005 \\ -0.02822 & 0.00036 \\ -0.05701 & 0.00123 \\ -0.08680 & 0.00280 \\ -0.11029 & 0.00466 \\ -0.12178 & 0.00576 \\ -0.111868 & 0.00461 \\ -0.00467 & 0.00003 \\ -0.01798 & 0.00018 \\ -0.03789 & 0.00059 \\ -0.06134 & 0.00141 \\ -0.08476 & 0.00266 \\ -0.10456 & 0.00415 \\ -0.11776 & 0.00539 \\ -0.12241 & 0.00573 \\ -0.11794 & 0.00441 \\ -0.10525 & 0.00082\end{array}$

$\begin{array}{rr}-0.00061 & 0.00000 \\ -0.00123 & 0.00000 \\ -0.00307 & 0.00000 \\ -0.00613 & 0.00000 \\ -0.01220 & 0.00001 \\ -0.02943 & 0.00016 \\ -0.05161 & 0.00108 \\ -0.06104 & 0.00265 \\ -0.05556 & 0.00357 \\ -0.03714 & 0.00183 \\ -0.01160 & -0.00401 \\ 0.01308 & -0.01347 \\ -0.02350 & 0.00008 \\ -0.04339 & 0.00057 \\ -0.05662 & 0.00161 \\ -0.06117 & 0.00286 \\ -0.05645 & 0.00357 \\ -0.04348 & 0.00271 \\ -0.02484 & -0.00053 \\ -0.00417 & -0.00640 \\ 0.01454 & -0.01419 \\ 0.02794 & -0.02227\end{array}$

(1)

$-0.00000$

$-0.00001$

$-0.00008$

$-0.00031$

$-0.00122$

$-0.00752$

$-0.02818$

$-0.05674$

-0.08578
-0.10763

$-0.11676$

$-0.11177$

0.00000

0.00000

0.00000

0.00000

0.00001

0.00009

0.00086

0.00347

0.00880

0.0163 .9

0.02410

0.02878

$\begin{array}{ll}-0.00467 & 0.00004\end{array}$

0.00037

$-0.03780 \quad 0.00151$

$\begin{array}{ll}-0.06101 & 0.00406\end{array}$

$\begin{array}{ll}-0.08382 & 0.00832\end{array}$

$-0.10247 \quad 0.01407$

$-0.11672 \quad 0.02587$

$\begin{array}{ll}-0.11097 & 0.02892 \\ -0.09880 & 0.02855\end{array}$

$\begin{array}{ll}-0.00061 & 0.00000 \\ -0.00123 & 0.00000 \\ -0.00307 & 0.00000 \\ -0.00614 & 0.00000 \\ -0.01228 & 0.00001 \\ -0.03070 & 0.00018 \\ -0.06132 & 0.00144 \\ -0.09169 & 0.00485 \\ -0.12139 & 0.01141 \\ -0.14972 & 0.02198 \\ -0.17560 & 0.03718 \\ -0.19759 & 0.05722 \\ & \\ -0.02411 & 0.00009 \\ -0.04819 & 0.00070 \\ -0.07218 & 0.00236 \\ -0.09595 & 0.00557 \\ -0.11926 & 0.01080 \\ -0.14177 & 0.01848 \\ -0.16299 & 0.02894 \\ -0.18229 & 0.04237 \\ -0.19892 & 0.05876 \\ -0.21204 & 0.07785\end{array}$


$\mathrm{C}(\mathrm{h}, 0)$

$\begin{array}{lll}0.01 & -0.00053 & 0.00000\end{array}$

$\begin{array}{lll}0.02 & -0.00107 & 0.00000\end{array}$

$\begin{array}{lll}0.05 & -0.00266 & 0.00000\end{array}$

$\begin{array}{lll}0.10 & -0.00532 & 0.00000 \\ 0.20 & -0.01059 & 0.00000\end{array}$

$0.50 \quad-0.02556 \quad 0.00001$

$\begin{array}{lll}1.00 & -0.04485 & 0.00006\end{array}$

$\begin{array}{lll}1.50 & -0.05317 . & -0.00004 \\ 2.00 & -0.04851 & -0.00078\end{array}$

$2.50-0.03212-0.00275$

$\begin{array}{rrr}3.00 & -0.00813 & -0.00627 \\ 3.50 & 0.01746 & -0.01099\end{array}$

$\begin{array}{lll}0.125 \pi & -0.02040 \quad 0.00001\end{array}$

$0.250 \pi \quad-0.03769 \quad 0.00004$

$0.375 \pi \quad-0.04924 \quad 0.00006$

$\begin{array}{lll}0.500 \pi & -0.05330 & -0.00009 \\ 0.625 \pi & -0.04928 & -0.00069\end{array}$

$\begin{array}{lll}0.625 \pi & -0.04928 & -0.00069 \\ 0.750 \pi & -0.03784 & -0.00203\end{array}$

$\begin{array}{lll}0.750 \pi & -0.03784 & -0.00203 \\ 0.875 \pi & -0.02078 & -0.00431\end{array}$

$\begin{array}{lll}0.000 \pi & -0.00076 & -0.00752\end{array}$

$\begin{array}{rrr}1.025 \pi & -0.00076 & -0.00752 \\ 1.125 \pi & 0.01911 & -0.01133\end{array}$

$1.250 \pi \quad 0.03582-0.01506$
$S(h, 0)$

$\begin{array}{ll}-0.00000 & 0.00000 \\ -0.00001 & 0.00000 \\ -0.00007 & 0.00000 \\ -0.00027 & 0.00000 \\ -0.00106 & 0.00001 \\ -0.00653 & 0.00008 \\ -0.02450 & 0.00041 \\ -0.04949 & 0.00122 \\ -0.07535 & 0.00254 \\ -0.09575 & 0.00397 \\ -0.10577 & 0.00459 \\ -0.10316 & 0.00318 \\ -0.00406 & 0.00005 \\ -0.01561 & 0.00022 \\ -0.03289 & 0.00064 \\ -0.05325 & 0.00138 \\ -0.07358 & 0.00243 \\ -0.09078 & 0.00359 \\ -0.10225 & 0.00445 \\ -0.10634 & 0.00445 \\ -0.10253 & 0.00297 \\ -0.09160 & -0.00045\end{array}$

$\frac{a}{\lambda}=4.5 \quad \frac{\alpha}{\beta}=0.01$

$E(h, 0)$

$\mathrm{C}(\mathrm{h}, \mathrm{h})$

$-0.00053 \quad 0.000 .00$

$-0.00107 \quad 0.00000$

$\begin{array}{ll}-0.00267 & 0.00000 \\ -0.00533 & 0.00000\end{array}$

$-0.01066 \quad 0.00000$

$-0.02666 \quad 0.00004$

$-0.05330 \quad 0.00031$

$\begin{array}{ll}-0.05330 & 0.00031 \\ -0.07991 & 0.00106\end{array}$

$-0.10646 \quad 0.00251$

$-0.13290 \quad 0.00489$

$\begin{array}{ll}-0.15916 & 0.00842 \\ -0.18513 & 0.01332\end{array}$

$-0.02094 \quad 0.00002$

$-0.04187 \quad 0.00015$

$\begin{array}{ll}-0.06278 & 0.00051\end{array}$

$\begin{array}{ll}-0.08367 & 0.00122 \\ -0.10453 & 0.00237\end{array}$

$-0.12531 \quad 0.00409$

$-0.14600 \quad 0.00649$

$-0.16655 \quad .0 .00966$

$\begin{array}{ll}-0.18690 & 0.01371 \\ -0.20698 & 0.01874\end{array}$
$\mathrm{S}(\mathrm{h}, \mathrm{h})$

$-0.00007 \quad 0.00000$

$-0.00027 \cdot 0.00000$

$\begin{array}{ll}-0.00106 & 0.00001\end{array}$

$-0.00652 \quad 0.00011$

$-0.02446 \quad 0.00084$

$\begin{array}{ll}-0.04924 & 0.00316\end{array}$

$\begin{array}{ll}-0.09341 & 0.01414\end{array}$

$-0.10140 \quad 0.02051$

$-0.09720$

$-0.00406$

$-0.01560$

$-0.03281$

-0.05294
-0.07273

-0.07273
-0.08892

$-0.0989$

$-0.10139$

$-0.09652$

$-0.08611$

0.02422

0.00006

0.00039

0.00144

0.00733

0.01220

0.01747

0.02194

0.02432
0.02379
$E(h, h)$

$\begin{array}{ll}-0.00053 & 0.00000 \\ -0.00107 & 0.00000\end{array}$

$-0.00267 \quad 0.00000$

$\begin{array}{ll}-0.00533 & 0.00000\end{array}$

$\begin{array}{ll}-0.01066 & 0.00001\end{array}$

$-0.02665 \quad 0.00016$

$-0.05323 \quad 0.00125$

$-0.07958 \quad 0.00421$

$-0.12989 \quad 0.01906$

$-0.15230 \quad 0.03222$

$-0.02093 \quad 0.00008$

$-0.04184 \quad 0.00061$

$-0.06266 \quad 0.00205$

$-0.08327 \quad 0.00483$

$\begin{array}{ll}-0.10349 & 0.00937\end{array}$

$-0.12300 \quad 0.01603$

$-0.14138 \quad 0.02509$

$\begin{array}{lll}-0.15809 & 0.03671\end{array}$

$\begin{array}{ll}-0.17247 & 0.05088 \\ -0.18380 & 0.06738\end{array}$

$$
\frac{a}{\lambda}=4.5 \quad \frac{\alpha}{\beta}=0.02
$$

$\begin{array}{ll}-0.00000 & 0.00000 \\ -0.00001 & 0.00000 \\ -0.00005 & 0.00000 \\ -0.00020 & 0.00000 \\ -0.00080 & 0.00002 \\ -0.00492 & 0.00011 \\ -0.01848 & 0.00047 \\ -0.03730 & 0.00115 \\ -0.05679 & 0.00207 \\ -0.07219 & 0.00287 \\ -0.07981 & 0.00284 \\ -0.07796 & 0.00115 \\ -0.00306 & 0.00006 \\ -0.01177 & 0.00027 \\ -0.02479 & 0.00067 \\ -0.04013 & 0.00127 \\ -0.05545 & 0.00200 \\ -0.06843 & 0.00269 \\ -0.07712 & 0.00301 \\ -0.08026 & 0.00257 \\ -0.07749 & 0.00096 \\ -0.06937 & -0.00209\end{array}$

$\begin{array}{ll}-0.00040 & 0.00000 \\ -0.00080 & 0.00000 \\ -0.00201 & 0.00000 \\ -0.00402 & 0.00000 \\ -0.00804 & 0.00000 \\ -0.02009 & 0.00003 \\ -0.04017 & 0.00024 \\ -0.06022 & 0.00080 \\ -0.08022 & 0.00189 \\ -0.10013 & 0.00368 \\ -0.11990 & 0.00634 \\ -0.13944 & 0.01003 \\ & \\ -0.01578 & 0.00001 \\ -0.03155 & 0.00011 \\ -0.04732 & 0.00039 \\ -0.06306 & 0.00092 \\ -0.07876 & 0.00179 \\ -0.09442 & 0.00308 \\ -0.10999 & 0.00489 \\ -0.12546 & 0.00727 \\ -0.14076 & 0.01032 \\ -0.15586 & 0.01410\end{array}$

$\begin{array}{rr}-0.00040 & 0.00000 \\ -0.00080 & 0.00000 \\ -0.00201 & 0.00000 \\ -0.00401 & 0.00000 \\ -0.00798 & 0.00001 \\ -0.01926 & 0.00008 \\ -0.03376 & 0.00052 \\ -0.03995 & 0.00120 \\ -0.03643 & 0.00130 \\ -0.02451 & -0.00031 \\ -0.00797 & -0.00435 \\ 0.00809 & -0.01033 \\ -0.01537 & 0.00004 \\ -0.02839 & 0.00028 \\ -0.03705 & 0.00077 \\ -0.04004 & 0.00127 \\ -0.03700 & 0.00134 \\ -0.02862 & 0.00039 \\ -0.01655 & -0.00201 \\ -0.00315 & -0.00591 \\ 0.00905 & -0.01077 \\ 0.01792 & -0.01556\end{array}$

$\begin{array}{ll}-0.00000 & 0.00000 \\ -0.00001 & 0.00000 \\ -0.00005 & 0.00000 \\ -0.00020 & 0.00000 \\ -0.00080 & 0.00002 \\ -0.00492 & 0.00013 \\ -0.01843 & 0.00079 \\ -0.03709 & 0.00261 \\ -0.05605 & 0.00599 \\ -0.07036 & 0.01052 \\ -0.07648 & 0.01484 \\ -0.07353 & 0.01711 \\ & \\ -0.00306 & 0.00007 \\ -0.01175 & 0.00040 \\ -0.02472 & 0.00127 \\ -0.03987 & 0.00299 \\ -0.05476 & 0.00569 \\ -0.06697 & 0.00916 \\ -0.07456 & 0.01282 \\ -0.07653 & 0.01577 \\ -0.07304 & 0.01715 \\ -0.06540 & 0.01643\end{array}$

$\begin{array}{ll}-0.00040 & 0.00000 \\ -0.00080 & 0.00000 \\ -0.00201 & 0.00000 \\ -0.00402 & 0.00000 \\ -0.00804 & 0.00001 \\ -0.02008 & 0.00012 \\ -0.04011 & 0.00094 \\ -0.05995 & 0.00317 \\ -0.07932 & 0.00744 \\ -0.09776 & 0.01433 \\ -0.11456 & 0.02419 \\ -0.12879 & 0.03716 \\ -0.01578 & 0.00006 \\ -0.03153 & 0.00046 \\ -0.04721 & 0.00154 \\ -0.06273 & 0.00364 \\ -0.07793 & 0.00705 \\ -0.09259 & 0.01205 \\ -0.10638 & 0.01885 \\ -0.11889 & 0.02756 \\ -0.12965 & 0.03816 \\ -0.13811 & 0.05047\end{array}$

0.05047 .

\begin{tabular}{|c|c|c|}
\hline $\begin{array}{l}0.01 \\
0.02 \\
0.05 \\
0.10 \\
0.20 \\
0.50 \\
1.00 \\
1.50 \\
2.00 \\
2.5 .0 \\
3.00 \\
3.50\end{array}$ & $\begin{array}{r}-0.00040 \\
-0.00080 \\
-0.00201 \\
-0.00401 \\
-0.00798 \\
-0.01926 \\
-0.03381 \\
-0.04008 \\
-0.03659 \\
-0.02427 \\
-0.00622 \\
0.01308\end{array}$ & $\begin{array}{r}0.00000 \\
0.00000 \\
-0.00000 \\
-0.00000 \\
-0.00000 \\
-0.00001 \\
-0.00007 \\
-0.00039 \\
-0.00129 \\
-0.00311 \\
-0.00597 \\
-0.00945\end{array}$ \\
\hline $\begin{array}{l}0.125 \pi \\
0.250 \pi \\
0.375 \pi \\
0.500 \pi \\
0.625 \pi \\
0.750 \pi \\
0.875 \pi \\
1.000 \pi \\
1.125 \pi \\
1.250 \pi\end{array}$ & $\begin{array}{r}-0.01538 \\
-0.02841 \\
-0.03712 \\
-0.04019 \\
-0.03717 \\
-0.02857 \\
-0.01574 \\
-0.00067 \\
0.01433 \\
0.02700 .\end{array}$ & $\begin{array}{l}-0.00000 \\
-0.00003 \\
-0.00014 \\
-0.00047 \\
-0.00119 \\
-0.00248 \\
-0.00441 \\
-0.00692 \\
-0.00969 \\
-0.01219\end{array}$ \\
\hline
\end{tabular}

$-0.13811$ 
$\begin{array}{lll}0.125 \pi & -0.01159 & -0.00001\end{array}$ $0.250 \pi \quad-0.02141-0.00007$ $0.375 \pi \quad-0.02798-0.00025$ $0.500 \pi-0.03030-0.00066$ $0.625 \pi-0.02804-0.00140$ $0.625 \pi-0.02857-0.00140$ $0.75 \pi \quad-0.02191-0.00258$ $1.000 \pi-0.00055-0.00616$

$1.000 \pi \quad-0.001075-0.00616$

$\begin{array}{lll}1.125 \pi & 0.01077 & -0.00818 \\ 1.250 \pi & 0.02039 & -0.00982\end{array}$

$\begin{array}{lr}-0.00000 & 0.00000 \\ -0.00001 & 0.00000 \\ -0.00004 & 0.00000 \\ -0.00015 & 0.00000 \\ -0.00060 & 0.00002 \\ -0.00371 & 0.00011 \\ -0.01391 & 0.00047 \\ -0.02811 & 0.00104 \\ -0.04281 & 0.00168 \\ -0.05444 & 0.00207 \\ -0.06024 & 0.00168 \\ -0.05893 & -0.00007 \\ -0.00231 & 0.00007 \\ -0.00887 & 0.00029 \\ -0.01868 & 0.00065 \\ -0.03025 & 0.00113 \\ -0.04180 & 0.00164 \\ -0.05160 & 0.00202 \\ -0.05818 & 0.00201 \\ -0.06060 & 0.00134 \\ -0.05859 & -0.00025 \\ -0.05255 & -0.00289\end{array}$

$\begin{array}{lllr}-0.00030 & 0.00000 & -0.00030 & 0.00000 \\ -0.00061 & 0.00000 & -0.00061 & 0.00000 \\ -0.00151 & 0.00000 & -0.00151 & 0.00000 \\ -0.00303 & 0.00000 & -0.00302 & 0.00000 \\ -0.00606 & 0.00000 & -0.00602 & 0.00000 \\ -0.01514 & 0.00002 & -0.01452 & 0.00005 \\ -0.03027 & 0.00018 & -0.02545 & 0.00030 \\ -0.04538 & 0.00060 & -0.03012 & 0.00063 \\ -0.06045 & 0.00142 & -0.02750 & 0.00046 \\ -0.07544 & 0.00277 & -0.01858 & -0.00099 \\ -0.09032 & 0.00477 & -0.00618 & -0.00415 \\ -0.10502 & 0.00755 & 0.00590 & -0.00856 \\ & & & \\ -0.01189 & 0.00001 & -0.01159 & 0.00002 \\ -0.02378 & 0.00009 & -0.02140 & 0.00017 \\ -0.03566 & 0.00029 & -0.02792 & 0.00043 \\ -0.04752 & 0.00069 & -0.03019 & 0.00086 \\ -0.05935 & 0.00135 & -0.02792 & 0.00051 \\ -0.07114 & 0.00232 & -0.02165 & -0.00040 \\ -0.08287 & 0.00368 & -0.01261 & -0.00235 \\ -0.09450 & 0.00548 & -0.00256 & -0.00532 \\ -0.10602 & 0.00777 & 0.00662 & -0.00837 \\ -0.11737 & 0.010 .01 & 0.01338 & -0.01224\end{array}$

$-0.00000$ $-0.0000$ $-0.0000$ $-0.00015$ $-0.00060$ $-0.00371$

$-0.0138$

$-0.02793$ $-0.04221$ $-0.05301$ $-0.05770$

$-0.05564$

$-0.00230$ $-0.00886$ $-0.01862$ $-0.03003$ $-0.05045$ $-0.05621$ $-0.05777$ $-0.0552$ $-0.04967$
0.00000 0.00000 0.00000 0.00000 0.00002 0.00013 0.0007 0.00214 0.00463 0.00783 0.01073 0.00008 0.00038 0.00110 0.00447 0.00689 0.00940 0.01131 0.01204 0.01127
0.00030 $-0.00061 \quad 0.00000$ $\begin{array}{ll}-0.00151 & 0.00000\end{array}$ $\begin{array}{ll}-0.00303 & 0.00000\end{array}$ $-0.01514 \quad 0.00001$ $-0.03023 \quad 0.00071$ $\begin{array}{ll}-0.04516 & 0.00239\end{array}$ $\begin{array}{ll}-0.05973 & 0.00560\end{array}$ $\begin{array}{ll}-0.07358 & 0.01077\end{array}$ $\begin{array}{ll}-0.08617 & 0.01817 \\ -0.09682 & 0.02787\end{array}$ $\begin{array}{ll}-0.01189 & 0.00004\end{array}$ $\begin{array}{lll}-0.02376 & 0.00035\end{array}$ $-0.03557 \quad 0.00116$ $\begin{array}{lll}-0.04725 & 0.00274\end{array}$ $\begin{array}{lll}-0.05869 & 0.00531\end{array}$ $\begin{array}{lll}-0.06970 & 0.00906\end{array}$ $-0.08004 \quad 0.01416$ $\begin{array}{ll}-0.08942 & 0.02068\end{array}$ $-0.09746 \quad 0.02861$ $\begin{array}{ll}-0.10378 & 0.03780\end{array}$

$\begin{array}{lll}0.01 & -0.00017 & 0.00000 \\ 0.02 & -0.00034 & -0.00000 \\ 0.05 & -0.00086 & -0.00000 \\ 0.10 & -0.00172 & -0.00000 \\ 0.20 & -0.00342 & -0.00000 \\ 0.50 & -0.00825 & -0.00002 \\ 1.00 & -0.01448 & -0.00019 \\ 1.50 & -0.01718 & -0.00063 \\ 2.00 & -0.01570 & -0.00145 \\ 2.50 & -0.01045 & -0.00267 \\ 3.00 & -0.00273 & -0.00415 \\ 3.50 & 0.00560 & -0.00556 \\ & & \\ 0.125 \pi & -0.00658 & -0.00001 \\ 0.250 \pi & -0.01217 & -0.00009 \\ 0.375 \pi & -0.01590 & -0.00030 \\ 0.500 \pi & -0.01722 & -0.00072 \\ 0.625 \pi & -0.01595 & -0.00137 \\ 0.750 \pi & -0.01229 & -0.00228 \\ 0.875 \pi & -0.00681 & -0.00339 \\ 1.000 \pi & -0.00034 & -0.00458 \\ 1.125 \pi & 0.00614 & -0.00565 \\ 1.250 \pi & 0.01171 & -0.00632\end{array}$

$$
\frac{\mathrm{a}}{\lambda}=4.5 \quad \frac{\alpha}{\beta}=0.05
$$

$\begin{array}{lr}-0.00000 & 0.00000 \\ -0.00000 & 0.00000 \\ -0.00002 & 0.00000 \\ -0.00009 & 0.00000 \\ -0.00034 & 0.00002 \\ -0.00211 & 0.00011 \\ -0.00791 & 0.00040 \\ -0.01597 & 0.00078 \\ -0.02434 & 0.00110 \\ -0.03099 & 0.00108 \\ -0.03435 & 0.00043 \\ -0.03370 & -0.00110 \\ & \\ -0.00131 & 0.00007 \\ -0.00504 & 0.00025 \\ -0.01061 & 0.00053 \\ -0.01718 & 0.00084 \\ -0.02376 & 0.00108 \\ -0.02936 & 0.00113 \\ -0.03314 & 0.00085 \\ -0.03458 & 0.00009 \\ -0.03351 & -0.00124 \\ -0.03016 & -0.00314\end{array}$

$\begin{array}{rr}-0.00017 & -0.00000 \\ -0.00034 & 0.00000 \\ -0.00086 & 0.00000 \\ -0.00172 & 0.00000 \\ -0.00342 & 0.00000 \\ -0.00825 & 0.00001 \\ -0.01446 & 0.00007 \\ -0.01712 & 0.00006 \\ -0.01566 & -0.00033 \\ -0.01066 & -0.00142 \\ -0.00368 & -0.00335 \\ 0.00317 & -0.00575 \\ & \\ -0.00558 & 0.00001 \\ -0.01215 & 0.00604 \\ -0.01586 & 0.00008 \\ -0.01716 & 0.00003 \\ -0.01590 & -0.00028 \\ -0.01238 & -0.00102 \\ -0.00731 & -0.00229 \\ -0.00164 & -0.00401 \\ 0.00358 & -0.00591 \\ 0.00751 & -0.00756\end{array}$

$\begin{array}{ll}-0.00000 & 0.00000 \\ -0.00000 & 0.00000 \\ -0.00002 & 0.00000 \\ -0.00009 & 0.00000 \\ -0.00034 & 0.00002 \\ -0.00210 & 0.00011 \\ -0.00788 & 0.00053 \\ -0.01585 & 0.00141 \\ -0.02396 & 0.00276 \\ -0.03012 & 0.00434 \\ -0.03287 & 0.00559 \\ -0.03187 & 0.00588 \\ & \\ -0.00131 & 0.00007 \\ -0.00503 & 0.00031 \\ -0.01057 & 0.00079 \\ -0.01704 & 0.00157 \\ -0.02341 & 0.00265 \\ -0.02865 & 0.00389 \\ -0.03197 & 0.00504 \\ -0.03295 & 0.00579 \\ -0.03168 & 0.00585 \\ -0.02865 & 0.00513\end{array}$

$-0.00017$ $-0.00034$ -0.00086
-0.00172 $-0.00172$ $-0.00344$ $-0.00860$ $-0.01716$ $-0.02563$ $-0.03387$ $-0.04168$ -0.04876
-0.05472 $-0.00675$ $-0.01349$ $-0.02019$ $-0.02681$ $-0.03949$ $-0.04532$ $-0.05058$ -0.05508
-0.05860 0.00000
0.00000
0.00000
0.00000
0.00000
0.00005
0.00040
0.00135
0.00317
0.00609
0.01024
0.01567
0.00002
0.00020
0.00066
0.00155
0.00300
0.00512
0.00800
0.01166
0.01609
0.02121 
Bh

$C(h, 0)$

$\begin{array}{lll}0.01 & -0.00010 & 0.00000 \\ 0.02 & -0.00020 & -0.00000 \\ 0.05 & -0.00049 & -0.00000 \\ 0.10 & -0.00098 & -0.00000 \\ 0.20 & -0.00194 & -0.00000 \\ 0.50 & -0.00469 & -0.00002 \\ 1.00 & -0.00823 & -0.00016 \\ 1.50 & -0.00977 & -0.00053 \\ 2.00 & -0.00894 & -0.00116 \\ 2.50 & -0.00595 & -0.00202 \\ 3.00 & -0.00155 & -0.00297 \\ 3.50 & 0.00323 & -0.00374 \\ & & \\ 0.125 \pi & -0.00374 & -0.00001 \\ 0.250 \pi & -0.00691 & -0.00008 \\ 0.375 \pi & -0.00904 & -0.00027 \\ 0.500 \pi & -0.00979 & -0.00060 \\ 0.625 \pi & -0.00907 & -0.00111 \\ 0.750 \pi & -0.00700 & -0.00176 \\ 0.875 \pi & -0.00388 & -0.00250 \\ 1.000 \pi & -0.00018 & -0.00322 \\ 1.125 \pi & 0.00354 & -0.00378 \\ 1.250 \pi & 0.00678 & -0.00402\end{array}$

0.0

$-0.00021-0.00000$

$\begin{array}{lll}0.10 & -0.00042-0.00000\end{array}$

$0.20 \quad-0.00083-0.00000$

$\begin{array}{lll}-0.00201 & -0.00001 \\ 1.00 \quad-0.00353 & -0.0001\end{array}$

$1.50 \quad-0.00419-0.0001$

$\begin{array}{lll}1.00 & -0.00349 & -0.000034\end{array}$

$-0.00255-0.00012$

$3.00 \quad-0.00065-0.00167$

$\begin{array}{rrr}3.00 & -0.00065 & -0.00167 \\ 3.50 & 0.00144 & -0.00198\end{array}$

$0.125 \pi \quad-0.00160-0.00001$

$0.250 \pi \quad-0.00296-0.00005$

$0.375 \pi \quad-0.00387 \quad-0.00017$

$\begin{array}{lll}0.500 \pi & -0.00420 & -0.00038\end{array}$

$0.625 \pi-0.00390-0.00068$

$0.750 \pi-0.00300-0.00105$

$\begin{array}{lll}0.875 \pi & -0.00166 & -0.00144 \\ 1.000 \pi & -0.00005 & -0.00178\end{array}$

$1.125 \pi \quad 0.00158=0.00199$
Sih, 0)

\begin{tabular}{|c|c|c|c|}
\hline $\begin{array}{l}-0.00000 \\
-0.00000 \\
-0.00001 \\
-0.00005 \\
-0.00019 \\
-0.00120 \\
-0.00449 \\
-0.00908 \\
-0.01385 \\
-0.01765 \\
-0.01961 \\
-0.01929\end{array}$ & $\begin{array}{r}0.00000 \\
0.00000 \\
0.00000 \\
0.00000 \\
0.00001 \\
0.00008 \\
0.00030 \\
0.00056 \\
0.00070 \\
0.00056 \\
-0.00005 \\
-0.00122\end{array}$ & $\begin{array}{l}-0.00010 \\
-0.00020 \\
-0.00049 \\
-0.00098 \\
-0.00195 \\
-0.00489 \\
-0.00977 \\
-0.01464 \\
-0.01949 \\
-0.02431 \\
-0.02909 \\
-0.03380\end{array}$ & $\begin{array}{l}C .00000 \\
C .00000 \\
c .00000 \\
c .00000 \\
c .00000 \\
C .00001 \\
c .00006 \\
c .00019 \\
C .00046 \\
0.00089 \\
c .00153 \\
c .00242\end{array}$ \\
\hline $\begin{array}{l}-0.00074 \\
-0.00286 \\
-0.00603 \\
-0.00977 \\
-0.01352 \\
-0.01672 \\
-0.01889 \\
-0.01975 \\
-0.01919 \\
-0.01731\end{array}$ & $\begin{array}{r}0.00005 \\
0.00019 \\
0.00039 \\
0.00059 \\
0.00070 \\
0.00064 \\
0.00032 \\
-0.00033 \\
-0.00133 \\
-0.00264\end{array}$ & $\begin{array}{l}-0.00384 \\
-0.00767 \\
-0.01150 \\
-0.01533 \\
-0.01914 \\
-0.02293 \\
-0.02670 \\
-0.03043 \\
-0.03411 \\
-0.03774 .\end{array}$ & $\begin{array}{l}0.00000 \\
0.00003 \\
0.00009 \\
0.00022 \\
0.00043 \\
0.00075 \\
0.00118 \\
0.00176 \\
0.000249 \\
0.00340\end{array}$ \\
\hline
\end{tabular}

$\frac{a}{\lambda}=4$

$\frac{\alpha}{\beta}=0.10$
$\mathrm{C}(\mathrm{h}, \mathrm{h})$

$\begin{array}{ll}-0.00010 & -0.00000 \\ -0.00020 & 0.00000 \\ -0.00049 & 0.00000 \\ -0.00098 & 0.00000 \\ -0.00194 & 0.00000 \\ -0.00468 & -0.00000 \\ -0.00821 & -0.00002 \\ -0.00973 & -0.00014 \\ -0.00892 & -0.00052 \\ -0.00611 & -0.00130 \\ -0.00217 & -0.00247 \\ 0.00174 & -0.00378 \\ & \\ -0.00374 & -0.00000 \\ -0.00690 & -0.00001 \\ -0.00901 & -0.00004 \\ -0.00976 & -0.00017 \\ -0.00906 & -0.00048 \\ -0.00708 & -0.00103 \\ -0.00422 & -0.00184 \\ -0.00101 & -0.00284 \\ 0.00197 & -0.00385 \\ 0.00426 & -0.00465\end{array}$

$S(h, h)$

$-0.00000$ $-0.00000$ $-0.00001$ $-0.00005$ $-0.00019$ $-0.00120$ $-0.00448$ $-0.00900$ $-0.01361$ $-0.01713$ $-0.01875$ $-0.01827$

\section{$-0.00074$} $-0.00286$ $-0.00600$ $-0.00968$ $-0.01329$ $-0.01820$ $-0.01882$ $-0.01816$ $-0.01652$

0.00000
0.00000
0.00000
0.00000
0.00001
0.00009
0.00038
0.00091
0.00164
0.00241
0.00289
0.00280
0.00005
0.00023
0.00054
0.00100
0.00159
0.00220
0.00270
0.00293
0.00277
0.00220

.00000 00000 00001 .00009 .00091 00241 .00005 0054 .00159 .00270 0.00277 0.00220
$E(h, h)$

$\begin{array}{ll}-0.00010 & 0.00000 \\ -0.00020 & 0.00000 \\ -0.00049 & 0.00000 \\ -0.00098 & 0.00000 \\ -0.00195 & 0.00000 \\ -0.00488 & 0.00003 \\ -0.00775 & 0.00023 \\ -0.01454 & 0.00077 \\ -0.01921 & 0.00180 \\ -0.02361 & 0.00344 \\ -0.02759 & 0.00578 \\ -0.03093 & 0.00881 \\ -0.00384 & 0.00001 \\ -0.00766 & 0.00011 \\ -0.01146 & 0.00037 \\ -0.01521 & 0.00088 \\ -0.01887 & 0.00170 \\ -0.02238 & 0.00290 \\ -0.02566 & 0.00451 \\ -0.02861 & 0.00657 \\ -0.03113 & 0.00905 \\ -0.03309 & 0.01190\end{array}$

\begin{tabular}{llllll}
-0.00004 & -0.00000 & -0.00000 & 0.00000 & -0.00004 & 0.00000 \\
-0.00008 & -0.00000 & -0.00000 & 0.00000 & -0.00008 & 0.00000 \\
-0.00021 & -0.00000 & -0.00001 & 0.00000 & -0.00021 & 0.00000 \\
-0.00042 & -0.00000 & -0.00002 & 0.00000 & -0.00042 & 0.00000 \\
-0.00083 & -0.00000 & -0.00008 & 0.00001 & -0.00084 & 0.00000 \\
-0.00201 & -0.00001 & -0.00051 & 0.00005 & -0.00209 & 0.00001 \\
-0.00352 & -0.00005 & -0.00192 & 0.00021 & -0.00417 & 0.00010 \\
-0.00417 & -0.00017 & -0.00385 & 0.00046 & -0.00622 & 0.00033 \\
-0.00384 & -0.00044 & -0.00583 & 0.00075 & -0.00820 & 0.00076 \\
-0.00265 & -0.00087 & -0.00736 & 0.00099 & -0.01007 & 0.00146 \\
-0.00097 & -0.00142 & -0.00809 & 0.00106 & -0.01174 & 0.00245 \\
0.00073 & -0.00195 & -0.00794 & 0.00085 & -0.01314 & 0.00372 \\
& & & & & \\
-0.00160 & -0.00000 & -0.00032 & 0.00003 & -0.00164 & 0.00001 \\
-0.00296 & -0.00002 & -0.00122 & 0.00013 & -0.00328 & 0.00005 \\
-0.00386 & -0.00008 & -0.00257 & 0.00029 & -0.00490 & 0.00016 \\
-0.00418 & -0.00020 & -0.00414 & 0.00050 & -0.00650 & 0.00038 \\
-0.00389 & -0.00041 & -0.00570 & 0.00073 & -0.00806 & 0.00072 \\
-0.00306 & -0.00073 & -0.00699 & 0.00094 & -0.00955 & 0.00123 \\
-0.00184 & -0.00113 & -0.00783 & 0.00106 & -0.01093 & 0.00191 \\
-0.00047 & -0.00158 & -0.00813 & 0.00103 & -0.01217 & 0.00278 \\
0.00083 & -0.00198 & -0.00790 & 0.00083 & -0.01322 & 0.00381 \\
0.00186 & -0.00224 & -0.00723 & 0.00048 & -0.01404 & 0.00500 \\
\hline & & & & &
\end{tabular}

0.00000

0.00000

0.00000

0.00000

0.00002

0.0002

0.00103

0.00000

0.0000

0.00004

0.00019

0.00032

0.0005

0.00106

0.00145 
$\frac{a}{\lambda}=4.5 \quad \frac{\alpha}{\beta}=0.20$

Bh

$\mathrm{C}(\mathrm{h}, 0)$

0.01

0.02

0.05

0.10

0.20

0.50

1.00

.00

.50

3.00

$0.125 \pi$
$0.250 \pi$

$0.375 \pi$

$0.500 \pi$

$0.625 \pi$

$0.875 \pi$

$1.000 \pi$

$1.125 \pi$

$1.250 \pi$
$S(h, 0)$

$\begin{array}{ll}-0.00000 & 0.00000 \\ -0.00000 & 0.00000 \\ -0.00000 & 0.00000 \\ -0.000 .00 & 0.00000 \\ -0.00000 & 0.00000 \\ -0.00003 & 0.00001 \\ -0.00011 & 0.00002 \\ -0.00023 & 0.00003 \\ -0.00036 & 0.00003 \\ -0.00047 & 0.00000 \\ -0.00053 & -0.00005 \\ -0.00053 & -0.00013 \\ & \\ -0.00002 & 0.00000 \\ -0.00007 & 0.00001 \\ -0.00015 & 0.00003 \\ -0.00025 & 0.00003 \\ -0.00035 & 0.00003 \\ -0.00044 & 0.00002 \\ -0.00050 & -0.00002 \\ -0.00053 & -0.00007 \\ -0.00052 & -0.00014 \\ -0.00047 & -0.00022\end{array}$

$E(h, 0)$

$\begin{array}{ll}-0.00000 & 0.00000\end{array}$

$-0.00000 \quad 0.00000$

$\begin{array}{ll}-0.00001 & 0.00000\end{array}$

$-0.00002 \quad 0.00000$

$\begin{array}{ll}-0.00005 & 0.00000 \\ -0.00012 & 0.00000\end{array}$

$-0.00025 \quad 0.00000$

$\begin{array}{ll}-0.00037 & 0.00000\end{array}$

$\begin{array}{ll}-0.00049 & 0.00001\end{array}$

$\begin{array}{ll}-0.00061 & 0.00002 \\ -0.00073 & 0.00004\end{array}$

$-0.000850 .00006$

$\begin{array}{ll}-0.00010 & 0.00000\end{array}$

-0.00019
-0.00029

$-0.00039 \quad 0.00001$

$-0.00048 \quad 0.00001$

$-0.00058 \quad 0.00002$

$-0.00067 \quad 0.00003$

$-0.00077 \quad 0.00004$

$-0.00086 \quad 0.00006$

$\begin{array}{ll}-0.00077 & 0.00004 \\ -0.00095 & 0.000006\end{array}$
$\mathrm{C}(\mathrm{h}, \mathrm{h})$

$\begin{array}{ll}-0.00000 & -0.00000 \\ -0.00002 & -0.00000 \\ -0.00001 & -0.00000 \\ -0.00002 & -0.00000 \\ -0.00005 & -0.00000 \\ -0.00012 & -0.00000 \\ -0.00021 & -0.00001 \\ -0.00025 & -0.00003 \\ -0.00023 & -0.00007 \\ -0.00016 & -0.00011 \\ -0.00006 & -0.00016 \\ 0.00005 & -0.00019 \\ & \\ -0.00009 & -0.00000 \\ -0.00018 & -0.00001 \\ -0.00023 & -0.00002 \\ -0.00025 & -0.00004 \\ -0.00023 & -0.00007 \\ -0.00018 & -0.00010 \\ -0.00011 & -0.00014 \\ -0.00002 & -0.00017 \\ 0.00006 & -0.00019 \\ 0.00014 & -0.00019\end{array}$

$S(h, h)$

$\begin{array}{lr}-0.00000 & 0.00000 \\ -0.00000 & 0.00000 \\ -0.00000 & 0.00000 \\ -0.00000 & 0.00000 \\ -0.00000 & 0.00000 \\ -0.00003 & 0.00001 \\ -0.00011 & 0.00002 \\ -0.00023 & 0.00004 \\ -0.00035 & 0.00005 \\ -0.00045 & 0.00005 \\ -0.00050 & 0.00003 \\ -0.00050 & -0.00002 \\ & \\ -0.00002 & 0.00000 \\ -0.00007 & 0.00001 \\ -0.00015 & 0.00003 \\ -0.00025 & 0.00004 \\ -0.00034 & 0.00005 \\ -0.00042 & 0.00006 \\ -0.00048 & 0.00004 \\ -0.00050 & 0.00002 \\ -0.00050 & -0.00002 \\ -0.00046 & -0.00007\end{array}$

$E(h, h)$

$\begin{array}{ll}-0.00000 & 0.00000 \\ -0.00000 & 0.00000 \\ -0.00001 & 0.00000 \\ -0.00002 & 0.00000 \\ -0.00005 & 0.00000 \\ -0.00012 & 0.00000 \\ -0.00025 & 0.00001 \\ -0.00037 & 0.00002 \\ -0.00048 & 0.00004 \\ -0.00059 & 0.00008 \\ -0.00068 & 0.00014 \\ -0.00076 & 0.00021 \\ & \\ -0.00010 & 0.00000 \\ -0.00019 & 0.00000 \\ -0.00029 & 0.00001 \\ -0.00038 & 0.00002 \\ -0.00047 & 0.00004 \\ -0.00056 & 0.00007 \\ -0.00064 & 0.00011 \\ -0.00071 & 0.00016 \\ -0.00076 & 0.00021 \\ -0.00081 & 0.00028\end{array}$

$$
\frac{a}{\lambda}=4.5 \quad \frac{\alpha}{\beta}=0.40
$$

$\begin{array}{ll}-0.00000 & 0.00000 \\ -0.00000 & 0.00000 \\ -0.00000 & 0.00000 \\ -0.00000 & 0.00000 \\ -0.00000 & 0.00000 \\ -0.00000 & 0.00000 \\ -0.00000 & 0.00000 \\ -0.00000 & 0.00000 \\ -0.00000 & 0.00000 \\ -0.00000 & -0.00000 \\ -0.00000 & -0.00000 \\ -0.00000 & -0.00000 \\ & \\ -0.00000 & 0.00000 \\ -0.00000 & 0.00000 \\ -0.00000 & 0.00000 \\ -0.00000 & 0.00000 \\ -0.00000 & 0.00000 \\ -0.00000 & 0.00000 \\ -0.00000 & -0.00000 \\ -0.00000 & -0.00000 \\ -0.00000 & -0.00000 \\ -0.00000 & -0.00000\end{array}$

$\begin{array}{ll}-0.00000 & 0.00000 \\ -0.00000 & 0.00000 \\ -0.00000 & 0.00000 \\ -0.00000 & 0.00000 \\ -0.00000 & 0.00000 \\ -0.00000 & 0.00000 \\ -0.00000 & 0.00000 \\ -0.00000 & 0.00000 \\ -0.00000 & 0.00000 \\ -0.00000 & 0.00000 \\ -0.00000 & 0.00000 \\ -0.00000 & 0.00000 \\ -0.00000 & 0.00000 \\ -0.00000 & 0.00000 \\ -0.00000 & 0.00000 \\ -0.00000 & 0.00000 \\ -0.00000 & 0.00000 \\ -0.00000 & 0.00000 \\ -0.00000 & 0.00000 \\ -0.00000 & 0.00000 \\ -0.00000 & 0.00000 \\ -0.00000 & 0.00000\end{array}$

$\begin{array}{lr}-0.00000 & 0.00000 \\ -0.00000 & 0.00000 \\ -0.00000 & 0.00000 \\ -0.00000 & 0.00000 \\ -0.00000 & 0.00000 \\ -0.00000 & 0.00000 \\ -0.00000 & 0.00000 \\ -0.00000 & 0.00000 \\ -0.00000 & 0.00000 \\ -0.00000 & 0.00000 \\ -0.00000 & -0.00000 \\ -0.00000 & -0.00000 \\ -0.00000 & 0.00000 \\ -0.00000 & 0.00000 \\ -0.00000 & 0.00000 \\ -0.00000 & 0.00000 \\ -0.00000 & 0.00000 \\ -0.00000 & 0.00000 \\ -0.00000 & 0.00000 \\ -0.00000 & -0.00000 \\ -0.00000 & -0.00000 \\ -0.00000 & -0.00000\end{array}$

$\begin{array}{ll}-0.00000 & 0.00000 \\ -0.00000 & 0.00000 \\ -0.00000 & 0.00000 \\ -0.00000 & 0.00000 \\ -0.00000 & 0.00000 \\ -0.00000 & 0.00000 \\ -0.00000 & 0.00000 \\ -0.00000 & 0.00000 \\ -0.00000 & 0.00000 \\ -0.00000 & 0.00000 \\ -0.00000 & 0.00000 \\ -0.00000 & 0.00000 \\ & \\ -0.00000 & 0.00000 \\ -0.00000 & 0.00000 \\ -0.00000 & 0.00000 \\ -0.00000 & 0.00000 \\ -0.00000 & 0.00000 \\ -0.00000 & 0.00000 \\ -0.00000 & 0.00000 \\ -0.00000 & 0.00000 \\ -0.00000 & 0.00000 \\ -0.00000 & 0.00000\end{array}$

.00000
.00000
.00000
.00000
.00000
.00000
.00000
.00000
.00000
.00000
.00000
.00000
.00000
.00000
.00000
.00000
.00000
.00000
.00000
.00000
.00000
.00000

$\begin{array}{lrl}0.01 & -0.00000 & -0.00000 \\ 0.02 & -0.00000 & -0.00000 \\ 0.05 & -0.00000 & -0.00000 \\ 0.10 & -0.00000 & -0.00000 \\ 0.20 & -0.00000 & -0.00000 \\ 0.50 & -0.00000 & -0.00000 \\ 1.00 & -0.00000 & -0.00000 \\ 1.50 & -0.00000 & -0.00000 \\ 2.00 & -0.00000 & -0.00000 \\ 2.50 & -0.00000 & -0.00000 \\ 3.00 & 0.00000 & -0.00000 \\ 3.50 & 0.00000 & -0.00000 \\ 0.125 \pi & -0.00000 & -0.00000 \\ 0.250 \pi & -0.00000 & -0.00000 \\ 0.375 \pi & -0.00000 & -0.00000 \\ 0.500 \pi & -0.00000 & -0.00000 \\ 0.625 \pi & -0.00000 & -0.00000 \\ 0.750 \pi & -0.00000 & -0.00000 \\ 0.875 \pi & -0.00000 & -0.00000 \\ 1.000 \pi & 0.00000 & -0.00000 \\ 1.125 \pi & 0.00000 & -0.00000 \\ 1.250 \pi & 0.00000 & -0.00000\end{array}$

$\begin{array}{rr}-0.00060 & -0.00000 \\ -0.00000 & -0.00000 \\ -0.00000 & -0.00000 \\ -0.00000 & -0.00006 \\ -0.00000 & -0.00000 \\ -0.00000 & -0.00000 \\ -0.00000 & -0.00000 \\ -0.00000 & -0.00000 \\ -0.00000 & -0.00000 \\ -0.00000 & -0.00000 \\ -0.00000 & -0.00000 \\ 0.00000 & -0.00000 \\ -0.00000 & -0.00000 \\ -0.00000 & -0.00000 \\ -0.00000 & -0.00000 \\ -0.00000 & -0.00000 \\ -0.00000 & -0.00000 \\ -0.00000 & -0.00000 \\ -0.00000 & -0.00000 \\ 0.00000 & -0.00000 \\ 0.00000 & -0.00000 \\ 0.00000 & -0.00000\end{array}$


$\beta \mathrm{h}$

0.01

0.02

0.05

0.10

0.20
0.50

1.00
1.50

1.50

2.00

3.00
3.50

$0.125 \pi$

$0.375 \pi$

$0.500 \pi$

$0.625 \pi$

$0.750 \pi$
$0.875 \pi$

$1.000 \pi$

$1.125 \pi$

$1.250 \pi$
$\mathrm{C}(\mathrm{h}, 0)$

$\begin{array}{rr}-0.00000 & -0.00000 \\ -0.00000 & -0.00000 \\ -0.00000 & -0.00000 \\ -0.00000 & -0.00000 \\ -0.00000 & -0.00000 \\ -0.00000 & -0.00000 \\ -0.00000 & -0.00000 \\ -0.00000 & -0.00000 \\ 0.00000 & -0.00000 \\ 0.00000 & -0.00000 \\ 0.00000 & 0.00000 \\ 0.00000 & 0.00000 \\ -0.00000 & -0.00000 \\ -0.00000 & -0.00000 \\ -0.00000 & -0.00000 \\ -0.00000 & -0.00000 \\ 0.00000 & -0.00000 \\ 0.00000 & -0.00000 \\ 0.00000 & 0.00000 \\ 0.00000 & 0.00000 \\ 0.00000 & 0.00000 \\ 0.000000 & 0.00000\end{array}$

$\mathrm{S}(\mathrm{h}, 0)$

$\begin{array}{rr}-0.00000 & 0.00000 \\ -0.00000 & 0.00000 \\ -0.00000 & 0.00000 \\ -0.00000 & 0.00000 \\ -0.00000 & 0.00000 \\ -0.00000 & 0.00000 \\ -0.00000 & 0.00000 \\ -0.00000 & 0.00000 \\ -0.00000 & -0.00000 \\ -0.00000 & -0.00000 \\ 0.00000 & -0.00000 \\ 0.00000 & -0.00000 \\ -0.00000 & 0.00000 \\ -0.00000 & 0.00000 \\ -0.00000 & 0.00000 \\ -0.00000 & 0.00000 \\ -0.00000 & -0.00000 \\ -0.00000 & -0.00000 \\ -0.00000 & -0.00000 \\ 0.00000 & -0.00000 \\ 0.00000 & -0.00000 \\ 0.00000 & -0.00000\end{array}$

$\frac{\mathrm{a}}{\lambda}=4.5 \quad \frac{\alpha}{\bar{\beta}}=0.70$

$E(h, 0)$

$\mathrm{C}(\mathrm{h}, \mathrm{h})$

$S(h, h)$

$E(h, h)$

$\begin{array}{ll}-0.00000 & 0.00000 \\ -0.00000 & 0.00000 \\ -0.00000 & 0.00000 \\ -0.00000 & 0.00000 \\ -0.00000 & 0.00000 \\ -0.00000 & 0.00000 \\ -0.00000 & 0.00000 \\ -0.00000 & 0.00000 \\ -0.00000 & 0.00000 \\ -0.00000 & 0.00000 \\ -0.00000 & 0.00000 \\ -0.00000 & 0.00000 \\ -0.000000 & 0.00000 \\ -0.00000 & 0.00000 \\ -0.00000 & 0.00000 \\ -0.00000 & 0.00000 \\ -0.00000 & 0.00000 \\ -0.00000 & 0.00000 \\ -0.00000 & 0.00000 \\ -0.00000 & 0.00000 \\ -0.00000 & 0.00000 \\ -0.00000 & 0.00000\end{array}$

$-0.00000-0.00000$

$-0.00000-0.00000$

$-0.00000-0.00003$

$-0.00000-0.00003$

$-0.00000-0.00000$

$-0.00000-0.00000$

$-0.00000-0.00000$

$0.00000-0.00000$

$0.00000-0.00000$

$0.00000 \quad 0.00000$

$0.00000 \quad 0.00000$

$-0.00000-0.00000$

$-0.00000-0.00000$

$-0.00000-0.00000$

$0.00000-0.00000$

$0.00000-0.00000$

$0.00000-0.00000$

$0.00000-0.00000$

$0.00000 \quad 0.00000$

$\begin{array}{ll}0.00000 & 0.00000 \\ 0.00000 & 0.00000\end{array}$

$\begin{array}{rr}-0.00000 & 0.00000 \\ -0.00000 & 0.00000 \\ -0.00000 & 0.00000 \\ -0.00000 & 0.00000 \\ -0.00000 & 0.00000 \\ -0.00000 & 0.00000 \\ -0.00000 & 0.00000 \\ -0.00000 & 0.00000 \\ -0.00000 & -0.00000 \\ -0.00000 & -0.00000 \\ 0.00000 & -0.00000 \\ 0.00000 & -0.00000 \\ -0.00000 & 0.00000 \\ -0.00000 & 0.00000 \\ -0.00000 & 0.00000 \\ -0.00000 & 0.00000 \\ -0.00000 & -0.00000 \\ -0.00000 & -0.00000 \\ -0.00000 & -0.00000 \\ 0.00000 & -0.00000 \\ 0.00000 & -0.00000 \\ 0.00000 & -0.00000\end{array}$

$-0.00000$

$-0.00000$

$-0.00000$

$-0.00000$

$-0.00000$

$-0.00000$

$-0.00000$

$-0.00000$

$-0.00000$

$-0.00000$

$-0.00000$

$-0.00000$

$-0.00000$

$-0.00000$

$-0.00000$

$-0.00000$

$-0.00000$

$-0.00000$

$-0.00000$

$-0.00000$

$-0.00000$
0.00000 0.00000 0.00000 0.00000 0.00000 0.00000 0.00000 0.00000 0.00000 0.00000 0.000000

0.00000 0.00000 0.00000 0.00000 0.00000 0.00000 0.00000 0.00000 0.00000 0.00000

$\begin{array}{lll}0.01 & -0.00000 & -0.00000 \\ 0.02 & -0.00000 & -0.00000\end{array}$

0.02

0.05

0.20

0.50

1.00

1.50

2.00
2.50

2.50
3.00

3.50

$-0.00000-0.00000$

$-0.00000-0.00000$

$-0.00000-0.00000$

$-0.00000-0.00000$

$-0.00000-0.00000$

$-0.00000-0.00000$

$\begin{array}{ll}0.00000 & -0.00000 \\ 0.00000 & -0.00000\end{array}$

$0.00000-0.00000$

$0.00000-0.00000$

$\begin{array}{lll}0.125 \pi & -0.00000 & -0.00000\end{array}$

$0.250 \pi \quad-0.00000-0.00000$

$0.375 \pi-0.00000-0.00000$

$0.500 \pi-0.00000-0.00000$

$0.625 \pi \quad 0.00000-0.00000$

$0.750 \pi \quad 0.00000-0.00000$

$0.875 \pi \quad 0.00000-0.00000$

$\begin{array}{lll}1.000 \pi & 0.00000 & -0.00000 \\ 1.125 \pi & 0.00000 & -0.00000\end{array}$

$$
\frac{a}{\lambda}=4 . E \quad \frac{\alpha}{\beta}=1.00
$$

$\begin{array}{rr}-0.00000 & 0.00000 \\ -0.00000 & 0.00000 \\ -0.00000 & 0.00000 \\ -0.00000 & 0.00000 \\ -0.00000 & 0.00000 \\ -0.00000 & 0.00000 \\ -0.00000 & 0.00000 \\ -0.00000 & 0.00000 \\ -0.00000 & -0.00000 \\ -0.00000 & -0.00000 \\ -0.00000 & -0.00000 \\ -0.00000 & -0.00000 \\ -0.00000 & 0.00000 \\ -0.00000 & 0.00000 \\ -0.00000 & 0.00000 \\ -0.00000 & 0.00000 \\ -0.00000 & -0.00000 \\ -0.00000 & -0.00000 \\ -0.00000 & -0.00000 \\ -0.00000 & -0.00000 \\ -0.00000 & -0.00000 \\ 0.00000 & -0.00000\end{array}$

$\begin{array}{ll}-0.00000 & 0.00000 \\ -0.00000 & 0.00000 \\ -0.00000 & 0.00000 \\ -0.00000 & 0.00000 \\ -0.00000 & 0.00000 \\ -0.00000 & 0.00000 \\ -0.00000 & 0.00000 \\ -0.00000 & 0.00000 \\ -0.00000 & 0.00000 \\ -0.00000 & 0.00000 \\ -0.00000 & 0.00000 \\ -0.00000 & 0.00000 \\ -0.00000 & 0.00000 \\ -0.00000 & 0.00000 \\ -0.00000 & 0.00000 \\ -0.00000 & 0.00000 \\ -0.00000 & 0.00000 \\ -0.00000 & 0.000 .00 \\ -0.00000 & 0.000 .00 \\ -0.00000 & 0.00000 \\ -0.00000 & 0.00000 \\ -0.00000 & 0.03000\end{array}$

$-0.00000-0.00000$ $-0.00000-0.00000$ $-0.00000-0.00000$ $-0.00000-0.00000$ $-0.00000-0.00000$ $-0.00000-0.00000$ $-0.00000-0.00000$ $-0.00000-0.00000$ $0.00000-0.00000$ $0.00000-0.00000$ $0.00000-0.00000$ $0.00000-0.00000$

$-0.00000-0.00000$ $-0.00000-0.00000$ $-0.00000 .-0.00000$ $-0.00000-0.00000$ $0.00000-0.00000$ $0.00000-0.00000$ $0.00000-0.00000$ $0.00000-0.00000$ $0.00000-0.00000$ $0.00000-0.00000$

$\begin{array}{lr}-0.00000 & 0.00000 \\ -0.00000 & 0.00000 \\ -0.00000 & 0.00000 \\ -0.00000 & 0.00000 \\ -0.00000 & 0.00000 \\ -0.00000 & 0.00000 \\ -0.00000 & 0.00000 \\ -0.00000 & 0.00000 \\ -0.00000 & -0.00000 \\ -0.00000 & -0.00000 \\ -0.00000 & -0.00000 \\ -0.00000 & -0.00000 \\ -0.00000 & 0.00000 \\ -0.00000 & 0.00000 \\ -0.00000 & 0.00000 \\ -0.00000 & 0.00000 \\ -0.00000 & -0.00000 \\ -0.00000 & -0.00000 \\ -0.00000 & -0.00000 \\ -0.00000 & -0.00000 \\ -0.00000 & -0.00000 \\ -0.00000 & -0.00000\end{array}$

$-0.00000$ $-0.00000$ $-0.00000$ $-0.00000$ $-0.00000$ $-0.00000$ $-0.00000$ $-0.00000$ $-0.00000$ $-0.00000$ $-0.00000$ $-0.00000$

$-0.00000$ $-0.00000$ $-0.00000$

$-0.00000$ $-0.00000$ $-0.00000$ $-0.00000$ $-0.00000$

$-0.00000$

0.00000 0.00000 0.00000 0.00000 0.00000 0.00000 0.00000 0.00000 0.00000 0.00000 0.00000 0.00000 0.00000 0.00000 0.00000 0.00000 0.00000 0.00000 0.00000 0.00000 0.00000 
$\mathrm{C}(\mathrm{h}, 0)$

0.0

0.02

0.05

0.20

0.50

1.00

1.50

2.50

3.00

3.50

$0.125 \pi$

$0.250 \pi$

$0.375 \pi$

$0.500 \pi$

$0.625 \pi$

$0.750 \pi$

$0.875 \pi$

$1.000 \pi$

$1.125 \pi$
$1.250 \pi$
$S(h, C)$

$0.00000-0.00000$ $0.00001-0.00000$ $\begin{array}{ll}0.00001 & -0.00000 \\ 0.00008 & -c .00000\end{array}$ $0.00032-0.00000$ $0.00127-0.00000$ $0.00779-0.00002$ $0.02926-10.00023$ $0.05912-0.00099$ $0.09003-0.00250$ $0.11440-0.00444$ $\begin{array}{ll}0.12629 & -0.00583 \\ 0.12296 & -0.00519\end{array}$

$0.00485-0.00001$ $0.01864-0.00009$ $0.03928-0.00042$ $0.06361-0.00116$ $0.08790-0.00236$ $0.10845-0.00388$ $0.12214-0.00529$ $0.12692-0.00592$ $0.12218-0.00504$ $0.10882-0.00201$

$\begin{array}{lrrrr}0.01 & 0.00054 & -0.00000 & 0.00000 & -0.00000 \\ 0.02 & 0.00109 & -0.00000 & 0.00001 & -0.00000 \\ 0.05 & 0.00272 & -0.00000 & 0.00007 & -0.00000 \\ 0.10 & 0.00543 & -0.00000 & 0.00027 & -0.00000 \\ 0.20 & 0.01081 & -0.00000 & 0.00108 & -0.00001 \\ 0.50 & 0.02608 & -0.00002 & 0.00666 & -0.00005 \\ 1.00 & 0.04577 & -0.00013 & 0.02500 & -0.00030 \\ 1.50 & 0.05426 & -0.00016 & 0.05052 & -0.00100 \\ 2.00 & 0.04949 & 0.00034 & 0.07693 & -0.00224 \\ 2.50 & 0.03270 & 0.00196 & 0.09777 & -0.00371 \\ 3.00 & 0.00813 & 0.00510 & 0.10797 & -0.00456 \\ 3.50 & -0.01811 & 0.00949 & 0.10520 & -0.00359 \\ 0.125 \pi & 0.02082 & -0.00001 & 0.00414 & -0.00003 \\ 0.250 \pi & 0.03847 & -0.00007 & 0.01593 & -0.00015 \\ 0.375 \pi & 0.05026 & -0.00016 & 0.03357 & -0.00048 \\ 0.500 \pi & 0.05440 & -0.00013 & 0.05436 & -0.00114 \\ 0.625 \pi & 0.05028 & 0.00027 & 0.07512 & -0.00214 \\ 0.750 \pi & 0.03856 & 0.00135 & 0.09268 & -0.00331 \\ 0.875 \pi & 0.02109 & 0.00333 & 0.10440 & -0.00428 \\ 1.000 \pi & 0.00058 & 0.00624 & 0.10852 & -0.00452 \\ 1.125 \pi & -0.01980 & 0.00981 & 0.10454 & -0.00343 \\ 1.250 \pi & -0.03694 & 0.01342 & 0.09320 & -0.00052\end{array}$

$E(h, 0)$

$\mathrm{C}(\mathrm{h}, \mathrm{h})$

$0.00064-0.00000$ $0.00127-0.00000$ $0.00318-0.00000$ $0.00637-0.00000$ $0.01273-0.00000$ $0.03183-0.00004$ $0.06365-0.00034$ $0.09544-0.00114$ 0.00270 $0.15883-0.00526$ $\begin{array}{ll}0.19031 & -0.00907 \\ 0.22152 & -0.01436\end{array}$

$0.02500-0.00002$ $0.04999-0.00016$ $0.07498-0.00055$ $0.07994-0.0013$ $0.12487-0.0025$ $0.14974-0.00440$ $0.17453-0.00698$ $0.19918-0.0104$ $0.22365-0.01478$ $0.24787-0.02022$

$-\frac{a}{\lambda}=5.0 \quad \frac{\alpha}{\beta}=0.005$

$\begin{array}{ll}0.00054 & -0.00000 \\ 0.00109 & -0.00000 \\ 0.00272 & -0.00000 \\ 0.00544 & -0.00000 \\ 0.01088 & -0.00000 \\ 0.02720 & -0.00004 \\ 0.05440 & -0.00029 \\ 0.08157 & -0.00097 \\ 0.10869 & -0.00230 \\ 0.13572 & -0.00449 \\ 0.16261 & -0.00775 \\ 0.18926 & -0.01227 \\ 0.02137 & -0.00002 \\ 0.04273 & -0.00014 \\ 0.06408 & -0.00047 \\ 0.08541 & -0.00112 \\ 0.10671 & -0.00218 \\ 0.12796 & -0.00376 \\ 0.14913 & -0.00597 \\ 0.17018 & -0.00889 \\ 0.19108 & -0.01263 \\ 0.21175 & -0.017 .27\end{array}$

$0.00054-0.00000$ $0.00109-0.00000$ $0.00272-0.00000$ $0.00543-0.00000$ $0.01081-0.00001$ $0.02608-0.00013$ $0.04573-0.00085$ $0.05412-0.00209$ $0.0492 .3-0.00280$ $0.03285-0.00137$ $\begin{array}{rr}0.00983 & 0.00341 \\ -0.01287 & 0.01125\end{array}$

$0.02082-0.00006$ $0.03845-0.00045$ $0.05019-0.00127$ $0.05424-0.00220$ $0.05006-0.00280$ $0.03852-0.00209$ $0.02181 \quad 0.00056$ $0.00306 \quad 0.00538$ $-0.01424 \quad 0.01186$
$S(h, h)$

$E(h, h)$

$0.00000-0.00000$ $0.00001-0.00000$ $0.00008-0.00000$ $0.00032-0.00000$ $0.00127-0.00000$ $0.00779-0.00005$ $0.02923-0.00069$ $0.05891-0.00309$ $0.08920-0.00813$ $0.11219-0.01548$ $0.12206-0.02312$ $0.11706-0.02797$

$0.00485-0.00002$ $0.01863-0.00027$ $0.03922-0.00128$ $0.06335-0.00363$ $0.08714-0.00767$ $0.10673-0.01322$ $0.11897-0.01943$ $0.12210-0.02492$ $\begin{array}{ll}0.11622 & -0.02812 \\ 0.10328 & -0.02789\end{array}$ $.00001-0.00000$ $0.00007-0.00000$ $0.00027-0.00000$ $0.00108-0.00001$ $0.00666-0.00007$ $0.02497-0.00069$ $0.05032-0.00279$ $0.07619-0.00705$ $0.09584-0.01314$ $0.10433-0.01934$ $0.10020-0.02310$

$0.00414-0.00004$ $0.01592-0.00030$ $0.03351-0.00122$ $0.05412-0.00326$ $0.07444-0.00667$ $0.09117-0.01128$ $0.10165-0.01636$ $0.10440-0.02077$

$\begin{array}{ll}0.09950 & -0.02321 \\ 0.08859 & -0.02278\end{array}$
$0.00064-0.00000$ $0.00127-0.00000$ $0.00318-0.00000$ $0.00637-0.00000$ $0.01273-0.00001$ $0.01273-0.00001$ $0.06359-0.00135$ $0.09515-0.00453$ $0.12617-0.01068$ $0.15603-0.02064$ $0.18382-0.03507$ $0.20828-0.05431$

$0.02500-0.00008$ $0.04997-0.00065$ $\begin{array}{lll}0.07487 & -0.00220\end{array}$ $0.09959-0.00520$ $0.12393-0.01011$ $0.14761-0.01734$ $0.17020-0.02723$ $0.19115-0.04003$ $\begin{array}{ll}0.20980 & -0.05581 \\ 0.22538 & -0.07444\end{array}$

$0.00054-0.00000$ $0.00109-0.00000$ $0.00272-0.00000$ $0.00544-0.00000$ $0.01088-0.00001$ $0.02720-0.00014$ $0.05434-0.00115$ $-0.00387$ $0.10778-0.00912$ $0.13327-0.01762$ $\begin{array}{ll}0.15696 & -0.02992 \\ 0.17779 & -0.04631\end{array}$

$0.02136-0.00007$ $0.04270-0.00056$ $0.06398-0.00188$ $0.08509-0.00444$ $0.10588-0.00864$ $0.12608-0.01480$ $0.14534-0.02324$ $0.16320-0.03415$ $0.17908-0.04758$ $0.19234-0.06344$ 
$\beta \mathrm{h}$

0.01

0.02

0.0

0.10

0.20

1.50

1.00

1.50

2.00

2.50
3.00

3.50

$0.125 \pi$ $0.250 \pi$

$0.500 \pi$

$0.625 \pi$

$0.750 \pi$

$.000 \pi$

$1.250 \pi$
$\mathrm{C}(\mathrm{h}, 0)$

$0.00046-0.00000$ $0.00093-0.00000$ $0.00232-0.00000$ $0.00464-0.00000$ $0.00924-0.00000$ $0.03912-0.00004$ $0.04637 \quad 0.00007$ $0.04231 \quad 0.00069$ $0.02798 \quad 0.00228$ $\begin{array}{ll}-0.01542 & 0.00878\end{array}$

$0.01779-0.00001$ $0.03288-0.00003$ $-0.00003$ $0.04649 \quad 0.00012$ $0.04298 \quad 0.00052$ $\begin{array}{ll}0.03298 & 0.00170\end{array}$ 0.00352 $\begin{array}{ll}0.02229-0.00001 \\ 0.03912 & -0.00004\end{array}$ $0.00700 \quad 0.00507$ $0.00055 \quad 0.00606$ $\begin{array}{ll}-0.01687 & 0.00905 \\ -0.03155 & 0.01195\end{array}$
$S(h, 0)$

$0.00000-0.00000$ $0.00023-0.00000$ $0.00093-0.00001$ $0.00093-0.00001$ $0.02137-0.00034$ $0.02137-0.00034$ $0.06574-0.00201$ $0.06574-0.002010$ $0.08331-0.00310$ $0.09001-0.00235$

$0.00354-0.00004$ $0.01362-0.00019$ $\begin{array}{ll}0.01362 & -0.00019 \\ 0.02869 & -0.00052\end{array}$ $0.04645-0.00111$ $0.06419-0.00193$ $0.07921-0.00282$ $0.07921-0.00282$ $0.00280-0.00341$ $0.08945-0.00218$ $\begin{array}{rr}0.08945 & -0.00218 \\ 0.07983 & 0.00057\end{array}$ $0.00001-0.00000$ $0.00006-0.00000$

$$
\frac{a}{\lambda}=5.0 \quad \frac{\alpha}{\beta}=0.01 \text { 、 }
$$

$E(h, C)$

$0.00046-0.00000$ $0.00093-0.00000$

$0.00232-0.00000$

$0.00930-0.00000$

$0.02325-0.00003$

$0.04649-0.00025$

$0.04649-0.00025$

$0.09288-0.00087$

$0.11597-0.00384$

$0.13894-0.00662$

$0.16169-0.01048$

$0.01826-0.00001$

$0.03651-0.00012$

$0.05476 \quad-0.00040$

$0.07299-0.00095$

$0.09119-0.00186$

$0.10934-5.00322$

$0.12742-i) .00510$

$0.14540-0.00759$

$\begin{array}{ll}0.16325 & -0.01079 \\ 0.18090 & -0.01475\end{array}$
$C(h, h)$

$0.00046-0.00300$ $0.05093-0.00300$ $0.00232-0.00100$ $0.00464-0.00000$ $0.00924-0.0000$ $0.02229-0.00010$ $0.03925-0.00166$ $0.04214-0.00199$ $0.02815-0.00058$ $0.02815-0.0005$

$-0.01082$

$0.01779-0.00025$ $0.03286-0.00035$ $0.04289-0.000 .77$ $0.04636-0.00170$ $0.04281-0.0020$ $0.03298-0.00125$ $874 \quad 0.00113$ $199 \quad 0.01072$ $-0.02298 \quad 0.01638$ $0.00277 \quad 0.00527$
$S(h, h)$

$0.00000-0.00000$ $0.00001-0.00000$ $0.00006-0.00000$ $0.00023-0.00000$ $\begin{array}{ll}0.00093 & -0.00001\end{array}$ $0.00569-0.00009$ $0.02134-0.00068$ $0.04299-0.00251$ $0.06508-0.0061$
0.0818 $\begin{array}{ll}0.08188 & -0.01115\end{array}$ $0.08919-0.01617$ $0.08577-0.01906$

$0.00354-0.00005$ $0.01361-0.00032$ $0.02863-0.00115$ $0.04623-0.00291$
$0.06359-0.00599$ $0.06359-0.00579$
$0.07789-0.00962$ $0.07789-0.00962$ $\begin{array}{ll}0.08687-0.01378 \\ 0.086928 & -0.01730\end{array}$ $0.08928-0.01730$ $0.08518-0.01914$ $0.07598-0.01857$
$E(h, h)$ $0.00093-0.00000$ $0.00232-0.00000$ $0.00465-0.00000$ $0.00930-0.00001$ $0.02324-0.00012$ $\begin{array}{ll}0.00098 & -0.00098\end{array}$ $0.06947-0.0033$ $0.09208-0.00779$ $0.11382-0.01504$
$0.13402-0.02553$ $0.13402-0.02553$ $0.15177-0.03949$ $0.03649-0.00048$ $0.05467-0.00161$ 0.00380
$0.07270-0.0038$ $0.09045-0.00738$ $0.10769-0.0126$ $0.12412-0.01984$ $0.15287-0.04057$ $0.16415-0.05406$
$0.00046-0.00000$

$0.01826-0.00008$

$\begin{array}{lrrrr}0.01 & 0.00034 & -0.00000 & 0.00000 & -0.00000 \\ 0.02 & 0.00068 & -0.00000 & 0.00001 & -0.00000 \\ 0.05 & 0.00170 & 0.00000 & 0.00004 & -0.00000 \\ 0.10 & 0.00339 & 0.00000 & 0.00017 & -0.00000 \\ 0.20 & 0.00675 & 0.00000 & 0.00068 & -0.00001 \\ 0.50 & 0.01628 & 0.00001 & 0.00416 & -0.00009 \\ 1.00 & 0.02858 & 0.00008 & 0.01561 & -0.00038 \\ 1.50 & 0.03388 & 0.00036 & 0.03153 & -0.00091 \\ 2.00 & 0.03092 & 0.00110 & 0.04802 & -0.00161 \\ 2.50 & 0.02048 & 0.00254 & 0.06105 & -0.00216 \\ 3.00 & 0.00518 & 0.00476 & 0.06750 & -0.00206 \\ 3.50 & -0.01121 & 0.00740 & 0.06590 & -0.00066 \\ & & & & \\ 0.125 \pi & 0.01300 & 0.00000 & 0.00259 & -0.00005 \\ 0.250 \pi & 0.02401 & 0.00003 & 0.00994 & -0.00023 \\ 0.375 \pi & 0.03137 & 0.00014 & 0.02095 & -0.00054 \\ 0.500 \pi & 0.03397 & 0.00043 & 0.03393 & -0.00100 \\ 0.625 \pi & 0.03141 & 0.00102 & 0.04689 & -0.00155 \\ 0.750 \pi & 0.02413 & 0.00205 & 0.05787 & -0.00204 \\ 0.875 \pi & 0.01325 & 0.00356 & 0.06522 & -0.00223 \\ 1.000 \pi & 0.00047 & 0.00549 & 0.06787 & -0.00182 \\ 1.125 \pi & -0.01227 & 0.00759 & 0.06550 & -0.00050 \\ 1.250 \pi & -0.02305 & 0.00944 & 0.05856 & 0.00190\end{array}$

$$
\frac{a}{\lambda}=5.0 \quad \frac{\alpha}{\beta}=0.02
$$

$0.00034-0.00000$ $\begin{array}{llll}0.00340 & -0.00000 & 0.00170 & -0.00000 \\ 0.00340 & -0.00000 & 0.00339 & -0.00000\end{array}$ $\begin{array}{llll}0.00679 & -0.00000 & 0.00339 & -0.00000\end{array}$ $\begin{array}{llll}0.01698 & -0.00002 & 0.01628 & -0.00006\end{array}$ $\begin{array}{llll}0.03395 & -0.00018 & 0.02855 & -0.00038\end{array}$ $\begin{array}{llll}0.05091 & -0.00061 & 0.03379 & -0.00085\end{array}$ $\begin{array}{llll}0.06782 & -0.00144 & 0.03082 & -0.00087\end{array}$ $\begin{array}{llll}0.08468 & -0.00280 & 0.02067 & 0.00043\end{array}$ $\begin{array}{llll}0.10143 & -0.00483 & 0.00643 & 0.0036\end{array}$ $0.11803-0.00764$

$0.01334-0.00001$ $0.02667-0.00009$ $0.03999-0.00029$ $0.05330-0.00070$ $0.06659-0.00136$ $0.07984-0.010235$ $0.10615-0.00554$ $0.11916-0.007 .87$ $0.13202-0.01076$ $0.09303-0.00372$ $\begin{array}{ll}-0.00767 & 0.00834\end{array}$

$0.01299-0.00003$ $0.03133-0.00055$ $0.03387-0.00090$ $0.03130-0.00090$ $0.02417-0.00012$ $\begin{array}{lll}0.01384 & 0.00177\end{array}$ $0.00223 \quad 0.00484$ $\begin{array}{ll}-0.00852 & 0.0086\end{array}$ $-0.01661 \quad 0.0125$ $0.02400-0.00020$
$0.00000-0.00000$ $\begin{array}{ll}0.00000 & -0.00000 \\ 0.00001 & -0.00000\end{array}$ $0.00004-0.00000$ $0.00017-0.0000$ $0.00068-0.0000$ $0.00416-0.00010$ $0.00415-0.00010$ $0.03138-0.00203$ $0.03738-0.00203$ $0.04750-0.00460$ $0.05978-0.00803$ $\begin{array}{ll}0.06519 & -0.01129 \\ 0.06286 & -0.01294\end{array}$

$0.00258-0.00006$ $0.00994-0.00032$ $0.02090-0.00100$ $0.03374-0.00232$ $0.04641-0.00437$ $0.05685-0.00700$ $0.06345-0.00976$ $0.06529-0.01198$ $0.06244-0.01296$ $0.05590-0.01226$
$0.00034-0.00000$ $0.00068-0.00000$ $0.00170-0.00000$ $0.00340-0.00000$ $0.00679-0.0000$ $0.01698-0.00009$ $0.03391-0.00072$ $0.05072-0.0024$ $0.06720-0.00568$ $0.08303-0.01096$ $0.09771-0.0185$ $0.11059-0.02871$

$0.01333-0.00004$ $0.02665-0.00035$ $0.03992-0.00117$ $0.05307-0.00277$ $0.06601-0.00538$ $0.07857-0.00921$ $0.09052-0.01445$ $0.10157-0.02120$ $0.11138-0.02950$ $0.11955-0.03927$ 
$\frac{a}{\lambda}=5.0 \quad \frac{\alpha}{\beta}=0.03$

$\beta \mathrm{h}$

$C(h, 0)$

$S(h, 0)$

$E(h, 0)$

$\mathrm{C}(\mathrm{h}, \mathrm{h})$

$S(h, h)$

$E(h, h)$

$0.00000-0.00000$

$0.00500-10.00000$

$0.00003-0.00000$

$0.00012-0.00000$

$0.00049-0.00001$

$0.00304-0.00009$

$0.02303-0.00081$

$0.03508-0.00127$

$0.04462-0.00151$

$\begin{array}{rr}0.04937 & -0.00112 \\ 0.04827 & 0.00029\end{array}$

$0.01499 \quad 0.00250$

0.00250
0.00424

0.00613

3.50

$0.125 \pi$

0.375

$0.500 \pi$

$0.625 \pi$

$0.750 \pi$

$1.000 \pi$

$1.125 \pi$

$-0.00817$

0.00001

0.00006

$0.02292 \quad 0.00022$

$0.02482 \quad 0.00056$

$0.02296 \quad 0.00116$

$0.00971 \quad 0.00332$

$0.00038 \quad 0.00478$

$1.250 \pi$

$\begin{array}{lll}0.00189 & -0.00006\end{array}$

$0.00726-0.00023$

$0.01530-0.00051$

$\begin{array}{ll}0.02478 & -0.00087\end{array}$

$0.03425-0.00124$

$0.04228-0.00148$

$0.04768-0.00142$

$0.04966-0.00084$

$0.04297 \quad 0.00248$
$0.00025 \quad-0.00000 \quad 0.00025-0.00000$

$0.00050-10.00000$

$0.00124-0.00000$

$0.00248-0.00000$

$0.00496-0.00000$

$0.01240-0.00002$

$0.02480-0.00013$

$0.04953-0.00105$

$0.06183-0.00205$

$\begin{array}{ll}0.08615 & -0.00558\end{array}$

$0.00974-0.00001$

$0.01948-0.00006$

$0.02921-0.00021$

$0.03893-0.00051$

$0.04863-0.00099$

$0.05830-0.00171$

$0.07749-0.00404$

$0.08698-0.00574$

$0.09635-0.00785$
$0.00000-0.00000$

$0.00000-0.00000$

$0.00003-0.00000$

$0.00012-0.00000$

$0.00049-0.00002$

$0.00304-0.00011$

$0.01133-0.00055$

$0.02291-0.00162$

$0.03467-0.00345$

$0.04365-0.00579$

$0.04766-0.00787$

$0.04608-0.00874$

$0.00189-0.00006$

$0.00726-0.00030$

$0.01526-0.00085$

$0.02463-0.00183$

$0.03387-0.00330$

$0.04151-0.00510$

$0.04635-0.00692$

$0.04777-0.00828$

$0.04578-0.00873$

$0.04112-0.00801$
$0.01140-0.00037$

$0.07405-0.00352$
$0.00050-0.00000$

$0.00124-0.00000$

$0.00248-0.00000$

$0.00493-0.00000$

$0.01189-0.00003$

$0.02085-0.00020$

$0.02468-0.00040$

$0.02254-0.0002$

$\begin{array}{lll}0.01517 & 0.00094\end{array}$

$\begin{array}{rr}0.00483 & 0.00336 \\ -0.00545 & 0.00674\end{array}$

$0.00949-0.00002$

$0.01753-0.00011$

$0.02288-0.00029$

$0.02474-0.0004$

$0.02289-0.00025$

$0.01772 \quad 0.00048$

$0.01021 \quad 0.00199$

$0.00177 \quad 0.00425$

$\begin{array}{ll}-0.01203 & 0.00961\end{array}$
$0.00025-0.00000$ $0.00050-0.00000$ $0.00124-0.00000$ $0.00248-0.00000$ $0.00496-0.00000$ $0.01240-0.00007$ $0.02476-0.00052$ $0.03703-0.00176$ $0.04904-0.00414$ $0.06057-0.00799$ $0.07124-0.01353$ $0.08058 \quad-0.02088$

$0.00974-0.00003$ $0.01946-0.00025$ $0.02915-0.00086$ $0.03875-0.00202$ $0.04818-0.00392$ $0.05732-0.00671$ $0.06601-0.01052$ $0.07404-0.01543$ $0.08116-0.02144$

$$
\frac{a}{\lambda}=5 . C \quad \frac{\alpha}{\beta}=0.05
$$

$\begin{array}{lrrrr}0.01 & 0.00013 & -0.00000 & 0.00000 & -0.00000 \\ 0.02 & 0.00026 & 0.00000 & 0.00000 & -0.00000 \\ 0.05 & 0.00066 & 0.00000 & 0.00002 & -0.00000 \\ 0.10 & 0.00132 & 0.00000 & 0.00007 & -0.00000 \\ 0.20 & 0.00263 & 0.00000 & 0.00026 & -0.00001 \\ 0.50 & 0.00634 & 0.00002 & 0.00162 & -0.00008 \\ 1.00 & 0.01114 & 0.00015 & 0.00608 & -0.00030 \\ 1.50 & 0.01321 & 0.00049 & 0.01229 & -0.00058 \\ 2.00 & 0.01207 & 0.00112 & 0.01873 & -0.00079 \\ 2.50 & 0.00802 & 0.00202 & 0.02385 & -0.00073 \\ 3.00 & 0.00206 & 0.00309 & 0.02643 & -0.00019 \\ 3.50 & -0.00438 & 0.00405 & 0.02591 & 0.00097 \\ 0.125 \pi & 0.00506 & 0.00001 & 0.00101 & -0.00005 \\ 0.250 \pi & 0.00936 & 0.00007 & 0.00388 & -0.00019 \\ 0.375 \pi & 0.01223 & 0.00024 & 0.00817 & -0.00040 \\ 0.500 \pi & 0.01325 & 0.00056 & 0.01323 & -0.00002 \\ 0.625 \pi & 0.01226 & 0.00106 & 0.01829 & -0.00078 \\ 0.750 \pi & 0.00944 & 0.00174 & 0.02259 & -0.00078 \\ 0.875 \pi & 0.00521 & 0.00254 & 0.02550 & -0.00053 \\ 1.000 \pi & 0.00021 & 0.00338 & 0.02660 & 0.00007 \\ 1.125 \pi & -0.00480 & 0.00411 & 0.02576 & 0.00103 \\ 1.250 \pi & -0.00911 & 0.00452 & 0.02314 & 0.000248\end{array}$

$\begin{array}{ll}0.00013 & -0.00000 \\ 0.00026 & -0.00000 \\ 0.00066 & -0.00000 \\ 0.00132 & -0.00000 \\ 0.00265 & -0.00000 \\ 0.00662 & -0.00001 \\ 0.01323 & -0.00007 \\ 0.01983 & -0.00024 \\ 0.02641 & -0.00056 \\ 0.03296 & -0.00109 \\ 0.03947 & -0.00188 \\ 0.04590 & -0.00297 \\ 0.00520 & -0.00000 \\ 0.01039 & -0.00003 \\ 0.01558 & -0.00011 \\ 0.02076 & -0.00027 \\ 0.02593 & -0.00053 \\ 0.03108 & -0.00091 \\ 0.03621 & -0.00145 \\ 0.04130 & -0.00215 \\ 0.04634 & -0.00306 \\ 0.05132 & -0.00417\end{array}$

$0.00013-0.00000$ $0.00026 \quad 0.00000$ $0.00066-0.00000$ $0.00132-0.00000$ $0.00132-0.00000$ $0.00634-0.00001$ $0.00634-0.0000$ $0.01310-0.00003$ 0.0131050 .00002 0.012050 .00034 0.002690 .00257 $-0.002690 .00257$

$0.00506-0.00000$ $0.00935-0.00002$ $0.01221-0.00003$ 0.013210 .00004 0.012240 .00030 $0.00554 \quad 0.00180$ $0.00554 \quad 0.00180$ $\begin{array}{ll}-0.00313 & 0.00441 \\ -0.00637 & 0.00561\end{array}$ $0.00107 \quad 0.00304$
$0.00000-0.00000$ $0.00000-0.00000$ $0.00002-0.00000$ $0.00007-0.00000$ $0.00026-0.00001$ $0.00162-0.00009$ $0.00607-0.00040$ $0.01221-0.00101$ $0.01848-0.00194$ $0.02329-0.00300$ $0.02550-0.00380$ $0.02477-0.00391$

$0.00101-0.00005$ $0.00387-0.00023$ $0.00814-0.00058$ $0.01313-0.00113$ $0.01806 .-0.00187$ $0.02214 \quad-0.0027$ $0.02476-0.00346$ $0.02558-0.00392$ $\begin{array}{ll}0.02463 & -0.00388 \\ 0.02225 & -0.00327\end{array}$

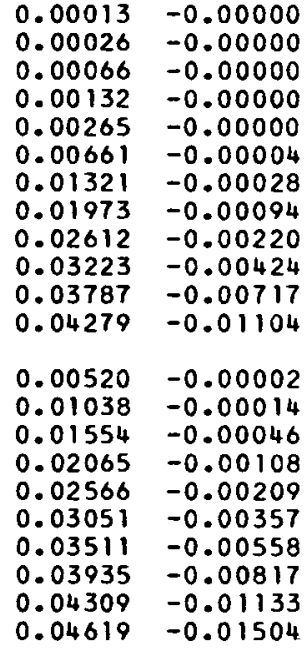


Bh

$\mathrm{C}(\mathrm{h}, 0)$

$\begin{array}{lll}0.01 & 0.00007 & 0.00000 \\ 0.02 & 0.00014 & 0.00000 \\ 0.05 & 0.00035 & 0.00000 \\ 0.10 & 0.00070 & 0.00000 \\ 0.20 & 0.00140 & 0.00000 \\ 0.50 & 0.00339 & 0.00002 \\ 1.00 & 0.00594 & 0.00012 \\ 1.50 & 0.00705 & 0.00039 \\ 2.00 & 0.00645 & 0.00084 \\ 2.50 & 0.00429 & 0.00145 \\ 3.00 & 0.00109 & 0.00209 \\ 3.50 & -0.00238 & 0.00258\end{array}$

$\begin{array}{lll}0.125 \pi & 0.00270 & 0.00001\end{array}$

$0.250^{-} \pi \quad 0.00499$

$0.375 \pi \quad 0.00653$

$0.500 \pi \quad 0.00707$

$0.625 \pi \quad 0.00655$

$0.750 \pi \quad 0.00505$

$0.875 \pi \quad 0.00278$

$1.125 \pi-0.00260$

$1.125 \pi \quad-0.00260$

\begin{tabular}{ll}
$-0.00495 \quad 0.00272$ \\
\hline
\end{tabular}
$S(h, 0)$

$\begin{array}{ll}0.00000 & -0.00000 \\ 0.00000 & -0.00000 \\ 0.00001 & -0.00000 \\ 0.00004 & -0.00000 \\ 0.00014 & -0.00001 \\ 0.00086 & -0.00066 \\ 0.00325 & -0.00021 \\ 0.00656 & -0.00039 \\ 0.01001 & -0.00048 \\ 0.01276 & -0.00035 \\ 0.01417 & 0.00011 \\ 0.01392 & 0.00096 \\ 0.00054 & -0.00004 \\ 0.00207 & -0.00014 \\ 0.00436 & -0.00028 \\ 0.00706 & -0.00041 \\ 0.00977 & -0.00048 \\ 0.01208 & -0.00041 \\ 0.01365 & -0.00016 \\ 0.01427 & 0.00031 \\ 0.01384 & 0.00103 \\ 0.01246 & 0.00195\end{array}$

$$
\frac{a}{\lambda}=5 . c \quad \frac{\alpha}{\beta}=0.07
$$

$E(h, 0)$

$C(h, h)$

$S(h, h)$

$0.00007-0.30000$ $0.00014-0.00000$ $0.00035-0.00000$ $0.00071-0.00000$ $\begin{array}{ll}0.00141 & -0.00000 \\ 0.00353 & -0.00000\end{array}$ $0.00753-0.0000$ $\begin{array}{ll}0.00706 & -0.100004 \\ 0.01058 & -0.00013\end{array}$ $0.01408-0.00030$ $\begin{array}{ll}0.01408 & -0.00030 \\ 0.01757 & -0.00058\end{array}$ $\begin{array}{ll}0.01757 & -0.00058 \\ 0.02104 & -0.00100\end{array}$ $\begin{array}{ll}0.02104 & -0.00100 \\ 0.02446 & -0.00158\end{array}$ $0.00277-0.00000$ $0.00554-0.00002$ $0.00831-0.00006$ $0.01107-0.00014$ $0.01383-0.00028$ $0.01657-0.00049$ $0.07930-0.00077$ 0.02469 .0 .00163 $\begin{array}{ll}0.02469 & -0.00163 \\ 0.02733 & -0.00222\end{array}$

$0.00007-0.00000$ $0.00014 \quad 0.00000$ $0.00035 \quad 0.00000$ $0.00070 \quad 0.00000$ $0.00338 \quad 0.00000$ $0.00593 \quad 0.00003$ $0.00703 \quad 0.00014$ $0.00645 \quad 0.00042$ $0.00439 \quad 0.00098$ $0.00439 \quad 0.00098$ $-0.00145 \quad 0.00267$

$0.00270 \quad 0.00000$ $0.00499 \quad 0.00001$ $0.00651 \quad 0.00005$ 0.006540 .00039 $0.00510 \quad 0.00079$ $0.00300 \quad 0.00135$ 0.000620 .00204 $-0.001630 .00273$ $\begin{array}{ll}-0.00163 & 0.00273 \\ -0.00340 & 0.00326\end{array}$
$0.00000-0.00000$ $0.00000-0.00000$ $0.00001-0.00000$ $0.00004-0.00000$ $0.00014-0.00001$ $0.00086-0.00006$ $0.00324-0.00026$ $0.00651-0.00062$ $0.00986-0.00109$ $0.01244-0.00156$ $0.01366-0.00182$

$0.00054 \quad-0.00004$ $\begin{array}{ll}0.00206 & -0.00016 \\ 0.00434 & -0.00037\end{array}$ $0.00700-0.00068$ $0.00963-0.00106$ $0.01182-0.00144$ $0.01324-0.00173$ $\begin{array}{lll}0.01372 & -0.00183\end{array}$ $0.01326 \cdot-0.00166$ $0.01204,-0.00121$
$E(h, h)$

$0.00007-0.00000$ $0.00014-0.00000$ $0.00035-0.00000$ $0.00071-0.00000$ $0.000353-0.00000$ $\begin{array}{ll}0.00353 & -0.00002 \\ 0.00704 & -0.00015\end{array}$ $0.01052-0.00050$ $\begin{array}{ll}0.01052 & -0.00050 \\ 0.01391 & -0.00117\end{array}$ $0.01391-0.00117$ $0.01715 \quad-0.00225$ $\begin{array}{ll}0.02013 & -0.00380 \\ 0.02272 & -0.00583\end{array}$

$0.00277-0.00001$ $0.00554-0.00007$ $0.00829-0.00024$ $0.01100-0.00057$ $0.01367-0.00111$ $0.01867-0.00296$ $0.01867-0.00296$ $0.02091-0.00433$ $0.02450-0.00794$

$$
\frac{a}{\lambda}=5.0 \quad \frac{\alpha}{\beta}=0.10
$$

$\begin{array}{lrl}0.01 & 0.00003 & 0.00000 \\ 0.02 & 0.00006 & 0.00000 \\ 0.05 & 0.00014 & 0.00000 \\ 0.10 & 0.00027 & 0.00000 \\ 0.20 & 0.00055 & 0.00000 \\ 0.50 & 0.00132 & 0.00001 \\ 1.00 & 0.00232 & 0.00007 \\ 1.50 & 0.00275 & 0.00022 \\ 2.00 & 0.00252 & 0.00047 \\ 2.50 & 0.00167 & 0.00078 \\ 3.00 & 0.00041 & 0.00107 \\ 3.50 & -0.00097 & 0.00126 \\ & & \\ 0.125 \pi & 0.00105 & 0.00000 \\ 0.250 \pi & 0.00195 & 0.00004 \\ 0.375 \pi & 0.00255 & 0.00012 \\ 0.500 \pi & 0.00276 & 0.00025 \\ 0.625 \pi & 0.00256 & 0.00045 \\ 0.750 \pi & 0.00197 & 0.00069 \\ 0.875 \pi & 0.00108 & 0.00093 \\ 1.000 \pi & 0.00002 & 0.00114 \\ 1.125 \pi & -0.00106 & 0.00126 \\ 1.250 \pi & -0.00202 & 0.00125\end{array}$

$\begin{array}{ll}0.00000 & -0.00000 \\ 0.00000 & -0.00000 \\ 0.00000 & -0.00000 \\ 0.00001 & -0.00000 \\ 0.00005 & -0.00001 \\ 0.00034 & -0.00003 \\ 0.00127 & -0.00011 \\ 0.00256 & -0.00020 \\ 0.00391 & -0.00022 \\ 0.00500 & -0.00011 \\ 0.00557 & 0.00017 \\ 0.00549 & 0.00063 \\ 0.00021 & -0.00002 \\ 0.00081 & -0.00008 \\ 0.00170 & -0.00015 \\ 0.00276 & -0.00021 \\ 0.00382 & -0.00022 \\ 0.00473 & -0.00016 \\ 0.00536 & 0.00001 \\ 0.00562 & 0.00028 \\ 0.00546 & 0.00067 \\ 0.00493 & 0.00113\end{array}$
$0.00006-0.00000$ $0.00014-0.00000$ $0.00028-0.00000$ $0.00055-0.00000$ $0.00138-0.000 .00$ $0.00275-0.00001$ $0.00412-0.00005$ $0.00548-0.00012$ $0.00684-0.00023$ $0.00819-0.00039$ $0.00951-0.00061$

$0.00108-0.00000$ $0.00216-0.0000$ $0.00324-0.0000$ $0.00431-0.00006$ $0.00539-0.0001$ $0.00645-0.00019$ $0.00751-0.00030$ $0.00856 .-0.00045$ $0.00960-0.00063$ $0.01063-0.00086$
$0.00003-0.00000$ $0.00006 \quad 0.00000$ $0.00014 \quad 0.00000$ $0.00027 \quad 0.00000$ $0.00055 \quad 0.00000$ 0.001320 .00000 0.002310 .00004 $0.00274 \quad 0.00013$ $0.00252 \quad 0.00031$ $0.00173 \quad 0.00059$ $0.00060 \quad 0.00094$ $-0.00056$

0.00105 0.00194 0.00254

0.00275

0.00256

0.002 .00

0.00119

0.00026

$-0.00136$

$\begin{array}{ll}0.00000 & -0.00000 \\ 0.00000 & -0.00000 \\ 0.00000 & -0.00000 \\ 0.00001 & -0.00000 \\ 0.00005 & -0.00001 \\ 0.00034 & -0.00003 \\ 0.00126 & -0.00013 \\ 0.00254 & -0.00029 \\ 0.00385 & -0.00046 \\ 0.00486 & -0.00058 \\ 0.00536 & -0.00059 \\ 0.00527 & -0.00043 \\ & \\ 0.00021 & -0.00002 \\ 0.00080 & -0.00008 \\ 0.00169 & -0.00018 \\ 0.00273 & -0.00031 \\ 0.00376 & -0.00045 \\ 0.00462 & -0.00056 \\ 0.00519 & -0.00061 \\ 0.00539 & -0.00056 \\ 0.00524 & -0.00041 \\ 0.00479 & -0.00016\end{array}$

$0.00003-0.00000$ $0.00006-0.00000$ $0.00014-0.00000$ $0.00028-0.00000$ $0.00055-0.00000$ $0.00137-0.00001$ $0.00274-0.00006$ $0.00409-0.00019$ $0.00541-0.00045$ $0.00666-0.00087$ $0.00780-0.00147$ $0.00879-0.00224$

$0.00108-0.00000$ $0.00216-0.00003$ $0.00323-0.00009$ $0.00428-0.00022$ $0.00531-0.00043$ $0.00631-0.00073$ $0.00724-0.00114$ $0.00810-0.00167$ $0.00885-0.00230$ $0.00947-0.00304$ 


$$
\frac{a}{\lambda}=5.0 \quad \frac{\alpha}{\beta}=0.20
$$

$\beta h$

$C(h, 0)$

$\mathrm{S}(\mathrm{h}, 0)$

$\begin{array}{lrllr}0.01 & 0.00000 & 0.00000 & 0.00000 & -0.00000 \\ 0.02 & 0.00000 & 0.00000 & 0.00000 & -0.00000 \\ 0.05 & 0.00001 & 0.00000 & 0.00000 & -0.00000 \\ 0.10 & 0.00001 & 0.00000 & 0.00000 & -0.00000 \\ 0.20 & 0.00002 & 0.00000 & 0.00000 & -0.00000 \\ 0.50 & 0.00006 & 0.00000 & 0.00001 & -0.00000 \\ 1.00 & 0.00010 & 0.00001 & 0.00006 & -0.00001 \\ 1.50 & 0.00012 & 0.00002 & 0.00011 & -0.00002 \\ 2.00 & 0.00011 & 0.00004 & 0.00017 & -0.00001 \\ 2.50 & 0.00007 & 0.00007 & 0.00022 & -0.00000 \\ 3.00 & 0.00001 & 0.00009 & 0.00025 & 0.00003 \\ 3.50 & -0.00006 & 0.00009 & 0.00025 & 0.00007 \\ 0.125 \pi & 0.00005 & 0.00000 & 0.00001 & -0.00000 \\ 0.250 \pi & 0.00008 & 0.00000 & 0.00003 & -0.00001 \\ 0.375 \pi & 0.00011 & 0.00001 & 0.00007 & -0.00001 \\ 0.500 \pi & 0.00012 & 0.00002 & 0.00012 & -0.00002 \\ 0.625 \pi & 0.00011 & 0.00004 & 0.00017 & -0.00001 \\ 0.750 \pi & 0.00009 & 0.00006 & 0.00021 & -0.00001 \\ 0.875 \pi & 0.00004 & 0.00008 & 0.00024 & 0.00001 \\ 1.000 \pi & -0.00001 & 0.00009 & 0.00026 & 0.00004 \\ 1.125 \pi & -0.00006 & 0.00009 & 0.00025 & 0.00007 \\ 1.250 \pi & -0.00011 & 0.00008 & 0.00023 & 0.00011\end{array}$

$E(h, 0)$

$\begin{array}{ll}0.00000 & -0.00000 \\ 0.00000 & -0.00000 \\ 0.00001 & -0.00000 \\ 0.00001 & -0.00000 \\ 0.00002 & -0.00000 \\ 0.00006 & -0.00000 \\ 0.00012 & -0.00000 \\ 0.00018 & -0.00000 \\ 0.00024 & -0.00000 \\ 0.00029 & -0.00001 \\ 0.00035 & -0.00002 \\ 0.00041 & -0.00003 \\ 0.00005 & -0.00000 \\ 0.00009 & -0.00000 \\ 0.00014 & -0.00000 \\ 0.00019 & -0.00000 \\ 0.00023 & -0.00000 \\ 0.00028 & -0.00001 \\ 0.00032 & -0.00001 \\ 0.00037 & -0.00002 \\ 0.00041 & -0.00003 \\ 0.00046 & -0.00004\end{array}$

$\mathrm{C}(\mathrm{h}, \mathrm{h})$

$0.00000 \quad 0.00000$ $0.00000 \quad 0.00000$ $0.00001 \quad 0.00000$ $0.00002 \quad 0.00000$ $0.00006 \quad 0.00000$ $0.00010 \quad 0.00001$ 0.000120 .00002

0.000110 .00003

$0.00008 \quad 0.00006$

$\begin{array}{ll}0.00008 & 0.00006 \\ 0.00002 & 0.00008\end{array}$ $\begin{array}{rr}0.000003 & 0.00009\end{array}$

\section{$0.00005 \quad 0.00000$}

$0.00008 \quad 0.000$

0.000120 .00001

0.000110 .0002

$0.00009 \quad 0.00005$

$0.00005 \quad 0.00007$ $0.00001 \quad 0.00008$ $\begin{array}{ll}-0.00003 & 0.00009 \\ -0.00007 & 0.00009\end{array}$
$S(h, h)$

$0.00000,-0.00000$ (1) $0.00000-0.00000$ $0.00000-0.00000$ $0.00000-0.00000$ $0.00001-0.00000$ $0.00011-0.00001$ $0.00017-0.00002$ $0.00022-0.00002$ $0.00024-0.00002$ $\begin{array}{rr}0.00024 & -0.00001 \\ 0.00024 & 0.00002\end{array}$

$0.00001-0.00000$ $0.00003-0.00001$ $0.00007-0.00001$ $0.00012-0.00002$ $0.00016-0.00002$ $0.00020-0.00002$ $0.00023-0.00002$ $0.00024-0.00000$ $0.00024 \quad 0.00002$ $0.00022 \quad 0.00004$
$E(h, h)$

$0.00000-0.00000$ $0.00000-0.00000$ $0.00001-0.00000$ $0.00001-0.00000$ $0.00002-0.00000$ $0.00006-0.00000$ $0.00012-0.00000$ $0.00018-0.00001$ $0.00023-0.00002$ $0.00028-0.00004$ $\begin{array}{ll}0.00033 & -0.00006 \\ 0.00037 & -0.00009\end{array}$

$0.00005-0.00000$ $0.00009-0.00000$ $0.00014-0.00000$ $0.00018-0.00001$ $0.00023-0.00002$ $0.00027-0.00003$ $0.00031-0.00005$ $0.00034-0.00007$ $0.00037-0.00010$ $0.00040-0.00012$

$$
\frac{\bar{\epsilon}}{\lambda}=5.0 \quad \frac{\alpha}{\beta}=0.40
$$

\begin{tabular}{|c|c|c|c|c|c|}
\hline $\begin{array}{l}0.00000 \\
0.00000 \\
0.00000 \\
0.00000 \\
0.00000 \\
0.00000 \\
0.00000 \\
0.00000 \\
0.00000 \\
0.00000 \\
0.00000 \\
0.00000\end{array}$ & $\begin{array}{l}-0.00000 \\
-0.00000 \\
-0.00000 \\
-0.00000 \\
-0.00000 \\
-0.00000 \\
-0.00000 \\
-0.00000 \\
-0.00000 \\
-0.00000 \\
-0.00000 \\
-0.000000\end{array}$ & $\begin{array}{r}0.000000 \\
0.00000 \\
0.00000 \\
0.00000 \\
0.00000 \\
0.000000 \\
0.00000 \\
0.00000 \\
0.00000 \\
0.00000 \\
0.00000 \\
-0.00000\end{array}$ & $\begin{array}{l}0.00000 \\
0.00000 \\
0.00000 \\
0.00000 \\
0.00000 \\
0.00000 \\
0.00000 \\
0.00000 \\
0.00000 \\
0.00000 \\
0.00000 \\
0.00000\end{array}$ & $\begin{array}{l}0.00000 \\
0.00000 \\
0.00000 \\
0.00000 \\
0.00000 \\
0.00000 \\
0.00000 \\
0.00000 \\
0.00000 \\
0.00000 \\
0.00000 \\
0.00000\end{array}$ & $\begin{array}{r}-0.00000 \\
-0.00000 \\
-0.00000 \\
-0.00000 \\
-0.00000 \\
-0.00000 \\
-0.00000 \\
-0.00000 \\
-0.00000 \\
-0.00000 \\
0.00000 \\
0.00000\end{array}$ \\
\hline $\begin{array}{l}0.00000 \\
0.00000 \\
0.00000 \\
0.00000 \\
0.00000 \\
0.00000 \\
0.00000 \\
0.00000 \\
0.00000 \\
0.00000\end{array}$ & $\begin{array}{l}-0.00000 \\
-0.00000 \\
-0.00000 \\
-0.00000 \\
-0.00000 \\
-0.00000 \\
-0.00000 \\
-0.00000 \\
-0.00000 \\
-0.00000\end{array}$ & $\begin{array}{r}0.00000 \\
0.00000 \\
0.00000 \\
0.00000 \\
0.00000 \\
0.00000 \\
0.00000 \\
-0.00000 \\
-0.00000 \\
-0.00000\end{array}$ & $\begin{array}{l}0.00000 \\
0.00000 \\
0.00000 \\
0.00000 \\
0.00000 \\
0.00000 \\
0.00000 \\
0.00000 \\
0.00000 \\
0.00000\end{array}$ & $\begin{array}{l}0.00000 \\
0.00000 \\
0.00000 \\
0.00000 \\
0.00000 \\
0.00000 \\
0.00000 \\
0.00000 \\
0.00000 \\
0.00000\end{array}$ & $\begin{array}{r}-0.00000 \\
-0.00000 \\
-0.00000 \\
-0.00000 \\
-0.00000 \\
-0.00000 \\
0.00000 \\
0.00000 \\
0.00000 \\
0.00000\end{array}$ \\
\hline
\end{tabular}

$\begin{array}{lrllr}0.01 & 0.00000 & 0.00000 & 0.00000 & -0.00000 \\ 0.02 & 0.00000 & 0.00000 & 0.00000 & -0.00000 \\ 0.05 & 0.00000 & 0.00000 & 0.00000 & -0.00000 \\ 0.10 & 0.00000 & 0.00000 & 0.00000 & -0.00000 \\ 0.20 & 0.00000 & 0.00000 & 0.00000 & -0.00000 \\ 0.50 & 0.00000 & 0.00000 & 0.00000 & -0.00000 \\ 1.00 & 0.00000 & 0.00000 & 0.00000 & -0.00000 \\ 1.50 & 0.00000 & 0.00000 & 0.00000 & -0.00000 \\ 2.00 & 0.00000 & 0.00000 & 0.00000 & -0.00000 \\ 2.50 & 0.00000 & 0.00000 & 0.00000 & 0.00000 \\ 3.00 & -0.00000 & 0.00000 & 0.00000 & 0.00000 \\ 3.50 & -0.00000 & 0.00000 & 0.00000 & 0.00000 \\ & & & & \\ 0.125 \pi & 0.00000 & 0.00000 & 0.00000 & -0.00000 \\ 0.250 \pi & 0.00000 & 0.00000 & 0.00000 & -0.00000 \\ 0.375 \pi & 0.00000 & 0.00000 & 0.00000 & -0.00000 \\ 0.500 \pi & 0.00000 & 0.00000 & 0.00000 & -0.00000 \\ 0.625 \pi & 0.00000 & 0.00000 & 0.00000 & -0.00000 \\ 0.750 \pi & 0.00000 & 0.00000 & 0.00000 & -0.00000 \\ 0.875 \pi & 0.00000 & 0.00000 & 0.00000 & 0.00000 \\ 1.000 \pi & -0.00000 & 0.00000 & 0.00000 & 0.00000 \\ 1.125 \pi & -0.00000 & 0.00000 & 0.00000 & 0.00000 \\ 1.250 \pi & -0.000 .00 & 0.00000 & 0.00000 & 0.00000\end{array}$

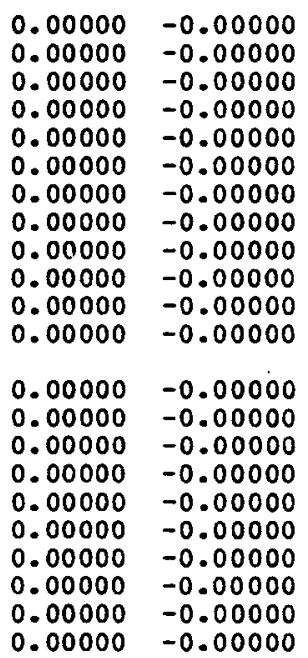


$\beta \mathrm{h}$

$C(h, 0)$

$\begin{array}{lrr}0.01 & 0.00000 & 0.00000 \\ 0.02 & 0.00000 & 0.00000 \\ 0.05 & 0.00000 & 0.00000 \\ 0.10 & 0.00000 & 0.00000 \\ 0.20 & 0.00000 & 0.00000 \\ 0.50 & 0.00000 & 0.00000 \\ 1.00 & 0.00000 & 0.00000 \\ 1.50 & 0.00000 & 0.00000 \\ 2.00 & -0.00000 & 0.00000 \\ 2.50 & -0.00000 & 0.00000 \\ 3.00 & -0.00000 & -0.00000 \\ 3.50 & -0.00000 & -0.00000 \\ & & \\ 0.125 \pi & 0.00000 & 0.00000 \\ 0.250 \pi & 0.00000 & 0.00000 \\ 0.375 \pi & 0.00000 & 0.00000 \\ 0.500 \pi & 0.00000 & 0.00000 \\ 0.625 \pi & -0.00000 & 0.00000 \\ 0.750 \pi & -0.00000 & 0.00000 \\ 0.875 \pi & -0.00000 & -0.00000 \\ 1.000 \pi & -0.00000 & -0.00000 \\ 1.125 \pi & -0.00000 & -0.00000 \\ 1.250 \pi & -0.00000 & -0.00000 \\ -1 & & \end{array}$

$S(h, 0)$

$0.00000-0.00000$ $0.00000-0.00000$ $0.00000-0.00000$ $0.00000-0.00000$ $0.00000-0.00000$ $0.00000-0.00000$ $0.00000-0.00000$ $0.00000-0.00000$ $0.00000 \quad 0.00000$ $0.00000 \quad 0.00000$ 0.000000 .0000 $-0.00000 \quad 0.00000$

$0.00000-0.00000$ $0.00000-0.00000$ $0.00000-0.00000$ $0.00000 \quad 0.00000$ $0.00000 \quad 0.00000$ $0.00000 \quad 0.00000$ $0.00000=0.00000$ $-0.00000-0.00000$ $\begin{array}{ll}-0.000000 & 0.00000 \\ -0.00000 & 0.00000\end{array}$ $\frac{\mathrm{a}}{\lambda}=5.0 \quad \frac{\alpha}{\beta}=0.70$

$E(h, 0)$

$C(h, h)$

$S(h, h)$

$E(h, h)$

$0.00000-0.00000$

$0.00000-0.00000$

$0.00000-0.00000$

$0.00000-0.50000$

$0.00000-0.00000$

$0.00000-0.00000$

$0.00000-0.00000$

$0.00000-0.0000$

$0.00000-0.30000$

$0.00000-0.30000$

$0.00000-0.130000$

$0.00000-0.00000$

$0.00000-0.00000$

$0.00000-0.00000$

$0.00000-0.00000$

$0.00000-0.00000$

$0.00000-0.00000$

$0.00000-0.00000$

$0.00000-0.00000$

$\begin{array}{ll}0.00000 & -0.00000 \\ 0.00000 & -0.00000\end{array}$
$0.00000-0.00000$ $0.00000-0.00000$ $0.00000-0.00000$ $0.00000-0.00000$ $0.00000-0.00000$ $0.00000-0.00000$ $0.00000-0.00000$ $0.00000 \quad 0.00000$ $0.00000 \quad 0.00000$ $-0.00000 \quad 0.00000$ $0.00000-0.00000$ $0.00000-0.00000$ $0.00000-0.00000$ $0.00000-0.00000$ $0.00000 \quad 0.00000$ $0.00000 \quad 0.00000$ $0.00000 \quad 0.00000$ $-0.00000 \quad 0.00000$ $-0.00000 \quad 0.00000$ $-0.00000 \quad 0.00000$ $0.00000-0.00000$ $\begin{array}{ll}0.00000 & -0.00000 \\ 0.00000 & -0.00000 \\ 0.00000 & -0.00000 \\ 0.00000 & -0.00000 \\ 0.00000 & -0.00000 \\ 0.00000 & -0.00000 \\ 0.00000 & -0.00000 \\ 0.00000 & -0.00000 \\ 0.00000 & -0.00000 \\ 0.00000 & -0.00000 \\ 0.00000 & -0.00000 \\ 0.00000 & -0.00000 \\ & \\ 0.00000 & -0.00000 \\ 0.00000 & -0.00000 \\ 0.00000 & -0.00000 \\ 0.00000 & -0.00000 \\ 0.00000 & -0.00000 \\ 0.00000 & -0.00000 \\ 0.00000 & -0.00000 \\ 0.00000 & -0.00000 \\ 0.00000 & -0.00000 \\ 0.00000 & -0.00000\end{array}$

$$
\frac{\mathrm{a}}{\lambda}=5.0 \quad \frac{\alpha}{\beta}=1.00
$$

$0.00000-0.00000$

$0.00000-0.00000$

$0.00000-0.00000$

$0.00000-0.00000$

$0.00000-0.00000$

$0.00000-0.00000$

$0.00000-0.00000$

$0.00000-0.00000$

$0.00000-0.0 .0000$

$0.00000-0.00000$

$0.00000-0.00000$

$0.00000-0.00000$

$0.00000-0.00000$

$0.00000-0.00000$

$0.00000-0.00000$

$0.00000-0.00000$

$0.00000-0.03000$

$0.00000-0.03000$

$0.00000-0.03000$

$0.00000-0.0 .3000$

$0.00000-0.02000$

$0.00000-0.00000$
$0.00000 \quad 0.00000$

$0.00000 \quad 0.00000$

$0.00000 \quad 0.00000$

$\begin{array}{ll}0.00000 & 0.00000 \\ 0.00000 & 0.00000\end{array}$

$0.00000 \quad 0.00000$

$0.00000 \quad 0.00000$

$0.00000 \quad 0.00000$

$\begin{array}{ll}-0.00000 & 0.00000\end{array}$

$\begin{array}{ll}-0.00000 & 0.00000\end{array}$

$\begin{array}{ll}-0.00000 & 0.00000\end{array}$

$0.00000 \quad 0.00000$

$0.00000 \quad 0.00000$

$0.00000 \quad 0.00000$

$-0.00000 \quad 0.00000$

$-0.00000 \quad 0.00000$

$-0.00000 \quad 0.00000$

$-0.00000 \quad 0.00000$

$-0.00000 \quad 0.00000$

$\begin{array}{ll}-0.00000 & 0.00000\end{array}$
$0.00000-0.00000$

$0.00000-0.00000$

$0.00000-0.00000$

$0.00000-0.00000$

$0.00000-0.00000$

$0.00000-0.00000$

$0.00000-0.00000$

$0.00000-0.00000$

$0.00000 \quad 0.00000$

$0.00000 \quad 0.00000$

$0.00000 \quad 0.00000$

$0.00000-0.00000$

$0.00000-0.00000$

$0.00000-0.00000$

$0.00000-0.00000$

$0.00000 \quad 0.00000$

$0.00000 \quad 0.00000$

$0.00000 \quad 0.00000$

$0.00000 \quad 0.00000$

$0.00000 \quad 0.00000$

0.00000 $\begin{array}{ll}0.00000 & -0.00000 \\ 0.00000 & -0.00000 \\ 0.00000 & -0.00000 \\ 0.00000 & -0.00000 \\ 0.00000 & -0.00000 \\ 0.00000 & -0.00000 \\ 0.00000 & -0.00000 \\ 0.00000 & -0.00000 \\ 0.00000 & -0.00000 \\ 0.00000 & -0.00000 \\ 0.00000 & -0.00000 \\ 0.00000 & -0.00000 \\ & \\ 0.00000 & -0.00000 \\ 0.00000 & -0.00000 \\ 0.00000 & -0.00000 \\ 0.00000 & -0.00000 \\ 0.00000 & -0.00000 \\ 0.00000 & -0.00000 \\ 0.00000 & -0.00000 \\ 0.00000 & -0.00000 \\ 0.00000 & -0.00000 \\ 0.00000 & -0.00000\end{array}$ 